Éditeurs scientifiques

C. Raimond, F. Sylvestre

D. Zakinet, A. Moussa

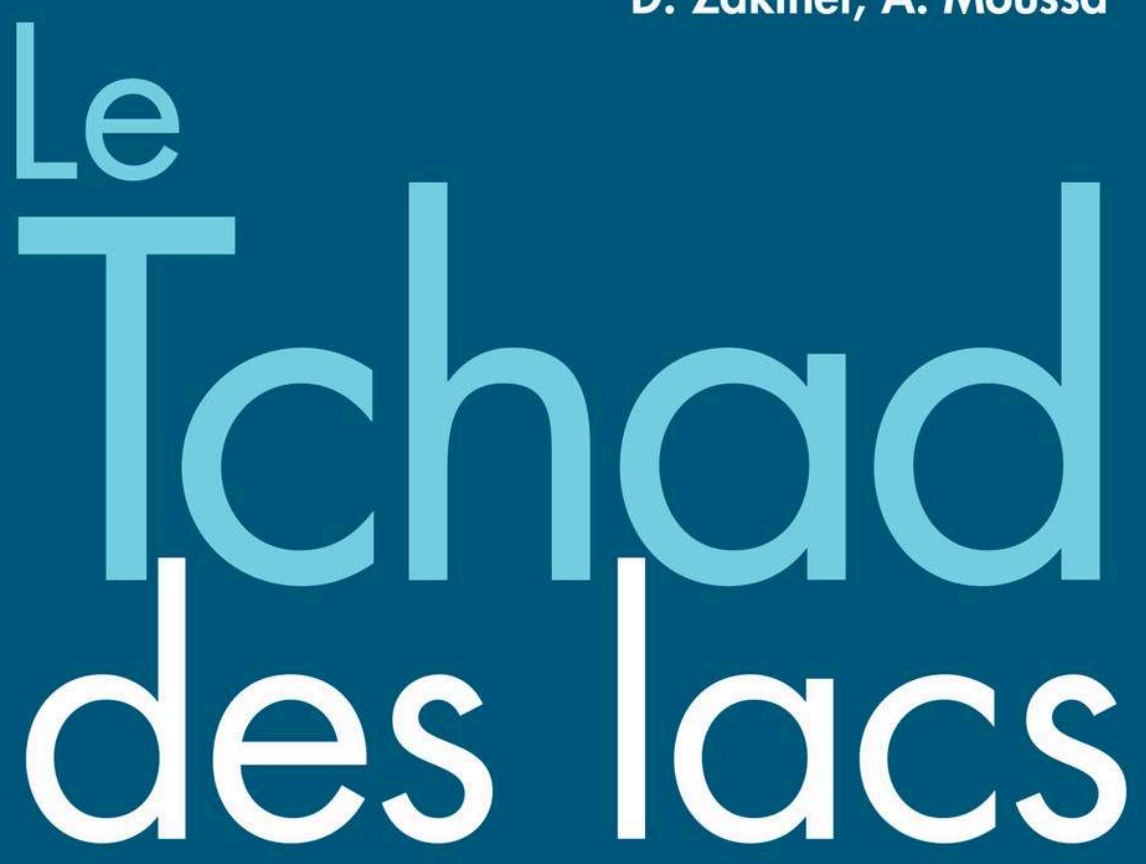

Les zones humides sahéliennes au défi du changement global

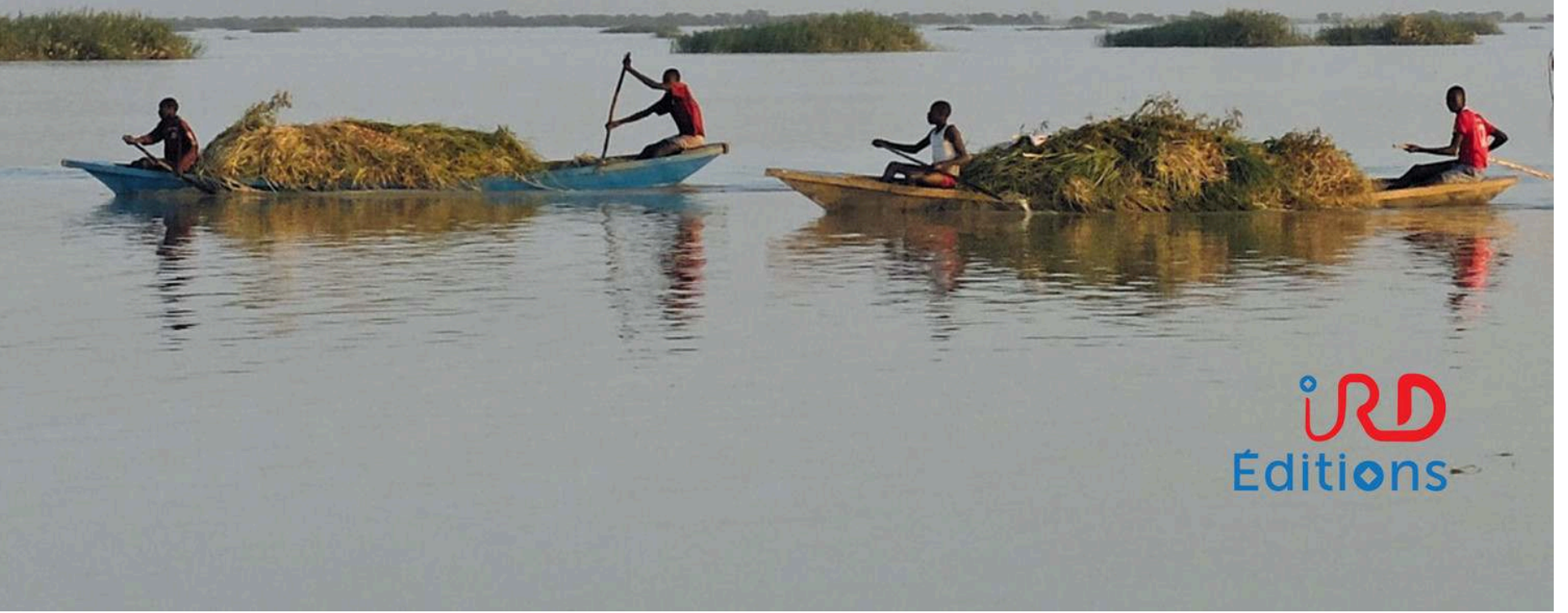




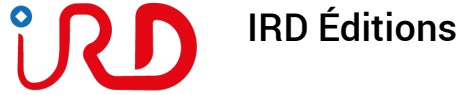 \\ Éditions}

\section{Le Tchad des lacs}

Les zones humides sahéliennes au défi du changement global

Christine Raimond, Florence Sylvestre, Dangbet Zakinet et Abderamane Moussa (éd.)

DOI : 10.4000/books.irdeditions.30450

Éditeur : IRD Éditions

Lieu d'édition : Marseille

Année d'édition : 2019

Date de mise en ligne : 29 août 2019

Collection : Synthèses

EAN électronique : 9782709927161

\section{OpenEdition}

\section{Books}

https://books.openedition.org

\section{Édition imprimée}

Date de publication : 1 juin 2019

EAN (Édition imprimée) : 9782709927154

Nombre de pages : 368

\section{Référence électronique}

RAIMOND, Christine (dir.) ; et al. Le Tchad des lacs : Les zones humides sahéliennes au défi du changement global. Nouvelle édition [en ligne]. Marseille : IRD Éditions, 2019 (généré le 29 janvier 2022). Disponible sur Internet : <http://books.openedition.org/irdeditions/30450>. ISBN 9782709927161. DOI : https://doi.org/10.4000/books. irdeditions.30450

Crédits de couverture

(c) CNRS/M. Schuster

(c) IRD Éditions, 2019

Conditions d'utilisation :

http://www.openedition.org/6540 


\section{RÉSUMÉS}

Reconnues comme hauts lieux de biodiversité (hot spots), les zones humides africaines sont sources de nombreux services écosystémiques. Les sociétés y mènent des activités de subsistance (pêche, agriculture, élevage, chasse, cueillette) intégrées au fonctionnement des écosystèmes. De plus en plus sollicités tout en restant très vulnérables, ces territoires jouent désormais un rôle moteur dans les économies locales et régionales, en exportant une large diversité de produits vers les villes. Or en ce début $\mathrm{du} \mathrm{XXI}^{\mathrm{e}}$ siècle, ils cumulent les défis de l'Anthropocène changement climatique, croissance démographique, urbanisation, mondialisation des échanges et ceux d'une crise profonde de gouvernance. Leur étude est donc devenue un enjeu scientifique et sociétal important.

En proposant plusieurs angles de lecture, cet ouvrage collectif contribue à une meilleure connaissance des zones humides sahéliennes à partir de l'étude des lacs du Tchad. Il articule les résultats des recherches à différents pas de temps : le temps long (géologie, archéologie), le temps moyen des dernières décennies (hydrologie, histoire, écologie, géographie) et le temps court de l'année et des saisons (géographie, anthropologie).

Étudiants, chercheurs, acteurs locaux et décideurs trouveront dans cet ouvrage un regard nouveau, pluridisciplinaire, sur ces socioécosystèmes lacustres. Il enrichit la vision des possibles pour les sociétés à venir et invite à un débat nécessaire sur la conception de politiques publiques intégrant les enjeux imbriqués de conservation, de préservation des services écosystémiques et de développement.

\section{CHRISTINE RAIMOND (ÉD.)}

Christine Raimond est géographe, directrice de recherche au CNRS (Prodig), spécialiste des mutations sociales et environnementales dans les territoires ruraux d'Afrique subsaharienne.

\section{FLORENCE SYLVESTRE (ÉD.)}

Florence Sylvestre est paléoclimatologue, directrice de recherche à l'IRD (Cerege), spécialiste de l'étude des lacs tropicaux dans les contextes de changements climatiques.

\section{DANGBET ZAKINET (ÉD.)}

Dangbet Zakinet est historien, enseignant-chercheur à l'Université de N’Djamena, spécialiste des conflits ruraux liés à l'accès aux ressources naturelles.

\section{ABDERAMANE MOUSSA (ÉD.)}

Abderamane Moussa est sédimentologue et enseignant-chercheur à l'Université de N’Djamena, spécialiste des dépôts lacustres, éoliens et des faciès sédimentaires des bassins tropicaux. 
NOTE DE L'ÉDITEUR

Ce volume a bénéficié du soutien financier de l'Ambassade de France au Tchad. 
Éditeurs scientifiques

C. Raimond, F. Sylvestre

D. Zakinet, A. Moussa

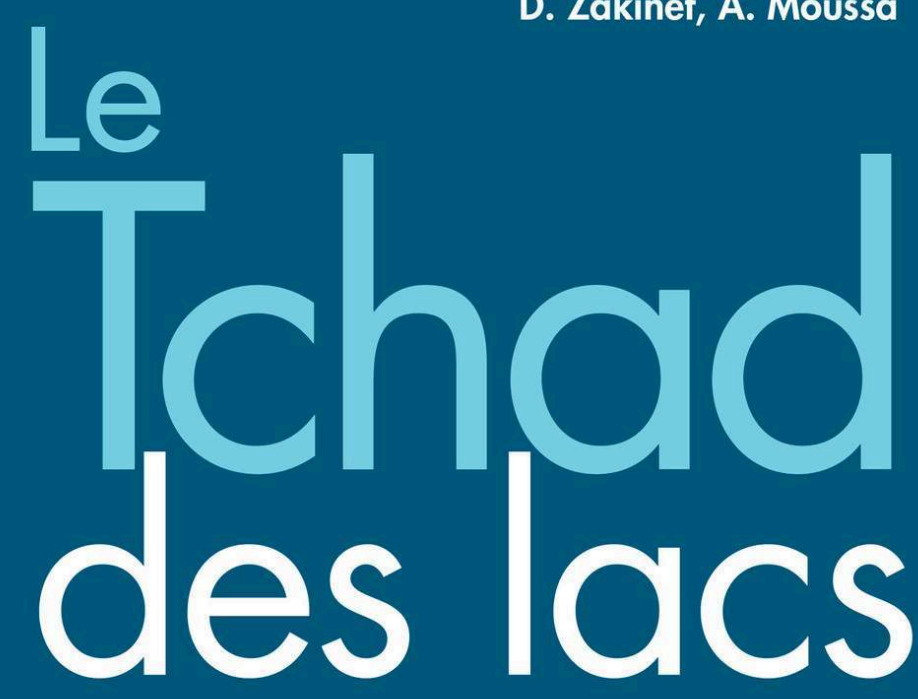

Les zones humides sahéliennes au défi du changement global

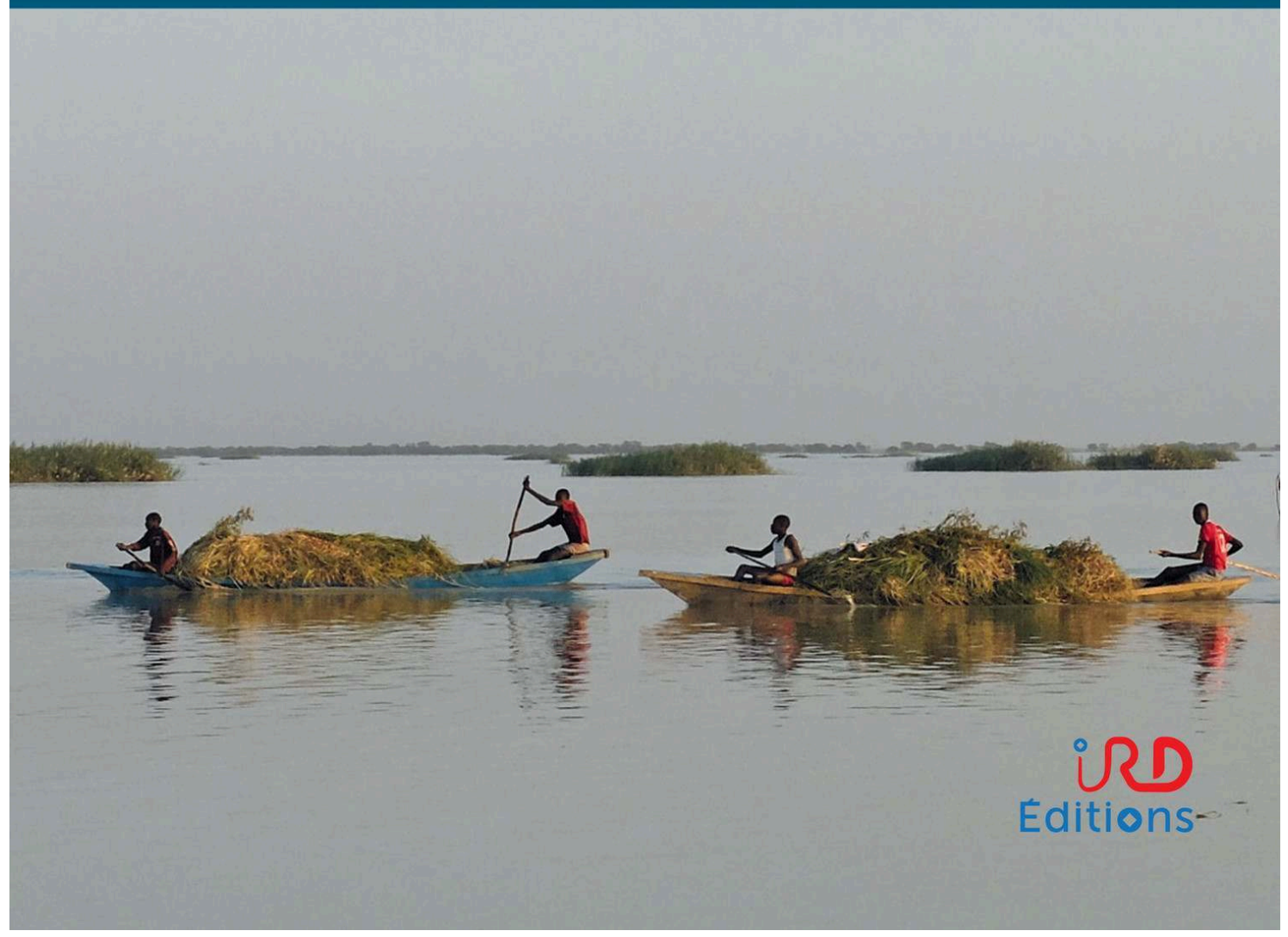




\section{Le Tchad des lacs}

Les zones humides sahéliennes au défi du changement global Christine Raimond, Florence Sylvestre, Dangbet Zakinet et Abderamane Moussa (éd.)

Éditeur : IRD Éditions

Lieu d'édition: Marseille

Année d'édition : 2019

Date de mise en ligne : 29 août 2019

Collection : Synthèses

\section{Oopertition \\ Qbooks}

http://books.openedition.org

\section{Édition imprimée}

Date de publication : 1 juin 2019

Nombre de pages : 368

Ce document vous est offert par OpenEdition Center USR 2004

\section{OpenEdition}

Référence électronique

RAIMOND, Christine (dir.) ; et al. Le Tchad des lacs : Les zones humides sahéliennes au défi du changement global. Nouvelle édition [en ligne]. Marseille : IRD Éditions, 2019 (généré le 29 août 2019). Disponible sur Internet : <http://books.openedition.org/irdeditions/30450>.

(c) IRD Éditions, 2019

Conditions d'utilisation :

http://www.openedition.org/6540 


\section{Le Tchad des lacs}

Les zones humides sahéliennes au défi du changement global 



\title{
Le Tchad des lacs
}

\section{Les zones humides sahéliennes au défi du changement global}

\author{
Éditeurs scientifiques \\ Christine RAIMOND, Florence SYLVESTRE, \\ Dangbet ZAKINET, Abderamane MOUSSA
}

Ce volume a bénéficié du soutien financier de l'Ambassade de France au Tchad.

\section{IRD Éditions}

INSTITUT DE RECHERCHE POUR LE DÉVELOPPEMENT

Collection Synthèses

Marseille, 2019 


\section{Coordination éditoriale}

Sylvie Hart

Préparation éditoriale

Stéphanie Quillon (34)

Mise en page

Desk (53)

Maquette de couverture

Michelle Saint-Léger

\section{Maquette intérieure}

Pierre Lopez

Photo de couverture

Livraison de fourrage à Yao. L'augmentation des populations sédentaires et transhumantes et de leur bétail accroît fortement la pression sur les ressources (Yao, février 2016).

(c) CNRS/M. Schuster

p. 4 de couverture

Multifonctionnalité de l'espace en bordure du lac Fitri (Gamsa, janvier 20|6).

Les jardins maraîchers et la pêche entravent le passage saisonnier des troupeaux vers les bourgoutières sur les îles.

(c) CNRS/C. Raimond

La loi du ler juillet 1992 (code de la propriété intellectuelle, première partie) n'autorisant, aux termes des alinéas 2 et 3 de l'article L. 122-5, d'une part, que les « copies ou reproductions strictement réservées à l'usage du copiste et non destinées à une utilisation collective » et, d'autre part, que les analyses et les courtes citations dans le but d'exemple ou d'illustration, « toute représentation ou reproduction intégrale ou partielle faite sans le consentement de l'auteur ou de ses ayants droit ou ayants cause, est illicite » (alinéa I er de l'article L. 122-4).

Cette représentation ou reproduction, par quelque procédé que ce soit, constituerait donc une contrefaçon passible des peines prévues au titre III de la loi précitée.

\section{(C) IRD, 2019}

ISBN : 978-2-7099-27|5-4 


\section{Composition du comité scientifique}

\section{Likius ANDOSSA}

paléontologue, université de N'Djamena, Tchad

\section{Ali Brahim BECHIR}

pastoraliste, Institut national d'élevage de Moussoro, Tchad

\section{Tchago Bouimon}

archéologue, université de N'Djamena, Tchad

\section{Mirjam de BruIJN}

anthropologue, université de Leyde, Pays-Bas

\section{Guillaume FaVREAU}

hydrogéochimiste, Institut de recherche pour le développement, Niger

\section{Abderamane HAMIT}

hydrogéochimiste, université de N'Djamena, Tchad

\section{Olivier LANGLOIS}

archéologue, Centre national de la recherche scientifique, France

\section{Jacques LemoalLe}

hydroécologue, Institut de recherche pour le développement, France

\section{Robert MADJIGOTO}

géographe, université de N'Djamena, Tchad

\section{Géraud MAgrin}

géographe, université Paris I Panthéon-Sorbonne, France

\section{Abderamane Moussa}

géologue, université de N'Djamena,Tchad

\section{Sougnabé Pabame}

socioéconomiste, Institut de recherche en élevage pour le développement, Tchad

\section{Christine RaImond}

géographe, Centre national

de la recherche scientifique, France

\section{Frédéric REOUNODJI}

géographe, ministère de l'Enseignement

supérieur et de l'Innovation, Tchad

\section{Mathieu Schuster}

géologue, Centre national de la recherche scientifique, France

\section{Florence SYLVESTRE}

paléoclimatologue, Institut de recherche pour le développement, France

\section{Christine Vallet-Coulomb}

hydrogéochimiste, Aix-Marseille Université, France

\section{Nicolas WaLdMANN}

paléoclimatologue, université Haifa, Israel

\section{Dangbet ZAKINET}

chef de mission, lac Fitri, historien, université de N'Djamena,Tchad 



\section{Sommaire}

Préface

Remerciements ....................................... 13

Introduction .......................................... 15

Christine Raimond, Florence Sylvestre, Dangbet Zakinet, Abderamane Moussa

\section{Partie I}

Les lacs tchadiens du Pléistocène à l'Anthropocène.

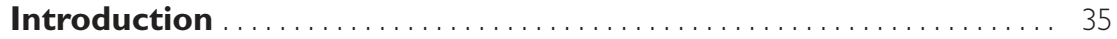

Christine Raimond, Florence Sylvestre

Chapitre I

Changements environnementaux et climatiques à la période humide africaine Holocène dans le bassin du lac Fitri (Tchad)

Mathieu Schuster, Florence Sylvestre, Christine Raimond, Abderamane Moussa,

Yassine Abakar, Tashi Yalikun

Chapitre 2

Paléoenvironnements et variations paléohydrologiques

du lac Tchad au cours des $\mathbf{1 2} 000$ dernières années.

Florence Sylvestre, Pierre Deschamps, Adoum Bari Sinine, Remadji Rirongarti,

Jean-Charles Mazur, Nicolas Waldmann, Paula do Amaral,

Camille Bouchez, Jean-Claude Doumnang

Chapitre 3

Des hommes et des lacs.

Peuplement des zones lacustres du Borkou (Tchad)

à l'Holocène

Yves Gauthier, Christine Gauthier 
Chapitre 4

Mission archéologique franco-tchadienne aux lacs d'Ounianga (Tchad). Principaux résultats des deux premières missions de terrain

Vincent Mourre, Guemona Djimet, Tchago Bouimon, Marie-Pierre Coustures,

Barbara Eichhorn, Jean Mbairo, Caroline Robion-Brunner, Margareta Tengberg

Chapitre 5

Variabilité des crues et des paysages du lac Fitri

depuis les grandes sécheresses des années 1970-1980

Tashi Yalikun, Christine Raimond, Angeline Kemsol Nagorngar, Dangbet Zakinet,

Mathieu Schuster, Florence Sylvestre

Partie 2

Dynamique des socioécosystèmes lacustres

Introduction

Christine Raimond, Florence Sylvestre

Chapitre 6

Les lacs d'Ounianga.

Mieux comprendre leur dynamique hydrologique

et écologique par une approche interdisciplinaire.

Thijs Van der Meeren, Pierre Deschamps, Florence Sylvestre, Baouyé Kemkong,

Abderamane Moussa, Mathieu Schuster, Dirk Verschuren

Chapitre 7

Étude de la dynamique hydrologique des lacs Iro et Fitri

par une approche isotopique.

Chloé Poulin, Bruno Hamelin, Christine Vallet-Coulomb, Guinbé Amngar,

Bichara Loukman, Jean-François Crétaux, Jean-Claude Doumnang, Abdallah

Mahamat Nour, Guillemette Menot, Florence Sylvestre, Pierre Deschamps

Chapitre 8

Caractérisation des paramètres physicochimiques

des eaux souterraines de la région du lac Iro.

Guinbé Amngar, Abderamane Hamit

Chapitre 9

Fluctuation des récoltes de sorgho repiqué et potentialités de culture.

Une analyse par télédétection dans la région du lac Fitri

Angeline Kemsol Nagorngar, Christine Raimond, Robert Madjigoto,

Valère Jofack Sokeng, Datoloum Djimassal, Joseph Libar, Fernand Kouamé Koffi 
Chapitre 10

La végétation pastorale du lac Fitri.

État des lieux et dynamique.

Ali Brahim Béchir, Koussou Mian-Oudanang, Alhassine Mahamat,

Tchoudiba Bourdjolbo

\section{Partie 3}

Gouvernance des territoires, logiques sociales

et hétérogénéité des acteurs

Introduction

Christine Raimond, Florence Sylvestre, Dangbet Zakinet

Chapitre II

Évolution technologique et gestion d'un espace halieutique dans la cuvette nord du lac Tchad

Hadiza Kiari Fougou, Jacques Lemoalle

Chapitre 12

De l'insécurité de Boko Haram

au conflit intercommunautaire dans le lac Tchad.

Le conflit Peul/Boudouma au Niger

Mahamadou Abdourahamani, Maman Waziri Mato

Chapitre 13

Pratiques et enjeux de la sécurisation foncière autour du lac Fitri.

Audrey Mbagogo Koumbraït

Chapitre 14

Économie des échanges au lac Fitri.

Un déficit récurrent en produits alimentaires

Blaise Bémadji, Ngaressem Goltob Mbaye

Chapitre 15

Les territoires d'eau ou comment gérer les ressources halieutiques dans les espaces lacustres?

Les lacs Fitri et Iro

Marguerite Saunier, Christine Raimond, Brahim Abba Ambar

\section{Partie 4}

Des territoires en mutation :

le défi du développement durable. 
Chapitre 16

L'avenir du lac Tchad : les échelles de l'incertitude.

Géraud Magrin, Jacques Lemoalle

Chapitre 17

Des communs non excluant ?

La gouvernance des ressources naturelles

dans la partie camerounaise du lac Tchad

Charline Rangé

Chapitre 18

Entre auto-organisation et planification dans la plaine

d'inondation du Logone (Cameroun)

Sarah Laborde, Aboukar Mahamat, Mark Moritz

Chapitre 19

Le lac Fitri, un modèle de gestion en crise ?

Ronan Mugelé

Conclusion

Pour un observatoire au lac Fitri.

Une exigence d'intégration de points de vue et d'enjeux différents

Christine Raimond, Dangbet Zakinet

Liste des auteurs 


\section{Préface}

Les écosystèmes lacustres concentrent de nombreux enjeux socioéconomiques et de biodiversité associés à de multiples services rendus : eau potable, irrigation, régulation hydrologique et bactériologique, ressources minérales, végétales et animales...

Ces zones humides sont aujourd'hui clairement menacées par les changements climatiques, l'intensification de l'agriculture, les pollutions diverses, les aménagements hydrauliques inadaptés et la forte pression anthropique. Une meilleure compréhension de leur fonctionnement complexe apparaît donc nécessaire, quasi vitale. Surtout au Sahel en général et au Tchad en particulier où elles représentent l'espoir à l'image des oasis surgissant du désert. S'agissant d'espaces plurifonctionnels, la démarche à suivre ne peut être que multidisciplinaire et combiner les apports des sciences dites dures et des sciences humaines. Telle avait été déjà l'approche retenue pour l'expertise collégiale réalisée par l'Institut de recherche pour le développement (IRD) dans le cadre du projet Préservation du lac Tchad et publiée en 2014. C'est tout l'intérêt du programme de recherche multidisciplinaire « Grands écosystèmes lacustres tchadiens » alias Gelt, financé par la coopération française pendant quatre ans, de l'avoir étendu à d'autres lacs de dimensions plus modestes. Après de nombreuses missions de terrain, ce projet s'est clôturé à N'Djamena du 25 au 27 avril 2017 par un colloque international dont les riches interventions sont rassemblées dans le présent ouvrage.

Dans cette somme imposante, les savoirs traditionnels ne sont pas oubliés, eux qui ont permis, depuis des millénaires, à des populations riveraines de ces lacs et aux nomades de s'adapter à un environnement en perpétuelle évolution. Ils ont en effet toute leur place à côté des modélisations mathématiques ou des analyses physicochimiques. Tel est notamment le message d'un magnifique ouvrage publié en 2006 par l'Organisation des Nations unies pour l'alimentation et l'agriculture intitulé Le futur est un ancien lac. Un appel poignant à des mesures scientifiques adaptées et à des politiques régionales adéquates. Le Gelt fournit, pour sa part, une contribution pour répondre à la première partie de ce diptyque.

Au-delà du recueil et de la formalisation des connaissances, ce programme Gelt a eu aussi des effets structurants et il a, en particulier, facilité la constitution 
d'écoles doctorales au Tchad. Forte des relations nouées entre chercheurs du Sud et du Nord, du dialogue des disciplines, de l'association recherche/formation à la recherche, du terrain avec le laboratoire, une communauté scientifique tchadienne est en train d'émerger. Cette évolution est porteuse d'espoir pour l'avenir au Tchad, terre de connaissance et berceau de l'humanité.

Philippe LACOSTE Ambassadeur de France au Tchad 


\section{Remerciements}

Les éditeurs scientifiques de cet ouvrage souhaitent remercier l'ensemble des acteurs du programme FSP Gelt " Grands écosystèmes lacustres tchadiens ", sans lesquels le corpus de travaux présenté dans les pages qui suivent n'aurait pu être possible.

Nous remercions l'Ambassade de France au Tchad et l'ensemble de ses personnels pour l'appui indéfectible tout au long de ces trois années du projet afin qu'il puisse être réalisé dans les meilleures conditions.

Nous remercions les institutions tchadiennes, le Centre national de la recherche pour le développement (CNRD) du Tchad et l'université de N'Djamena, pour leur engagement, leur soutien, tant pour la réalisation des missions de terrain que pour la disponibilité des enseignants-chercheurs et l'accompagnement des étudiants dans la réalisation de leurs travaux.

Nous remercions également les institutions françaises et les unités de recherche des universités d'Aix-Marseille, de Paris 1 Panthéon-Sorbonne, de Strasbourg, l'IRD, le CNRS ainsi que l'université de Gand en Belgique pour leur accompagnement, leur soutien pendant le programme, mais aussi dans son prolongement afin que l'ensemble des données collectées puisse être valorisé sous la forme de cet ouvrage et des nouveaux projets qu'il motive.

Tous les articles présentés dans cet ouvrage ont été révisés par les membres du comité scientifique constitué à cet effet, qui rassemble le comité scientifique du programme Gelt élargi à des collègues extérieurs spécialistes des thématiques abordées. Qu'ils en soient remerciés ici.

Enfin, nous tenons à remercier les autorités nationales et locales, et toutes les populations lacustres qui ont accueilli les équipes de chercheurs et d'étudiants, nombreux, curieux, exigeants. Qu'ils trouvent dans ces pages une petite partie de ce qu'ils nous ont donné, mais surtout les informations et arguments pour construire ensemble une connaissance partagée et un projet d'avenir. 



\section{Introduction}

Christine RAIMOND, Florence SYLVESTRE,

Dangbet ZAKINET, Abderamane MousSA

$\mathrm{Au}$ Sahel, les zones humides couvrent plus de $10 \%$ du territoire. Cinq plaines d'inondation figurent parmi les plus importantes : la vallée du Sénégal, le delta intérieur du Niger, les plaines d'inondation reliées au lac Tchad, les marais de Sudd au Sud-Soudan et de Gambella en Éthiopie. Ces milieux présentent des particularités exceptionnelles : saisonnières ou permanentes, encore humides alors que s'assèchent les régions à l'entour en saison sèche, moins étendus lors des périodes de sécheresse, la végétation y reste verte une grande partie de l'année et offre refuge et ressources aux animaux et aux humains. Ils concentrent une forte biodiversité et fournissent en abondance des ressources en eau, poissons, produits de cueillette (combustible, vannerie, pharmacopée et aliments), terres et pâturages de décrue pour l'agriculture. Au début du XXI ${ }^{\mathrm{e}}$ siècle, ces zones à haut potentiel productif sont devenues de véritables poumons économiques fournissant les produits alimentaires aux populations rurales et urbaines qui en dépendent.

Les enjeux écologiques et économiques de ces zones humides sahéliennes sont reconnus, d'abord par les sociétés qui les occupent depuis des périodes plus ou moins anciennes, mais aussi par la communauté scientifique qui a très tôt cherché à en comprendre le fonctionnement complexe et a proposé des options pour leur aménagement (GALlAIS, 1967 ; GouROU, 1982 ; ADAMS, 1993). Dès les premières études régionales, l'organisation sociale et l'analyse culturelle sont associées à celle des processus hydroécologiques pour rendre compte des systèmes retenus par les sociétés et les accompagner dans leur développement.

Pourtant, les aménagements proposés vont souvent à l'encontre des principes de mobilité, multiactivité des exploitations et multifonctionnalité des espaces, à la base de la résilience de ces socioécosystèmes caractérisés par une très grande variabilité environnementale et étroitement dépendants du climat. L'artificialisation 


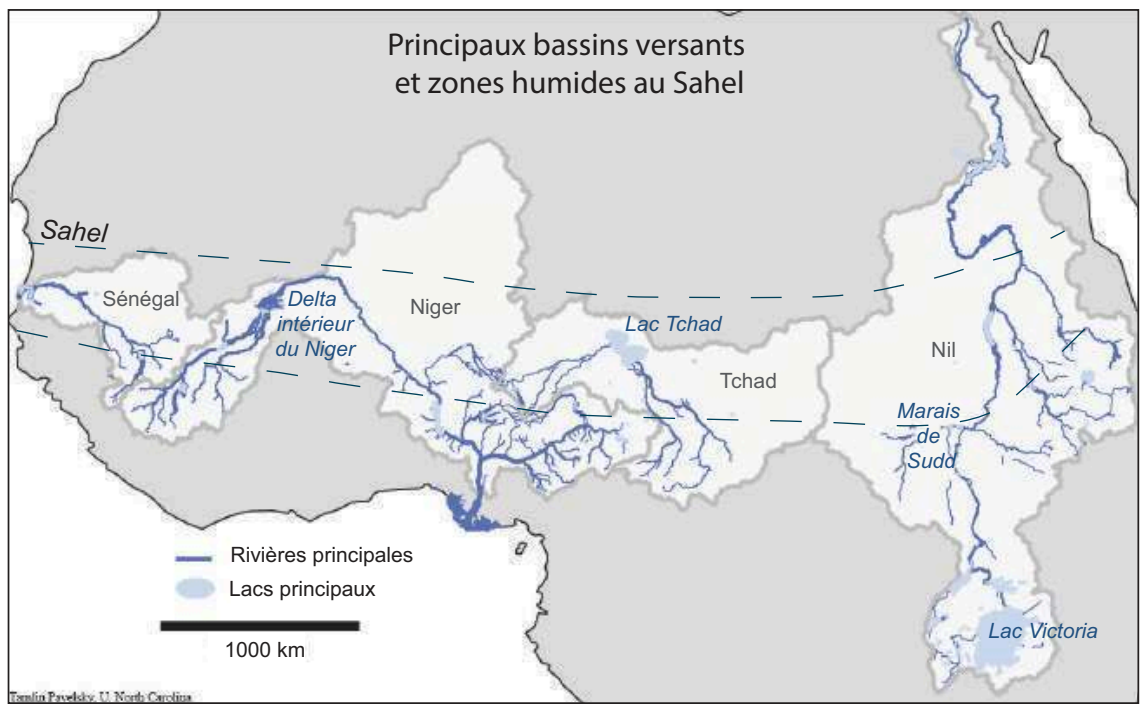

Figure I

Lacs et zones humides au Sahel.

Les ressources aquatiques saisonnières ou permanentes, comme les lacs qui perdurent toute l'année, revêtent de ce fait un intérêt vital pour la faune, la flore et les sociétés riveraines. Source : Nasa Swot.

Réalisation :Tilman Pavelsky, université North Carolina.

du milieu, mise en place dans les grands projets de périmètres irrigués des années 1970-1980, impose un investissement lourd en capital, une technicité et une organisation sociale de partage de l'eau nouvelles, ainsi qu'une redistribution des terres et de la main d'œuvre familiale qui figurent parmi les nombreux facteurs d'échec de la plupart de ces grands aménagements (RAISON et MAGRIN, 2009 ; Bertoncin et PASE, 2012).

En marge de ces aménagements localisés et peu nombreux, les attraits de l'eau et des ressources multiples fournies par les zones humides sahéliennes n'ont cessé d'attirer des populations de plus en plus nombreuses. Qu'elles soient installées de façon permanente, usagères temporaires ou saisonnières, elles déploient et concentrent leurs activités dans des milieux impactés par la péjoration climatique, au risque de briser l'équilibre des socioécosystèmes, dans un contexte concurrentiel et conflictuel.

L'importance et le rôle stratégique des services rendus par ces zones humides ont été rappelés au début des années 1990 dans le mouvement international en faveur de la protection de la biodiversité (SCOONES, 1991 ; RAMSAR, 2010). Le constat de la mise en danger de ces zones à haute biodiversité et fragiles, du fait de leur localisation sous des latitudes au climat semi-aride alternant saisons humides et sèches, s'appuie sur les inventaires réalisés en Afrique (BURGIS et Simoens, 1987 ; BernacseK et al., 1992 ; TAYlor et al., 1995 ; Olivry et al., 
1996 ; ZWARTS et al., 2009). Les dispositifs de protection dans le cadre de la Convention Ramsar s'inscrivent dans une démarche plus large de gestion des ressources naturelles et de participation des usagers à un développement durable (GEPIS, 2000 ; BORRINI et al., 2010). En reconnaissant la priorité donnée à la valorisation économique des ressources naturelles et à la participation de la conservation à la lutte contre la pauvreté, les réflexions sont orientées vers le renforcement des interrelations entre dynamiques de conservation et développement économique (SENE et al., 2006) dans le cadre d'une gestion intégrée adaptée aux problématiques des zones humides africaines (ORANGE et al., 2002). Cependant, les dispositifs peinent à se mettre en place en raison non seulement des lourdeurs institutionnelles nécessitant l'intervention d'une multiplicité d'acteurs intervenant aux échelles locales à régionales, mais aussi des lenteurs de définition de politiques publiques innovantes dans le contexte post-ajustement structurel et un cadre législatif inadapté (BARRIÈRE et BARRIÈRE, 2002).

Le début $\mathrm{du} \mathrm{XXI}^{\mathrm{e}}$ siècle voit un intérêt renouvelé pour les zones humides sahéliennes. La mise à l'agenda du changement climatique à l'échelle internationale identifie la vulnérabilité du continent africain et de ses ressources en eau. Ainsi, zones humides sahéliennes et lacs sont considérés comme autant d'indicateurs environnementaux qu'il faut surveiller dans la durée. En même temps, ces zones à haute productivité sont convoitées par de nombreux investisseurs, étrangers et urbains nationaux, qui veulent augmenter les productions agricoles pour l'exportation et pour le marché régional en croissance. La relance des politiques publiques par des États qui ont pris conscience, à la fin des années 2000, de leur vulnérabilité face aux marchés internationaux tout puissants (GRÉGOIRE et al., 2018) ravive la controverse entre agriculture familiale et entreprise agricole (RANGÉ et COCHET, 2018).

Face aux enjeux productifs, à la conflictualité de l'accès aux ressources naturelles et au défi de l'alimentation et de la croissance démographique, les décisions politiques se cantonnent aux options technicistes proposées dans la période post-indépendance qui n'ont pourtant pas fait la preuve de leur efficacité. La crise terroriste qui touche le Sahel depuis les années 2000 ajoute son poids sur l'avenir de ces zones humides, particulièrement dans le lac Tchad qui est redevenu la zone d'insécurité qu'elle était au XIX ${ }^{\mathrm{e}}$ siècle. Les liens entre changement climatique, pauvreté et insécurité sont souvent évoqués pour expliquer les origines de la crise ; de ce point de vue, la préservation et la réhabilitation des zones humides dans leur fonctionnement productif sont un enjeu pour réguler les répercussions sur les migrations internationales, notamment celles à destination de l'Europe (Madgwick et Pearce, 2017). Même si ces analyses sont à nuancer en intégrant le fonctionnement des systèmes régionaux et de politiques publiques inadaptées (MAGrin et PÉROUSE DE MontClos, 2018), elles soulignent l'importance des zones humides sahéliennes dans la géopolitique de l'Afrique subsaharienne.

La formulation des questions posées sur les zones humides sahéliennes s'est modifiée au cours des dernières décennies, mais pas leurs fondements. Les 
mêmes principes de fonctionnement prévalent et les mêmes enjeux de gouvernance s'expriment en fonction de stratégies contradictoires d'une diversité d'acteurs qui s'accroît. Le défi du changement global, caractérisé par l'évolution climatique, la croissance démographique, l'urbanisation et la demande alimentaire urbaine, les migrations, le développement de l'économie de marché, impose cependant d'intégrer le temps long des siècles au temps court de la décennie, de l'année et de la saison considérées jusque-là. Les sociétés changent aussi et renouvellent leurs priorités, leurs besoins, leurs rapports de force en interne et avec l'extérieur. La définition de la citoyenneté et de la démocratie évolue également. Tous ces éléments nécessitent d'être pris en compte dans une démarche interdisciplinaire pour comprendre les interrelations entre la dynamique des écosystèmes, les sociétés et les systèmes de gouvernance afin de renouveler les actions à mener dans ces territoires.

L'immensité des plaines d'inondation au Sahel rend difficile l'appréhension des socioécosystèmes dans leur ensemble et de la gouvernance à repenser pour intégrer les échelles de décisions, du terroir à l'État et l'organisation régionale correspondante. De taille plus petite, spatialement circonscrits et en eau toute l'année, les lacs concentrent les mêmes caractéristiques. Ils fournissent de bons indicateurs des dynamiques sociales et environnementales à l'œuvre, et des verrous scientifiques à lever pour penser leur avenir dans le cadre d'un développement durable.

\section{Les lacs tchadiens, un bien commun et des enjeux de connaissance à définir}

Le Tchad est particulier en Afrique par la diversité de ses lacs, de tailles, d'origines et de fonctionnements différents, et représentatifs des problématiques propres aux zones humides africaines sous pressions multiples.

Les lacs ont par le passé occupé une très grande place dans le paysage, notamment de 10000 à 5000 ans lorsque les conditions climatiques ont permis à la zone de convergence intertropicale d'apporter des pluies de mousson en quantité plus importante pendant la saison estivale (GASSE, 2000). À cette période, on évalue que les lacs occupaient au moins $10 \%$ du Sahel actuel (QuADE et al., 2018) et, à l'image des témoignages archéologiques, on perçoit toute l'importance que ces étendues d'eau ont joué pour l'évolution des sociétés néolithiques (KUPER et KRÖPELIN, 2006). Le lac Tchad couvrait une superficie de plus de $350000 \mathrm{~km}^{2}$ et a joué un rôle crucial dans l'évolution de ces écosystèmes.

Après cette période, la région connaît une aridification progressive au cours de laquelle se mettent en place les conditions que nous connaissons aujourd'hui. Des lacs relictuels de la période antérieure, les lacs Tchad, Ounianga et Fitri ont 
perduré et se sont progressivement imposés comme des espaces vitaux pour les populations. Cet intérêt des sociétés contemporaines pour les zones lacustres dans le bassin tchadien est relativement récent, hormis pour les lacs Ounianga, car jusque dans les années 1950 et 1960 elles étaient considérées comme un obstacle à l'agriculture et au développement, avec des sols gorgés d'eau difficiles à cultiver, des conditions de vie et de travail impossibles en raison de l'insalubrité, de l'insécurité hydrique due à la variabilité des dates et des niveaux d'inondation, et des difficultés d'accès et de transport des récoltes vers les marchés. Seul l'élevage puis la pêche s'y étaient développés à l'instar des autres eaux continentales africaines (CHAUVEAU et al., 2000).

Ce sont les sécheresses des années 1970 et 1980 qui ont révélé le caractère stratégique de ces zones lacustres tchadiennes pour l'agriculture et l'élevage car un grand nombre des ménages y ont installé leurs activités devenues impossibles ailleurs par manque de pluies. Depuis cette date, les populations ont adapté leurs systèmes de production entre les zones lacustres et l'extérieur grâce à une grande mobilité des activités et de la main d'œuvre, réglée sur le rythme et l'ampleur des crues saisonnières (RAIMOND et al., 2014).

Les cinq lacs du Tchad occupent une place croissante dans l'économie régionale par leurs potentialités productives et leur attractivité pour les populations. Le lac Tchad, partagé entre Tchad, Niger, Nigeria et Cameroun, est le plus connu et le plus médiatisé de par sa taille (près de $200 \mathrm{~km} \mathrm{du}$ nord au sud, soient $25000 \mathrm{~km}^{2}$ en périodes de hautes eaux) et les débats contradictoires sur sa potentielle disparition (MAGRIN, 2016). Les lac Fitri, Léré, Iro sont beaucoup moins médiatisés et étudiés, plus petits aussi puisqu'ils couvrent entre 200 et $800 \mathrm{~km}^{2}$. Les lacs Ounianga au nord du pays, nés de la résurgence de la nappe phréatique en plein désert, désignent un ensemble de 18 petits lacs interconnectés (20 $\mathrm{km}^{2}$ au total) répartis sur deux sites, Ounianga Kebir et Ounianga Serir. Le caractère spectaculaire de ces sites en fait une image emblématique du tourisme au Tchad et a motivé leur classement au patrimoine mondial de l'Unesco en 2012.

Malgré leurs caractéristiques contrastées dans des environnements désertique à soudanien, les lacs du Tchad partagent une histoire similaire du point de vue des politiques publiques : grandes oubliées des politiques agricoles (hors les essais de poldérisation au lac Tchad), ces zones lacustres ne font pas l'objet de grands aménagements et sont restées longtemps enclavées. Des dispositifs de protection environnementale sous Convention Ramsar sont mis en place à partir des années 1990 : le lac Fitri devient réserve de biosphère en octobre 1989 (décret 773/PR/MTE/8), la partie tchadienne du lac Tchad site Ramsar en août 2001, le lac Léré en novembre 2005 en même temps que les dépressions Toupouri, le lac Iro en mai 2006 avec la plaine d'inondation du Salamat. Ces labels ne sont pas accompagnés de mesures politiques spécifiques de protection et de développement, si bien que ces lacs ont poursuivi une trajectoire de développement endogène. 


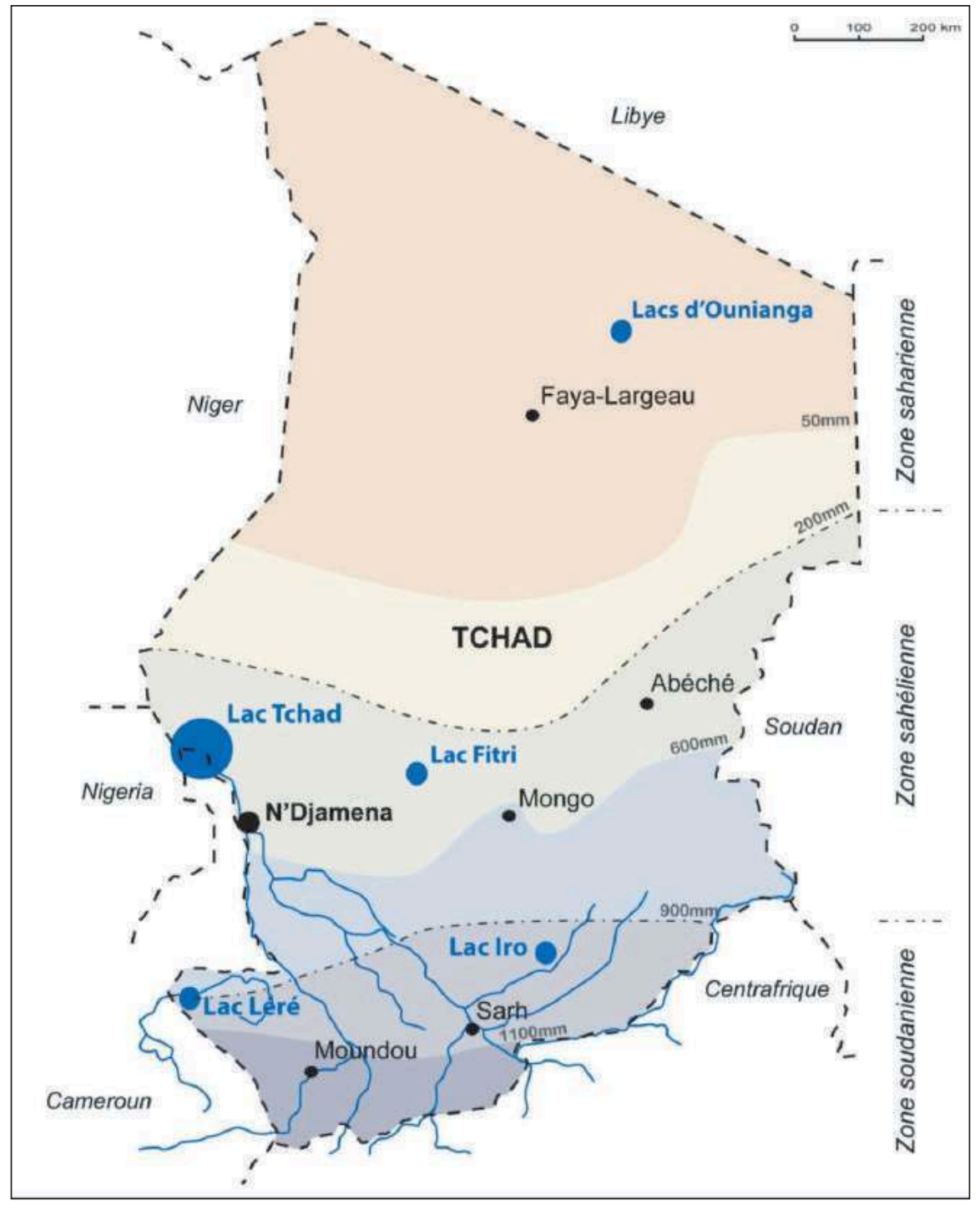

Figure 2

Les cinq grands lacs du Tchad avec des caractéristiques,

potentialités et fonctionnements contrastés de la zone soudanienne jusqu'au Sahara.

Réalisation : Chloé Leconte.

Comme dans l'ensemble du Sahel, les zones humides fluviales et fluviolacustres tchadiennes sont devenues des secteurs à forte productivité, d'où sont exportées les productions agricoles et halieutiques pour l'alimentation des métropoles régionales, voire vers les débouchés méridionaux urbains et portuaires. Pour le lac Tchad, le désenclavement des rives sud d'abord au Nigeria puis au Tchad 
est vecteur d'un développement rapide dont les premiers bénéficiaires sont les sociétés lacustres. Pour les lacs Fitri, Léré et Iro occupés de plus longue date, ce sont les cheptels qui ont considérablement augmenté et tirent parti, en complément de l'agriculture et de la pêche, des pâturages lacustres devenus indispensables dans les systèmes de production. La croissance démographique pèse également sur les ressources naturelles. Des conflits à dimensions multiples émergent, que les institutions locales peinent à réguler, tout comme les règlements administratifs qui ne tiennent pas compte de la spécificité de ces milieux et de ces sociétés.

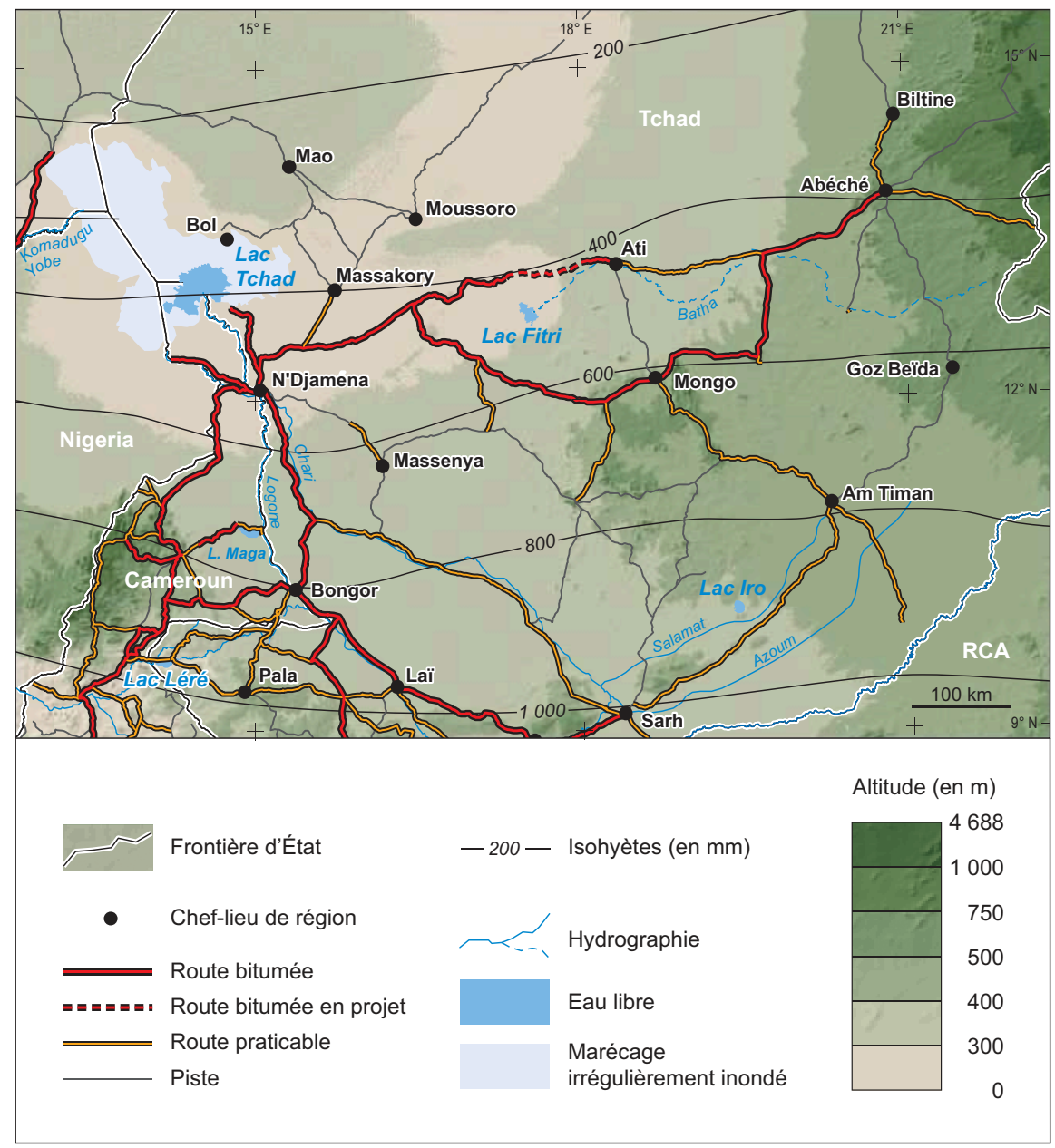

Figure 3

Le réseau des routes et pistes : un désenclavement partiel des lacs Tchad, Fitri, Léré et Iro. Sources : SRTM30, USGS, BDD Plantadiv/Prodig, carte annuelle des pluies IRD 2012.

Réalisation : C.Valton, IRD, UMR Prodig, 2019. 
Les violences perpétrées depuis 2009 par le groupe djihadiste Boko Haram et la répression militaire qui s'en est suivie ont particulièrement affecté les zones humides du lac Tchad et de ses affluents, redevenues zones d'insécurité et désertées volontairement ou manu militari dans le cadre des dispositifs de l'état d'urgence. Cette situation modifie profondément la trajectoire de développement de la région et particulièrement de la zone lacustre, mais aussi les relations entre les sociétés et leur environnement, et les options d'aménagement reformulées par la redistribution des populations réfugiées. Les autres zones lacustres sont indirectement impactées, soit par le déplacement de populations (pêcheurs du lac Tchad vers le lac Fitri par exemple), soit par la disparition de débouchés économiques (notamment le marché nigérian pour le bétail que les éleveurs du Fitri ne peuvent plus exporter), soit par les changements des itinéraires des troupeaux qui augmentent la pression sur les terroirs (comme au lac Léré où se reportent les itinéraires du bétail sur pied exporté vers le sud du Cameroun et du Nigeria, qui passaient auparavant par N'Djamena). Le lac Iro est indirectement concerné par la crise de la RCA et les populations d'éleveurs réfugiées.

Dans le contexte du changement global et de la montée des insécurités au Sahel, la pression sur les zones humides éprouve fortement la résilience des socioécosystèmes lacustres. Les objectifs de protection environnementale sont peu relayés par des mesures concrètes et localement légitimées. L'expérience de cogestion réussie au lac Léré dans les années 1990 (DAGOU et al., 2005) n’a pas trouvé écho au Tchad alors qu'elle aurait pu faire jurisprudence pour le renouvellement des stocks halieutiques et la promotion d'une activité de pêche durable. Plus largement, ces milieux lacustres restent particulièrement méconnus alors qu'ils sont de plus en plus convoités par une grande diversité d'acteurs dont les objectifs divergent et se concurrencent. Pour penser les options de protection environnementale et de développement durable, renforcer la connaissance de ces zones humides constitue un enjeu scientifique à part entière.

\section{Le programme Gelt}

Cet ouvrage constitue une synthèse des recherches réalisées dans le cadre du programme Grands écosystèmes lacustres tchadiens (Gelt), soutenu par un Fonds de solidarité prioritaire piloté par l'ambassade de France à N'Djamena de 2013 à 2017. Ce programme est né d'une idée originale des chercheurs impliqués dans la recherche au Tchad qui ont perçu l'effet structurant de ces milieux sur les sociétés agropastorales, renforçant à la fois leur capacité d'adaptation et leur résilience face aux crises multiples du Sahel.

Les recherches sur ces milieux se sont très tôt focalisées sur le lac Tchad. Dès le début $\mathrm{du} \mathrm{XX}^{\mathrm{e}}$ siècle, les expéditions scientifiques ont relaté de nombreuses observations, soulignant sa très forte variabilité rythmée par les fluctuations du 
climat (Tılho, 1910 ; 1925 ; 1928). Puis dans les années 1950 à 1970, les équipes de l'Orstom s'investissent dans l'observation et la description de ce lac, livrant un corpus de données qui fait encore référence sur son fonctionnement hydrologique, chimique, géochimique, sédimentaire et sa biodiversité (RocHE, 1973 ; Dupont, 1968 ; Couty et Duran, 1968 ; Louis, 1970 ; Servant et Servant, 1970 ; Fontes et al., 1970 ; CARmouze, 1976 ; Gac, 1980 ; Maley, 1981 ; CARMOUZE et al., 1983 ; Olivry et al., 1983 ; LeMOALle, 1991). BouQueT (1990) livre une étude régionale très détaillée sur le lac Tchad et ses sociétés.

$\mathrm{Au}$ fil des années, le lac Tchad s'est imposé comme le lac de référence pour l'étude de la ressource en eau au Sahel. La menace de sa disparition mobilise la communauté scientifique, les acteurs politiques et les gestionnaires de l'environnement. Il a fait, notamment, l'actualité de la COP 21 (MAGRIN, 2016). Plusieurs synthèses rassemblant un large panel de disciplines ont été publiées et soulignent toutes les difficultés d'appréhension et de gestion d'un espace grand comme la Belgique, partagé entre quatre pays, à haute variabilité environnementale, caractérisé par une très grande diversité ethnolinguistique et la violence armée de la dernière décennie (BERTONCIN et PASE, 2012 ; LEMOALLE et Magrin, 2014 ; Magrin, Lemoalle, Pourtier, 2015 ; Magrin et Pérouse DE Montclos, 2018).

À partir de cette base de connaissances qui continue à s'étoffer sur le lac Tchad, le programme Gelt s'est focalisé sur l'étude des autres lacs tchadiens, plus petits, peu ou pas documentés, encore accessibles dans le contexte d'insécurité croissante dans la région. Il n'a pas été possible de s'investir sur le lac Léré situé à la frontière camerounaise, où peu de nouvelles recherches ont été entreprises depuis les années 2000 - même si les actions de gestion intégrée se sont poursuivies dans le cadre de la coopération allemande, qu'il serait intéressant de capitaliser. Ce sont donc trois lacs situés dans les trois zones climatiques du Tchad qui ont concentré les travaux : Ounianga, Fitri et Iro.

Du point de vue physique, ces lacs ont été peu ou pas décrits. Avant le programme Gelt, il n'existait aucune donnée hydrologique sur le lac Iro hormis une description sommaire datant de 1987 (BuRGIS et SimoENS, 1987). Les lacs d'Ounianga avaient fait l'objet d'une monographie (CAPOT-REY, 1961) et d'une étude portant sur la reconstitution des paléoenvironnements aquatiques au cours des 6000 dernières années (KRÖPELIN et al., 2008). Les lacs Léré et Fitri étaient documentés à l'issue d'un programme interuniversitaire Campus entre 2001 et 2003 et de travaux de thèse (RAIMOND, 1999 ; Moupeng, 2004 ; PAssinRInG, 2005). Deux ouvrages sur le lac Iro (PAIRAULT, 1966 et 1994) et plusieurs études sur le pastoralisme sur le lac Fitri (COUREL et al., 1996 ; AuBAGUE et al., 2007 ; MARTY et al., 2012) complètent la bibliographie.

Trois axes ont structuré les recherches selon les problématiques propres à chaque lac : l'étude du fonctionnement physique ; les dynamiques démographiques et leurs influences sur les activités humaines ; la gouvernance et les relations de pouvoir. Il n'a pas été prévu de démarche comparative entre les lacs tant les situations sont contrastées. Les lacs d'Ounianga sont isolés en plein désert et 
témoignent plus du temps long des changements, des routes et carrefours transsahariens et de l'insécurité des populations oasiennes que des problématiques de concentration des activités, de surexploitation et de gouvernance qui caractérisent les zones lacustres méridionales. Le lac Fitri, dont le fonctionnement hydrologique, l'organisation des activités et le rôle de zone refuge s'apparentent au lac Tchad, s'en différencie par la présence d'un pouvoir coutumier encore présent qui régule l'accès aux ressources lacustres. Le lac Iro est une particularité physique héritée probablement d'un impact de météorite (Doumnang J. C., Rochette P., com. pers.), qui fonctionne au rythme saisonnier de la plaine d'inondation de la rivière Salamat. Cependant, plusieurs thématiques se font écho qui sont reprises dans cet ouvrage : les temporalités des changements, le rôle vital des lacs pour les sociétés qui les occupent, l'enclavement facteur limitant du développement, les crises de gouvernance face à la multiplication des acteurs du territoire.

Au-delà des activités de recherche, le programme Gelt a eu pour ambition de renforcer les capacités de formation par la recherche des étudiants et enseignantschercheurs tchadiens. Adossé à trois masters de géographie, d'anthropologie et d'hydrologie et système d'information géographique (Master Hydro-SIG) de l'université de N'Djamena, ce programme a permis la formation de six promotions d'étudiants par une approche complémentaire terrain-laboratoire, organisée autour de missions de terrain associant les chercheurs du Nord et du Sud. Six missions ont été organisées par le programme Gelt : deux sur chaque lac à un an d'intervalle. La première campagne de terrain s'est déroulée en 2015 en février (lac Fitri), en avril (lac Iro) et en décembre (lacs d'Ounianga). La seconde campagne a eu lieu en 2016 au cours des mêmes périodes sur les mêmes sites. Ces campagnes de terrain ont mobilisé des étudiants et des enseignantschercheurs des universités de N'Djamena, de Sarh, d'Ati, d'Aix-Marseille, de Paris 1 Panthéon-Sorbonne et de Gand (Belgique) et des chercheurs de l'IRD et du CNRS.

\section{L'exigence d'une approche multidisciplinaire}

Pour mieux comprendre et appréhender les problématiques révélées par ces socioécosystèmes lacustres, l'approche privilégiée a été multidisciplinaire afin d'intégrer les points de vue différents en sciences de la terre (géologues, hydrologues, hydrogéologues, géochimistes, micropaléontologues), de la nature (écologues, botanistes) et en sciences humaines et sociales (géographes, anthropologues, historiens). L'objectif de ce large dispositif est de croiser les regards disciplinaires, les méthodes et les connaissances sur un même objet-lac 
pour renouveler les questionnements scientifiques d'une part, les contributions aux réflexions sur l'aménagement et la gouvernance d'autre part.

Une attention toute particulière a été accordée au cadre théorique et à l'organisation de cette recherche, que ce soit pour les questions de recherche identifiées à traiter collectivement, pour les champs disciplinaires concernés, la production de connaissance et leur mobilisation sur des enjeux environnementaux. Ainsi, l'un des points forts du programme Gelt a été le partage des questions, méthodes et observations de terrain lors des missions communes. Là se sont affinés les sujets des étudiants en master et doctorat, se sont partagées les données de référence sur les lacs et les thématiques, se sont construites les méthodologies par la mutualisation de certaines phases répétitives de terrain et se sont croisés les regards disciplinaires.

\section{Présentation de l'ouvrage}

Les vingt chapitres rassemblés dans cet ouvrage ont été présentés lors du colloque international «Recherches croisées sur les écosystèmes tchadiens » qui a eu lieu à N'Djamena du 25 au 27 avril 2017 et dont l'objectif était de confronter les principaux résultats du programme Gelt à ceux issus d'autres zones lacustres d'Afrique subsaharienne. Ils couvrent les résultats des recherches récentes, offrant une vision originale du Tchad à travers le prisme de ses lacs. Les prospections menées contribuent à la reconstitution des environnements sur les derniers millénaires et apportent une perspective temporelle aux données actuelles sur le fonctionnement hydrologique et l'évolution des environnements et des sociétés pour réfléchir sur l'avenir.

L'ouvrage s'articule autour de quatre parties étroitement liées, retraçant les temporalités des dynamiques de ces socioécosystèmes lacustres complexes, leur adaptation au changement global et leur gouvernance. Au total, 62 auteurs, acteurs du programme Gelt et extérieurs, du Sud et du Nord, sont réunis dans cet ouvrage. Tous témoignent par l'écriture de la façon dont une conception pluridisciplinaire de la recherche sur un objet commun - Le Tchad des lacs apporte une cohérence à l'activité scientifique et enrichit la production de connaissances.

\section{Partie I \\ Les lacs tchadiens du Pléistocène à l'Anthropocène}

Le temps court des crises, telles que vécues actuellement dans le bassin du lac Tchad, s'inscrit dans le temps long des changements climatiques à l'origine de mutations paysagères majeures auxquelles ont dû s'adapter les sociétés humaines. L'histoire des lacs du Tchad s'écrit depuis au moins sept millions d'années, dans 
les archives sédimentaires et archéologiques qui attestent des périodes humides (le « Sahara vert ») et des périodes sèches, et de son occupation par des humains et des animaux de différentes espèces. Berceau de l'humanité attesté par la découverte des premiers hominidés, le lac Tchad témoigne aussi des installations, à différentes périodes, de pasteurs et de pêcheurs sur ses rives. Celles-ci marquent autant l'extension passée des lacs que l'ancienneté des sociétés humaines, des systèmes de production et de leurs interactions avec les milieux.

La première partie déroule cette histoire sur le temps long à la lumière des nouvelles découvertes dans les domaines de la géologie, des paléoenvironnements et de l'archéologie. En renseignant plus précisément les processus à l'œuvre lors de la dernière grande période d'optimum climatique à l'Holocène, ces travaux précisent les conditions de formation des lacs Tchad, Ounianga et Fitri, reliques du Mégalac Tchad. Ils documentent aussi un processus d'assèchement et ses conséquences sur les écosystèmes, équivalent à celui que l'on risque de connaître dans le cadre du changement climatique marqué par l'Anthropocène. L'échelle du temps court de la perception par les hommes est proposée en conclusion pour le lac Fitri, en retraçant le rythme et l'ampleur des crues depuis les grandes sécheresses de la moitié du $\mathrm{XX}^{\mathrm{e}}$ siècle. C'est en effet sur la combinaison de ces différentes temporalités, temps longs des changements, temps courts des perceptions et des politiques, que repose un double enjeu : celui de la connaissance sur la variabilité de ces zones lacustres liée au climat, mais aussi celui de l'aménagement et de la gouvernance de ces zones lacustres dont dépendent un nombre croissant de personnes.

\section{Partie 2 \\ Dynamique des socioécosystèmes lacustres}

Du Sahara à la zone soudanienne, les lacs du Tchad constituent des zones humides associant plans d'eau libre et marécages caractérisés par leur richesse en biodiversité et fournissant des services multiples pour les sociétés qui les habitent. Appréhender la variabilité environnementale de ces milieux dépend de notre compréhension du fonctionnement de l'hydrosystème et des interactions entre eau pluviale, fluviale et souterraine, des échanges avec l'atmosphère, mais aussi des actions des sociétés lacustres.

Les cinq textes qui composent cette partie montrent comment l'analyse du fonctionnement hydrologique actuel contribue à la reconstitution des effets des changements climatiques passés d'une part, et à l'anticipation des évolutions futures d'autre part. Ces résultats nuancent, pour la période actuelle, l'importance $\mathrm{du}$ changement climatique en cours par rapport aux facteurs anthropiques. Ils appellent à une analyse approfondie de ces systèmes complexes pour évaluer l'évolution des ressources lacustres tenant compte des temporalités de la variabilité, de l'adaptation des systèmes de production, de la croissance démographique et de l'augmentation des besoins des populations. 


\section{Partie 3 \\ Gouvernance des territoires, logiques sociales et hétérogénéité des acteurs}

Si l'ancienneté de l'occupation humaine des zones humides fluviolacustres n'est pas partout équivalente en Afrique, celles-ci se caractérisent toutes aujourd'hui par une grande diversité d'acteurs qui interagissent sur le territoire, et par des systèmes de production basés sur la mobilité, la pluriactivité et la multifonctionnalité des espaces. Hormis dans les lacs d'Ounianga où la mise en valeur des eaux douces souterraines relève des systèmes oasiens, pour les autres lacs l'agriculture, l'élevage et la pêche tirent profit des rythmes de la crue et sont menés alternativement sur les mêmes espaces. Que le territoire lacustre soit maîtrisé par une seule société hiérarchisée (lacs Fitri et Léré par exemple), partagé par plusieurs pouvoirs coutumiers comme au lac Tchad, ou par des sociétés acéphales comme au lac Iro, ces eaux et ressources lacustres sont régies par un ensemble de règles plus ou moins anciennes, en évolution permanente et remises en question par l'arrivée de nouveaux acteurs, la mise en place des services déconcentrés de l'État et la décentralisation.

Les zones lacustres constituent ainsi des arènes où les acteurs agissent en fonction de stratégies propres et d'héritages historiques qui pèsent entre les groupes, mais qui constituent aussi des opportunités pour penser de nouvelles formes de gouvernance. Plusieurs éclairages sont apportés à partir des activités de pêche, d'élevage et d'agriculture dans les lacs Tchad, Fitri et Iro. Face aux conflits qui se multiplient entre les acteurs, ces nouvelles études mettent en relief la nécessité de tenir compte des conditions écologiques locales et de leur variabilité pour penser de nouvelles règles de cogestion entre les usagers, les pouvoirs locaux, les collectivités territoriales et les services de l'État.

\section{Partie 4 \\ Des territoires en mutation : le défi du développement durable}

Quelles sont les trajectoires de développement des zones lacustres du Tchad? C'est à cette vaste question que répond la quatrième partie. Plusieurs pistes sont explorées qui éprouvent le concept du développement durable, tant pèsent la croissance démographique et la montée des insécurités, et tant les interactions et rétroactions entre développement économique, viabilité écologique et sociale sont grandes au sein des systèmes complexes étudiés. L'importance des jeux d'échelles, du local à l'international, de l'individuel au collectif, est rappelée et mobilisée pour appréhender les trajectoires possibles. Partout, les questions de l'aménagement et du désenclavement sont récurrentes et impactent le fonctionnement des socioécosystèmes qui a fait la preuve de leur résilience sur le temps long. Les modalités d'accès aux ressources sont au cœur des débats. Elles peinent à être tranchées par des législations nationales inadaptées à la notion de bien commun que constituent les ressources lacustres pour les usagers. 
Localement, les sociétés innovent selon des principes d'auto-organisation qu'il est important d'identifier et qui pourraient faire jurisprudence. Inversement, l'ancien système de gouvernement du lac Fitri par le sultanat bilala trouve ses limites. Comprendre ces mutations est un enjeu fondamental pour la conservation des zones humides sahéliennes et la viabilité des systèmes mis en place. C'est aussi une contribution majeure aux éclairages locaux indispensables à toute prise de décision en matière de gestion territoriale d'une part, et à la conception de politiques publiques et lois nationales adaptées aux réalités locales d'autre part.

Le chapitre conclusif défend l'idée d'un observatoire du changement global, sur le lac Fitri qui a bénéficié des regards de la plus grande diversité de disciplines et qui présente, en 2019, le meilleur potentiel d'innovation dans les domaines de l'environnement, du développement et des institutions. Une maison des Sciences, telle qu'il en existe en d'autres lieux pour mettre en relation directe scientifiques, acteurs du territoire et usagers, fournirait dans la durée un dispositif et des outils pour intégrer dans une analyse commune les points de vue et enjeux contrastés exprimés au sein de cet ouvrage. L'ambition est de reconnaître la nécessité d'une hybridation des savoirs et de concilier la climatologie, les sciences de l'environnement et les sciences humaines pour la connaissance et pour sauver un patrimoine en péril. Un observatoire, donc, pour reconnaître que se comprendre, plus encore se comprendre pour agir, quand on vient de mondes aussi différents que les paléoenvironnements, l'archéologie, l'anthropologie, les sciences de la conservation, le développement et la société civile, ne se fait pas sans volonté commune ni outils partagés.

\section{Bibliographie}

ADAMs W. M., 1993

Indegenous use of wetlands and sustainable development in West Africa. The Geographical Journal, 159-2 : 209-218.

Augague S., Djimadoum D., Ali Adoum M., 2007

Le Fitri : diagnostic pastoral. Ante-Iram pour le programme d'hydraulique pastorale au Tchad Central «Almy Al Afia » (AFD), 91 p.

Barreteau D., Jungraithmayr H., SEIBERT U., 1997

L'homme et l'eau dans le bassin du lac Tchad. Paris, Orstom, 487 p.
BARrière O., BARRIÈre C., 2002

Un droit à inventer. Foncier et environnement dans le delta intérieur du Niger. IRD Éditions, À Travers Champs, 387 p.

Bernacsek G. M., Hugues J. S., Hugues R. H., 1992

Répertoire des zones humides d'Afrique, IUCN, Unep, WCNC, 808 p.

Bertoncin M., Pase A., 2012

Autour du lac Tchad. Enjeux et conflits pour le contrôle de l'eau. Paris, L'Harmattan, $354 \mathrm{p}$. 
Borrini-Feyerabend G., Pimbert M., Farvar M. T., Kothari A., Renard Y., 2010 Partager le pouvoir : cogestion des ressources naturelles et gouvernance partagée de par le monde. Iied ; IUCN, 498 p.

\section{Bouquet C., 1990}

Insulaires et riverains du lac Tchad. 2 tomes, L'Harmattan, Paris, 412 et 463 p.

Burgis M. J., Symoens J. J., éd., 1987 African wetlands and shallow water bodies = Zones humides et lacs peu profonds d'Afrique. Paris : Orstom, coll. Travaux et documents, 211, $650 \mathrm{p}$.

\section{CAPOT-ReY R., 1961}

Borkou et Ounianga. Étude de géographie régionale. Institut de recherches sahariennes, vol. 5,182 p.

\section{CARMouze J.-P., 1976}

La régulation hydrogéochimique du lac Tchad. Contribution à l'analyse biogéodynamique d'un système endoréique en milieu continental. Paris, Orstom, coll. Travaux et documents, 58, $428 \mathrm{p}$.

\section{Carmouze J.-P., Durand J.-R., LévêQue C., 1983 \\ Lake Chad: ecology and productivity} of a shallow tropical ecosystem. Junk Publishers, The Hague. 575 p.

\section{Chauveau J.-P. Jul-Larsen E., Chaboud C., 2000}

Les pêches piroguières en Afrique de l'Ouest: dynamiques institutionnelles : pouvoirs, mobilités, marchés. Paris, Karthala, 397 p.

\section{Courel M-F, Morin S., Raimond C., 1997}

« Intégration modèle ou modèle d'intégration. La gestion de l'environnement au lac Fitri (Tchad) ». In : Singaravelou (éd.) : Gestion de l'environnement dans les pays tropicaux, Bordeaux, Dymset, Cret, p. 311-326.

\section{Couty P., Duran P., 1968}

Le commerce du poisson au Tchad. Orstom, Paris, $252 \mathrm{p}$.

Dagou P., Mustapha M. A., Mbaye N. G., Passinring K., Marabe N.-O., 2005

«La pêche dans les lacs Fitri et Léré au Tchad. Techniques de capture, conservation des produits et enjeux de protection ». In : Raimond C., Garine E., Langlois O., éd. : Ressources vivrières et choix alimentaires dans le bassin du lac
Tchad. IRD, coll. Colloques et séminaires, p. 87-112.

\section{Dugan P., 1993}

Wetlands in danger: a world conservation atlas. New York, New York, Oxford University Press, 1993. 192 p.

\section{DUPONT B., 1968}

Étude sédimentologique du lac Tchad. Premiers résultats. Orstom, Fort-Lamy, 76 p.

\section{Fontes J. C., Gonfiantini R., Roche M. A., 1970}

Deutérium et Oxygène-18 dans les eaux du lac Tchad. Isotopes in Hydrology, IAEA-SM-129/23, p : 387-404. http://www.documentation.ird.fr/hor/ fdi:04791.

Gallais J., 1967

Le delta intérieur du Niger, étude de géographie régionale. Dakar, Ifan, $621 \mathrm{p}$.

\section{GAC J. Y., 1980}

Géochimie du bassin du lac Tchad. Bilan de l'altération, de l'érosion et la sédimentation. Paris, Orstom, coll. Travaux et documents, 123, $251 \mathrm{p}$.

\section{Gasse F., 2000}

Hydrological changes in the African tropics since the Last Glacial Maximum. Quaternary Science Reviews, 19 : 189-211.

GEPIS, 2000

Vers une gestion durable des plaines d'inondation sahéliennes. UICN-Brao, 214 p.

\section{GoURou P., 1982}

Terres de bonne espérance. Le monde tropical. Paris, Plon, 455 p.

Grégoire E., Kobiané J.-F., LAnge M.-F., 2018

L'État réhabilité en Afrique. Réinventer les politiques publiques à l'ère néolibérale. Paris, Karthala, coll. Hommes et sociétés, 356 p.

Kröpelin S., Verschuren D., LéZine A.-M., Eggermont H., Cocquyt C., Francus P., Cazet J.-P., Fagot M., Rumes B.,

RusSElle J. M., 2008

Climate-driven ecosystem succession in the Sahara: the past 6000 years. Science, 320 : 765-768.

Kuper R., Kropelin S., 2006

Climate-Controlled Holocene Occupation 
in the Sahara: Motor of Africa's Evolution. Science, 313 : 803-807.

\section{LEMOALLE J., 1991}

The hydrology of Lake Chad during a drought period / Eléments d'hydrologie du lac Tchad au cours d'une période de sécheresse (1973-1989). FAO Fisheries Reports, 445 : 54-61.

Lemoalle J., Magrin G., dir., 2014

Le développement du lac Tchad : situation actuelle et futurs possibles. CBLT, N'Djamena, Marseille, Expertise collégiale IRD, AFD-FFEM, rapport de synthèse, $67 \mathrm{p}$. + traduction anglaise et contributions intégrales des experts (CD, 20 chapitres, 620 p.).

\section{LOUIS P., 1970}

Contribution géophysique à la connaissance géologique du bassin du lac Tchad. Mémoire, Orstom, Paris, 42, 311 p.

\section{Madgwick F. J., Pearce F., 2017}

Water shocks. Wetlands and human migration in the Sahel. Wetlands International, the Netherlands. https://www.wetlands.org/ publications/water-shocks-wetlands-humanmigration-sahel/

\section{MAGRIN G., 2016}

The disappearance of Lake Chad: history of a myth. Journal of Political Ecology 23: 204-222.

Magrin G., Lemoalle J., Pourtier R., 2015 Atlas du lac Tchad. Paris, IRD Éditions/Passages, $225 \mathrm{p}$.

\section{Magrin G., Pérouse de Montclos M. A., dir., 2018}

La région du lac Tchad à l'épreuve de Boko Haram. Crise et enjeux de développement. Études de l'AFD, 291 p.

\section{MaLey J., 1981}

Études palynologiques dans le bassin du lac Tchad et paléoclimatologie de l'Afrique Nord tropicale, de 30000 ans à l'époque actuelle. Thèse en sciences, université de Montpellier, Paris, Orstom, coll. Travaux et documents, 129, $586 \mathrm{p}$.

\section{Marty A., Zakinet D., Khamis D. D.,} BERNARD C., 2012

Almy al Afia 2. Analyse de l'évolution des ressources dans le département du Fitri. Document principal. République du Tchad, programme d'hydraulique pastorale au Tchad central, Phase II. Antea-Iram, 128 p.

\section{Moupeng B., 2006}

Le lac Fitri : Dynamique du système hydrographique quaternaire et actuel (Sahel tchadien). Thèse de doctorat, université de Provence, Avignon, 171 p.

\section{Olivry J. C., Chouret A., Vuillaume G., Lemoalle J., Bricquet J. P., 1996 \\ Hydrologie du lac Tchad. Paris, Orstom, 266 p.}

\section{Orange D, Arfi R, Kuper M, Morand P,} Poncet Y., éd., 2002

Gestion intégrée des ressources naturelles en zones inondables tropicales. IRD, 997 p.

\section{Pairault C., 1966}

Boum-le-Grand, village d'Iro. Paris, institut d'ethnologie, travaux et mémoires, LXXIII, $325 \mathrm{p}$.

\section{Pairault C., 1994}

Retour au pays d'Iro, chronique d'un village au Tchad. Paris, Karthala, 292 p

\section{Passinring K., 2005}

Milieux naturels et paysages du bassin versant des lacs de Léré (département de Léré, Tchad). Thèse de géographie, université Aix-Marseille 1.

Quade J., Dente E., Armon M., Bem Dor Y., Morin E., Adam O., EnZel Y., 2018

Megalakes in the Sahara? A review. Quaternary research, 1-23.

\section{RAIMOND C., 1999}

Terres inondées et sorgho repiqué. Évolution des espaces agricoles et pastoraux dans le bassin du lac Tchad. Doctorat de géographie, université Paris 1,543 p.

\section{RaImond C, RAngé C., Guérin H., 2014} « La multiactivité et la multifonctionnalité, principes d'un développement pour le Lac ? ». In : Lemoalle J., Magrin G., dir., 2013. Le développement du lac Tchad : situation actuelle et futurs possibles. CBLT, N'Djamena, Marseille, Expertise collégiale IRD, AFD-FFEM, p. 423-474 (clé USB).

\section{Raison J. P., Magrin G., éd., 2009}

Des fleuves entre conflits et compromis. Essais d'hydropolitique africaine. Karthala, 299 p.

\section{RAMSAR, 2010}

Wise use of wetlands: Concepts and approaches for the wise use of wetlands. Ramsar handbooks 
for the wise use of wetlands, 4th edition, vol. 1.

Ramsar Convention Secretariat, Gland,

Switzerland. www.ramsar.org/sites/default/files/ documents/library/hbk4-01.pdf

\section{Rangé C., Cochet H., 2018}

Multiusage familial et agriculture de firme sur les rives du lac Tchad : une comparaison des performances économiques. Natures Sciences Sociétés, 26, 1, 33-48.

\section{Roche M. A., 1973}

Traçage naturel salin et isotopique des eaux du système hydrologique du lac Tchad. Thèse de doctorat ès Sciences. Université Paris-VI, Orstom, 398 p., 131 fig.

\section{SCOONES I., 1991}

Wetlands in Drylands. Key resources

for agricultural and pastoral production in Africa. Ambio, 20 : 366-371.

Sene M., Thiaw I., Lamizana-Diallo B., 2006 Gestion des zones humides en milieux arides : leçons d'expérience. UICN, Programme de renforcement des capacités institutionnelles pour la gestion des ressources des zones humides en Afrique de l'Ouest, 86 p.
Servant M., Servant S., 1970

Les formations lacustres et les diatomées du Quaternaire récent du fond de la cuvette tchadienne. Revue de Géographie physique et de géologie dynamique, vol. XII-1, p. 63-76.

\section{Tilho J., 1910}

Documents scientifiques de la Mission Tilho : 1906-1909. Larose, 3 vol., 7 cartes.

\section{Tilho J., 1925}

Sur l'ordre de grandeur des variations de profondeur et d'étendue du lac Tchad. Comptes rendus hebdomadaires des séances de l'Académie des sciences, Tome 180: 238-260.

\section{Tilho J., 1928}

Variations et disparition possible du Tchad. Annales de géographie, vol 2017, p. 238-260.

Taylor A. R. D., Howard G. W., BegG G. W., 1995

Developping wetland inventories in Southern Africa : a review. Vegetatio, 118 : 57-79.

ZWARts L., BiJlsma R. G., VAN der KAMP J., Wymega E., 2009

Living in the edge. Wetlands and birds in a changing Sahel. KNNV Publishing, 564 p. 



\section{Partie I}

\section{Les lacs tchadiens du Pléistocène à l'Anthropocène}

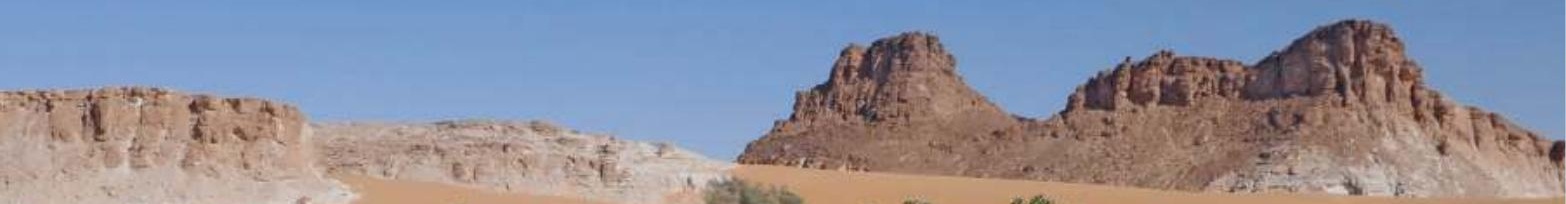

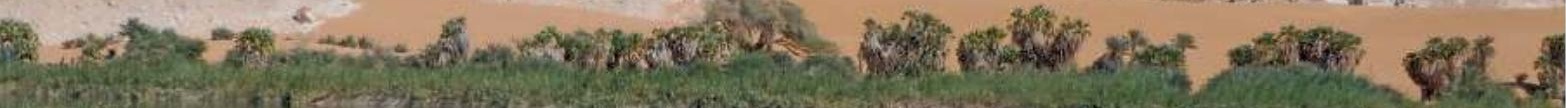

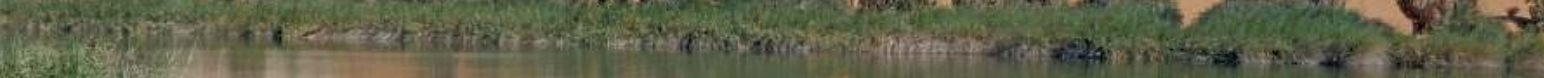
(1) 
Les lacs tchadiens hérités d'une longue histoire hydrogéologique et paléoclimatique ont été de tout temps des lieux de vie pour les sociétés

(lac Bokou, Ounianga Serir, octobre 2016).

(C) IRD/P. Deschamps. 


\section{Introduction}

Christine RAIMOND, Florence SYLVESTRE

Le climat sahélien est caractérisé par une alternance de périodes humides et de périodes sèches. Aussi loin que l'on puisse remonter dans le temps, les reconstitutions montrent qu'aux périodes humides et fastes pour les écosystèmes succèdent des phases de péjoration climatique ayant des conséquences parfois dramatiques sur les environnements et les êtres vivants associés. Cette succession concerne des périodes qui ont été très longues, d'autres très courtes. Elle a été vécue encore très récemment au Sahel avec la sécheresse des années 1970 et 1980, qui a succédé à la phase relativement humide des années 1950. Depuis le début des années 2000 , une tendance à l'augmentation des précipitations est observée en réponse à une accélération du cycle hydrologique et à l'augmentation globale des températures. Cependant, pour le futur immédiat, tous les modèles climatiques ne s'accordent pas et ajoutent de l'incertitude en prédisant des tendances contraires d'une simulation à l'autre sur l'évolution des précipitations. Une tendance qui semble néanmoins faire l'unanimité est une augmentation de la variabilité interannuelle de la pluviométrie, renforçant la difficulté de la prédiction du changement climatique de la région.

La validation de ces modèles climatiques établis à l'échelle globale repose en partie sur leur capacité à reproduire la variabilité passée. En ce sens, l'histoire du Sahara et du Sahel offre une opportunité exceptionnelle de tester les réponses des écosystèmes aux forçages externes et aux rétroactions internes du système climatique. En effet, cette région a connu des périodes beaucoup plus humides que celle observée pendant le $\mathrm{XX}^{\mathrm{e}}$ siècle et on sait que cette région n'a pas toujours été la vaste étendue aride balayée par les vents que nous connaissons aujourd'hui. L'une des périodes qui a particulièrement attiré la communauté scientifique au cours de ces dernières décennies se situe entre 12000 et 5000 ans pendant l'Holocène : la région était alors caractérisée par un paysage verdoyant, couvert de prairies et d'arbres, parsemé de nombreux lacs et incisé par de grands réseaux fluviatiles. De nombreux travaux se sont intéressés à caractériser la fin 
de cette période humide et la transition avec la période sèche qui a suivi, puisque cela permet de tester un analogue sur les récurrences des périodes sèches, dramatiques pour les populations.

La reconstitution de cette histoire climatique est possible grâce à l'étude d'archives sédimentaires, en particulier celles accumulées au fond des lacs. Les lacs sont d'excellents enregistreurs de la dynamique du cycle hydrologique à l'échelle de leur bassin versant. Par leur localisation topographique en fond de cuvette, l'ensemble des particules drainées sur leur bassin enregistrent les caractéristiques des événements climatiques. De même, au sein de la masse d'eau, des organismes conservent une signature de ces événements. L'observation, la mesure et l'analyse de ces informations sédimentées au fond des lacs permettent de retracer leur histoire hydrologique, reflet d'une partie de l'histoire climatique régionale.

Dès les premières explorations géologiques sur le territoire tchadien, il a été mis en évidence des témoignages sédimentaires de l'histoire hydrologique et climatique dans le bassin du lac Tchad. L'évolution du lac Tchad a été retracée sur des périodes très anciennes en mettant en évidence des phases de transgression et de régression. Lors de la dernière phase de transgression, il y a 10000 ans, le Mégalac Tchad aurait atteint une superficie 25 fois plus grande que sa taille moyenne actuelle, recouvrant une superficie de plus de $350000 \mathrm{~km}^{2}$.

Si les données sur l'histoire du lac Tchad sont de plus en plus fournies, très peu sont disponibles sur les autres lacs tchadiens. Le lac Fitri a fait l'objet de descriptions rapides inscrites dans le Mégalac Tchad. Les lacs Léré et Iro, localisés à l'extérieur de cet ensemble, sont peu ou pas décrits. Seul le lac Yoa à Ounianga Kebir a fait l'objet d'un carottage en 2008 mettant en évidence l'histoire paléohydrologique de cette région au cours des 6000 dernières années. Cet enregistrement a apporté des données importantes sur la fin de la période humide Holocène, qui encore aujourd'hui attise le débat sur les mécanismes responsables de cette transition climatique. Des enregistrements complémentaires, couvrant les derniers siècles, mettent en évidence la dynamique paléohydrologique des lacs d'Ounianga dans le contexte d'aridité actuelle.

Les travaux du programme Gelt complètent cette histoire des lacs du Tchad en consacrant une attention particulière aux archives sédimentaires, archéologiques et hydrologiques.

Une étude portant sur les archives morphosédimentaires autour du lac Fitri et son contexte géomorphologique met en évidence le potentiel particulièrement intéressant de ce lac pour renforcer la connaissance paléohydrologique et paléoclimatique à l'échelle de l'ensemble de la région. Son intérêt se situe aussi, pour la période actuelle, dans sa capacité à rendre compte des dynamiques du climat sahélien parce qu'il est principalement alimenté par la rivière Batha qui draine un bassin versant situé exclusivement dans la bande sahélienne, alors que le lac Tchad informe plus spécifiquement sur la dynamique tropicale du climat de la région. Plusieurs autres axes de recherche ont été ouverts qui viendront à terme compléter cette histoire sédimentaire et climatique régionale. 
Une autre étude portant sur une carotte prélevée dans la cuvette sud du lac Tchad apporte des données originales sur l'évolution du lac entre 11000 et 5000 ans. Si de nombreux travaux ont quantifié l'extension maximale du lac au cours de cette période, peu ont reconstitué les variations du lac en continu au cours du temps. Ces résultats précisent la chronologie de la période humide Holocène, en particulier sa terminaison et la transition avec la période aride à partir de 5000 ans.

Les civilisations anciennes et les vestiges qu'elles ont laissés localement témoignent aussi d'un environnement particulier et de son évolution. L'archéologie complète intimement l'histoire régionale, même si les vestiges sont épars et difficiles à repérer dans l'immensité régionale. Il est évident que la dynamique hydrologique a marqué considérablement l'histoire des peuplements et des sociétés, en particulier au cours du Néolithique.

Le Sahara tchadien recèle de nombreux témoignages des sociétés pastorales notamment au nord du Tchad lorsque le lac Tchad atteignait son extension maximale au cours de l'Holocène. Les populations repoussées sur les contreforts de l'Ennedi et du Tibesti ont laissé des traces de leur vie, que nous retrouvons aujourd'hui grâce à des gravures et des peintures sur roche, des outils taillés ou encore des structures d'habitat organisé autour des paléorivages. Pour la première fois, dans la région du Borkou, une représentation d'activités de pêche et de navigation est mise en évidence. Cette activité est mise en relation avec la dynamique hydrologique du lac Tchad dont elle marque la localisation des rivages à certaines périodes et suggère, notamment avec les représentations des pirogues et de transport de marchandises, une forme de sédentarisation des populations. La prospection archéologique réalisée dans la région d'Ounianga, peu ou pas étudiée compte tenu des conditions très contraignantes de son climat et de sa difficulté d'accès, révèle également de nombreux témoignages archéologiques et la présence de populations depuis le Paléolithique et le Néolithique. Les gravures rupestres confirment la diversité de la faune sauvage typique des zones soudaniennes (éléphants, girafes, antilopes) et du bétail domestiqué au Néolithique. Pour la première fois aussi, est mentionnée une activité métallurgique inédite à ces latitudes. La qualité de conservation des sites d'habitation précoloniaux témoigne d'une histoire locale particulièrement mouvementée pour des sociétés lacustres soumises aux questions d'insécurité depuis au moins plusieurs siècles. Elle révèle aussi une économie de subsistance très adaptée aux conditions de la sécheresse, où ce n'est pas l'eau de surface du lac, mais celle des résurgences et puits dans la nappe d'eau douce qui fait ressource.

Les questions liées à la variabilité contemporaine de la ressource en eaux sont étudiées ici à partir de l'exemple du lac Fitri, où les rythmes de crue et de décrue conditionnent les activités anthropiques. Face à l'absence de suivis hydrométriques de surface, les méthodes issues de la télédétection spatiale ont été utilisées pour estimer la superficie du lac sur la période 1972-2015, en tenant compte de la variabilité intra et interannuel du régime hydrologique. Cet exemple souligne 
les différences de perception de la variabilité lacustre selon les temporalités envisagées dans cette première partie : celles de l'ère géologique, du changement climatique, de la mémoire des hommes et femmes, de l'année, de la saison. Si l'expérience des uns peut renseigner le devenir des autres, leur appréhension au sein d'un même système relève d'une réalité complexe difficile à saisir. Toutefois, la prise en compte de ces temporalités constitue un enjeu majeur pour la connaissance et l'action dans ces zones lacustres. 


\title{
hangements environnementaux
} et climatiques à la période humide africaine Holocène dans le bassin

\section{du lac Fitri (Tchad)}

\author{
Mathieu SCHUSTER, Florence SYLVESTRE, \\ Christine RAIMOND, Abderamane MousSA, \\ Yassine ABAKAR, Tashi YALKUN
}

\section{Introduction}

Le lac Fitri, centré sur $12^{\circ} 50^{\prime} \mathrm{N}$ et $17^{\circ} 30^{\prime} \mathrm{E}$, est le plus grand lac s'étendant uniquement sur le territoire du Tchad (fig. 1 et 2). Ce lac et sa zone humide associée jouent un rôle majeur dans cette partie du Sahel pour les activités humaines (agriculture, élevage, pêche, transhumance, pastoralisme, commerce) et comme refuge pour la biodiversité (oiseaux migrateurs, faune sauvage, végétation) (RAIMOND et al., 2017).

Le lac Fitri reste mal connu ainsi que le souligne clairement LEMOALLE (1987) : « On possède peu d'informations sur ce lac. L'essentiel des données provient d'une courte campagne réalisée en décembre 1973 et de documents non publiés du service d'hydrologie du Centre Orstom de N'Djamena. », possiblement parce que l'essentiel de l'attention a été jusqu'à présent porté sur le lac Tchad. Ces deux grands lacs partagent un passé commun puisqu'ils ont fait partie à l'Holocène du Mégalac Tchad, avant de s'individualiser à la fin de l'optimum climatique dit de la « période humide africaine » ou du « Sahara vert » (SCHUSTER, 2002). Ils ont ensuite connu des évolutions distinctes, dans un contexte global commun mais avec des spécificités propres liées à des physiographies différentes. Tous deux sont endoréiques, occupent un bassin à la topographie/bathymétrie 
très peu prononcée (profondeur très faible en comparaison avec la taille du plan d'eau, s'apparentant ainsi à la catégorie des wind-driven waterbodies définie par NuTz et al., (2018) et dépendent très fortement des précipitations de la mousson ouest-africaine et des régimes de vent saisonniers dont l'empreinte hydrodynamique ancienne a été évaluée par BoucheTTE et al. (2010). Toutefois, le Fitri, avec son bassin de drainage occupant la bande sahélosaharienne, est totalement dépendant de la profondeur de pénétration vers le nord de la zone

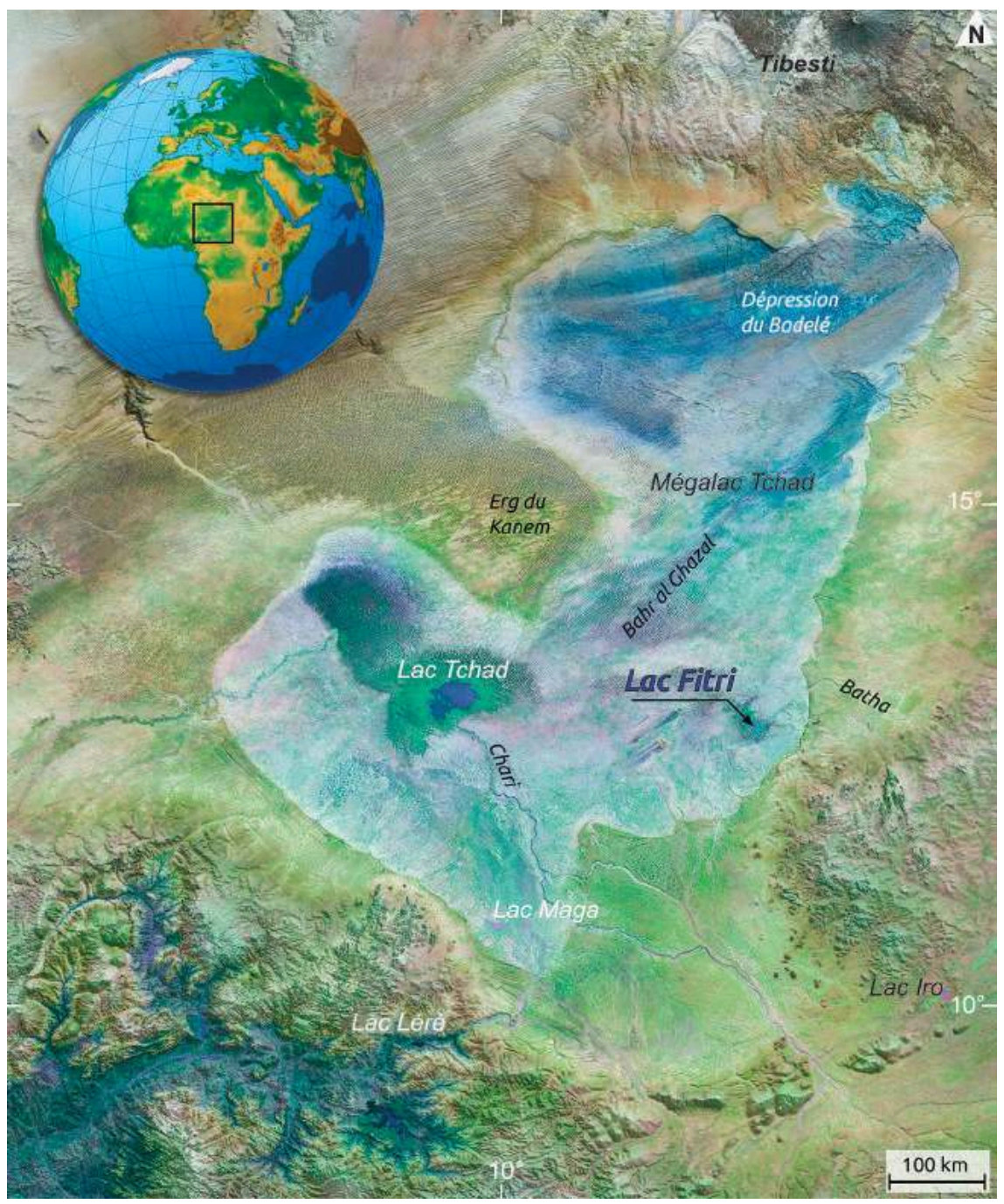

Figure I

Localisation du site d'étude montrant le lac Fitri et le lac Tchad dans le contexte du Mégalac Tchad.

Source : fusion Landsat et STRM. Réalisation : Claude Roquin, IPG-Strasbourg. 


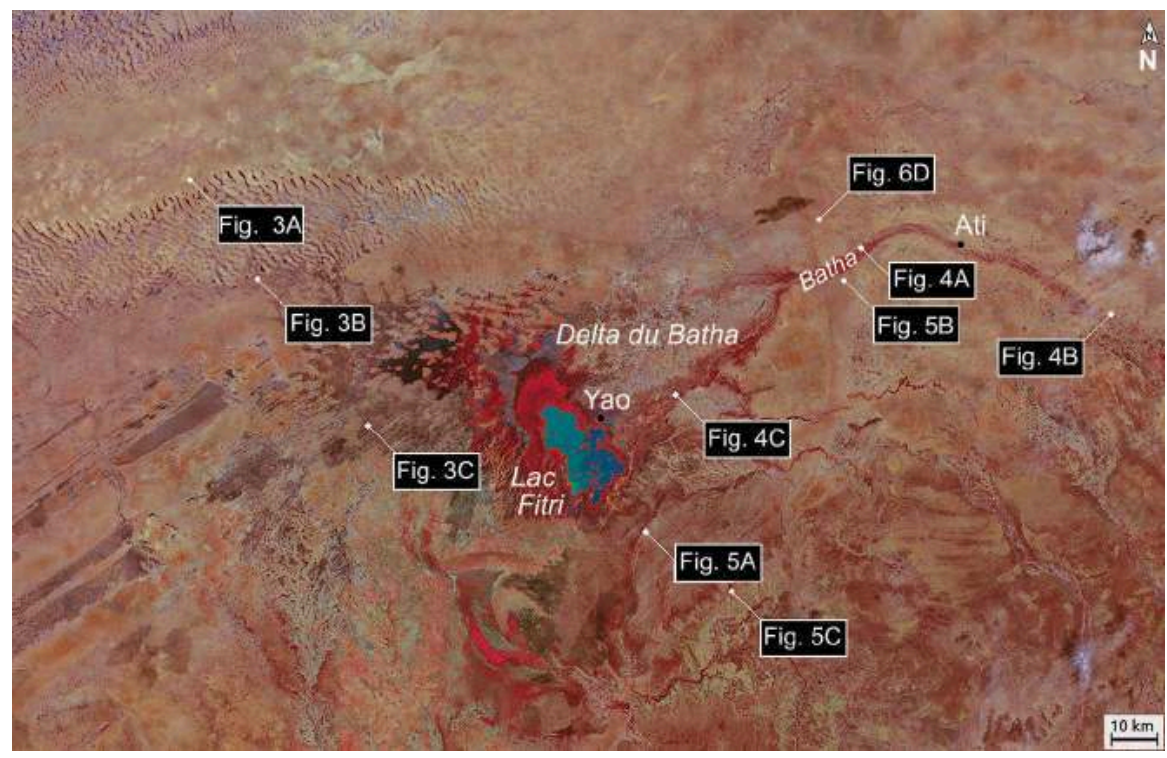

Figure 2

Vue satellite du lac Fitri et d'une partie de son bassin montrant la nette dominance des environnements de dépôts sableux et localisant les différentes figures illustrant cet article.

Source : mosaïque d'images Spot de 2010 (Sidrat) en fausses couleurs faisant apparaître la végétation en rouge.

de convergence intertropicale (ZCIT). La variabilité de la mousson ouestafricaine s'exprime au lac Fitri par des fluctuations de grande amplitude de la superficie des eaux libres et de la profondeur du lac, à toutes les échelles de temps (de la saison à plusieurs millénaires) (YALIKUN et al., ce volume).

Ainsi, le lac Fitri, avec son caractère sahélien fortement marqué, est un lac terminal qui constitue un enregistreur sensible et privilégié de la variabilité de la mousson et de son impact sur les paysages et les écosystèmes passés. Malgré cet intérêt majeur pour la recherche scientifique, les archives sédimentaires du lac Fitri et de son bassin n'ont fait pour le moment l'objet que de rares études (e.g. Lemoalle, 1987 ; Moupeng, 2006). Pour exemple, il aura fallu attendre 2016 pour que, grâce au programme Gelt, soient prélevés les premiers échantillons de sédiments des fonds du lac (bennes et carottes) et qu'une première caractérisation de sa bathymétrie soit entreprise.

La présente contribution est à considérer comme une première étape d'identification des principaux environnements et processus de dépôts présents dans le bassin. Réalisée principalement à partir de l'interprétation géomorphologique sur images stellitaires, cette étude a pu être complétée par des reconnaissances ponctuelles sur le terrain. Les grands types d'environnements sédimentaires (fluviatiles, deltaïques, lacustres et éoliens) reflètent des conditions de mise en place différentes souvent liées au forçage par le climat, tandis que leurs relations spatiales (emboîtement, superposition, érosion, remaniement) traduisent une chronologie relative de mise en place. Ainsi, un premier scénario 
d'évolution des environnements et du climat dans cette région peut être proposé. Dans un futur proche, celui-ci pourra être nettement amélioré, évidemment par une étude du même type plus approfondie incluant un effort important de datations absolues, mais aussi en intégrant les données d'études complémentaires initiées dans le cadre du programme Gelt (i.e. étude de la variabilité spatiale du lac pour la période observée par l'imagerie aérienne et satellite, analyse des archives lacustres prélevées par carottage et possiblement données de l'imagerie sismique des fonds du lac).

\section{Méthodologie}

L'approche retenue ici est simple puisqu'elle repose principalement sur la photointerprétation des grandes structures morphosédimentaires caractéristiques des environnements de dépôts à partir de l'observation d'images satellitaires selon une méthode éprouvée (SCHUSTER et al., 2005 et 2014 ; PAILlou et al., 2009 ; Nutz et Schuster, 2016 ; SCHUSTER et Nutz, 2018). La principale source d'images est Google Earth Pro (pour des raisons pratiques et économiques) dont la pertinence pour la recherche scientifique et notamment les géosciences n'est plus à démontrer (SCHEFFERS et al., 2012). Dans la région du lac Fitri, le faible couvert végétal et la bonne préservation des structures morphosédimentaires s'avèrent un atout pour appliquer ce type d'approche.

La chronologie relative retenue permettra ensuite d'orienter les futures actions visant à apporter des datations absolues. Elle est basée sur les principes simples, mais robustes, de la stratigraphie qui permet de définir les relations temporelles entre des couches géologiques, notamment le principe de recoupement (i.e. les couches sont plus anciennes que les structures ou les roches qui les recoupent). Deux jalons temporels sont apportés par deux événements climatiques majeurs marqués par des changements environnementaux drastiques, à savoir :

- l'épisode de très forte aridité de la fin du dernier maximum glaciaire (DMG) qui a favorisé une forte expansion du désert du Sahara comme en témoignent des systèmes de dunes et d'ergs dunaires fossiles qui se sont développés loin vers le sud, bien au-delà des lacs Tchad et Fitri ;

- l'épisode de très forte humidité de l'Holocène dit de la « période humide africaine » ou du « Sahara vert » (ca. 11 500-5 000 ans BP), qui s'accompagne à l'échelle du Sahara de la réactivation des réseaux hydrographiques, du développement de lacs, de l'installation d'une végétation et d'une faune de savane et de l'occupation humaine (e.g. De MEnocal et Tierney, 2012 ; BARD, 2013) et qui se traduit au Tchad notamment par l'installation du Mégalac Tchad (SCHUSTER, 2002 ; Schuster et al., 2014). 


\section{Résultats préliminaires}

Les principaux environnements de dépôts silicoclastiques identifiés dans le bassin du lac Fitri à partir de la reconnaissance géomorphologique sur images satellitaires sont les systèmes éoliens, fluviatiles au sens large et lacustres (principalement littoraux).

Hormis des pointements de roches du socle (granitoïdes), le paysage est très fortement dominé par d'anciens champs de dunes attribués au dernier maximum glaciaire. Les dunes éoliennes présentent diverses morphologies (transverses, linéaires, barchanoïdes) et dimensions (longueur, largeur, hauteur et longueur d'onde) (fig. 3). Cette diversité traduit des conditions d'écoulement des vents contrastées bien que les directions soient assez constantes (du NE vers le SO) suggérant des phases de mise en place différentes. Ces dunes sont antérieures aux autres milieux de dépôts identifiés comme en témoignent des incisions fluviatiles, des remaniements partiels par la dynamique littorale lacustre, le remplissage de dépressions interdunaires par des dépôts lacustres et palustres, et l'ennoiement partiel de dunes par les eaux du lac Fitri. Il convient de noter que la morphologie ainsi que l'organisation spatiale de certaines dunes diffèrent des modèles classiques ; il pourrait s'agir d'accumulations sableuses sousaquatiques liées à l'hydrodynamique dans le Mégalac Tchad (e.g. barres d'avant-côte).

Le principal système fluviatile est représenté par le Batha dont le cours est, selon les endroits, soit à faible sinuosité (rivière en tresses à barres longitudinales et transverses) soit à forte sinuosité (rivière en méandres à barres d'accrétion latérales ou point bars) (fig. 4 et 6). Dans sa partie distale, il s'étale en plusieurs branches pour former un delta de type fluvial fan (STANISTREET et MCCARTHY, 1993) ou distributary fluvial system (HARTLEY et al., 2010) pouvant rappeler le delta de l'Okavango (Botswana) (fig. 2 et 4). De nombreuses portions de chenaux à méandres abandonnés peuvent aussi être identifiées dans la région, notamment en relation avec des systèmes dunaires, et témoignent d'anciens réseaux hydrographiques qui restent à caractériser. Le cours actuel du Batha incise très fortement le cordon littoral du Mégalac Tchad (à l'est du Fitri, en aval de Ati), tandis que vers le sud, le cordon littoral du Mégalac Tchad est également affecté par des incisions fluviatiles plus discrètes (fig. 5).

Le pourtour du Mégalac Tchad (fig. 1) est marqué à l'échelle de tout le bassin par un cordon sableux qui montre des grandes structures littorales typiques (e.g. crêtes de plages, flèches sableuses, deltas) permettant de définir l'ancienne ligne de rivage de cet immense paléolac (SCHUSTER et al., 2005). Au sud-est du Fitri, de grandes rides arquées et organisées concentriquement depuis le rivage du Mégalac Tchad jusqu'au rivage du lac Fitri (fig. 5) marquent également d'anciens rivages intermédiaires et enregistrent la formation du proto-lac Fitri, initiée à la fin de l'optimum climatique de l'Holocène. 

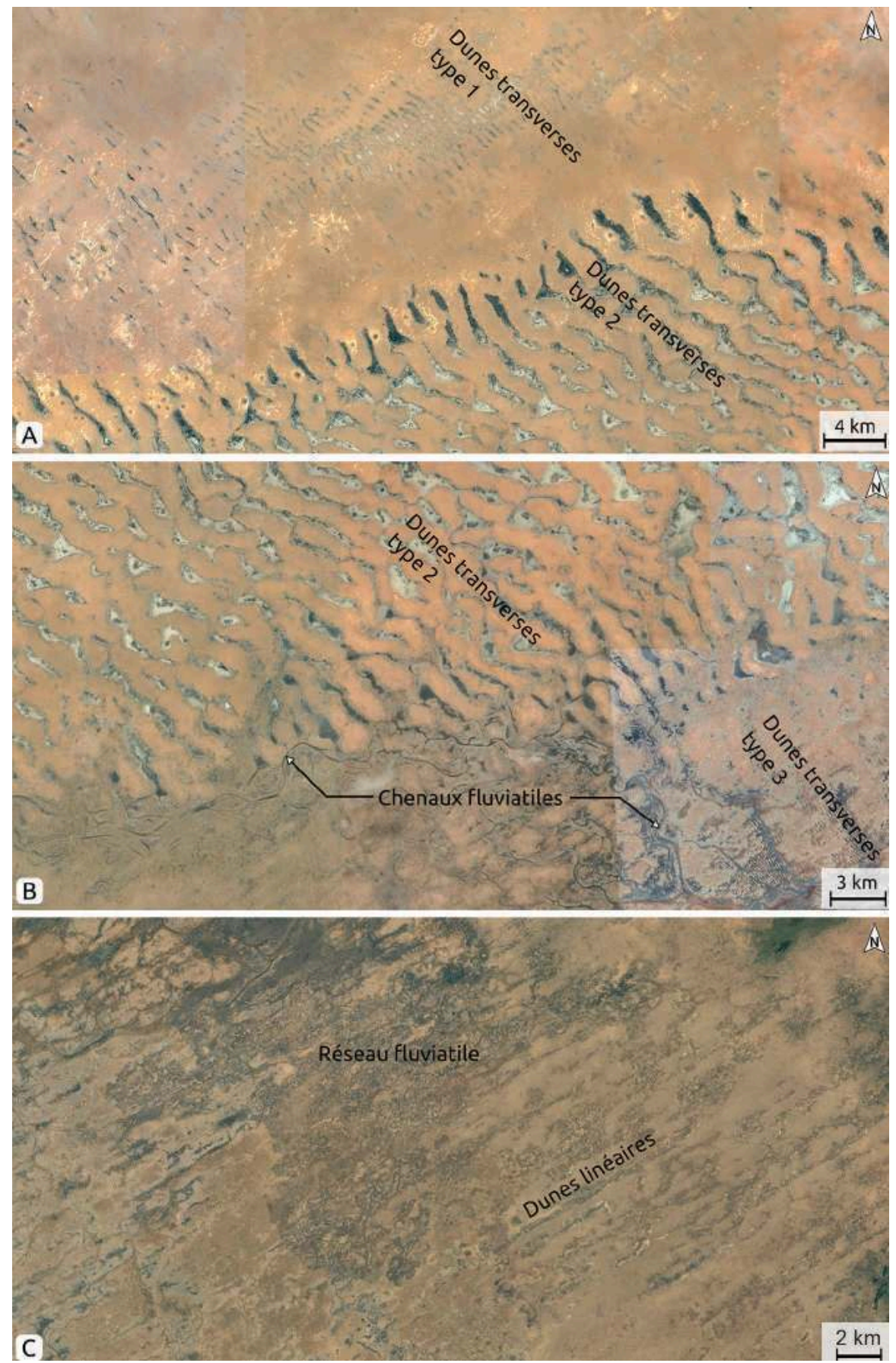

Figure 3

Vues satellite montrant certaines morphostructures éoliennes présentant diverses interactions avec des réseaux fluviatiles, voire avec des milieux lacustres et palustres au sein des dépressions interdunaires. Les divers types de dunes témoignent de directions de vents issus du NE-ENE.

$\boldsymbol{A}$ et $\mathbf{B}:$ Trois types de dunes transverses présentant trois longueurs d'onde distinctes.

C : Dunes linéaires partiellement altérées par un réseau hydrographique postérieur à leur mise en place.

Source : Google Earth Pro. 

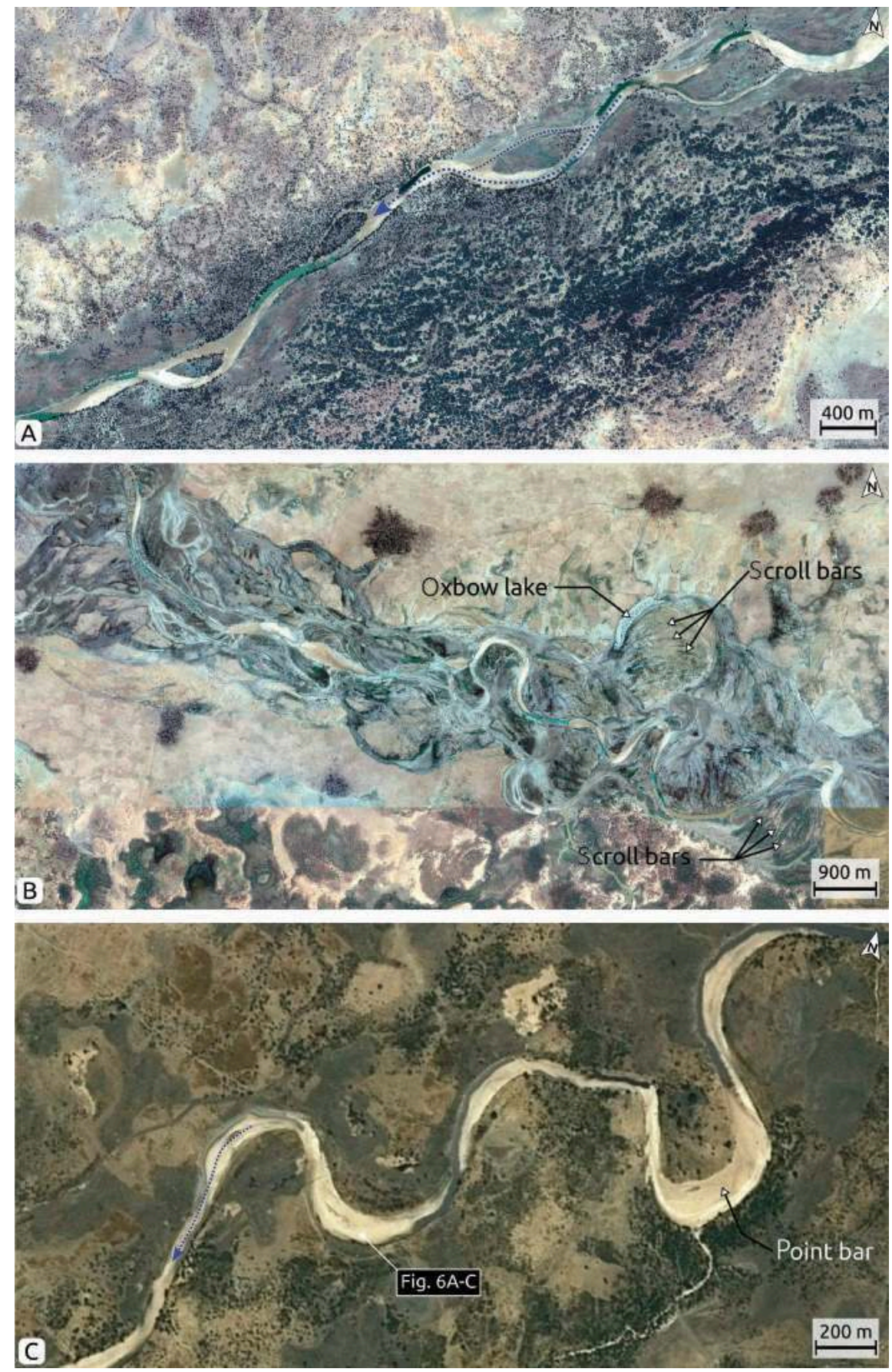

Figure 4

Vues satellite montrant certaines morphostructures fluviatiles associées à la rivière Batha.

A : Portion à faible sinuosité (aval d'Ati) montrant des barres fluviatiles longitudinales végétalisées et transverses dans le chenal. B : Portion à forte sinuosité (amont d'Ati) montrant des boucles de méandres abandonnées (oxbow lakes) et de grandes rides arquées (scroll bars) témoignant de la migration des méandres par accrétion latérale. $\boldsymbol{C}$ : Série de boucles de méandres dans le delta du Batha, montrant la zone en accrétion (accumulation de sable sous forme de mégarides point bars sur la berge convexe) et la zone en érosion (berge concave). 

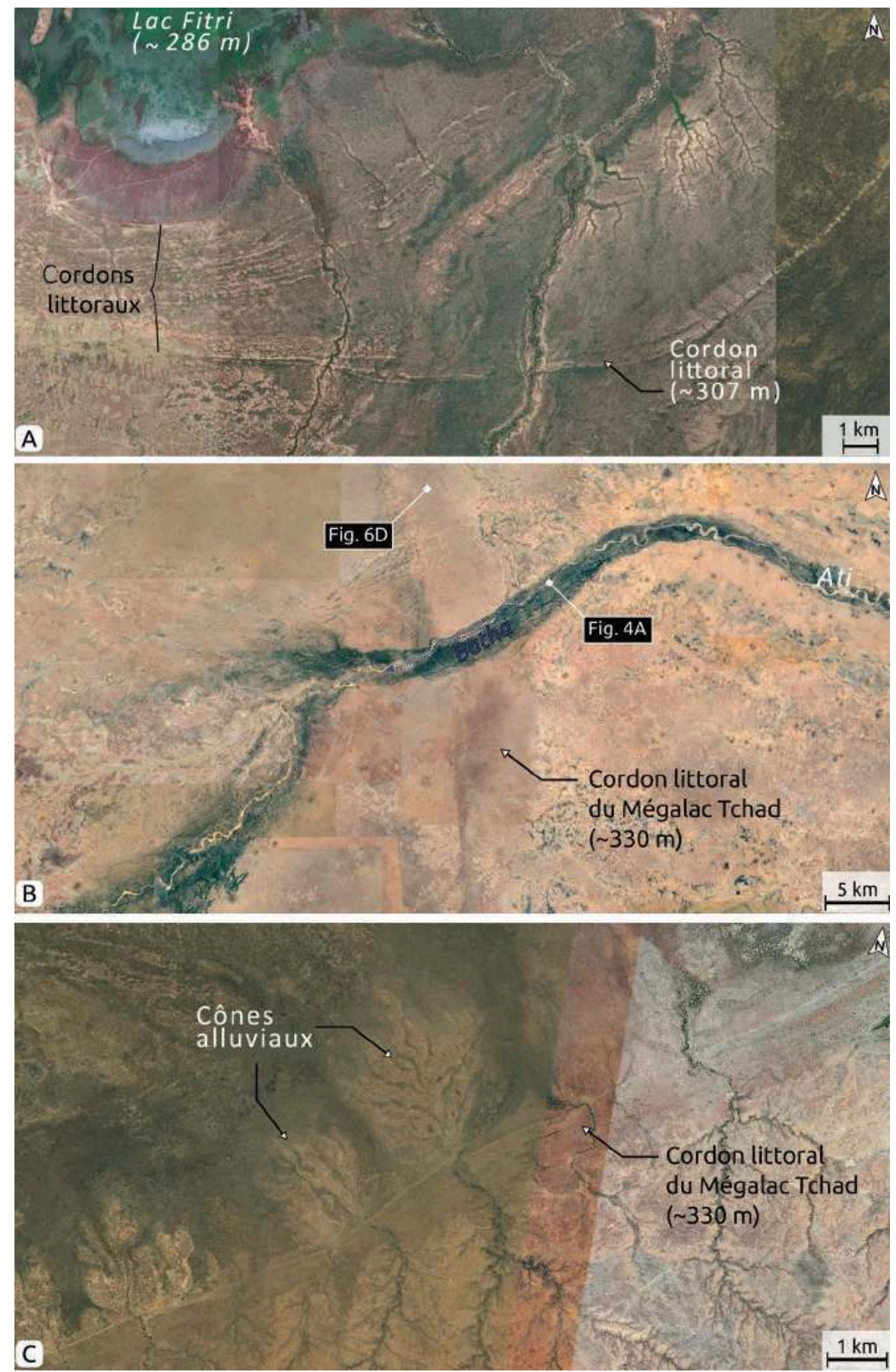

Figure 5

Vues satellite montrant certaines morphostructures littorales des anciens rivages du Mégalac Tchad et du proto-lac Fitri. A : Séquence de crêtes de plages intermédiaires entre le haut niveau du Mégalac et le niveau actuel du lac Fitri. B : Cordon littoral du Mégalac Tchad fortement incisé par la rivière Batha. À noter le chenal actif, les terrasses fluviatiles associées et les traces d'anciens chenaux. $\boldsymbol{C}$ : Cordon littoral du Mégalac Tchad vers le sud, affecté par l'érosion alluviale post-régression.

Source: Google Earth Pro. 

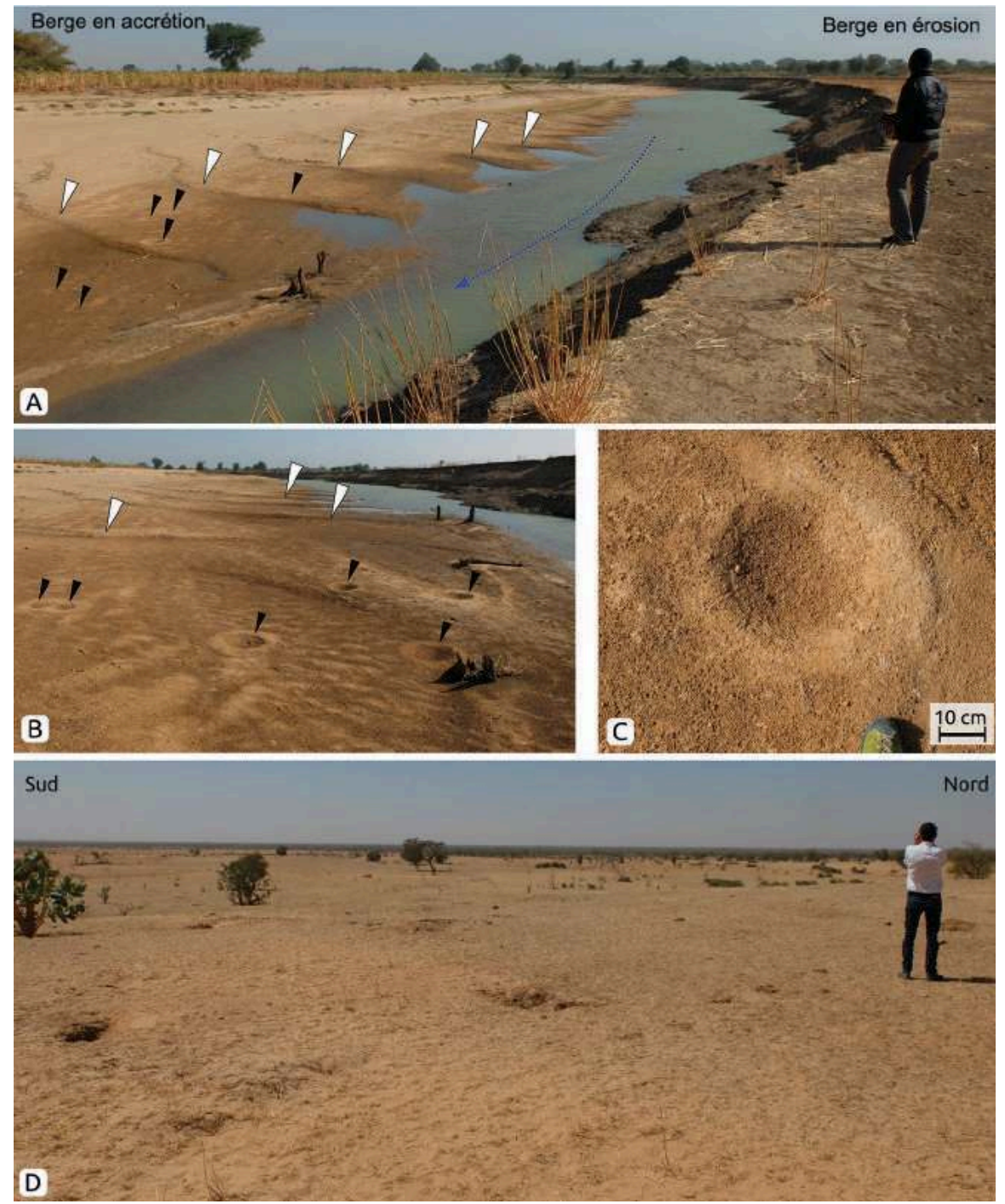

Figure 6

Illustrations de terrain, janvier 2016. A, B et C : Barre de méandre (point bar)

en cours de formation dans le Batha. Cette barre composite est constituée de mégarides amalgamées (flèches blanches : crêtes des mégarides) dont la forte asymétrie résulte du transport du sable par le courant d'eau qui les a générées. La surface des mégarides est affectée par des structures biosédimentaires (Piscichnus) en forme de cratère (flèches noires) interprétées comme des nids de poissons (Tilapias). D : Vue plongeante depuis le sommet du cordon littoral principal du Mégalac Tchad (ouest d'Ati); l'arrière-plan correspond au fond du paléolac et était, dans le passé, totalement inondé.

Se dégage alors un premier scénario de mise en place des paysages sédimentaires dans la région du Fitri (fig. 7). À l'Holocène inférieur, les ergs dunaires mis en place au Pléistocène et notamment à la fin du dernier maximum glaciaire sont ennoyés et se développe le Mégalac Tchad dans le contexte de l'optimum climatique de la période humide africaine. Le contour de l'actuel Fitri est inclus dans celui du Mégalac Tchad. Suite à la péjoration climatique, le Mégalac Tchad 
est en phase régressive (i.e. migration de la ligne de rivage vers le centre du bassin, diminution de la superficie et de la profondeur du lac) et apparaît un proto-lac Fitri (à l'instar du lac Tchad et d'un éphémère lac dans la dépression du Bodélé) qui s'individualise pour progressivement acquérir sa configuration actuelle.

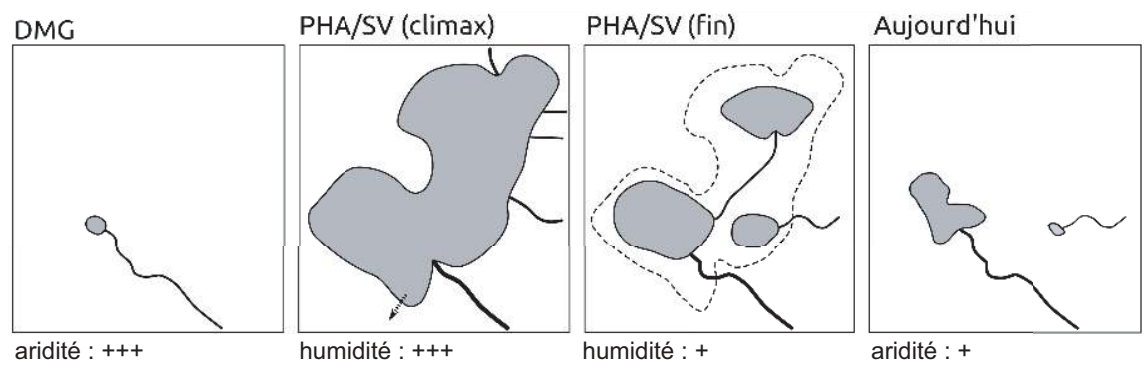

Figure 7

Modèle simplifié d'évolution des environnements lacustres dans le bassin du Tchad depuis la fin du dernier maximum glaciaire (DMG) jusqu'à aujourd'hui, en passant par la période humide africaine ou l'épisode dit du « Sahara vert » (PHA/SV).

\section{Perspectives}

Cette étude préliminaire souligne un solide potentiel de recherche (et de formation) en sédimentologie et en géomorphologie qui présente des implications académiques et appliquées fortes : restitution des paléoenvironnements et paléoclimats, systèmes sédimentaires analogues actuels pour décrypter des séries géologiques anciennes notamment du Tchad (i.e. paléoenvironnements de Toumaii), évaluation du potentiel réservoir (eau et hydrocarbures) de grands corps sédimentaires silicoclastiques, ou encore pour la typologie des stocks de sables. Par exemple, pour ce qui concerne la sédimentologie de faciès, le bassin du lac Fitri offre une remarquable diversité d'environnements sédimentaires continentaux actuels et subactuels facilement observables; il constitue à ce titre un cas d'étude exemplaire pour comprendre les interactions entre les milieux et processus de dépôts éoliens, fluviatiles, deltaïques et lacustres, tout en intégrant l'impact de la pédogénèse et de la bioturbation sur les dépôts primaires.

Les deux efforts majeurs à fournir dans le domaine de la restitution de l'évolution des paysages successifs au Fitri pour les années à venir concerneront d'une part la cartographie géomorphologique et la caractérisation sédimentologique (i.e. faciès sédimentaires, structures internes et architecture stratigraphique) détaillée des formations de surface à partir de l'imagerie satellite, à partir de l'observation directe sur le terrain et de la prospection géophysique de proche surface (Ground Penetrating Radar, résonance magnétique protonique), et d'autre part 
l'établissement d'un calendrier précis de mise en place des différents systèmes morphosédimentaires par des méthodes de datations absolues, en particulier la méthode par OSL (Optically Stimulated Luminescence ; PreusSER et al., 2008) parfaitement adaptée à ce contexte (e.g. datation des divers types de dunes, des cordons littoraux, des terrasses lacustres).

Les défis à relever seront d'arriver à collaborer avec les autres disciplines qui étudient le lac Fitri afin d'intégrer les diverses échelles spatiales et temporelles d'observation (i.e. carottages et imagerie sismique des fonds du lac, variabilité du lac par imagerie satellite), d'aboutir à une étude comparée de l'évolution des lacs Tchad et Fitri afin de mettre en avant les caractéristiques communes et identifier les différences, pour arriver à déconvoluer les forçages liés au climat et ceux liés aux particularités physiographiques des deux bassins. Enfin, une approche multidisciplinaire permettrait de contribuer au développement par une valorisation des connaissances acquises avec un transfert vers des domaines plus applicatifs comme la gestion des ressources en eau (e.g. cartographie hydrogéologique).

Cette étude permet de caractériser comment et à quelle vitesse réagissent les systèmes sédimentaires éolofluviolacustres, voire les écosystèmes associés, face aux forçages par le climat, avec des amplitudes et des durées variables. Cette question est fondamentale pour toute étude des environnements et climats du passé. Aujourd'hui, dans le contexte du défi global que représente le changement climatique, elle révèle toute sa modernité et son adéquation avec des questions sociétales. En effet, elle est essentielle pour anticiper le devenir des écosystèmes (i.e. résilience et adaptation) les plus sensibles à la variabilité du climat, tels que les zones humides du Sahel, où la pression anthropique va vraisemblablement continuer de s'accroître.

\section{Remerciements}

Nous remercions très chaleureusement le programme pluridisciplinaire Gelt, et à travers lui toutes les personnes et organismes tchadiens et français qui l'ont imaginé, qui lui ont donné vie, qui l'ont rendu possible et qui ont contribué à son bon déroulement, depuis les missions sur le terrain jusqu'au colloque de restitution. Nous remercions deux reviewers anonymes pour leur relecture pertinente de ce travail. Mathieu Schuster remercie Jean-François Ghienne et Claude Roquin pour les discussions sans fin. Cet article est une contribution à l'initiative MALaBaR : Modern and Ancient Lake Basins Research. 


\section{Bibliographie}

\section{BARD E., 2013}

Out of the African Humid Period. Science, 342 : 808-809.

\section{Bouchette F., Schuster M., Ghienne J.-F., Denamiel C., Roquin C., Moussa A., DURINGER P., 2010 \\ Hydrodynamics in the Holocene Lake Mega- Chad. Quaternary Research, 73 : 226-236.}

De Menocal P. B., Tierney J. J., 2012 Green Sahara: African Humid Periods Paced by Earth's Orbital Changes. Nature Education Knowledge, 3 (10) : 12.

Hartley A. J., Weissmann G. S., Nichols G. J., WARWICK G. L., 2010 Large Distributive Fluvial Systems: Characteristics, Distribution, and Controls on Development. Journal of Sedimentary Research, 80 : 167-183.

\section{Lemoalle J., 1987}

« Lac Fitri ». In : Burgis M. J., Symoens J. J., éd. : African Wetlands and Shallow Water Bodies. Zones humides et lacs peu profonds d'Afrique. Paris, Orstom, coll. Travaux et documents, 221, $650 \mathrm{p}$.

\section{Moupeng B., 2006}

Le lac Fitri : dynamique du système hydrographique quaternaire et actuel (Sahel tchadien). Thèse de doctorat, université de Provence, Avignon, 171 p.

\section{Nutz A., Schuster M., 2016}

Stepwise drying of Lake Turkana at the end of the African Humid Period: A forced regression modulated by solar activity variations? Solid Earth, 7 : 1609-1618.

\section{Nutz A., Schuster M., Ghienne J.-F., Roquin C., Bouchette F., 2018}

Wind-driven waterbodies: a new category of lake within an alternative sedimentologically-based lake classification. Journal of Paleolimnology, 59 : 189-199.

Paillou P., Schuster M., Farr T., Tooth S., Rosenqvist A., Lopez S., 2009

Mapping of a Major Paleodrainage System in Eastern Libya using Orbital Imaging Radar: The Kufrah River. Earth and Planetary Science Letters, 277 : 327-333.
Preusser F., Degering D., Fuchs M., Hilgers A., Kadereit A., Klasen N., KrbetscheK M., Richter D., Spencer J., 2008

Luminescence dating: Basics, methods and applications. E\&G Quaternary Science Journal, 57 : 95-149.

Raimond C., Zakinet D., Mugelé R., Kemsol Nagorngar A., Mbagogo A., YALIKUN T., BRAHIM BÉCHIR A., Madjigoto R., Schuster M., Sylvestre F., DESCHAMPS P., 2017

«Les nouveaux enjeux pour le lac Fitri, entre variabilité environnementale, croissance démographique et conflits d'usage ». In : Livre des résumés étendus du colloque international Recherches croisées sur les écosystèmes lacustres tchadiens, N'Djamena, Tchad, 25-27 avril 2017, $601 \mathrm{p}$.

SCheffers A. M., Scheffers S. R., Kelletat D. H., 2012

The Coastlines of the World with Google Earth: Understanding our Environment. Coastal Research Library 2, Springer, Dordrecht, 293 p.

SCHUSTER M., 2002

Sédimentologie et paléoécologie des séries à vertébrés du paléolac Tchad depuis le Miocène supérieur. Thèse de doctorat, université Louis Pasteur de Strasbourg, 152 p.

Schuster M., Nutz A., 2018

Lacustrine wave-dominated clastic shorelines: modern to ancient littoral landforms and deposits from the Lake Turkana Basin (East African Rift System, Kenya). Journal of Paleolimnology, 59 : 221-243.

Schuster M., Roquin C., Durand A., Moussa A., Ghienne J.-F., Allenbach B., DURinger P., Bouchette F., 2014

Shorelines of the Holocene Megalake Chad (Africa, Sahara) investigated with very high resolution satellite imagery (Pléiades): example of the Goz Kerki paleo-spit. Revue française de photogrammétrie et télédétection, 208 : 63-68.

Schuster M., Roquin C., Duringer P., Brunet M., Fontugne M., Mackaye H. T., VignAud P., GHIENNE J.-F., 2005

Highlighting Holocene Lake Mega-Chad 
paleoshorelines from space. Quaternary Science Reviews, 24 : 1821-1827.

Stanistreet I. G., MCCARThy T. S., 1993

The Okavango Fan and the classification

of subaerial fan systems. Sedimentary Geology, 85 : 115-133.
YALIKUN T., RAIMOND C., Kemsol Nagorngar A., Zakinet D., Schuster M., Sylvestre F.

« Variabilité des crues et des paysages du Fitri depuis les grandes sécheresses des années 19701980 ». Ce volume. 



\title{
aléoenvironnements
}

\section{et variations paléohydrologiques du lac Tchad au cours des 12000 dernières années}

\author{
Florence SYLVESTRE, Pierre DESCHAMPS, \\ Adoum Bari SININE, Remadji RIRONGARTI, \\ Jean-Charles MAZUR, Nicolas WaLdMANN, \\ Paula do Amaral, Camille Bouchez, \\ Jean-Claude Doumnang
}

\section{Introduction}

L'Afrique subsaharienne, au cours des 20000 dernières années, a connu une évolution du climat sensiblement différente de celle qu'elle connaît aujourd'hui. En effet, après des conditions extrêmement arides au cours du dernier maximum glaciaire (DMG) il y a 20000 ans, des conditions plus humides et plus chaudes se sont mises en place pendant la phase de déglaciation et l'Holocène moyen, permettant l'extension des nappes d'eau et de la végétation (GASSE, 2000 ; Hoelzmann et al., 2004). Entre environ 11000 et 5000 ans, les sociétés néolithiques évoluent dans un Sahara verdoyant, alimenté par des cours d'eau et des lacs permanents, accompagné par une recharge des aquifères (KUPER et KRÖPELIN, 2006).

Ces conditions climatiques ont été possibles grâce à une configuration orbitale optimale qui a favorisé la migration vers le nord de la zone de convergence intertropicale, entraînant le front de mousson jusqu'aux latitudes sahariennes (BRACONNOT et al., 2000). Cette circulation de mousson est renforcée par 
l'augmentation de l'insolation d'été sur l'hémisphère Nord, induite par le cycle orbital de précession (BERGER et LOUTRE, 1991).

Toutefois les modèles climatiques montrent que des rétroactions positives sont nécessaires pour rendre compte de l'amplitude des changements observés et notamment de la rapidité du retour aux conditions arides (CLAUSSEN et al., 1999). En effet, malgré ce consensus sur la description de l'évolution paléoclimatique au cours de cette période, de nombreuses questions restent encore débattues. La durée, l'intensité et la transition entre ces phases climatiques associées à la réponse des écosystèmes sont très discutées (BARD, 2013). Par exemple, la fin de la période humide et le retour aux conditions arides actuelles il y a 5000 ans font l'objet de nombreux débats car certains enregistrements montrent une transition extrêmement abrupte (DE MENOCAL et al., 2000), alors que d'autres montrent un passage progressif des conditions humides à la phase aride (KRÖPELIN et al., 2008). Ces transitions sont d'autant plus discutées qu'elles nous renseignent sur la réponse des écosystèmes aux modifications du climat, notamment dans un contexte de changements des paramètres globaux : l'insolation qui, en Afrique subsaharienne, détermine la position de la zone de convergence intertropicale et du front de mousson, et par conséquent des précipitations.

Dans ce contexte de changements climatiques, le lac Tchad offre l'opportunité de reconstituer l'évolution des paléoenvironnements et du climat. Dans ce lac, ont été obtenues des archives sédimentaires continues couvrant la période humide holocène et la transition vers la période aride actuelle.

Dans cette présentation, nous allons décrire les reconstitutions des paléoenvironnements à partir de l'analyse des assemblages de diatomées fossiles. En effet, les diatomées sont reconnues comme d'excellents bio-indicateurs de la variabilité des milieux aquatiques (BATTARBEE et al., 2001). Elles renseignent sur la variabilité des niveaux lacustres ainsi que sur la chimie de l'eau (e.g. salinité) dans laquelle elles se développent. Constituées d'un squelette siliceux, elles se préservent particulièrement bien dans les sédiments, et leur ubiquité à l'échelle de la planète permet de connaître avec précision leurs affinités écologiques. En outre, dans le lac Tchad elles sont reconnues comme le groupe du phytoplancton le plus développé (LemOALLE, 1979).

\section{Sites d'étude}

Le lac Tchad est une très vaste étendue d'eau douce, située à la limite entre Sahara et Sahel entre $12^{\circ} 20^{\prime}-14^{\circ} 20^{\prime} \mathrm{N}$ et $13-15^{\circ} 20^{\prime} \mathrm{E}$ à $280 \mathrm{~m}$ d'altitude au-dessus du niveau de la mer (fig. 1). Il est constitué de deux cuvettes, l'une au nord, l'autre au sud, séparées par une zone de hauts fonds appelée « la grande 
barrière ». Une troisième zone située à l'est, appelée « archipels » correspond à une zone de dunes plus ou moins ennoyées saisonnièrement en fonction des apports en eau reçus par le lac. Reconnu comme le quatrième lac du continent africain, le lac Tchad connaît une très forte variabilité à différentes échelles de temps, saisonnière, annuelle, pluridécennale à millénaire. En effet, au cours des quarante dernières années, il est passé de $25000 \mathrm{~km}^{2}$ dans les années 1950-1960 à moins de $2500 \mathrm{~km}^{2}$ à la fin des années 1980 pour revenir à une superficie de plus de $10000 \mathrm{~km}^{2}$ depuis le début des années 2000 (LemOALle et MAGRIN, 2014).

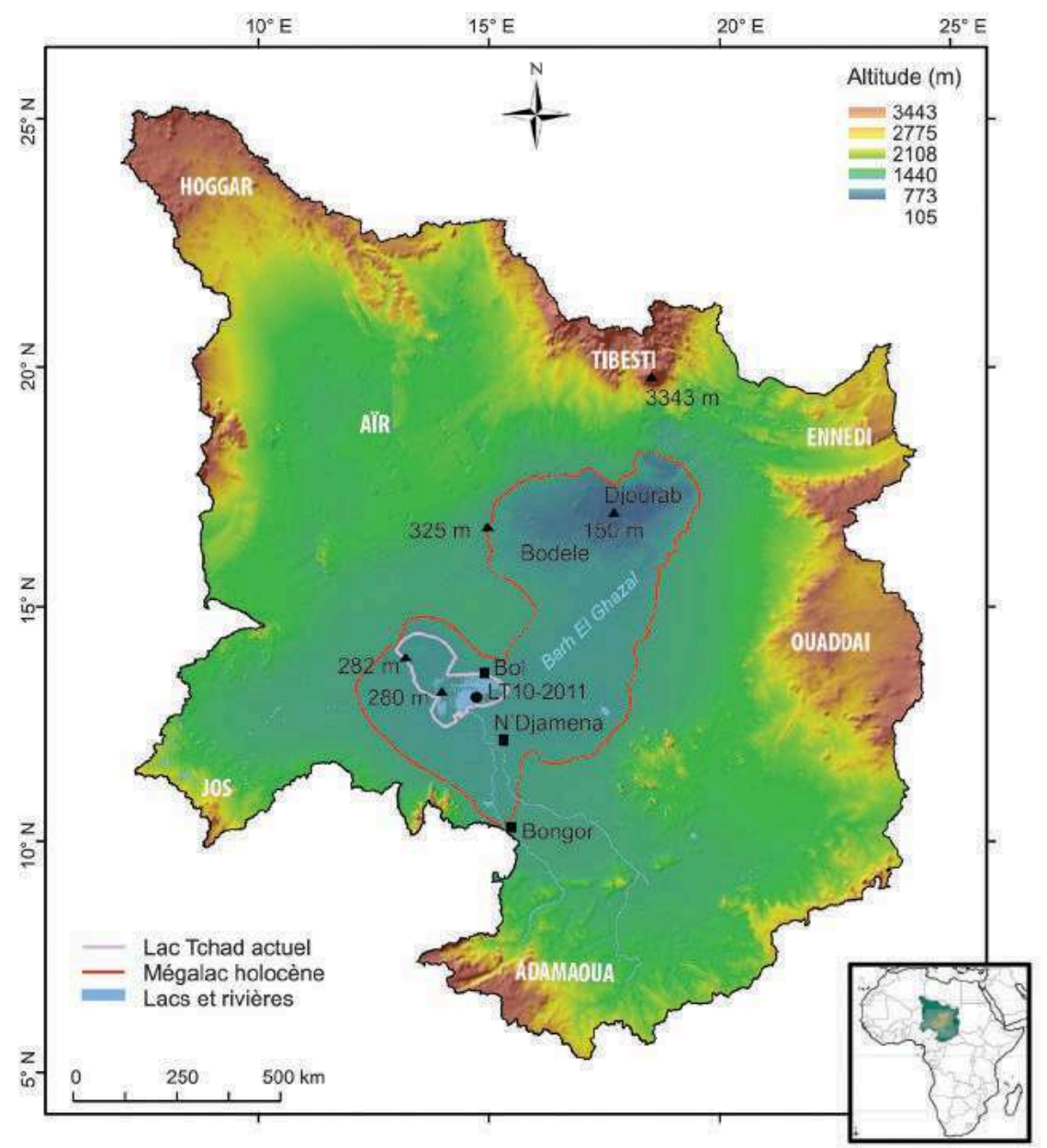

Figure I

Bassin versant du lac Tchad et localisation de la carotte LTI0-20II.

Le trait rouge correspond à l'extension maximale du lac Tchad pendant l'Holocène moyen ; le trait rose délimite la position moyenne du lac Tchad actuel.

Source : USGS. Figure réalisée avec la MNT GTopo 3 et les données Hydrolk. 
Son bassin versant couvre une superficie de $2400000 \mathrm{~km}^{2}$, représentant $8 \%$ $\mathrm{du}$ continent africain. Les parties hydrologiques actives aujourd'hui sont, au sud, le bassin du Chari (610 $000 \mathrm{~km}^{2}$ ) avec son affluent principal le Logone, à l'ouest le bassin de la Komadougou Yobé (174 $\left.000 \mathrm{~km}^{2}\right)$, auxquels s'ajoutent les petits bassins de l'El Beïd, du Yedseram et Ngadda au Nigeria (environ $30000 \mathrm{~km}^{2}$ ). L'alimentation en eau de surface est assurée à $85 \%$ par le fleuve Chari, le reste des apports provenant des tributaires secondaires et des précipitations reçues directement à la surface du lac (OLIVRY et al., 1996). Sa superficie et son niveau résultent principalement de la pluie sur le bassin du Chari. Lorsque la pluie diminue de $10 \%$ sur ce bassin, le débit du fleuve varie de $30 \%$, ce qui induit une variation proportionnelle de la superficie du lac (Lemoalle et al., 2012). Les pertes en eau du lac se font essentiellement par évaporation, estimée en moyenne à $2 \mathrm{~m}$ par an et par infiltration estimée à $300 \mathrm{~mm}$ par an (Bouchez et al., 2016).

\section{Matériel}

Une carotte sédimentaire a été réalisée dans le lac Tchad en 2011 à l'aide d'un carottier Wright installé sur une barge ancrée sur deux bateaux (photo 1). La carotte LT10-2011 a été prélevée dans la cuvette sud du lac (fig. 1). Elle mesure $5,81 \mathrm{~m}$ de long et présente une lithologie relativement homogène composée d'argiles et de limons sur la totalité de sa longueur, à l'exception de sa base qui est constituée de sables fins. En effet, cette carotte atteint un niveau de sables correspondant à un ancien champ de dunes recouvert par les sédiments lacustres holocène, identifiés grâce à une campagne d'acquisition sismique opérée en même temps que le prélèvement de la carotte LT10-2011.

\section{Méthodes}

L'enregistrement sédimentaire obtenu a fait l'objet de datations par le radiocarbone pour établir sa chronologie absolue. Treize datations sur matière organique totale ont été réalisées sur la carotte LT10-2011. Seule une datation à la base du sondage a été réalisée sur des microcharbons extraits à partir du sédiment.

Toutes les datations sont exprimées en âge calendaire (ans cal BP), converti via le logiciel Calib 6.0 (REIMER et al., 2004). Le modèle d'âge à partir d'interpolation linéaire a été réalisé à l'aide du logiciel $R$. 


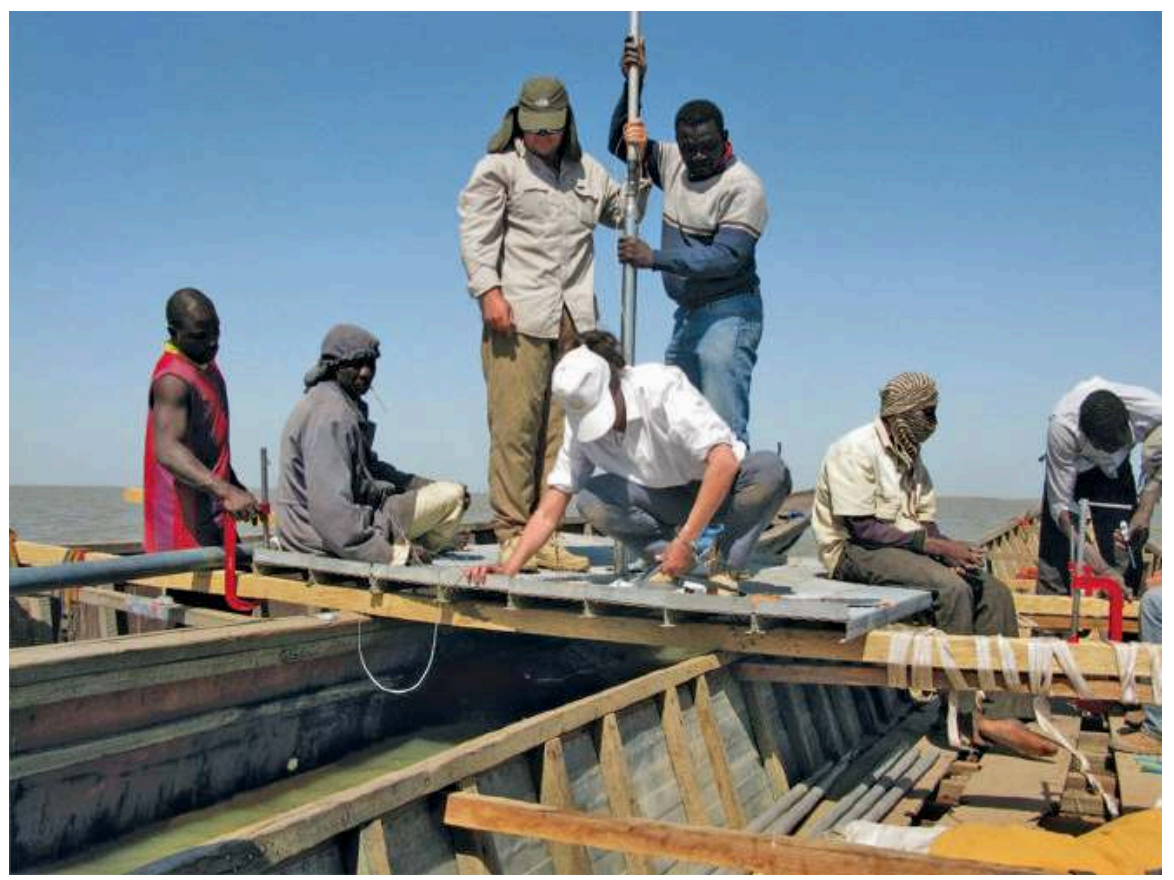

Photo I

Photographie illustrant l'opération de carottage de LTI0-20I I dans le lac Tchad en 20 I I.

(c) Camille Bouchez.

La carotte LT10-2011 a fait l'objet de nombreuses analyses sédimentologiques, géochimiques, palynologiques (AMARAL et al., 2013). Nous présentons ici uniquement les résultats obtenus à partir de l'étude des diatomées fossiles contenues dans les sédiments.

Les diatomées fossiles ont été analysées dans 36 échantillons à partir de 0,5 à 1 gr de sédiment. L'extraction des diatomées a été réalisée avec la méthode classiquement utilisée qui consiste à extraire uniquement le contenu siliceux du sédiment par des traitements chimiques $\left(30 \% \mathrm{H}_{2} \mathrm{O}_{2}, 10 \% \mathrm{HCl}\right)$ et physiques (séparation des phases sédimentaires par décantation en colonne) (BATTARBEE et al., 2001). À partir du résidu siliceux contenant les diatomées, un montage entre lame et lamelle est réalisé pour son observation en microscopie optique photonique (Nikon Eclipse 80i, grossissement $1000 \mathrm{x}$ avec interférentiel Nomarski, n.a. = 1,32). L'unité de comptage étant la valve de diatomées, un minimum de 500 valves est déterminé par échantillon. Les déterminations taxonomiques sont réalisées à partir d'un grand nombre d'ouvrages, dont les plus consultés sont SERVANT-VILDARY (1978) et GASSE (1986). La systématique utilisée repose sur celle établie par KRAMMER et LANGE-BERTALOT (1986, 1988, 1989, 1991). Toutefois des synonymies ont été introduites sur la base de la révision de la taxonomie introduite par Round et al. (1990). 
L'interprétation écologique des diatomées est basée d'une part sur la connaissance de l'autoécologie de chaque espèce à partir de la littérature, et d'autre part sur un référentiel actuel composé d'observations réalisées sur les lacs tchadiens (RIRONGARTI et SYLVESTRE, données non publiées ; RIRONGARTI, 2014) et africains (GASSE et al., 1995).

Les résultats des comptages sont exprimés en abondance relative (\% du total de valves comptées). Une extraction des espèces dominantes composant les assemblages a été réalisée à partir d'analyses statistiques multivariées (analyse factorielle par correspondance, classification hiérarchique) à l'aide du logiciel XLStat 2012.

\section{Résultats}

Les 13 datations par radiocarbone obtenues sur la carotte LT10-2011 montrent une cohérence stratigraphique de la base jusqu'à $236 \mathrm{~cm}$. Les âges obtenus sur les premiers $236 \mathrm{~cm}$ indiquent une remobilisation des sédiments. Ces phénomènes syn- ou post-sédimentaires s'observent très fréquemment dans les grands lacs lorsqu'ils atteignent une profondeur relativement faible et sont soumis à un régime de vent fréquent (VERSCHUREN, 1999), comme c'est le cas pour le lac Tchad (Olivry et al., 1996). De plus, après 5000 ans cal BP, le lac Tchad a connu de nombreuses phases d'assèchement (SERVANT et SERVANT-VILDARY, 1980), ce qui a pu également conduire à des hiatus dans la sédimentation. Par conséquent, nous présentons les résultats entre $236 \mathrm{~cm}$ - datés à 4700 ans cal BP - et la base de la carotte datée à 11900 ans cal BP.

Cinquante espèces de diatomées réparties en 23 genres ont été identifiées dans la carotte. Les assemblages de diatomées sont caractérisés par des espèces benthiques indicatrices de bas niveaux lacustres, des espèces planctoniques caractérisant à l'inverse de hauts niveaux du lac et/ou des surfaces d'eau libres, et des formes tychoplanctoniques qui vont marquer des phases de plus grande variabilité du niveau lacustre, notamment pendant des phases de transgression et/ou de régression. De plus, la détermination des espèces de diatomées dans la carotte LT10-2011 montre une remarquable stabilité au niveau des conditions de salinité du lac. Toutes les espèces sont indicatrices de milieu d'eau douce.

La figure 2 montre la succession des diatomées fossiles dominantes en fonction du temps. La base de l'enregistrement est dominée par des espèces benthiques. Très rapidement, elles sont remplacées par des espèces tychoplanctoniques, dominées par Fragilaria construens à $90 \%$. À partir de 10800 ans cal BP, ces espèces sont remplacées par des formes planctoniques du genre Aulacoseira qui domine les assemblages jusqu'à 5000 ans cal BP. Toutefois à partir de 8000 ans cal BP, on observe une augmentation de l'espèce Pantoscekiella ocellata jusqu'à 


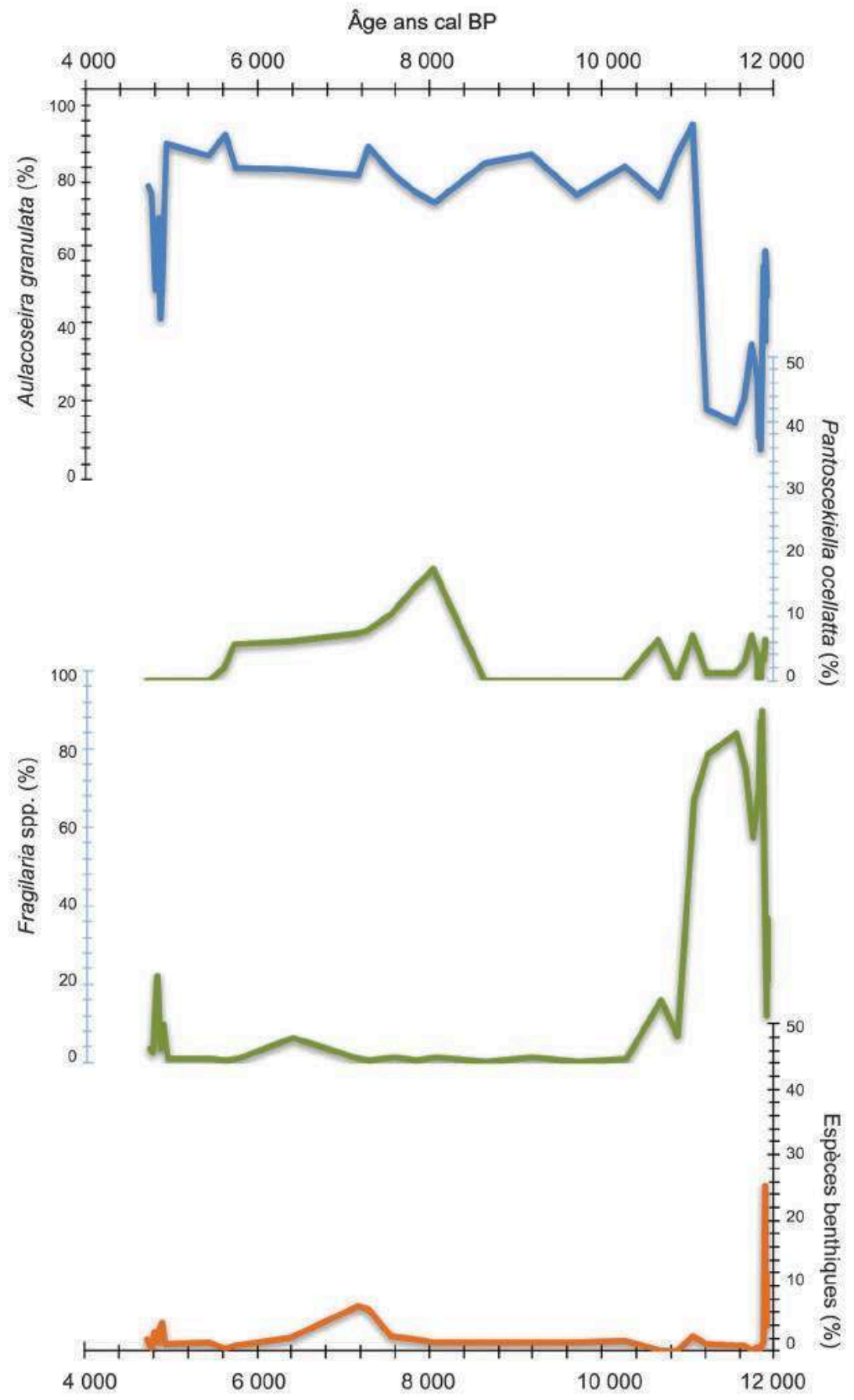

Figure 2

Abondance relative (\%) des espèces dominantes de diatomées composant les assemblages fossiles dans le lac Tchad. 
5700 ans cal BP associée à des formes benthiques. À partir de 4800 ans cal BP, les espèces du genre Aulacoseira diminuent et sont associées à des espèces benthiques et tychoplanctoniques.

\section{Reconstitution des paléoenvironnements lacustres}

Après une phase extrêmement aride enregistrée au cours du DMG et de la dernière déglaciation, la carotte LT10-2011 montre que le lac Tchad s'est remis en eau à partir de 11900 ans cal BP. Les assemblages de diatomées, dominés par des espèces benthiques indiquent une phase de bas niveau à la base de la carotte correspondant à la remise en eau du lac. Très rapidement, les apports en eau se sont intensifiés, indiqués par la dominance d'espèces tychoplanctoniques d'eau douce suggérant une reprise des apports en provenance du Chari-Logone. Ces apports se sont poursuivis jusqu'à 10800 ans cal BP, date à partir de laquelle les assemblages de diatomées montrent que le lac a dû atteindre un stade de haut niveau lacustre relativement stable, permettant ainsi le développement des espèces planctoniques du genre Aulacoseira. Toutefois, à partir de 8000 ans cal BP, Pantoscekiella ocellata, indicatrice d'une plus forte variabilité du plan d'eau et de faible profondeur, souvent associée à de la végétation (e.g. phragmites ; SERVANT-VILDARY, 1978) apparaît dans les assemblages. Elle est suivie par une augmentation des espèces benthiques à 7000 ans cal BP et de la présence des diatomées tychoplanctoniques à 6300 ans cal BP. À partir de 5700 ans cal BP, un plan d'eau relativement stable s'est à nouveau installé, avant de connaître une diminution plus marquée à partir de 4800 ans cal BP.

\section{Implications paléoclimatiques}

Cette reconstruction montre une remise en eau du lac dès la fin de la dernière déglaciation, immédiatement après l'épisode froid du Younger Dryas (12 50011900 ans cal BP), qui semble être caractérisé en Afrique subsaharienne par une période de sécheresse (GASSE, 2000). D'après cet enregistrement, il ne semble pas que le lac ait enregistré une remise en eau antérieure, par exemple au cours du Bölling-Alleröd (14 700-12 700 ans cal BP), comme cela est observé dans d'autres sites (DE MENOCAL et al., 2000 ; GASSE, 2000 ; LÉzINE et al., 2011). Cet enregistrement montre également que les apports en eau ont été très importants entre 12000 et 10800 ans cal BP. Il est généralement admis que le 
développement des conditions humides au début de l'Holocène en Afrique subsaharienne a été possible grâce à une configuration orbitale optimale dès la fin de la dernière déglaciation (BRACONNOT et al., 2007). Ces conditions ont permis une augmentation des températures sur les latitudes tropicales, favorisant une progression vers le nord de la zone de convergence intertropicale sur le continent, entraînant ainsi une pénétration de la mousson. De plus, cette progression de la mousson a été favorisée par un gradient de température entre le continent et l'océan et des effets rétroactifs entre l'état de surface continentale et l'atmosphère (CLAUSSEN et al., 2006). Ces conditions optimales se sont traduites par une augmentation des précipitations à l'échelle de l'Afrique subsaharienne qui ont conduit à une élévation des nappes d'eau comme l'atteste l'enregistrement sédimentaire du lac Tchad à partir de 11900 ans cal BP. La phase de plus hauts niveaux lacustres semble atteinte à partir de 10800 ans cal $\mathrm{BP}$ et serait restée stable jusqu'à 5000 ans cal BP, en accord avec les données sur la partie Nord du bassin du lac Tchad (ARMitage et al., 2015) et de l'ensemble de l'Afrique subsaharienne (LÉZINE et al., 2011). Pendant cette phase, le lac Tchad aurait atteint sa phase d'extension maximale, appelé ainsi « Mégalac » avec une superficie de $350000 \mathrm{~km}^{2}$ (SCHUSTER et al., 2005).

Toutefois, il semblerait que la fin de la période humide holocène dans le lac Tchad ait débuté plus tôt que généralement admis. En effet, on date la fin de cette période vers 5000 ans cal BP (DE MeNOCAL et al., 2000) alors que le lac semble amorcer une diminution de son niveau dès 8000 ans cal BP, indiquée par la présence d'espèces benthiques et tychoplanctoniques. Cette phase de diminution des niveaux lacustres s'accélère dès 4900 ans cal BP. Une diminution progressive du niveau du lac Tchad a déjà été mise en évidence par des données polliniques réalisées sur un carottage voisin de celui présenté dans cette étude (AMARAL et al., 2013). Les grains de pollen ainsi qu'une reconstitution des précipitations à partir de ces données ont montré qu'une mise en place progressive des conditions arides a débuté dès 6700 ans cal BP.

\section{Conclusion}

Les diatomées fossiles préservées dans les sédiments du lac Tchad mettent en évidence les grandes phases de l'histoire hydrologique de l'Afrique subsaharienne à partir de la dernière déglaciation et jusqu'à l'Holocène moyen. Ces données originales contribuent à préciser leur chronologie, en mettant notamment en évidence que la fin de la période humide en Afrique subsaharienne a certainement été amorcée plus précocement vers 8000 ans cal BP et de manière progressive. Après 5000 ans cal BP, les environnements lacustres semblent avoir évolué plus rapidement vers des conditions de plus forte aridité. 


\section{Remerciements}

Ce travail a été effectué dans le cadre des accords de coopération entre l'université de N'Djamena, Aix-Marseille Université et l'IRD et de la convention de recherche entre le Centre national de recherche et de développement tchadien (CNRD) et l'IRD. Nous remercions l'IRD pour son soutien dans le cadre du projet « Lac Tchad ». Nous remercions le CNRD et l'université de N'Djamena pour leur aide logistique au cours des missions de terrain sur le lac Tchad. Nous remercions également le Cerege pour son appui pour la partie analytique.

\section{Bibliographie}

Amaral P. G. C., Vincens A., Guiot J., Buchet G., Deschamps P., Doumnang J. C., Sylvestre F., 2013

Palynological evidence for gradual vegetation and climate changes during the African humid period termination at 13 degrees $\mathrm{N}$ from a MegaLake Chad sedimentary sequence. Climate of the Past, 9 : 223-241.

\section{Armitage S., Bristow C., Drake N., 2015}

West African monsoon dynamics inferred from abrupt fluctuations of Lake Mega-Chad. PNAS 112 : 8543-8548.

Battarbee R. W., Jones V. J., Flower R. J., Cameron N. G., Bennion H., 2001

« Diatoms ». In : Smol J. P. et al., éd. : Tracking Environmental Change using Lake Sediments, The Netherlands, Dordrecht, Kluwer Academic Publishers : 155-202.

\section{BARD E., 2013}

Out of the African Humid Period. Science, 342 : 808-809.

\section{Berger A., Loutre M.-F., 1991}

Insolation values for the climate of the last 10 millions of years. Quaternary Science Reviews, 10 : 297-317.

Bouchez C., Goncalves J., Deschamps P., Vallet-Coulomb C., Hamelin B., Doumnang J. C., Sylvestre F., 2016 Hydrological, Chemical, and Isotopic Budgets of Lake Chad: A Quantitative Assessment of Evaporation, Transpiration and Infiltration Fluxes. Hydrology and Earth System Sciences. 20 (4) : 1599-1619. https://doi.org/10.5194/ hess-20-1599-2016.

Braconnot P., Marti O., Joussaume S., LECLAINCHE Y., 2000

Ocean Feedback in Response to $6 \mathrm{Kyr}$ BP Insolation, Journal of Climate, 13 : 1537-1553.

Braconnot P., Otto-Bliesner B., Harrison S., Joussaume S., Peterchmitt J.-Y., Abe-Ouchi A., Crucifix M., Driesschaert E., Fichefet Th., Hewitt C. D., Kageyama M., Кitoh A., Loutre M.-F., Marti O., Merkel U., Ramstein G., Valdes P., Weber L., Yu Y., ZhAO Y., 2007

Results of PMIP2 coupled simulations of the Mid-Holocene and Last Glacial Maximum. Part 1: experiments and large scale features. Climate of the Past, 3 : 261-277.

Claussen M., Kubatzki C., Brovkin V., Ganopolski A., Hoelzmann P., Pachur H. J., 1999

Simulation of an abrupt change in Saharan vegetation in the mid-Holocene. Geophysical Research Letters, 26 : 2037-2040.

Claussen M., Fohlmeister J., Ganopolski A., Brovkin V., 2006 Vegetation dynamics amplifies precessional forcing. Geophysical Research Letters, 33 : L09709, 1-4. 
De Menocal P., Ortiz J., Guilderson T., Adkins J., SARnthein M., BAKer L., YARUSINSKY M., 2000

Abrupt onset and termination of the African Humid Period: rapid climate responses to gradual insolation forcing. Quaternary Science Reviews, $19: 347-36$.

\section{Gasse F., 1986}

East African diatoms: taxonomy and ecological distribution. Bibliotheca Diatomologica,

Band 11. Cramer, Stuttgart, 201 p.

GASSE F., 2000

Hydrological changes in the African tropics since the Last Glacial Maximum. Quaternary Science Reviews, 19 : 189-211.

Gasse F., Juggins S., Ben Khelifa L., 1995 Diatom-based transfer functions for inferring past hydrochemical characteristics of African lakes. Palaeogeography, Palaeoclimatology,

Palaeoecology, 117 : 31-54.

Hoelzmann P., Gasse F., Dupont L. M.,

Salzmann U., Staubwasser M.,

Leuchner D. C., Sirocko F., 2004

Palaeoenvironmental changes in the arid and subarid belt (Sahara-Sahel-Arabian Peninsula) from $150 \mathrm{ka}$ to present. In : Battarbee R. W. et al., éd. : Past Climate through Europe and Africa, Dordrecht, Springer, 219-256.

Kröpelin S., Verschuren D., LéZine A.-M., Eggermont H., Cocquyt C., Francus P., Cazet J.-P., Fagot M., Rumes B., Russell J. M., Conley D. J., Schuster M., Von Suchodoletz H., Engstrom D. R., 2008 Climate-driven ecosystem succession in the Sahara: the last 6000 years. Science, 320 : 765-768.

Krammer K., Lange-Bertalot H., 1986

Susswasserflora von Mitteleuropa.

Bacillariophyceae 1. Teil: Naviculaceae.

Gustav Fischer Verlag, Jena. 876 p.

Krammer K., Lange-Bertalot H., 1988

Susswasserflora von Mitteleuropa.

Bacillariophyceae 2. Teil: Bacillariaceae, Epithemiaceae, Surirellaceae. Gustav Fischer Verlag, Jena. 596 p.

Krammer K., Lange-Bertalot H., 1989 Achnanthes. Eine monographie der Gattung. Cramer J., Berlin-Stuttgart, 393 p.
Krammer K., Lange-Bertalot H., 1991

Susswasserflora von Mitteleuropa.

Bacillariophyceae 3. Teil: Centrales, Fragilariaceae, Eunotiaceae. Gustav Fischer Verlag, Jena. 576 p.

KUPER R., KRöPELIN S., 2006

Climate-Controlled Holocene Occupation in the Sahara: Motor of Africa's Evolution. Science, 313 : 803-807.

\section{Lemoalle J., 1979}

Biomasse et production phytoplanctoniques du lac Tchad (1968-1976). Relations

avec les conditions de milieu. Thèse de doctorat, université Paris 6, 287 p.

\section{Lemoalle J., Bader J.-C., LeblanC M.,} SEDICK A., 2012

Recent changes in Lake Chad: Observations, simulations and management options (19732011). Global and Planetary Change, 80-81 : 247-254.

Lemoalle J., Magrin G., DIR., 2014 Le développement du lac Tchad : situation actuelle et futurs possibles. CBLT, N'Djamena, Marseille, Expertise collégiale IRD, AFD-FFEM, rapport de synthèse, $67 \mathrm{p}$. + traduction anglaise et contributions intégrales des experts $(\mathrm{CD}$, 20 chapitres, 620 p.).

\section{Lézine A.-M., Hély C., Grenier C., Braconnot P., Krinner G., 2011 \\ Sahara and Sahel vulnerability to climate changes, lessons from Holocene hydrological data. Quaternary Science Review, 30 : 3001-3012.}

Olivry J.-C., Chouret A., Vuillaume G., LemoAlle J., BRICQUeT J.-P., 1996 Hydrologie du lac Tchad. Paris, Orstom éditions, $265 \mathrm{p}$.

Reimer P. J., Baillie M. G. L., Bard E., Bayliss A., BeCK J. W., Bertrand C. J. H., Blackwell P. G., Buck C. E., Burr G. S., Cutler K. B., Damon P. E., Edwards R. L., Fairbanks R. G., Friedrich M., Guilderson T. P., Hogg A. G., Hughen K. A., Kromer B., McCormac G., Manning S., Ramsey C. B., Reimer R. W., Remmele S., Southon J. R., Stuiver M., Talamo S., Taylor F. W., Van Der Plicht J., WeyhenMeyer C. E., 2004 IntCal04 terrestrial radiocarbon age calibration, 0-26 cal kyr BP. Radiocarbon, 46 : 1029-1058. 


\section{RIRONGARTI R., 2014}

Étude de la dynamique algaire dans le lac Tchad : le cas des diatomées. Mémoire de master 2, université de N'Gaoundéré, Cameroun, $30 \mathrm{p}$.

Round F. E., Crawford R. M., Mann D. G., 1990

The Diatoms, Biology \& Morphology of the genera. Cambridge University Press, $747 \mathrm{p}$.

Schuster M., Roquin C., Duringer P., Brunet M., Caugy M., Fontugne M., Mackaye Taïsso H., Vignaud P., GHienne J. F., 2005

Holocene Lake Mega-Chad palaeoshorelines from space. Quaternary Science Review, 24 : 1821-1827.

\section{Servant M., Servant-Vildary S., 1980}

L'environnement quaternaire du bassin du Tchad. In : Williams M. A. J., Faure H., éd. : The Sahara and the Nile. Quaternary Environments and prehistoric occupation in northern Africa, Balkema, Rotterdam, The Netherlands, p. 113-162.

\section{SERVANT-VILdARY S., 1978}

Étude des diatomées et paléolimnologie du bassin tchadien au Cénozoïque supérieur. Paris, Orstom, coll. Travaux et documents, 84, $346 \mathrm{p}$.

\section{VERSCHUREN D., 1999}

Sedimentation controls on the preservation and time resolution of climate-proxy records from shallow fluctuating lakes. Quaternary Science Reviews, 18 : 821-837. 


\title{
Des hommes et des lacs
}

\section{Peuplement des zones lacustres du Borkou (Tchad) à l'Holocène}

\author{
Yves GAUTHIER, \\ Christine GAUTHIER
}

\section{Introduction}

Avec la récente mise à disposition des images satellite en haute résolution sur l'ensemble de la planète, l'archéologie a fait un bond qualitatif et quantitatif, et cela plus particulièrement pour les zones désertiques du fait de l'absence de couvert végétal. En effet, il est maintenant possible de détecter des structures en pierres sèches de 2 à $3 \mathrm{~m}$ d'envergure et ainsi d'inventorier la plupart des monuments (funéraires ou non) et restes d'habitats (désignés sous le terme « enclos » dans la suite du document) et cela sur la quasi-totalité du Sahara auquel nos recherches sont dédiées.

Aux dizaines ou quelques centaines de constructions lithiques connues jusque-là par des études de terrain, des dizaines de milliers d'autres sont venues s'ajouter, ouvrant la voie à des études plus détaillées et à grande échelle. Ces images (localisées sur les fig. 6 et 7) permettent en particulier d'établir des cartes de distribution de constructions datant essentiellement de la seconde moitié de l'Holocène. Parallèlement, ces données satellitaires offrent la possibilité de reconstituer l'étendue des centaines, voire des milliers de paléolacs ou marais qui parsemaient alors le Sahara, principalement dans sa moitié sud.

Par ailleurs, gravures et peintures rupestres nous renseignent sur bien des particularités des populations et notamment sur des aspects souvent indiscernables 
dans les autres restes archéologiques. Dans ce travail, nous montrons comment la mise en relation des données de ces diverses sources d'information conduit à une meilleure compréhension du peuplement de la région du Borkou, de la distribution des enclos et de l'évolution des modes de subsistance sous l'effet de la péjoration climatique qui a entraîné la disparition de la quasi-totalité des surfaces en eau.

\section{Pêche et navigation}

Les peintures relevées dans les massifs septentrionaux présentent majoritairement des scènes d'élevage depuis les phases les plus anciennes jusqu'aux plus récentes (Bailloud, 1997 ; Gauthier et Gauthier, 2017). De nombreuses scènes de chasse, pour certaines publiées, sont par ailleurs connues (BAILlOUD, 1997). En revanche, celles qui évoquent un milieu aquatique restent rarissimes.

De fait, à l'échelle du Sahara, on ne connaît qu'une cinquantaine de sites, dont cinq au Tchad, qui ont livré des images de poissons (GAUTHIER et GAUTHIER, 2011). De probables Clarias sp. sont illustrés à Fofoda au nord-est du Tibesti (BocCAzZI et BocCAZzI, 1999). Depuis 2012, nous avons nous-mêmes eu l'occasion d'en observer sur quatre autres sites. Deux de ces représentations, découvertes respectivement à Gaora Hallagana et à proximité d'Archeï (fig. 1a et $1 \mathrm{~b}$ ), apparaissent indépendamment de tout élément de contexte. En revanche, les poissons de Tohil (fig. 2c) et d'Elikéo (fig. 1c) figurent dans des scènes qui illustrent deux méthodes différentes de pêche. Si leur identification n'est pas aisée, on peut néanmoins penser qu'il s'agit d'espèces fréquentes dans les paléolacs du nord du Tchad : Lates niloticus, Clarias sp., Hydrocynus sp. ou Tilapia sp. comme pourraient l'indiquer les taches du poisson de la figure 1b.

Tout aussi rares sont les images attestant de la navigation au Sahara à l'Holocène. Seuls 24 sites ont révélé des images de bateaux. Sept d'entre eux illustrent des voiliers ayant sillonné les océans vers le début de notre ère et ne concernent donc pas la navigation en milieu saharien (GAUTHIER et GAUTHIER, 2010). Les 17 sites restant ont livré 29 images de pirogues. Huit de ces sites se trouvent au Tchad, pays qui totalise plus des deux tiers des représentations de barques connues (20 sur 29). Les plus nombreuses (8) proviennent de la falaise d'Artchana (HuARD et MASSIP 1964a; GAUthIER et GAUTHIER, 2018). Six autres, dont trois seulement étaient auparavant connues (ARCANGIOLI, 1996), ont été peintes dans l'abri de Tohil qui s'ouvre directement sur un paléolac. Les autres sont dispersées dans différents abris : à Bodhoué (JACQUET, 2001), à Orori et deux autres lieux de toponyme inconnu (fig. 2) à Taïtroa vers Faya et dans un autre site du nord-est de l'Ennedi (peintures inédites). Les barques sont parfois assez grandes pour embarquer cinq passagers (fig. 2b). 

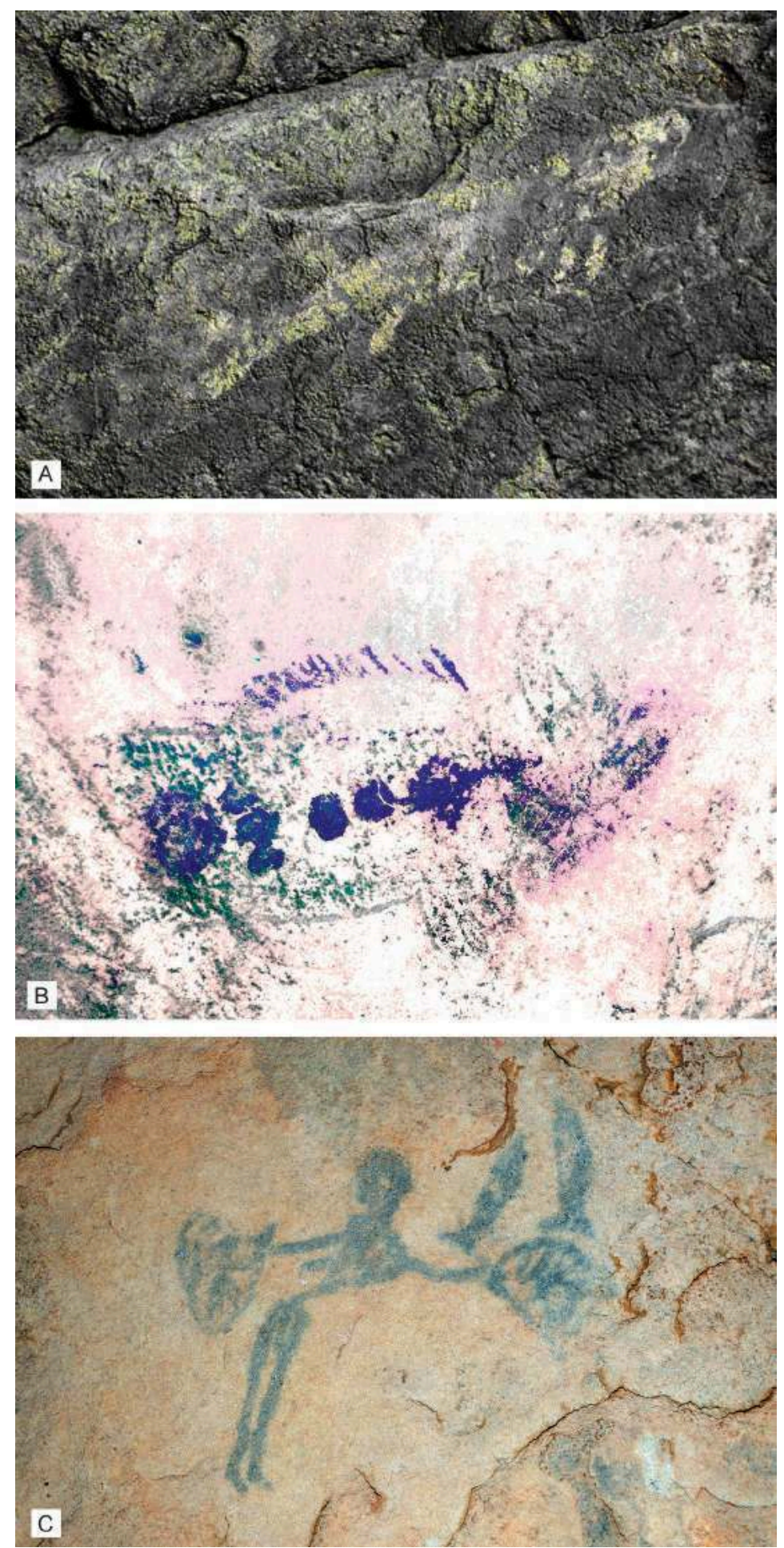

Figure I

Poissons (Ennedi). a) Gaora Hallagana ;

b) Archeï, probable Tilapia ; c) Elikéo, nasses et poissons. 

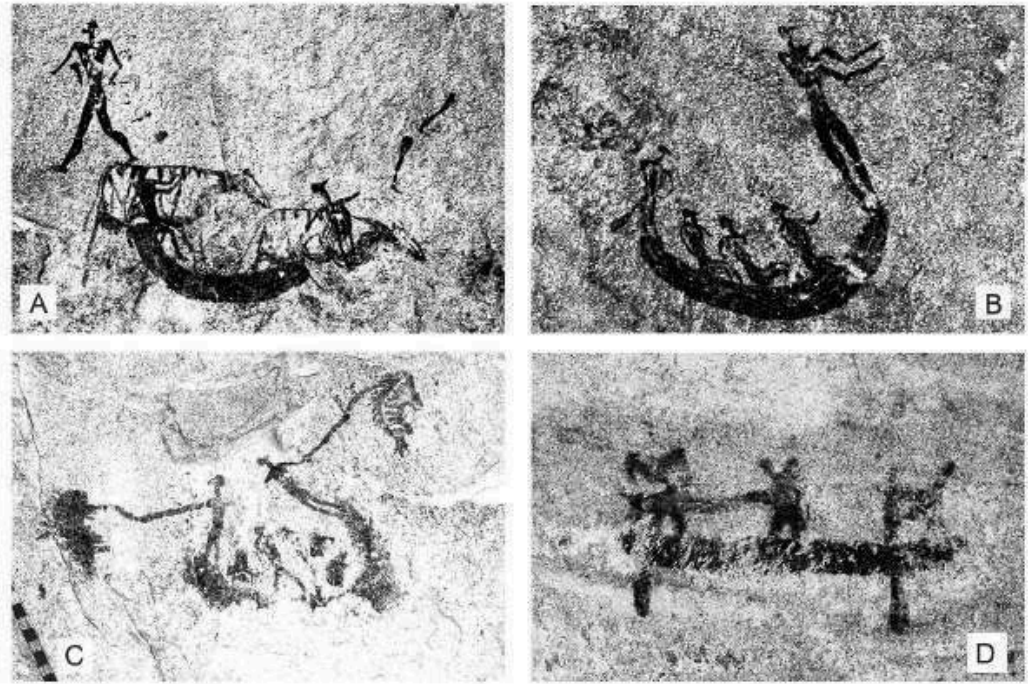

Figure 2

Barques et pêche à la ligne. a-c) Tohil, (Borkou); d) Orori (Borkou). À noter, les deux poissons au bout des lignes (c).

Les dimensions, proportions et formes des coques sont très variables : les plus plates peuvent correspondre à des barques monoxyles, comme celle de Dufuna (BREUNIG, 1996) ; les plus arquées pourraient être constituées de roseaux comme sembleraient l'indiquer les liens transversaux sur la pirogue de Bodhoué qui évoquent les embarcations utilisées au début du $\mathrm{Xx}^{\mathrm{e}}$ siècle sur le lac Tchad (BIDAULT, 1945). Rien ne permet toutefois d'exclure d'autres modes de fabrication, à base d'écorce, de planches cousues ou d'ambatch (Aeschynomene elaphroxylon). En dépit de leur nombre restreint, ces documents attestent la diversité des techniques de construction et la maîtrise de la navigation au cours du Néolithique.

Les embarcations ne servaient pas qu'au transport. À leur bord, les utilisateurs pratiquaient la pêche ainsi que le détaillent les peintures de Tohil. Sur trois des barques représentées, on constate la présence d'individus qui se tiennent debout une ligne à la main. Pour se convaincre qu'il s'agit bien de scènes de pêche, il suffit de remarquer les poissons remontés par certains protagonistes (fig. 2c).

À Artchana, une peinture suggère que la pêche se pratiquait aussi à l'épervier (GAuthier et Gauthier, 2018). Soit en complément d'un autre dispositif, soit comme instrument principal, la nasse a également fait partie des techniques de pêche : à Elikéo, des poissons apparaissent au-dessus des deux nasses manipulées par un personnage (fig. 1c).

L'abri d'Artchana II est très riche puisqu'on y relève des pêcheurs équipés de harpons à deux rangs de barbelures (ibid. et fig. 3). La pêche au harpon est confirmée par la découverte de plusieurs pièces à proximité d'Artchana, près du rivage du Mégalac Tchad lorsque celui-ci atteignait son plus haut niveau et plus généralement dans la région de Faya (HuARD et MASsIP, 1964b). Certains 
de ces harpons ont été trouvés mêlés à des ossements de poissons (COURTIN, 1964a, 1964b, 1965). Toujours à Artchana, un personnage tient une foène d'un côté et une épuisette de l'autre. Compte tenu du contexte aquatique (harpons, foène, épuisette, barques), il n'est pas aberrant de penser que, sur la figure 3, les lignes parallèles qui bordent la scène représentent un barrage à poissons. À noter qu'à ce jour, aucune scène de pêche au filet n'a été reconnue ni au Tchad ni ailleurs au Sahara.

Comme en Égypte pharaonique, les harpons servaient pour la chasse aux animaux aquatiques, une activité elle aussi décrite à Artchana (GAUTHIER et GAUTHIER, 2018 : fig. 22). Installé à la proue d'une barque, un chasseur tente de harponner un hippopotame. Ce type d'activité est rare dans l'iconographie saharienne. La peinture d'Artchana atteste là encore de la maîtrise de la navigation qu'avaient ces populations.

Quoique moins exceptionnelles, les figurations de sauriens sont rares. Particulièrement extraordinaire est la scène de chasse au crocodile représentée à Tohil. Un de ces sauriens y apparaît cerné par des personnages qui lui ont transpercé la tête avec un harpon ou une lance (ARCANGIOLI, 1996). Couché devant l'animal, l'un des protagonistes tente de le museler, probablement à l'aide d'un anneau de corde (fig. 4).
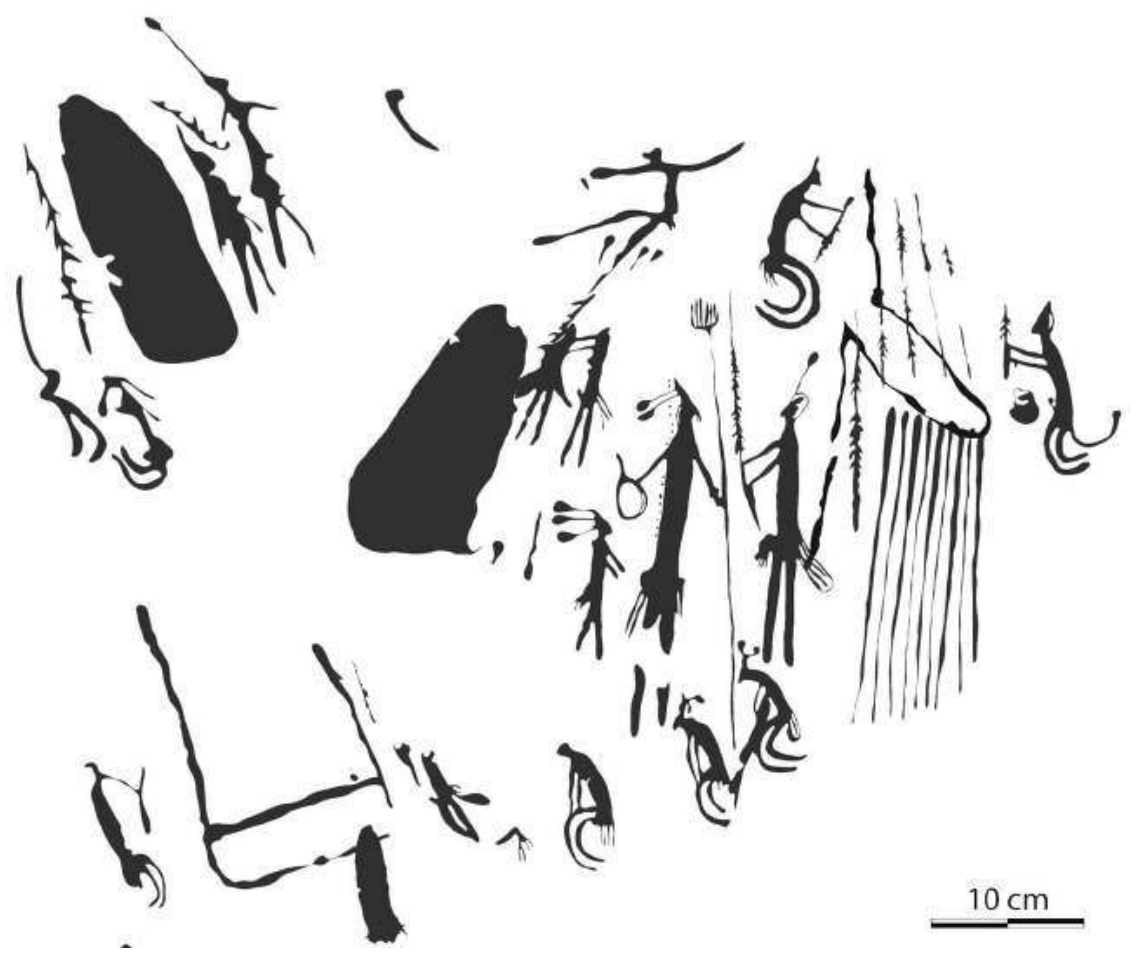

Figure 3

Artchana II. Pêcheurs munis de harpons, d'une foène et d'une épuisette devant un possible barrage à poissons. Voir localisation sur la figure 9. Relevé d'après photo. 


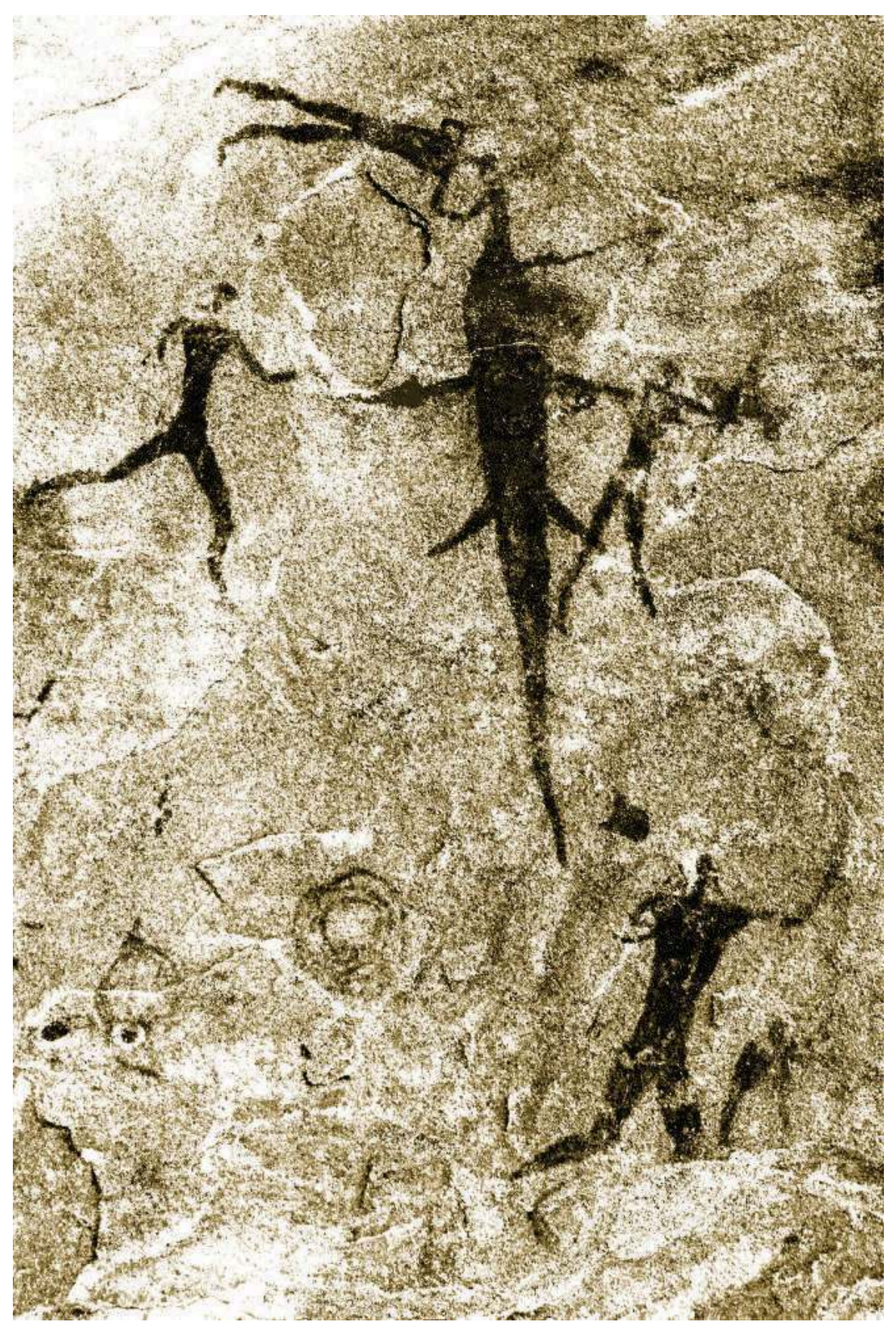

Figure 4

Tohil. Chasse au crocodile. 
Toutes ces illustrations rendent compte d'une exploitation variée des milieux aquatiques. Couplée à la présence de harpons et d'ossements de poissons sur les sites archéologiques ${ }^{1}$, cette documentation iconographique témoigne du fait que les lacs et des zones humides participaient de manière majeure à la subsistance de certaines populations sahariennes du milieu de l'Holocène. L'intérêt de ces populations pour les milieux aquatiques et les zones humides peut s'apprécier d'une tout autre manière.

\section{Archéologie des enclos}

\section{Caractéristiques et fonction des enclos}

Les populations qui se sont succédé durant l'Holocène ont laissé de multiples traces de leur passage. L'une d'elle n'a pas encore fait l'objet d'une exploitation optimale. Dans la littérature relative au BET (Borkou, Ennedi, Tibesti), sont mentionnés des cercles de pierres répartis sur une quinzaine de sites (HUARD et Massip, 1967). Ces structures, dont les diamètres vont de quelques mètres à $120 \mathrm{~m}$, présentent des caractéristiques diverses. Alors que certaines ne sont constituées que d'un simple contour de pierres (maximum $\sim 20 \mathrm{~cm}$ ), d'autres forment une double ligne ou sont délimitées par des blocs de grande taille $(\geq 50 \mathrm{~cm}$ ). Seule cette dernière catégorie va nous intéresser ici.

Quelques exemples permettent de mieux cerner les formes, les dimensions et le mode de construction de ces structures (fig. 5a). La première structure, qui a été vue au sol, est située près du village de Yéné (fig. 9). Les autres ont été reconnues sur des images satellitaires (fig. 5b-c). Plutôt que « cercles de pierres », il nous semble préférable de dénommer ces structures « enclos » car, si certaines sont subcirculaires, beaucoup sont elliptiques ou subrectangulaires, voire plus complexes.

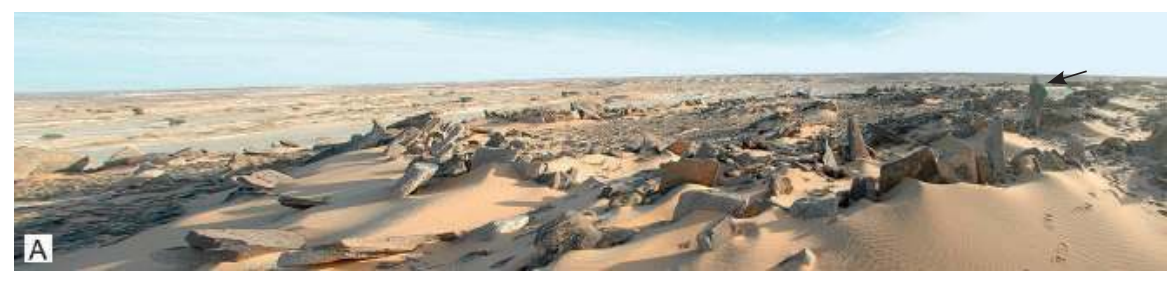

Figure 5a

Borkou. Enclos sur promontoire, délimité par un muret en plaques.

En arrière-plan, le fond du Mégalac Tchad. La flèche localise le personnage qui donne l'échelle.

I. C'est vrai à Délebo I où des céramiques wavy line et dotted wavy line sont accompagnées d'os d'hippopotames et de poissons (BAILLOUD, 1997). 

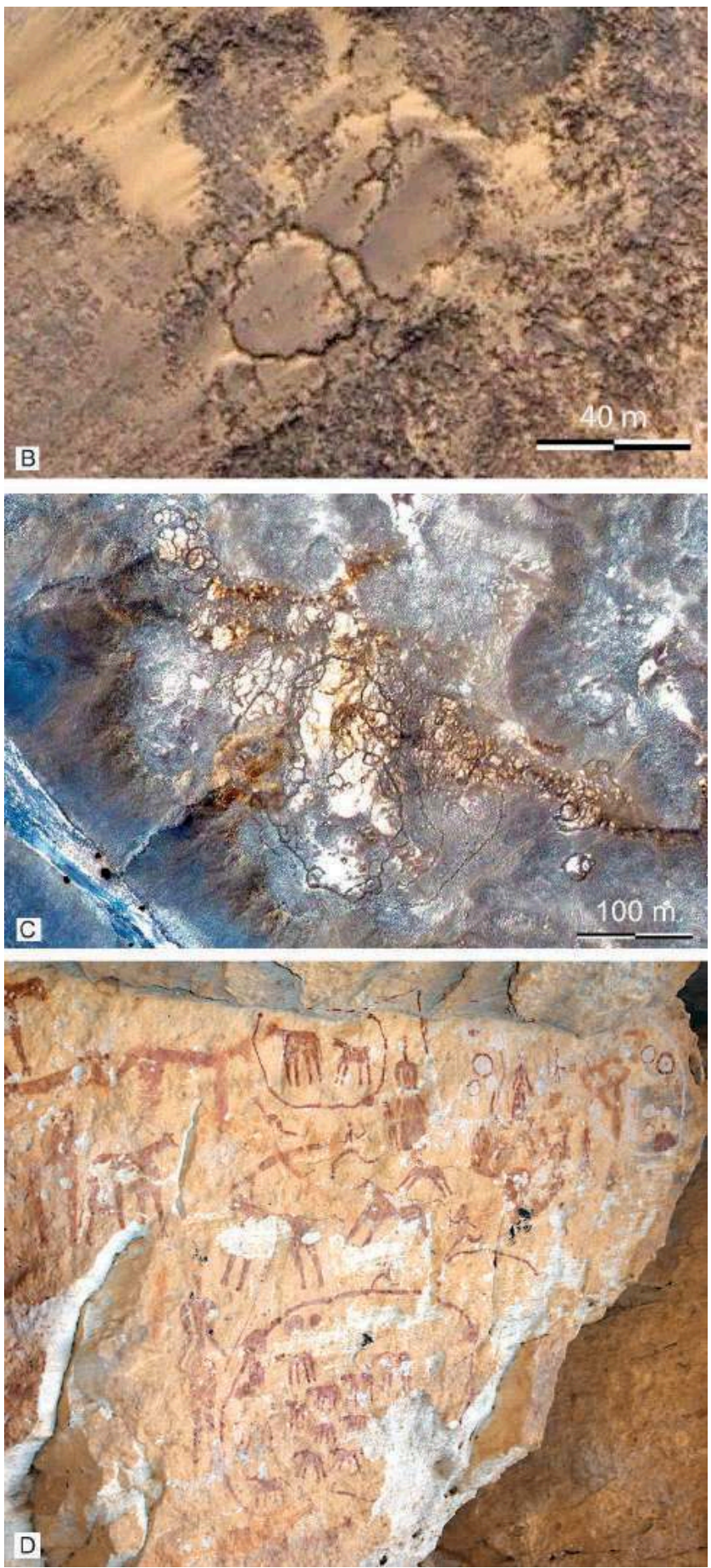

Figure 5 (suite)

b, c) Borkou et Tibesti.Vue aérienne de différents enclos, compartimentés ou non. d) Béchiké (Ennedi). Bovins confinés dans des enclos. 
L'extension maximale des enclos varie de 10 à $750 \mathrm{~m}$. S'ils sont fréquemment d'un seul tenant, se résumant à un muret d'enceinte, beaucoup sont compartimentés, délimitant des espaces de surface très variables dans lesquels on trouve, parfois, des cercles plus petits $(\leq 5-6 \mathrm{~m})$. Le schéma rappelle celui des kraals d'Afrique du Sud, avec des espaces dédiés aux animaux, d'autres réservés à l'habitat. Il est tout à fait vraisemblable que ces enclos et les kraals aient eu des fonctions similaires. Compte tenu de leur dimension imposante, ils paraissent plutôt destinés à parquer des bovins, les espaces plus petits étant dédiés aux ovins et aux caprins. Les cercles de quelques mètres de diamètre peuvent être interprétés comme la trace au sol des habitats alors que les cercles plus petits $(\leq 2 \mathrm{~m})$ pourraient correspondre à des constructions servant au confinement et à la protection des chevreaux et agneaux, comme cela se pratique chez les Touareg. L'utilisation d'espaces clos partagés par les bovins et leurs propriétaires est d'ailleurs illustrée par plusieurs peintures (fig. 5d). Un des enclos figurés est à double compartiment et les animaux semblent cohabiter avec un humain assis sur la gauche de l'enclos, à proximité de poteries, sacs ou ustensiles. Ailleurs, une habitation est incluse dans le périmètre d'un enclos occupé par des animaux domestiques. Bien entendu, les peintures ne permettent pas d'identifier les matériaux employés. Si les données archéologiques attestent de l'utilisation des pierres, l'usage d'épineux ou de haies végétales, très répandu en Afrique de nos jours, était certainement déjà commun.

En résumé, ces enclos en pierres sont les traces les plus évidentes laissées par des populations de pasteurs. Leur distribution, très hétérogène, apporte des informations qui laissent envisager des modes de vie et de subsistance ouverts à des activités complémentaires.

Si nombre d'enclos sont isolés, d'autres forment de véritables villages. Au Tchad, pays auquel nous limitons la discussion - il en existe des milliers ailleurs - nous en avons répertorié plus de 7 000, nombre qui cumule les enclos observés au sol et ceux, la majorité, repérés sur les images satellite. En réalité, ils sont beaucoup plus nombreux : 1) nous n'avons pas exploré toute la région ; 2) la résolution des images n'est pas toujours suffisante pour les détecter ; 3) certains se confondent avec le terrain ou sont ensablés. Néanmoins les zones explorées sont suffisamment vastes pour une première analyse qui réserve quelques surprises. Au-delà des regroupements en villages susmentionnés, on constate, sur la carte de distribution, d'importantes concentrations et des zones à densité très faible (fig. 6). Les enclos sont ainsi très rares (moins de 15) en Ennedi et peu fréquents dans les régions sableuses à l'exception des bordures de reliefs. Les plus nombreux sont au Tibesti où de fortes disparités sont constatées selon les lieux. Il y en a environ 2000 au Borkou (fig. 7), et on note deux centres particulièrement importants à proximité de Faya et Kaortchi. Quelques concentrations de moindre densité sont observées vers Gouro et autour du cratère d'Aorounga.

Si de multiples facteurs interviennent dans le choix d'un lieu d'implantation, le climat et l'environnement sont des clés essentielles pour interpréter correctement cette distribution. 


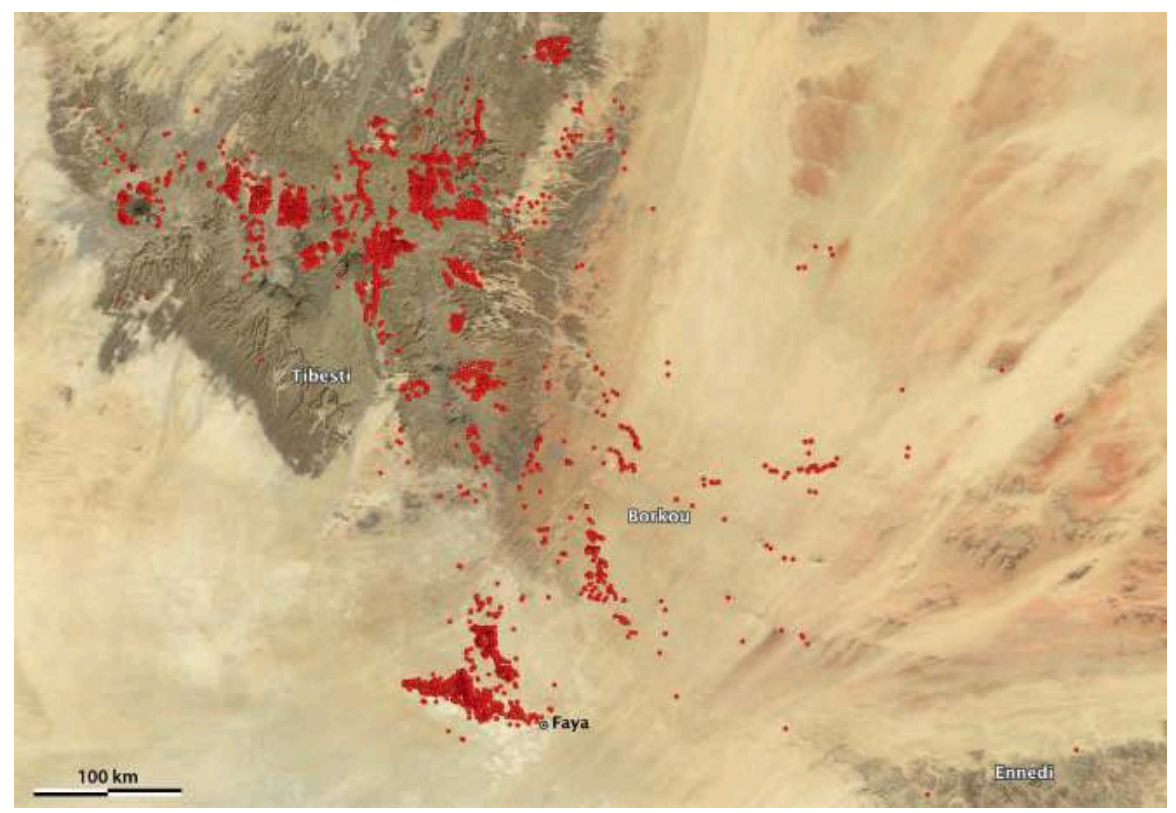

Figure 6

Distribution des enclos au Tibesti et au Borkou. À noter, la faible densité dans les régions sableuses. Source : Google Earth.

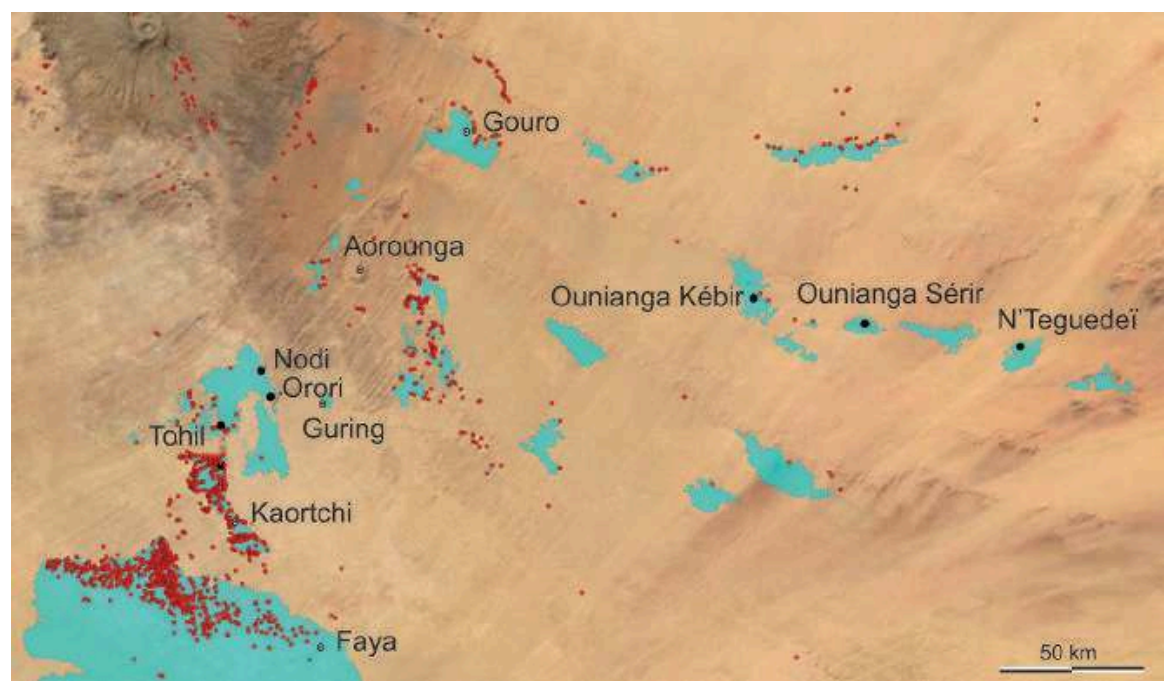

Figure 7

Reconstitution des principaux paléolacs du Borkou et distribution des enclos. Les points noirs figurent les (petits) lacs actuellement en eau. En bas, le nord du Mégalac Tchad (niveau 329 m). Sont indiqués les principales localités et le cratère d'impact d'Aorounga. Source : Google Earth. 


\section{Les lacs du Borkou}

Les contours du Mégalac Tchad à son maximum (niveau $329 \mathrm{~m}$ ) sont assez bien connus (Armitage et al., 2015). Avec une surface de près de $360000 \mathrm{~km}^{2}$, il s'étendait jusqu'aux falaises d'Artchana, au nord de Faya (fig. 9). Quand on parcourt le Borkou, il n'est pas inhabituel de traverser de nombreux paléolacs, aux rivages bien marqués dont les fonds blancs sont facilement identifiables sur les images satellitaires. Ces lacs, dont il reste encore des vestiges (Nodi, Orori, Tohil, Bedo), formaient des chapelets entre Faya et le sud de l'Emi Koussi, mais aussi entre Gouro et les Erdis à l'est ou encore dans la région du cratère d'Aorounga. Nous avons reconstitué nombre d'entre eux, dont ceux de Gouro $\left(240 \mathrm{~km}^{2}\right)$, Kaortchi $\left(230 \mathrm{~km}^{2}\right)$ et Tohil-Yarda $\left(660 \mathrm{~km}^{2}\right)$ (fig. 7). Comme les actuels lacs d'Ounianga, les mares résiduelles sont alimentées essentiellement par des sources qui tirent leur origine de la proximité avec le Tibesti voisin. Ces surfaces, en eau au Néolithique, ont joué un rôle primordial dans la répartition des populations, notamment de celles qui ont bâti ces enclos dont il va maintenant être question (fig. 7 à 9).

\section{Les enclos de Kaortchi}

À son optimum, le lac de Kaortchi s'étendait sur $40 \mathrm{~km}$, au-delà du lac résiduel de Bedo. Environ 720 enclos sont répartis autour, reproduisant le rivage du lac à la cote $322 \mathrm{~m}$ (fig. 8). Dès que l'on s'éloigne du lac, les enclos disparaissent, ce qui témoigne de la forte attractivité des plans d'eau. Une fraction des enclos, décroissante avec la régression du lac, se situe en aval du paléorivage, témoignant d'une poursuite de la construction de ce type de structures postérieurement au retrait du lac. Pour la cote $314 \mathrm{~m}$, une vingtaine d'enclos seulement est localisée dans le périmètre du lac. Pour le niveau $310 \mathrm{~m}$, ils ne sont plus que deux, signalant que la fin de la phase culturelle liée à ces constructions est contemporaine de la disparition du lac.

En d'autres termes, la distribution comporte une dimension chronologique, les populations se rapprochant du lac à mesure de son retrait. Ce type d'enclos a probablement perduré sur une assez longue période. Toutefois, dans leur grande majorité, ces structures semblent contemporaines d'un niveau élevé de remplissage (322-318 m), donc d'une période assez humide. Avec la disparition du lac, les populations ont migré, se sont regroupées autour des cuvettes résiduelles, et/ou ont changé de lieu et de mode de vie.

\section{Les enclos au nord du Mégalac Tchad}

Comme à Kaortchi, la densité d'enclos tend vers zéro dès qu'on s'éloigne du rivage du Mégalac Tchad à son niveau maximum. S'il a été un facteur attractif pour les populations (fig. 9), il a manifestement constitué une barrière de diffusion vers le sud : hormis les quelques exemplaires de l'Ennedi, on ne recense plus aucun enclos en pierre aux latitudes basses, au sud de Faya. L'essentiel des 
1110 enclos répertoriés est localisé entre les niveaux 329 m (maximum) et $250 \mathrm{~m}$. Là encore, on peut postuler une utilisation de ces constructions sur une assez longue période, avec migration progressive (ici vers le sud) pour suivre les rives du lac en phase de régression, ainsi qu'en témoignent les enclos situés en aval, sur le fond de la cuvette. Les densités les plus élevées sont observées dans la zone actuellement la plus humide, autour de Yéné et Gourma, deux villages entourés de palmeraies et jardins.

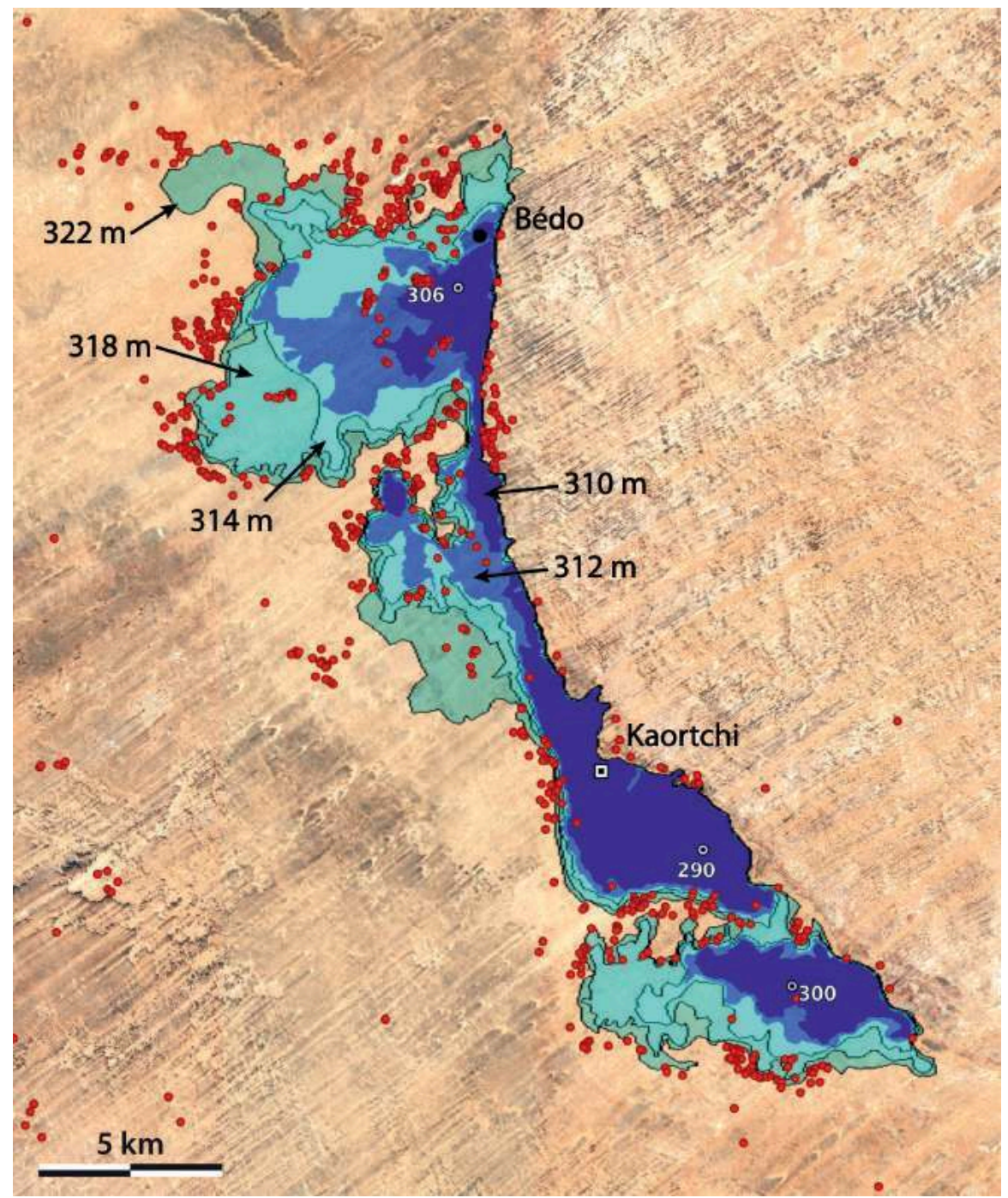

Figure 8

Distribution des 720 enclos autour du paléolac de Kaortchi, détail de la fig. 7 .

Cinq niveaux sont représentés : $322 \mathrm{~m}$ (vert), $318 \mathrm{~m}$ (bleu-vert), $314 \mathrm{~m}$ (bleu clair), $312 \mathrm{~m}$ (bleu foncé), et $310 \mathrm{~m}$ (violet). Les points jaunes figurent le fond de chaque cuvette, le point noir localise les mares résiduelles de Bédo. 


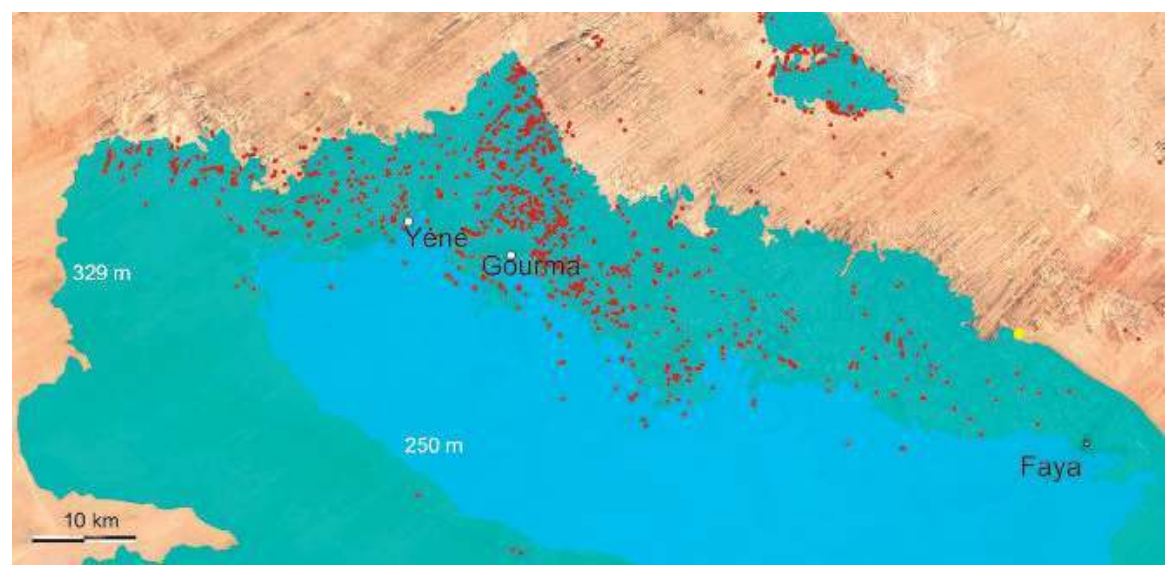

Figure 9

Distribution des enclos dans le nord du Mégalac Tchad.

Deux niveaux sont indiqués : $329 \mathrm{~m}$ (maximum en vert) et $250 \mathrm{~m}$ (bleu clair).

Le site aux barques d'Artchana II (point jaune) est localisé au nord-ouest de Faya.

Les enclos sont souvent localisés sur les buttes laissées par l'érosion éolienne ou juste à leur pied. Ces buttes allongées selon un axe nord-est-sud-ouest ont été modelées par l'harmattan et constituent la fin du système de crêtes et couloirs qui couvre une large portion du Borkou (GAUTHIER et GAUTHIER, 2017 ; fig. 6-9).

\section{Les enclos de Gouro et Aorounga}

Le village de Gouro est établi au bord de la cuvette d'un paléolac limitée au nord par une falaise qui borde le plateau sur des dizaines de kilomètres (fig. 7). À flanc de falaise et au pied de celle-ci, nous avons dénombré 25 enclos, tous alignés sur la même courbe de niveau (à quelques mètres près). Comme à Kaortchi, ces enclos dessinent, pour partie, le rivage du lac au bord duquel ils ont été construits. Sur le même niveau, on trouve aussi des tumulus et d'autres monuments funéraires.

À quelques kilomètres au nord, on observe une autre ligne de falaises délimitant un deuxième niveau du plateau. La blancheur des sédiments donne à penser qu'il y avait un autre lac ou une zone palustre dont les contours sont, une fois encore, balisés par un alignement d'enclos situés à la même altitude.

De même, autour du cratère de météorite d'Aorounga, les enclos apparaissent essentiellement dans les zones lacustres et palustres. Ils soulignent, ici encore, la tendance qu'ont eu ces populations à coloniser préférentiellement les bords de lacs et, pour le Tibesti, les grandes vallées (fig. 6).

\section{Quel âge pour ces enclos ?}

La distribution très particulière des enclos dans le nord du Mégalac Tchad (fig. 9) apporte une première réponse à cette question. Presque tous ces enclos se trouvant 
dans l'aire occupée par le lac à son maximum, il est clair qu'ils sont postérieurs au dernier épisode de hautes eaux : les structures ne seraient pas préservées comme elles le sont si le lac était remonté et les avait ennoyées. De plus, les tempêtes, qui pouvaient se développer sur un lac de la dimension du Mégalac Tchad, auraient détruit une majeure partie des enclos lors d'une transgression. Selon SERVANT-VILDARY (1978), le lac est resté à son plus haut niveau jusque vers $5500 \mathrm{BP}$, date post quem pour les enclos. Plus récemment, ARMITAGE et al. (2015) ont repris l'étude de l'évolution du niveau du lac. Ils proposent une fluctuation autour du niveau maximum $(335 \mathrm{~m})$ entre $\sim 11500$ et $\sim 5000$ ans (dates calendaires) avec un cluster de dates pour des paléorivages à $329 \mathrm{~m}$, niveau retenu ici pour la discussion (fig. 10). Selon ces auteurs, après une première régression le lac, descendu à $\sim 220 \mathrm{~m}$, serait remonté au-dessus de $300 \mathrm{~m}$ avant de connaître une régression finale assez abrupte. Ce « rebond » ne fait cependant pas l'unanimité et, peut-être, serait-il à reconsidérer pour le secteur nord du Mégalac Tchad. L'absence d'enclos aux altitudes basses $(<250 \mathrm{~m})$ indique l'abandon de ce type de structures avec le retrait des eaux et l'assèchement de la cuvette de Faya. Sur le diagramme d'évolution de la hauteur d'eau du Mégalac Tchad (fig. 10), les niveaux $329 \mathrm{~m}$ et $250 \mathrm{~m}$ bornent l'époque de construction qui se situerait donc entre $\sim 5000$ et 2300 BP pour les enclos localisés entre Faya et Yéné. Il ne s'agit là que d'une estimation chronologique grossière, une première approche qui doit être confirmée par des études de terrain et supportée par des datations directes. On notera que la limite inférieure correspond approximativement à l'époque d'introduction du dromadaire en

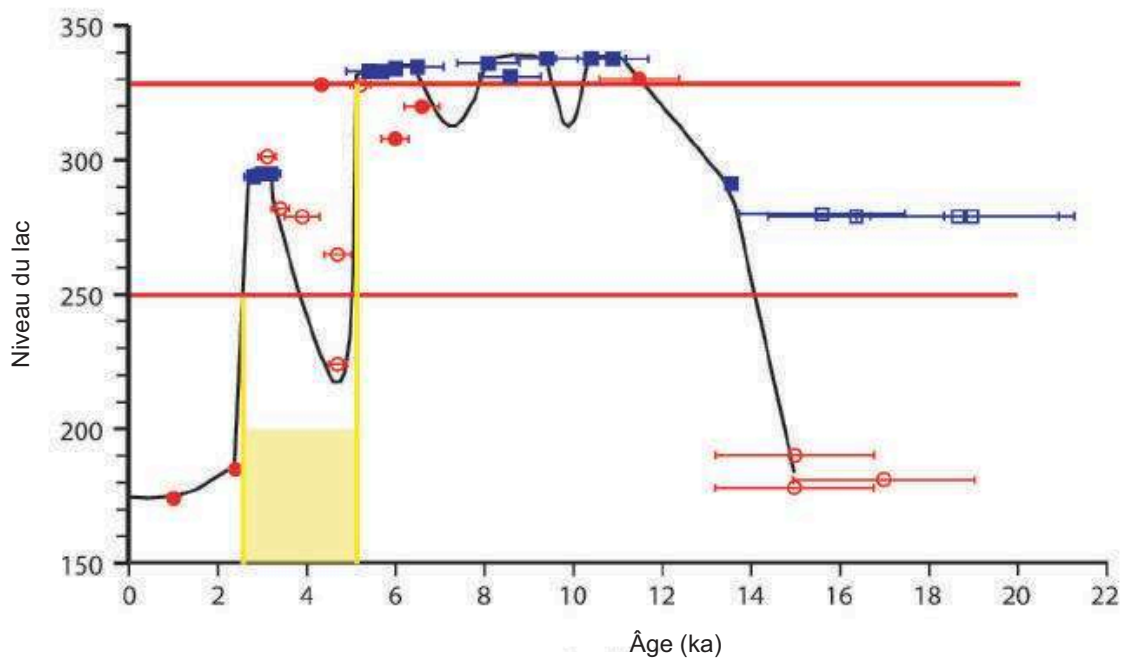

Figure 10

Évolution du niveau du Mégalac Tchad au cours de l'Holocène (adaptation de ARMITAGE et al., 2015). Lignes rouges : indication des niveaux 329 et 250 m qui délimitent la fourchette d'existence des enclos dans la région de Faya. 
Afrique, un phénomène qui confirme la dégradation du climat et laisse envisager une modification drastique des modes de vie.

\section{Des peuples de pêcheurs?}

Avec l'élargissement de la zone d'étude, d'autres exemples viendront à terme étayer ce schéma chronologique et confirmer l'importance des lacs et marais dans le choix des lieux d'installation. Ceci concerne les enclos en pierre mais aussi d'autres phénomènes anthropiques comme des monuments funéraires de type compas graeber (GABRIEL, 1970), qui sont vraisemblablement l'œuvre d'un autre groupe culturel, ou encore, dans un certain nombre de cas, l'art rupestre.

Ainsi qu'on l'observe pour les enclos, les sites ornés de la région de Faya restent cantonnés au nord de la cuvette du Mégalac Tchad : ils sont moins nombreux au nord des falaises d'Artchana et disparaissent vers le sud, la limite avoisinant la cote $260 \mathrm{~m}$. Les sites comportent d'autant plus d'images de chameaux qu'ils sont aux altitudes les plus basses, sur des espaces colonisés suite au retrait du Mégalac Tchad. Pour moitié environ, les sites rupestres occupent les reliefs d'Artchana qui dominaient le rivage du lac à son maximum holocène.

Le cas le plus emblématique est celui d'Artchana II (fig. 9), un abri localisé dans la partie supérieure d'un piton qui se dresse à la cote $328 \mathrm{~m}$. Comme indiqué supra, ARMitAgE et al. (2015) placent le niveau maximum du Mégalac Tchad à $329 \mathrm{~m}$ : à ce moment-là, le bas du piton, où se trouve un auvent lui aussi orné, était donc très certainement sous l'eau ou submergé lors des tempêtes.

Dans l'abri supérieur, les scènes où apparaissent les pêcheurs et leurs barques (fig. 3) ne comportent aucun bovin domestique. On ne trouve ni barque ni pêcheur sous l'auvent orné creusé à la base du piton. De plus, les rares bovidés qui sont peints sur les autres panneaux n'ont aucun rapport stylistique, ni technique, avec ces pêcheurs et rien ne permet de leur attribuer un statut domestique (GAUthier et GaUthier, 2018). Enfin, ces pêcheurs n'ont rien de commun avec les personnages qui accompagnent les bovins de l'abri inférieur.

Le scénario - abri supérieur hors d'eau et abri inférieur non accessible - reste à valider mais on peut postuler que les scènes aquatiques et les personnages de l'abri supérieur ont été réalisés lors d'un des épisodes de hautes eaux du lac (le dernier ?), à une date antérieure à la domestication des bovins. Les sujets, dont les bovins, de l'abri inférieur auraient été réalisés ultérieurement, lors de la phase de régression du Mégalac Tchad.

La situation est différente pour le site de Tohil (fig. 2). Deux des barques sont en effet surchargées par des bovins domestiques alors même que les passagers des barques sont différents, tant stylistiquement que techniquement, des pasteurs qui 
accompagnent ces bovins. Ces pasteurs, bicolores, ont des coiffures et des vêtements bien marqués alors que ces attributs n'apparaissent pas sur les figurations de pêcheurs, seulement représentés en aplat monochrome. La séquence reproduit en apparence celle d'Artchana, avec deux phases successives d'occupation, par des pêcheurs d'abord et par des pasteurs ensuite. Le parallèle entre les deux sites est souligné par le fait que l'abri de Tohil domine lui aussi un lac qui s'étendait sur $140 \mathrm{~km}^{2}$.

Un autre point mérite d'être souligné : au Borkou, les abris avec figurations de barques (Artchana, Tohil, Orori et Taïtroa) sont situés en bordure ou à proximité d'un paléolac, dans des zones où il n'y a pas le moindre enclos.

Sans qu'il soit possible de lier les bovins, l'art rupestre et les constructions, on peut proposer qu'il y a eu, dans la région des lacs, au moins deux époques : d'une part des pêcheurs qui sillonnaient les lacs en pirogue et, plus tardivement, des pasteurs à l'origine des enclos. Ces derniers étaient-ils seulement des pasteurs ? On peut se poser la question quand on observe la distribution des enclos du Borkou qui reproduisent parfaitement le contour des paléolacs, alors que leur densité est quasi nulle ailleurs. Postuler qu'ils tiraient une partie de leur subsistance de ces lacs tombe sous le sens, de même qu'il est légitime de voir en eux des navigateurs. On ne peut toutefois exclure que ces pasteurs n'aient exploité les ressources aquatiques que de manière subsidiaire. Quelques dizaines seulement d'enclos ont été visités, essentiellement dans la région de Yéné et l'observation (trop) rapide ne nous a pas permis d'y observer des restes anthropiques susceptibles de nous renseigner sur les modes de vie. À ce stade, il faut rappeler que la région est soumise à une forte érosion éolienne, que les enclos visités étaient généralement ensablés (fig. 5) et que, hors stratigraphie, les arêtes et les os, y compris ceux utilisés pour les harpons, sont rarement préservés. Seuls trois sites de la cuvette de Faya, c'est-à-dire sur plus de 100 km, ont livré des harpons (COURTIN, 1965). Quant à l'élevage, thème illustré par des milliers d'images, les preuves se résument, pour le Tchad, à une unique mâchoire de bovins datée de 630 ans cal BP. (KeDING et al., 2007). La probabilité pour trouver des harpons ou des os est donc très faible. En l'état actuel des connaissances, nous ne pouvons prouver ni la fonction pastorale des enclos, ni l'hypothèse d'une alimentation basée partiellement sur la pêche. Mais, une fois encore, l'absence de preuve n'est pas la preuve de l'absence et les hypothèses formulées devront impérativement être (in)validées par des études de terrain.

Partout au Sahara, l'art rupestre nous enseigne que la chasse était pratiquée et le BET ne fait pas exception en la matière. Il est fort probable que le gibier complétait la diète des pêcheurs d'Artchana et de Tohil et des pasteurs qui occupaient les enclos. Les contributions à cette dernière de la chasse, de la pêche et de l'élevage étaient inévitablement variables avec la géographie et l'époque : c'est ce qui différencie les deux groupes culturels mis en évidence et, au sein du groupe des pasteurs bâtisseurs d'enclos, ceux du Tibesti en contexte montagneux de ceux du Borkou qui vivaient au bord des lacs. 


\section{Ancienneté de la pêche et de la navigation, émergence de la domestication}

$\mathrm{Au}$ Borkou, en l'état actuel des connaissances, les peintures se rapportant à la pêche et à la navigation ne comportent jamais de bovins domestiques. De plus, les rares superpositions (fig. 2) vont dans le sens d'une antériorité des activités aquatiques sur l'élevage. Une impression similaire est donnée par les peintures d'Artchana II. Là, les pêcheurs au harpon et les barques ont été peints dans des scènes dépourvues de bovin et à une époque de hautes eaux du Mégalac Tchad, probablement avant l'introduction de la domestication dans la région.

En Ennedi, les figurations de barques et celle du pêcheur avec nasses et poissons (fig. 1) s'insèrent dans un horizon archaïque voire dans la phase la plus ancienne des peintures du massif. Néanmoins, les études les plus récentes montrent que quelques bovins domestiques ont probablement été peints antérieurement (GAuthier et GaUthier, 2017).

$\mathrm{Au} \mathrm{BET}$, en première approche, pêche et navigation semblent globalement précéder la domestication. Toutefois, compte tenu de la présence de vaches sous les étages anciens de l'Ennedi, on peut penser que les activités aquatiques ont perduré après l'introduction de cet animal. En aucun cas, cependant, ces activités ne subsistent dans les étages récents de l'art pariétal.

Les images rupestres illustrant des activités aquatiques sont rares. Néanmoins celles que nous connaissons s'agencent logiquement avec ce qui est connu de l'évolution climatique et de la régression du Mégalac Tchad et des autres lacs du Borkou. Pour le Borkou et la zone des lacs au moins, on peut penser que, dans un premier temps, pêche et chasse ont été, avec la cueillette, les sources principales de nourriture. L'introduction des bovins, ovins et caprins viendra ensuite et l'élevage prendra une place d'autant plus importante que les lacs régressent. La disparition des hippopotames, des crocodiles, des éléphants et des rhinocéros, présents dans les phases anciennes de l'iconographie et absents de toutes les phases récentes ${ }^{2}$, est une autre évidence de la péjoration climatique. Celle-ci se verra encore soulignée par la part croissante faite aux chevaux et chameaux : ces derniers marquent l'établissement définitif de l'aridité et l'assèchement presque total des surfaces en eau libre du Tchad septentrional.

Mais faut-il s'étonner de l'ancienneté relative de la pêche et de la navigation au Tchad septentrional, des activités que l'iconographie ferait remonter au moins au dernier épisode de hautes eaux qui se termine il y a 5000 à 5500 ans ? En 1987, une barque monoxyle a été exhumée des sédiments à Dufuna, près de la Komadougou Yobé (Nigeria), une rivière qui, de nos jours encore, alimente le lac Tchad. Cette pirogue, l'une des plus anciennes de la planète, est âgée d'environ 8000 ans (Breunig, 1996). L'auteur précise que la technique de 
construction est si développée qu'il ne peut s'agir d'une première tentative et que la navigation en Afrique, et par conséquent sur le Mégalac Tchad, est certainement plus ancienne.

\section{Conclusion}

Les apports récents en matière d'art rupestre et plus largement d'archéologie et de paléoenvironnement dans le nord du Tchad viennent bousculer les idées reçues et la croyance en une évolution classique de populations de chasseurs progressivement remplacés par des pasteurs.

Au plan environnemental, la nouveauté vient de la reconstitution d'une multitude de lacs qui parsemaient le Borkou mettant l'accent sur le potentiel des ressources aquatiques de cette région, potentiel sans équivalent au Sahara central, à l'Holocène. Il y a peu de doute que les zones humides dépassaient de loin en surface celles occupées par les eaux vives. Sur une grande partie du Borkou, entre Faya et le Tibesti, le terrain est peu accidenté et le très faible dénivelé, sur de grandes distances, a pu favoriser le développement de vastes marais, lors des phases transgressives de l'Holocène. Si cette vision est correcte, cela pourrait expliquer le caractère discontinu de diverses traces anthropiques au premier rang desquelles figurent l'art rupestre ${ }^{3}$, l'habitat ou encore les monuments funéraires qui sont absents dans ce secteur et/ou restent confinés à des zones de faible étendue.

Parallèlement, les conditions climatiques en vigueur à l'Holocène moyen ont favorisé l'émergence de groupes culturels qui tiraient leur subsistance de ces zones humides et ont laissé des traces de leurs activités aquatiques sur les parois.

De nouvelles images de barques et quelques scènes de pêche comptent parmi les avancées notables. Mais, nous insisterons surtout sur le caractère inédit des milliers d'enclos détectés au BET, un type de structure apparu probablement peu après le début de la régression du Mégalac Tchad qui a débuté il y a 5500 à 5000 ans.

Si l'on prend en considération le nombre et la concentration de ces enclos au nord-ouest de Faya et au Tibesti, il est surprenant de constater qu'ils n'ont attiré ni l'attention des militaires, qui sont pourtant à l'origine de bien des découvertes faites au Sahara, ni celle des chercheurs. Avec les monuments en forme de compas (GABRIEL, 1970), guère plus étudiés que nos enclos, et qui se comptent eux aussi par milliers, ils constituent pourtant, avec l'art rupestre, la documentation archéologique la plus importante du Sahara tchadien. Nous appuyant sur leur distribution étonnante, nous avons pu mettre en évidence une implantation

3. Cette zone est parsemée d'abris et rochers susceptibles d'être peints ou gravés. 
préférentielle de ces structures sur les bords de lacs. Si leurs bâtisseurs étaient manifestement des pasteurs, il est plus que vraisemblable qu'ils ont aussi exploité les ressources halieutiques. Étaient-ils les descendants de ces groupes de pêcheurs de l'Holocène moyen ou venaient-ils d'ailleurs, apportant avec eux le concept de domestication? Nous ne sommes pas en mesure de le dire.

Nous ne présentons ici qu'une analyse préliminaire et il faudra assurément étudier ces structures en pierre sur le terrain pour en apprendre davantage sur l'évolution du peuplement du Tchad septentrional. Quoi qu'il en soit, le climat et son évolution drastique, qui a abouti au climat très aride d'aujourd'hui, ont eu une influence déterminante sur le sort des occupants de ces régions. Les bouleversements qu'ils ont entraînés sur les modes de vie et sur les lieux d'implantation en témoignent et nous interpellent en même temps.

\section{Bibliographie}

\begin{abstract}
ArCangioli S., 1996
« Tohil ». In : Arte rupestre nel Ciad: Borku, Ennedi, Tibesti. Milano, Edizione Pyramids : 63-65.
\end{abstract}

Armitage S., Bristow C., Drake N., 2015

West African monsoon dynamics inferred from abrupt fluctuations of Lake Mega-Chad. PNAS 112 : 8543-8548.

\section{BAILlOUd G., 1997}

Art rupestre en Ennedi. Paris, Sépia, 154 p.

\section{Boccazzi A., Boccazzi D., 1999}

La fauna selvaggia nelle pitture della regione di Ouri (Tibesti orientale), Sahara, $11: 130$.

\section{Bidault J., 1945}

Pirogues et pagaies. Paris, Éditions J. Susse, 293 p.

\section{BReUNig P., 1996}

" The 8000-year-old dugout canoe from Dufuna (NE Nigeria) ». In : Aspects of African Archaeology. $10^{\text {th }}$ Congress of the PanAfrican Association for Prehistory and Related Studies, Harare, University of Zimbabwe Publications, Harare : 461-468.

\section{Courtin J., 1964a}

Sites préhistoriques des environs de Largeau, Tchad, BSPF, 61 (5) : 108-109.

\section{CourTin J., 1964b}

Sites préhistoriques du Borkou (Nord Tchad), $B S P F, 61$ (6) : 128-131.

\section{Courtin J., 1965}

Découverte de harpons en os au Borkou, République du Tchad, BSPF, 62 (2) : 70-75.

\section{GABRIEL B., 1970}

Bauelement präislamischer gräbertypen im Tibesti-Gebirge (Zentral Ostsahara), Acta Praehistorica et Archaeologia, 1 : 1-28.

\section{Gauthier Y., Gauthier C., 2010}

De la navigation chez les Têtes Rondes : peintures des confins algéro-libyens, Les Cahiers de l'AARS, 14 : 143-168.

\section{Gauthier Y., Gauthier C., 2011}

Des poissons chez les Têtes Rondes?, Les Cahiers de l'AARS, 15 : 119-132.

\section{Gauthier Y., Gauthier C., 2017}

Éole, le mauvais élève de la classe ? Remarques sur les peintures dites « archaïques » en Ennedi, Les Cahiers de l'AARS, 19 : 95-130.

\section{Gauthier Y., Gauthier C., 2018}

« Nouveaux documents sur les milieux aquatiques au Sahara tchadien : le site d'Artchana II revisité ». In : Whatever happened to the People?, Humans and Anthropomorphs in the rock art of Northern Africa, International Conference, Brussels, 17-19 septembre 2015. 
Huard P., Massip J.-M., 1964a

Les peintures rupestres de la falaise d'Artiena (Borkou), BSPF, 61 (1) : 10.

\section{Huard P., Massip J.-M., 1964b}

Harpons en os et céramique à décor en vague au Sahara tchadien, BSPF, 61 : 105-123.

\section{Huard P., Massip J.-M., 1967}

Monuments du Sahara nigéro-tchadien : 1. Grands cercles et pierres levées, BIFAN, XXIX, série B (1-2) : 1-27.

\section{JACQUeT G., 2001}

Piste oubliée en Haut-Ennedi (Tchad). Sahara, 12 : 141-149.

\section{Keding B., Lenssen-Erz T., Pastoors A.,} 2007

Pictures and pots from pastoralists. Investigations into the prehistory of the Ennedi highlands in NE Chad. Sahara, $18: 23-46$.

\section{SERVANT-VILDARY S., 1978}

Étude des diatomées et paléolimnologie du bassin tchadien au Cénozoïque supérieur, Paris. Orstom, coll. Travaux et documents, 84, 346 p. 


\title{
Mission archéologique franco-tchadienne aux lacs d'Ounianga (Tchad) Principaux résultats
} des deux premières missions de terrain

\author{
Vincent MOURRE, Guemona DIIMET, \\ Tchago BOUIMON, Marie-Pierre COUSTURES, \\ Barbara EICHHORN, Jean MBAIRO, \\ Caroline ROBION-BRUNNER, Margareta TENGBERG
}

\section{Présentation de la mission}

Le projet Archéologie des régions lacustres du Tchad (ArRéLat) est né de la signature en juillet 2013 d'une convention de collaboration scientifique entre l'université de N'Djamena (Tchad), la direction du patrimoine du Tchad, l'université Toulouse Jean-Jaurès et le laboratoire Traces de Toulouse (France). Un avenant signé en 2016 a permis d'associer à cette convention le Centre national de recherche pour le développement tchadien (ex Cnar) et l'Institut national de recherches archéologiques préventives français.

Le projet vient concrétiser les liens établis entre ces partenaires afin notamment de développer des projets de recherche et de mettre en place une formation en archéologie. Dans ce cadre, deux étudiants tchadiens sont d'ores et déjà inscrits à l'université Toulouse Jean-Jaurès, l'un en master 2 Préhistoire et Protohistoire Europe/Afrique et l'autre en thèse de doctorat sur l'histoire et l'archéologie de la métallurgie du fer.

Le projet ArRéLat a reçu le soutien de la commission des fouilles du ministère des Affaires étrangères en 2015. L'ambassade de France à N'Djamena apporte un 
soutien significatif en accordant des bourses à des étudiants tchadiens pour qu'ils puissent compléter leur formation à l'université de Toulouse Jean-Jaurès.

\section{La région des lacs d'Ounianga, présentation sommaire et historique des recherches}

La région des lacs d'Ounianga nous a très tôt semblé particulièrement propice d'un point de vue archéologique dans la mesure où des sites relevant de différentes périodes y avaient été identifiés et pour partie cartographiés dans le cadre de travaux antérieurs.

Les 18 lacs d'Ounianga se trouvent dans le désert du Sahara, dans la région de l'Ennedi (département de l'Ennedi Ouest), dans le nord du Tchad. Ils se répartissent en deux groupes distants d'une quarantaine de kilomètres. Le groupe d'Ounianga Kebir (litt. " le grand Ounianga ») comprend 4 lacs dont le plus important est le lac Yoa. Le groupe d'Ounianga Teli (ou Ounianga Serir, « le petit Ounianga ») comprend 14 lacs dont le plus important est le lac Teli. Certains de ces lacs sont salés, voire hyper salés, tandis que d'autres sont constitués d'eau douce. Des phénomènes complexes de pompage naturel des nappes aquifères permettent en effet de compenser dans certains cas l'intense évaporation. Le lac Yoa présente ainsi la particularité d'avoir livré une sédimentation continue et non perturbée pour tout l'Holocène, offrant ainsi une source d'information particulièrement précieuse pour reconstituer le paléoclimat récent de la région saharienne (KRÖPELIN, 2007 ; FRANCUS et al., 2013).

L'ensemble des lacs d'Ounianga a été inscrit sur la liste du patrimoine mondial de l'Unesco au titre des critères naturels. Dans le rapport de l'Union internationale pour la conservation de la nature (UICN) ayant déterminé ce classement, il est indiqué que ces lacs forment « le plus grand complexe connu de lacs en milieu hyperaride, avec des lacs de volume important d'eau, de structure et de composition différentes (lacs salés, hyper salés et d'eau douce) » (Collectif, 2012). Parmi les mesures consécutives au classement, l'UICN souligne également la nécessité d'élargir les recherches scientifiques dans le secteur.

D'un point de vue archéologique et paléontologique, plusieurs missions anciennes ont porté sur la région des lacs d'Ounianga. Dès les années 1930, des ossements de grands mammifères quaternaires ont été recueillis à proximité $d u$ lac Yoa par le lieutenant Pâris de Bollardière. Étudiés par Léonce Joleaud et Jean Lombard, ils incluent des ossements d'Hippopotame (Hippopotamus amphibius L.), de proboscidiens (Elephas sp., Paleoloxodon iolensis) et de sanglier de forte taille (Sus scrofa L.). Des vertèbres correspondant à un grand 
silure (Lates niloticus L.) sont également signalées (JOLEAUD et LOMBARD, 1933). Aucune trace d'activité anthropique associée à ces ossements n'a été mentionnée par les auteurs.

En 1957, Anthony Jean Arkell identifie des sites préhistoriques dans le cadre de la British Ennedi Expedition (ARKELL, 1959, 1960, 1964). Cet auteur signale en particulier la présence d'une industrie atérienne près du lac Yoa (ARKELL, 1962a), étudiée par la suite par Thierry Tillet (1983).

Plusieurs sites acheuléens ont également été identifiés par Jean Courtin dans les années 1960 (COURTIN, carnets de terrain inédits). Les industries qu'ils ont livrées ont été rapprochées de l'Acheuléen évolué sur la base de critères morphologiques et typologiques (TILLET, 1983). Par ailleurs, la présence de sites néolithiques a été mentionnée (ARKELL, 1962a, 1962b ; CourTin, carnets de terrain inédits).

\section{Principaux résultats}

Une première mission exploratoire de prospection et sondages sur le terrain a eu lieu entre le 22 novembre et le 21 décembre 2015. Elle a permis d'explorer ou d'inventorier 40 sites archéologiques, dont 32 inédits, 7 précédemment décrits par A. J. Arkell (1964) et 1 découvert par J. Courtin (carnets inédits). 1885 échantillons archéologiques ont été recueillis, dont 1364 vestiges lithiques, 423 fragments de céramique, 88 restes fauniques et 10 vestiges divers (éléments de parure, bracelets, perles). Une deuxième mission a eu lieu entre le 16 octobre et le 13 novembre 2016. Durant celle-ci, 30 entités archéologiques ont été explorées ou inventoriées, dont 26 inédites, 2 précédemment décrites par A. J. Arkell et 2 découvertes l'année précédente. 1297 échantillons archéologiques ont été recueillis, dont 1111 vestiges lithiques, 160 fragments de céramique, 23 restes fauniques et 3 vestiges divers, sans compter de nombreux échantillons paléométallurgiques. Toutes les découvertes ont été géoréférencées au sein d'un système d'information géographique (fig. 1).

\section{Des industries paléolithiques de surface}

Les vestiges lithiques hors contexte stratigraphique sont omniprésents dans le secteur. Parmi les découvertes les plus significatives, il convient de mentionner plusieurs sites acheuléens, dont un probable site de production, mais aussi plusieurs ensembles témoignant de l'importante variabilité régionale du Middle Stone Age. En effet, dans un même contexte géographique sont associés :

- des pièces pédonculées typiquement atériennes, ce faciès trouvant ici l'une de ses expressions les plus sud-orientales (SCERRI, 2013); 
- des nucléus et pointes relevant de modalités de débitage de type nubien (photo 1);

- des lames retouchées sur supports robustes débités à la pierre dure (fig. 3) pour lesquelles des éléments de comparaison sont à rechercher dans le sud de la Libye (CANCELLIERI et DI LERNIA, 2013).

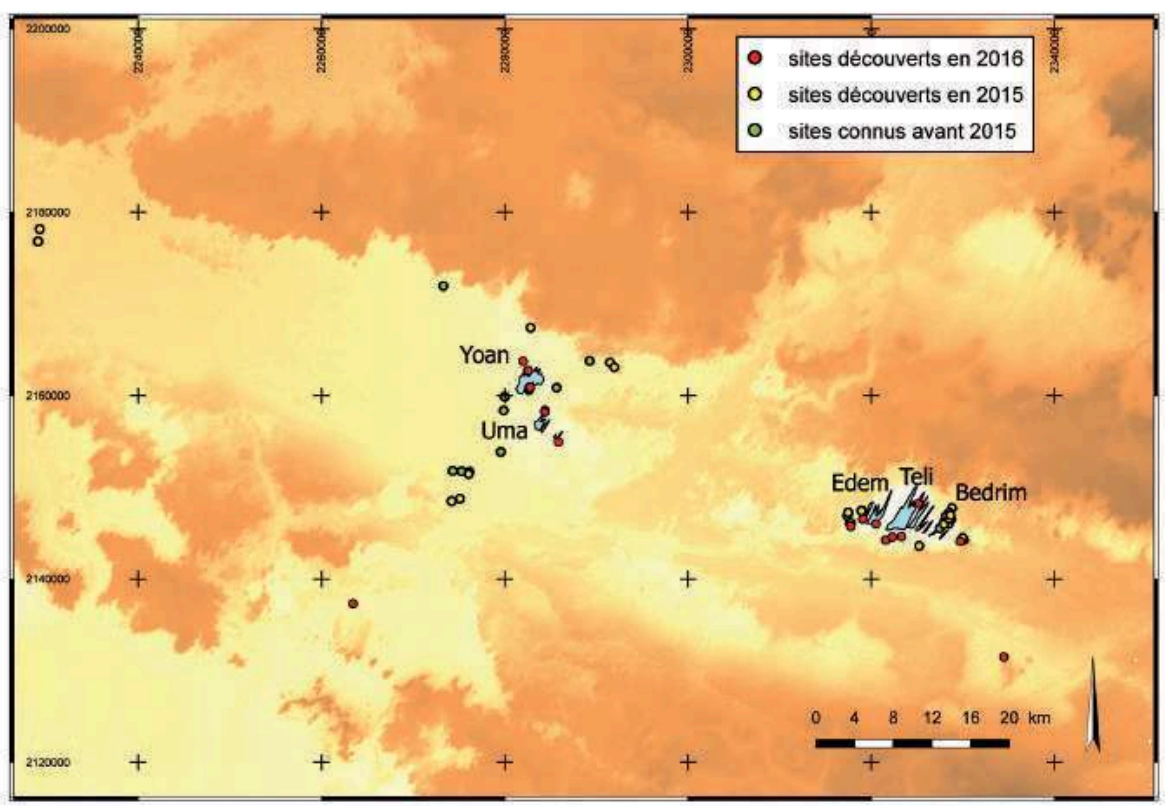

Figure I

Répartition des sites archéologiques explorés dans le cadre des missions archéologiques franco-tchadiennes 2015 et 2016 dans la région des lacs d'Ounianga.

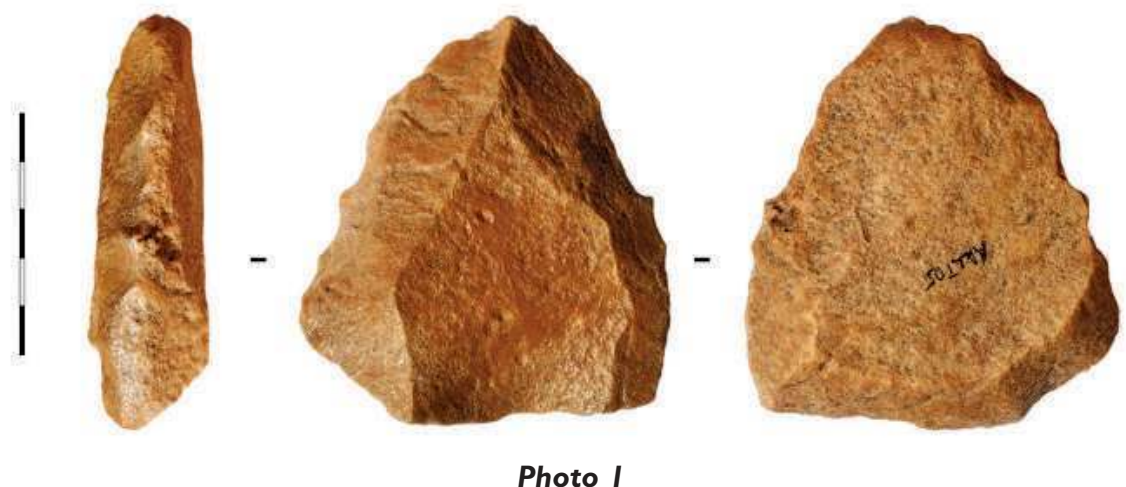

Nucléus Levallois à pointe de type nubien (Middle Stone Age). L'échelle représente $5 \mathrm{~cm}$ (C) V. Mourre. 

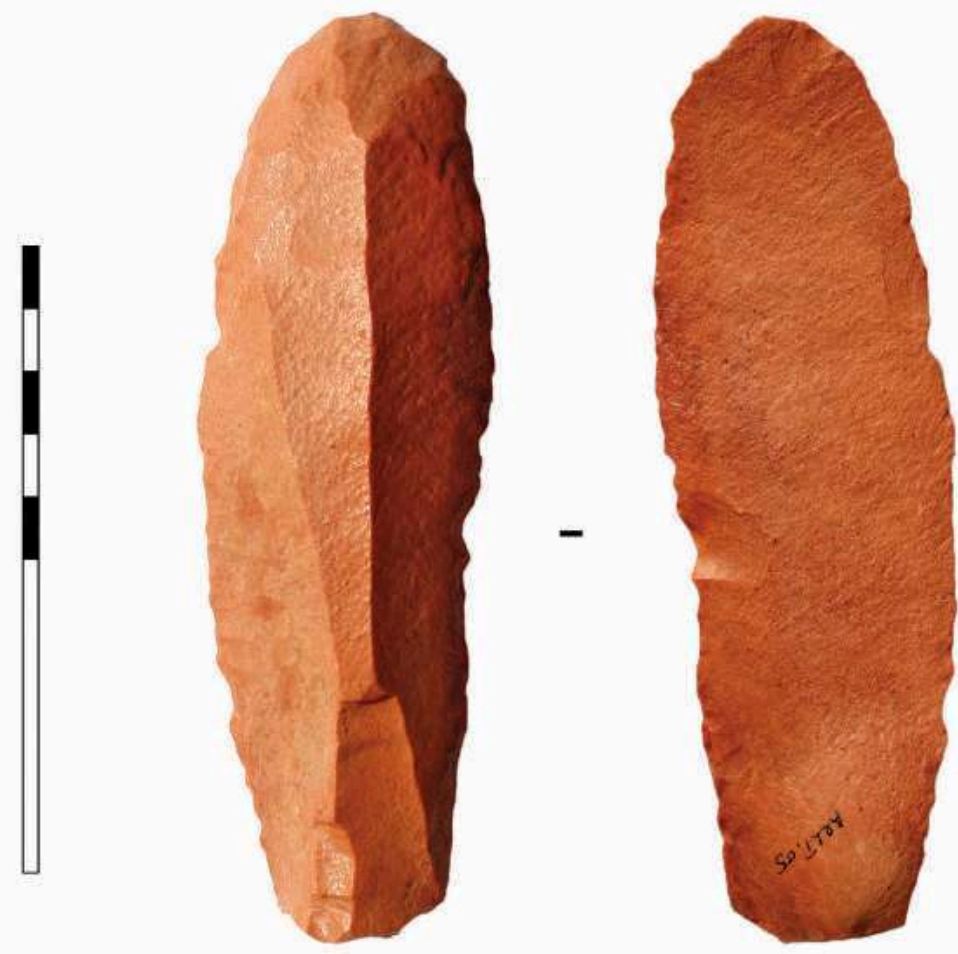

Photo 2

Lame robuste débitée au percuteur dur (Middle Stone Age). L'échelle représente $10 \mathrm{~cm}$. Lac Yoan (Ounianga Kebir).

(c) V. Mourre.

\section{Un site néolithique}

De manière générale, nous nous sommes heurtés à une difficulté classique en contexte saharien, à savoir celle d'identifier des séquences stratigraphiques en place qui témoignent d'un recouvrement sédimentaire garantissant une homogénéité et une représentativité des ensembles archéologiques. Lors d'un sondage réalisé en 2016 près du lac Yoa (site ARLT49), nous avons tout de même pu mettre en évidence une petite séquence stratigraphique d'environ $1 \mathrm{~m}$ de puissance, dans une dépression située entre deux buttes témoins de grès de Nubie (photo 3 et fig. 2).

D'un point de vue archéologique, le mobilier mis au jour dans le niveau archéologique unique semble homogène. Les éléments les plus diagnostiques d'un point de vue chronoculturel sont les fragments de céramique décorés de cannelures et d'impressions et les 14 segments et 3 fragments de segments, permettant de rattacher l'ensemble au Néolithique. La plupart des vestiges fauniques sont des fragments non déterminables, à l'exception d'un fragment 


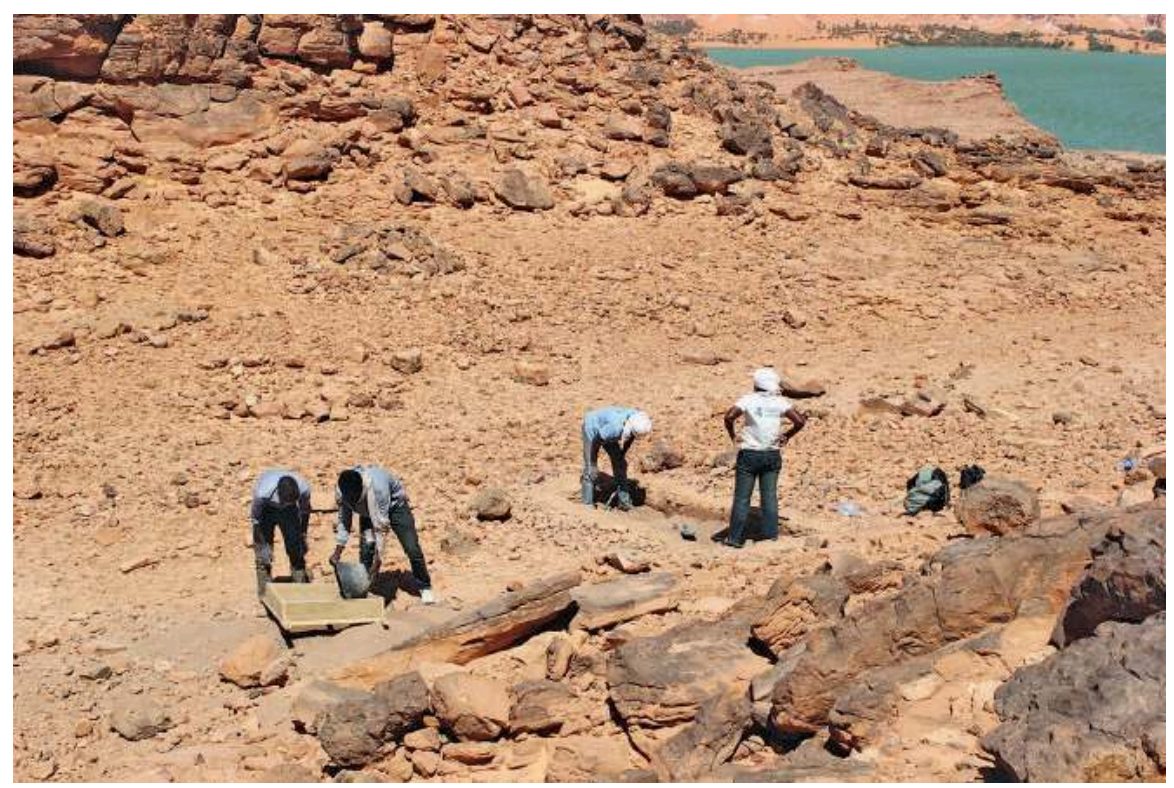

Photo 3

Sondage archéologique (ARLT49) réalisé entre deux buttes témoins de grès de Nubie au sud-est du lac Yoan (Ounianga Kebir).

(c). Mourre.

5

6

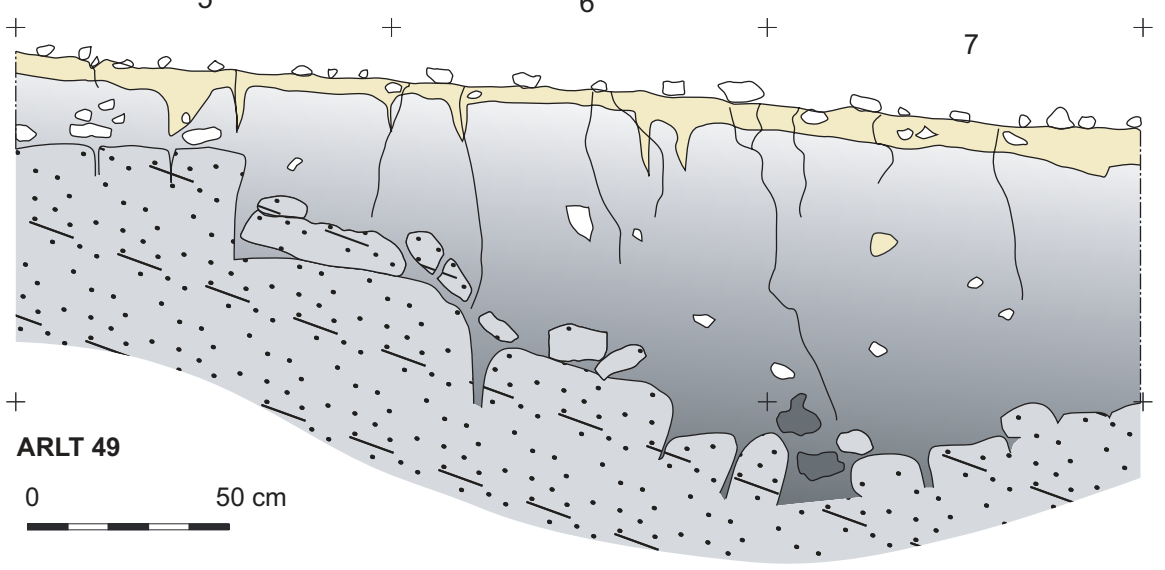

Figure 2

Relevé de la coupe du sondage archéologique ARLT49.

Réalisation : Vincent Mourre. 
de phalange et de deux fragments de dents d'herbivores de taille moyenne. Une datation par le radiocarbone sur un charbon de bois (Acacia type) provenant de la passe comprise entre 60 et $80 \mathrm{~cm}$ sous le niveau actuel du sol, à la base du niveau archéologique, a donné un âge de $3440 \pm 40$ ans BP (Poz-96683), soit un âge calibré de $3726 \pm 72$ ans cal BP${ }^{1}$. La présence d'un fragment de perle en cauri (Monetaria moneta) dans la première passe de décapage est également à signaler puisqu'elle rend compte d'une fréquentation beaucoup plus récente du lieu.

\section{Art rupestre néolithique}

La mission 2016 a été marquée par l'exploration de deux sites à gravures rupestres dans la région d'Ounianga Teli.

Le premier nous a été signalé par Pierre Deschamps (ARLT61) et est situé sur la butte témoin d'Ishwele (également transcrit Isweri dans ARKELL, 1964). En dazaga, ce terme désigne un diastème prononcé entre les incisives supérieures, communément surnommé « les dents du bonheur », et il fait de toute évidence référence aux deux pics saillants et légèrement espacés à son sommet. La butte est un piton de grès d'une soixantaine de mètres de hauteur, complètement isolé dans le paysage et visible de très loin. Il présente plusieurs panneaux de gravures ainsi que des figures isolées.

L'un des principaux panneaux (panneau des éléphants) est situé à mi-hauteur de cette butte sur sa face nord-ouest. D'environ $3 \mathrm{~m}$ de long pour $1 \mathrm{~m}$ de hauteur, il comporte de nombreuses figures animales explicites dont des représentations d'espèces de la grande faune africaine, notamment des éléphants et des girafes. Quelques figures anthropomorphes sont également présentes; elles sont caractérisées par des membres inférieurs très longs et aux extrémités effilées qui leur confèrent une silhouette fantomatique. Les traits sont émoussés et caractérisés par une patine sombre prononcée, qui tranche avec le trait clair des gravures de dromadaires très fréquentes dans la région.

Un deuxième ensemble important de figures se trouve au sud-est, à la base de la butte (panneau des girafes; photo 4). D'environ $2 \mathrm{~m}$ de long pour $1 \mathrm{~m}$ de hauteur, il s'organise autour de 2 figures principales de girafes, l'une rayée et l'autre hachurée. Les membres inférieurs de ces girafes se confondent avec les membres inférieurs de 2 personnages anthropomorphes. Ces derniers semblent tenir des objets qu'il est tentant d'interpréter comme des boucliers et des armes. Des bovidés sont également représentés, dont un probable bovin et deux probables oryx (cornes longues et droites, pointant vers l'arrière). À proximité, d'autres figures de girafes sont présentes.

À mi-hauteur de la butte, un autre ensemble comporte également des figurations de girafes associées à des figures potentiellement plus récentes, notamment des 


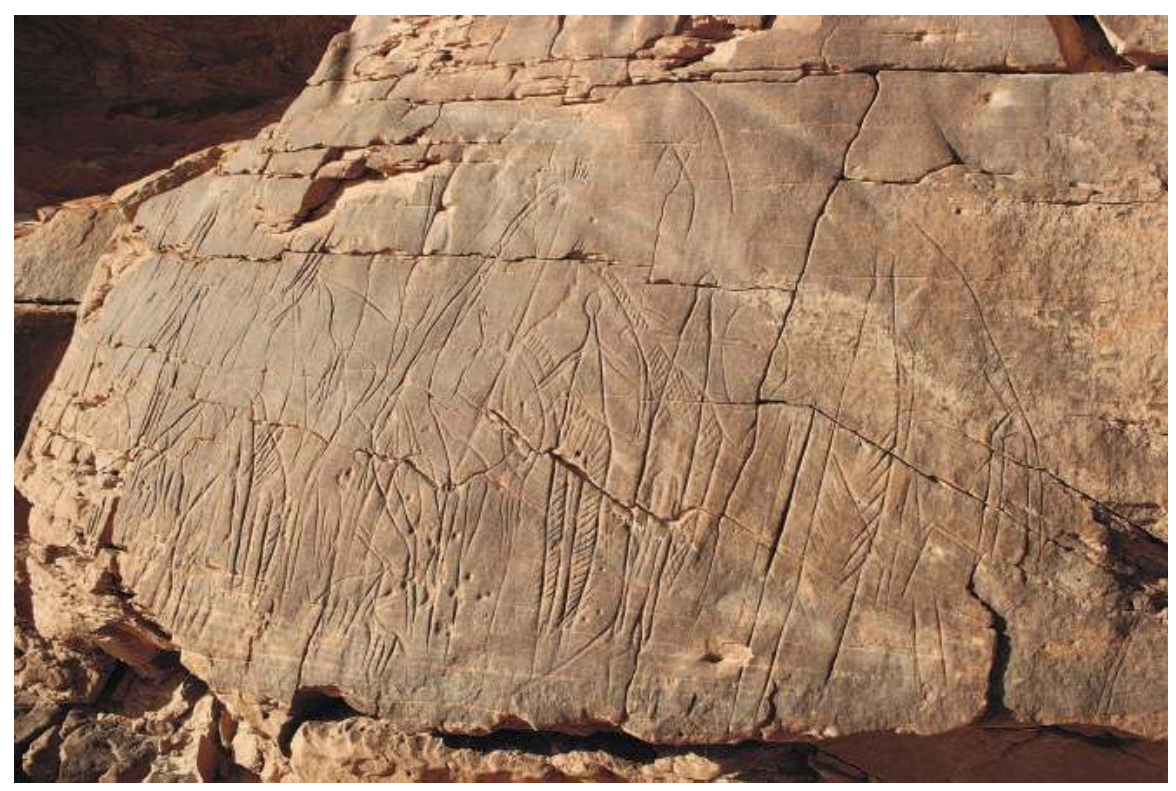

Photo 4

Panneau gravé néolithique découvert en 2016 au sud du lac Teli (Ounianga Serir).

(C) V. Mourre.

représentations schématiques de sandales au sol. Des figures animales gravées (girafes notamment) sont présentes dans la partie supérieure de la butte, jusqu'au sommet. Certains blocs de grès anciennement effondrés portent des gravures encore visibles, mais non identifiables.

Le deuxième site à gravures rupestres a été identifié par Djimet Guemona et Christine Raimond à proximité de l'extrémité sud du lac Ardjou, le plus occidental d'Ounianga Teli. Des figures gravées sont présentes en abondance sur les pentes d'un éperon orienté nord-est/sud-ouest et culminant à une dizaine de mètres au-dessus du niveau du lac le plus proche. Parmi les figures animales explicites, on compte de nombreux bovidés, quelques girafes ainsi que 2 probables autruches. Des figures géométriques symétriques sont également présentes. Cet ensemble pourrait correspondre à celui signalé par A. J. Arkell (1964, p. 10) que nous avions recherché en vain en 2015. Le secteur compte de nombreux amas de pierres correspondant probablement à des sépultures, parfois démantelées.

À ce stade, concernant la chronologie de ces sites, nous en sommes réduits à proposer des terminus assez vagues. Les progrès récents concernant la périodisation et la chronologie de l'art saharien semblent montrer que cet art ne précède guère les débuts du pastoralisme, vers 5000 ans cal BC (LE QuELLEC, 2013). Par ailleurs, la grande faune africaine a progressivement décliné durant les $4^{\mathrm{e}}$ et $3^{\mathrm{e}}$ millénaires pour disparaître lors de la dégradation climatique de 2200 ans BC. Un examen approfondi des œuvres sera nécessaire pour tenter de préciser leurs affinités stylistiques, en particulier avec celles relevant des styles de Tazina et du Mesak. 


\section{Sites à structures de stockage excavées}

Plusieurs sites à structures excavées ont été découverts, dont 3 dans la région d'Ounianga Serir et 1 dans la région d'Ounianga Kebir. Ces structures sont creusées dans des formations sédimentaires carbonatées très tendres et sont à l'heure actuelle comblées par des sédiments sableux (photo 5). Leurs ouvertures circulaires sont visibles à la surface du sol actuel et leur nombre varie d'une dizaine à plus de 150 pour un même site. Quatre de ces structures ont été fouillées et relevées : d'une profondeur comprise entre 30 et $80 \mathrm{~cm}$ pour un diamètre à l'ouverture pouvant atteindre $70 \mathrm{~cm}$ et un diamètre maximal pouvant atteindre $1,20 \mathrm{~m}$, elles présentaient un profil en ampoule et évoquaient des silos destinés au stockage alimentaire. Les 4 structures fouillées étaient pauvres en mobilier archéologique mais deux d'entre elles ont livré des carporestes qui ont fait l'objet d'une détermination (M.T.) : dans un cas (ARTLT18), il s'agit de noyaux de palmier dattier Phoenix dactylifera et dans l'autre (ARLT28), il s'agit de graines d'une graminée de la sous-famille des Panicoideae, groupe dans lequel on trouve par exemple Panicum turgidum, une espèce courante dans les milieux désertiques, dans le Sahara comme dans la péninsule arabique.

Des datations par le radiocarbone ont été réalisées pour ces carporestes : les noyaux de palmier dattier ont donné un âge de $395 \pm 30$ ans BP (Poz-97033), soit $429 \pm 69$ ans cal BP, et les graines de Panicoideae un âge de $1645 \pm 30$ ans BP (Poz-97032), soit $1552 \pm 34$ ans cal BP. Ces âges très différents apportent plus de questions que de réponses et il est en l'état actuel des recherches difficile

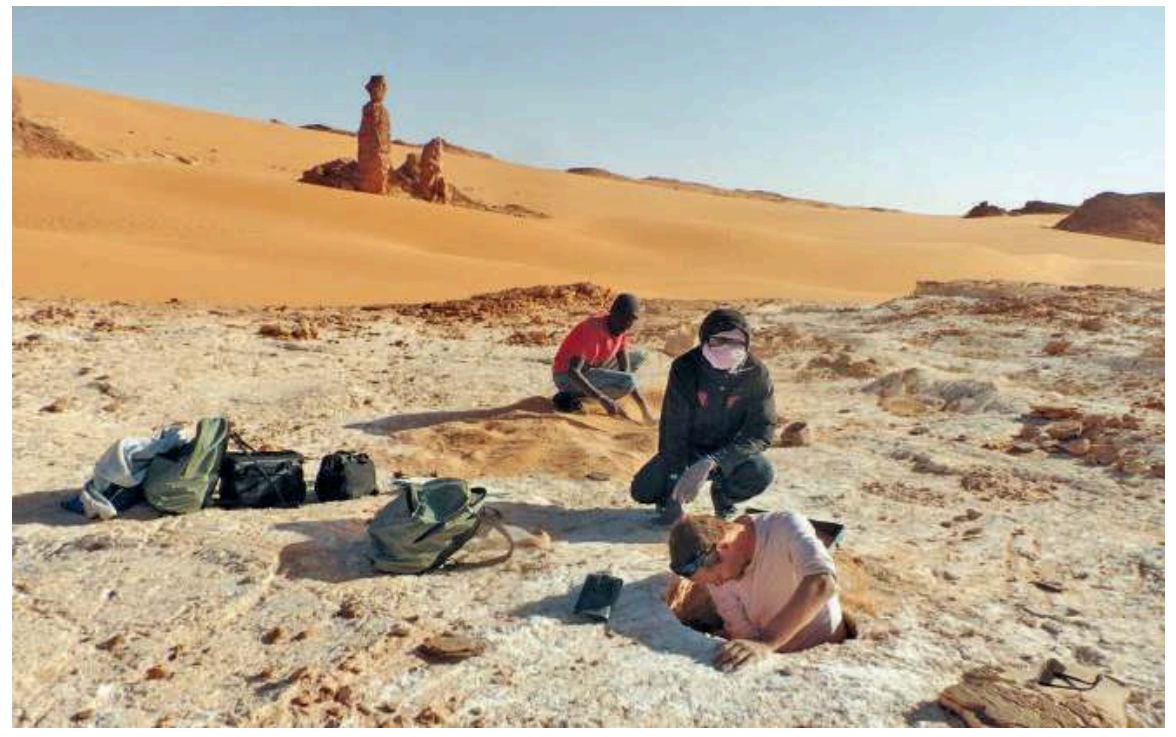

Photo 5

Fouille d'une structure de stockage excavée dans des formations carbonatées (Ounianga Serir). () J. Mbairo. 
de proposer une interprétation globale pour ces sites à structures excavées. Des structures préhistoriques liées au stockage physique sont documentées dans la zone sahélienne (GrONENBORN, 1997) et dans la vallée du Nil (HiLDEBRAND et Schilling, 2016) mais nous n'avons à ce jour pas identifié d'élément de comparaison dans la zone saharienne.

\section{Activités métallurgiques traditionnelles}

L'un des résultats les plus significatifs de la mission 2016 est la mise en évidence d'une activité de métallurgie traditionnelle dans la région d'Ounianga. Suite aux enquêtes orales, les prospections ont permis d'identifier des scories isolées, des amas de scories, des zones d'épandage de scories mais aussi des bases de fours de réduction. Sept sites ont été identifiés, dont 4 à Ounianga Kebir, à proximité des lacs Uma et Forodone, et 3 à Ounianga Teli. L'amas ARLT51, près du lac Uma, a livré des scories présentant des caractéristiques liées aux activités de forge (photo 6). Quatre datations par radiocarbone ont livré des résultats proches de 400 ans cal BP pour trois d'entre elles et de 500 ans cal BP pour la dernière. À proximité, le site ARLT53 a livré 4 bases de fours liés à la transformation du minerai en métal. L'épandage de scories ARLT62, proche du lac Ardjou dans la région d'Ounianga Teli, a donné une datation par radiocarbone proche de 1000 ans cal BP (tabl. 1).

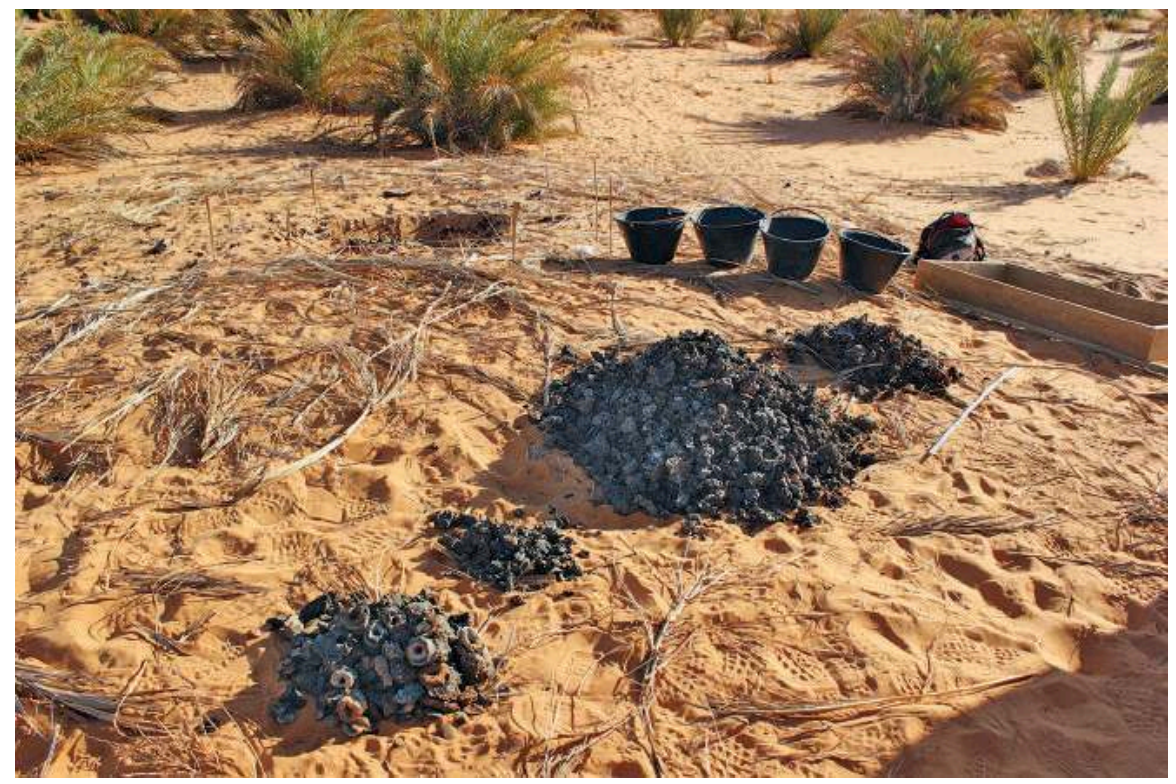

Photo 6

Fragments de tuyères et scories caractéristiques d'activités métallurgiques découverts dans un amas à proximité du lac Uma (Ounianga Kebir).

(C). Mourre. 
Tableau I

Datations des sites métallurgiques ARLT5 I et ARLT62 (datations sur charbons d'Acacia type)

\begin{tabular}{|llll|}
\hline Échantillon & Code laboratoire & Âge I4C & Âge cal BP \\
\hline ARLT5I niv.0I & Poz-96684 & $425 \pm 30 \mathrm{BP}$ & $493 \pm$ I9 cal BP \\
\hline ARLT5I niv.02 & Poz-96685 & $320 \pm 30 \mathrm{BP}$ & $387 \pm 53 \mathrm{cal} \mathrm{BP}$ \\
\hline ARLT5I niv.03 & Poz-96687 & $330 \pm 30 \mathrm{BP}$ & $393 \pm 55 \mathrm{cal} \mathrm{BP}$ \\
\hline ARLT5I niv.04 & Poz-96688 & $340 \pm 30 \mathrm{BP}$ & $399 \pm 58 \mathrm{cal} \mathrm{BP}$ \\
\hline ARLT62 lotI amas I & Poz-96689 & $1095 \pm 30 \mathrm{BP}$ & $1009 \pm 37 \mathrm{cal} \mathrm{BP}$ \\
\hline
\end{tabular}

\section{Sites d'habitat précoloniaux}

Au rang des données nouvelles figure également la redécouverte d'un passé précolonial, qui doit énormément aux enquêtes orales initiées en 2016 par nos collègues chercheurs en sciences humaines, en particulier Christine Raimond et Zakinet Dangbet. Nous avons notamment pu visiter deux sites d'habitat historique perchés sur des éperons rocheux.

Le premier (Egnoa; ARLT57; photo 7) se trouve au nord du lac Yoan, à environ $2 \mathrm{~km}$ du fort d'Ounianga Kebir. La tradition rapporte que ces lieux étaient habités

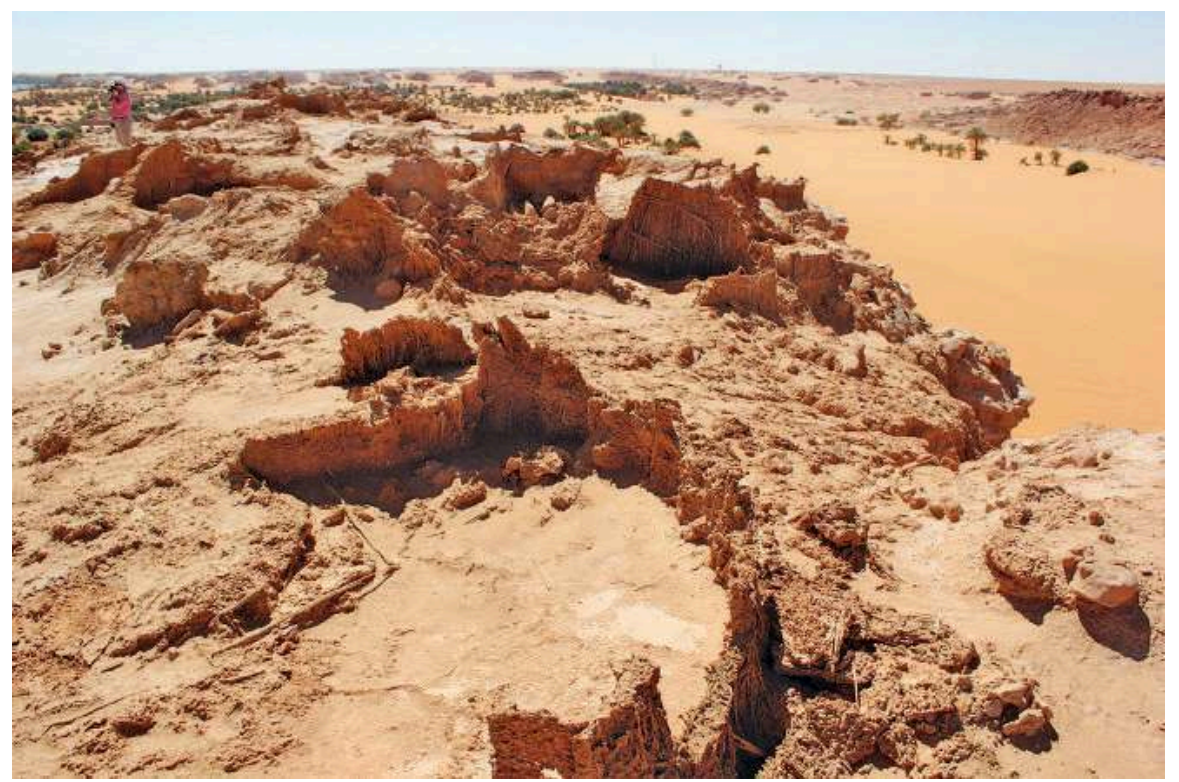

Photo 7

Vue générale des restes de quelques structures dans l'un des habitats historiques perchés sur des éperons rocheux au nord du lac Yoan, à environ $2 \mathrm{~km}$ du fort d'Ounianga Kebir (Egnoa ; ARLT57).

(C) V. Mourre. 
avant l'arrivée des colons français en 1913 et l'implantation d'une garnison au fort. La position naturellement fortifiée de l'éperon aurait permis aux populations de se prémunir contre les razzias dont elles étaient régulièrement victimes. L'accès aux habitations n'est possible qu'à une extrémité de l'éperon, par un passage étroit et très escarpé. Au sommet de l'éperon sont visibles les bases d'environ 65 structures circulaires. Certaines comportent encore une partie de leurs élévations, conservées sur 0,50 à $1 \mathrm{~m}$ et composées d'un torchis de terre crue sur des clayonnages en palmes de dattier, voire de briques en terre crue ou de blocs de grès assemblés avec un mortier de terre crue. Les diamètres des structures varient de 1 à $2 \mathrm{~m}$, ce qui traduit probablement la présence conjointe d'habitations et de structures de stockage. Le fond des structures se présente sous forme de taches rougeâtres, parfois accompagnées de foyers clairement visibles.

Le second site perché (Ambra ; ARLT58) que nous avons visité est plus proche du lac Yoa. Il correspond à l'emplacement de l'ancienne chefferie. Des structures maçonnées, accolées aux parois de l'éperon, nous ont été présentées comme des greniers utilisés pour le stockage de dattes. Sur l'éperon lui-même, des bases de structures circulaires sont présentes, mais en nombre plus réduit qu'à Egnoa. Leur état de conservation est également moindre.

\section{Perspectives}

Avec une soixantaine d'entités archéologiques explorées ou inventoriées en seulement quelques semaines effectives de travail sur le terrain, le bilan scientifique des missions Ounianga 2015 et 2016 est évidemment très positif. Notre souhait est de poursuivre les recherches sur le terrain lors d'une prochaine mission dans la région des lacs d'Ounianga.

\section{Remerciements}

La mission archéologique franco-tchadienne est soutenue par le ministère de l'Europe et des Affaires étrangères, par le service de coopération et d'action culturelle de l'ambassade de France à N'Djamena et par la direction des activités internationales de l'Inrap. Les missions archéologiques Ounianga 2015 et 2016 n'auraient pas été possibles sans le soutien du programme Gelt et en particulier sans l'aide d'Ibrahim Chitou, de Moussa Abderamane et de Kelly Nkouka. Les auteurs remercient les étudiants tchadiens qui ont pris part efficacement aux 
missions de terrain à Ounianga : Abakar Abanga, Pierrot Mbaïhondoum et Sephora Milamem Ngaodanbe. Merci également aux collègues chercheurs Zakinet Dangbet, Pierre Deschamps, Christine Raimond, Mathieu Schuster, Thomas Stieglitz, Florence Sylvestre et Thijs Van der Meeren pour les nombreux échanges enrichissants. Vincent Mourre remercie très chaleureusement Jean Courtin pour les documents inédits qu'il lui a communiqués ainsi que pour son enthousiasme et son intérêt pour ces nouveaux travaux de recherche au Tchad.

Les résultats du projet et les rapports de mission sont disponibles sur http:// arrelat.eklablog.com/.

\section{Bibliographie}

\section{ARKell A. J., 1959}

Preliminary report of the Archaeological results of the British Ennedi Expedition 1957, Kush, VII, (Khartoum) : 15-26.

\section{Arkell A. J., 1960}

Report of the Archaeological Results of the British Ennedi Expedition, 1957, Man, $60: 40$

\section{Arkell A. J., 1962a}

The Aterian of Great Wanyanga (Ounianga Kebir). In : Mortelmans G., éd. : Actes $d u I^{e}$ congrès panafricain de préhistoire et de l'étude du Quaternaire, Léopoldville 1959, Musée royal de l'Afrique centrale, Tervuren (Belgique), $\mathrm{n}^{\circ} 40$ (section III) : 233-242.

\section{Arkell A. J., 1962b \\ The distribution in central Africa of one early Neolithic Ware (Dotted wavy line pottery) and its possible connection with the beginning of pottery. In : Mortelmans G., éd. : Actes $d u I V^{e}$ congrès panafricain de préhistoire et de l'étude du Quaternaire, Léopoldville 1959, Musée royal de l'Afrique centrale, Tervuren (Belgique), $\mathrm{n}^{\circ} 40$ (section III) : 283-287.}

\author{
ARKell A. J., 1964 \\ Wanyanga and an Archaeological \\ Reconnaissance of the South-West Libyan Desert: \\ The British Ennedi Expedition 1957, Londres, \\ Oxford University Press, 24 p. + pl. \\ Cancellieri E., Di Lernia S., 2013 \\ Middle Stone Age human occupation \\ and dispersals in the Messak plateau (SW Libya,
}

central Sahara), Quaternary International, vol. $300: 142-152$.

\section{Collectif, 2012}

Candidature au patrimoine mondial, évaluation technique de l'UICN, lacs d'Ounianga (Tchad), rapport d'évaluation de l'UICN, 22 p., multigr.

Francus P., Von Suchodoletz H., Dietze M., Donner R. V., Bouchard F., RoY A.-J., FAgot M., Verschuren D., KRöPelin S., 2013 Varved sediments of Lake Yoa (Ounianga Kebir, Chad) reveal progressive drying of the Sahara during the last 6100 years. Sedimentology 60, 911-934.

\section{GRONENBORN D., 1997}

An ancient storage pit in the SW Chad Basin, Nigeria, Journal of Field Archaeology, 24 (4) : 431-439.

Hildebrand E. A., Schilling T. M., 2016 Storage amidst early agriculture along the Nile: Perspectives from Sai Island, Sudan, Quaternary International, 412 (Part B) : 81-95.

Joleaud L., Lombard J., 1933

Mammifères quaternaires d'Ounianga Kebir (Tibesti sud-oriental), Compte rendu de l'académie des Sciences, 196 (7) : 497-499.

KRÖPELIN S., 2007

High-resolution climate archives in the Sahara (Ounianga, Chad). In : Bubenzer O., Bolten A., Darius F., éd. : Atlas of Cultural and Environmental Change in Arid Africa, Cologne, 21, Heinrich-Barth-Institut : 56-57. 


\section{Le Quellec J.-L., 2013}

Périodisation et chronologie des images rupestres du Sahara central, Préhistoires Méditerranéennes, 4, mis en ligne le 14 octobre 2013. [En ligne].

\section{SCERRI E. M. L., 2013}

The Aterian and its place in the North African Middle Stone Age, Quaternary International, $300: 111-130$.

\section{TILlet TH., 1983}

Le Paléolithique du bassin tchadien septentrional (Niger-Tchad), Paris, CNRS, 319 p. 


\section{ariabilité des crues}

\section{et des paysages du lac Fitri} depuis les grandes sécheresses des années 1970-1980

Tashi YalikUn, Christine RaIMOND, Angeline KEMSOL NAGORNGAR, Dangbet ZAKINET, Mathieu SCHUSTER, Florence SYLVESTRE

\section{Introduction}

L'importance des zones humides tropicales est reconnue à l'échelle internationale, tant pour leur biodiversité, leur rôle écosystémique, que pour les ressources qu'elles fournissent aux sociétés (MADGWICK et PEARCE, 2017). À cette échelle, un constat de déclin s'affirme aussi bien en superficie qu'en qualité (GARDNER et al., 2015 ; CREED et al., 2017). Au Sahel, les zones humides sont vitales pour les populations, notamment pendant la saison sèche. Si les chercheurs démontrent le caractère réversible de l'extension de certains lacs, comme le lac Tchad qui se stabilise à un état de «Petit lac » après les sécheresses des années 1970-1980 (MAGRIN et al., 2015), les études peinent à prendre en compte la variabilité inter et intra-annuelle qui caractérise les régimes hydrologiques des lacs sahéliens. « À ce rythme d'exploitation et sans changer le mode actuel d'utilisation [...] la perte d'habitats et de biodiversité, mais aussi de systèmes productifs et de ressources vitales pour la survie de la population, est inévitable " : cette conclusion alarmiste de l'évaluation de la zone de biosphère du Fitri par le BEGC (2016) ne tient pas compte de l'évolution des ressources du lac, liée à celle de la pluviométrie dans son bassin versant. L'objectif de cet article est de préciser cette variabilité inter et intra-annuelle. L'acquisition d'images satellitaires entre 
1972 et 2015 fournit un suivi annuel pour cette période ; un suivi mensuel est réalisé pour l'année 2015. Une méthodologie originale a été expérimentée pour discriminer les eaux libres et la végétation marécageuse qui composent la zone humide. L'analyse des résultats contribue à mieux comprendre le fonctionnement du lac sur le court et le moyen terme, ainsi que l'évolution des surfaces de la zone humide où se concentrent les ressources disponibles en saison sèche.

Une étude aux objectifs similaires existe pour le lac Tchad (LEBLANC et al., 2011), à partir d'une méthodologie qui utilise les données thermiques (Tmax) du satellite Météosat pour identifier les eaux couvertes de végétation aquatique et fournit une série chronologique mensuelle d'estimations de la superficie totale inondée pour le lac Tchad entre 1986 et 2001. Cette technique tire parti de la résolution temporelle élevée et du capteur thermique du satellite géosynchrone pour cartographier les eaux libres et les eaux couvertes de végétation aquatique dans de grandes masses d'eau comme le «Petit» lac Tchad dont la superficie varie entre 3000 et $14000 \mathrm{~km}^{2}$. Elle est inadaptée pour un plan d'eau de taille relativement petite, en raison de la faible résolution spatiale des données Météosat qui est de $5 \mathrm{~km}^{2}$. Nous proposons une nouvelle technique qui profite de la haute résolution spatiale pour une bonne répétitivité des données de télédétection optique. Elle permet de cartographier avec une précision plus élevée les zones humides de régions lacustres en zone aride et semi-aride pour suivre leurs variations à court et à long terme.

\section{Délimitation et caractéristiques de la zone d'étude}

Centré sur $12^{\circ} 50^{\prime} \mathrm{N}$ et $17^{\circ} 30^{\prime} \mathrm{E}$, le lac Fitri est situé à $300 \mathrm{~km}$ à l'est de N'Djamena dans une plaine alluviale à la topographie peu prononcée. Il se situe, tout comme le lac Tchad, dans les limites du Mégalac Tchad holocène (LEBLANC et al., 2006 ; SCHUSTER et al., ce volume) (fig. 1). D'un point de vue administratif, il fait partie du département de Fitri dans la région du Batha et son chef-lieu, Yao est également le siège du sultanat bilala. Il est situé sur une presqu'île à proximité de l'unique pointement rocheux de la région. Le lac Fitri est labellisé zone Ramsar depuis le 13 juin 1990.

Le climat est marqué par l'alternance d'une saison des pluies de quatre à cinq mois (fig. 2), centrée sur le mois d'août, et d'une saison sèche de six à sept mois, en partie hivernale de décembre à février avec des températures comprises entre 11 et $22^{\circ} \mathrm{C}$. Les maxima de température $\left(40-41^{\circ} \mathrm{C}\right)$ sont généralement observés au mois d'avril. Les précipitations sont marquées par une forte irrégularité dans leur répartition tant spatiale que temporelle (NIEL et al., 2005) (fig. 3). 


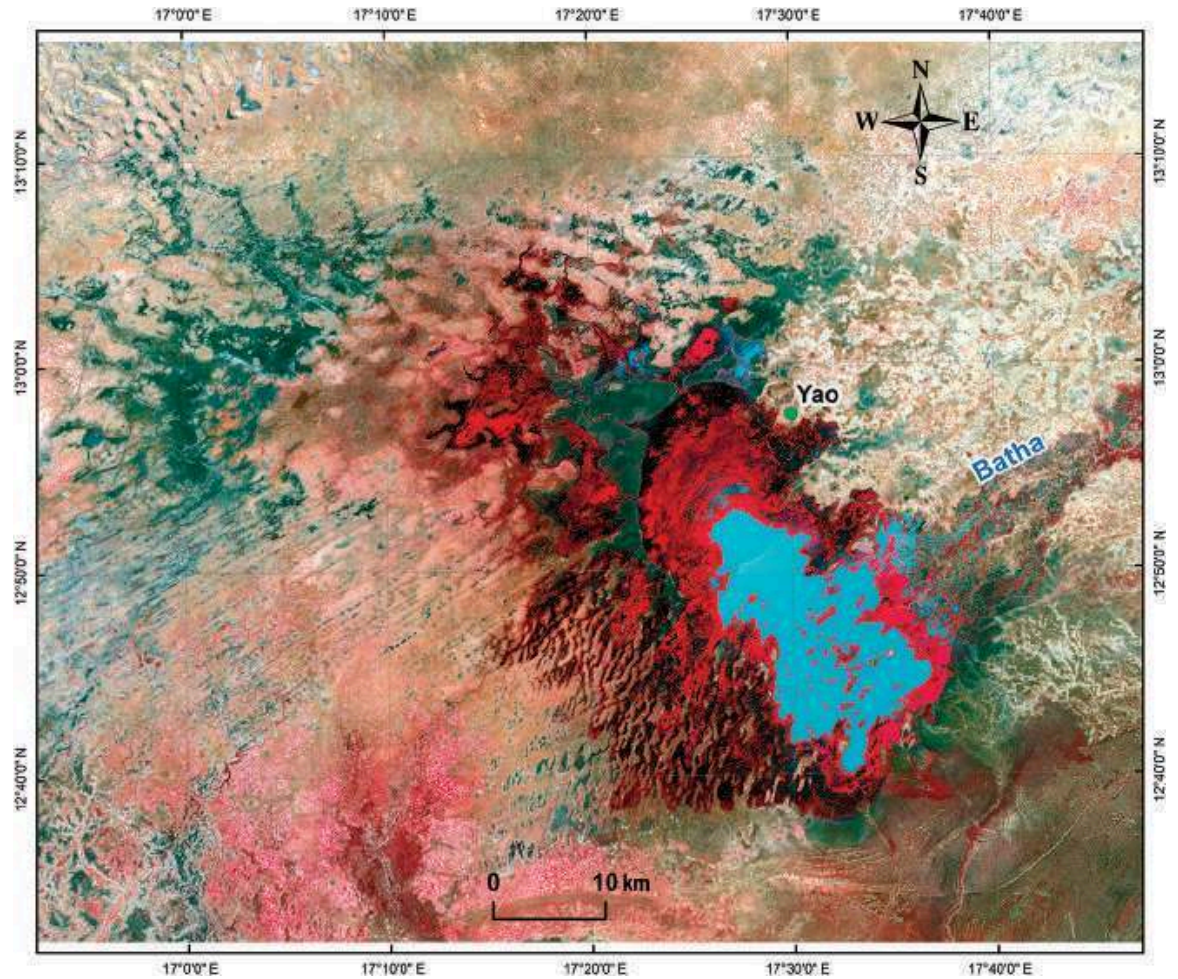

Figure I

Zone d'étude. Image Landsat 8 OLI du /3//0/20/5.

Source : Landsat 8.

\section{Précipitations et températures mensuelles à Yao}

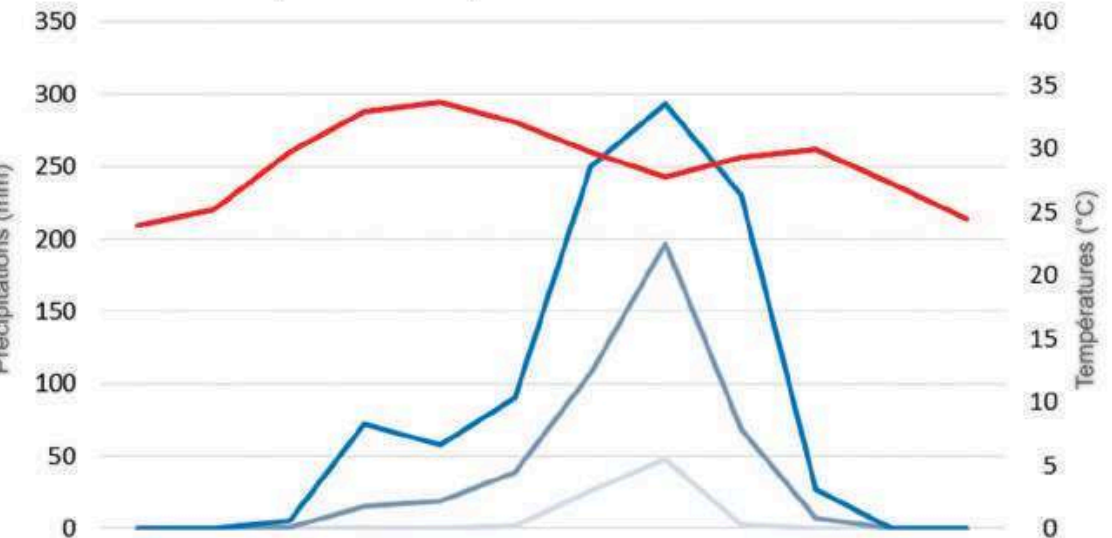

jan. févr. mars avr. mai juin juill. août sept. oct. nov. déc.

$\longrightarrow \min \longrightarrow \operatorname{moy} . \max \longrightarrow$ température

Figure 2

Graphique ombrothermique pour la station de Yao représentant les moyennes mensuelles de précipitations et de températures de 1983 à 2013.

Source : Drem. 
Cette irrégularité s'explique par la distribution du flux de la mousson guinéenne, principale source d'humidité. Il a plu en moyenne $400 \mathrm{~mm}$ à Yao entre 1970 et 2013 avec des variations interannuelles allant jusqu'à $669 \mathrm{~mm}$ en 2005 contre seulement $121 \mathrm{~mm}$ en 1975.

L'évolution saisonnière de l'humidité relative de l'air est identique à celle des précipitations, le maximum se situe en juillet et août, de l'ordre de 85 à $90 \%$. L'évaporation annuelle est très élevée, en moyenne comprise entre 3775 et $4000 \mathrm{~mm}$ (BEGC, 2016).

Le réseau hydrographique est constitué de plusieurs cours d'eau intermittents dont le plus important est le fleuve Batha (60\% des apports), avec ses affluents le Melmélé, le Zilla, le Zerzer et l'Abourda (BIEP, 1989). Il est complété par les rivières venant du massif central tchadien. La superficie moyenne du lac de $800 \mathrm{~km}^{2}$ (LEMOALLE, 1979) est en fait très variable d'une année à l'autre et d'une saison à l'autre. Le lac s'est complètement asséché en 1901, 1973 et 1985. Il aurait aussi atteint une superficie maximale de $1300 \mathrm{~km}^{2}$ en 1970 (LEMOALLE, 1979).

La végétation s'organise en auréoles autour des eaux libres. Au-delà de la cuvette entièrement végétalisée, plusieurs grandes plaines sont inondées par la crue du lac ou des principaux cours d'eau, dont les plaines de Manga (rives nord du lac), Dogo (rives nord-est du lac) et Gamsa (delta du Batha). Elles sont cultivées en sorgho repiqué (berbéré, Sorghum bicolor). Les rives sud-ouest apparaissent plus hétérogènes du fait d'avancées lacustres à l'intérieur d'une série sableuse ancienne constituée par des dunes fixées, orientées nord-est/sud-ouest. Ces dunes, où sont installés les villages, surplombent les dépressions de 3 à $4 \mathrm{~m}$ où les vertisols à boisement dense d'Acacia seyal sont cultivés, après défrichage, en berbéré.

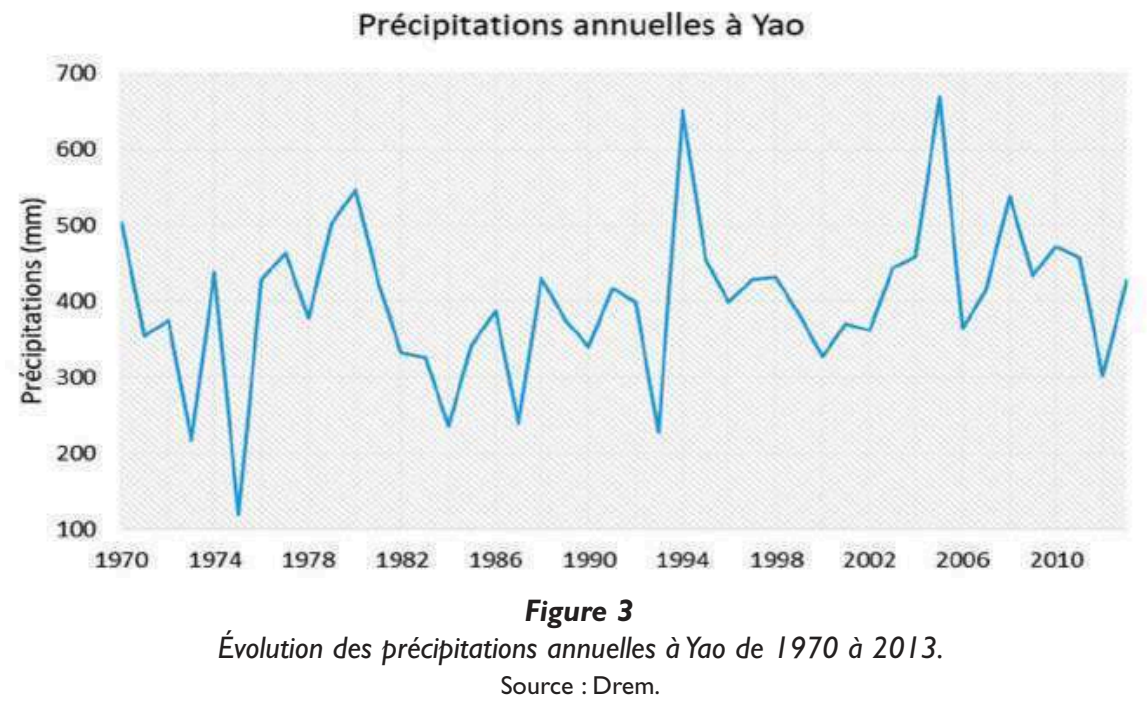


À l'extérieur de ce système, les cuvettes argileuses alimentées par l'eau des pluies et les nappes affleurantes sont également repiquées en sorgho. Autour, la steppe sahélienne présente une strate arborée plus ou moins dense en fonction de la disponibilité en eau. Elle se dessèche très vite à la fin de la saison pluvieuse. Les prairies à l'ouest ne sont pas cultivées et principalement exploitées par les éleveurs. Le nord-est est plus peuplé et composé d'une mosaïque de villages, de champs de saison des pluies et de pâturages. Les éleveurs les exploitent avant les récoltes de berbéré, puis mènent les troupeaux dans les pâturages aquatiques.

Il est devenu courant au Fitri d'entendre dire que « les eaux du lac se vident à l'ouest ", alors que ce n'était pas observé auparavant (com. pers., enquêtes de terrain 2015). Pour vérifier cette observation, nous avons choisi de délimiter une zone d'étude qui couvre les mares situées au nord-ouest du lac, occasionnellement inondées. Ainsi, la zone d'étude est comprise entre $12^{\circ} 32^{\prime} 10^{\prime \prime} \mathrm{N}-13^{\circ} 15^{\prime} 55^{\prime \prime} \mathrm{N}$ et $16^{\circ} 52^{\prime} 20^{\prime \prime} \mathrm{E}-17^{\circ} 48^{\prime} 06^{\prime \prime} \mathrm{E}$. Elle s'étend sur $8135 \mathrm{~km}^{2}$ et couvre le lac Fitri et les plaines inondables environnantes (fig. 1). Fortement inondable, cette zone reste enclavée plus de trois mois dans l'année à partir du mois d'août.

\section{Méthodologie}

\section{Collection de données}

Nous avons collecté au total 42 images des satellites Landsat (38 scènes) et $\operatorname{Spot}^{1}$ (4 scènes) entre 1972 et 2015 (tabl. 1). Les images Landsat viennent des capteurs MSS (Multispectral Scanner, résolution : $80 \mathrm{~m}, 1$ scène), TM (Thematic Mapper, résolution : 30 m, 5 scènes), ETM+ (Enhanced Thematic Mapper Plus, résolution : $30 \mathrm{~m}, 8$ scènes) et OLI (Operational Land Imager, résolution : $30 \mathrm{~m}$, 24 scènes). La sélection des images suit plusieurs critères : une par année depuis les premiers satellites au moment de la crue (octobre) pour avoir des images comparables, et une image par mois pour un suivi mensuel sur l'année 2015, qui correspond aux observations de terrain.

Les images Landsat apportent une couverture quasi synoptique de très grandes surfaces (chaque scène couvre $32400 \mathrm{~km}^{2}$ ), une faible déformation géométrique, une bonne répétitivité et des données multispectrales pour une meilleure différenciation des composants du milieu. Les images Spot sont utilisées pour compléter la série pendant les années où les images Landsat ne sont pas disponibles. Malgré une résolution spectrale plus faible (4 canaux) et une couverture au sol contraignante $\left(60 \mathrm{~km} \times 60 \mathrm{~km}\right.$, soient $\left.3600 \mathrm{~km}^{2}\right)$, les hautes et très hautes résolutions spatiales des images Spot $(20 \mathrm{~m}$ à 2,5 m) s'avèrent 
très utiles pour préciser les lignes de rivages, les transitions souvent progressives des hauteurs d'eau, les sédiments et le sol humide, ou bien entre l'eau, les macrophytes aquatiques et une couverture végétale dense sur terrain sec.

Tableau I

Images satellitaires collectées sur le lac Fitri.

\begin{tabular}{|c|c|c|c|c|c|c|c|c|c|c|c|c|c|}
\hline Année & janv. & févr. & mars & avr. & mai & juin & juill. & août & sept. & oct. & nov. & déc. & Satellite \\
\hline 1972 & & & & & & & & & & 30 oct. & & & Landsat I MSS \\
\hline 1986 & & 18 févr. & & & & & & & & 13 oct. & & & Landsat 5 TM \\
\hline 1987 & & & 6 mars & & & & & & & 16 oct. & & & Landsat 5 TM \\
\hline 1995 & 27 janv. & & & & & & & & & & & & Spot 3 \\
\hline 1998 & & & & & & & & & & & & fer déc. & Landsat 5 TM \\
\hline 1999 & & & & & & & & & & & & 12 déc. & Landsat 7 ETM+ \\
\hline 2000 & & 14 févr. & & & & & & & & 27 oct. & 28 nov. & & Landsat 7 ETM+ \\
\hline 2001 & & & & & 7 mai & & & & & & & & Landsat 7 ETM+ \\
\hline 2002 & & & & & & & & & & & 18 nov. & & Landsat 7 ETM+ \\
\hline 2003 & & & & II avr. & 13 mai & & & & & & & & Landsat 7 ETM+ \\
\hline 2006 & 17 janv. & & & & & & & & & & & & Spot 5 \\
\hline 2008 & & & & & & & & & & & & & Landsat 7 ETM+ \\
\hline 2009 & & & & & & & & & 16 sept. & & & & Spot 4 \\
\hline 2011 & & & & & & & & & & 9 oct. & & & Spot 5 \\
\hline 2013 & & & & & 16 mai & 17 juin & & & & 23 oct. & 8 nov. & & Landsat 8 OLI \\
\hline 2014 & II janv. & 12 févr. & & & 19 mai & 20 juin & & & 24 sept. & 26 oct. & 27 nov. & 29 déc. & Landsat 8 OLI \\
\hline 2015 & I 4 janv. & 15 févr. & 19 mars & 20 avr. & 22 mai & 23 juin & 9 juill. & 10 août & 27 sept. & 13 oct. & 14 nov. & 16 déc. & Landsat 8 OLI \\
\hline
\end{tabular}

\section{Traitement des images}

\section{Composition colorée}

Les compositions colorées proche infrarouge (PIR), rouge (R) et vert (V) des 12 images Landsat 8 de 2015 montrent les variations mensuelles du paysage en périodes de crue et de décrue (fig. 4). Les eaux libres se distinguent facilement en raison de la faible luminance de l'eau dans le PIR et prennent des couleurs allant du bleu au turquoise. Leur étendue maximale se situe au mois d'août, en saison des pluies, tandis que les mois d'avril, mai et juin correspondent aux périodes sèches. Autour, une végétation marécageuse dense et verte, donc en pleine activité chlorophyllienne, se caractérise par sa forte luminance dans le canal PIR et s'affiche en rouge. Dans ces secteurs végétalisés, il est difficile d'estimer la profondeur de l'eau, qui peut varier de 0 à $4 \mathrm{~m}$. La végétation de marécage contraste facilement avec la végétation des pourtours du lac, plus sèche et moins dense, même durant la saison des pluies. 


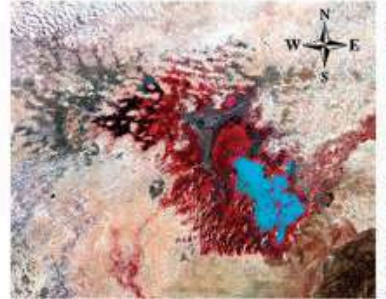

Janvier

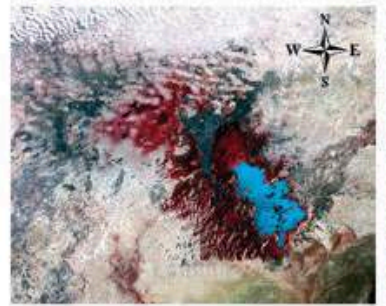

Avril

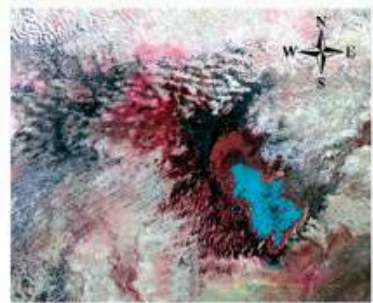

Juillet

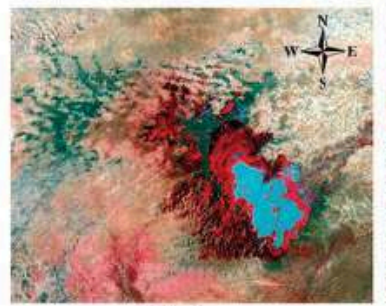

Octobre

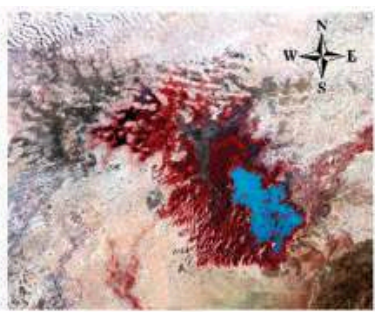

Février

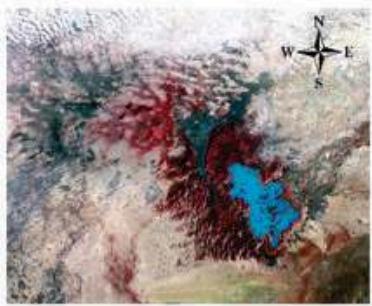

Mai

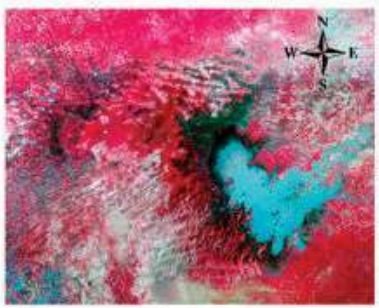

Août

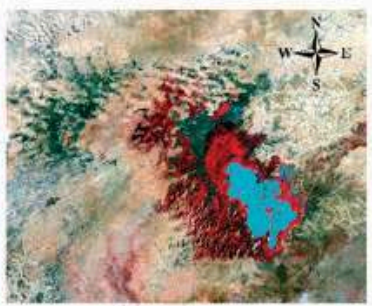

Novembre

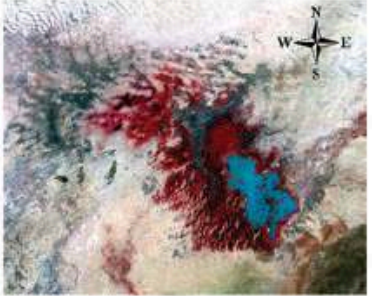

Mars

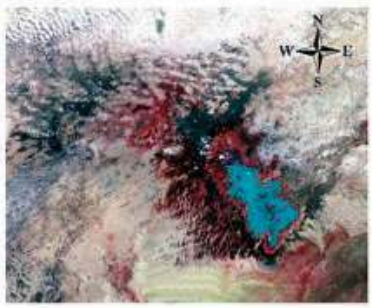

Juin

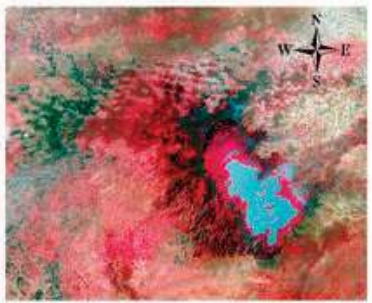

Septembre

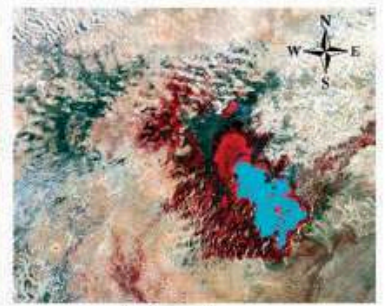

Décembre

0 $10 \quad 20 \mathrm{~km}$

Figure 4

Images Landsat 8 des mois de 2015 en composition colorée proche infrarouge, rouge et vert. Source : Landsat 8.

\section{Classification}

Un schéma général de classification est élaboré pour classer et comparer les résultats entre images multispectrales et multicapteurs (fig. 5). Le flux de travail commence par une classification en 10 classes par IsoData (Tou et GonZALEZ, 
1974). Cette méthode de classification non supervisée examine les signatures spectrales de tous les pixels de l'image et détermine des groupements selon un nombre de classes fixé. Ici, 10 classes thématiques cohérentes différencient les principaux composants du milieu, qui sont ensuite combinées en 2 classes : zones inondées (eaux libres et marécages) et non inondées, validées par notre connaissance du terrain (2015-2016). En cas de résultat non satisfaisant, un masque est créé sur la classe "zones inondées » pour exclure les sources de confusion. L'image sous masque est reclassée en 10 classes, qui sont ensuite regroupées en zones inondées et non inondées. Les zones exclues par le masque s'ajoutent à la classe de zones non inondées. Cette procédure est répétée autant de fois que nécessaire pour correspondre à la vérité terrain. Lorsque le résultat

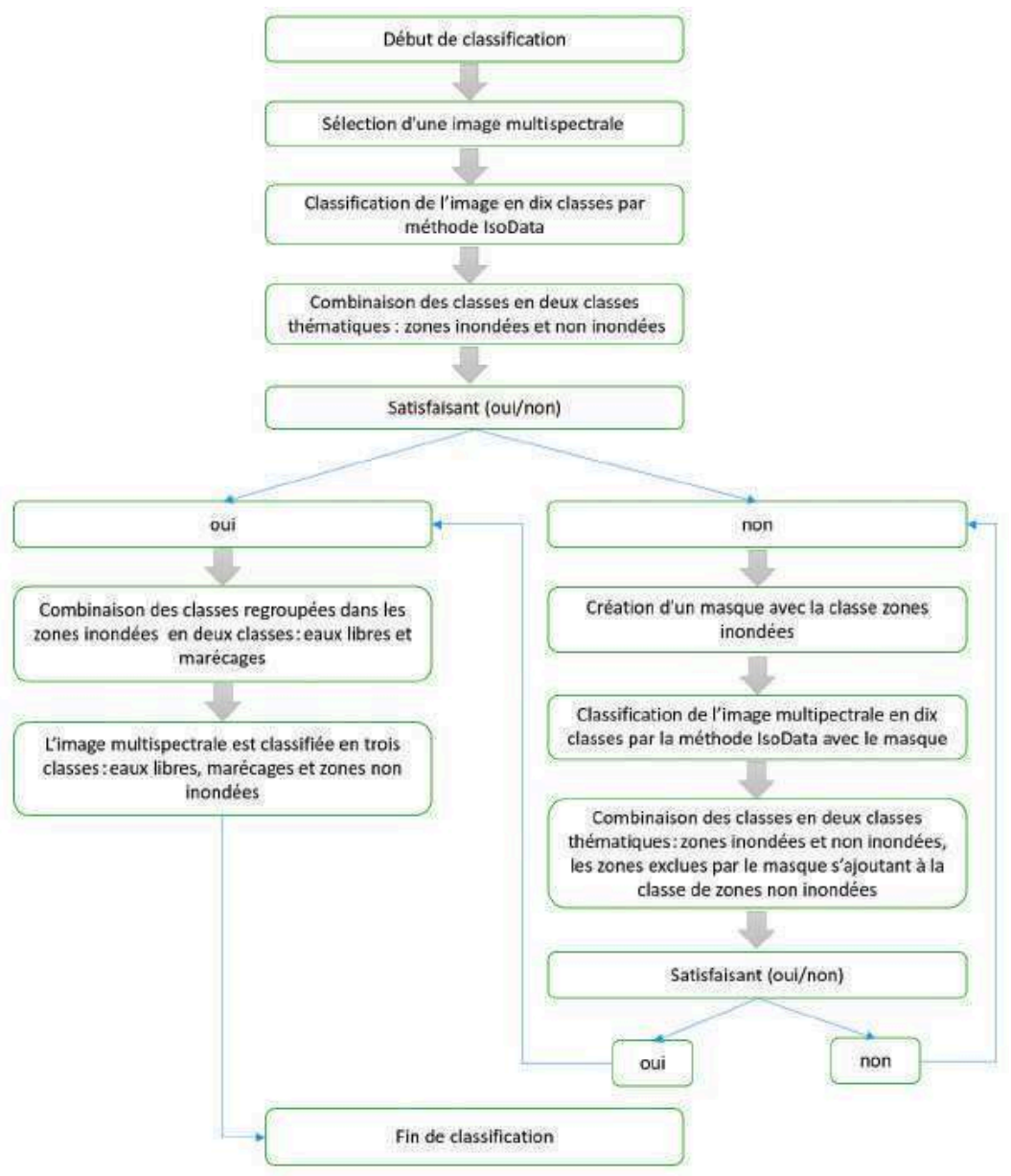

Figure 5

Méthodologie de classification d'images satellite en 3 classes : eaux libres, marécages et zones non inondées. 
est satisfaisant, les zones inondées sont divisées en 2 classes : eaux libres et marécages.

Plusieurs approches de validation et d'évaluation de la qualité des résultats d'une classification sont disponibles (CONGALTON, 1991). Pour les images du Fitri, les coefficients Kappa ${ }^{2}$ (COHEN, 1960) sont compris entre 0,63 et 0,87, avec des précisions globales (le nombre de pixels bien classés divisé par le nombre de pixels total) des classifications comprises entre 76,52\% et 91,41\% (tabl. 2). Les résultats sont donc «bons » à « excellents ». Les données de terrain, cartes thématiques et l'imagerie satellite à très haute résolution (Spot, Google Earth) ont été utilisées pour générer des points de contrôle et ont servi comme deuxième outil qualitatif de validation des résultats.

Tableau 2

Précision globale de classification et coefficient Kappa.

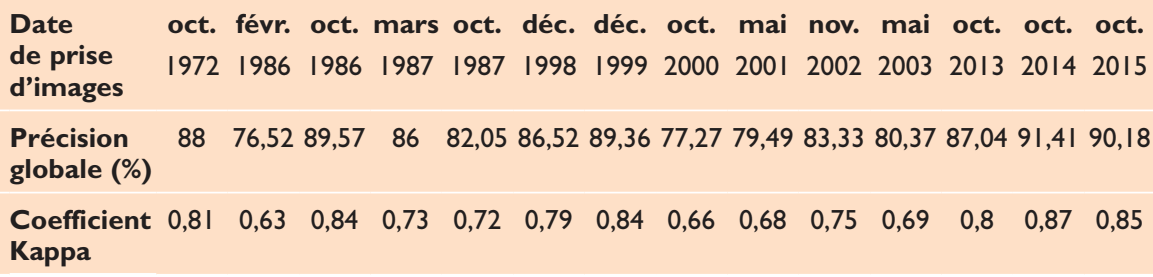

La figure 6 montre en six étapes une implémentation de la méthodologie pour l'image du Landsat 7 ETM+ du 12/12/1999. Un seul masque a été créé dans ce cas, mais le nombre de masques à créer peut varier selon la résolution spectrale (nombre de canaux), la qualité de l'image et les conditions atmosphériques (nuages). Ainsi, 0 à 1 masque a été créé pour les images du Landsat 8 OLI, 3 masques pour l'image Landsat MSS, 1 à 2 masques pour les autres capteurs.

La classification a été appliquée d'abord sur les images de 2015, car les données de terrain nombreuses (point GPS, cartes, notes de terrain), la haute qualité et la bonne fréquence des images du Landsat 8 couvrant tous les mois de l'année ont assuré une bonne interprétation des données. Cette base de comparaison a limité les risques d'erreur d'interprétation pour les classifications des images des autres années.

2. Le coefficient Kappa est une approche quantitative qui utilise tous les éléments de la matrice de confusion et tient compte à la fois des erreurs de commission et d'omission. C'est un nombre réel compris entre -I et I : l'accord est d'autant plus élevé que la valeur de Kappa est proche de I. LANDIS ET KOCH (1977) proposent les interprétations suivantes : kappa $<0,0$ très mauvais ; $0,0-0,2$ mauvais ; $0,21-0,4$ médiocre ; $0,41-0,6$ modéré ; $0,6 \mathrm{I}-0,80$ bon $; \geq 0,8$ l excellent. 


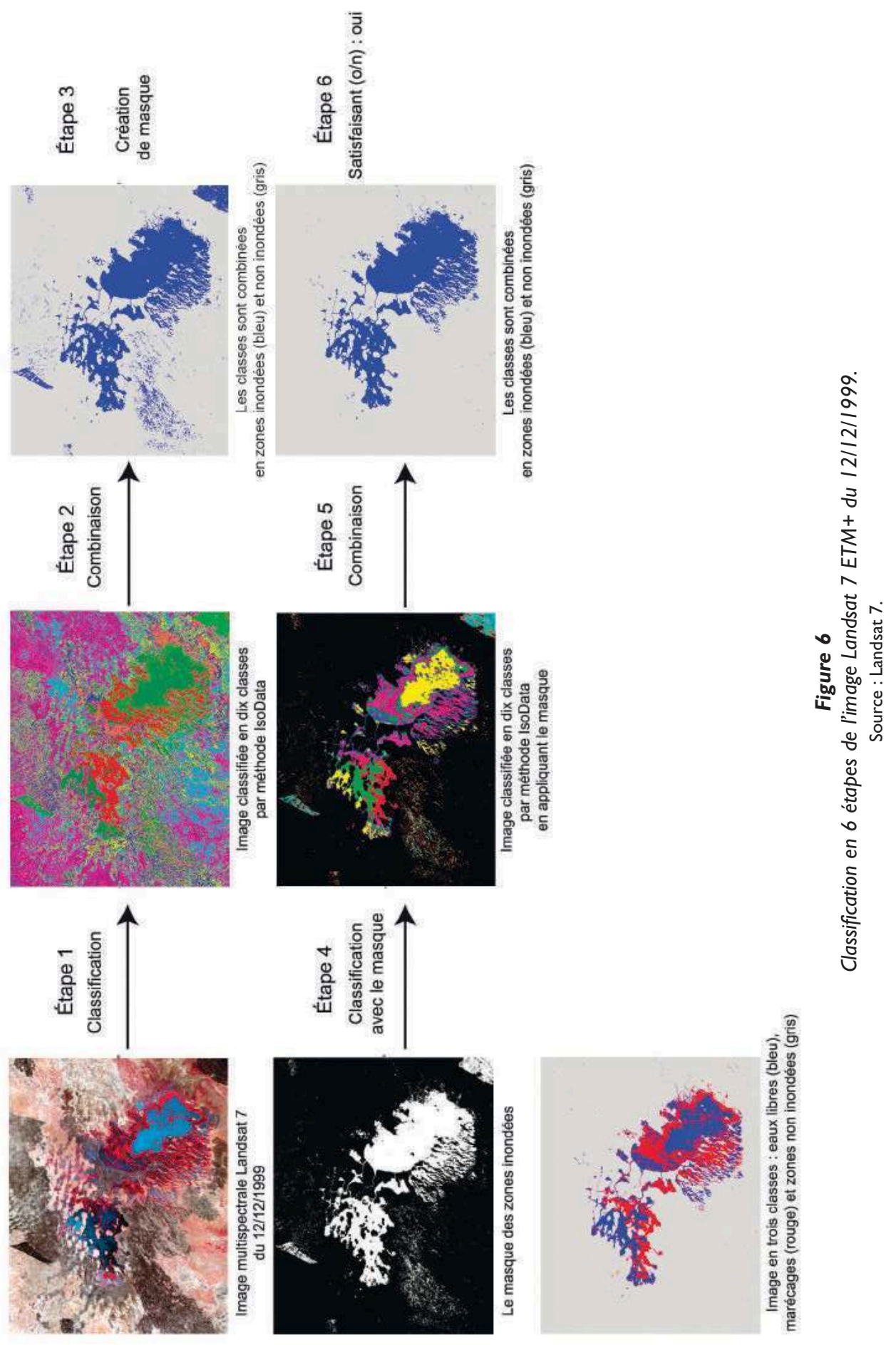




\section{Suivi mensuel de la crue de 2015}

La figure 7 montre les résultats de la classification en 2 classes (zones inondées et non inondées) des images Landsat 8 de l'année 2015. Ici, les eaux libres et les marécages ne sont pas distingués. Les fluctuations de la zone humide suivent

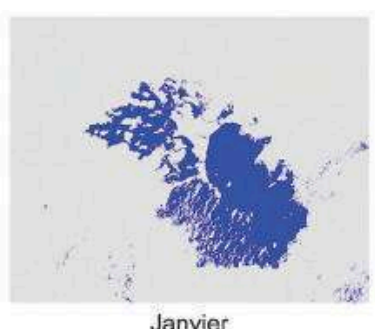

Janvier

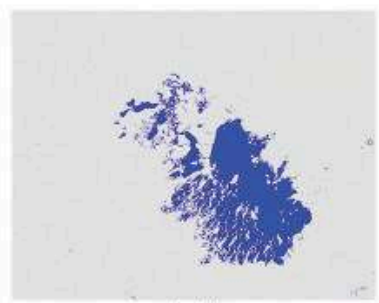

Avril

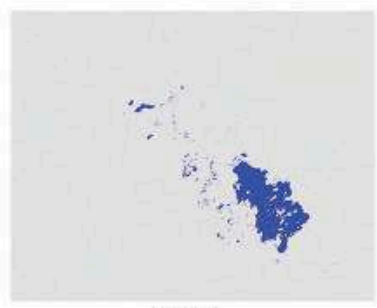

Juillet

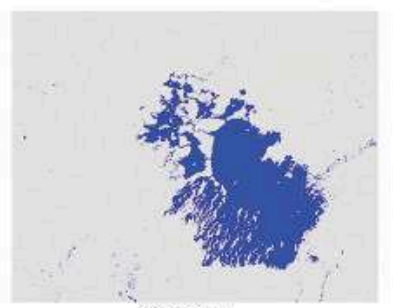

Octobre

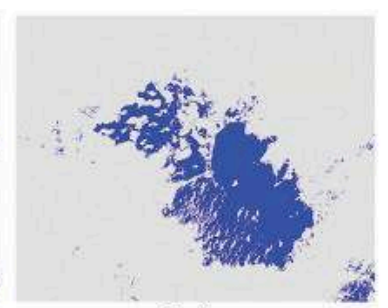

Février

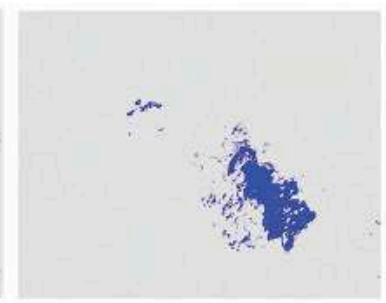

Mai

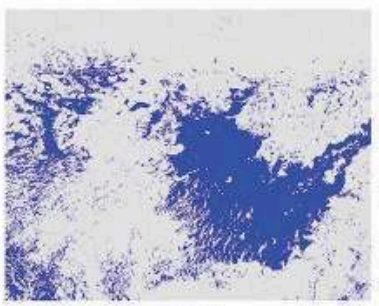

Août

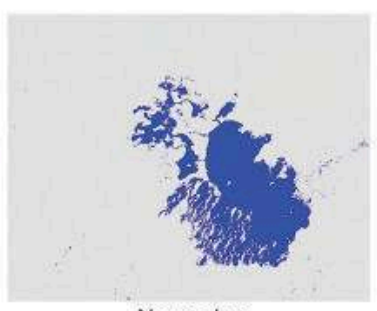

Novembre

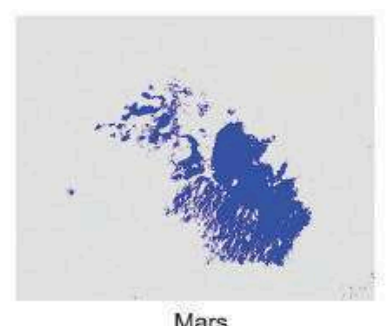

Mars

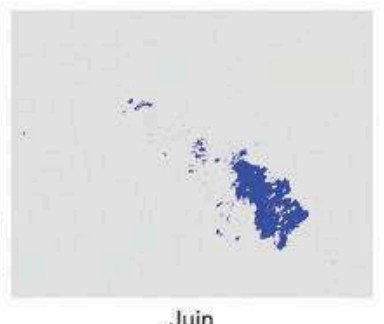

Juin

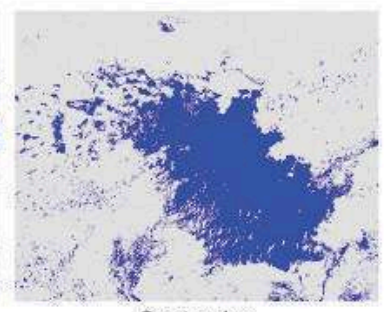

Septembre

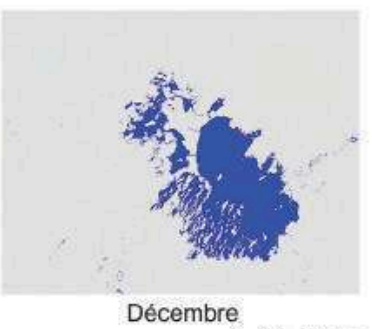

$0 \quad 1020 \mathrm{~km}$

Figure 7

Variations mensuelles de la zone humide en 2015 .

Source : Images Landsat 8. 
les rythmes connus en zone sahélienne (fig. 2). À l'étiage, en mai et juin, la zone humide est quasiment réduite à la superficie des eaux libres, soient $220 \mathrm{~km}^{2}$ (tabl. 3). La crue intervient en août et septembre : pendant ces deux mois, la superficie de la zone humide est multipliée par huit et dépasse $1700 \mathrm{~km}^{2}$. Les eaux du Batha contribuent à la crue dès le mois d'août : les rives de cette rivière très humides pendant ce mois s'assèchent en septembre quand son cours se tarit. Les eaux progressent en ennoyant toute la cuvette nord et les espaces interdunaires au sud-ouest.

\section{Tableau 3}

Superficies de la zone humide $\left(\mathrm{km}^{2}\right)$ d'après la classification des images de 2015 .

$\begin{array}{lcccccccccccc}\text { mois } & \text { janv. } & \text { févr. } & \text { mars } & \text { avr. } & \text { mai } & \text { juin } & \text { juill. } & \text { août } & \text { sept. } & \text { oct. } & \text { nov. } & \text { déc. } \\ \text { Surface } & \text { I } 04 \mathrm{I} & \mathrm{I} 075 & 797 & 726 & 265 & 215 & 22 \text { I } & 782 & \text { I } 767 & \text { I } 008 & 889 & 853\end{array}$

À cette période, les villages sont abandonnés pour plusieurs mois. Les habitants se déplacent vers les villages des zones exondées où ils cultivent le mil et le sorgho. Ils ne reviennent qu'au moment de la décrue, en octobre ou novembre (fig. 8) afin de repiquer le berbéré. C'est après cette période que les troupeaux des éleveurs transhumants arrivent pour la saison sèche ; ils repartent dans les pâturages sahéliens avec l'arrivée des pluies et avant que la région ne soit à nouveau ennoyée.

Le delta du Batha et le secteur de Yao restent inondés le plus longtemps. Au nord, les vertisols absorbent très rapidement l'eau, alors que la végétation marécageuse s'installe plus durablement à l'intérieur d'une ancienne ligne de rivage du lac Fitri ${ }^{3}$. Celle-ci se remarque à peine sur le terrain mais signe une courbe très nette vue du ciel, au travers de laquelle plusieurs chenaux déversent l'eau dans les mares situées plus à l'ouest. Au sud-est, la décrue fait émerger progressivement le relief dunaire modelant le fond du lac.

La même analyse réalisée pour l'année 2014, avec les 7 images Landsat disponibles, confirme le rythme et le rapport des superficies entre étiage en juin et crue en septembre (fig. 9). Ces résultats de l'analyse par télédétection sont cohérents avec les données de l'échelle de crue installée à Yao en 1986. La crue correspond donc bien au cumul des eaux de pluies sur le bassin versant, apportées par le Batha, et celles qui tombent localement à Yao $(25 \%$ des apports d'après COUREL et al., 2005). Il reste à confirmer la superficie particulièrement élevée de la zone humide calculée à $3500 \mathrm{~km}^{2}$ pour septembre 2014 contre $1750 \mathrm{~km}^{2}$ en septembre 2015. C'est aussi pour le mois d'août que la confusion avec la végétation active, qui ne dépend pas directement de la zone inondable, est la plus grande. Il est donc possible que les valeurs obtenues pour ce mois soient surestimées. 


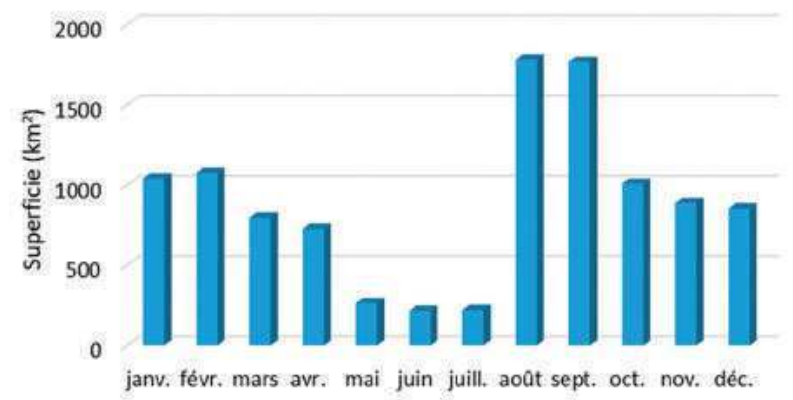

Figure 8

Variations mensuelles des superficies de la zone humide du lac Fitri en 2015.

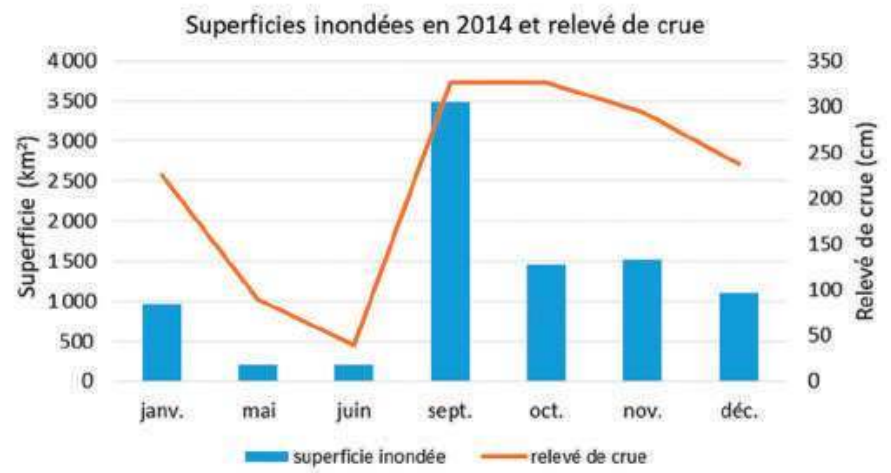

Figure 9

Comparaison entre la superficie de la zone humide en 2014 calculée par télédétection et la hauteur de crue à Yao aux mêmes dates.

Sources : Landsat 8, Drem.

\section{Une zone humide en expansion de 1972 à 2015}

La classification en 3 classes - eaux libres, marécages et zones non inondées des images Landsat et Spot montre la variabilité des crues du lac Fitri entre 1972 et 2015 (fig. 10).

L'année 1972 correspond aux premières images Landsat. C'est aussi une année de grande sécheresse au Sahel. Au Fitri, les faibles pluies accumulées dans le bassin versant ont tellement réduit l'extension de la zone humide que le lac s'est asséché au cours de la saison sèche (LEMOALLE, 1979). Nous manquons d'images pour documenter la période sèche qui a suivi, mais les périodes sèches suivantes, en 1986 et 1987, montrent une zone humide réduite avec des eaux libres qui serpentent dans les zones les plus profondes du lac. Pendant la décennie 19751985 , la localisation des eaux libres est très variable, disparaissant plus ou moins sous la végétation aquatique. La zone deltaïque est logiquement la plus 


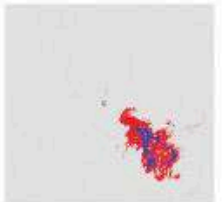

30-10-1972

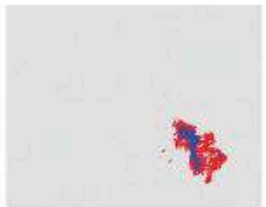

06-03-1987

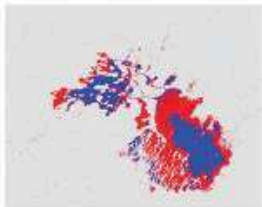

01-12-1998

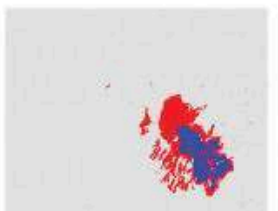

27-10-2000
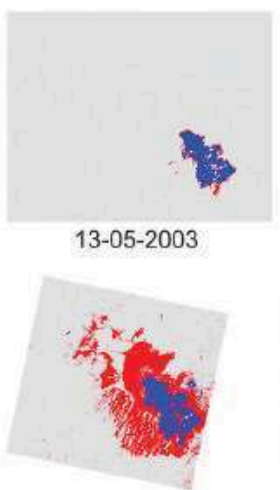

09-10-2011

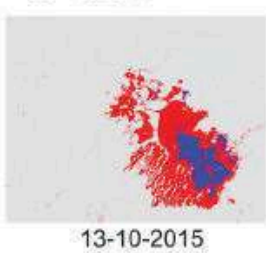

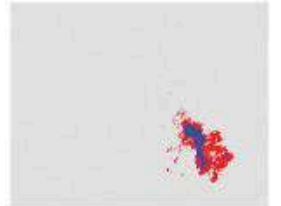

18-02-1986

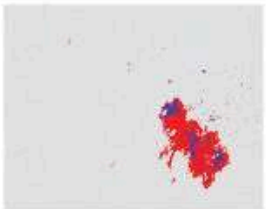

16-10-1987

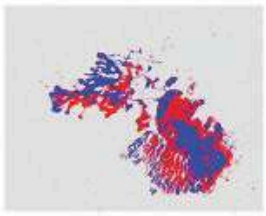

12-12-1999

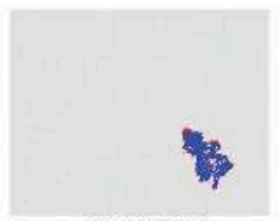

07-05-2001
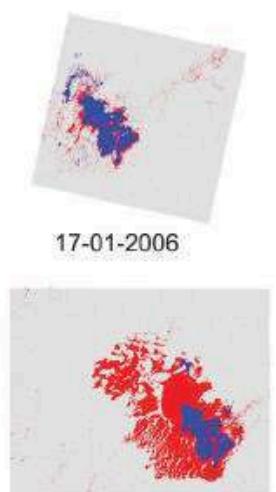

23-10-2013
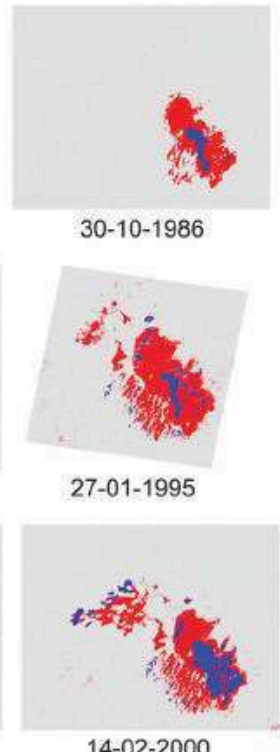

$14-02-2000$

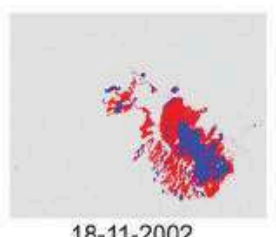

$18-11-2002$
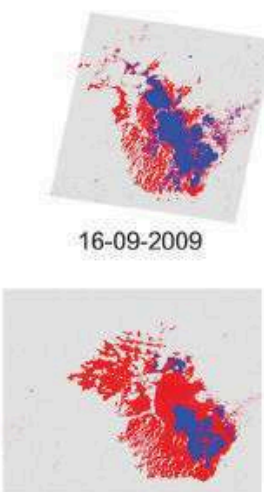

26-10-2014

\section{$01020 \mathrm{~km}$}

Figure 10

Variations interannuelles des eaux libres (en bleu) et des marécages (en rouge) entre 1972 et 2015. 
marécageuse. Suite à la baisse du niveau de l'eau, de nombreuses îles sont dégagées. À l'étiage en 1985, un véhicule pouvait traverser le lac dans sa largeur.

L'année 1994 est une année pluviométrique exceptionnelle. À la station météorologique de Yao, on mesurait $650 \mathrm{~mm}$ après quinze années comprises entre 250 et $400 \mathrm{~mm}$ (fig. 3). Ces pluies abondantes sur l'ensemble du bassin versant se traduisent par un remplissage du lac beaucoup plus important, comme le montre l'image de janvier 1995. Les eaux s'étendent dans la cuvette jusqu'à l'ancienne ligne de rivage à la courbe bien marquée au nord du lac (fig. 1) et aux espaces interdunaires au sud-est, où la végétation aquatique s'installe. Les eaux parviennent également aux mares du nord-est à travers les chenaux. Les eaux libres toutefois restent peu étendues et toujours limitées aux parties les plus profondes du lac (fig. 10).

Entre 1972 et 1995, les eaux libres s'étendent sur moins de 100 km² (tabl. 4). Ce n'est qu'à partir de janvier 1998 qu'elles prennent l'extension qu'on leur

\section{Tableau 4}

Surfaces d'eau libre et de zones marécageuses $\left(\mathrm{km}^{2}\right)$.

\begin{tabular}{|lccc|}
\hline Date de prise d'images & Eau libre & Marécages & Total \\
\hline oct. 1972 & 106 & 370 & 476 \\
\hline févr. 1986 & 52 & 148 & 201 \\
\hline oct. 1986 & 56 & 342 & 398 \\
\hline mars 1987 & 57 & 140 & 196 \\
\hline oct. 1987 & 54 & 309 & 363 \\
\hline janv. 1995 & 79 & 556 & 635 \\
\hline déc. 1998 & 451 & 551 & 1002 \\
\hline déc. 1999 & 585 & 530 & 1115 \\
\hline févr. 2000 & 262 & 568 & 830 \\
\hline oct. 2000 & 188 & 351 & 539 \\
\hline nov. 2000 & 192 & 341 & 533 \\
\hline mai 2001 & 153 & 19 & 172 \\
\hline nov. 2002 & 293 & 480 & 773 \\
\hline avr. 2003 & 190 & 46 & 236 \\
\hline mai 2003 & 157 & 29 & 186 \\
\hline janv. 2006 & 204 & 104 & 308 \\
\hline sept. 2009 & 325 & 364 & 689 \\
\hline oct. 2011 & 187 & 607 & 794 \\
\hline oct. 2013 & 200 & 950 & 1150 \\
\hline oct. 2014 & 239 & 1078 & 1317 \\
\hline oct. 2015 & 214 & 788 & 1002 \\
\hline
\end{tabular}


connaît actuellement, entre 200 et $350 \mathrm{~km}^{2}$ entre octobre et février (fig. 10). Les deux images de décembre 1998 et 1999 fournissent les superficies d'eaux libres maximales, respectivement 540 et $585 \mathrm{~km}^{2}$, notamment en raison du remplissage des mares au nord-ouest : non inondées depuis de nombreuses années (près de trente ans d'après nos résultats), celles-ci se remplissent d'abord d'eau puis se végétalisent progressivement, fournissant les ressources exploitées depuis par la pêche et l'élevage. C'est aussi dans ce secteur qu'une magnifique doumeraie s'est développée, qui fournit les noix pour une filière rentable de combustibles vers N'Djamena (GuEdon, 2016).

Si l'on exclut les 3 images correspondant à l'étiage (mai 2001, avril et mai 2003), nous observons depuis le début des années 2000 une certaine stabilisation de la superficie du lac à un niveau nettement plus haut que celui des années sèches entre 1972 et 1995 . Cette superficie reste très variable, avec des années sèches (2006) ou plus humides (2014).

Les variations des superficies de la zone humide correspondent assez fidèlement à celles observées pour la pluviométrie à Yao (fig. 2). Les décalages s'expliquent par une pluviométrie plus importante dans d'autres secteurs du bassin versant : les années 1998 et 1999 n'apparaissent pas particulièrement pluvieuses à Yao mais sont caractérisées par une forte crue. La tendance de la pluviométrie annuelle est à l'augmentation à Yao depuis les années 1970 (fig. 3), ainsi que celle des données de l'échelle de crue de Yao (fig. 12). Nos résultats sur l'extension des crues du lac Fitri calculés par télédétection apparaissent donc cohérents avec ces tendances générales. Ils montrent une expansion de la zone humide qui a doublé depuis les années sèches de la décennie 1970.

Les données de hauteur d'eau à Yao confirment la stabilisation du niveau du lac depuis le début des années 2000. On observe également une réduction de sa variabilité interannuelle, particulièrement importante entre 1986 et 1996 (fig. 12). Le remplissage des mares au nord-ouest du lac semble avoir lieu lorsque la hauteur d'eau à Yao dépasse la cote 350 .

\section{Une augmentation des ressources disponibles}

Comme le lac Tchad, qui après un stade «Petit lac sec » dans les années 1970 s'est stabilisé à un niveau « Petit lac » (MAgrin et al., 2015), le lac Fitri n'est pas dans une dynamique régressive. Nos résultats montrent que depuis les grandes sécheresses sahéliennes, le lac Fitri a retrouvé un régime hydrique plus important. Il est difficile de comparer nos chiffres d'estimation de la superficie du lac à celui évalué par J. LemoALle (1979) qui indique une superficie de $1300 \mathrm{~km}^{2}$ en 1970, car l'auteur ne précise pas s'il s'agit des 


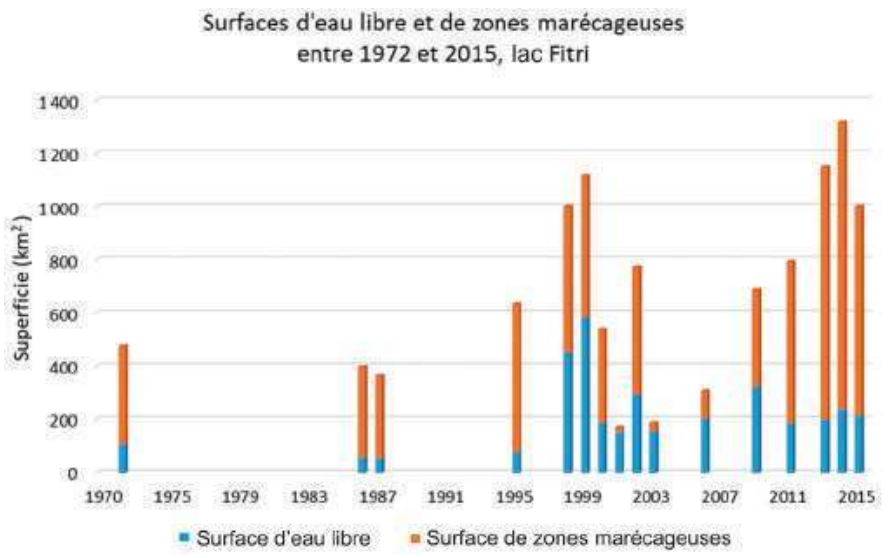

Figure II

Variations des surfaces d'eau libre et de zones marécageuses de 1972 à 2015.

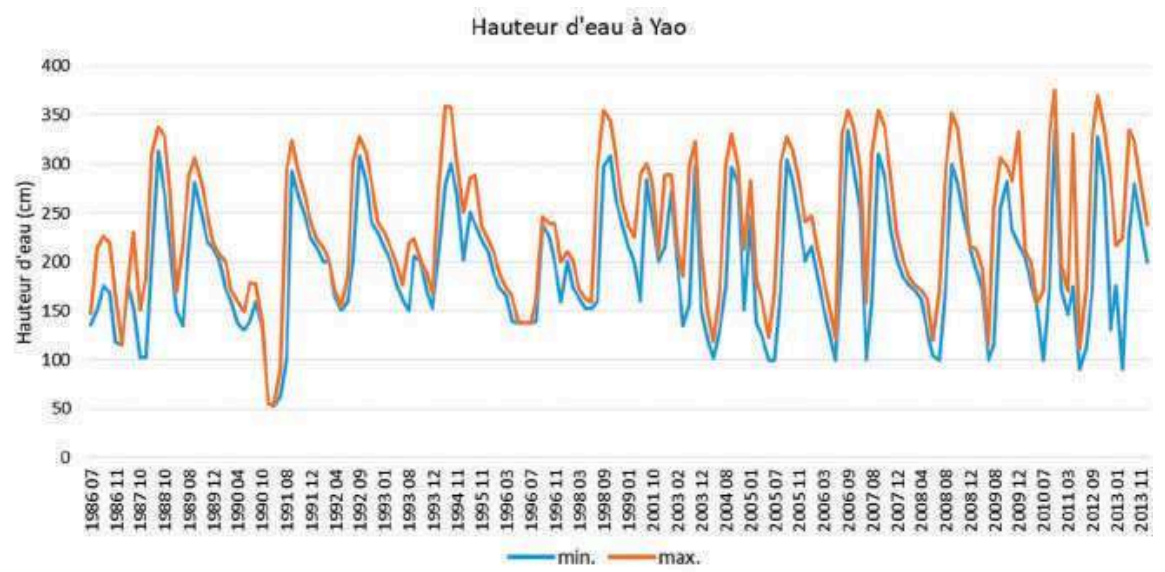

Figure 12

Hauteur d'eau en période de crue et d'étiage de 1986 à 201 I à Yao.

Sources : échelle de crue de Yao, Drem.

eaux libres seulement, auquel cas le lac aurait été beaucoup plus grand qu'aujourd'hui.

Les activités anthropiques - agriculture, élevage, pêche - pratiquées au lac Fitri pendant la saison sèche se localisent essentiellement dans la zone humide. Contrairement à la perception de dégradation des ressources, qui s'appuie sur le discours généralisé sur la désertification, mais aussi localement sur un constat de baisse des prises de poisson par pêcheur, de réduction de la taille des prises et de dégradation des ressources pastorales (MARTY et al., 2012 ; BEGC, 2016), nous pouvons affirmer que les ressources apportées par la zone humide sont stabilisées et en extension depuis les années 1990.

Les eaux libres, où se pratique la pêche, sont aujourd'hui deux fois plus étendues que dans les années 1970-1980 (fig. 11). Elles ont recouvert la cuvette principale du lac qui était très végétalisée jusqu'à la moitié des années 1990, repoussant 
la végétation marécageuse vers l'extérieur. L'augmentation de la production halieutique du lac est difficile à estimer, car elle dépend de l'association entre eaux libres et marécages, où se multiplient les espèces, grandissent les alevins et se nourrissent les adultes. L'absence de données ne permet pas de conclure à une baisse de la productivité.

Les surfaces marécageuses couvraient entre 300 et $400 \mathrm{~km}^{2}$ dans les années 19701980, entre 600 et $900 \mathrm{~km}^{2}$ pour les années 2011-2015 (l'année 2014, avec $1000 \mathrm{~km}^{2}$, restant exceptionnelle pour la période récente ; tabl. 4). Elles comprennent des peuplements de Echinochloa stagnina et de Phragmites sp. sur les îles et en bordure d'eau, puis dans les zones inondées plus temporairement, des forêts d'Acacia nilotica. Leur localisation a fortement évolué depuis les années 1970-1980 (fig. 11). À partir de la moitié des années 1990, cette végétation s'étale pour coloniser l'ensemble de la cuvette principale du lac, les mares au nord-ouest et les espaces interdunaires du sud-ouest. Elle s'est ainsi déplacée de plusieurs centaines de mètres. Cette observation est confirmée par les agriculteurs de sorgho qui désignent l'emplacement des anciens champs.

Pour le lac Tchad, où l'on trouve le même type de pâturage aquatique avec une utilisation complémentaire des sous-produits de l'agriculture par le bétail, la capacité de charge animale théorique est estimée à $1 \mathrm{UBT} / \mathrm{ha}^{4}$ pour du bétail restant douze mois sur place, 2 UBT/ha s'il ne reste que six mois (GUÉRIN et al., 2014). Avec les mêmes chiffres, la capacité d'accueil serait au Fitri, pour du bétail restant six mois sur place, de 60 à 80000 UBT dans les années 1970 et de 120 à 180000 UBT $^{5}$ depuis le début des années 2000. Si la pression exercée par le bétail sur l'environnement du Fitri est de plus en plus perceptible au cours de la dernière décennie, marquée par un élagage des arbres appétés par le bétail et la disparition du pâturage sec en quelques semaines après l'arrivée des troupeaux transhumants, la forte augmentation de la disponibilité fourragère a permis l'accueil de troupeaux plus nombreux, ainsi que leur temps de résidence saisonnier plus long dans le lac.

\section{Conclusion}

Le suivi des extensions de la crue annuelle du lac Fitri depuis 1972 montre la difficulté de mesurer un lac peu profond : sa superficie varie d'un facteur 1 à 8 au cours d'une année (exemple de 2015), et elle double entre 1972 et 2015

\footnotetext{
4. UBT : Unité de bétail tropical correspondant à un bovin standard de $250 \mathrm{~kg}$ de poids vif ; les effectifs des bovins d'autres poids et des autres espèces sont évalués avec cette unité en appliquant des coefficients.

5. Ces chiffres théoriques sont à retenir pour leur tendance, car ils semblent très sous-estimés par rapport au cheptel recensé dans le Fitri : 800000 bovins, 400000 ovins et 750000 caprins fréquentaient le Fitri entre 2007 et 201 I (MARTY et al., 2012) pendant des saisons plus ou moins longues et en complément des ressources extérieures à la zone humide.
} 
en ne considérant que les mois d'octobre. Cette situation rend la cartographie de cette région très difficile, ce dont témoigne la diversité des formes cartographiées pour ce lac depuis les années 1960 (carte IGN 1978 ; BIEP, 1989 ; BEGC, 2015 ; RAIMOND et al., à paraître).

Nos résultats confirment les caractéristiques propres à un lac endoréique en zone semi-aride. L'ampleur de la crue dépend étroitement de la pluviométrie de son bassin versant situé exclusivement en zone sahélienne, puis de la forte évaporation liée aux températures élevées de la saison chaude. Les fluctuations des crues suivent assez bien le rythme d'années sèches et humides enregistré par le pluviomètre proche du lac (station de Yao). Contrairement au lac Tchad, avec lequel il est couramment comparé, le pic de crue du lac Fitri, en août et septembre, est presque concomitant avec celui de la pluviométrie locale, alors que le pic de crue du lac Tchad est lié à celui du Chari et intervient plus tard dans l'année, en novembre et décembre et plus tard encore dans la cuvette nord.

Nous montrons que les superficies des eaux libres et des marécages ont doublé depuis les sécheresses des années 1970-1990. Elles se sont stabilisées depuis le début des années 2000, et fournissent des ressources plus abondantes à une population humaine en forte croissance (la population a doublé entre 1989 et 2009, soient 110400 habitants selon le recensement national de la population). Nos résultats contribuent à expliquer les chiffres obtenus par le récent recensement de l'élevage au Tchad, qui estiment que le bétail a doublé depuis 1976 (Ministère DE L'ÉleVAGE, 2016). Si la pression anthropique est de plus en plus visible dans la région du Fitri, cette augmentation des populations humaines et animales a été rendue possible grâce à un " reverdissement » et un meilleur approvisionnement en eau du lac, conformément à ce qui est observé dans d'autres zones sahéliennes (DARDEL et al., 2014). Un suivi régulier de la crue du lac Fitri, en plus de la pluviométrie, fournirait un excellent indicateur des tendances du climat dans cette région très peu étudiée, mais aussi des tensions sur les ressources dans le cadre de la croissance démographique.

\section{Bibliographie}

BEGC, 2016

Étude diagnostique de la diversité biologique de la réserve de biosphère du lac Fitri.

République du Tchad, ministère de

l'Environnement et des Pêches, FSE, 81 p.

\section{BIEP, 1989}

Étude de développement rural intégré du lac Fitri. République du Tchad, ministère de l'Agriculture, Banque islamique de développement, Cedrat-SA, Biep, document de synthèse et annexes, 61 p., multigr.

\section{CoHen J., 1960}

A coefficient of agreement for nominal scales. Education Psychology Measurement, 20 : 37-46.

\section{Congalton R. G., 1991}

A review assessing the accuracy of classifications of remotely sensed data. Remote Sensing of Environment, 37 : 35-46. 
Courel M. F., Bachimon P., Riser J., Dagou P., Mustapha M. A., Raimond C., 2005 Géosystèmes lacustres, étude des régions des lacs Léré et Fitri, Tchad. Ministère des Affaires étrangères, France, rapport scientifique, projet Campus 5 : 97319 107, fac $n^{\circ} 99002600$. Corus (MAE), 318 p.

I. F. Creed, C. R. Lane, J. N. Serran, L. C. Alexander, N. B. Basu,

A. J. K. Calhoun, J. R. Christensen, M. J. Cohen, C. Craft, E. D'Amico, E. DeKeyser, L. Fowler, H. E. Golden, J. W. JaWitz, P. Kalla, L. K. Kirkman, M. LANG, S. G. Leibowitz, D. B. LeWIS, J. Marton, D. L. McLaughlin,

H. RaAnAN-Kiperwas, M. C. Rains, K. C. Rains, L. SMith, 2017

Enhancing protection for vulnerable waters. Nature geoscience, vol. 10 : 809-815. DOI: 10.1038/NGEO3041

Dardel C., Kergoat L., Hiernaux P., Mougin E., Grippa M., TuCKer C. J., 2014 Re-greening Sahel: 30 years of remote sensing data and field observations (Mali, Niger). Remote Sensing of Environment, 140 : 350-364.

Gardner R. C., Barchiesi S., Beltrame C., Finlayson C. M., Galewski T., Harrison I., Paganini M., Perennou C., Pritchard D. E., Rosenqvist A., WAlpole M., 2015 State of the World's Wetlands and their Services to People: A compilation of recent analyses. Gland, Switzerland, Ramsar Briefing Note, 7 , $21 \mathrm{p}$.

\section{GUÉDON J., 2016}

Origine et évolution de la filière des noix de doum du Fitri : nécessité alimentaire et opportunité énergétique. Master 1 de géographie, université Paris 1 PanthéonSorbonne, $152 \mathrm{p}$.

Guérin H., RéounodJi F., RANGÉ C., 2014 « Les dynamiques d'élevage ». In : Lemoalle J., Magrin G., dir. : Le développement du lac Tchad : situation actuelle et futurs possibles. CBLT, N'Djamena, Marseille, Expertise collégiale IRD, AFD-FFEM, rapport de synthèse, 67 p. + traduction anglaise et contributions intégrales des experts (CD, 20 chapitres, 620 p.), 423-474

Niel H., Leduc C., Dieulin C., 2005

Caractérisation de la variabilité spatiale et temporelle des précipitations annuelles sur le bassin du lac Tchad au cours du $20^{\mathrm{e}}$ siècle. Hydrological Sciences Journal, 50 : 2-243.
LANDi J. R., Koch G. G., 1977

The Measurement of Observer Agreement for Categorical Data. Biometrics, 33 : 159-174.

Leblanc M., Favreau G., Maley J., Nazabou Y., Leduc C., Stagnitti F., Van Oevelen P. J., DelcauX F., Lemoalle J., 2006

Reconstruction of Megalake Chad using february 2000 shuttle radar topographic mission data.

Palaeogeography, Palaeoclimatology,

Palaeoecology, 239 : 16-27.

Leblanc M., Lemoalle J., Bader J.-C, Tweed S., Mofor L., 2011

Thermal remote sensing of water under flooded vegetation: New observations of inundation patterns for the "Small" Lake Chad. Journal of Hydrology, 404 : 87-98.

\section{Lemoalle J., 1979}

Étude des potentialités du bassin conventionnel du lac Tchad. Hydrobiologie: Utilisation de la télédétection pour l'évaluation des surfaces inondées. Paris, Orstom, 62 p.

\section{Madgwick F. J ., Pearce F., 2017}

Water shocks. Wetlands and human migration in the Sahel. Wetlands International, the Netherlands. https:/www.wetlands.org/ publications/water-shocks-wetlands-humanmigrationsahel/

Magrin G., Pourtier R., Lemoalle J., 2015

Atlas du lac Tchad. Paris, IRD Éditions/Passages, $225 \mathrm{p}$.

\section{Marty A., Zakinet D., Khamis D. D.,}

BERNARD C., 2012

Almy al Afia 2. Analyse de l'évolution des ressources dans le département du Fitri. Document principal. République du Tchad, programme d'hydraulique pastorale au Tchad central, Phase II. Antea-Iram, 128 p.

\section{Ministère de L'ÉleVAGe, 2018}

Recensement général de l'élevage (RGE 20122015). Principaux résultats définitifs. République du Tchad, 78 p.

\section{Raimond C., Mbagogo A., Madjigoto R.,} ZAKINET D., à paraître

Repenser la gouvernance d'une zone humide autour d'un pouvoir local fort : l'exemple du lac Fitri (Tchad) face à la montée des insécurités. Colloque Méga-Tchad Les insécurités dans le bassin du lac Tchad, Nice, XVII ${ }^{\mathrm{e}}$ colloque Méga-Tchad, 14-16 juin 2017. 
Schuster M., Sylvestre F., Raimond C., Moussa A., Abakar Y., Yalikun T.

«Changements environnementaux et climatiques à la période humide africaine Holocène dans le bassin du lac Fitri (Tchad) ». Ce volume.
Tou J. T., Gonzalez R. C., 1974

Pattern Recognition Principles. Addison-Wesley Publishing Company, Reading, Massachusetts, $377 \mathrm{p}$. 

Partie 2

\section{Dynamique des socioécosystèmes lacustres}

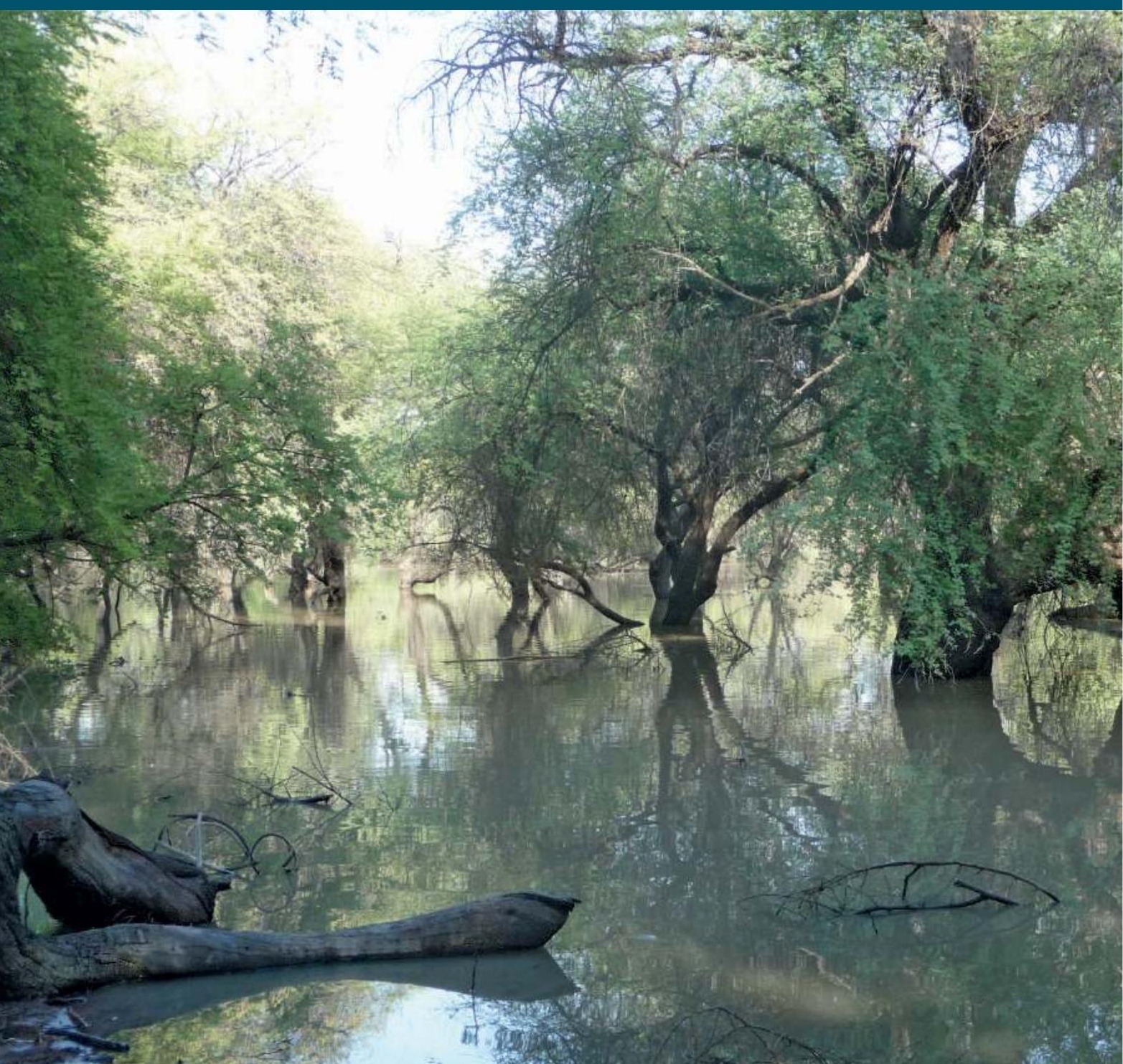


Forêt d'Acacia nilotica saisonnièrement inondée au lac Fitri (Galo, février 20I5). Ces secteurs trop inondés pour l'agriculture de décrue sont exploités successivement pour la pêche puis le pâturage.

(c) CNRS/C. Raimond. 


\section{Introduction}

Christine RAIMOND, Florence SYLVESTRE

Un système lacustre est parfois très complexe et ne se limite pas à une nappe d'eau de surface. Un lac est le résultat d'un bilan qui se compose d'apports et de pertes. Les apports sont représentés par les précipitations, les eaux de ruissellement, les eaux souterraines et les eaux des rivières. Les pertes sont composées de l'évaporation, l'évapotranspiration dans le cas d'un lac végétalisé et des infiltrations vers les aquifères. Les caractéristiques morphologiques d'un lac peuvent générer une amplification plus ou moins importante des événements climatiques. Généralement, les lacs fermés sans exutoire de surface en zone aride à semi-aride font partie des sites les plus sensibles aux variations climatiques.

Dans certains cas, les eaux souterraines jouent un rôle primordial sur le bilan hydrologique des lacs qui s'affranchissent alors en partie des aléas climatiques. En effet, la réponse d'un lac à un apport en précipitation n'est pas linéaire si une partie des apports s'infiltre dans un réservoir souterrain et est donc soustraite de la surface. Réciproquement, un lac recevant un apport par résurgence d'eau souterraine n'est pas en phase avec les changements climatiques tamponnant les fluctuations du plan d'eau.

Ces deux situations sont actuellement observées dans les lacs tchadiens. Nous savons que le lac Tchad repose sur un double aquifère couvrant la quasi-totalité de son bassin versant. Le premier, appelé l'aquifère quaternaire, a une profondeur moyenne d'une centaine de mètres sur une superficie de $500000 \mathrm{~km}^{2}$ et est actuellement rechargé par infiltration des eaux de surface du lac; le second plus profond, appelé le Continental terminal, est aujourd'hui confiné et n'alimente que certaines zones, notamment l'oasis de Faya-Largeau, par remontées artésiennes. Nous supposons que les lacs Fitri et Iro, situés dans le bassin du lac Tchad, peuvent se comporter comme le lac Tchad, mais des recherches approfondies sur ces deux hydrosystèmes sont nécessaires pour les caractériser 
et apprécier leur comportement respectif. Dans les systèmes oasiens sahariens, en particulier les lacs d'Ounianga, ce sont les eaux souterraines en résurgence de l'aquifère des grès de Nubie qui les alimentent tout au long de l'année, même dans les conditions actuelles d'aridité. Cette situation exceptionnelle de lac en plein désert leur confère d'ailleurs leur caractère unique et leur a valu d'être classés au patrimoine mondial de l'Unesco en 2012.

Ces eaux souterraines représentent un atout de premier ordre pour les populations sahélosahariennes car elles constituent des réservoirs d'eau considérables. Aujourd'hui dans le bassin du lac Tchad, l'aquifère quaternaire constitue une ressource en eau privilégiée pour l'eau potable, et tous les autres usages domestiques après que les eaux de surface (pluies, zones humides, lacs et autres mares) ont disparu en saison sèche. C'est en effet dans cette nappe superficielle disponible par pompage tout au long de l'année indépendamment des fluctuations climatiques, que les populations puisent de l'eau pour leurs activités. L'évaluation du réservoir et les modalités de sa recharge sont donc essentielles, surtout dans le contexte actuel d'aridification du climat et de pressions croissantes dues à une augmentation des usages.

Le fonctionnement d'un écosystème lacustre dépend du bilan hydrique, mais aussi de nombreuses autres caractéristiques à prendre en compte, dont l'action anthropique. Lorsqu'il est de faible profondeur, comme les lacs Tchad et Fitri, l'extension du lac fluctue fortement en fonction des saisons qui rythment le réapprovisionnement des zones humides et la disponibilité en ressources (eau, poisson, végétation, terre agricole). La baisse et le retrait des eaux à la suite d'une longue période plus sèche pour un très vaste lac, comme cela a été le cas pour le lac Tchad des années 1970 à 1990, ne signifient pas forcément une perte en ressources naturelles : les vastes terres de décrue dégagées ont au contraire permis l'extension des marécages, de la pêche, des pâturages et des cultures, et l'installation d'une population plus nombreuse sur ses rives. Les travaux du Gelt montrent que sur la même période, les conséquences de l'assèchement du climat sur le lac Fitri, beaucoup plus petit, ont été beaucoup plus difficiles à vivre pour les populations riveraines qui ont vu les eaux libres disparaître sous la végétation marécageuse, les pâturages se réduire à la cuvette lacustre, les terres argileuses périphériques non inondées devenir incultivables. Seul le retour à des conditions hydrologiques plus favorables rétablit l'extension antérieure des ressources et relance les systèmes de production. Ce fonctionnement étroitement dépendant des variations climatiques est tamponné dans la zone soudanienne, comme pour les lacs Léré et Iro, en raison d'une pluie plus abondante et d'un approvisionnement fluviatile plus régulier.

Cette deuxième partie apporte des connaissances nouvelles sur le fonctionnement des socioécosystèmes lacustres en précisant les bilans hydriques pour les lacs Ounianga, Fitri et Iro, et en soulignant les conséquences pour les sociétés du lac en termes de potentiel productif pour l'élevage et l'agriculture. Les données collectées permettront, à terme, de fournir une synthèse du fonctionnement des hydrosystèmes des lacs Fitri et Iro. 
Si la finalité des recherches présentées sur les lacs d'Ounianga porte, dans la continuité de la partie 1, sur la reconstitution paléohydrologique dans ces milieux aux conditions climatiques extrêmes, les nouvelles données apportées sur les propriétés physicochimiques de la colonne d'eau sont inédites et éclairent notre compréhension du fonctionnement de ces hydrosystèmes lacustres quasiment inconnus. Elles sont, en outre, essentielles pour la compréhension des informations livrées par les indicateurs et traceurs analysés dans leurs archives sédimentaires et feront référence dans ce domaine.

Pour les lacs Fitri et Iro, une première approche du bilan hydrologique est mise en œuvre sur la base d'une méthodologie de géochimie isotopique appuyée par l'hydrologie satellitale. Ce travail pionnier pour ces deux lacs dresse une première description de ces deux hydrosystèmes et met en évidence un fonctionnement plus complexe qu'attendu avec de fortes connexions entre eaux souterraines et eaux de surface. Ces données sont particulièrement utiles pour le lac Iro qui est de tous les lacs au Tchad celui pour lequel nous n'avions quasiment aucune observation de son environnement physique.

Les effets de la variabilité lacustre interannuelle et de l'action anthropique au lac Fitri sont mis en évidence en observant les fluctuations des espaces mis en culture depuis les grandes sécheresses des années 1984-85 d'une part, les évolutions de la végétation pastorale d'autre part. Ces deux contributions montrent l'importance de l'analyse systémique pour l'étude de ces zones lacustres dans leur complexité, intégrant les fonctionnements hydrologiques et écologiques $\mathrm{du}$ milieu en interaction avec les sociétés qui en tirent leur subsistance. L'évolution de la disponibilité en ressources naturelles dépend non seulement des effets du climat mais aussi des pressions anthropiques croissantes qui par leurs prélèvements et ceux de leur bétail provoquent une dégradation environnementale. Celle-ci est imputée à tort à un changement climatique dont les effets à long terme sont probablement sous-estimés, mais qui, sur la période des deux dernières décennies, est plutôt favorable à l'extension des zones humides et des services écosystémiques associés. Dans ce contexte de croissance démographique et des cheptels, des concurrences entre acteurs et activités remettent en question les principes de mobilité, de multifonctionnalité des espaces et de relations entre les acteurs qui fondaient la résilience de ces socioécosystèmes. 

Les lacs d'Ounianga Mieux comprendre leur dynamique hydrologique et écologique par une approche interdisciplinaire

\author{
Thijs VAN DER MEEREN, Pierre DESCHAMPS, \\ Florence SYLVESTRE, Baouyé KEMKONG, \\ Abderamane MOUSSA, Mathieu SCHUSTER, \\ Dirk VERSCHUREN
}

\title{
Introduction
}

Les lacs d'Ounianga inscrits au patrimoine mondial de l'Unesco depuis 2012 sont situés dans le nord-est du Tchad. Ils abritent une grande diversité d'habitats aquatiques, sources, lacs et zones humides (voir fig. 1, page 16), ainsi qu'une salinité très contrastée, allant de l'eau douce à des eaux hypersalines (CNAR ${ }^{1}$, 2011). Ces systèmes lacustres extrêmement rares présentent non seulement l'originalité d'être caractérisés par cette grande diversité d'habitats aquatiques mais aussi d'être concentrés sur une zone géographique bien définie au cœur du Sahara central. Ces lacs sont les reliques de lacs plus vastes et plus profonds, qui se sont développés au début de la période humide africaine ( 10 000 ans) comme en témoignent de nombreux affleurements de dépôts lacustres. La transition vers les systèmes de lacs actuels n'est que partiellement comprise et a eu un impact profond sur les populations humaines de cette région par le biais des changements majeurs de ces lacs. Aujourd'hui, nous disposons de peu 
d'informations sur la variabilité naturelle à long terme pour déterminer et discuter de l'utilisation durable des services écosystémiques que fournissent ces lacs. Bien que l'impact environnemental des activités humaines dans les bassins d'Ounianga semble relativement modeste et localisé, étant données la grande fragilité des écosystèmes en zone aride et la rareté des ressources en eau douce dans le Sahara, les enjeux sont élevés.

La recherche interdisciplinaire, combinant les études limnologiques et écologiques des systèmes actuels avec des analyses paléolimnologiques, micropaléontologiques, géochimiques et paléohydrologiques sur les archives sédimentaires, améliore la compréhension de la dynamique de l'écosystème aquatique à long terme, notamment de l'impact du changement climatique sur les environnements et les sociétés. Les sédiments au fond des lacs enregistrent les changements environnementaux des lacs eux-mêmes et de leur bassin versant à des échelles de temps allant de l'année à plusieurs millénaires. Compte-tenu de la rareté des archives naturelles dans le Sahara (anneaux de croissance des arbres, dépôts de grotte par exemple), les sédiments lacustres des bassins d'Ounianga contiennent des informations paléoenvironnementales uniques. Les lacs se sont maintenus, malgré le fort déficit en humidité du climat désertique (i.e. une forte évaporation et pratiquement pas de pluie), par l'apport continu des eaux souterraines fossiles provenant de l'aquifère des grès de Nubie. Différents indicateurs contenus dans les sédiments lacustres fournissent des renseignements clés sur les changements passés : la limnologie (la profondeur des lacs, leur salinité, leur productivité aquatique), les processus terrestres (l'érosion, la couverture végétale, le flux de poussières) et le changement climatique (l'aridité relative, l'activité éolienne).

Cette contribution présente un certain nombre d'axes de recherche suivis par le programme Gelt afin d'améliorer la compréhension de la dynamique à long terme du fonctionnement de l'écosystème aquatique des lacs d'Ounianga, du contexte paléoclimatologique de la région et de l'histoire de son occupation humaine.

\section{Caractérisation du fonctionnement limnologique}

Connaître le fonctionnement limnologique des lacs et en particulier l'intensité de la fréquence du mélange de leur colonne d'eau permet de mieux comprendre les conséquences écologiques des perturbations passées et futures causées par le changement climatique et l'activité humaine. Par exemple, pendant la première moitié de l'Holocène où le lac Yoa était d'eau douce (KRÖPELIN et al., 2008 ; EGGERMONT et al., 2008), les conditions ont pu être plus favorables pour la vie benthique (FrANCUS et al., 2013) du fait que les conditions d'eau douce auraient 
favorisé le mélange et l'oxygénation de la colonne d'eau, en dépit de sa plus grande profondeur à l'époque. De plus, des perturbations équivalentes dans des contextes climatiques identiques mais avec des propriétés physiques différentes (taille, superficie) peuvent provoquer des réponses écologiques contrastées dans les lacs.

Lors des campagnes sur le terrain qui ont débuté à partir de 2003, des données sur les propriétés physiques et hydrochimiques ont été recueillies sur différents plans d'eau d'Ounianga. Compte tenu de l'éloignement du site et des conditions climatiques contraignantes, il est particulièrement difficile d'obtenir des données sur ces milieux et ainsi d'en comprendre les variations saisonnières. Jusqu'à présent, tous les travaux sur le terrain ont été réalisés au cours de la fenêtre temporelle la plus propice, c'est-à-dire entre les mois d'été chaud et les mois d'hiver froid et venteux de l'hémisphère nord, et ont laissé la plus grande partie de l'année sans observation in situ. Pourtant, le cycle saisonnier dans la région d'Ounianga est bien contrasté, avec des températures moyennes mensuelles allant de $15^{\circ} \mathrm{C}$ en janvier à plus de $30^{\circ} \mathrm{C}$ au mois de juillet (CAPOT-REY, 1961 ; FRANCUS et al., 2013). Ces amplitudes de température sont renforcées par des changements également importants de l'intensité du vent.

Dans ce contexte, les enregistreurs automatiques de température de la colonne d'eau fournissent des renseignements clés sur le cycle saisonnier du mélange et de la stratification de la colonne d'eau des lacs à température contrôlée.

Les colonnes d'eau des lacs Yoa et Bokou, qui sont les lacs respectivement les plus profonds de Ounianga Kebir et de Ounianga Serir (fig. 1), ont été mesurées en automatique (enregistreur Vemco Minilog-II) à plusieurs profondeurs entre novembre 2010 et janvier 2012 (fig. 2). La température de l'eau à $5 \mathrm{~m}$ de profondeur, correspondant à la couche supérieure de l'eau (épilimnion) mélangée quotidiennement par le vent et les courants, a suivi les tendances moyennes mensuelles de la température de l'air, bien que l'eau se réchauffe et se refroidisse avec un léger retard par rapport à la température de l'air, en raison de sa capacité thermique élevée. La température de l'eau à proximité du fond, correspondant à la couche profonde plus froide (hypolimnion) mélangée une seule fois par an n'a pas varié en fonction de la température de l'air, mais reflète clairement le cycle annuel du mélange et de la stratification de la colonne d'eau.

En se basant sur la présence de conditions isothermes tout au long de la colonne d'eau (supérieure et inférieure), ces données révèlent que le mélange pendant la saison froide dans le lac Bokou s'est produit de façon continue de début octobre à janvier, et de façon intermittente jusqu'à début avril. Dans le lac Yoa, le mélange complet s'est produit de façon continue à partir du début du mois de novembre jusqu'à janvier (fig. 2). À compter de janvier, l'eau de surface se réchauffe. Cette situation crée un écart de température de plus en plus important (thermocline) entre la couche supérieure chaude (qui se mélange) et la couche inférieure froide (qui ne se mélange pas), même si le transfert de chaleur par diffusion à travers cette thermocline semble également provoquer un réchauffement progressif de la couche inférieure (fig. 2). Dans les deux lacs, la 
stratification de température la plus prononcée (c'est-à-dire le contraste de température le plus important entre la surface et le fond) coïncide avec un réchauffement maximum en surface au mois de juillet. Le refroidissement des eaux de surface à partir de septembre, induite par la diminution de la température de l'air et l'augmentation du vent, réduit simultanément ce contraste de
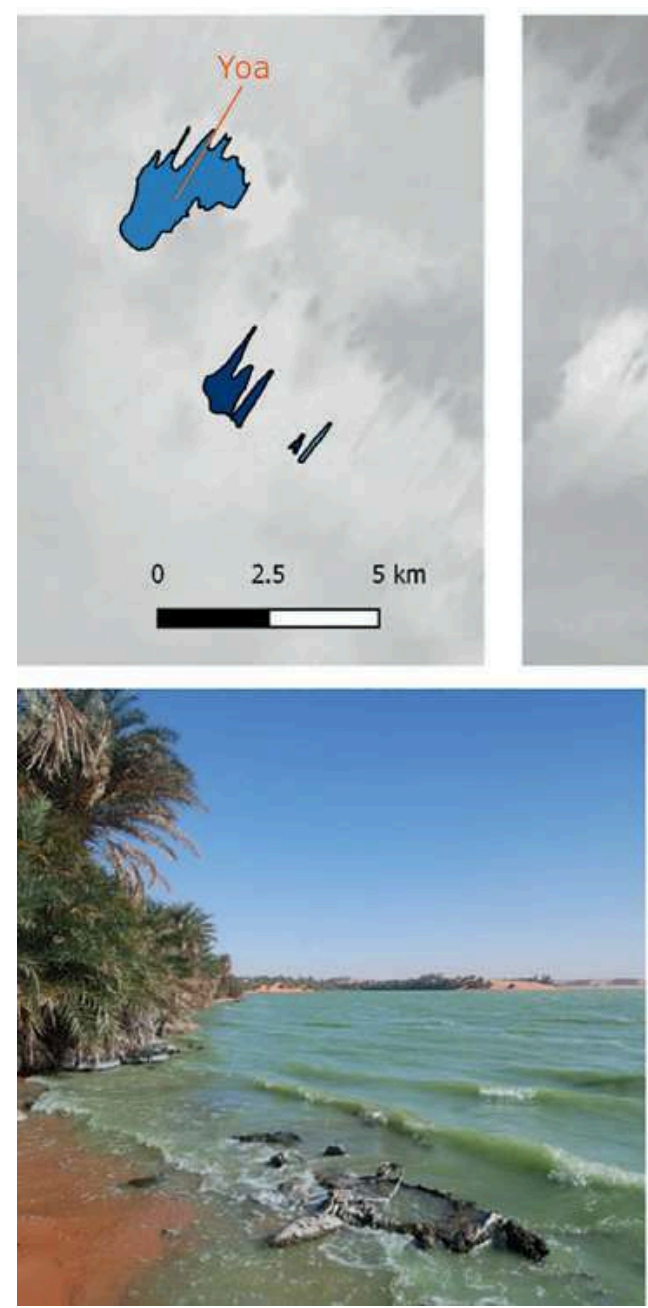
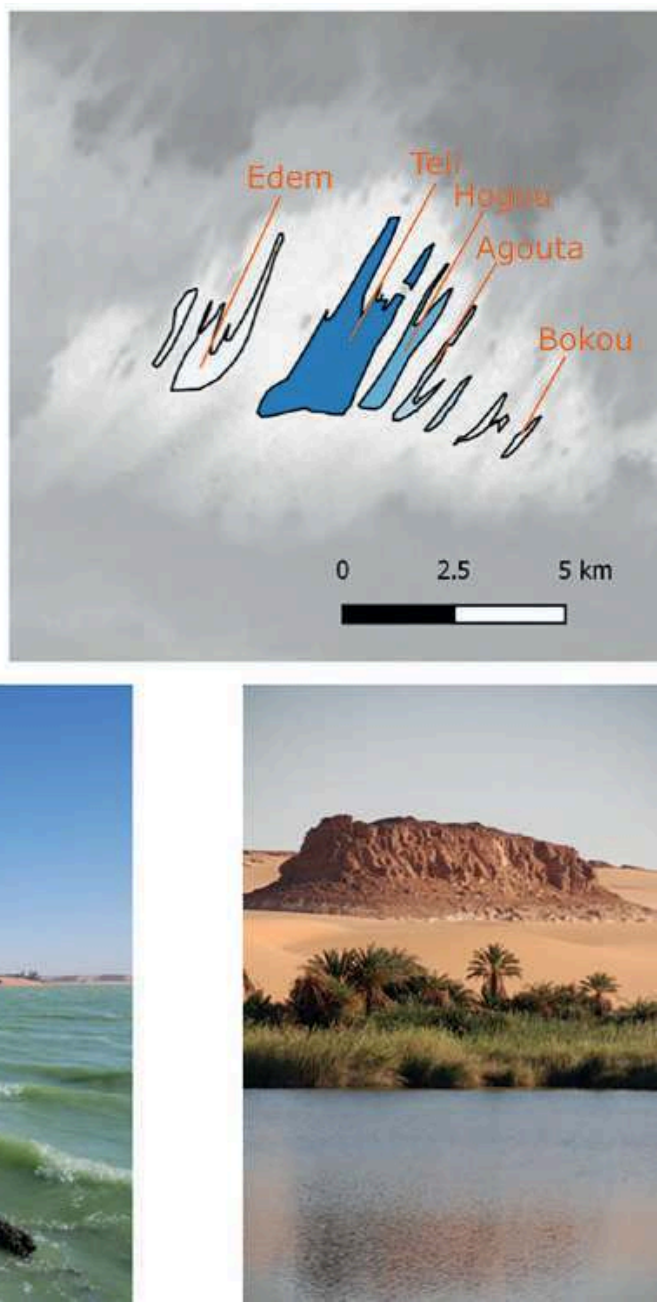

Figure I

En haut : Carte de localisation des lacs à Ounianga Kebir (à gauche) et Serir (à droite), avec la topographie relative indiquée en niveaux de gris et la coloration des lacs représentant la gamme de salinité (du blanc pour les lacs d'eau douce au bleu foncé pour les lacs hypersalés), les noms des lacs mentionnés dans le texte sont indiqués ; les tapis de roseaux flottants

(phragmites) pouvant couvrir partiellement les lacs d'eau douce ne sont pas représentés sur ces schémas. En bas : Photographies prises à partir de la rive ouest du lac Yoa (à gauche) et du lac Bokou (à droite), en regardant respectivement vers le nord-est et vers l'est. 
température et diminue progressivement la thermocline jusqu'au début du mélange en saison froide. À cette période, la colonne d'eau redevient isotherme à toutes les profondeurs.

Pendant le mélange complet d'octobre (novembre) jusqu'en janvier, la température de l'eau diminue considérablement, respectivement de 25 à $15^{\circ} \mathrm{C}$ dans le lac Bokou et de 21 à $13{ }^{\circ} \mathrm{C}$ dans le lac Yoa. Le fait que la stratification estivale dans ces lacs soit interrompue dès le milieu de l'automne, c'est-à-dire bien avant que les températures de l'air hivernales les plus basses soient atteintes, témoigne de l'importance du mélange turbulent induit par le vent, par rapport au mélange convectif induit par la température, en " relançant » le mélange saisonnier dans ces lacs. De plus, en raison d'une plus grande contrainte du vent à la surface du lac Yoa $\left(5,1 \mathrm{~km}^{2}\right.$ par rapport à $0,2 \mathrm{~km}^{2}$ pour le lac Bokou), les fluctuations de température quotidiennes pendant la saison de stratification sont beaucoup plus importantes à Yoa, pour des profondeurs d'eau données.

La durée saisonnière de la stratification et du mélange de la colonne d'eau peut avoir des répercussions considérables sur l'état écologique des lacs, principalement par son effet sur l'oxygénation de l'hypolimnion, et sur l'apport en éléments nutritifs pour le phytoplancton responsable de la productivité aquatique dans l'épilimnion. Cependant, les régimes de mélange saisonnier similaires peuvent donner lieu à des réactions biologiques très différentes dès lors que les lacs présentent des caractéristiques physiques et chimiques différentes (fig. 3). C'est le cas pour les lacs Bokou et Yoa. Le premier est un petit lac d'eau douce

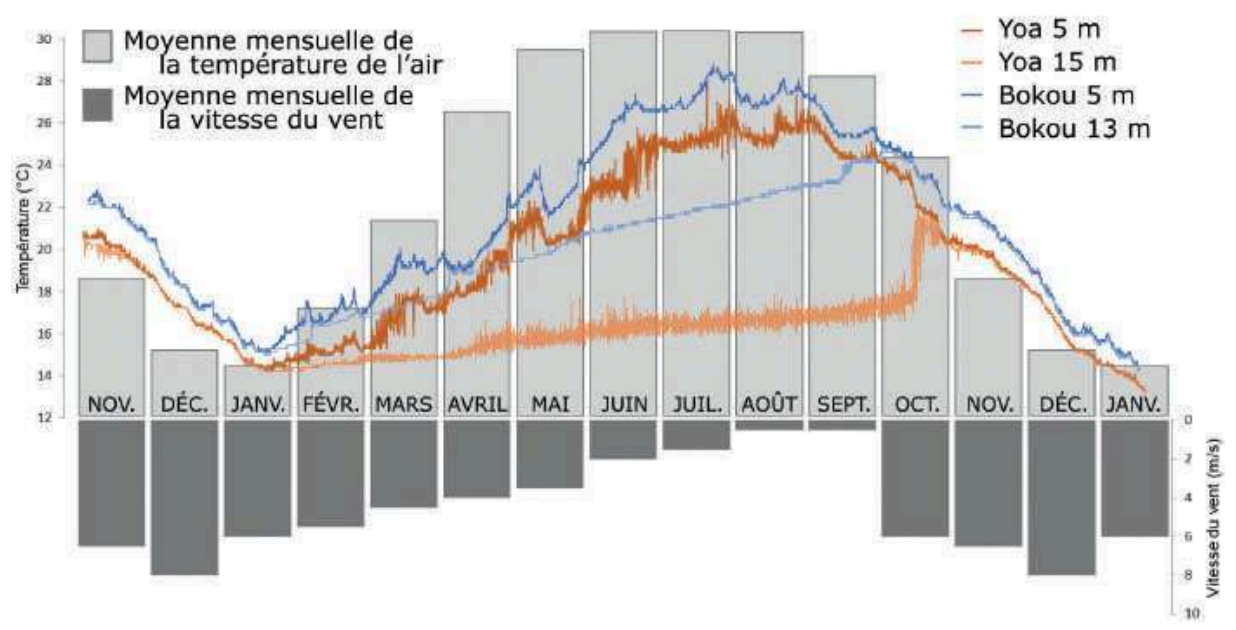

Figure 2

Série chronologique de la température de l'eau à des profondeurs données dans les lacs Yoa et Bokou (courbes, enregistreur automatique des températures toutes les deux heures) comparée aux moyennes mensuelles de la température de l'air mesurée entre 1970 et 2010 (barres gris clair, données de Francus et al., 2013) et aux moyennes mensuelles de la vitesse du vent entre 1957 et 1996 (barres gris foncé sur l'axe inversé, données de WASHINGTON et al., 2006). 
(320 $\mu \mathrm{S} / \mathrm{cm}$ ) peu productif, le second est un grand lac hypersalin (env. $70000 \mu \mathrm{S} / \mathrm{cm}$ ) plus productif. Les mesures de l'oxygène dissous de la colonne d'eau lors de la saison du mélange (décembre 2003 et janvier 2012) montrent que la colonne d'eau du lac Yoa est complètement anoxique pendant le premier mois de mélange, même si c'est le moment où l'oxygène atmosphérique dissous dans les eaux de surface pourrait potentiellement être injecté en profondeur dans la colonne d'eau.

Cette anoxie est une conséquence de la conjugaison de trois facteurs. Tout d'abord, le lac Yoa hypersalin permet que la concentration en oxygène dissous en équilibre avec l'atmosphère ne soit que d'environ $60 \%$ de ce qu'elle serait en eau douce à des températures similaires. Deuxièmement, la densité spécifique élevée de cette eau salée (environ $1,055 \mathrm{~g} / \mathrm{ml}$, comparativement à $0,999 \mathrm{~g} / \mathrm{ml}$ pour l'eau douce à température similaire) nécessite considérablement plus d'énergie pour qu'un mélange se fasse à une profondeur donnée. Enfin, la présence d'un grand volume d'eau de fond anoxique avec de fortes concentrations en éléments chimiques réduits l'emporte sur la couche d'eau superficielle relativement peu profonde qui est en contact direct avec l'atmosphère. Les odeurs de soufre autour du lac Yoa au cours (début) de la saison du mélange illustrent de manière significative les processus de réduction qui dominent les eaux de fond pendant environ neuf mois d'isolement de l'atmosphère. La demande élevée en oxygène des éléments chimiques (et des matières organiques en décomposition) dans ces eaux de fond prive temporairement la couche de

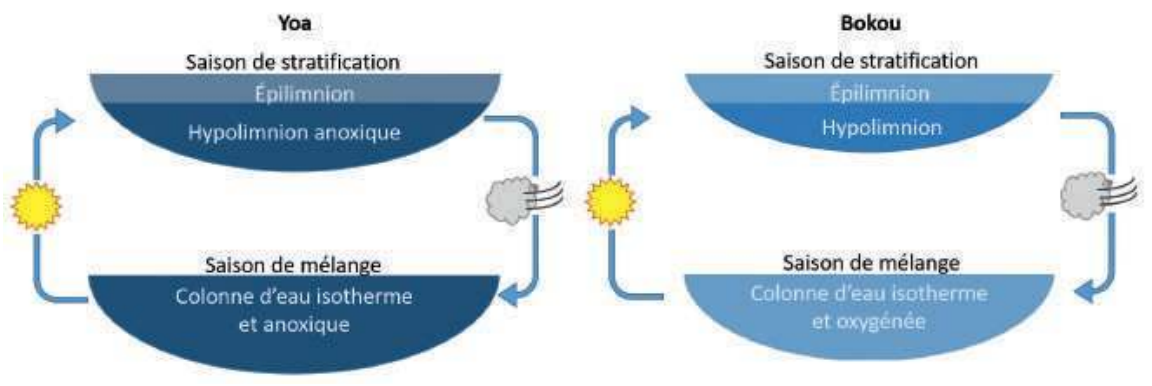

Figure 3

Modèle conceptuel de mélange saisonnier (eaux profondes et eaux de surface) dans un lac et implication sur les conditions écologiques en fonction des conditions morphologique et chimique différentes. Des conditions réduites et anoxiques dans la colonne d'eau inférieure volumineuse (hypolimnion) du lac Yoa salin, plus productif durant la stratification, privent l'eau de surface en oxygène pendant une partie de la saison de mélange, alors que l'hypolimnion du lac Bokou, dilué et moins productif, reste probablement oxygéné tout au long de l'année, ce qui n'appauvrit pas la surface de l'eau en oxygène au début du mélange. Dans ce système, l'augmentation de l'insolation de janvier jusqu'à juillet est le principal facteur favorisant la stratification des eaux du lac, tandis que les forts vents d'automne, alliés à la diminution de l'ensoleillement, jouent un rôle important dans la répartition de cette stratification. Les différences en termes de propriétés physiques y contribuent également : l'hypolimnion du lac Yoa est relativement plus important que celui du lac Bokou. 
surface de tout oxygène pendant la majeure partie de la saison du mélange, sauf apparemment lors de sa phase finale lorsqu'un faible niveau d'oxygène dissous a été enregistré (données de janvier 2012). Cependant, ce peu d'oxygène ayant été injecté est consommé rapidement après le renouvellement du début de la stratification en février. Par conséquent, le fond du lac Yoa est certainement dénué de tous organismes benthiques qui pourraient causer la bioturbation des sédiments récemment déposés.

Dans le lac Bokou, au contraire, la colonne d'eau est complètement oxygénée pendant la saison de mélange. La dissolution de l'oxygène dans ce lac d'eau douce est optimale, et le mélange des couches plus profondes est plus efficace que dans le lac Yoa. Étant donné la nature moins productive du lac Bokou, beaucoup moins d'algues sédimentent vers l'hypolimnion, ce qui se traduit par une moindre demande en oxygène au cours du processus de décomposition. Enfin, en raison du volume relatif moins important de son hypolimnion, le mélange avec l'eau de surface affecte à peine les niveaux d'oxygène dans ce dernier. Ainsi, bien que les cycles de mélange saisonnier pour ces deux lacs soient très semblables (et tous deux clairement dépendants du climat), leur impact écologique est très différent. Le biote du lac Yoa est confronté à un tout autre environnement hydrochimique saisonnier (hypersalinité constante aggravée par l'anoxie complète pendant au moins un mois) que le biote du lac Bokou (eau douce en permanence, avec l'épilimnion oxygéné en permanence). Les données actuellement disponibles ne permettent pas de déterminer si l'oxygène hypolimnique dans le lac Bokou s'épuise au cours de la saison de stratification.

En complément de la caractérisation physique et hydrochimique des divers plans d'eau à Ounianga Serir et Ounianga Kebir, une série d'échantillons prélevés au filet a été recueillie contenant à la fois des organismes planctoniques et benthiques, et des échantillons de sédiments contenant à la fois des matières vivantes et subfossiles. En mettant l'accent sur ces groupes d'invertébrés aquatiques dont les restes sont bien préservés même dans les sédiments (e.g. les chironomes, les mollusques, les ostracodes, les cladocères), l'analyse de leur répartition entre les lacs permettra de mieux comprendre les exigences écologiques de chaque groupe et de chaque espèce, et donc de promouvoir l'interprétation paléoenvironnementale des assemblages fossiles déposés au cours de certaines périodes dans le passé.

\section{Archives sédimentaires}

Les lacs ont la capacité d'enregistrer fidèlement les changements climatiques et environnementaux dans leurs sédiments, mais seulement si cette archive naturelle est continue et non perturbée. La nature stratifiée fine et régulière (e.g. varves) des sédiments du lac Yoa (KRÖPELIN et al. 2008 ; Francus et al. 
2013) est la preuve de l'accumulation immédiate et continue non perturbée de sédiments fins dans ce lac au cours des 6200 dernières années, démontrant à la fois sa stabilité hydrologique à long terme et les conditions anoxiques permanentes de son environnement en profondeur, tout au long de cette période. De tels enregistrements paléoenvironnementaux continus et avec une résolution annuelle sont probablement inégalés pour l'ensemble du Sahara. Pourtant, en raison de la taille et du fonctionnement hydrologique du lac Yoa, les signatures de certains événements climatiques passés peuvent être sur ou sous-accentuées dans ses enregistrements sédimentaires. Des carottages réalisés dans les lacs Edem, Teli et Agouta à Ounianga Serir fournissent désormais un moyen de comparer directement l'histoire environnementale de la fin de l'Holocène pour chaque lac, et ils pourront révéler des événements climatiques passés à l'échelle régionale qui n'auront peut-être pas été bien enregistrés dans les enregistrements sédimentaires du lac Yoa. En outre, les sédiments finement stratifiés du lac Teli, hypersalin $(112000 \mu \mathrm{S} / \mathrm{cm})$, apportent une archive sédimentaire non perturbée et continue. La qualité de cet enregistrement est comparable à celle du lac Yoa sur au moins le dernier millénaire, bien que la profondeur du lac Teli n'excède pas 4,5 m (en 2016) contre $25 \mathrm{~m}$ dans le lac Yoa. Cela signifie que la salinité plus importante du lac Teli, et également probablement l'abri au vent du lieu de carottage dans la partie nord de ce lac, compensent sa faible profondeur : une plus forte densité de son eau hypersaline $(>1,100 \mathrm{~g} / \mathrm{ml})$ permet d'éviter les turbulences dues au vent qui pourraient remettre en suspension ou perturber les sédiments accumulés. De même, toute forme de bioturbation est absente dans les sédiments récents du lac Teli, ce qui reflète une eau de fond anoxique en permanence. Comme attendu, les carottes de sédiments provenant du lac Edem $(670 \mu \mathrm{S} / \mathrm{cm})$ et du lac Agouta $(1780 \mu \mathrm{S} / \mathrm{cm})$ bien plus dilués et mieux oxygénés ne montrent pas de stratifications fines sur ce même intervalle de temps, bien qu'elles aient été prélevées à des hauteurs d'eau identiques.

L'analyse des indicateurs sédimentologiques reflétant la composition et la texture de la masse de sédiments permet de déduire les variations de profondeur du lac, le régime de mélange et la chimie de l'eau. Les images obtenues par scanner tomographique $(\mathrm{CT})$ à rayons $\mathrm{X}$ haute résolution donnent un aperçu de la texture et des gradients de densité des sédiments, et ce même sans ouvrir les carottes. Les variations de la susceptibilité magnétique fournissent des informations sur la nature et l'origine de la fraction minérale clastique arrivant au lac soit par le vent, soit par le ruissellement dans le bassin. L'analyse par la perte au feu (DEAN, 1974) permet de déterminer la teneur en eau, la porosité, et les constituants organiques et inorganiques. L'analyse de la granulométrie permet une estimation de la texture des sédiments.

De plus, les fossiles du biote aquatique sont conservés et utilisés comme des indicateurs biologiques pour déduire les changements passés en termes de répartition de l'habitat aquatique et d'hydrologie. Les mollusques fossiles, qui sont des indicateurs de substrats spécifiques, apparaissent en abondance dans les affleurements des dépôts lacustres du début de l'Holocène, à la fois dans les bassins de Kebir et de Serir (VAn BoxlaER et al., 2011). Une analyse combinée 
approfondie des assemblages des chironomidés et des diatomées récents et fossiles permet de procéder à des déductions quantitatives de la salinité du passé (e.g. KRÖPELIN et al., 2008 ; EgGermont et al., 2008). Enfin, l'analyse des ostracodes fossiles contribue aux reconstructions environnementales et paléohydrologiques à travers l'analyse de l'assemblage des espèces et de l'empreinte géochimique (analyse des isotopes stables) (e.g. VAN DER MEEREN et al., 2011).

La connaissance du fonctionnement actuel du lac et une combinaison des données résultant des analyses sédimentologiques, paléoécologiques et géochimiques, améliorent la reconstitution fiable des changements environnementaux au cours du temps et du lien entre ces variations hydroclimatiques et les dynamiques des sociétés à l'échelle régionale.

\section{Comprendre la dynamique écologique à long terme}

\section{Facteurs de changement}

L'aquifère des grès de Nubie (ou NSAS pour Nubian Sandstone Aquifer System), qui a vraisemblablement été rechargé pour la dernière fois au milieu de l'Holocène (Voss et Soliman, 2014), alimente en permanence les lacs d'Ounianga Kebir et Serir en eau douce (env. $200 \mu \mathrm{S} / \mathrm{cm}$ ). Faisant partie des plus grandes nappes aquifères au monde $\left(>2000000 \mathrm{~km}^{2}\right)$, il se compose d'un certain nombre de bassins interconnectés latéralement et verticalement en Égypte, en Libye, au Soudan et au Tchad (SALEM et PALlas, 2004). En raison de sa forte connexion avec la décharge d'eau souterraine de l'aquifère NSAS, la réponse de l'équilibre hydrique à la variation du régime hydrologique climatique est supposée être relativement faible, sauf pour les échelles de temps les plus longues (i.e. Holocène et Quaternaire) (KRÖPELIN et al., 2008 ; EGGERMONT et al., 2008). Bien que l'analyse des enregistrements sédimentaires couvrant les derniers siècles des lacs Edem, Agouta et Hogou (CREUTZ et al., 2016) semble indiquer que la sensibilité hydrologique de ces lacs désormais exclusivement alimentés par des eaux souterraines est certes relativement modeste, d'autres éléments suggèrent une certaine sensibilité au changement climatique à des échelles de temps relativement courtes.

D'un point de vue hydrogéologique, les bassins d'Ounianga sont situés à proximité de la marge de l'aquifère des grès de Nubie et sont relativement proches des anciennes régions d'alimentation à haute altitude telles que les imposants reliefs volcaniques du Tibesti, résultant des voies d'écoulement des eaux souterraines relativement courtes (Voss et Soliman, 2014). Au niveau de 
cette situation périphérique, l'étendue verticale de l'aquifère est également beaucoup plus limitée que dans les zones à basse altitude situées au nord, où le temps de déplacement avant la décharge des eaux souterraines à la surface peut être beaucoup plus long (supérieur à 1 million d'années ; Voss et Soliman, 2014). Les connexions locales d'eau souterraine entre les lacs peuvent amplifier la concentration par évaporation, ce qui constitue une deuxième raison importante selon laquelle les lacs d'Ounianga peuvent présenter une sensibilité hydrologique plus importante que prévue.

Dans les deux bassins d'Ounianga, il existe un écoulement souterrain (entrée et sortie) entre les lacs, ce qui se traduit par des équilibres hydriques contrastés contrôlés de manière distincte par l'évaporation et la décharge des eaux souterraines. À l'échelle locale, il en résulte une amplification des changements de l'humidité au cours du temps. Dans le bassin de Serir, ce mécanisme est bien exprimé dans la partie centrale du lac hypersalin Teli qui est aligné à une série de lacs plus dilués (George, 1999 ; VAn BocXlaer et al., 2011 ; Creutz et al., 2016). Une troisième raison pour laquelle la sensibilité à la variabilité hydrique liée au climat ne peut pas être négligeable est la variation saisonnière des niveaux d'eau des lacs à Ounianga (CAPOT-REY, 1961) : on ne s'attendrait pas à une telle variabilité si les lacs reflétaient simplement le niveau d'un grand réservoir d'eaux souterraines fossiles, sans surimposition climatique locale contemporaine. Quelques preuves de cette surimposition climatique peuvent également être trouvées dans la subtile mais notable augmentation du niveau du lac Teli entre 1984 et 2016, observée par images satellite Landsat (USGS/Nasa).

\section{La résilience et les services écosystémiques}

Bien que la grande incertitude des résultats des modélisations du climat pour l'Afrique (e.g. Rowell et al., 2016) interdise une prévision climatique claire pour de nombreuses régions, les précipitations moyennes pour la plupart des régions arides subtropicales et aux latitudes tempérées comme le Sahara central devraient diminuer (IPCC, 2014). Tandis que la performance des modèles climatiques s'améliore constamment, par exemple par une meilleure intégration des retours en termes de surface terrestre et de végétation-climat, la fiabilité des projections climatiques régionales pour le nord du Tchad va également probablement augmenter. Toutefois, une grande partie de l'adaptation de la région au changement climatique et de son adéquation avec les politiques de gestion des ressources naturelles dépend largement de la bonne compréhension de la dynamique de ces écosystèmes terrestres et aquatiques. Sans connaissances suffisantes de la sensibilité climatique à long terme des ressources des eaux de surface, des projections climatiques régionales, même très précises, laisseraient les décideurs dans le doute quant à la politique appropriée à adopter.

Pour la douzaine de lacs, les sources et les habitats des zones humides associés dans la région d'Ounianga, les connaissances actuelles sur la résilience écologique à long terme et la sensibilité du climat sont en grande partie limitées au lac Yoa, le plus profond et le plus volumineux d'entre eux, et donc aussi le 
moins sensible à la variabilité du régime hydrique liée au climat. Certaines informations sont disponibles pour les lacs Edem, Agouta et Hogou dans le bassin de Serir (CREUTz et al., 2016), mais elles sont pour le moment limitées aux derniers siècles. Sur la base des informations actuellement disponibles, il est très difficile de tirer des conclusions sur la résilience des écosystèmes aquatiques de l'ensemble des lacs à l'échelle des siècles ou des millénaires. En l'état, nous ne disposons pas des éléments nécessaires sur la variabilité naturelle à long terme pour déterminer et discuter de l'utilisation durable des services écosystémiques que fournissent ces lacs.

Les lacs sahariens d'Ounianga représentent un patrimoine naturel et culturel unique, reconnu au patrimoine mondial de l'Unesco. Les populations ont utilisé ces territoires comme habitat temporaire ou permanent depuis de nombreux millénaires. L'art rupestre montrant des mammifères qui sont aujourd'hui localement éteints, les villages abandonnés sur les falaises escarpées bordant les lacs Yoa et Teli, les vestiges anciens de structures d'habitat, tous illustrent les changements importants en termes de climat, d'environnement et de culture auxquels cette région désertique a dû faire face au cours du temps. Une meilleure compréhension de l'histoire environnementale et de la résilience écologique de ces lacs peut aider à mieux se préparer aux changements globaux qui sont à venir.

\section{Remerciements}

Nous remercions le gouvernement du Tchad et son Centre national de recherche pour le développement pour l'autorisation des recherches, et le programme Gelt pour l'organisation interdisciplinaire des travaux sur le terrain en 2015 et 2016. Nous remercions l'administration locale d'Ounianga Kebir et d'Ounianga Serir pour le soutien apporté à ces missions. Nous remercions également la National Geographic Society (USA) pour le soutien financier aux travaux conduits sur le terrain en 2016 grâce à la subvention NGEF186-16 attribuée à Thijs Van der Meeren. 


\section{Bibliographie}

CAPOT-Rey R., 1961

Borkou et Ounianga. Étude de géographie régionale. Alger, université d'Alger (Institut de recherches sahariennes), vol. 5, 182 p.

\section{CNAR, 2011}

Proposition d'inscription des lacs d'Ounianga sur la Liste du patrimoine mondial, N'Djamena, 327 p., multigr.

\section{Creutz M., Van Bocxlaer B.,} Abderamane M., Verschuren D., 2016

Recent environmental history of the desert oasis lakes at Ounianga Serir, Chad. Journal of Paleolimnology, 55: 167-183.

\section{DEAN W. E. J., 1974}

Determination of carbonate and organic matter in calcareous sediments and sedimentary rocks by loss on ignition: comparison with other methods. J Sediment Petrol, 44: 242-248.

\section{Eggermont H., Verschuren D., Fagot M.,} Rumes B., Van BocXlaer B., Kröpelin S., 2008

Aquatic community response in a groundwaterfed desert lake to Holocene desiccation of the Sahara. Quaternary Science Reviews, 27: 2411-2425.

Francus P., Von Suchodoletz H., Dietze M., Donner R. V., Bouchard F., Roy A. J., Fagot M., Verschuren D., KröPelin S., 2013 Varved sediments of Lake Yoa (Ounianga Kebir, Chad) reveal progressive drying of the Sahara during the last 6100 years. Sedimentology, 60: 911-934.

\section{GEORGE U., 1999}

Entdeckungen im Herzen der Leere. Geo, 10: 20-50.

IPCC (éd. Pachauri R. K. and Meyer L. A.), 2014

Climate Change 2014: Synthesis Report.

Contribution of Working Groups I, II and III to the Fifth Assessment Report of the Intergovernmental Panel on Climate Change. Geneva, IPCC, 151 p.

Kröpelin S., Verschuren D., Lezine A. M., Eggermont H., Cocquyt C., Francus P.,
Cazet J. P., Fagot M., Rumes B., Russell J. M., Darius F., Conley D. J., Schuster M., Von Suchodoletz H., ENGSTROM D. R., 2008

Climate-driven ecosystem succession in the Sahara: The past 6000 years. Science, 320: 765-768.

\section{Rowell D. P., Senior C. A., Vellinga M., GraHAM R. J., 2016}

Can climate projection uncertainty be constrained over Africa using metrics of contemporary performance? Climatic Change, 134: 621-633.

\section{Salem O., Pallas P., 2004}

"The Nubian Sandstone Aquifer System (NSAS) ». In : Appelgren B., éd.: Managing shared aquifer resources in Africa, Paris, Unesco (ISARM-Africa; Series on Groundwater $\mathrm{n}^{\circ} 8$ ), $238 \mathrm{p}$.

\section{Van BocXlaer B., Verschuren D.,} Schettler G., KRöPelin S., 2011

Modern and early Holocene mollusc fauna of the Ounianga lakes (northern Chad): implications for the palaeohydrology of the central Sahara. Journal of Quaternary Science, 26: 433-447.

VAn der Meeren T., Ito E., Verschuren D., Almendinger J. E., Martens K., 2011

Valve chemistry of Limnocythere inopinata (Ostracoda) in a cold arid environment Implications for paleolimnological interpretation. Palaeogeography, Palaeoclimatology, Palaeoecology, 306: 116-126.

\section{Voss C. I., Soliman S. M., 2014}

The transboundary non-renewable Nubian Aquifer System of Chad, Egypt, Libya and Sudan: classical groundwater questions and parsimonious hydrogeologic analysis and modeling. Hydrogeology Journal, 22: 441-468.

Washington R., Todd M. C., Engelstaedter S., Mbainayel S., Mitchell F., 2006

Dust and the low-level circulation over the Bodélé Depression, Chad: Observations from BoDEx 2005. J. Geophys. Res., 111 (D03): doi:10.1029/2005JD006502 


\title{
Etude de la dynamique hydrologique des lacs Iro et Fitri par une approche isotopique
}

\author{
Chloé Poulin, Bruno HameliN, \\ Christine Vallet-Coulomb, Guinbé Amngar, \\ Bichara LOUKMAN, Jean-François CRÉTAUX, \\ Jean-Claude DOUmnang, Abdallah MaHAmat Nour, \\ Guillemette MENOT, Florence SYLVESTRE,
} Pierre DESCHAMPS

\section{Introduction}

Au Sahel, les effets combinés de l'augmentation de la population, de la dégradation des terres et du changement de régime pluviométrique ont entraîné une détérioration des sols et de la ressource en eau (UNEP et ICRAF, 2006). Depuis le XIX siècle, le Sahel a enregistré successivement des épisodes de sécheresses et des périodes plus humides (Nicholson, 2013). En plus de cette variabilité naturelle, il faut désormais prendre en compte l'impact du changement climatique sur le régime pluviométrique. À ce jour, aucun consensus sur l'évolution des précipitations au $\mathrm{XXI}^{\mathrm{e}}$ siècle n'a été trouvé (DRUYAN, 2011 ; DEFRANCE et al., 2017). Ces incertitudes sur les précipitations se répercutent sur l'évolution des systèmes lacustres sahéliens. En effet, une grande partie de ces lacs sont des systèmes endoréiques qui forment des réservoirs pendant la saison sèche. Leur vulnérabilité face aux conditions climatiques, combinée à leur grande importance sociétale, rend la compréhension de leur fonctionnement hydrologique incontournable. 
L'étude de ces systèmes se heurte au peu de données disponibles dû à un faible suivi des variables hydroclimatiques à l'échelle de leurs bassins versants (SivaPAlan et al., 2003). Pour étudier un cycle hydrologique complet, il faut $a$ minima des données représentatives des saisons sèches et des pluies. Or le terrain en zone sahélienne s'avère bien souvent impraticable en saison des pluies rendant les zones inondées plus difficilement accessibles. Dans ce contexte, les données géochimiques, et plus particulièrement les isotopes stables, se sont révélés utiles dans la détermination des bilans hydrologiques lacustres en raison de leur caractère intégrateur des processus hydrologiques se produisant au sein du lac et de son bassin versant.

Nous proposons ici d'établir un premier bilan hydrologique des lacs Iro et Fitri situés dans le bassin du lac Tchad, en utilisant les isotopes stables de la molécule d'eau. Le fonctionnement d'un lac est caractérisé par ses apports (appelés « Entrées » en figure 1) représentés par les précipitations $(\partial \mathrm{P})$, le ruissellement et l'infiltration $(\partial \mathrm{l})$, mais aussi par ses sorties correspondant à l'évaporation $(\partial \mathrm{E})$, les écoulements de surface et l'infiltration $(\partial \mathrm{L})$ (fig. 1). La transpiration n'a pas été prise en compte dans cette étude en raison de la forte fluctuation saisonnière de la végétation et de l'absence de données mensuelles. Le terme « infiltration » désigne ici l'apport des eaux souterraines au lac dans le cas des entrées et l'infiltration des eaux du lac vers le souterrain dans le cas des sorties. La quantification de ces flux entrants et sortants est indispensable à la compréhension du fonctionnement hydrologique d'un système lacustre et permet de rendre compte des interactions entre la surface et le souterrain pour une gestion intégrée de la ressource.

Entrées

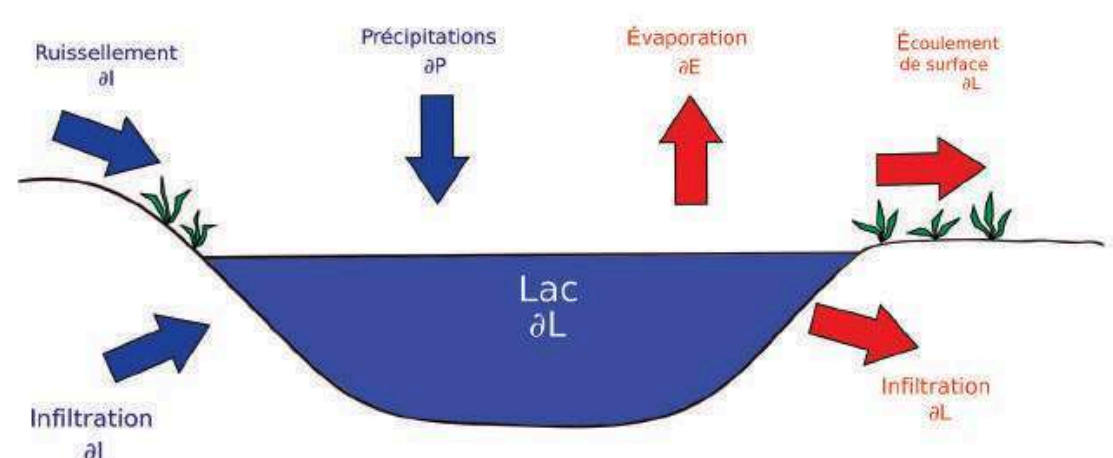

Figure I

Schéma simplifié du fonctionnement hydrologique d'un système lacustre adapté au cas d'étude. Les flux représentés par les flèches bleues correspondent aux flux entrants (précipitations, ruissellement et infiltration). Les flux représentés par les flèches rouges correspondent aux flux sortants (évaporation, écoulement de surface et infiltration). Les notations $\partial$ indiquent la composition isotopique des différents flux. 
Cette démarche, initiée par GoNFIANTINI (1986), a déjà été utilisée par plusieurs auteurs pour caractériser les flux évaporatoires d'une chaîne de lacs (GIBSON et EDWARDS, 2002), pour calculer le rapport flux évaporatoire/flux d'entrée (MAYR et al., 2007), ou encore déterminer la composition isotopique du signal d'entrée (YI et al., 2008). Ces études portent toutes sur des lacs situés en climat tempéré froid dans lequel l'évaporation ne joue pas un rôle important dans leurs bilans hydrologiques. Sur le lac Tchad, les récents travaux de Bouchez et al. (2016) permettent de quantifier ces différents flux par isotopie contrairement aux travaux antérieurs (FONTES et al., 1970 ; RocHE, 1980) qui se sont concentrés sur le fonctionnement hydrodynamique du lac sans caractérisation de flux. Nous proposons ici d'adapter cette méthode isotopique en climat sahélien à partir de données récoltées lors de deux missions Gelt successives en 2015 sur les lacs Iro et Fitri.

\section{Contexte général des lacs Iro et Fitri}

Situés au cœur du bassin du lac Tchad, les lacs Iro et Fitri appartiennent à deux bassins versants distincts (fig. 2). Le bassin versant du Bahr Salamat (195000 km²) est un sous bassin du Chari-Logone ; prenant sa source au Darfour, il alimente le lac Iro en saison des pluies via un défluent dont le sens s'inverse en décrue (BILlON et al., 1974). Le lac Fitri est situé dans le bassin versant du Batha (96 $000 \mathrm{~km}^{2}$ ) dont l'intégralité se situe en zone sahélienne.

Les enregistrements montrent une pluviométrie annuelle de $765 \mathrm{~mm}$ par an à la station d'Am Timan en amont du lac Iro, contre $360 \mathrm{~mm}$ par an à Ati en amont du lac Fitri (moyenne annuelle entre 1960 et 2014, Drem). Les précipitations sont apportées par la mousson africaine entre juin et septembre qui est liée à un déplacement vers le nord de la zone de convergence intertropicale (ZCIT). Les températures moyennes annuelles sont respectivement de $27^{\circ} \mathrm{C}$ et $28{ }^{\circ} \mathrm{C}$ pour les lacs Iro et Fitri, tandis que leur humidité relative moyenne annuelle est de $50 \%$ et $40 \%$ respectivement (données Drem, stations d'Am Timan entre 1966 et 1976 pour le lac Iro, et station d'Ati entre 1960 et 2004 pour le lac Fitri [Boyer et al., 2006]). L'évaporation Piche a été enregistrée entre 1961 et 1988 à la station de Birao (en Centrafrique, fig. 2) qui se situe à la même latitude que le lac Iro. Le flux moyen est de 1,8 m par an.

Les débits des rivières sont issus de relevés réalisés sur le Bahr Azoum (bassin du Bahr Salamat) entre 1953 et 1966 (BILLON et al., 1974) ; ils sont complétés ensuite par la Drem jusqu'en 1973 (BOYER et al., 2006). Dans le bassin versant du Batha, les données de débit ont été relevées entre 1955 et 1993 (BOYER et al., 2006). 


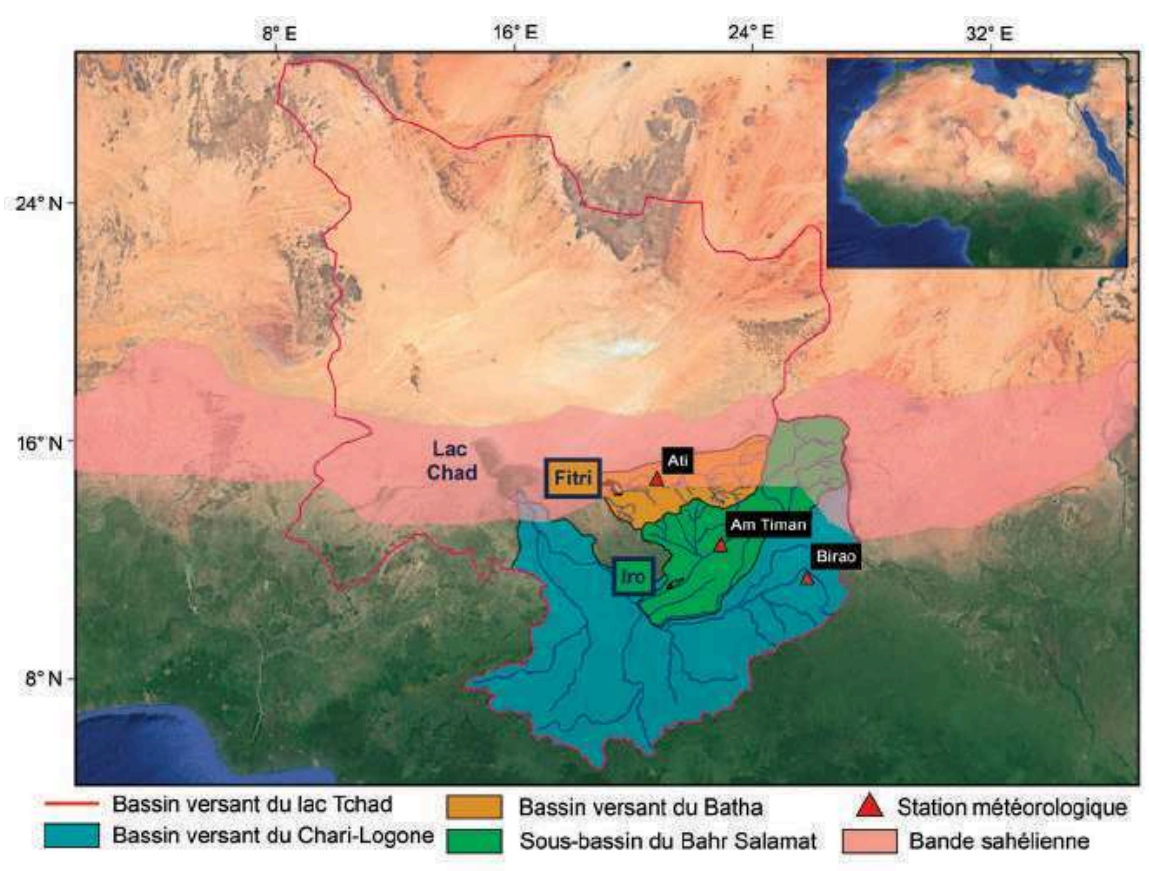

Figure 2

Localisation des bassins versants et des lacs étudiés. Les triangles rouges représentent la position des stations météorologiques utilisées dans cette étude.

Source : Google Earth.

Les rivières s'écoulent sur des dépôts d'âge quaternaire de la cuvette tchadienne : ce sont des dépôts fluviolacustres ou éoliens qui varient entre sables et argiles. Autour de ces deux lacs, quelques intrusions granitiques apparaissent sous forme d'inselbergs, laissant parfois place à des cuirasses latéritiques (notamment autour du lac Iro). Les aquifères des lacs Iro et Fitri sont localisés dans ces dépôts sédimentaires sablo-argileux : peu profonde autour du lac Iro (de 5 à $30 \mathrm{~m}$ ), la surface piézométrique de la nappe autour du lac Fitri s'approfondit des bords du Batha (10-15 m à Ati) vers le nord où elle peut atteindre $50 \mathrm{~m}$ de profondeur en une vingtaine de kilomètres (SCHNEIDER, 2004).

\section{Matériels et méthodes}

Nous disposons de trois types de données : des données géochimiques mesurées à partir d'échantillons récoltés sur le terrain, des données météorologiques des différentes stations présentes sur le bassin et des données satellitaires. 
Les données géochimiques ont été collectées lors de deux campagnes de terrain organisées par le programme Gelt : la première sur le lac Fitri, fin février 2015, et la seconde sur le lac Iro en avril 2015 (fig. 3 et 4). À chaque fois, les paramètres physicochimiques ont été mesurés et des échantillons d'eaux souterraine et de surface (lac Iro et lac Fitri) ont été collectés (directement sur les lacs et à partir de puits) pour l'analyse des isotopes stables, des ions majeurs et du ${ }^{36} \mathrm{Cl}$. $\mathrm{Au}$ total, 33 points d'eau souterraine ont été prélevés (14 autour du lac Iro et 19 autour du lac Fitri) et 4 échantillons d'eau lacustre ( 2 pour chaque lac). Le Bahr Salamat a également été échantillonné juste avant le défluent menant au lac Iro, ainsi que le Chari en amont de la confluence avec le Bahr Salamat. En revanche, le Batha étant à sec à cette période, il n'a pu être échantillonné.

Les isotopes stables de la molécule d'eau ont été analysés au Cerege soit par spectromètre laser (Picarro L1102-i) pour les échantillons ayant une salinité faible $(<1000 \mu \mathrm{S} / \mathrm{cm})$, soit pour ceux de plus forte salinité $(>1000 \mu \mathrm{S} / \mathrm{cm})$, par spectromètre de masse Delta Plus, après équilibration au $\mathrm{CO}_{2}(10 \mathrm{~h}$ à $291 \mathrm{~K})$ ou $\mathrm{H}_{2}(2 \mathrm{~h}$ à $291 \mathrm{~K}$ avec des catalyseurs platine $)$ - respectivement pour $\partial^{18} \mathrm{O}$ et $\partial^{2} \mathrm{H}-$ via une unité d'équilibration automatisée HDO Thermo Finnigan. Les rapports isotopiques sont reportés en pour mille (\%o) versus Smow (Standard Mean Ocean Water), normalisés à 1'échelle VSMOW2-SLAP2, en utilisant 3 standards internes suivant les recommandations de la feuille de référence de l'IAEA (IAEA, 2009). Toutes les analyses ont été a minima dupliquées. L'incertitude est inférieure à $\pm 0,15 \%$ o $(1 \sigma)$ et à $\pm 1 \%$ o $(1 \sigma)$ respectivement pour les données de $\partial^{18} \mathrm{O}$ et $\partial^{2} \mathrm{H}$.

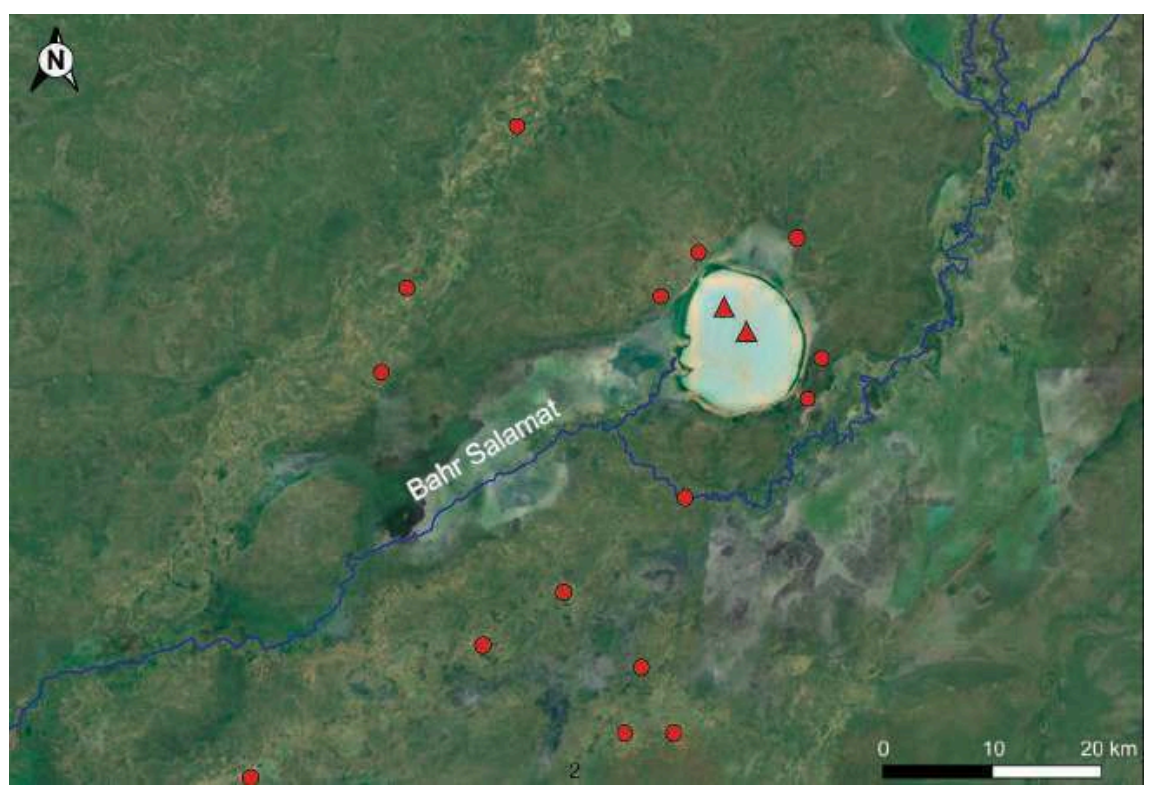

Figure 3

Localisation des prélèvements des eaux de surface et souterraines autour du lac Iro.

Les triangles rouges représentent les eaux du lac.

Source : Google Earth 2018, relevés GPS. 


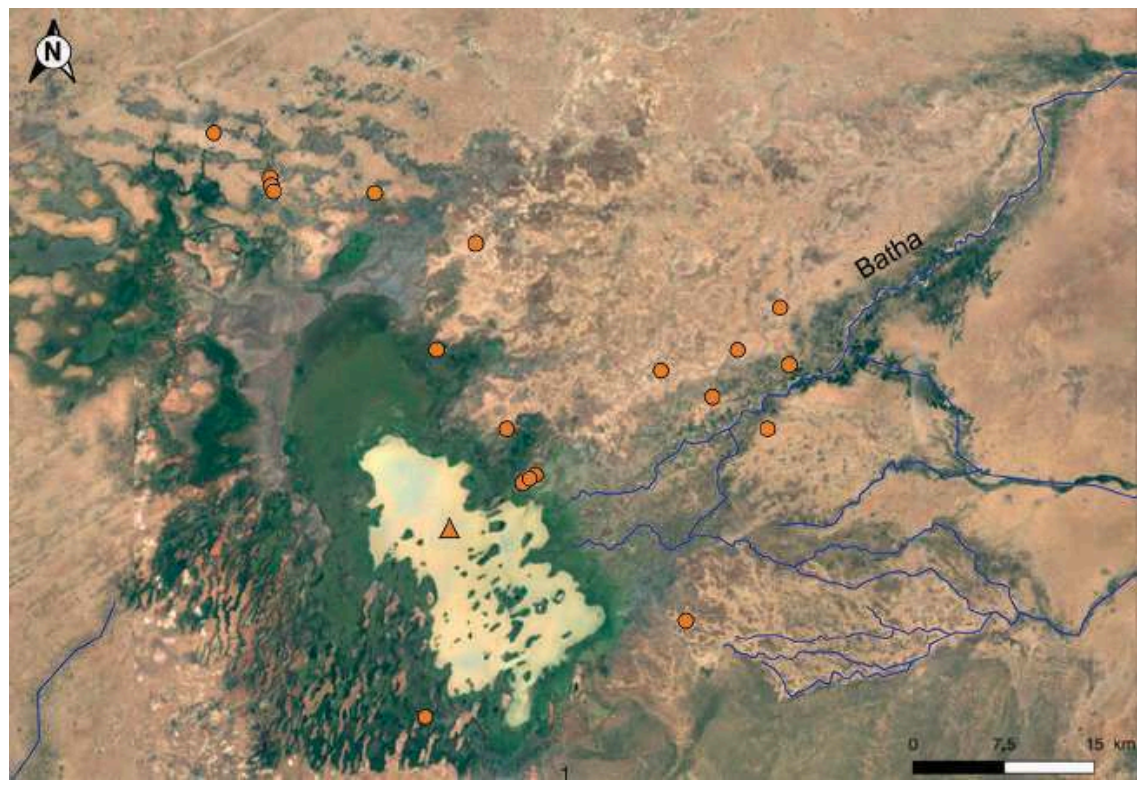

Figure 4

Localisation des prélèvements des eaux de surface et souterraines autour du lac Fitri.

Les triangles orange représentent les eaux du lac.

Source : Goolgle Earth 2018, relevés GPS.

Les variations du niveau lacustre ont été estimées par altimétrie satellite (CRÉTAuX et al., 2016). Les données altimétriques utilisées dans cette étude proviennent de l'altimètre AltiKa embarqué sur le satellite Saral, et ont permis de reconstituer les variations des niveaux des lacs Iro et Fitri entre mars 2013 et avril 2016. L'absence de données sur le milieu de l'année 2015 pour les deux lacs, puis pour la fin d'année 2015 sur Iro, est liée à une dérive de l'orbite du satellite, entraînant une dérive au sol de la trace qui est sortie de la zone des lacs.

\section{Résultats}

\section{Chimie des eaux}

Les lacs Iro et Fitri partagent avec le lac Tchad (Bouchez et al., 2016) la caractéristique d'être des lacs d'eau douce, malgré les forts taux d'évaporation enregistrés dans cette région. Les données de conductivité mesurées pendant la saison sèche présentent une valeur de $170 \mu \mathrm{S} / \mathrm{cm}$ pour le lac Iro et $140 \mu \mathrm{S} / \mathrm{cm}$ pour le lac Fitri, avec un $\mathrm{pH}$ commun proche de 8. 
$\mathrm{Au}$ contraire, les eaux souterraines autour des lacs sont plus salées et sont caractérisées par une grande variabilité spatiale. Autour du lac Iro, la conductivité varie entre 65 et $1012 \mu \mathrm{S} . \mathrm{cm}^{-1}$, contre 705 et $14000 \mu \mathrm{S} . \mathrm{cm}^{-1}$ pour l'aquifère autour du lac Fitri. Les pH sont plutôt acides autour du lac Iro $(5,0<\mathrm{pH}<7,0)$ et plutôt basiques autour du lac Fitri $(6,5<\mathrm{pH}<8,5)$.

\section{Isotopes stables de la molécule d'eau}

Concernant les isotopes stables, les données mesurées dans les deux lacs Iro et Fitri sont respectivement de $+3,11 \%$ et $+2,04 \%$ en $\partial^{18} \mathrm{O}$, et $+12,3 \%$ et $+5,8 \%$ o en $\partial^{2} \mathrm{H}$, caractéristique attendue d'une signature d'évaporation marquée $\left(\mathrm{d}_{\text {excess }}\right.$ de -12 et $-10 \%$ respectivement; $\mathrm{d}_{\text {excess }}=\partial^{2} \mathrm{H}-8 . \partial^{18} \mathrm{O}$, et traduit l'écart à la Droite météorique mondiale, DMM). Pour ce qui est des mesures effectuées sur les eaux prélevées dans les aquifères, les variations de composition isotopique entre les deux systèmes sont similaires (entre $-5 \%$ et $0 \%$ pour le $\partial^{18} \mathrm{O}$ autour du lac Iro et entre $-5 \%$ et $+2 \%$ pour le Fitri).

Interprétées dans un diagramme de corrélation entre les valeurs mesurées en $\partial^{2} \mathrm{H}$ et en $\partial^{18} \mathrm{O}$, les données des lacs Iro et Fitri s'organisent en 2 droites de pente proche (appelées droites d'évaporation locales dans la suite du texte) mais significativement distinctes (fig. 5). Comparées à la droite météorique mondiale qui a été calculée à partir d'un ensemble d'échantillons de précipitation à l'échelle du globe, elles présentent une pente plus faible, traduisant des systèmes hydrologiques affectés par l'évaporation. Les données les plus appauvries, et donc les moins évaporées, sont les plus proches de la DMM. Les valeurs trouvées à l'intersection de la DMM et des droites d'évaporation (respectivement $-5,83 \%$, $-36,6 \%$ en $\partial^{18} \mathrm{O}$ et $\partial^{2} \mathrm{H}$ pour Iro, et $-7,21 \%$, $-47,7 \%$ pour Fitri) représentent la composition isotopique pondérée théorique de l'eau entrant dans ces systèmes.

Enfin, et de manière très remarquable, les données des deux lacs, Iro et Fitri, se reportent toutes les deux exactement sur la droite d'évaporation d'Iro, suggérant un système aquifère fortement connecté aux eaux de surface dans ce cas (fig. 5). En revanche, cela traduit une situation plus complexe dans le cas du lac Fitri.

La moyenne pondérée des précipitations calculée entre 1963 et 1978 (données enregistrées par l'IAEA à la station de N'Djamena [IAEA WMO, 2017]) $\left(-3,53 \% 0,-18,4 \%\right.$ en $\partial^{18} \mathrm{O}$ et $\left.\partial^{2} \mathrm{H}\right)$ est plus enrichie que les valeurs trouvées à l'intersection de la DMM et des droites d'évaporation locales.

La figure 5 replace également nos données en perspective de celles publiées dans la littérature pour le lac Tchad, dans la cuvette nord (FONTES et al., 1970), et dans la cuvette sud (Bouchez et al., 2016). L'ensemble de ces points est localisé au-dessus de la droite que nous avons obtenue pour le lac Iro : les points de la cuvette nord sont très enrichis et sont compris entre $-0,8 \%$ et $+15 \%$ pour $\partial^{18} \mathrm{O}$ et entre $-2,8 \%$ et $+77 \%$ pour $\partial^{2} \mathrm{H}$. Les points de la cuvette sud sont plus appauvris : entre $-3,3 \%$ et $-1,5 \%$ o pour le $\partial^{18} \mathrm{O}$ et entre $-26 \%$ et $-10 \%$ pour le $\partial^{2} \mathrm{H}$ et se rapprochent des données isotopiques observées sur les systèmes Iro 
et Fitri. Ces données sur le lac Tchad sont compatibles avec les données de précipitations de N'Djamena. Cet enrichissement progressif traduit un système de plus en plus évaporé depuis la cuvette sud vers la cuvette nord.

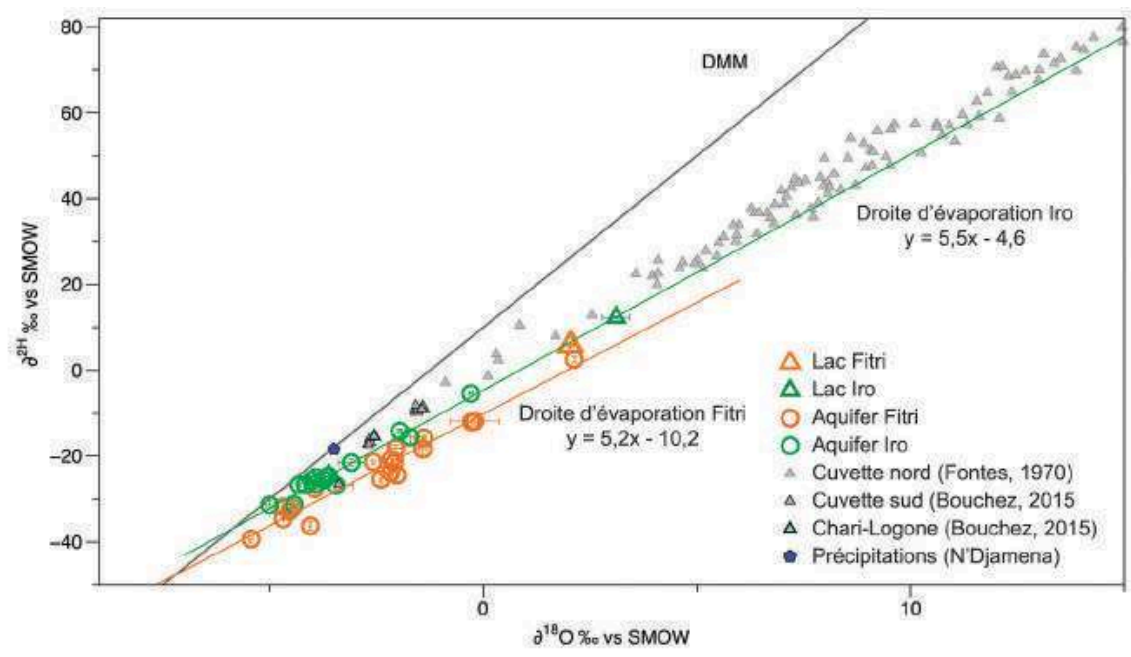

Figure 5

Relation $\partial^{2} \mathrm{H} / \partial^{18} \mathrm{O}$ pour les eaux échantillonnées autour des lacs Iro et Fitri. Les triangles verts et orange représentent respectivement la composition isotopique des lacs Iro et Fitri, tandis que

les cercles représentent les données des eaux souterraines des aquiferes de ces deux lacs.

Les triangles gris représentent les données des eaux du lac Tchad (cuvettes nord et sud),

et le triangle bleu, la composition isotopique du Chari-Logone. Le pentagone bleu foncé est la composition isotopique moyenne pondérée des pluies à N'Djamena. Les données des eaux souterraines et lacustres du lac Iro s'alignent selon une même droite d'évaporation de pente 5,5.

Les données des eaux souterraines du lac Fitri, plus appauvries, s'alignent

sur une deuxième droite d'évaporation de pente 5,2. On remarque que les eaux du lac Fitri s'alignent sur la même droite d'évaporation que celles du lac Iro.

\section{Altimétrie spatiale}

Les données d'altimétrie spatiale montrent une variation de niveau d'eau comprise entre 2 et $3 \mathrm{~m}$ sur le lac Iro, respectivement pour 2013 et 2014 (l'année 2015 étant incomplète), avec un maximum atteint aux mois d'août et septembre et un minimum atteint en juin et en mai pour ces deux années. Les variations sur le lac Fitri pour ces deux mêmes années sont de $2 \mathrm{~m}$ avec un maximum atteint en août et un minimum en juin (fig. 6).

Les fluctuations des lacs observées grâce aux images Landsat 7 et 8 confirment ces fortes variations à l'échelle intra-annuelle. La superficie du lac Iro est multipliée par 4 entre les périodes de basses et de hautes eaux. D'une superficie d'environ $100 \mathrm{~km}^{2}$ en basses eaux, il peut atteindre jusqu'à $400 \mathrm{~km}^{2}$ en hautes eaux. D'une profondeur de $2 \mathrm{~m}$ en moyenne mesurée lors de la campagne de terrain en avril 2015, le lac peut donc atteindre $5 \mathrm{~m}$ de profondeur en période de hautes eaux. 
Le lac Fitri connait également de grandes variations de superficie : sa surface passe de plus de $1000 \mathrm{~km}^{2}$ en saison des pluies, à moins de $200 \mathrm{~km}^{2}$ en saison sèche. Sa bathymétrie mesurée pendant la campagne de terrain de février 2016 montre une profondeur maximale de $2,5 \mathrm{~m}$ dans sa partie ouest et peut donc atteindre $4 \mathrm{~m}$ de profondeur en saison de hautes eaux.
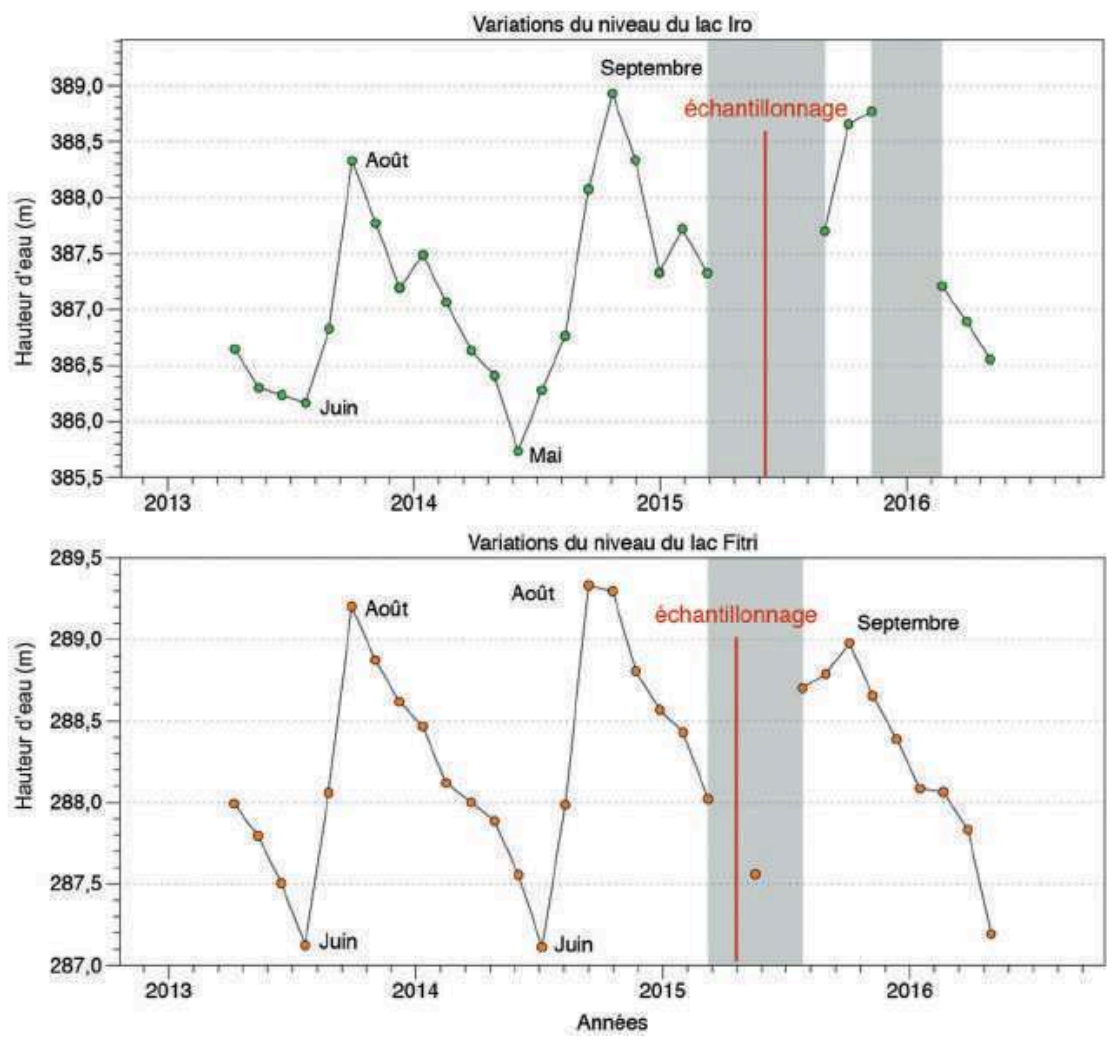

Figure 6

Fluctuations du niveau lacustre à partir des données d'altimétrie spatiale (satellite Saral) pour le lac Iro (en vert) et le lac Fitri (en orange). Les données couvrent la période 2013-2016, les zones grises représentent une absence de données et les barres rouges la période d'échantillonnage pour les deux lacs. Les variations du niveau du lac Iro montrent un maximum de 3,5 m atteint en 2014 et un maximum de 2,5 m pour le lac Fitri. Les hauteurs d'eau maximales sont observées entre août et septembre pour les deux lacs, tandis que le minimum est atteint entre mai et juin. 


\section{Discussion}

Afin d'avoir une première compréhension du fonctionnement hydrologique des systèmes Iro et Fitri, nous proposons de calculer un rapport Évaporation/Entrée à partir de la composition isotopique des lacs. Ce rapport est généralement utilisé sur des lacs dont la composition isotopique est suivie régulièrement. Mais nous allons montrer qu'il peut être utilisé en première approximation d'un bilan hydrologique à partir d'un point de fin de saison sèche. Puis, pour plus de précision, nous nous rapprocherons d'une composition isotopique moyenne annuelle à partir de l'estimation des variations de volume du lac obtenues par les données d'observation satellitaires. Cette méthode a été testée et validée au préalable sur un lac tropical (Ihotry, Madagascar) (Poulin et al., 2018).

\section{Estimation du bilan hydrologique des lacs}

Le bilan hydrologique d'un lac homogène à l'état stationnaire est donné par :

$$
\frac{\text { Evaporation }}{\text { Entrée }}=\frac{\partial_{I}-\partial_{L}}{\partial_{E}-\partial_{L}}
$$

Équation 1

Avec «Entrée »: le flux entrant dans le lac (de surface et/ou souterrain) et « Évaporation » : le flux évaporatoire ; $\partial_{\mathrm{I}}$ et $\partial_{\mathrm{L}}$ sont les compositions isotopiques respectives du flux entrant et du lac, et peuvent être mesurées (au moins pour les flux de surface). $\partial_{\mathrm{E}}$ est la composition isotopique de l'eau évaporée à partir du lac. Ce flux est appauvri en isotopes lourds par rapport au lac et dépend principalement du fractionnement cinétique. Difficilement mesurable, il est classiquement calculé d'après le modèle de Craig et Gordon (C\&G) (CRAIG et GordOn, 1965).

$$
\partial_{E}=\frac{\frac{\partial_{L}-\varepsilon^{*}}{\alpha}-h \partial a-\varepsilon_{k}}{1-h+\varepsilon_{k}}
$$

Équation 2

$\partial_{\mathrm{E}}$ est ainsi exprimé en fonction des facteurs de fractionnements à l'équilibre $\left(\alpha, \varepsilon^{*}\right)$ ou cinétique $\left(\varepsilon_{\mathrm{K}}\right)$, déjà discutés par de nombreux auteurs (e.g. MAJOUBE, 1971 ; Merlivat, 1978 ; Gonfiantini, 1986 ; Horita et WeSOlOWSKI, 1994 ; GAT, 1996, 1995). L'humidité relative $h$, varie saisonnièrement et est obtenue à partir des stations météorologiques locales. La composition isotopique atmosphérique $\partial \mathrm{a}$ peut être mesurée directement sur le terrain à l'aide de piège cryogénique (FONTES et al., 1970 ; KRABBENHOFT et al., 1990 ; SALAMALIKIS et al., 2015), ou récemment par spectrométrie laser (TREMOY et al., 2012).

Comme ces mesures restent à l'heure actuelle relativement peu répandues, une alternative consiste à supposer en première approximation l'équilibre liquide/ vapeur entre les précipitations et la vapeur d'eau atmosphérique :

$$
\begin{aligned}
& \partial a=\frac{\partial_{P}-\varepsilon^{*}}{\alpha} \text { Où } \partial_{\mathrm{P}} \text { est la composition isotopique des précipitations. } \\
& \partial a \approx \partial_{P}-\varepsilon^{*}
\end{aligned}
$$


Calculé à partir des valeurs d'état stationnaire du système ou à partir de valeurs intégrées sur les variations saisonnières, le rapport Évaporation/Entrée permet de distinguer les lacs "fermés », où l'évaporation est la seule sortie d'eau (Évaporation/Entrée $=1$ ), de ceux qui constituent un réservoir intermédiaire d'un flux d'eau en transit de surface ou souterrain (Évaporation/Entrée $<1$ ). Le dernier cas où Évaporation/Entrée $>1$ correspond à un lac au bilan hydrique négatif, et donc en cours d'assèchement progressif.

Cette approche a été largement utilisée dans la littérature (ZUBER, 1983 ; GIBSON et al., 1993 ; GiBSON, 2002 ; MAYR et al., 2007 ; Yi et al., 2008 ; BROCK et al., 2009), en particulier pour des intercomparaisons des bilans hydriques de réseaux de lacs à l'échelle d'une région. Dans les régions où les contrastes saisonniers entre saisons sèche et humide sont importants, comme pour les lacs tchadiens, il est par contre indispensable de disposer d'une évaluation des valeurs intégrées sur le cycle annuel pour appliquer l'équation 1, et donc d'un suivi d'observation régulier des variations de volume et de composition isotopique du lac, et de mesures sur les flux entrants et sortants.

Néanmoins, nous montrons ci-dessous que, même pour des lacs où le monitoring n'est pas encore envisageable pour des raisons logistiques, une première estimation du bilan hydrique peut être encadrée à partir des mesures isotopiques de saison sèche, et de contraintes obtenues par données satellitaires sur les variations de niveau.

En effet, pour un lac pour lequel les valeurs régionales de $\partial \mathrm{a}$, h et $\partial_{\mathrm{I}}$ peuvent être considérées comme connues ou estimées, il existe une valeur de composition isotopique caractéristique de lac "fermé », $\partial_{\mathrm{L}-c l o s e d}$, obtenue en combinant l'équation 2 avec la condition d'état stationnaire, $\partial_{\mathrm{E}}=\partial_{\mathrm{I}}$ :

$$
\partial_{L-\text { closed }}=\left(\partial_{I} \cdot\left(1-h+\varepsilon_{K}\right)+h \partial a+\varepsilon_{K}\right) \cdot \alpha+\varepsilon^{*} \quad \text { Équation } 4
$$

La composition isotopique mesurée en saison sèche, $\partial_{\mathrm{L}-\text { dry }}$, peut alors être comparée directement à cette valeur de $\partial_{\mathrm{L}-\text { closed }}$. En particulier, pour tout lac tel que $\partial_{\mathrm{L}-\text { dry }}<\partial_{\mathrm{L}-\text { closed }}$ on en déduit que la valeur moyenne annuelle de $\partial_{\mathrm{L}}$ (nécessairement inférieure à $\partial_{L_{d} \text { dry }}$ ), elle aussi inférieure à $\partial_{\text {L-closed, et donc que }}$ ce lac est nécessairement " ouvert » et possède un écoulement de sortie. Une valeur maximale du rapport Évaporation/Entrée peut ainsi être calculée à l'aide de l'équation 1.

Afin d'encadrer plus précisément la valeur de Évaporation/Entrée, on peut également estimer une valeur modèle de $\partial_{\mathrm{L}_{\text {w wet }}}$ correspondant à la composition $\mathrm{du}$ lac en fin de saison humide. De cette manière, il est possible de calculer une valeur de $\partial_{\mathrm{L}}$ approchant une valeur moyenne annuelle : on fait l'hypothèse ici que la moyenne de $\partial_{\mathrm{L}-w e t}$ et $\partial_{\mathrm{L}-\text { dry }}$ est proche de la valeur annuelle moyenne de $\partial_{\mathrm{L}}$. Le cas le plus simple est celui d'un lac fermé, lorsque l'évaporation est suffisamment faible pour être négligée par rapport aux entrées d'eau pendant la saison où le lac se remplit. Dans ce cas : 


$$
\partial_{L-w e t}=y \partial_{I}+(1-y) \partial_{L-d r y}
$$

Équation 5

avec $y=\frac{V_{w e t}-V_{d r y}}{V_{w e t}}$

Où $\mathrm{V}_{\text {wet }}$ représente le volume maximal du lac et $\mathrm{V}_{\text {dry }}$ le volume de fin de saison sèche.

\section{Bilan hydrologique des lacs Iro et Fitri}

Cette démarche a été appliquée pour les lacs Iro et Fitri, dont les résultats sont illustrés sur la figure 7. En fonction des différentes hypothèses possibles pour les valeurs des paramètres $\partial_{\mathrm{I}}$ (nous faisons l'hypothèse ici que $\partial_{\mathrm{I}}=\partial_{\mathrm{P}}$ pris comme étant la valeur du point d'intersection entre la DMM et la droite d'évaporation de Iro ; fig. 7) et da (données de TREMOY et al., 2012 pouvant être extrapolées au Sahel), les valeurs de $\partial_{\mathrm{L}-c l o s e d}$ peuvent varier entre $+3 \%$ et $+6 \%$ en $\partial^{18} \mathrm{O}$ et entre $+4 \%$ et $+28 \%$ en $\partial^{2} \mathrm{H}$ pour le lac Iro. Pour le lac Fitri on obtient une gamme de valeurs comprises entre $+5 \%$ et $+7 \%$ en $\partial^{18} \mathrm{O}$ et entre $+13 \%$ et $+33 \%$ en $\partial^{2} \mathrm{H}$. Sur la figure 7 une valeur moyenne de $\partial_{\mathrm{L} \text {-closed }}$ est représentée pour chacun des lacs. Ces valeurs sont, dans les deux cas, significativement supérieures à nos mesures de saison sèche sur ces deux lacs. On est donc ici dans la situation où $\partial_{\mathrm{L}-d r y} \leq \partial_{\mathrm{L}-c l o s e d}$, caractéristique de lacs ouverts. Les valeurs maximales de Évaporation/Entrée qui en découlent sont pour Iro Évaporation/ Entrée $=0,6$, calculé à la fois pour $\partial^{18} \mathrm{O}$ et $\partial^{2} \mathrm{H}$. Cette valeur maximale, associée à la valeur de $\Delta \mathrm{V} / \mathrm{V}_{\text {dry }}$ estimée à partir des données satellitaires, permet de calculer la gamme des valeurs de $\partial_{\text {wet }}$ basée sur l'équation 5, en gardant en mémoire qu'il ne s'agit que d'une approximation de premier ordre, basée sur des hypothèses simplificatrices (évaporation négligée). La moyenne arithmétique entre $\partial_{\mathrm{L}-\mathrm{dry}}$ et $\partial_{\mathrm{L} \text {-wet }}$ fournit enfin une valeur moyenne de la composition isotopique du lac, à partir de laquelle on calcule un rapport Évaporation/Entrée $=0,4$.

Pour le lac Fitri, nous trouvons une valeur Évaporation/Entrée $=0,5$ qui se rapproche d'une valeur moyenne puisque l'échantillonnage a eu lieu au milieu de la saison sèche. Ces rapports indiquent une évaporation de l'ordre de $50 \%$ sur le bilan hydrique de sortie des deux lacs, contre $70 \%$ sur le lac Tchad (Bouchez et al., 2016).

Ces chiffres peuvent être comparés aux informations hydrologiques disponibles sur les systèmes étudiés (récapitulatif tabl. 1). Pour Iro, en nous basant sur l'estimation du flux d'évaporation de 1,8 m par an (BOYER et al., 2006), et en faisant l'hypothèse d'une surface moyenne de $350 \mathrm{~km}^{2}$, nous obtenons un flux moyen d'évaporation sur le lac (E) de $5.10^{8} \mathrm{~m}^{3}$. an ${ }^{-1}$. Le flux entrant est compris entre 22 et $41 \mathrm{~m}^{3} \cdot \mathrm{s}^{-1}$ (en enlevant les précipitations directes sur le lac), et le flux de sortie entre $11 \mathrm{~m}^{3} \cdot \mathrm{s}^{-1}$ et $21 \mathrm{~m}^{3} \cdot \mathrm{s}^{-1}$. Ces résultats sont cohérents avec le débit moyen du Bahr Azoum à la station d'Am Timan mesuré entre 1953 et 1975. Avec les données disponibles actuellement, il n'est possible ni de déterminer la part du souterrain dans les flux d'entrées ni la part infiltrée. Pour cela, un suivi 


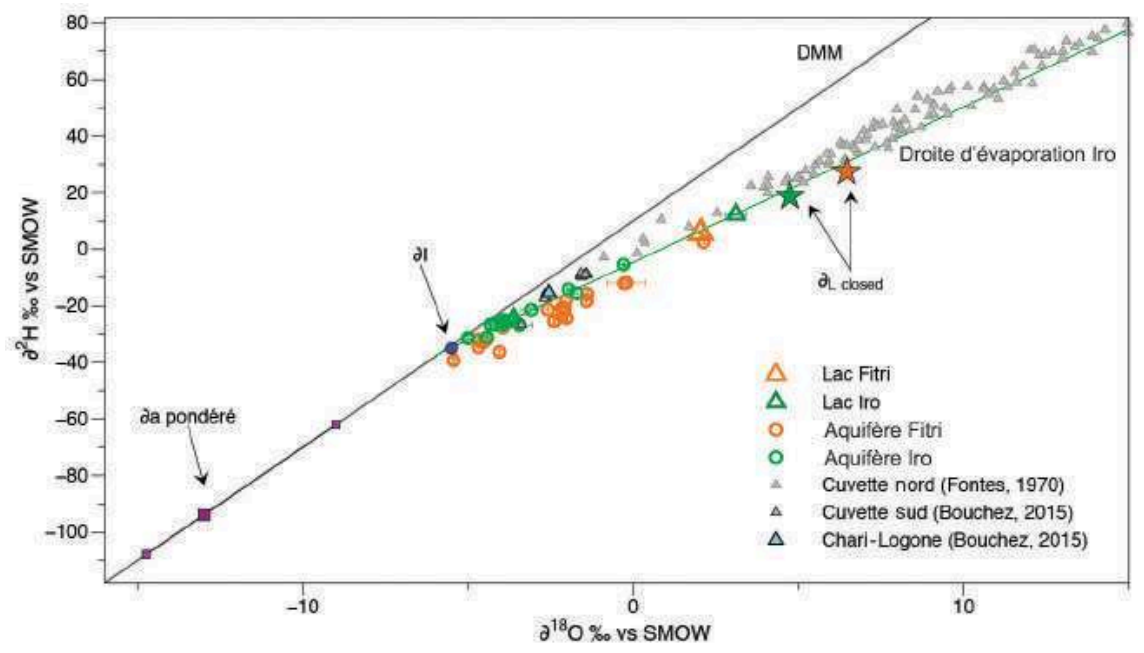

Figure 7

Résultats théoriques obtenus pour les lacs Iro et Fitri. Les étoiles vertes et orange correspondent aux valeurs de $\partial_{\text {Lclosed }}$ en fonction de $\partial a, h$, et $\partial_{p}$ pour les lac Iro et Fitri respectivement.

On observe que dans les deux cas, $\partial_{\text {Lclosed }}$ est supérieur ou égal à la composition isotopique des lacs en fin et en milieu de saison sèche, démontrant ainsi l'ouverture de ces lacs avec un flux de sortie en surface ou souterrain.

Tableau 1

Valeurs des flux d'entrée et de sortie obtenues pour les lacs Iro et Fitri.

$\begin{array}{llll} & \text { Évaporation m/an } & \mathbf{Q}_{\text {entrant }} \mathbf{m}^{3} / \mathbf{s} & \mathbf{Q}_{\text {sortant }} \mathbf{m}^{3} / \mathbf{s} \\ \text { Iro } & \mathrm{I}, 8 & 22<\mathrm{Q}_{\text {entrant }}<4 I & 1 \mid<\mathrm{Q}_{\text {sortant }}<2 \mathrm{I} \\ \text { Fitri } & 2,0 & 43<\mathrm{Q}_{\text {entrant }}<110 & 14<\mathrm{Q}_{\text {sortant }}<36\end{array}$

isotopique mensuel du lac et de l'aquifère est indispensable ainsi qu'un relevé mensuel du débit de la rivière.

Pour le lac Fitri, en prenant une évaporation d'environ $2 \mathrm{~m} \cdot \mathrm{an}^{-1}$ (similaire à celle utilisée sur le lac Tchad [Bouchez et al., 2016]), on obtient un flux de l'ordre de $16.10^{8} \mathrm{~m}^{3} \cdot \mathrm{an}^{-1}$, un flux entrant entre 43 et $110 \mathrm{~m}^{3} \cdot \mathrm{s}^{-1}$. Le débit moyen annuel maximum enregistré à la station d'Ati entre 1956 et 1993 est de $66 \mathrm{~m}^{3} \cdot \mathrm{s}^{-1}$ et correspond aux pluies records ( $571 \mathrm{~mm}$ par an) enregistrées à cette même station la même année (Drem). A contrario, le Batha n'a pas été fonctionnel pendant les années 1980. Cependant, les débits enregistrés à Ati ne sont pas représentatifs du total des flux entrants dans le lac, car une multitude de petites rivières difficilement quantifiables alimentent le lac, contribuant ainsi aux flux calculés. Les flux de sorties sont compris entre 14 et $36 \mathrm{~m}^{3} . \mathrm{s}^{-1}$ et en l'absence d'exutoire de surface visible, viennent probablement alimenter la partie ouest de l'aquifère. En effet, nous avons vu que dans la partie est du lac, où l'échantillonnage des eaux souterraines a été réalisé, la composition isotopique des eaux souterraines 
et des eaux de surface forment deux droites d'évaporations distinctes : les eaux n'ont pas la même signature isotopique signifiant une déconnexion entre l'aquifère et le lac pour cette partie est du système hydrologique.

\section{Conclusion}

Notre étude illustre la possibilité d'obtenir une évaluation semi-quantitative des flux hydrologiques sur des lacs peu documentés en milieu intertropical à partir de données ponctuelles de fin de saison sèche, couplées à des données satellitaires pour l'estimation des fluctuations lacustres.

Les résultats obtenus montrent le caractère « ouvert» des lacs Iro et Fitri comme le laissait penser la faible salinité de ces lacs. Le rapport E/I de 0,4 et 0,5 obtenu respectivement sur ces deux lacs a permis d'une part de quantifier un flux d'entrée $\left(22<\mathrm{I}_{\text {Iro }}<41 \mathrm{~m}^{3}\right.$. $\mathrm{s}^{-1}$ et $43<\mathrm{I}_{\text {Fitri }}<110 \mathrm{~m}^{3}$. $\left.\mathrm{s}^{-1}\right)$ mais également un flux de sortie $\left(11<\mathrm{Q}_{\text {Iro }}<21 \mathrm{~m}^{3} \cdot \mathrm{s}^{-1}\right.$ et $\left.14<\mathrm{Q}_{\text {Fitri }}<36 \mathrm{~m}^{3} \cdot \mathrm{s}^{-1}\right)$. Il est néanmoins difficile, avec les données actuelles, de déterminer s'il existe une sortie vers le souterrain pour le lac Iro. En revanche les flux de sortie pour le lac Fitri, endoréique, se font nécessairement en direction de l'aquifère, dans sa partie ouest suivant l'écoulement régional : les données isotopiques de la partie est ont montré une déconnexion avec le lac.

Ce travail préliminaire doit être complété par une étude de terrain plus approfondie : un suivi mensuel du niveau des lacs et des mesures de débits sur les rivières sont indispensables pour aller plus loin dans l'étude de ces systèmes lacustres. Il en va de même pour les aquifères où un suivi piézométrique doit être réalisé pour étudier les écoulements régionaux mais aussi les fluctuations saisonnières de la nappe.

\section{Bibliographie}

Billon B., Guiscafre J., Herbaud J., OBerlin G., 1974

« Le bassin du fleuve Chari ». Monographies

Hydrologiques $\mathrm{n}^{\circ} 2$, Orstom éditions : 441-450.

Bouchez C., Goncalves J., Deschamps P., VAllet-Coulomb C., Hamelin B., Doumnang J.-C., Sylvestre F., 2016

Hydrological, Chemical, and Isotopic Budgets of Lake Chad: A Quantitative Assessment of Evaporation, Transpiration and Infiltration Fluxes. Hydrology and Earth System Sciences. 20 (4) : 1599-1619.

Boyer J. F., Dieulin C., Rouche N., Cres A., Servat E., Paturel J. E., Mahe G., 2006 SIEREM an Environnmental Information System for Water Resources. In Climate Variability 
and Change-Hydrological Impacts, IAHS Publ., 308 edition, 5th World Friend Conference, La Havana, Cuba, 2006.

Brock Bronwyn E., Yi Y., Clogg-Wright K. P., EdWards T. W. D., WOLFE B. B., 2009

Multi-Year Landscape-Scale Assessment of Lakewater Balances in the Slave River Delta, NWT, Using Water Isotope Tracers. Journal of Hydrology, 379 (1-2) : 81-91.

\section{Craig H., Gordon L. I., 1965}

« Stable Isotopes in Oceanographic Studies and Paleotemperatures », Edited by E. Tongiorgi, (V. Lischi e Figli, Pisa, 1965), 9-130.

Cretaux J.-F., Abarca-del-Rio R., Berge-Nguyen M., Arsen A., Drolon V., Clos G., Maisongrande P., 2016 Lake Volume Monitoring from Space. Surveys in Geophysics 37 (2) : 269-305.

Defrance D., Ramstein G., Charbit S., Vrac M., Moïse Famien A., Sultan B., Swingedoum D., Dumas C., Gemenne F., Alvarez-Solas J., Vanderlinden J. P., 2017 Consequences of Rapid Ice Sheet Melting on the Sahelian Population Vulnerability. Proceedings of the National Academy of Sciences, 114 (25) : 6533-38.

\section{DRUYAN L. M., 2011}

Studies of 21st-Century Precipitation Trends over West Africa. International Journal of Climatology, 31 (10) : 1415-1424.

\section{Fontes J. C., Gonfiantini R., Roche M. A., 1970}

Deutérium et Oxygène-18 dans les eaux du lac Tchad. Isotopes in Hydrology, IAEA-SM-129/23, p 387-404.

\section{GAT J. R., 1996}

Oxygen and Hydrogen Isotopes

in the Hydrologic Cycle. Annual Review of Earth and Planetary Sciences, 24 (1) : 225-262.

\section{GAT J. R., 1995}

Stable Isotopes of Fresh and Saline Lakes. In : Physics and Chemistry of Lakes, 139-165. Springer.

\section{GibSON J. J., 2002}

Short-Term Evaporation and Water Budget Comparisons in Shallow Arctic Lakes Using Non-Steady Isotope Mass Balance. Journal of Hydrology, 264 (1) : 242-261.
Gibson J. J., Edwards T. W. D., 2002

Regional Water Balance Trends and EvaporationTranspiration Partitioning from a Stable Isotope Survey of Lakes in Northern Canada: regional water balance using stable isotopes. Global Biogeochemical Cycles, 16 (2) : 10-1-10-14.

Gibson J. J., Edwards T. W. D., Bursey G. G., Prowse T. D., 1993

Estimating Evaporation Using Stable Isotopes: Quantitative Results and Sensitivity Analysis for Two Catchments in Northern Canada. Hydrology Research, 24 (2-3) : 79-94.

\section{GONFIANTINI R., 1986}

Environmental Isotopes in Lake Studies. Handbook of Environmental Isotope Geochemistry. Amsterdam, The Netherlands.

\section{Horita J., Wesolowski D. J., 1994}

Liquid-Vapor Fractionation of Oxygen and Hydrogen Isotopes of Water from the Freezing to the Critical Temperature. Geochimica et Cosmochimica Acta, 58 (16) : 3425-3437.

IAEA, 2009

Reference Sheet for VSMOW2 and SLAP2 International Measurement Standards. Issued 2009-02-13, International Atomic Energy Agency, Vienna, 5 p., edition. http://curem.iaea. org/catalogue/SI/pdf/VSMOW2_SLAP2.pdf.

IAEA WMO, 2017

Global Network of Isotopes in Precipitation. The GNIP Database.2017 : http://Www.Iaea.Org/ Water.

Krabbenhoft D. P., Bowser C. J., Anderson M. P., VAlley J. W., 1990 Estimating Groundwater Exchange with Lakes: 1. The Stable Isotope Mass Balance Method. Water Resources Research, 26 (10) : 2445-53.

\section{MaJoube M., 1971}

Fractionnement en Oxygene-18 et en Deuterium entre l'eau et sa vapeur. Journal de Chimie Physique 68 (10) : 1423-1436.

\section{Mayr C., Lücke A., Stichler W., Trimborn P., Ercolano B., Olivia G., Ohlendorf C., Soto J., Fey M., Haberzettl T., Janssen S., Schabitz F., Schleser G. H., Wille M., ZolitschKa B., 2007}

Precipitation origin and evaporation of lakes in semi-arid Patagonia (Argentina) inferred 
from stable isotopes $\left(\Delta^{18} \mathrm{O}, \Delta^{2} \mathrm{H}\right)$. Journal of Hydrology, 334 (1-2) : 53-63.

\section{Merlivat L., 1978}

Molecular Diffusivities of H2 16O, HD16O, and H2 $18 \mathrm{O}$ in Gases. Journal of Chemical Physics, 69 : 2864-2871.

\section{NichOLSON S. E., 2013}

The West African Sahel: A Review of Recent Studies on the Rainfall Regime and Its Interannual Variability. International Scholarly Research Notices Meteorology, 2013 : 1-32.

Poulin C., Hamelin B., Vallet-Coulomb C., Amngar G., Loukman B., Cretaux J. F., Doumnang J.-C., Mahamat Nour A., Menot G., Sylvestre F., Deschamps P., 2018 Unraveling the Hydrological Budget of Isolated and Seasonally Contrasted Sub-Tropical Lakes. Hydrology and Earth System Science.

Discussion. 1-32.

\section{RoCHE A., 1980}

Hydrogéologie des côtes du lac Tchad à No, Tchingam et Soro (Kanem). Orstom, coll. Travaux et documents, 89-116.

\section{Salamalikis V., Argiriou A. A., Dotsika E.,} 2015

Stable Isotopic Composition of Atmospheric Water Vapor in Patras, Greece: A Concentration Weighted Trajectory Approach. Atmospheric Research, Atmospheric Processes in the Mediterranean, 152 : 93-104.

\section{SCHNEIDER J. L., 2004}

Géologie, Archéologie, Hydrogéologie de la République du Tchad. 2 volumes, 693 p.

Sivapalan M., Takeuchi K., Franks S. W., GuPTA V. K., Karambiri H., LAKShMi V., Liang X., McDonnel J. J., Mendiondo E. M., O'Connel P. E., OKi T., Pomeroy J. W., Schertzer D., Uhlenbrook S., Zehe E., 2003 IAHS Decade on Predictions in Ungauged Basins (PUB), 2003-2012: Shaping an Exciting Future for the Hydrological Sciences.

Hydrological Sciences Journal 48 (6) : 857-80.

Tremoy G., Vimeux F., Mayaki S., Souley I., Cattani O., Risi C., Favreau G., Oi M., 2012 A 1-Year Long $\delta^{18} \mathrm{O}$ record of water vapor in Niamey (Niger) reveals insightful atmospheric processes at different timescale. Geophysical Research Letters 39 (8) : L08805.

KandJi S. T., Mackensen J., Verchot L., 2006 Climate Change and Variability in the Sahel Region : Impacts and Adaptation Strategies in the Agricultural Sector. Unep, Icraf, 58 p.

Yi Y., Brock B. E., Falcone M. D., Wolfe B. B., Edwards T. W. D., 2008 A Coupled Isotope Tracer Method to Characterize Input Water to Lakes. Journal of Hydrology, 350 (1-2) : 1-13.

\section{ZUBER A., 1983}

« On the Environmental Isotope Method for Determining the Water Balance Components of Some Lakes ». Journal of Hydrology 61 (4) : 409-27. 


\section{aractérisation des paramètres} physicochimiques des eaux souterraines de la région du lac Iro

Guinbé AMNGAR, Abderamane HAMIT

\section{Introduction}

Dans les bassins hydrogéologiques tchadiens, d'importantes ressources en eaux souterraines sont exploitables. Les ressources renouvelables annuellement sont estimées à environ 20 milliards de $\mathrm{m}^{3}$ (BRGM, 1987). Ces ressources ont de multiples fonctions et usages. Si les eaux de surface sont essentielles pour la préservation de la biodiversité, pour l'agriculture, la pêche et l'élevage, les eaux souterraines constituent une ressource qui, du fait de sa plus forte résilience à la variabilité climatique, est d'une grande importance durant les périodes de sécheresse. Cette ressource en eau souterraine est localisée soit dans des grands systèmes aquifères sédimentaires continus couvrant $75 \%$ du territoire, soit dans des aquifères discontinus localisés dans les formations superficielles latéritiques ou de socle (Ministère de L'EnVironnement et DEs EAUX DU TCHAD, 2003 ; SCHNEIDER, 2001a et 2001b).

La mobilisation de la ressource stockée dans ces aquifères discontinus, qui sont caractérisés par de fortes hétérogénéités spatiales et une variabilité temporelle des processus de recharge, est souvent problématique. En atteste le faible pourcentage de réussite des forages obtenus par les programmes d'hydraulique villageoise en zone de socle ou latéritique (VINCENT, 2010). Mobiliser ces ressources exige au préalable des études hydrologiques, géochimiques et géophysiques permettant une meilleure connaissance de leur fonctionnement et des processus de recharge. 
L'objectif de cet article est d'étudier le fonctionnement du système aquifère dans la zone du lac Iro et sa région, qui se trouve confrontée localement à d'énormes difficultés d'accessibilité aux eaux souterraines. Il s'agira en particulier de décrire le système hydraulique de son bassin versant et de caractériser les eaux de l'aquifère en effectuant des analyses hydrogéochimiques.

\section{Site d'étude}

La région du lac Iro s'étend entre $9^{\circ} 33^{\prime}$ et $10^{\circ} 31^{\prime}$ ' de latitude Nord et entre $18^{\circ} 20^{\prime}$ et $19^{\circ} 40^{\prime}$ de longitude Est, couvrant une superficie d'environ 8590 km ${ }^{2}$ avec 119085 habitants (RGPH2, 2009 ; fig. 1). L'économie de la région est principalement basée sur des activités agropastorales. Cette région est soumise au climat de types sahélien et soudanien, recevant des précipitations comprises entre 600 et $1300 \mathrm{~mm}$ par an. Le climat est caractérisé par deux saisons nettement différenciées : une saison sèche qui s'étend de novembre à mars, suivie d'une saison des pluies (BILlon et GuISCAFre, 1969). Ces variations climatiques

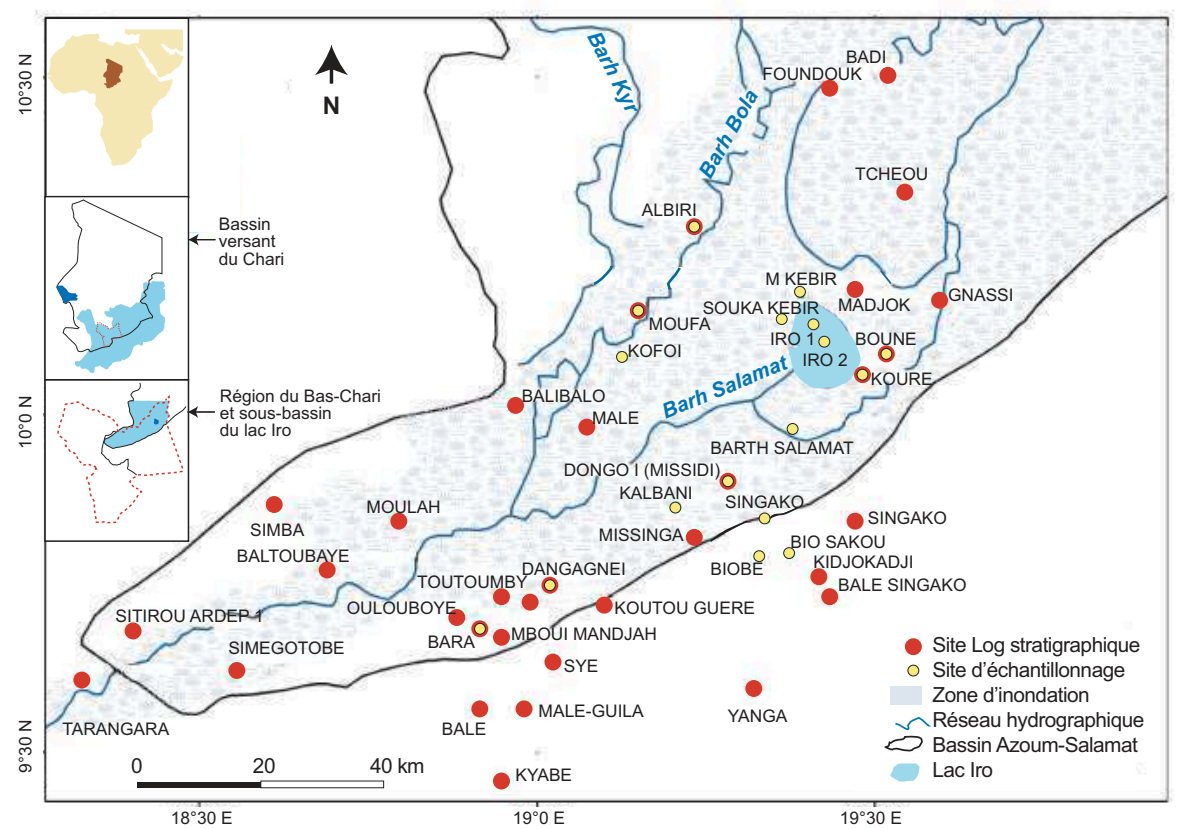

Figure I

Localisation de la zone d'étude.

Source : BERTHELOT, 1958.

Réalisation : Guinbé Amngar, septembre 2018. 
s'expliquent essentiellement par les déplacements saisonniers de la zone de convergence intertropicale. Pour la période comprise entre 1940 et 1964, la quantité de précipitations annuelle a été estimée à $1011 \mathrm{~mm}$ (BILLON et GUISCAFU, 1969), alors que de 1984 à 2014, elle a été de 943,5 mm (Source : Services d'exploitation et des applications météorologiques du Tchad, 2015) enregistrant une diminution de $67,5 \mathrm{~mm}$, soit $6,7 \%$.

Les mesures de l'évaporation avec la méthode Piche varient entre 1700 à $2000 \mathrm{~mm}$ avec une forte valeur annuelle moyenne de $1840,2 \mathrm{~mm}$ (Source : Services d'exploitation et des applications météorologiques du Tchad, 2015). La température moyenne mensuelle observée est de $28,3^{\circ} \mathrm{C}$ avec une température maximale variant de $31{ }^{\circ} \mathrm{C}$ au mois d'août à $39,1{ }^{\circ} \mathrm{C}$ au mois de mars et une température minimale de $25,6^{\circ} \mathrm{C}$ au mois d'avril à $16,7^{\circ} \mathrm{C}$ au mois de décembre. Les humidités relatives maximales varient de 45,3\% en février à $84,7 \%$ en août-septembre et les minimales de $11,2 \%$ en février à $61,5 \%$ en août.

\section{Contexte géologique et hydrogéologique}

La zone d'étude repose sur le socle précambrien composé de granit calcoalcalin, de gneiss, de schistes et de quartzites (LouIs, 1970). Ce socle affleure sous la forme de pointements rocheux $(470$ à $700 \mathrm{~m})$ et d'inselbergs plus ou moins érodés. Le socle est recouvert par des formations sédimentaires, non métamorphisées, d'âge s'étendant du Tertiaire au Quaternaire (BILloN et GUISCAFRE, 1969). En effet, le socle précambrien est recouvert d'abord par des formations sédimentaires continentales de nature sableuse à gréseuse avec la présence de cuirasses latéritiques. Ce sont des dépôts fluviolacustres correspondant aux séries inférieures et moyennes des " grès et sables paléotchadiens »(WACRENIER, 1953). Les formations quaternaires viennent recouvrir une partie de ces formations et se prolongent par les formations du sous-bassin Keïta (SCHNEIDER et WOLF, 1992a et 1992b). Ces formations correspondent à une série de dépôts fluviolacustres argilo-sableux à nodules calcaires avec localement la présence de cuirasses ferrugineuses subactuelles à actuelles, observées sur le pourtour du lac Iro. La plaine d'inondation (zone marécageuse) présente une très légère pente vers le sud-ouest $\left(5 \times 10^{-4}\right.$ et $\left.3 \times 10^{-4}\right)$ et n'a pour tout relief que les cordons sableux. L'ensemble de la plaine est inondé en saison des pluies sous des hauteurs d'eau variables, en moyenne de 0,80 à $1,20 \mathrm{~m}$.

Le socle cristallin affleure directement ou apparaît peu profond sous une couverture d'étendues sablo-argileuses. Les ressources en eau souterraine sont très limitées et liées aux fractures ouvertes du socle et à la frange d'altération qui résulte de la décomposition des roches sous l'action des agents climatiques, surtout à l'aplomb des fractures du socle. Une zone de transition «biseau sec » 
qui relie en pente douce le piémont des inselbergs sous un recouvrement argilosableux est peu propice à la recharge bien qu'elle jouisse des précipitations et des eaux d'inondation. Les formations du Continental terminal recèlent de puissantes couches sableuses avec une capacité de réserves exploitables estimées entre 72,5 et $145 \mathrm{Gm}^{3}$ (BRGM, 1987). Ces ressources sont renouvelables : la lame d'eau infiltrée moyenne théorique est estimée à $83 \mathrm{~mm}$ et le volume renouvelable chaque année à $13000 \mathrm{~mm}^{3}$ (BRGM, 1987). Une nappe phréatique générale est drainée dans les fonds de vallées par les cours d'eau (SCHNEIDER, 2001). La nappe phréatique du Quaternaire est peu profonde, à moins de $10 \mathrm{~m}$ en saison sèche. Elle est alimentée par les précipitations et principalement par les eaux du Barh Azoum-Salamat qui inonde l'ensemble de la dépression (PiAS, 1970a et 1970b). Les nappes alluviales des vallées principales ont la puissance des alluvions qui peut atteindre 10 à $30 \mathrm{~m}$. Ces nappes sous-fluviales sont réalimentées lorsque le lit mineur de la vallée réussit à traverser la couche limoneuse superficielle ou si, à l'occasion de fortes crues, l'eau de surface inonde l'ensemble de la vallée et réussit à s'infiltrer sur ses bordures.

\section{Matériels et méthodes}

Les mesures et les prélèvements sur le terrain ont été effectués du 4 au 12 avril 2015 sur les différents sites d'étude dans le cadre du programme Gelt. Dix-huit échantillons ont été prélevés dans l'aquifère quaternaire (notés IRO-AQ dans le tableau 1, dans le lac (IRO-Lac) et la rivière (IRO-Riv) pour l'analyse des éléments majeurs (anions et cations).

La filtration et les différentes fractions aliquotes des éléments majeurs et l'élément trace le strontium $\left(\mathrm{Sr}^{2+}\right)$ ont été effectuées au laboratoire Cerege en France. L'analyse chimique des eaux a été réalisée par chromatographie ionique (modèle de la machine ICS1000 Dionex) au laboratoire $\mathrm{HSM}^{1}$ en France. Elle n'est représentative que lorsque la balance ionique est inférieure ou égale à $7 \%$. La température, le $\mathrm{pH}$, la conductivité électrique $\left(\mathrm{EC}\right.$ à $25^{\circ} \mathrm{C}$ ) et le potentiel redox (Eh) ont été mesurés sur le terrain au moment des prélèvements. Les mesures de l'alcalinité ont été effectuées par titration des espèces carbonatées $\left(\mathrm{HCO}_{3}{ }^{-} ; \mathrm{CO}_{3}{ }^{2-}\right)$ par un acide fort $\left(\mathrm{H}_{2} \mathrm{SO}_{4}\right)$, après ajout de l'indicateur coloré (vert de bromocrésol).

Les unités hydrogéologiques (zone de socle et zone sédimentaire) sont identifiées et caractérisées. Il y a eu quelques relevés piézométriques des forages et des puits existants. Nous avons également enrichi notre étude avec la base de données Siteau (Système d'information tchadien sur l'eau) du ministère chargé de l'eau au Tchad comprenant des données météorologiques et hydrologiques. 


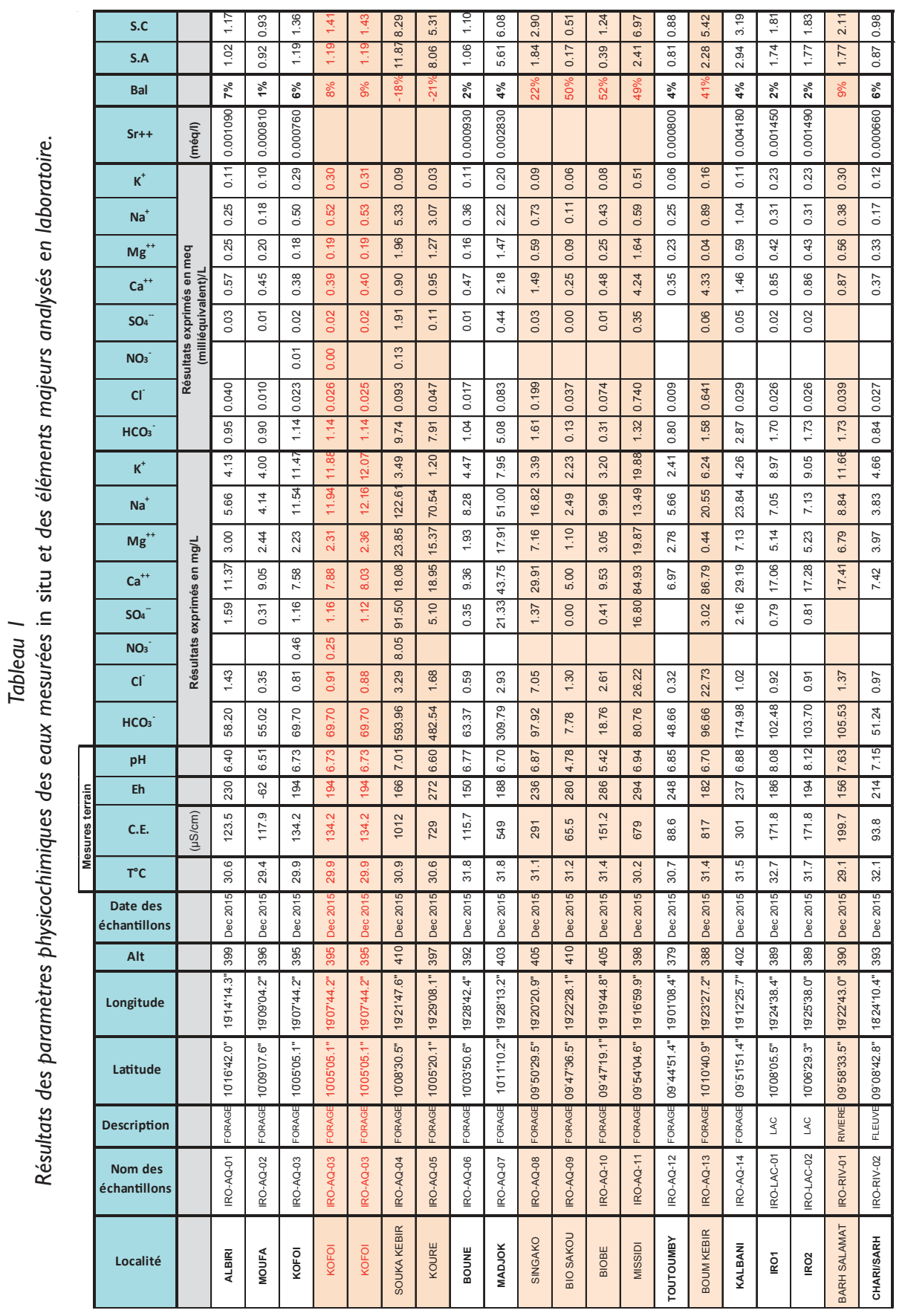


De plus, nous nous sommes servis de la description lithologique des « cuttings » prélevés tous les mètres des sédiments traversés par les forages réalisés en 2010 dans le cadre du Programme d'hydraulique villageoise dans le Salamat et le lac Iro (PHVSLI), de leurs données des essais de débit et des connaissances géologiques et hydrologiques existantes (fig. $2 \mathrm{a}$ et $2 \mathrm{~b}$ ). Ces observations ont permis une reconstitution des successions lithologiques des unités hydrogéologiques (mise en évidence du socle cristallin et ses dépôts continentaux) et de déterminer quelques caractéristiques hydrodynamiques. À défaut d'un altimètre permettant d'avoir les altitudes réelles des points d'eau, nous avons utilisé des images SRTM (année 2001 à une résolution de $90 \mathrm{~m}$ ) pour extraire les cotes du sol (Z) des différents points d'ouvrage par le logiciel ArcGIS10 et en déduire les cotes piézométriques. Les valeurs de celles-ci ont été calculées par le logiciel Surfer en utilisant une méthode de krigeage pour en déduire les courbes hydro-isohypses et la vue 3D du bassin hydrogéologique.

Enfin, les cartes de distribution de teneur en ions ont été réalisées à l'aide des résultats d'analyse chimique des éléments majeurs à balance ionique inférieure à $7 \%$ avec le logiciel libre Quantum GIS.

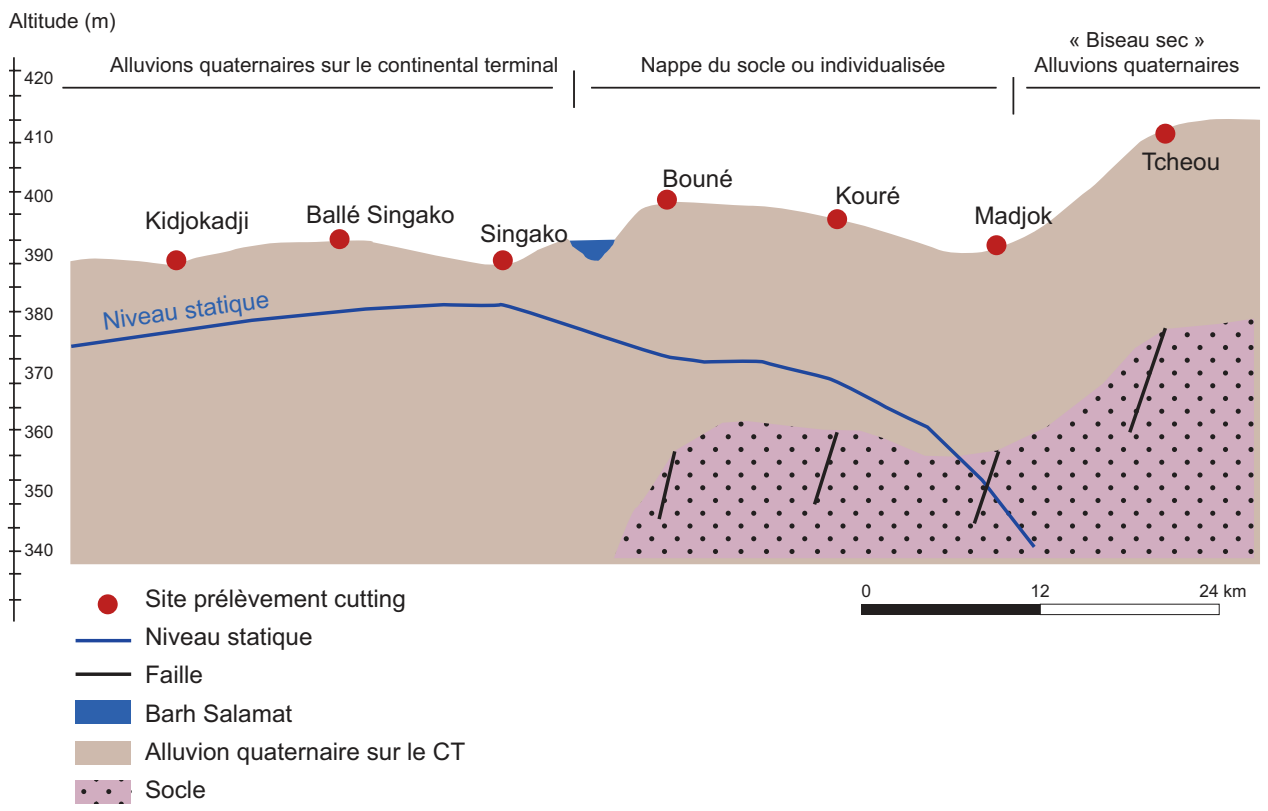

Figure $2 a$

Log stratigraphique des unités hydrogéologiques.

Source : Base de données Siteau, 2014.

Réalisation : Guinbé Amngar, septembre 2015. 
PROFIL SUD-OUEST ET NORD-EST

Est

Ouest / Sud-Ouest

Nord-Est

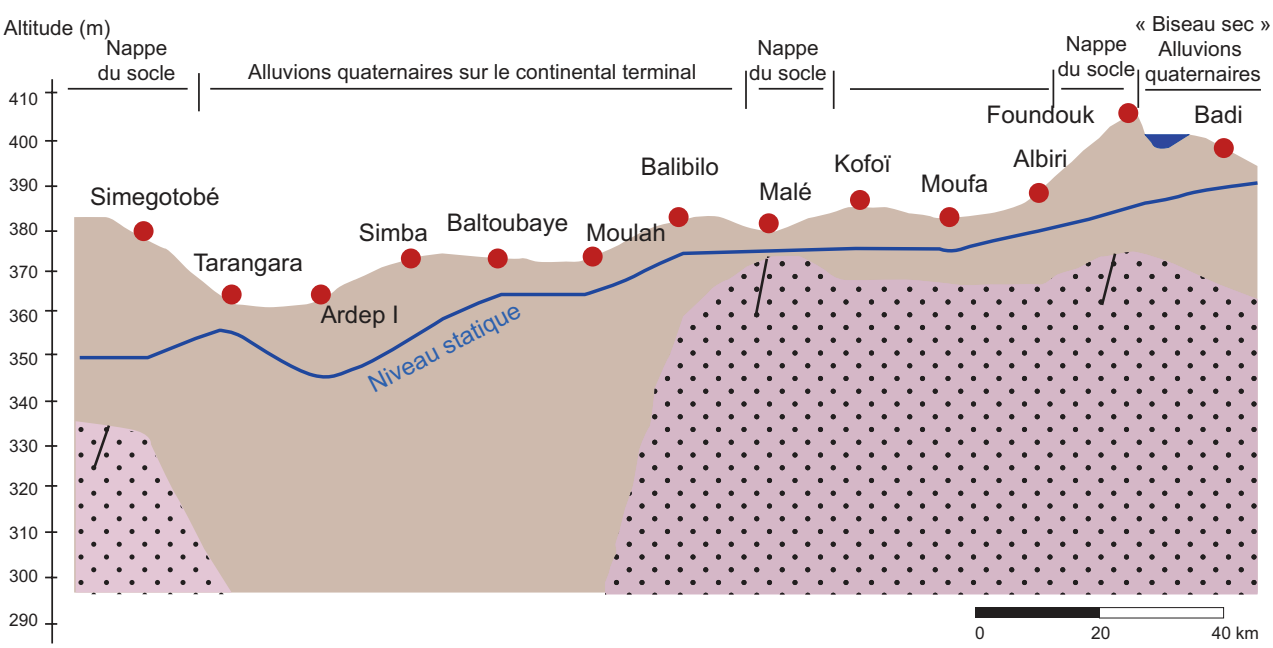

Site prélèvement cutting

Niveau statique

Faille

Barh Bola

Alluvion quaternaire sur le CT

$\therefore$ Socle

\section{Figure $\mathbf{2 b}$}

Log stratigraphique des unités hydrogéologiques.

Source : Base de données Siteau, 2014.

Réalisation : Guinbé Amngar, septembre 2015.

\section{Résultats}

\section{Unités hydrogéologiques et caractéristiques hydrauliques des nappes}

Les coupes lithologiques relevées dans les différents sites de la zone ont conduit à l'établissement des différents profils et ont permis la mise en évidence de 3 types de nappes : les nappes généralisées, les nappes alluviales et les nappes individualisées (fig. 3). La profondeur de la surface des nappes généralisées est minimale (7,5 $\mathrm{m}$ à Tarangara/Baltoubaye) dans les plaines alluviales. Elle augmente quand on s'éloigne des vallées en direction du plateau du Continental terminal qui, portant le nom de « Koro » de Kyabé, présente une nappe dépassant 
60 à $80 \mathrm{~m}$ de profondeur (SCHNEIDER, 2001) (117 m à Ballé/Kyabé mais avec un niveau hydrostatique à $380 \mathrm{~m}$ ). Schneider (2001) indique que la productivité dans les formations du Continental terminal montre une forte hétérogénéité lithologique liée aux conditions de sédimentation fluviolacustre. Par contre, les forages de Ballé/Kyabé montrent des écarts plus faibles et certains secteurs sont caractérisés par des perméabilités relativement homogènes mais faibles à très faibles, par exemple à Tarangara et Guilako.

La profondeur de la surface des nappes alluviales varie de 5 à $15 \mathrm{~m}$ dans les zones d'alimentation de bordure. Elle dépasse $25 \mathrm{~m}$ à Biobé/Singako. Par ailleurs, le lac Iro apparaît comme une vaste mare sans rôle notable dans l'alimentation de la nappe : un puits creusé sur sa bordure nord à Boum Kebir montre un niveau d'eau à $26 \mathrm{~m}$. La productivité présente une forte hétérogénéité lithologique à travers les débits spécifiques des forages réalisés. C'est l'exemple des forages de la localité de Moufa/Boum Kebir qui ont chacun un débit spécifique de 11,29 et 22,58 $\mathrm{m}^{3} / \mathrm{h} / \mathrm{m}$. Ceux de Singako ont aussi chacun des débits de 5,81, 20,22 et $24,32 \mathrm{~m}^{3} / \mathrm{h} / \mathrm{m}$. Les débits spécifiques des nappes alluviales des vallées principales se situent entre 8,50 à $14,50 \mathrm{~m}^{3} / \mathrm{h} / \mathrm{m}$.

Pour les nappes du socle, la profondeur des puits est en général de 42 à $55 \mathrm{~m}$ pouvant atteindre $69 \mathrm{~m}$ par exemple à Boum Kebir. La productivité est très faible comprise entre 0,04 à $0,59 \mathrm{~m}^{3} / \mathrm{h} / \mathrm{m}$. La zone « biseau sec » est un espace

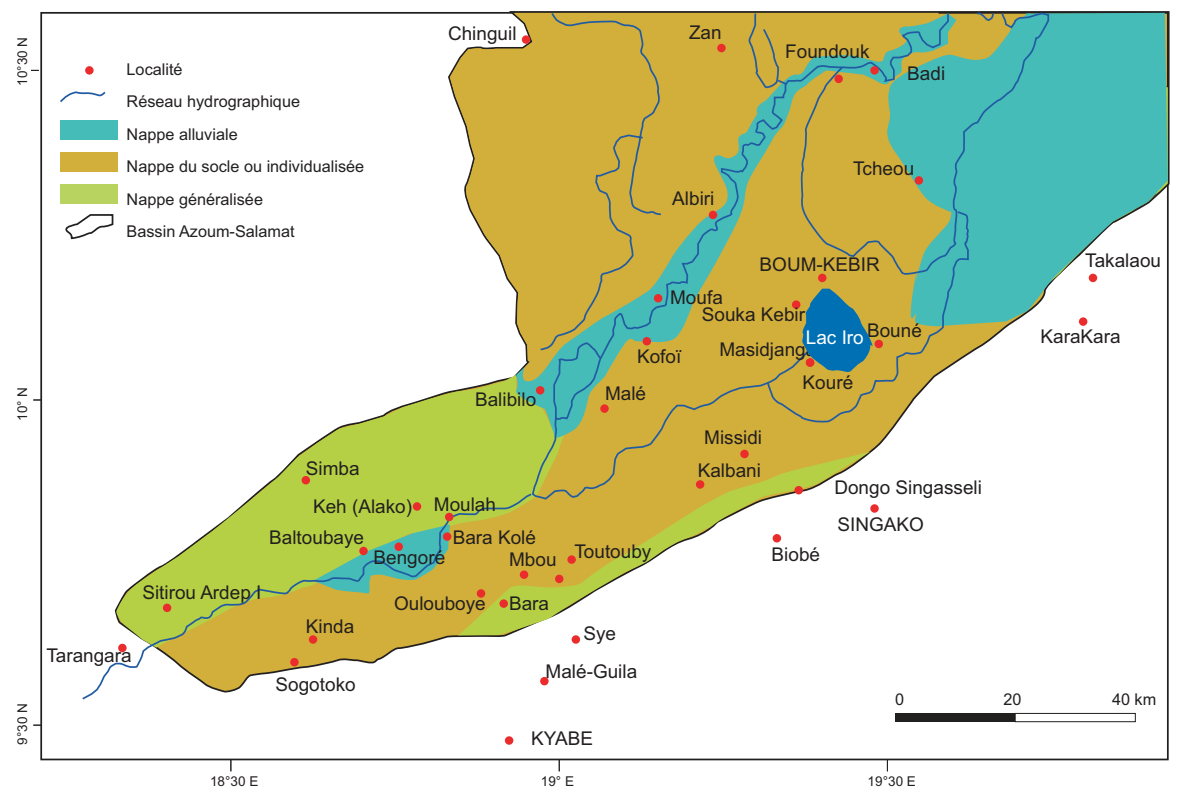

Figure 3

Répartition spatiale des unités hydrogéologiques de la zone d'étude.

Sources : Projet Almy Bahaïm 1995-2009 ; BERTHELOT, 1958.

Réalisation : Guinbé Amngar, juin 2018. 
hydrogéologiquement défavorable où l'eau est à rechercher dans les fissures du socle, parfois dans des petites nappes perchées.

\section{Paramètres hydrodynamiques}

Les niveaux statiques des ouvrages (de 3,56 à 117,03 m) et les cotes du sol extraites des images SRTM (comprises entre 360 et $526 \mathrm{~m})^{2}$ ont conduit à déterminer les cotes piézométriques (comprises entre 348,95 et 435,70 m) permettant d'établir la carte piézométrique (fig. 4). Les différentes variations des altitudes des courbes hydro-isohypses, l'orientation des lignes de courant et la carte en 3D mettent en évidence le sens de l'écoulement des eaux souterraines, les dépressions et les dômes piézométriques (fig. 5). Trois dépressions sont localisées dans la partie exutoire de ce bassin. Ces dépressions se trouvent à Boum Kebir juste au nord du lac Iro, à Missidi/Singako et à Gnassi/ Salamat.

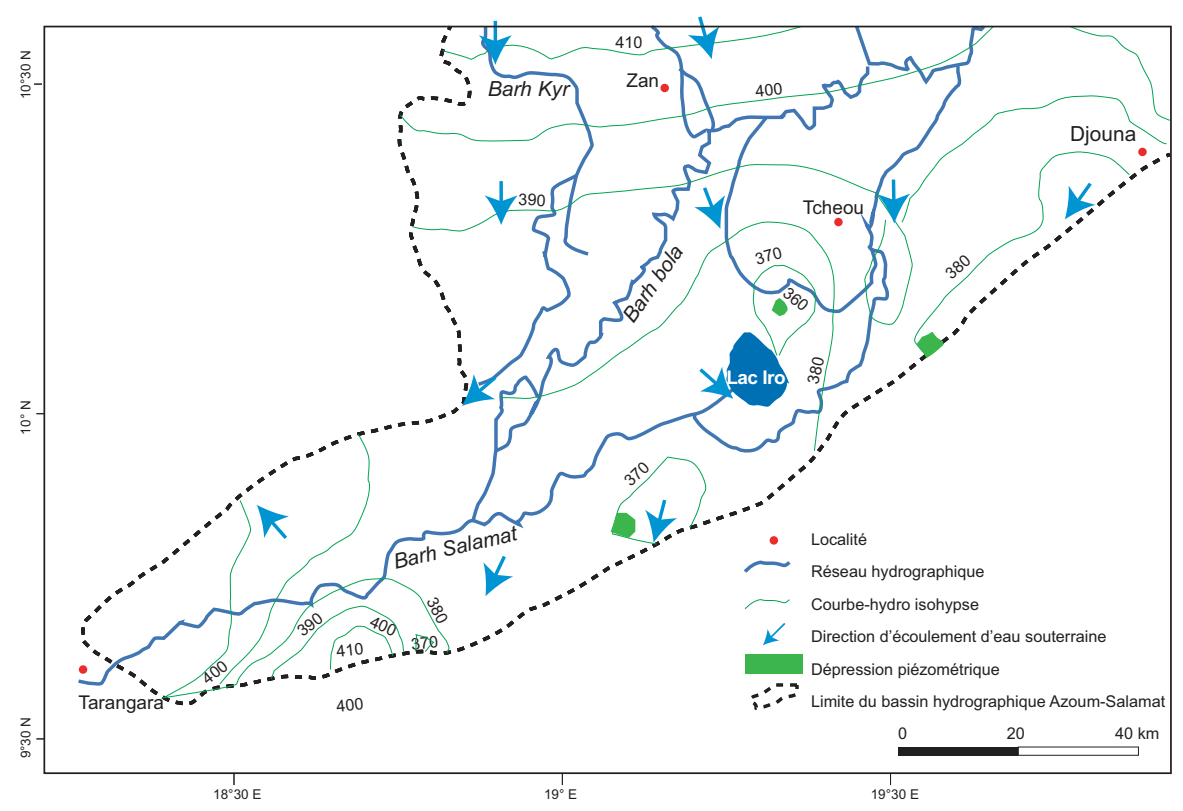

Figure 4

Carte piézométrique autour du lac Iro.

Sources : SRTM 200I, résolution 90 m ; BERTHELOT, 1958 ; base de données Siteau, 2014.

Réalisation : Guinbé Amngar, septembre 2015. 


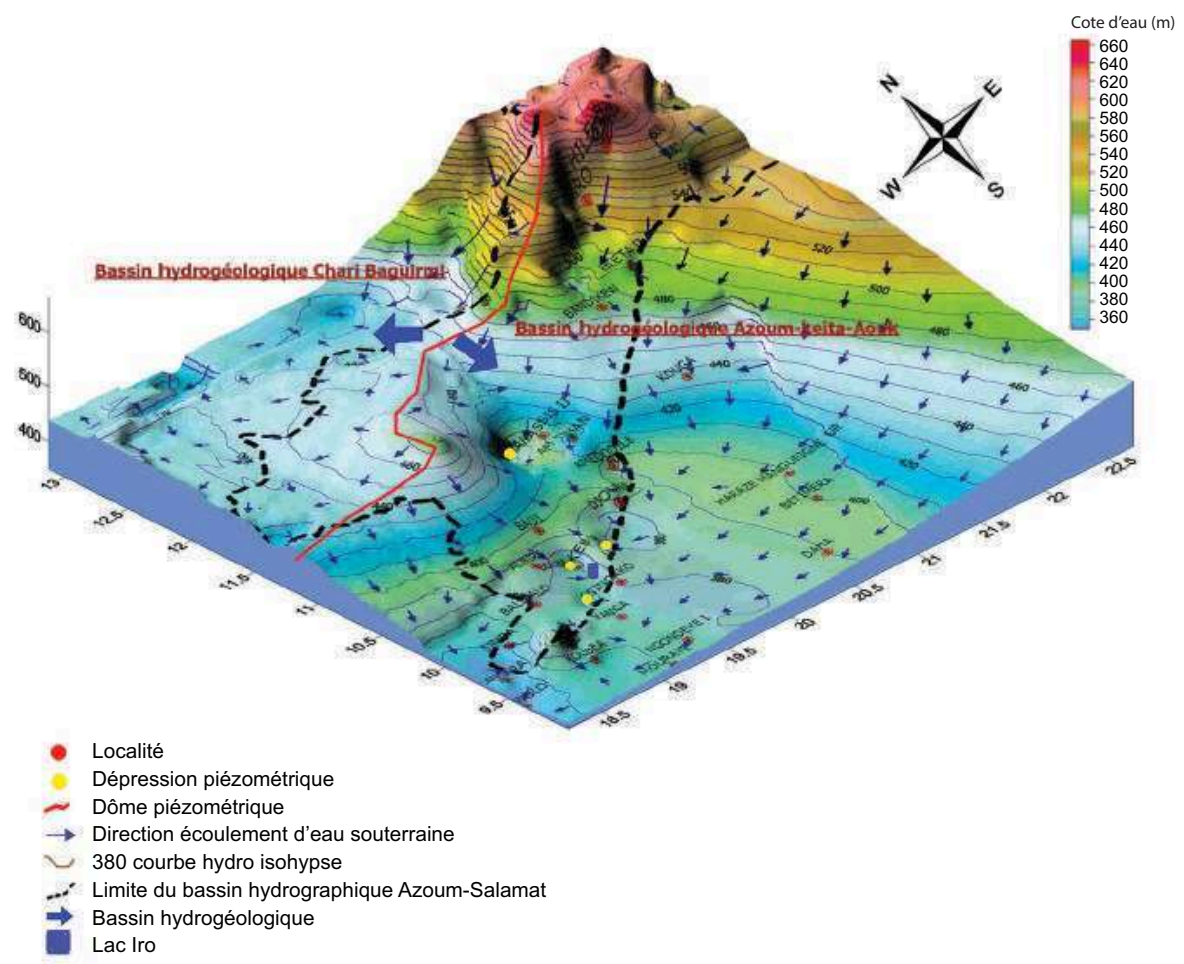

Figure 5

Le bassin hydrogéologique Azoum-Salamat en 3D.

Sources : SRTM 200I, résolution 90 m ; BERTHELOT, 1958 ; base de données Siteau, 2014.

Réalisation : Guinbé Amngar, septembre 2015.

\section{Caractérisations physicochimiques des eaux souterraines}

Les résultats des analyses physicochimiques des échantillons prélevés sont présentés dans le tableau 1. La moitié des échantillons analysés sont en bon équilibre ionique. La température des eaux analysée varie entre $29,4{ }^{\circ} \mathrm{C}$ et $32,7{ }^{\circ} \mathrm{C}$, avec une moyenne de $30,8^{\circ} \mathrm{C}$. Ces valeurs sont très proches de la température moyenne atmosphérique qui est de $28,3^{\circ} \mathrm{C}$. Le $\mathrm{pH}$ varie entre 6,40 et 6,88. La moyenne des valeurs pour les eaux souterraines est de 6,69. Globalement, les eaux souterraines présentent des valeurs de $\mathrm{pH}$ presque neutres. Les conductivités électriques mesurées sont comprises entre 88,60 et $549 \mu \mathrm{S} /$ $\mathrm{cm}$, avec une moyenne d'environ 204,27 $\mu \mathrm{S} / \mathrm{cm}$. Le potentiel redox (Eh) indique logiquement des conditions oxydantes pour les eaux souterraines. Seul un forage (village Moufa) indique des conditions réductrices $(-62 \mathrm{mV})$. Les alcalinités mesurées varient de 48,80 à 309,88 mg/l avec une moyenne de $111,37 \mathrm{mg} / 1$ pour les eaux souterraines. Les valeurs les plus élevées sont localisées entre les deux dépressions de Boum Kebir et de Gnassi/Salamat au nord-est du lac Iro. 
Les teneurs en calcium sont comprises entre $6,97 \mathrm{mg} / \mathrm{l}$ et $43,75 \mathrm{mg} / \mathrm{l}$ avec une moyenne de $16,75 \mathrm{mg} / \mathrm{l}$. Les concentrations en potassium des eaux souterraines de la zone d'étude présentent des valeurs relativement faibles, qui varient de $2,41 \mathrm{mg} / \mathrm{l}$ à 11,47 mg/l avec une teneur moyenne de 5,53 mg/l. La concentration maximale admissible est de $12,06 \mathrm{mg} / \mathrm{l}$. Les eaux souterraines ont une concentration en $\mathrm{Na}^{+}$qui varie de $4,14 \mathrm{mg} / \mathrm{l}$ à $51 \mathrm{mg} / \mathrm{l}$ dont la moyenne est de $15,73 \mathrm{mg} / \mathrm{l}$, la concentration maximale admissible par la norme de l'OMS étant égale à $200 \mathrm{mg} / \mathrm{l}$. Les teneurs en $\mathrm{Mg}^{2+}$ sont en dessous de la concentration maximale admissible qui est de 49,32 mg/l. Elles varient de 1,93 mg/l à 17,91 mg/l avec une moyenne de $5,35 \mathrm{mg} / \mathrm{l}$. Les teneurs en $\mathrm{Cl}^{-}$varient de $0,32 \mathrm{mg} / \mathrm{l}$ à $2,93 \mathrm{mg} / \mathrm{l}$ avec une moyenne de $1,06 \mathrm{mg} / \mathrm{l}$. Les teneurs en $\mathrm{SO}_{4}{ }^{2-}$ varient de $0,31 \mathrm{mg} / 1$ à $21,33 \mathrm{mg} / 1$ avec une moyenne de 4,48 mg/l. Un seul échantillon (Kofoï) a fait l'objet de l'analyse de cet ion avec une teneur en $\mathrm{NO}_{3}$ - de $0,46 \mathrm{mg} / \mathrm{l}$.

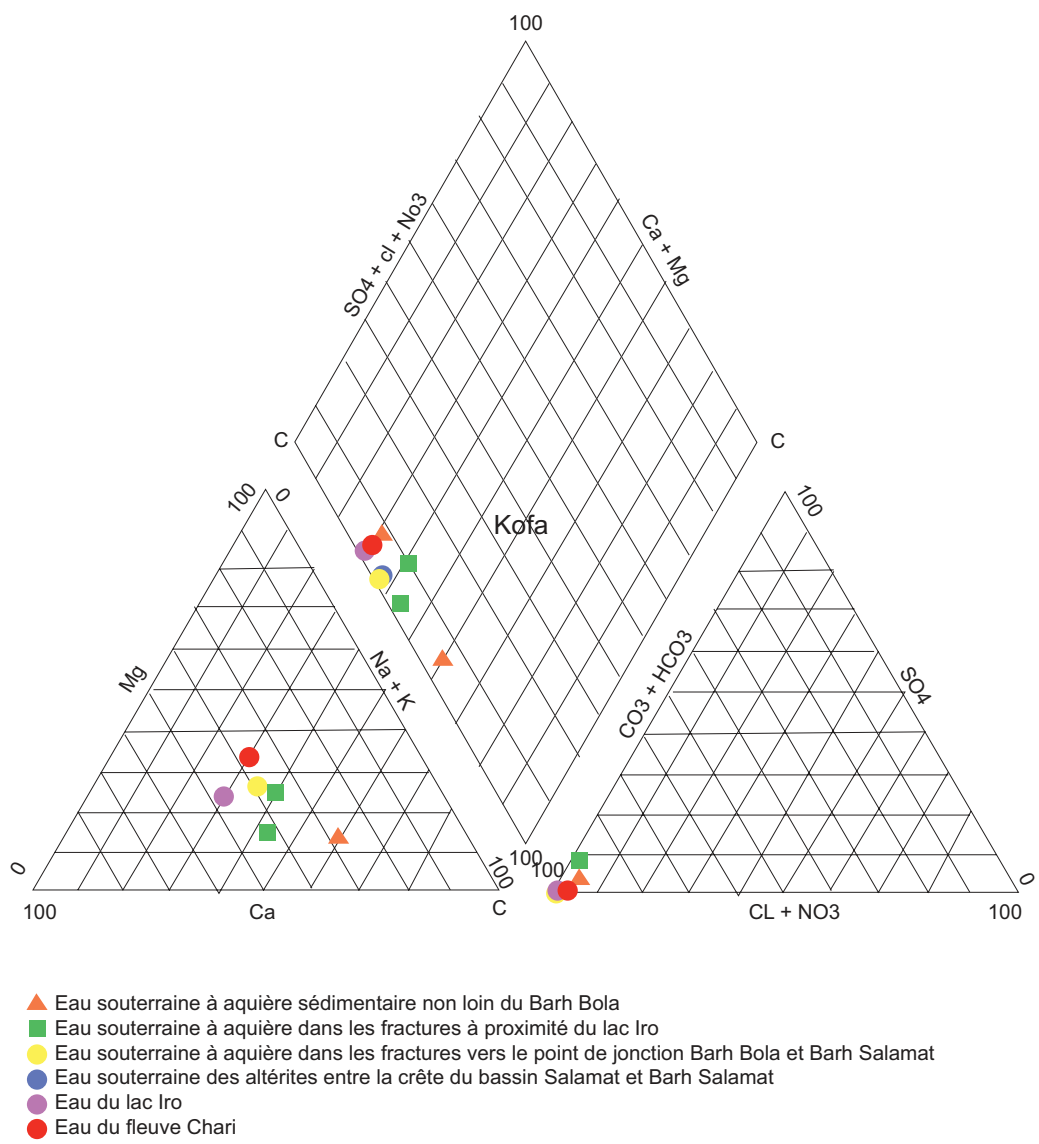

Figure 6

Diagramme de Piper des eaux de la zone du lac Iro et son bassin versant. 
Les résultats montrent donc que les eaux sont dominées par des faciès bicarbonatés calciques et magnésiens et des faciès bicarbonatés sodiques et potassiques (fig. 6). Le faciès bicarbonaté calcique se rencontre dans la quasitotalité des eaux des nappes. Ce faciès pourrait être influencé par les eaux de surface dominées par les carbonates.

Les eaux bicarbonatées sodiques (et potassiques) représentent $10 \%$ des eaux analysées. Elles se rencontrent dans la zone marécageuse de la zone d'étude.

\section{Discussion}

\section{Paramètres physicochimiques}

On observe que les forages captant des eaux circulant dans les roches du socle et sur le pourtour du lac Iro présentent les valeurs de conductivité les plus élevées (549 à $1012 \mu \mathrm{S} / \mathrm{cm}$ ). Cela suggère que cette variation de la conductivité pourrait être issue d'une dissolution des arènes granitiques. Les valeurs les plus faibles $(65,5$ à $199,7 \mu \mathrm{S} / \mathrm{cm})$ sont observées dans les forages captant les aquifères qui pourraient être alimentés par l'infiltration des eaux météoriques et/ou les eaux de surface. L'évolution de l'alcalinité en fonction de la conductivité électrique montre une tendance normale dans ce type d'environnement marqué par l'absence de formations évaporitiques (fig. 7).

\section{Distribution des teneurs des ions}

La figure 8 présente la distribution pour chaque cation dans le bassin versant. On observe que les valeurs les plus élevées en calcium sont mesurées dans les forages des nappes du socle et dans les dépressions piézométriques. Cette teneur en ion calcium suggère, en se basant sur la carte piézométrique, que le sens de l'écoulement des eaux est orienté vers les dépressions et la minéralisation en $\mathrm{Ca}^{2+}$ pourrait se faire au cours du cheminement soit par l'altération des matériaux éluviaux (résidus des roches désagrégées) ou des plagioclases calcosodiques (les argiles), soit par la dissolution de carbonate de calcium à travers les nodules calcaires. Les plus fortes teneurs en potassium sont plus largement représentées en amont du bassin versant et seraient fonction du degré d'altération des minéraux des roches intrusives. Les concentrations en sodium sont importantes autour du lac Iro et dans la zone de la dépression piézométrique. Cette prédominance pourrait résulter de l'altération accentuée de matériaux à forte concentration en fer possédant des plagioclases sodiques (albite) et des orthoses sodipotassiques (anorthose). Les fortes concentrations en strontium sont observées au centre des dépressions piézométriques dans les forages des nappes du socle. Ces concentrations en $\mathrm{Sr}^{2+}$ nous laissent penser que quand l'écoulement se fait exclusivement dans des matériaux détritiques de façon relativement lente, la 


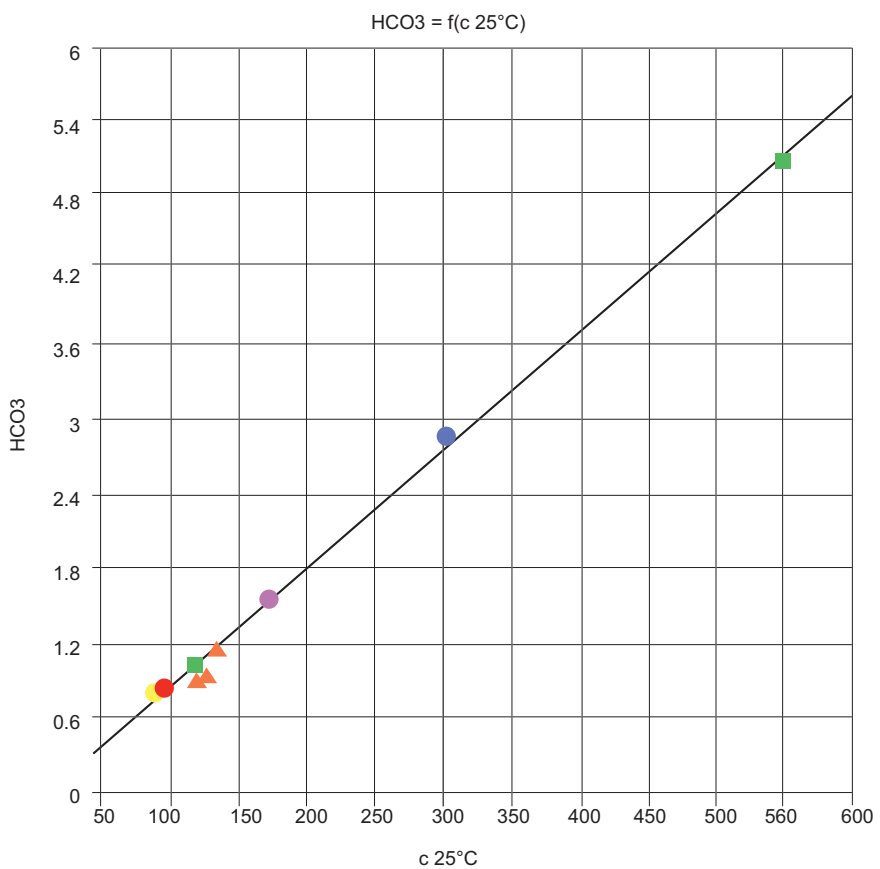

Figure 7

Évolution des ions bicarbonates $\mathrm{HCO}_{3}^{-}$en fonction de la conductivité électrique (voir légende des couleurs figure 6).

teneur en $\mathrm{Sr}^{2+}$ est faible. Cependant dans les zones d'altération des roches intrusives où l'écoulement est relativement rapide, il y a une plus forte teneur en $\mathrm{Sr}^{2+}$. Cela suggère que cet élément peut avoir comme origine l'altération des formations granitiques (ABDERAMANE, 2012).

Cette concentration pourrait aussi être accentuée par le phénomène d'évaporation. La figure 9 montre la distribution de l'ion $\mathrm{HCO}_{3}-$. On observe que plus la concentration en $\mathrm{HCO}_{3}$ - est élevée, plus la conductivité électrique est importante et inversement. L'analyse de la carte d'isovaleurs en bicarbonate révèle qu'elle est superposable à celle des conductivités électriques. Elle permet aussi d'indiquer une augmentation de la teneur du sud-ouest vers le centre de la dépression (nord-est). L'enrichissement des eaux souterraines en $\mathrm{HCO}_{3}$ - pourrait être lié à la typologie des eaux météoriques dont l'apport des $\mathrm{HCO}_{3}$ - serait dû à l'infiltration météorique, à l'altération des plagioclases calciques, à la dissolution de carbonate de calcium des nodules calcaires et/ou à la dissolution d'hydrogénocarbonate de sodium (ou bicarbonate de soude : natron). Les fortes teneurs en magnésium sont localisées dans la zone de la dépression piézométrique (fig. 8). Les valeurs les plus élevées pour le chlore sont localisées au nord-est à proximité de la dépression au puits de Madjok $(0,44 \mathrm{meq} / \mathrm{l})$. La carte d'isovaleurs en sulfate montre les teneurs les plus faibles dans la quasi-totalité de la zone d'étude où elles sont inférieures à 0,05 meq/l. (fig. 10). Dans le sens 

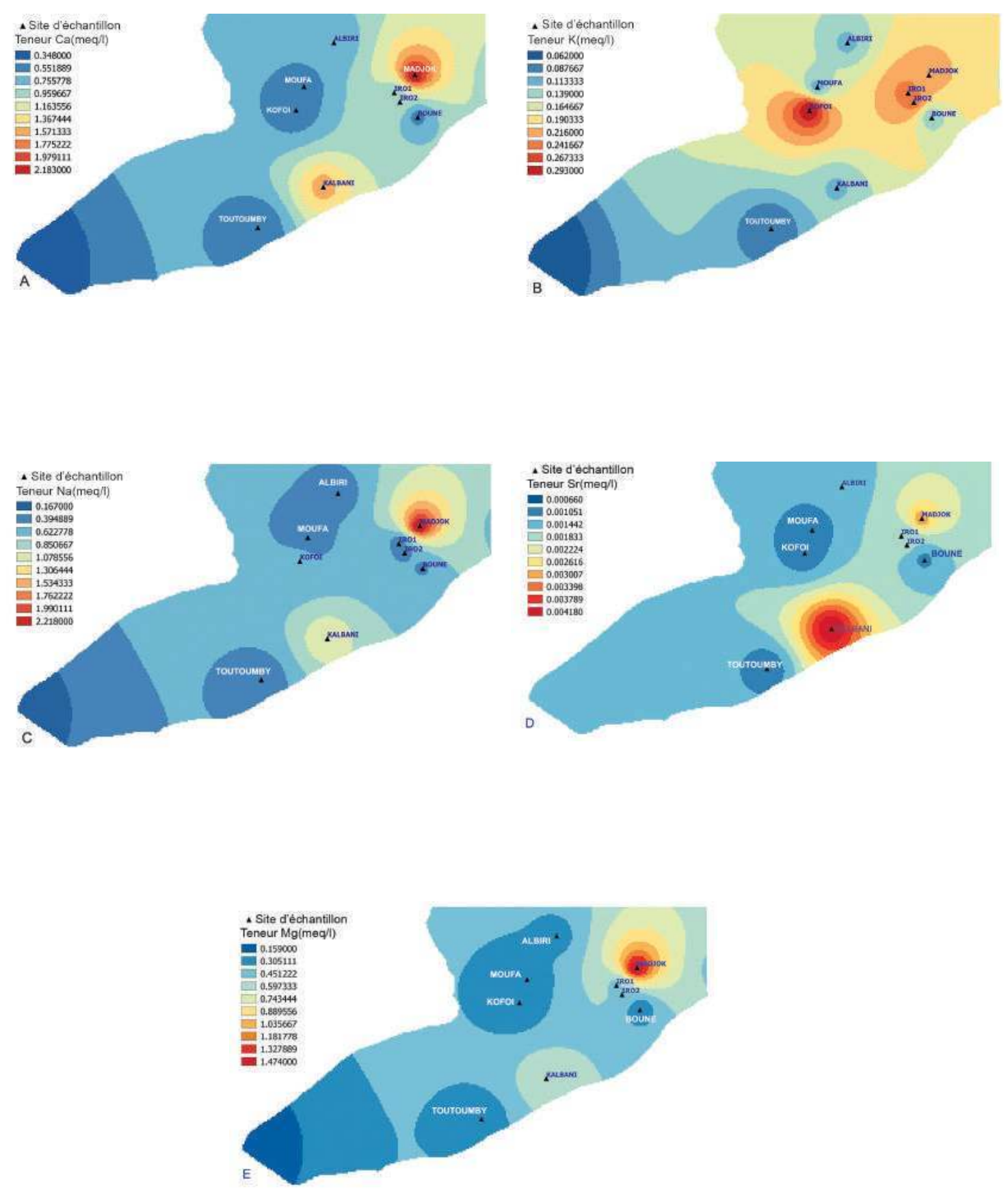

Figure 8

Répartition spatiale de la teneur en cation : $\mathrm{Ca}, \mathrm{K}, \mathrm{Na}, \mathrm{Sr}, \mathrm{Mg}$ 

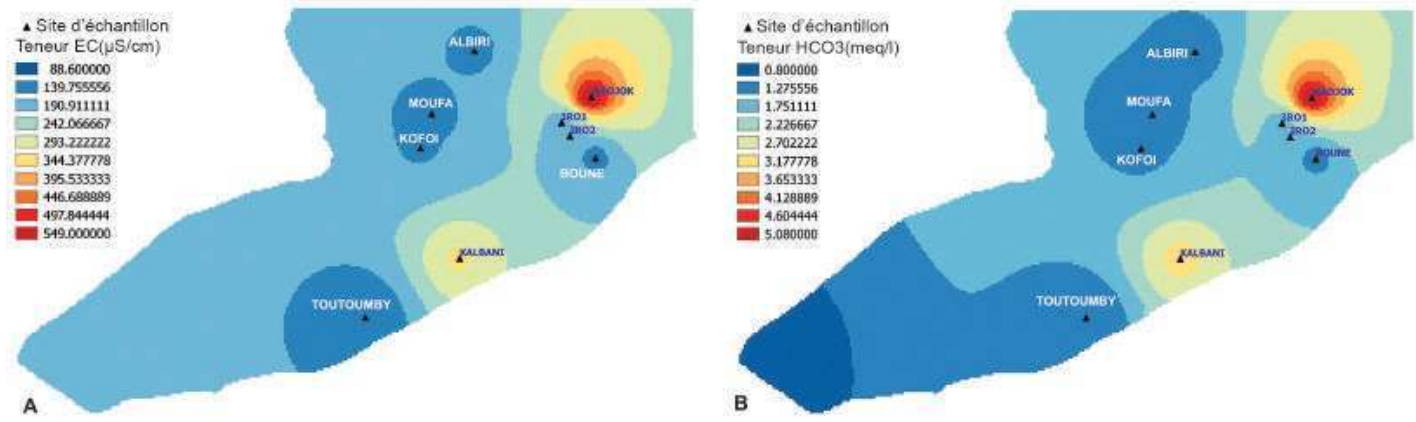

Figure 9

Répartition spatiale des teneurs en bicarbonate et de la conductivité électrique.
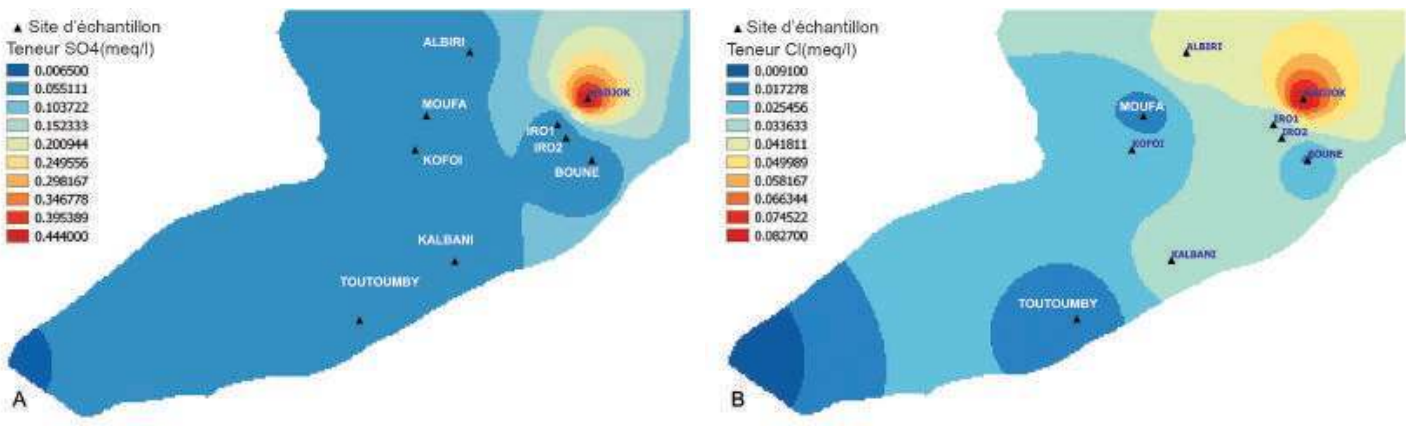

Figure 10

Répartition spatiale des teneurs des ions chlorure et sulfate.

de l'écoulement des eaux vers les dépressions, la minéralisation en $\mathrm{Mg}^{2+}, \mathrm{Cl}^{-}$et $\mathrm{SO}_{4}{ }^{2-}$ pourrait se faire au cours du cheminement. Cette concentration pourrait aussi être accentuée par le phénomène d'évaporation.

\section{Conclusion}

Les résultats de cette étude apportent des données originales contribuant à la compréhension du fonctionnement hydrodynamique et hydrochimique du système aquifère du lac Iro et de sa région. Les cartes piézométriques réalisées en $2 \mathrm{D}$ et en 3D du bassin hydrogéologique ont mis en évidence les zones de dépression piézométrique (Boum Kebir, Missidi et Gnassi) et le dôme 
piézométrique. L'évolution du gradient hydraulique a une direction nord-est vers les dépressions, suggérant un temps de résidence relativement long dans l'aquifère favorisant ainsi sa minéralisation. Cette étude montre aussi que la position perchée du lac Iro et du recouvrement argilo-sableux de sa zone recevant les précipitations et les eaux d'inondation par rapport à la nappe d'eau souterraine ne constitue pas leur pôle de recharge directe.

L'étude hydrochimique fait ressortir une zone de mélange des eaux souterraines au lac Iro et ses environs où les eaux minéralisées sont supposées être issues d'une part de la dissolution des minéraux contenus dans la matrice des roches encaissantes de l'aquifère, et d'autre part, de l'évaporation des eaux provenant de la partie amont au nord-est du bassin hydrogéologique. Les eaux faiblement minéralisées se rencontrent au sud-ouest de la zone d'étude dans la partie aval du bassin hydrogéologique. Le recouvrement de cette partie est essentiellement constitué des matériaux détritiques (sableux, sablo-argileux) bien arrosés par les précipitations, les eaux d'inondation et les eaux des vallées. Ce sont ces secteurs qui constituent les pôles de recharge directe.

\section{Bibliographie}

\begin{abstract}
Abderamane H., 2012
Étude du fonctionnement hydrogéochimique $d u$ système aquifère du Chari Baguirmi (République du Tchad). Thèse de doctorat, université de Poitiers, 288 p.
\end{abstract}

Billon B., Guiscafre J., 1969

Monographie hydrologique du Chari.

Première partie : Les facteurs conditionnels du régime. Paris, Orstom, 114 p.

\section{BERThelot R., 1958}

Étude hydrologique du bahr Azoum. Orstom, 38 p. multigr.

Billon B., Guiscafre J., Herbaud J., 1974 « Le bassin du fleuve Chari ». Monographies Hydrologiques $\mathrm{n}^{\circ}$ 2, Paris, Orstom, $450 \mathrm{p}$.

\section{BRGM, 1987}

Actualisation des connaissances sur les ressources en eau de la République du Tchad. Deuxième partie: Synthèse des données hydrogéologiques et carte à 1/1 $500000^{e}, 116 \mathrm{p}$. Troisième partie : Synthèse des données géologiques et carte à $1 / 1500000^{e}, 104 \mathrm{p}$.

\section{LouIS P., 1970}

Contribution géophysique à la connaissance géologique du bassin du lac Tchad. Orstom, $\mathrm{n}^{\mathrm{o}} 42,311 \mathrm{p}$.

\section{Ministère de L'EnVIRonnement et de L'EAU, 2003}

Schéma directeur de l'eau et de l'assainissement : ressources en eau et environnement (2003-2020). République du Tchad, HCNE, MEE, ONU-DAES, Pnud, 147 p., multigr.

\section{PIAS J., 1970a}

Notice explicative. Carte pédologique du Tchad à 1/1000 000 . Paris, Orstom, $\mathrm{n}^{\circ} 41,167 \mathrm{p}$.

\section{PIAs J., 1970b}

Les formations sédimentaires tertiaires et quaternaires de la cuvette tchadienne et les sols qui en dérivent. Paris, Orstom, $\mathrm{n}^{\circ} 43$, $407 \mathrm{p}$.

\section{RGPH2, 2009}

Deuxième recensement général de la population et de l'habitat, résultats définitifs par souspréfecture, mars 2012. Inseed, ministère du Plan, de l'Économie et de la Coopération internationale, annexes : 44-164. 
SCHNEIDER J. L., WOLF J. P., 1992a

Carte géologique et carte hydrogéologique au 1/1 $500000^{e}$ de la République du Tchad. Mémoire explicatif. BRGM n 209, vol. 1, 415 p.

SCHNEIDER J. L., WOLF J. P., 1992b

Carte géologique et carte hydrogéologique au 1/1 $500000^{e}$ de la République du Tchad.

Mémoire explicatif. BRGM no 209, vol. 2, 531 p.

SCHNEIDER J. L., 2001a

Géologique, archéologie, hydrogéologie. BRGM, vol. 1, $462 \mathrm{p}$.

\section{SCHNEIDER J. L., 2001b}

Géologique, archéologie, hydrogéologie. BRGM, vol. 2, $706 \mathrm{p}$.

\section{Simler R., 2013}

Manuel pour Diagrammes, logiciel

d'hydrochimie du Laboratoire d'Hydrogéologie.

Université d'Avignon, 35 p.

\section{VinCENT P. M., 2010}

Synthèse hydrogéologique. Projet Almy Bahaïm, Tchad, 29 p., multigr.

\section{Wacrenier Ph., 1953}

Lai-Garoua. Rapport de fin de mission 1953.

Rapport DMG Brazzaville, inéd. ${ }^{\circ}$ (W)-T 12 du 29/11/53. 5 p. 

Fluctuation des récoltes de sorgho repiqué et potentialités de culture Une analyse par télédétection
dans la région du lac Fitri

Angeline KEMSOL Nagorngar, Christine RaImond,

Robert MADJIGOTO, Valère JOFACK SOKENG,

Datoloum DJIMASSAL, Joseph LIBAR,

Fernand KOUAMÉ KOFFI

\section{Introduction}

Zone humide située au cœur du Sahel, le lac Fitri devient un objet de convoitise et attire nombre d'hommes et d'animaux venus de zones plus défavorisées. Selon MARTY et al. (2012), cette zone lacustre dispose d'un potentiel agricole, halieutique et pastoral très élevé et est considérée comme un grenier céréalier et une zone de refuge aussi bien pour les agriculteurs, les pêcheurs que pour les transhumants et la faune sauvage, principalement en période sèche. Les évaluations réalisées par le Bureau interministériel d'étude et de programmation (BIEP, 1989) et les différentes phases des projets du Fonds international de développement agricole (FIDA, 2006), montrent aussi les potentialités élevées des ressources naturelles du Fitri pour contribuer efficacement à atteindre de la sécurité alimentaire, non seulement au Fitri mais aussi dans une grande partie de la bande sahélienne du Tchad. Malheureusement, cette zone connaît une crise alimentaire récurrente. Pourquoi cette zone lacustre, que les politiques publiques appellent à devenir une zone de haute productivité agricole pouvant contribuer à la sécurité alimentaire nationale, notamment pour l'approvisionnement des villes, est-elle régulièrement reconnue comme déficitaire en céréales ? Nous 
posons l'hypothèse que, en plus de la variabilité climatique qui détermine l'extension des cultures de décrue, l'afflux récent et rapide de populations extérieures, qui ne cultivent pas, absorbe une grande partie des productions locales et contribue fortement à l'insécurité alimentaire de la région.

Cette communication présente les résultats de l'analyse diachronique des images satellitaires du Fitri sur trente ans, afin de suivre l'évolution de l'occupation des sols en relation avec la demande alimentaire locale. Ce travail s'appuie aussi sur les données collectées lors de la campagne de terrain de 2016 organisée et financée par le programme Gelt.

Nous retenons trois objectifs pour cette présentation :

- analyser la dynamique spatiale des différentes unités du paysage du Fitri entre 1985 et 2015 par l'imagerie satellitaire pour suivre l'évolution des superficies cultivées ;

- évaluer la contribution du sorgho repiqué en décrue, appelé « berbéré » au Tchad, à la sécurité alimentaire dans le Fitri ;

- déterminer les potentialités des zones de cultures de décrue du Fitri par l'analyse multicritère.

\section{Matériel et méthodes}

La zone d'étude couvre une superficie de $3360 \mathrm{~km}^{2}$ (336 000 ha) dans le département du Fitri. Elle prend ainsi en compte les zones inondables du lac Fitri potentiellement cultivables en berbéré (fig. 1).

Le climat de la zone est de type sahélien avec deux saisons contrastées : la saison sèche s'étend d'octobre à mai et la saison de pluie de juin à septembre. Les cumuls pluviométriques enregistrés entre 1970 et 2016 à Yao font ressortir une moyenne annuelle de $400 \mathrm{~mm}$. Cinq types de sols se distinguent : les vertisols, les sols steppiques, les sols halomorphes, les sols hydromorphes et les sols minéraux bruts.

La méthodologie repose d'une part sur le traitement des images satellitaires et l'analyse multicritère et d'autre part sur les enquêtes socioéconomiques auprès des principaux acteurs de la filière agricole du Fitri.

\section{Données satellitaires et enquêtes de terrain}

Pour l'analyse de l'évolution des unités du paysage, nous exploitons les images satellitaires Landsat de 1985 à 2016, disponibles sur le site http://glovis.usgs.gov. 


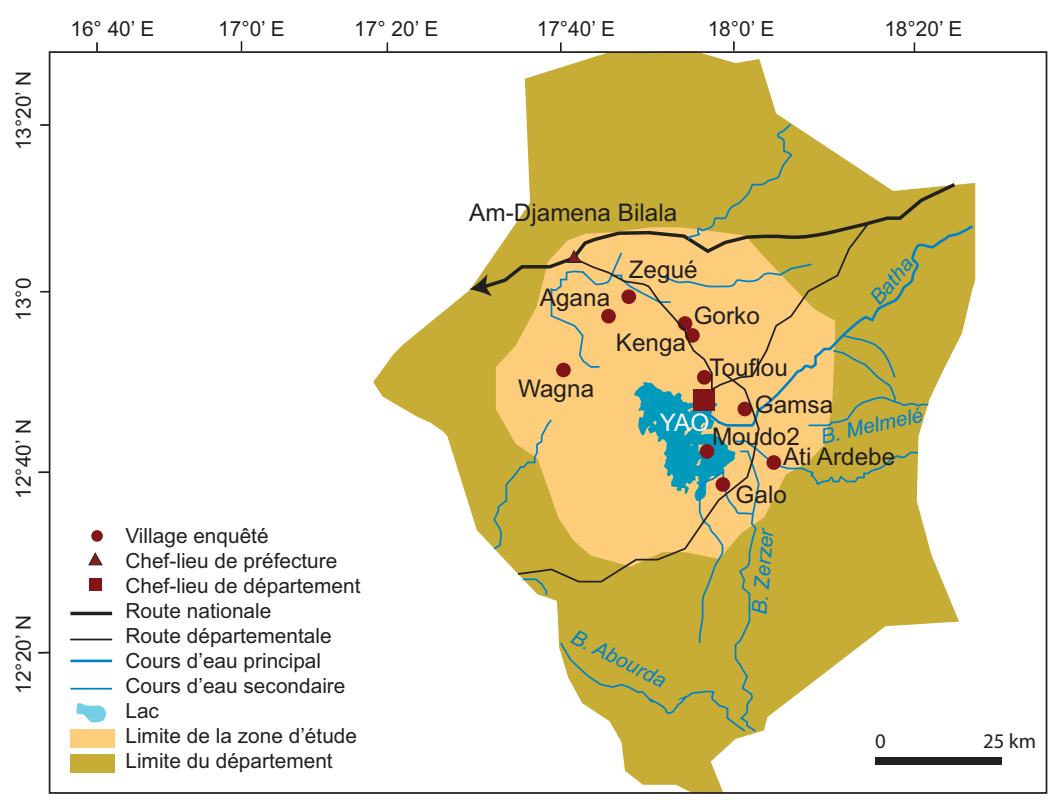

Figure I

Localisation de la zone d'étude et des villages enquêtés dans le département du Fitri.

Dix scènes sont utilisées et représentent les couples d'images crue/décrue correspondant à une campagne agricole. Les dates des images analysées sont :

$-13 / 10 / 1986$ et $01 / 01 / 1987$

- 11/08/2001 et $19 / 02 / 2002$

$-26 / 10 / 2011$ et $15 / 02 / 2012$

$-24 / 09 / 2014$ et $14 / 01 / 2015$

- 11/09/2015 et $17 / 01 / 2016$

Le programme Gelt a financé l'achat de 5 images $\operatorname{Spot}^{1}$. Ces images datent respectivement de 27/01/1995, 17/01/2006, 16/09/2009, 09/10/2011 et 06/02/2015. Elles ont servi à la validation de la classification des images Landsat. Les cartes de sols et végétation (PIAS, 1970) ont été géoréférencées et vectorisées. Les logiciels Envi et ArcGis ont servi au traitement des données. Les données de terrain sont constituées :

- de points GPS localisant les unités du paysage pour valider l'interprétation des images satellitaires;

- des enquêtes socioéconomiques auprès de 25 producteurs de berbéré, dont

9 focus groupes, dans les campements de berbéré ou les villages (fig. 1) ; 
- des entretiens auprès des autorités administratives et traditionnelles (préfet, sous-préfet, notables représentants du sultan), des agents de l'Office national de développement rural, des ONG et des associations et groupements du Fitri.

\section{Méthodes}

\section{Établissement des cartes d'occupation du sol}

Les cartes d'occupation du sol se basent sur la classification des images satellitaires Landsat après corrections radiométriques et atmosphériques. La difficulté de discriminer les cultures de décrue dans une seule image, en raison des faibles densités de repiquage (TRIBOULET, 1995), a conduit à mettre en place une méthode contextuelle exploitant des paires d'images crue (août-septembre)/ décrue (croissance maximale du berbéré en janvier-février) sous masques successifs (KeMsol NAGORNGAR et al., 2018). La première image sert à cartographier l'inondation afin de créer un masque radiométrique des zones inondées, qui sera utilisé dans la classification de l'image de décrue pour extraire les zones cultivées en berbéré. À l'issue de la classification, l'évolution des unités d'occupation du sol est calculée par différents taux : le taux moyen d'expansion annuelle, le taux de changement global et la matrice de transition. Ces taux proposés par la FAO en $1963^{2}$ sont les plus utilisés pour déterminer les états de changement de l'occupation des sols (NoyOlA-MEDRANO et al., 2009, Arouna 2012, Kpedenou et al., 2016).

L'année 1987 constitue la date de départ de l'analyse. Elle est marquée par une faible crue et une pluviométrie à Yao enregistrée en 1986 de $387 \mathrm{~mm}$, et se situe après plusieurs années sèches. En 1984 et 1985 notamment, le lac s'est asséché. En 1987, les enquêtes mentionnent une timide reprise de l'agriculture de décrue.

L'analyse se termine en 2015, année où l'inondation a été particulièrement bonne. Les enquêtes et les observations directes soulignent une exploitation maximale en berbéré du point de vue des superficies.

Huit classes d'occupation des sols définies par la télédétection et validées sur le terrain sont discriminées :

- la forêt correspond à la végétation ligneuse dense de différentes formations végétales : la forêt à Acacia nilotica en bordure du lac, les forêts galeries bordant les cours d'eau et la doumeraie (Hyphaene thebaica) localisée au nord-ouest du lac ;

2. Taux moyen annuel d'expansion spatiale : $T c=\left[(S 2 / S I)^{\frac{1}{t}}-I\right] * 100$

Taux de changement global : $T g=\frac{S 2-S I}{S I} * 100$

Avec SI : superficie de l'unité à la date TI; T : nombre d'années entre TI et T2 ; S2 : superficie de l'unité à la date $\mathrm{T} 2$. 
- la savane regroupe une formation arborée moins dense que la forêt avec pratiquement les mêmes espèces en plus d'une strate de hautes herbes pérennes. Elle domine dans le paysage et se maintient notamment au sud-ouest et en périphérie du lac ;

- la steppe renferme une végétation arbustive très clairsemée, séparée par de grandes plages de sols nus et une végétation herbeuse annuelle. Cette classe regroupe aussi les vieilles jachères ;

- la prairie marécageuse représente la végétation herbeuse inondée (Echinochloa stagnina) sur le pourtour du lac. Elle est en contact direct avec les eaux libres et constitue un lieu privilégié pour le pâturage ;

- le plan d'eau concentre les eaux libres du lac et des cuvettes alentour ;

- la culture pluviale représente les superficies mises en culture pendant la saison pluvieuse et dénudées en saison sèche ;

- la culture de décrue concerne les parcelles repiquées en sorgho en novembre et récoltées en février-mars. En raison de leur petite taille, les parcelles de maraîchage sont également prises en compte dans cette classe ;

- le sol nu regroupe en majorité les sols noirs très argileux situés au nord du lac, où aucune végétation ne peut se maintenir. Connus localement sous le nom de kosso en bilala, ces sols s'incrustent parfois à l'intérieur des vertisols productifs exploités en berbéré. Il existe également des espaces dénudés, communément appelés naga qui sont surtout localisés dans les steppes, mais assez difficiles à discriminer dans les images Landsat.

\section{Analyse de la sécurité alimentaire}

L'analyse de la sécurité alimentaire a été réalisée à partir des résultats de l'enquête socioéconomique, la prise en compte de la norme de consommation moyenne de céréales estimée à $159 \mathrm{~kg} /$ pers par an au Tchad (CILSS, 2004), complétés par les résultats de la dynamique de l'occupation des sols. L'enquête a aussi permis de connaître les techniques culturales du sorgho repiqué et les stratégies d'adaptation des ménages à l'insécurité alimentaire. Les entretiens avec les autorités, responsables d'ONG et associations ont abordé la sécurité alimentaire à l'échelle départementale.

\section{Estimation des potentialités agricoles des zones de décrue}

Les potentialités des zones de culture de décrue sont déterminées par la méthode d'analyse multicritère, qui vise à expliciter une famille cohérente de critères pour concevoir, justifier et transformer les préférences au sein d'un processus de décision (CAILlET, 2003, JofACK, 2016). Son but est de considérer tous les paramètres pour leur attribuer un poids lié à leur importance relative et à leur action par rapport à ces critères, ce qui doit aboutir à une agrégation des résultats. Les critères utilisés pour définir les potentialités agricoles des zones de décrue sont : les types de sols, le relief (modèle numérique de terrain), la densité de drainage, la carte d'occupation du sol et l'indice d'humidité calculés sur l'image 
de 2015, et les zones inondables extraites de l'image de crue de 2014 (KEMSOL NAGORNGAR, 2018).

\section{Résultats et discussion}

\section{Évolution de l'occupation du sol au Fitri entre 1987 et 2015}

Les précisions globales de 85,5\% (1987), 94,9\% (2002) et 81,1\% (2015) valident la classification. Les cartes d'occupation du sol sont présentées figure 2.

L'évolution des unités du paysage est établie entre 1987 et 2015 (fig. 3) et précisée par la matrice de transition (tabl. 1). Sur ce tableau, la diagonale contient les valeurs des superficies des unités qui sont restées stables entre les deux dates et les conversions se font des lignes vers les colonnes. Hors de la diagonale se trouvent les valeurs des superficies des unités ayant subi des modifications entre les deux dates.

Tableau I

Matrice de transition des unités d'occupation du sol au Fitri entre 1987 et 2015.

\begin{tabular}{|c|c|c|c|c|c|c|c|c|c|}
\hline \multirow[b]{2}{*}{$\begin{array}{l}\text { Unité } \\
\text { en } 1987\end{array}$} & \multicolumn{9}{|c|}{ Unité en 2015} \\
\hline & CD & CP & Sol nu & PM & PE & Steppe & Savane & Forêt & $\begin{array}{l}\text { Sup. } \\
\text { totale en } \\
\text { I } 987 \text { (ha) }\end{array}$ \\
\hline$C D$ & 1901 & & & 275 & 31 & & 773 & & 2979 \\
\hline $\mathrm{CP}$ & 2081 & 5767 & & 873 & & 4289 & 3983 & 7 & 17001 \\
\hline Sol nu & 5573 & & 2150 & 687 & & 76 & 1515 & & 10001 \\
\hline PM & & & 282 & 13531 & 14360 & 420 & 3906 & & 32499 \\
\hline PE & & & & 2 & 6002 & & I & & 6005 \\
\hline Steppe & 9539 & 21646 & & 125 & & 63025 & 7713 & & 102048 \\
\hline Savane & 36644 & 17287 & 571 & 7833 & 3536 & 24185 & 63186 & 9761 & 163001 \\
\hline Forêt & 269 & 300 & & 674 & 74 & 7 & 926 & 254 & 2504 \\
\hline $\begin{array}{l}\text { Sup. totale } \\
\text { en } 2015 \\
\text { (ha) }\end{array}$ & 56005 & 44999 & 3002 & 24000 & 24003 & 92002 & 82003 & 10022 & \\
\hline
\end{tabular}

CD : Culture de décrue, $C P$ : Culture pluviale, $P M$ : Prairie marécageuse, PE : Plan d'eau.

Quatre unités de paysage connaissent une évolution fortement progressive au cours de la période considérée. Les plans d'eau se sont étendus avec un taux moyen d'expansion de $5 \%$ par an sur toutes les unités de paysage en périphérie, principalement dans les prairies marécageuses (60\% de la superficie en eaux 


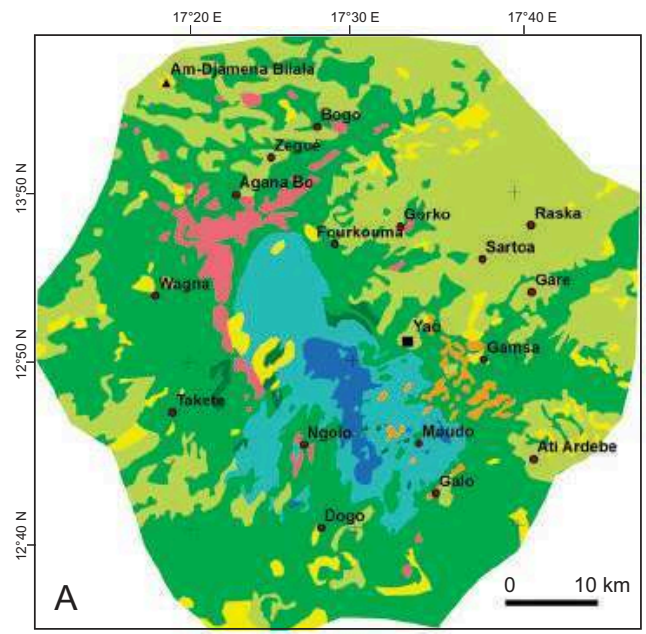

Carte d'occupation du sol au lac Fitri en 1987

Source : image satellitaire Landsat TM

P183 r 051 du 1er janvier 1987 et points GPS terrains

Réalisation septembre 2017

Kemsol Nagorngar A.

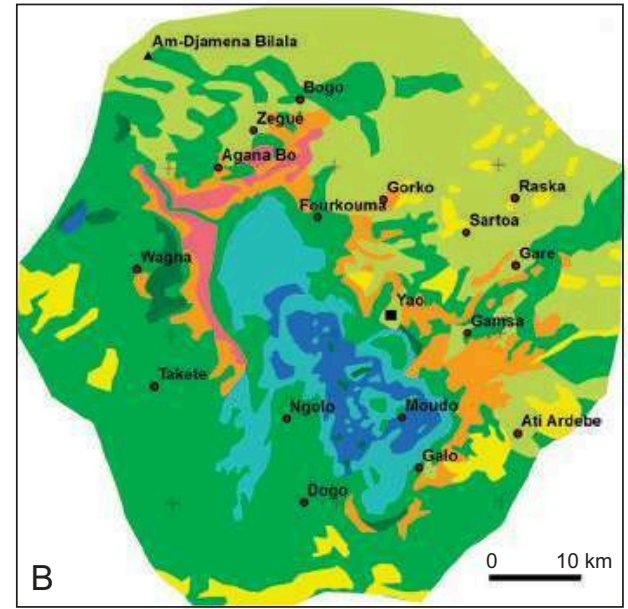

Carte d'occupation du sol au lac Fitri en 2002

Source : image satellitaire Landsat ETM

P183 r 051 du 19 février 2002 et points GPS terrains

Réalisation septembre 2017

Kemsol Nagorngar A.

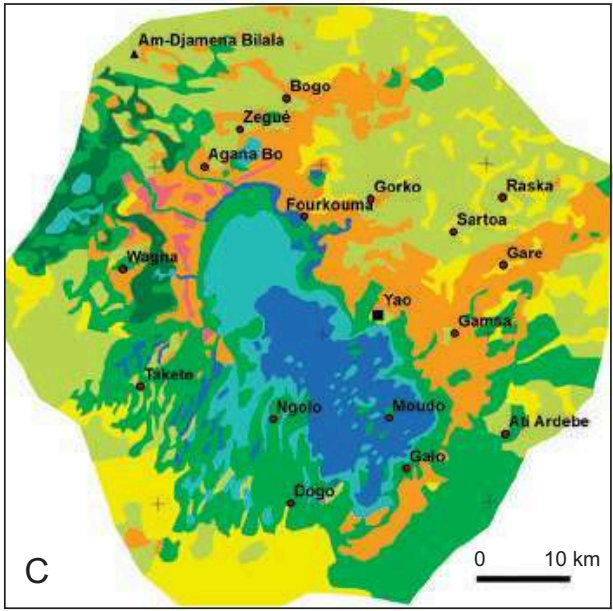

Carte d'occupation du sol au lac Fitri en 2015

Source : image satellitaire Landsat OLI

P183 r 051 du 14 janvier 2015 et points GPS terrains Réalisation septembre 2017

Kemsol Nagorngar A.

Figure 2

Cartes d'occupation du sol du Fitri en 1987 (a), 2002 (b) et 2015 (c). 


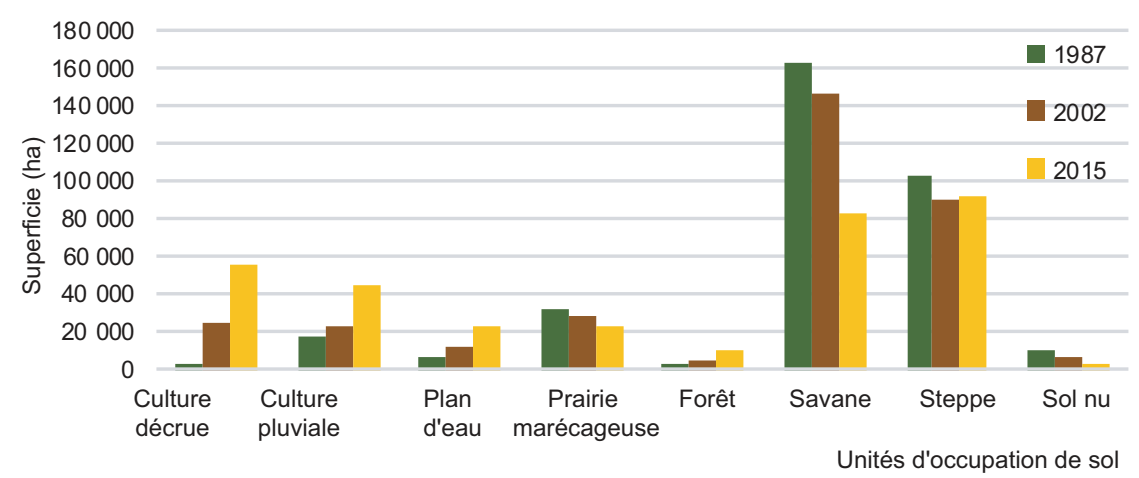

Figure 3

Occupation du sol au Fitri entre 1987 et 2015

libres de 2015) et la savane (15\%). Leur superficie passe de 6000 ha en 1987 à 24000 ha en 2015, soit un taux global d'évolution de $300 \%$.

Avec la progression des eaux libres et le retour des pluies, les cultures de décrue ont connu une forte expansion : de 3000 ha en 1987 à 56000 ha en 2015, leur taux global d'évolution est de $1780 \%$ (11\% par an). Seules $64 \%$ des superficies cultivées en 1987 le sont encore en 2015, et elles ne représentent que $3 \%$ des superficies repiquées en 2015. Le reste s'est transformé en savane (26\%), prairie marécageuse $(9 \%)$ et plan d'eau $(1 \%)$. L'extension des zones cultivées en décrue est faite sur les zones à nouveau touchées par la crue, sur la savane $(66 \%$ des superficies cultivées en 2015), la steppe (17\%), les sols nus (10\%) et les cultures pluviales $(4 \%)$.

Les cultures pluviales se sont aussi fortement étendues, avec un taux global d'évolution de $165 \%$ en vingt-huit ans (4\% par an). Les surfaces qu'elles occupaient en 1987 ont été remplacées par la steppe (25\%), la savane (23\%), les cultures de décrue (12\%) et la prairie marécageuse $(5 \%)$ en 2015 . Sur les surfaces cultivées en 2015, la proportion des anciennes zones de cultures ne représente que $13 \%$, les autres apports proviennent essentiellement des steppe (48\%), savane (38\%) et forêt (1\%).

L'amélioration des conditions hydriques favorise aussi la progression de la forêt, même si sa superficie reste modeste. La forêt à proximité des marécages en 1987 a disparu sous la pression anthropique (défrichages agricoles, pastoralisme), alors qu'une nouvelle forêt d'Hyphaene thebaica se développe au nord-ouest du lac où une succession de cuvettes draine les débordements de la crue du lac. Le taux global d'évolution des forêts est de $300 \%$ sur la période (5\% par an).

La progression des eaux libres et des surfaces cultivées s'est principalement faite au détriment :

- des prairies marécageuses, en grande partie recouvertes par les eaux libres et qui n’ont conservé que $42 \%$ de leur superficie de 1987. Elles se sont étendues 
en moindre proportion sur les forêts, sol nu, cultures pluviales et savane. Leur taux global d'évolution se situe à - $26 \%$;

- des sols nus qui régressent de $70 \%$ (- $4 \%$ par an) et se localisent en 2015 dans des secteurs très différents de ceux de 1987. Ils sont en grande partie recultivés en berbéré (56\% des superficies de sol nu en 1987) ou recouverts par la savane $(19 \%)$ ou les marécages $(9 \%)$;

- des savanes avec un taux global d'évolution de seulement - 50 \% (- $2 \%$ par an), mais cette régression représente la plus grande perte de superficie : elles passent de 163000 ha à 83000 ha, soit 80000 ha perdus entre 1987 et 2015 au profit de toutes les autres unités de paysage. Cette dynamique régressive reflète la pression anthropique croissante dans la région et masque la dynamique climatique favorable à la reprise de la végétation au cours de la période, que l'on observe cependant par la reprise, dans les secteurs où la population est moins dense, de la savane sur la steppe (7 713 ha), la prairie (3 900 ha) et les cultures pluviales (3 $983 \mathrm{ha}$ ).

La steppe est l'unité de paysage qui apparaît la plus stable du point de vue des superficies, avec un taux global d'évolution de - $10 \%$ (- 0,4\% par an). Elle a pourtant beaucoup évolué dans le paysage, en ne conservant que $62 \%$ de sa superficie de 1987 en 2015 au profit des cultures pluviales et de décrue et de la savane. Son extension est faite sur les mêmes unités et marque une surexploitation des ressources dans ces espaces, qui semble s'accentuer depuis 2002 (fig. 3).

De ces résultats il faut retenir un retour à des conditions climatiques favorables depuis les sécheresses des années 1970-1980, qui a permis une augmentation considérable des ressources naturelles disponibles dans la région du Fitri, également soulignée par YALIKUN et al. (ce volume). Cependant, la pression démographique croissante, marquée dans les cartes d'occupation du sol par une augmentation spectaculaire des superficies cultivées et le maintien de grandes superficies de steppes entretenues par le pâturage des animaux, limite la régénération de la végétation.

\section{Berbéré et sécurité alimentaire}

Le Fitri dispose de ressources considérables dont le potentiel agricole devrait apporter la sécurité alimentaire à la population, avec le berbéré comme principal moteur. Les besoins céréaliers sont estimés par le Cilss à $159 \mathrm{~kg} /$ pers par an au Tchad. Les rendements moyens de berbéré sont estimés à $900 \mathrm{~kg} / \mathrm{ha}$ selon les enquêtes (confirmé par BIEP, 1989). La superficie exploitée en berbéré est estimée à 56000 ha en 2015 (selon le traitement des images satellitaires, tabl. 1), ce qui donne une production de $50000 \mathrm{t}$ de sorgho en 2015 (900 kg/ha x $56000 \mathrm{ha})$. Or la population du département du Fitri en 2015 est estimée à 163396 personnes selon le recensement de 2009. Pour couvrir les besoins de cette population en céréales, il faudrait donc $159 \mathrm{~kg} /$ personne x 163396 personnes, c'est-à-dire 26000 t. Ainsi en 2015, exceptionnellement favorable, les excédents ont été 
vendus ou échangés avec les éleveurs transhumants ou exportés à l'extérieur de la zone, mais cela ne reflète pas la situation habituelle du Fitri. L'année suivante, par exemple, a connu une crise alimentaire qu'il a fallu compenser par des importations de céréales.

\section{Les facteurs de l'irrégularité de la production céréalière}

Cette variabilité de la production n'est pas seulement due à l'irrégularité de la crue, même si elle explique la variabilité des superficies de berbéré. Nous avons vu par les cartes d'occupation du sol entre 1987 et 2015 que le retour à de meilleures conditions hydriques a permis l'augmentation régulière des superficies repiquées en sorgho. Mais de plus faibles crues interviennent régulièrement. Ainsi, 2015-2016 a entraîné une diminution importante du berbéré, dont les superficies sont passées de 56000 ha en 2015 à 37000 ha en 2016 (résultat du traitement des images Landsat).

La croissance démographique explique aussi une augmentation des besoins alimentaires locaux. Selon les deux recensements officiels, la population du département du Fitri est passée de 77000 habitants en 1993 à 110400 en 2009, et les arrivées s'accélèrent depuis. Cette croissance concerne aussi bien les sédentaires que les éleveurs transhumants et les pêcheurs. Certains sont à la recherche de meilleures conditions de vie, d'autres fuient l'insécurité, ou l'aridité de leur milieu d'origine. Si l'augmentation des cultures de décrue et pluviales a suivi le rythme des crues du lac et de la croissance démographique, l'accélération de celle-ci depuis le début des années 2000 aggrave les déficits alimentaires locaux, notamment en cas de crue moins étendue. L'arrivée de plus de 40000 orpailleurs entre janvier 2016 et avril 2017 (estimation des autorités administratives du Fitri) vient aggraver la situation. Même si en mai 2017, l'État avait réussi à les chasser, l'attraction qu'exerce l'orpaillage sur les populations est telle que le risque qu'elles reviennent est très grand.

Les ennemis de culture sont nombreux dans cette zone humide et peuvent anéantir une récolte. Le gardiennage des champs contre les oiseaux granivores est obligatoire pendant la fructification et mobilise femmes et enfants durant plusieurs semaines à partir de janvier, avec des moyens rudimentaires utilisés pour protéger les panicules. L'accroissement du cheptel fréquentant la région augmente la pression sur les cultures de décrue, ce qui engendre des conflits plus fréquents entre agriculteurs et éleveurs (OulONA, 2016). De plus, le Fitri accueille une population mobile d'éléphants qui causent des dégâts énormes lorsqu'ils entrent dans les champs, notamment aux alentours de la forêt de Wagna où ils séjournent saisonnièrement; les phacochères sont redoutables dans la zone de Galo.

\section{Un système agraire adapté aux irrégularités de la production}

Face à la variabilité des ressources en eau, une organisation foncière particulière est observée. Ainsi, les producteurs disposent d'au moins deux parcelles situées dans des zones plus au moins proches du lac. Si l'une est trop, ou pas assez 
inondée, l'autre peut être mise en culture. Les agriculteurs peuvent aussi emprunter des parcelles à ceux qui ont de vastes superficies, ou recourir aux réserves foncières du sultan en cas d'année très déficitaire. Cette fluidité du système foncier permet aux agriculteurs d'accéder à une parcelle quel que soit le profil de la crue (MBAGOGO, ce volume).

Une autre adaptation à la variabilité des productions est la pratique de la pluriactivité, qui apporte des produits et revenus complémentaires au berbéré (BÉmADJi, 2016) : la culture pluviale, la pêche (SAUNIER, 2016), le petit élevage, le maraîchage, la cueillette des noix de doum (GUÉDON, 2016) et d'autres produits alimentaires (creb, nénuphar, riz sauvage), ainsi que le travail temporaire à Am Djamena-Bilala, Ati ou N'Djamena.

Cependant, l'accroissement de la demande locale et l'augmentation des ventes de céréales au moment de la récolte pour couvrir des besoins sociaux et/ou pour rembourser des dettes sont une entrave croissante à la sécurité alimentaire locale et à la constitution de stocks à l'échelle des ménages et des villages. La pratique de l'usure est répandue dans la zone d'Am Djamena-Bilala : les commerçants prêtent des produits de première nécessité (céréales, sucre, savon, huile, sel) en période de soudure et se font rembourser en nature à la récolte du berbéré avec un taux d'intérêt qui double ou triple la valeur du produit emprunté.

\section{L'aide alimentaire}

L'appui technique et matériel de l'ONDR ainsi que des ONG telles que Adra ${ }^{3}$, Solidarité internationale ${ }^{4}$, Parsat $^{5}$ ou Pads $^{6}$ aident les producteurs à diversifier et intensifier l'agriculture, notamment le marâ̂chage qui fournit un complément alimentaire non négligeable. Ces organismes apportent aussi une aide alimentaire préventive ou dans l'urgence à travers l'Onasa (Office national de sécurité alimentaire).

L'alerte famine est déclenchée par le sultan lorsque le Batha ne coule pas pendant trois mois d'affilée après le début de la saison des pluies. L'alerte suit une procédure hiérarchique auprès du sous-préfet, du préfet puis du gouverneur à Ati, qui saisit l'Onasa à N'Djamena. Ainsi, en juillet 2016, 1000 sacs de $100 \mathrm{~kg}$ $(100 \mathrm{t})$ de mil pénicillaire ont été envoyés au Fitri et revendus au prix subventionné de $11000 \mathrm{~F} / \mathrm{sac}$. Les sacs sont répartis par village et quartier selon la taille de la population. Dans les zones enquêtées, chaque famille a reçu un sac pour 4 personnes. L'Onasa a encore envoyé en mars 2017, 500 sacs de $100 \mathrm{~kg}$ de riz au prix subventionné de $13500 \mathrm{~F} / \mathrm{sac}$, répartis selon les mêmes critères aux populations du Fitri.

3. Agence adventiste d'aide et de développement, au Fitri depuis 20I2, financement Usaid.

4. Au Fitri depuis 2012, financement Union européenne.

5. Projet d'amélioration de la résilience des systèmes agricoles au Tchad, au Fitri depuis 20I5, financement Fida.

6. Projet d'appui au district sanitaire, au Fitri depuis 2016, financement Coopération suisse. 


\section{Potentialités agricoles des zones de décrue au lac Fitri}

Peut-on augmenter les superficies cultivées en décrue dans la zone lacustre ? L'analyse multicritère, réalisée à partir des poids générés pour chaque critère, produit la carte de potentialités des cultures de décrue au lac Fitri (fig. 4). Pour la crue de 2014-2015, les superficies des zones favorables à très favorables

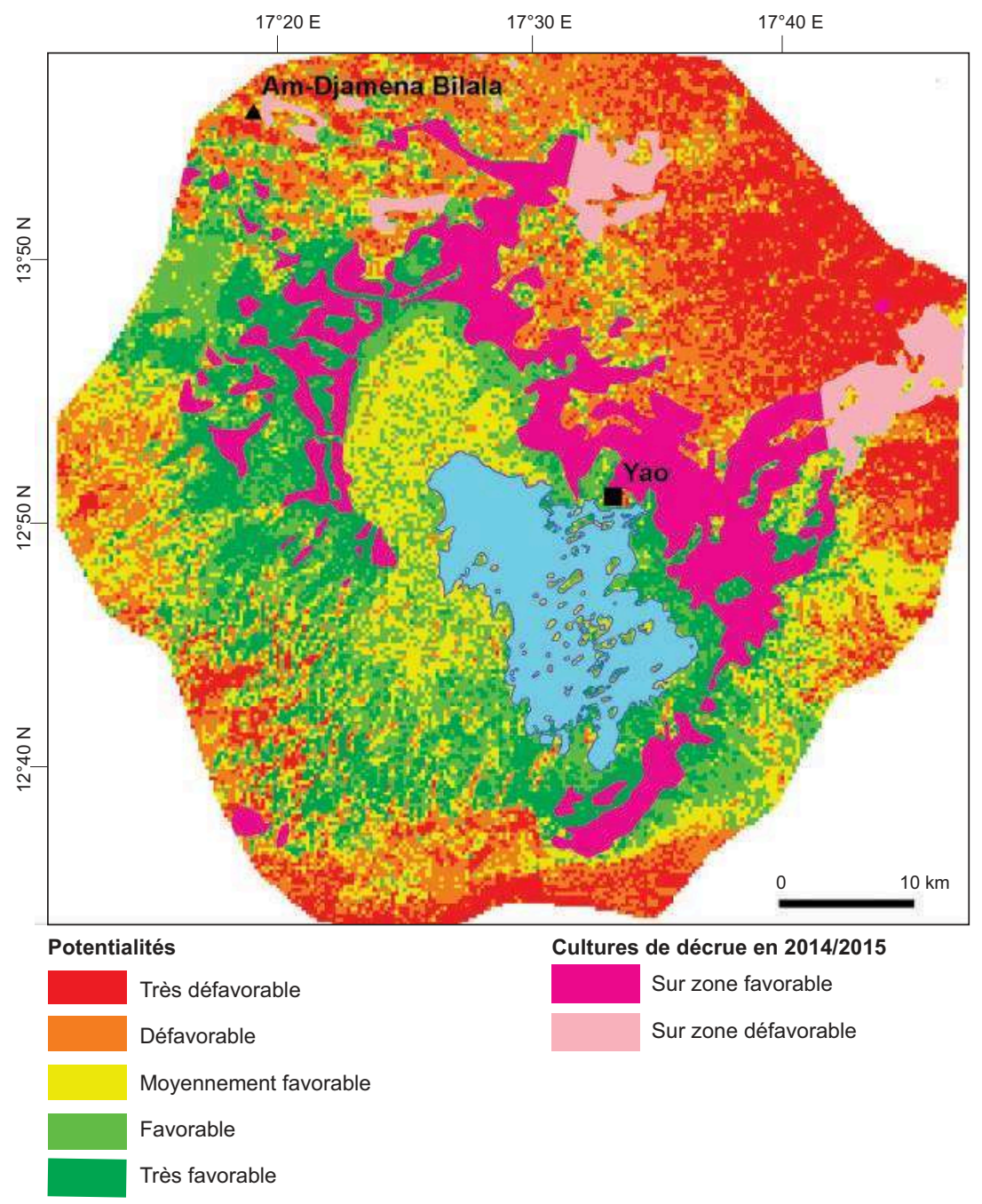

Figure 4

Exploitation et potentialités des zones de cultures de décrue au lac Fitri.

Carte issue d'une analyse multicritères.

Réalisation :Angeline Kemsol Nagorngar, septembre 2017. 
s'élèvent à 108900 ha, celles de zones moyennement favorables à 108000 ha et celles des zones défavorables à 119600 ha.

Les zones les plus favorables sont mises en valeur pour le berbéré. Pendant la campagne 2014-2015, elles ont accueilli 44400 ha de cultures de décrue, soit $41 \%$ de leurs potentialités. 11000 ha sont cultivés dans les zones moyennement favorables, soit seulement $10 \%$ de leurs potentialités. Il reste donc $59 \%$ de terres favorables qui n'ont pas été cultivées. Les principales raisons sont liées à la restriction environnementale qui interdit de couper des arbres au Tchad (loi 14 de 2008 portant régime des forêts, de la faune et des ressources halieutiques), mais aussi à l'inaccessibilité de certaines zones : ainsi, il n’y a pas de village ni de route à l'ouest du lac, où les troupeaux sont plus nombreux, et donc peu de surfaces cultivées.

Aucun aménagement n'a été entrepris au Fitri pour sécuriser l'alimentation en eau des parcelles et étendre les superficies cultivables. Les aménagements du PNSA (Programme national de sécurité alimentaire) en 2012 pour la riziculture se sont soldés par un échec, car mal adaptés au système multifonctionnel du lac Fitri. Un schéma d'aménagement à l'échelle du département est indispensable pour stabiliser la production céréalière, gérer les ressources pastorales et surtout préserver l'équilibre écosystémique de cette réserve de biosphère désignée « zone humide d'importance internationale » placée sous le régime de la Convention de Ramsar (décret no 773/PR/MTE/89 du 2 octobre 1989).

\section{Conclusion}

L'étude de la dynamique de l'occupation du sol au Fitri depuis les grandes sécheresses de 1970-1980 révèle une forte augmentation des zones de cultures (de décrue et pluviale) et de la taille du lac et des forêts, ainsi qu'une forte régression de la savane et une stabilité de la steppe. Malgré ses multiples ressources favorables à la pêche, la cueillette, aux cultures pluviales et de décrue et à l'élevage, le Fitri a des difficultés à s'autosuffire en céréales en raison de la variabilité des ressources en eau, de la pression croissante des cheptels sur les cultures et du nombre de plus en plus important de résidents permanents et temporaires. Cette région exporte rarement ses récoltes, sauf dans le cas d'année exceptionnelle comme en 2014-2015.

La cartographie des potentialités de culture du berbéré révèle que $41 \%$ des zones favorables aux cultures de décrue dans les conditions de l'année 20142015 ont été mises en culture ; le reste ne peut faire l'objet d'exploitation sans friction avec les autres secteurs d'activités, essentiellement l'élevage, ou occasionnerait une destruction du couvert arboré et une dégradation de la biodiversité. La stabilisation et l'augmentation des productions céréalières 
passent désormais par la réalisation des aménagements hydroagricoles adaptés à ce système plurifonctionnel. Ces aménagements qui doivent être proposés sur les bases d'études approfondies devraient permettre de sécuriser l'alimentation en eau des parcelles de décrue existantes et d'étendre l'inondation à de nouveaux secteurs favorables tout en respectant la conduite des autres activités.

\section{Remerciements}

Les auteurs expriment toute leur gratitude au programme Gelt qui a financé la collecte des données sur le terrain, au SCAC qui a financé la formation doctorale d'Angeline Kemsol Nagorngar en Côte d'Ivoire et au CNRD pour son appui. Nous remercions aussi l'UMR 8586 Prodig pour son accueil et Mr Tashi Yalikun pour son appui dans les traitements des images satellitaires.

\section{Bibliographie}

\begin{abstract}
Arouna O., 2012
Cartographie et modélisation prédictive des changements spatiotemporels de la végétation dans la commune de Djidja au Bénin : implications pour l'aménagement $d u$ territoire. Thèse de doctorat, université d'Abomey-Calavi, 246 p.
\end{abstract}

\section{BÉMADJI B., 2016}

Économie des échanges de produits alimentaires autour du lac Fitri. Master 2 de géographie, université N'Djamena, 168 p.

\section{BIEP, 1989}

Étude de développement rural intégré du lac Fitri. République du Tchad, ministère de l'Agriculture, Banque islamique de développement, Cedrat-SA, Biep, document de synthèse et annexes, 61 p., multigr.

\section{Caillet R., 2003}

Analyse multicritère : Étude et comparaison des méthodes en vue d'une application en analyse de cycle de vie. Cirano, série scientifique. Montréal, $52 \mathrm{p}$.

\section{CILSS, 2004}

Normes de consommation des principaux produits alimentaires dans les pays du Cilss. Rapport d'étude, juillet 2004. 67 p.

FIDA, 2006

Tchad : Projet de développement rural dans la région du Batha. Rapport de préévaluation, document de travail III. Développement de l'agriculture, de l'élevage et des ressources naturelles. Fonds international de développement agricole, $35 \mathrm{p}$.

\section{GuÉDON J., 2016}

Origine et évolution de la filière des noix de doum du Fitri : nécessité alimentaire et opportunité énergétique. Master 1 de géographie, université Paris 1 PanthéonSorbonne, $152 \mathrm{p}$.

\section{JofaCK S. V., 2016}

Cartographie des potentialités en eaux souterraines dans les hauts plateaux de l'OuestCameroun : contribution de la télédétection (optique et radar), des systèmes d'information géographique et des réseaux de neurones. 
Thèse de doctorat, université Félix-HouphouëtBoigny d'Abidjan-Cocody.

\section{KPEDEnOU K. D., BoukPessi D., TAnZidani T., TCHAMIE K., 2016}

Quantification des changements de l'occupation du sol dans la préfecture de Yoto (Sud-Est Togo) à l'aide de l'imagerie satellitaire Landsat. Sciences de l'environnement, laboratoire de recherches biogéographiques et d'études environnementales, université de Lomé, $\mathrm{p}$ : 137-156.

Kemsol Nagorngar A., Jofack S. V., Madjigoto R., Raimond C., RiRABÉ D., LIBAR J., 2018

Discrimination des cultures de décrue par la classification semi-automatique des images Landsat au lac Fitri (Tchad). International Journal of Engineering Science Invention, vol. 7, issue $2: 32-42$.

\section{Kemsol Nagorngar A., 2018}

Dynamique des cultures de décrue dans les zones lacustres soudano-sahéliennes de 1985 à 2015 : cas des lacs Fitri et Iro au Tchad. Thèse de doctorat, université Félix-Houphouët-Boigny d'Abidjan-Cocody, 178 p.

\section{Mbagogo Koumbrait A.}

«Pratiques et enjeux de la sécurisation foncière autour du lac Fitri ». Ce volume.

\section{Marty A., Zakinet D., Khamis D. D., BERNARD C., 2012}

Almy al Afia 2. Analyse de l'évolution des ressources dans le département du Fitri. Document principal. République du Tchad, programme d'hydraulique pastorale au Tchad central, Phase II. Antea-Iram, 128 p.

\author{
Noyola-Medrano C., Mering C., \\ Beltran R., Antonio M., 2009 \\ Évaluation du changement de l'occupation
}

du sol à l'aide des images Landsat et Spot: Champ volcanique de la Sierra Chichinautzin (Mexique). 24 ${ }^{\text {th }}$ Internacional Cartography Conference: The World's Geo-Spatial Solutions, ICC, 12 p.

\section{OulONA E., 2016}

Les conflits d'usage des ressources naturelles dans le système lacustre du Fitri : résultante d'une multiactivité et d'une multifonctionnalité de l'espace? Master 2 de géographie, université de N'Djamena, $110 \mathrm{p}$.

\section{Pias J., 1970}

La végétation du Tchad, ses rapports avec les sols. Variations paléoclimatiques au Quaternaire récent. Paris, Orstom, coll. Travaux et documents, 6, $49 \mathrm{p}+1$ carte.

\section{SAUNIER M., 2016}

Les territoires d'eau : enjeux et jeux de pouvoir pour la gestion des ressources halieutiques dans les lacs Fitri et Iro au Tchad. Mémoire de master 2 de géographie, université Paris 1 Panthéon-Sorbonne, 128 p.

\section{Triboulet C., 1995}

Les transformations des paysages du Diamaré et du bassin de la Bénoué (Nord-Cameroun). Étude à l'aide de l'imagerie Spot. Thèse de doctorat de l'École des hautes études en sciences sociales, spécialité géographie, $695 \mathrm{p}$.

YALIKUN T., RAIMOND C., Kemsol Nagorngar A., Zakinet D., Schuster M., Sylvestre F.

«Variabilité des crues et des paysages du Fitri depuis les grandes sécheresses des années 1970$1980 »$. Ce volume. 

La végétation pastorale du lac Fitri

\section{État des lieux et dynamique}

Ali Brahim BÉChIR, Koussou Mian-Oudanang, Alhassine MAHAMAT, Tchoudiba BOURDJOLBO

\section{Introduction}

En système d'élevage extensif tel que pratiqué dans le Fitri, l'essentiel de l'alimentation du bétail est assuré par les pâturages naturels. Cependant, ces écosystèmes pastoraux sont soumis à une forte dégradation en raison d'une part, de la péjoration climatique et d'une double pression agricole et pastorale et, d'autre part, à cause de la forte augmentation de la population humaine et des effectifs du cheptel due à une sédentarisation des pasteurs et au développement de l'agroélevage. En effet, l'homme par ses pratiques locales d'utilisation des terres agit sur la dynamique des phytocénoses (HigGINGS et al., 1999). Il provoque ainsi une transformation des écosystèmes qui s'amplifie au rythme de la croissance démographique. Face à cette situation, une gestion conservatoire de ces parcours s'impose. Cela exige une bonne connaissance de leur structure et de leur dynamique ainsi que des moyens d'évaluation à court terme de leurs potentialités. Au Tchad, peu de travaux ont été réalisés sur la végétation pastorale (GASTON et al., 1975 ; TOUTAIN et al., 2000) et plus particulièrement celle de la zone du Fitri (PIAS, 1970). C'est pourquoi, les connaissances biologiques et écologiques (structure, diversité et dynamique) sur les parcours naturels du Fitri sont insuffisantes et très anciennes. Cela constitue une limite et un grand handicap si l'on veut élaborer des programmes d'aménagement en vue d'une gestion 
conservatoire. Face à cette situation et à la menace qui pèse sur les parcours de la zone, la présente étude se propose d'identifier les différents types de pâturages, d'apprécier simultanément leur diversité floristique, leur structure ainsi que leur dynamique. La connaissance de la composition et de la structure de la végétation pastorale devrait ainsi permettre de renseigner sur les variations qualitatives et quantitatives des fourrages disponibles pour le bétail. La finalité est d'accompagner les acteurs vers une gestion raisonnée des ressources fourragères afin de répondre aux différents besoins, opportunités et contraintes.

\section{Matériels et méthodes}

\section{Présentation de la zone d'étude}

Le département du Fitri est situé au sud-ouest dans la région du Batha, au centre du Tchad entre les $12^{\mathrm{e}}$ et $13^{\mathrm{e}}$ parallèles Nord et les $17^{\mathrm{e}}$ et $18^{\mathrm{e}}$ méridiens Est (fig. 1). Il fait partie de la grande zone bioclimatique sahélienne, comprise entre les isohyètes $200 \mathrm{~mm}$ et $700 \mathrm{~mm}$. Le régime thermique est marqué par une longue saison sèche de neuf mois dont le plus chaud est le mois d'avril (40 à $41^{\circ} \mathrm{C}$ ) et celle relativement froide allant de décembre à février $\left(11\right.$ à $\left.22^{\circ} \mathrm{C}\right)$. La végétation est de type steppique.

\section{Méthode d'étude de la végétation}

\section{Choix des zones de relevés}

Les sites d'inventaires ont été choisis sur l'image satellitaire Landsat ETM+ de 2013 à une résolution de $28 \mathrm{~m}$. Le choix des zones de relevés a été guidé par le souci de couvrir une plus grande partie de la zone jugée représentative et de couvrir un nombre relativement important de types de végétation également représentatifs. Les collectes des données effectuées dans les différentes zones l'ont été selon une même méthodologie. Les données ont été ensuite croisées avec le type d'occupation de sol en présence : zones de pâturages, zones mixtes et zones agricoles exclusives. Chacune des zones de relevés retenues a fait l'objet d'une étude selon la méthode classique de relevé de végétation.

\section{Inventaire floristique}

Les relevés phytosociologiques ont été effectués selon la méthode de BRAUNBlanquet (1932) dans des placeaux ${ }^{1}$ de 30 x 30 m pour la strate ligneuse et 10 x 10 m pour la strate herbacée. À l'intérieur de chaque placeau, l'inventaire 


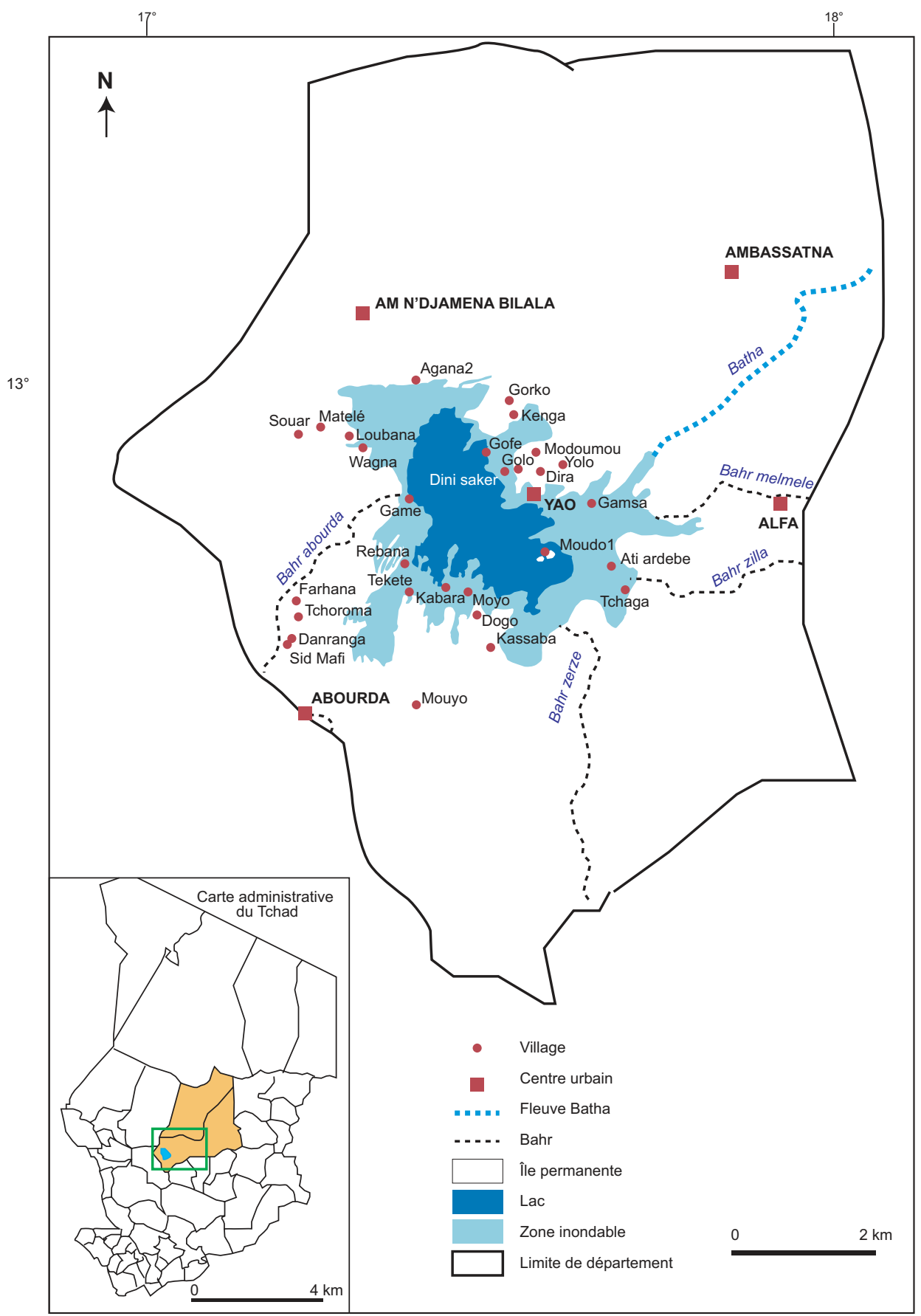

Figure I

Carte de localisation de la zone d'étude.

Source : P-Sidrat.

Réalisation :Tchoudiba Bourdjolbo, projection WGS84, 0I/03/20I7. 
des ligneux a concerné les arbres, arbustes et lianes dont le diamètre à 1,30 $\mathrm{m}$ du sol (D1, 30) est supérieur ou égal à $5 \mathrm{~cm}$. La circonférence du tronc à $1,3 \mathrm{~m}$ de hauteur (D1, 3) a été mesurée avec un ruban gradué. Le diamètre (D) a été ensuite calculé par la formule : $\mathrm{D}=$ circonférence/ $\pi$. Au total 298 relevés ont été effectués, dont 196 pour la strate herbacée et 102 pour la strate ligneuse.

La nomenclature adoptée est celle de African Plants Database du conservatoire et jardin botaniques de la ville de Genève ${ }^{2}$. D'autres observations ont pris en compte 5 aspects : la composition floristique, le recouvrement de la végétation, la géomorphologie, la nature du sol et la proportion de sol nu.

\section{Analyse et traitement des données}

La richesse floristique et les fréquences ont été déterminées sur la base de la liste floristique générale des différents relevés. Les fréquences spécifiques ont permis d'exprimer l'occurrence des espèces : les espèces fréquentes sont celles qui ont été recensées dans au moins $50 \%$ des relevés; les espèces peu fréquentes ont été celles qui sont présentes dans 25 à $49 \%$ de relevés et les espèces rares sont celles présentes dans moins de $25 \%$ de relevés. Les familles, les types biologiques de chaque espèce ont été représentés à l'aide des spectres bruts et pondérés. Le spectre pondéré est le rapport entre le recouvrement cumulé des espèces d'un type biologique et le recouvrement cumulé des espèces de tous les types biologiques (Sinsin et OuMOROU, 2000 ; FAYE, 2010). Ces calculs ont été effectués sur la base des recouvrements moyens (RM) correspondants à chaque classe d'abondance/dominance.

L'indice de Shannon (H), d'équitabilité (E) de PIÉLOU (1966) et le coefficient de similitude de SORENSEN (1948) ont été utilisés pour caractériser la diversité floristique des différentes unités de végétation, décrire la répartition des effectifs des différentes espèces des peuplements et évaluer la ressemblance floristique entre les différentes unités de végétation identifiées.

\section{Le spectre biologique ou forme biologique}

Pour apprécier la réaction d'un pâturage aux contraintes du milieu, il est important de classer les plantes selon la classification de RAUNKIÆR (1934). Adaptée par plusieurs auteurs (AKÉ Assi, 2002 ; MBAYNGONE et al., 2008) pour étudier des formations végétales tropicales, cette classification tient essentiellement compte de la position des bourgeons et de la taille de l'individu : les thérophytes (Th), les hémicryptophytes (He), les géophytes (Ge), les chaméphytes (Ch) et les phanérophytes $(\mathrm{Ph})$. 


\section{Résultat et discussion}

\section{Richesse floristique}

Au total, 372 relevés phytosociologiques ont été effectués, dont 204 pour la strate ligneuse et 168 pour la strate herbacée. L'examen global de la flore des différents sites a montré 187 espèces dont 108 herbacées et 79 ligneux réparties en 139 genres et 68 familles. Les plantes fourragères ont représenté 39,03\% de la flore totale. Les familles les plus importantes ont été les Poacées, les Mimosacées, les Capparidacées, les Fabacées et les Amaranthacées (fig. 2).

Les familles recensées n'ont pas toutes la même importance agrostologique. La forte proportion des Poacées et des légumineuses (Mimosacées, Césalpiniacées et Fabacées) confère une bonne valeur agrostologique aux différents types de pâturage du Fitri. La forte représentativité des plantes appartenant à la famille des Poacées et des légumineuses (Mimosacées, Fabacées et Césalpiniacées) sur ces parcours leur confère un intérêt agrostologique particulier. Ils constituent la zone de repli par excellence pour le bétail transhumant en saison sèche.

\section{La fréquence spécifique}

L'analyse des relevés montre que seulement 13 espèces (soit 28,32\%) sont fréquentes sur les différents sites d'étude. En effet, elles ont été recensées dans au moins $50 \%$ des relevés. Il s'agit d'Acacia nilotica $(78,55 \%)$, Balanites aegyptiaca (67,45\%), Acacia tortilis (58,74\%), Hyphaene thebaica (49,76\%), Acacia senegal (46,54 \%), Schoenefeldia gracilis (37,65\%), Cenchrus biflorus

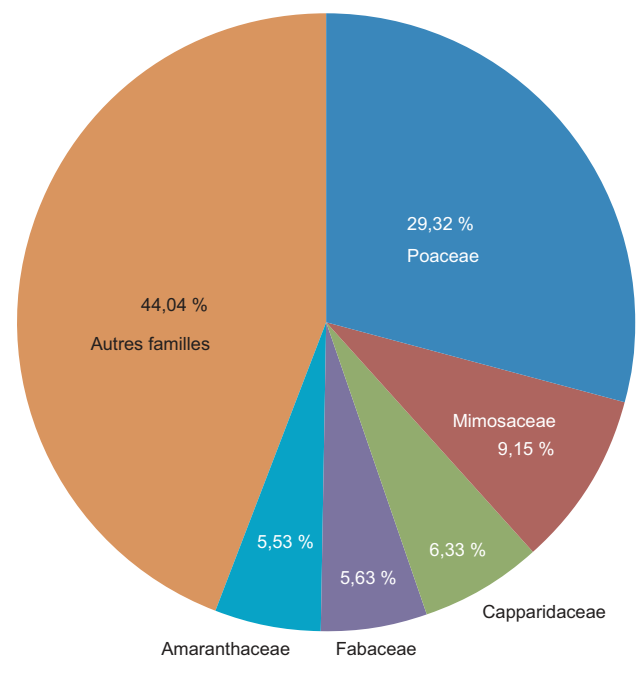

Figure 2

Spectre des familles. 
(57,20\%), Calotropis procera (52,21\%), Boscia senegalensis (43,65\%). La faible proportion d'espèces fréquentes observée traduit l'homogénéisation de la végétation décrite par plusieurs auteurs (JAUFFRET et LAVOREL, 2003 ; TARHOUNI et al., 2007). 23 espèces (16,20\%) sont peu fréquentes car recensées dans moins de $40 \%$ des relevés : Cordia sinensis, Ziziphus mauritiana, Pennisetum pedicellatum, Brachiaria deflexa, Dalbergia melanoxylon, Salvadora persica, Leptadenia pyrotechnica, Capparis separia, Tribulis terrestris, Zornia glochidiata, Alysicarpus ovalifolius et Chrozophora senegalensis.

Les espèces rares recensées représentent $12 \%$ de l'effectif global. Parmi celles-ci on compte un grand nombre de ligneux (Tamarindus indica, Piliostigma reticulatum, Kigelia africana, Stereospermum kunthianum, Diospyros mespiliformis) et d'herbacées (Ludwigia stolonifera, Panicum maximum, Andropogon gayanus...) très appréciés par le bétail. Ces fréquences montrent que seulement très peu d'espèces sont largement distribuées contre un grand nombre peu répandues sinon rares. En effet, des graminées vivaces comme Andropogon gayanus, Sporobolus helvolus, Panicum maximum, Cymbopogon schoenantus et autres herbacées très recherchées par les pasteurs telles que Blepharis linearifolia, Ludwigia stolonifera... ont complètement disparu.

\section{Spectres biologiques}

L'analyse des types biologiques a montré une prééminence des thérophytes ( $68,43 \%$ de spectre brut et $49,12 \%$ de spectre pondéré) et des phanérophytes ( $26,13 \%$ de spectre brut et $58,12 \%$ de spectre pondéré) sur les autres formes biologiques. Ils sont suivis de très loin par les hémicryptophytes $(7,22 \%$ de spectre brut et $5,41 \%$ de spectre pondéré), les chaméphytes $(5,18 \%$ de spectre brut et $1,38 \%$ de spectre pondéré) et les géophytes $(3,21 \%$ de spectre brut et $0,97 \%$ de spectre pondéré) (fig. 3). Cette dominance des thérophytes traduit une prévalence des formations savanicoles (MBAYNGONE et al., 2008).

La forte proportion des phanérophytes constitués majoritairement d'espèces ligneuses fourragères comme Maerua crassifolia, Acacia tortilis, Leptadenia pyrotechnica, Anogeissus leiocarpus, Balanites aegyptiaca et Acacia nilotica constitue un important apport fourrager pour le cheptel surtout pendant la saison sèche ou période de soudure. La prééminence des thérophytes est l'expression d'un climat sahélien à longue saison sèche et du substrat sableux et témoigne de leur adaptation aux stress climatiques (TARHOUNI et al., 2007). La présence des hémicryptophytes même en faible proportion, indique une stabilité des sols et des conditions d'humidité relativement acceptable (FoURNIER et al., 2000).

Les parcours peu ou pas fréquentés sont colonisés par des espèces phanérophytes ou des géophytes très peu appréciées par les animaux. L'impact de la présence animale sur la végétation est très important aussi bien sur le plan quantitatif que qualitatif. En outre, du point de vue pastoral, les associations végétales dominées par des annuelles et hémicryptophytes sont généralement plus appréciées par les animaux (RAKOTOARIMANANA et al., 2008). 


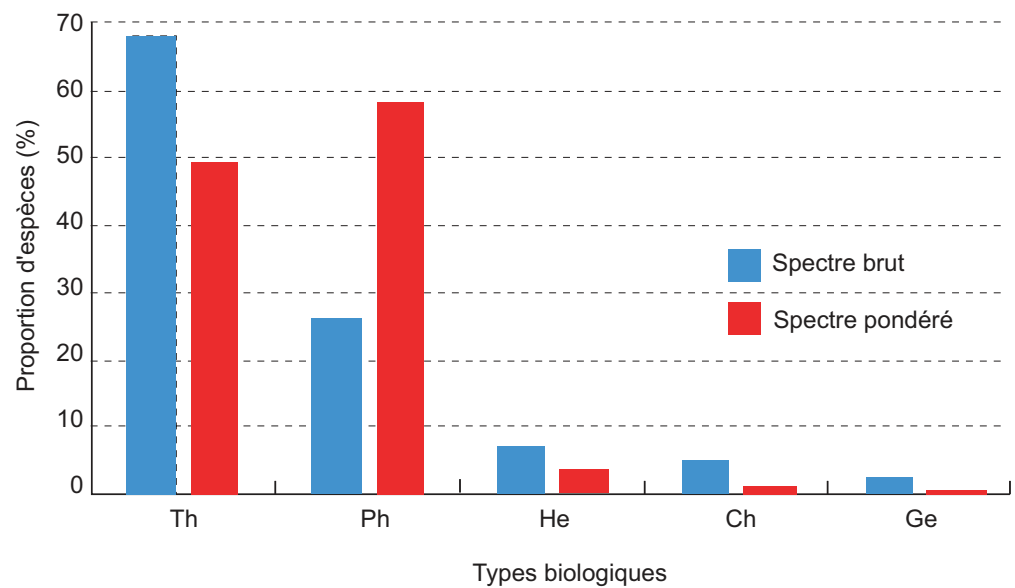

Figure 3

Spectres brut et pondéré des types biologiques observés dans les différents sites.

\section{Répartition des principales espèces ligneuses par classe de diamètre}

La figure 4 montre la répartition des espèces ligneuses selon les classes de diamètre. Toutes les espèces ont présenté des classes de diamètre significativement différentes. Avec un diamètre moyen de l'ordre de 7,8 $\pm 0,62 \mathrm{~cm}$, la végétation ligneuse de la zone montre un peuplement relativement équilibré bien qu'une forte proportion $(63,36 \%)$ soit représentée par des individus de diamètre compris entre $[0-5 \mathrm{~cm}]$. La présence massive de jeunes individus dans la première classe de diamètre serait favorisée par les conditions hydriques du sol (HIGGINs et al., 1999). La distribution des peuplements baisse à partir de classes de [20-25 cm]. Cette situation s'explique par le fort taux de pertes d'individus entre les classes [20-25 cm] et [>75 cm] comme cela a été observé par BÉCHIR et KABORÉZOUNGRANA (2012).

Pris individuellement, les peuplements d'Acacia nilotica et de Balanites aegyptiaca apparaissent plus équilibrés. La régénération est bonne chez ces espèces qui se sont distinguées des autres par une forte représentation dans des classes de diamètres inférieurs $[0-20 \mathrm{~cm}]$, traduisant une bonne dynamique de reconstitution.

En effet, les peuplements relativement stables d'Acacia nilotica et de Balanites aegyptiaca sont caractérisés par leur bonne représentation dans toutes les classes de diamètre. En revanche, les peuplements d'Acacia seyal ont montré un recrutement moyen dans les classes de diamètres inférieurs [10-15 cm] et [20$25 \mathrm{~cm}$ ] et ont été très peu représentées dans les classes de diamètres intermédiaires [25-30 cm] à [45-50 cm]. Ils étaient complètement absents dans les classes $[50-55 \mathrm{~cm}]$ jusqu'aux classes [>75 cm]. L'absence d'individus de l'espèce 


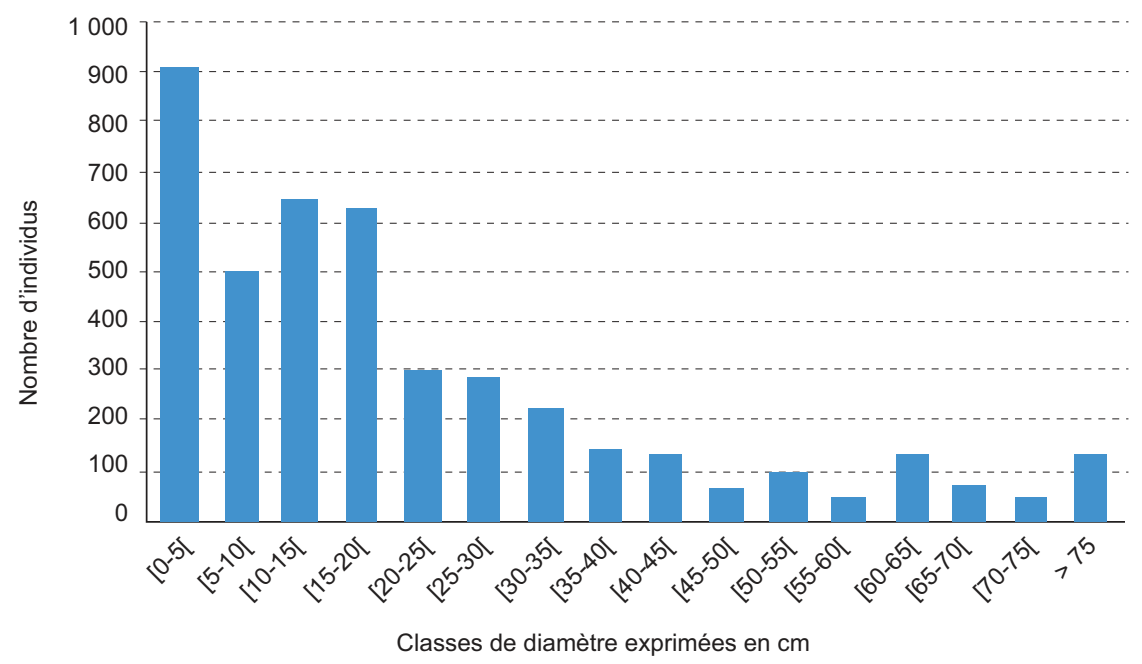

Figure 4

Répartition de l'ensemble des espèces par classe de diamètre.

appartenant aux classes de régénération et aux classes supérieures est un indice de perturbation en raison de leur surexploitation et de la pression auxquelles se trouvent soumis les individus de l'espèce (BÉCHIR et KABORÉ-ZOUNGRANA, 2012). En effet, le surpâturage autour des individus reproducteurs provoque l'épuisement des réserves racinaires au niveau des souches ou des plantules et entraîne une absence totale de régénération (LEJJU et al., 2001).

\section{Les principales unités pastorales identifiées}

Les pâturages du département du Fitri se subdivisent en 6 unités pastorales en fonction des facteurs physique (géomorphologie), biologique et anthropique (jachères et cultures).

\section{Unités pastorales I}

Les pâturages des savanes boisées sont localisés au sud du lac sur des substrats d'origine sédimentaire dans une zone de transition entre les sols ferrugineux tropicaux au sud et les sols bruns steppiques au nord décrits par PIAS (1970). La végétation marque une transition entre les steppes à épineux du domaine sahélien et les savanes à Combrétacées du domaine soudanien. Ce sont des savanes boisées très clairsemées. La strate ligneuse est dominée par Sclerocarya birrea, Combretum glutinosum, Anogeissus leiocarpus, Acacia senegal, Albizzia amara, Balanites aegyptiaca, Sterculia setigera, Celtis toka et Albizzia amara. On observe aussi des peuplements d'Hyphaene thebaica très dégradés sur sols steppiques en bordure du lac. En raison de la présence d'un nombre assez limité de plantes couvrant très peu le sol, ces unités pastorales ont le plus souvent un 
recouvrement faible à moyen (25 à $50 \%$ ). En effet, les espèces présentes y forment une étendue assez discontinue de prairies où sont associées plusieurs espèces. La strate herbacée dominée par Cenchrus biflorus, Eragrostis tremula, Schoenefeldia gracilis, Hyparrhenia rufa, Aristida stipoides, Chloris prieurii est très lâche et donc moins fournie. Elle a varié localement selon l'importance d'Hyparrhenia rufa ou Cenchrus biflorus qui n'ont pas une distribution régulière. Lorsque ces espèces sont très bien distribuées, des faciès à Eragrostis tremula, des variantes à Schoenefeldia gracilis et Aristida stipoides et enfin des faciès à Chloris prieurii apparaissent.

\section{Unités pastorales II}

Les végétations pastorales sur sols des $n a g a^{3}$ sont une variante beaucoup plus clairsemée des unités de savanes boisées et se localisent sur les bourrelets et dans les zones d'épandage, à l'arrière du cordon sableux. Elles se rencontrent également au sud-ouest et à l'ouest du lac où elles forment une mosaïque entre les sols steppiques sableux et les dépressions argileuses. Ce sont des savanes sur sols argilo-limoneux ou vertisols à inondation non excessive au voisinage des cours d'eau où elles alternent avec les pâturages des naga. Elles sont aussi observées à proximité du lac dans la zone deltaïque du Batha et sur la bordure sud-ouest où de grandes superficies de savanes ont été défrichées au profit des cultures de sorgho repiqué berbéré. La strate arbustive très clairsemée est dominée par Balanites aegyptiaca à laquelle se trouvent associées Dalbergia melanoxylon, Boscia senegalensis, Acacia seyal, Capparis decidua, Maerua crassifolia, Capparis corymbosa, Bauhinia rufescens, Anogeissus leiocarpus, Calotropis procera, Acacia trotilis, Lannea humilis... Des unités de végétation à Hyphaene thebaica très dégradées et occupant les sols steppiques bordent le nord-ouest du lac vers les villages de Gallo et Goffé. La strate herbacée discontinue et de petite taille est dominée dans la partie la plus sèche par Cenchrus biflorus, Schoenefeldia gracilis, Aristida mutabilis, Brachiaria sp., Eragrostis tremula et dans les zones les plus inondées par Panicum laetum, Brachiaria laeta, Sorghum arundinaceum, vetiveria nigritana, Cymbopogon giganteus et Hyparrhenia rufa. Parmi les légumineuses herbacées présentes, on peut citer Alysicarpus ovalifolius, Zornia glochidiata, Aeschynomes indica...

\section{Unités pastorales III}

Les steppes herbeuses à arbustives des ensablements et dunes sont localisées sur les plateaux sableux à morphologie dunaire ou aplanie dominante au nord de la zone. La strate ligneuse arbustive quelquefois rabougrie et très éparse est très basse (hauteur moyenne 2 à $3 \mathrm{~m}$ ) et ne se rencontre que dans les dépressions et certaines zones où les conditions d'alimentation du sol en eau sont assez

3. Le terme naga est un mot arabe tchadien, équivalent de hardé en peul. II désigne une végétation caractérisée par quelques ligneux très épars et à très faible recouvrement et une forte proportion de sol nu. Cette végétation n'est pas la résultante des facteurs climatiques mais au contraire elle est due à des facteurs pédologiques particuliers. Le sol est de couleur grise ou noire en surface avec par endroit des étendues sableuses claires et de faible épaisseur. 
bonnes. Les principales espèces ligneuses sont : Balanites aegyptiaca, Acacia tortilis, Acacia senegal, Acacia laeta, Acacia mellifera et Leptadenia pyrotechnica. Parmi les essences ubiquistes, on trouve assez couramment Salvadora persica, Ziziphus mauritiana, Bauhinia rufescens, Cordia sinensis, Boscia senegalensis et Calotropis procera, Asclépiadacée pionnière de la reconquête des zones sablonneuses les plus dégradées. La strate ligneuse est quelquefois marquée par la présence d'Acacia seyal, Mimosacée arbustive épineuse pouvant constituer de vastes peuplements sur des sols hydromorphes. On peut également citer des espèces compagnes comme Sclerocarya birrea, Combretum glutinosum, Anogeissus leiocarpus... La strate herbacée est formée d'un cortège assez varié d'espèces annuelles dominées par des graminées dont les plus fréquentes sont: Cenchrus biflorus, Aristida funiculata, Aristida adscensonis, Aristida mutabilis, Eragrostis ciliaris, Eragrostis tremula et Schoenefeldia gracilis.

\section{Unités pastorales IV}

Les parcours des savanes à Acacia seyal sur sols argilo-limoneux sont localisés sur des vertisols à inondation non excessive au voisinage des cours d'eau. Ces unités pastorales se montrent comme des variantes de la savane boisée mais très éclaircies. Elles ne résultent pas des effets climatiques mais au contraire elles seraient dues à des facteurs pédologiques particuliers. La monotonie de ces types de pâturages très clairsemés est quelquefois interrompue par des formations pastorales assez denses en bordure des cours d'eau ou des mares temporaires. En raison des contraintes édaphiques, la strate ligneuse est essentiellement dominée par Acacia seyal. Sur les berges le long des cours d'eau, la strate ligneuse est dominée par Acacia nilotica, Balanites aegyptiaca, Acacia tortilis auxquelles se trouvent associées Capparis decidua, Capparis corymbosa, Maerua crassifolia, Dalbergia melanoxylon, Boscia senegalensis, Lannea himils, Calotropis procera et Cordia sinensis. La strate herbacée de petite taille, discontinue et éparse est dominée dans la partie exondée plus sableuse par Schoenefeldia gracilis, Cenchrus biflorus, Aristida stipoides, Eragrostis tremula et Aristida mutabilis et quelques rares reliques de Cymbopogon giganteus en bordure des dépressions argileuses.

\section{Unités pastorales $V$}

Les pâturages aquatiques ou prairies marécageuses se développent principalement sur sols argileux très hydromorphes et inondés sur de vastes surfaces presque toute l'année. Ils occupent également les zones à hydromorphie temporaire en bordure du lac où ils occupent de vastes surfaces ainsi que les méandres créés par les crues. La strate herbacée graminéenne semi-aquatique est dominée par des espèces comme Hyparrhenia rufa, Andropogon gayanus, Setaria pumila et Setaria sphacelata. En bordure de l'eau sur les endroits moins inondés, on note la présence de Vetiveria nigritana, Ipomea aquatica, Aeschynomene elaphroxylon, et dans les zones les plus inondées Echinochloa pyramidalis, Echinochloa stagnina (bourgou), Panicum laetum, Ludwigia stolonifera, Oryza Barthii, Aeschynomene elaphroxylon, Pistia stratioides auxquelles se trouvent associées Polygonum sp., Cyperus spp. 
En eau profonde, le plan d'eau est colonisé par Nymphaea Lotus, Vossia cuspidata, Oryza barthii, Nymphea aquatica, Ludwigia stolonifera, Aeschynomene elaphroxylon... Sur la terre ferme en bordure du lac, des espèces arbustives ou arborées comme Acacia sieberiana, Piliostigma reticulatum, Hyphaene thebaica, Ficus platiphylla, Ficus gnaphalocarpa, Acacia albida, Mimosa sensitiva, Acacia seyal, Acacia nilotica, Balanites aegyptiaca, Ziziphus mauritiana sont observées.

\section{Unités pastorales $\mathrm{VI}$}

Les parcours des forêts galeries en bordure du lac, cours d'eau et mares sont situés dans le prolongement des affluents du lac Fitri. Ils augmentent de superficie lorsque les sols deviennent alluvionnaires, argilo-limoneux ou argilo-sableux. Ces sols sont généralement cultivés quand ils sont faiblement inondés. Ces types d'unités pastorales sont composés de grands arbres et d'un sous-bois bien fourni. En plus des espèces des prairies marécageuses, on peut citer Acacia nilotica, Tamarindus indica, Balanites aegyptiaca, Acacia albida, Anogeissus leiocarpus, Ficus platyphylla et Ficus gnaphalocarpa. Parmi les arbustes on note Ziziphus mauritiana, Albizzia chevalerie, Acacia ataxacantha, Bauhinia rufescens, Boscia senegalensis, Capparis tomentosa, Capparis corymbosa... La strate herbacée très lâche est représentée par Achyrentes aspera, Dicliptera verticilata, Panicum laetum...

La très forte concentration des animaux et leur séjour plus ou moins prolongé a provoqué un envahissement des parcours par des espèces appétées comme Cenchrus biflorus, Zornia glochidiata et Dactyloctenium aegyptium et par des refus comme Calotropis procera, Chrozophra senegalensis, Cassia obtusifolia et Glinus latioides. Cela constitue un signe d'une dynamique régressive de la végétation pastorale. Les espaces peu fréquentés par les animaux sont en revanche colonisés par Aristida mutabilis, Schoenefeldia gracilis, Tephrosia bracteolata. Ce résultat est similaire à celui rapporté dans la même région par BÉCHIR et MopATÉ (2015).

\section{Diversité floristique des différentes unités pastorales identifiées}

La diversité floristique est caractérisée à travers les critères décrits ci-après.

\section{Richesse spécifique et équitabilité de Piélou}

D’une manière générale, toutes les unités végétales identifiées ont montré un indice d'équitabilité de Piélou élevé compris entre 0,88 et 0,90 (tabl. 1). Ce résultat montre qu'il existe un certain équilibre entre les espèces. Les plus grandes valeurs d'indice d'équitabilité ont été observées dans les unités I et IV, ce qui signifie que leurs flores respectives ont été réparties de façon plus homogène et équitable. La plus faible richesse floristique a été observée au sein de l'unité II (13 espèces seulement). En revanche, les unités I, IV et VI ont présenté une importante richesse floristique (tabl. 1). Elles ont aussi enregistré les plus faibles indices de diversité. Ces résultats montrent qu'une grande 
diversité et une richesse floristique ne sont pas synonymes d'un pâturage de bonne valeur fourragère car les espèces non appétées peuvent y être dominantes.

Tableau 1

Richesses spécifiques, indices de diversité de Shannon et d'équitabilité de Piélou des différentes unités pastorales.

\begin{tabular}{|llll|}
$\begin{array}{l}\text { Unités } \\
\text { pastorales }\end{array}$ & $\begin{array}{l}\text { Richesse } \\
\text { spécifique }\end{array}$ & $\begin{array}{l}\text { Indice de diversité } \\
\text { de Shannon (en Bits) }\end{array}$ & $\begin{array}{l}\text { Équitabilité } \\
\text { de Piélou }\end{array}$ \\
\hline I & 5 I & $2,52 \pm 0,55$ & $0,90 \pm 0,009$ \\
\hline II & 13 & $2,86 \pm 026$ & $0,89 \pm 0,07$ \\
\hline III & 24 & $2,84 \pm 0,38$ & $0,89 \pm 0,06$ \\
\hline IV & 49 & $2,73 \pm 0,36$ & $0,90 \pm 0,05$ \\
\hline V & 19 & $2,84 \pm 0,39$ & $0,88 \pm 0,06$ \\
\hline VI & 46 & $2,96 \pm 0,55$ & $0,89 \pm 0,05$ \\
\hline
\end{tabular}

Tableau 2

Comparaison des coefficients de similitude de Sorensen des différentes unités pastorales identifiées.

\begin{tabular}{|lllllll|}
\hline Unités pastorales & I & II & III & IV & V & VI \\
\hline I & & & & & \\
\hline II & 0,23 & & & & \\
III & 0,27 & 0,41 & & & \\
\hline IV & 0,43 & 0,25 & 0,32 & & \\
\hline V & 0,37 & 0,26 & 0,29 & 0,24 & \\
VI & 0,25 & 0,25 & 0,26 & 0,28 & 0,26 \\
\hline
\end{tabular}

\section{Indice de Shannon}

L'indice de diversité de Shannon $(\mathrm{H})$ a été plus élevé pour l'unité pastorale VI qui fait partie des formations les plus pâturées en raison de leur proximité avec les points d'eau et aussi à cause de l'ombrage qu'elles offrent. Ce résultat est en accord avec ceux rapportés par KOUASSI et al., (2010) et BÉCHIR et MoPATÉ (2015) dans des zones agro-écologiques similaires. En effet les plus grandes diversités floristiques sont liées à l'hétérogénéité environnementale ou à la diversité des habitats (MBAYNGONE et al., 2008).

\section{Indice de similitude de Sorensen}

Le tableau 2 donne les valeurs d'indices de similitude de Sorensen entre les différentes formations pastorales. On observe qu'elles ont été toutes inférieures à $50 \%$. Les unités I et IV ont montré la plus forte similarité $(0,43)$, suivies des unités II et III $(0,41)$. La plus faible similitude a été observée entre les unités I 
et II, et IV et V (0,23 et 0,24 respectivement). La forte variabilité observée au niveau de l'indice de similarité entre les différentes unités pastorales identifiées serait liée à la durée et à l'intensité de leur exploitation plutôt qu'aux conditions écologiques et à leur localisation géographique (RAKOTOARIMANANA et al., 2008).

\section{Conclusion}

Cette étude a permis de faire un état des lieux de la dynamique de la flore et de la végétation pastorale en relation avec les conditions écologiques ou anthropiques. L'étude a montré une succession d'unités pastorales à importance agrostologique très variée. Mais en raison de leur exploitation intensive liée au maintien prolongé des troupeaux sur les aires de pâture, la richesse spécifique de la strate herbacée a considérablement diminué. La dynamique de la strate herbacée est marquée par la prédominance d'herbacées annuelles et la raréfaction des vivaces. Les prairies aquatiques se trouvent également menacées en raison de la forte colonisation agricole des espaces pastoraux. Cette compétition rend très difficile et conflictuel l'accès aux ressources pastorales et la mobilité du bétail. La présente étude constitue une étape dans l'établissement d'une base des données sur la flore et la végétation pastorales du lac Fitri.

\section{Bibliographie}

AKÉ Assi L., 2002

Flore de la Côte d'Ivoire : catalogue systématique, biogéographique et écologique II. Boissiera 58, $401 \mathrm{p}$.

BÉChIR A. B., Mopaté L. Y., 2015

Analyse de la dynamique des pâturages autour des ouvrages hydrauliques des zones pastorales du Batha ouest au Tchad. Afrique Sciences, 11 (1) : 212-226.

BÉChir A. B., Kaboré-Zoungrana C., 2012 Fourrages ligneux des savanes du Tchad: structure démographique et exploitations pastorales. Cameroon Journal of Experimental Biology, vol. $8 \mathrm{n}^{\circ}$ 1, 35-46.
Braun-Blanquet J., 1932

Plant Sociology. The study of plant communities (traduction anglaise de «pflazensoziologie Fuller »), GD, Conard High School, éd. université de Chicago, Chicago, USA, 439 p.

FAYe E., 2010

Diagnostic partiel de la flore et de la végétation des Niayes et du bassin arachidier du Sénégal : application des méthodes floristiques, phytosociologique, ethnobotanique et cartographique. Thèse de doctorat, université libre de Bruxelles, 253 p.

Fournier A., Yoni M., Zombré P., 2000 Les jachères à Adropogon gayanus en savane soudanienne dans l'ouest du Burkina Faso : 
flore, structure, déterminants et fonction dans l'écosystème. Étude Flore Végétation. Burkina Faso, 5 : 3-32.

Garba I., Touré I., ICKOWICZ A., 2012 «Évolution historique de la pluviosité ». In : Système d'information sur lepastoralisme au Sahel. Atlas des évolutions des systèmes pastoraux au Sahel 1970-2012. FAO et Cirad, éd. : 8-11.

Gaston A., Peyre de Fabrègues B., KLein H. D., Dulieu D., 1975

Pâturages du sud-ouest du Tchad. Tome 1 : Milieu, végétation, bromatologie. Tome 2 : Notice des cartes des ensembles pastoraux. Tome 3 : Cartes Cirad-IEMVT, 365 et 62 p.

Higgings I. S., Shackleton M. C., Robinson R. E., 1999

Changes in woody community structure and composition under constrasting lan use systems in semi-arid savanna. South Africa, Journal of biogeography, 26 : 219-627.

JaUfFret S., LAVOREL S., 2003

Are plant functional types relevant to describe degradation in arid, southern Tunisian steppes? Journal of Vegetation Science, 14 : 399-408.

Kouassi A. F., Adou C. Y., Ipou I. J., KAMANZI K., 2010

Diversité floristique des zones côtières pâturées de la Côte d'Ivoire : cas du cordon littoral Port-Bouët-Grand-Bassam (Abidjan). Sciences \& nature, 7 (1) : 69-86.

\section{LeJJU J. B., ORYem-Origa H.,}

KASEnene J. M., 2001

Regeneration of indigenous trees in Mgahinga Gorilla National Park, Uganda. African Journal of Ecology, 39 : 65-73.

Mbayngone E., Thiombiano A., Hahn-Hadjali K., Guinko S., 2008 Caratéristiques écologiques de la végétation ligneuse du sud-est du Burkina Faso (Afrique de l'Ouest) : cas de la réserve de Pama. Candollea, $63: 17-33$.

\section{Pias J., 1970}

La végétation du Tchad, ses rapports avec les sols. Variations paléoclimatiques au Quaternaire récent. Paris, Orstom, coll. Travaux et documents, $6,49 \mathrm{p}+1$ carte.

\section{PIÉLOU E. C., 1966}

Species diversity and pattern diversity in the study of ecological succession. J. Theoret. Biol. 10: 370-383.

\section{Rakotoarimanana V., Gondard H.,} Ranaivoarivelo N., Carrière S., 2008 Influence des pâturages sur la diversité floristique, la production et la qualité fourragère d'une savane des hautes terres malgaches (région de Fianarantsoa). Sécheresse, 19 (1) : 39-46.

\section{RAUNKIÆR C., 1934}

The Life Forms of Plants and Statistical Plant Geography, Oxford University Press, p. 2-104.

Shannon C. E., 1948

A mathematical theory of communication. Bell System Technical Journal, 27 : 379-423.

\section{Sinsin B., OUMOrou M., 2000}

Étude de la diversité spécifique du groupement à Cochlospermum tinctorium A. Rich.

des savanes arbustives du Nord-Benin. Acta Bot. Gallica, 147 (4) : 345-360.

\section{SORENSEN C. E., 1948}

A method of establishing groups of equal amplitude in plant sociology based on similarity of species content. K. Danske vidensk. Selsk, 5 : 1-34.

\section{Tarhouni M., Ben Salem F., Ouled}

Belgacem A., Henchi B., Neffati M., 2007

Variation de la richesse floristique en fonction du gradient de pâturage au voisinage de points d'eau. Sécheresse, 18 (4) : 9-234.

Toutain B., Touré O., RÉounodJi F., 2000 Étude prospective de la stratégie nationale de gestion des ressources pastorales au Tchad. Rapport final, République du Tchad, Cirad, rapport emvt 00-28, $102 \mathrm{p}$. 
Partie 3

\section{Gouvernance des territoires, logiques sociales et hétérogénéité des acteurs}

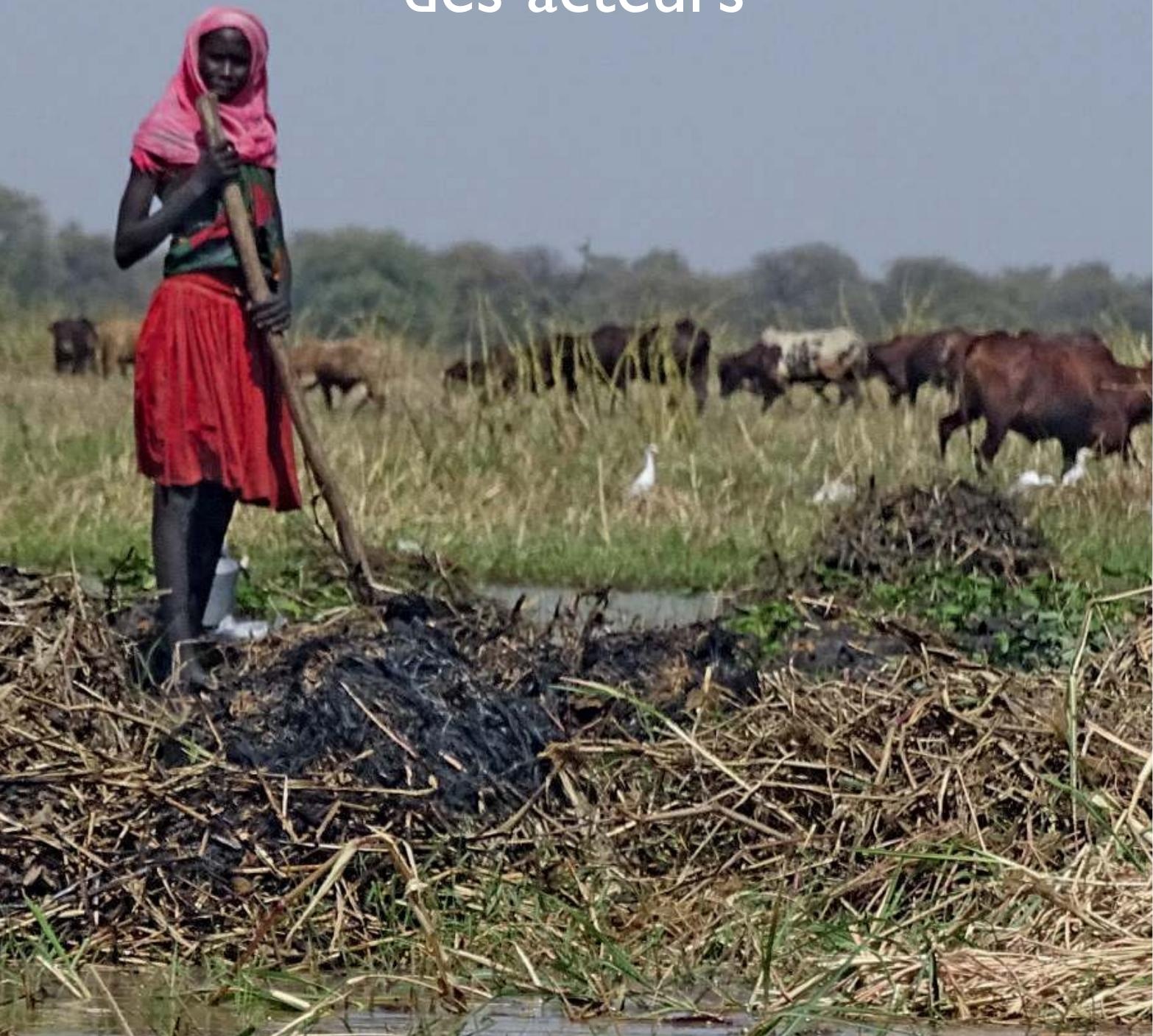


Multifonctionnalité de l'espace en bordure du lac Fitri (Gamsa, janvier 2016). Les jardins maraîchers et la pêche entravent le passage saisonnier des troupeaux vers les bourgoutières sur les îles.

(c) CNRS/C. Raimond. 


\section{Introduction}

Christine RAIMOND, Florence SYLVESTRE,

Dangbet ZAKINET

Les ressources en eau et en végétation qui caractérisent les lacs d'Afrique subsaharienne en font des lieux de vie privilégiés pour les sociétés humaines. L'ancienneté de leur occupation est variable en fonction de l'histoire et des nuisances à maîtriser pour s'adapter à un milieu parfois inhospitalier. L'absence de sociétés « hydrauliques » est remarquable dans le bassin du lac Tchad jusqu'au milieu du $\mathrm{XX}^{\mathrm{e}}$ siècle, où s'observaient dans les zones humides fluvio-lacustres de faibles densités de population et peu de grands aménagements. Les sociétés lacustres ont au contraire cherché à s'adapter à un environnement favorable mais souvent fluctuant, en suivant la ressource en eau au gré des saisons et en partageant l'espace entre plusieurs activités. La gouvernance territoriale et la régulation de l'accès aux ressources naturelles reposent sur ces interrelations étroites entre processus hydro-écologiques, organisation sociale et cultures.

Les lacs tchadiens diffèrent autant par leurs caractères physiques et les sociétés qui les habitent que par les modes de gouvernance. Les lacs Fitri $\left(800 \mathrm{~km}^{2}\right)$, Léré $\left(40 \mathrm{~km}^{2}\right)$ et Tréné $\left(7 \mathrm{~km}^{2}\right)$ sont intégrés dans un territoire plus vaste maitrisé par une chefferie centralisée et hiérarchisée assurant le gouvernement du territoire et la régulation des droits d'accès. Le sultanat bilala est installé au Fitri depuis le $\mathrm{XVI}^{\mathrm{e}}$ siècle par droit de conquête. Il assure depuis lors l'administration de l'ensemble des populations installées dans son territoire, rassemblé depuis la colonisation française jusqu'en 2016 en un seul canton. Une alliance ancienne avec les Arabes transhumants Djaatne leur octroie un droit d'usage saisonnier, selon des règles d'accès négociées et arbitrées par le sultanat. La chefferie moundang dirigée par le Gong de Léré est moins ancienne et s'appuie, elle aussi, sur une hiérarchie de chefs de villages. Ces deux chefferies existent encore aujourd'hui, même si leur puissance et leur légitimité diminuent face à la diversification des acteurs du territoire et aux réformes administratives successives. 
Les autres lacs tchadiens ne disposent pas de cette unité de gestion territoriale, maîtrisée par une seule institution coutumière. Le lac Tchad $\left(10000 \mathrm{~km}^{2}\right.$ depuis le début des années 2000) s'étend sur quatre pays frontaliers et partage son territoire entre plusieurs groupes. Les insulaires Boudouma occupent les îles de la cuvette nord du lac et dans les archipels, et ont été pendant longtemps les seuls à habiter l'espace lacustre. De grands empires se sont succédé sur les rives : sultanats Kotoko, empire du Kanem-Bornou, Mobber et Arabes constituent autant de modes de vie et de gouvernance différents qui se sont déployés à l'écart du lac considéré comme une vaste zone d'insécurité et d'insalubrité. Ce n'est qu'avec le retrait des eaux du lac à la suite des sécheresses des années 1970 à 1980 que cet espace est devenu attractif pour une population aux origines très diverses et qui s'est rapidement densifiée. Si les chefferies établies sur les rives ont tenté de suivre la progression de la création des villages à l'intérieur $\mathrm{du}$ lac, les règles d'accès et leur régulation y sont nouvelles et en constante redéfinition. Le retour de l'insécurité, marqué par les exactions du groupe Boko Haram et les mesures exceptionnelles liées à l'état d'urgence, remet tout le système en question.

De taille beaucoup plus modeste, le lac Iro est occupé par une société acéphale qui n'a pas d'institution de régulation de l'accès aux ressources naturelles et les activités s'y déroulent selon un système d'auto-organisation. L'appropriation des ressources en eau douce des lacs Ounianga, au cœur du Sahara, s'est faite au gré des guerres et des conquêtes. Un système complexe d'héritages familiaux transmet les palmeraies de génération en génération, mais ni les lacs ni les sources, dont l'emplacement varie en fonction de l'ensablement, ne sont appropriés individuellement ni gérés par une institution collective. La situation change avec l'attribution du label Patrimoine mondial de l'Unesco et la création d'un comité de gestion des lacs en 2012.

Dans tous ces lacs, l'eau et les ressources liées sont considérées comme un bien commun indivisible et inaliénable régies par un ensemble de règles plus ou moins anciennes, qui évoluent avec l'arrivée de nouveaux acteurs localement et les nouveaux cadres administratifs et politiques. La multiplication des conflits dans les zones lacustres, où se concentrent les activités de contre-saison dans un contexte de forte vulnérabilité, et les enjeux d'un retour « au développement» dans le lac Tchad, imposent de mieux comprendre ces systèmes de régulation endogènes. C'est l'objectif de cette troisième partie, qui approfondit notre connaissance du fonctionnement des socioécosystèmes en analysant les pratiques et les conflits entre acteurs, révélateurs des évolutions en cours.

Les cinq chapitres qui composent cette partie proposent une lecture du socioécosystème des lacs Tchad, Fitri et Iro à partir des principales activités qui y sont pratiquées : l'agriculture, l'élevage, la pêche et le commerce. Ils précisent les systèmes de production, les modalités de l'accès aux ressources et le rôle des institutions locales de gouvernance.

Deux contributions portent sur une région peu connue du lac Tchad, dans la partie nigérienne de la cuvette nord. Située au nord de la grande barrière, qui 
constitue un seuil de végétation à franchir pour la propagation de la crue venue du Chari, la cuvette nord est très vulnérable aux variations climatiques, marquées par plusieurs phases d'assèchement au cours du $\mathrm{Xx}^{\mathrm{e}}$ siècle. Ces conditions contraignent fortement les activités, qui ont connu un nouvel essor à partir de la fin des années 1990. Depuis 2013 et les débordements des actions de Boko Haram au Niger, cette zone est aussi très impactée par la crise sécuritaire. Pour mieux contrôler le lac Tchad où une partie du groupe Boko Haram s'était réfugiée, le gouvernement nigérien a expulsé la population et interdit toute activité dans le lac, créant de facto une zone de non droit où se recomposent les alliances et rapports de force entre éleveurs, mais aussi entre pêcheurs dont les activités ont repris avec un statut illégal. L'analyse de l'émergence de nouveaux conflits intercommunautaires entre des groupes de pasteurs qui n'ont jamais été en conflit auparavant démontre les enjeux de l'accès aux ressources pastorales lacustres devenues inaccessibles et les conséquences de la crise sécuritaire régionale.

Les autres contributions s'extraient de cette actualité brûlante pour replacer les systèmes de gouvernance dans leur évolution sur le temps long. Pour les activités de pêche et d'agriculture, qui s'inscrivent dans le même rythme saisonnier dépendant des crues, l'analyse des techniques, de la gestion des ressources et des conflits qui en découlent ne peut se faire sans mobiliser une connaissance fine du fonctionnement hydrologique et distinguer les pratiques en fonction des paysages contrastés à l'intérieur des lacs. Ainsi, sont analysées au lac Fitri les formes coutumières de la régulation foncière et la mise sous tension contemporaine de cette régulation sous l'effet de la pression démographique et de la montée en puissance de l'État au niveau local. La précision des informations apportées sur les pratiques permet de mieux comprendre les enjeux fonciers et l'influence de la chefferie traditionnelle pour ce lac. Les problèmes posés par l'émergence de l'État dans la régulation des ressources sont replacés dans un contexte politique national caractérisé par ses très faibles capacités institutionnelles et par la politisation - au service d'un système clientéliste - de la régulation des ressources naturelles. Ce chapitre souligne aussi la diversification des acteurs et la crise de légitimité du sultanat bilala, ce qui remet en question et en profondeur le système de gouvernance ancien.

Un apport original sur les logiques contrastées entre acteurs est celui de l'économie des échanges et de la contribution des zones lacustres à la sécurité alimentaire. Le bilan en produits agricoles (notamment céréaliers) de la région du lac Fitri replace la dynamique de développement par rapport à la problématique de l'enclavement et des limites à l'exportation qu'il représente. Que ce soit pour le lac Iro ou Fitri, et encore dans de nombreux secteurs du lac Tchad, l'enclavement constitue un frein principal à l'essor d'une agriculture commerciale pourvoyeuse de revenus pour les producteurs et de produits alimentaires pour les villes. Au Fitri, le désenclavement en cours par la construction de la route nationale qui rejoint Ngoura à Ati accélère le développement de nouvelles filières commerciales, comme le poisson frais et la noix de doum, en s'appuyant sur un réseau de commerçants préexistant. Toutefois, le commerce reste encore très contraint par la variabilité environnementale et la précarité des conditions de 
commercialisation des produits, ce qui explique l'alternance entre années excédentaires et déficitaires en produits céréaliers notamment.

Le dernier texte revient sur la question de la pêche avec une mise en perspective croisée entre les lacs Fitri et Iro sous l'angle des institutions et des pratiques permettant (ou non) sa régulation, en interrogeant la capacité des institutions coutumières à servir de support à des conventions locales modernes. Il fait écho au chapitre sur la pêche dans le lac Tchad sur la question de cogestion de la ressource halieutique, avec une mise en perspective par rapport à d'autres travaux sur la pêche continentale africaine. C'est en faisant l'analyse précise des pratiques locales de pêche, des conflits et des tensions en cours que sont identifiées des pratiques d'exclusion et définis de nouveaux territoires dans l'eau, à l'intérieur d'un espace théoriquement indivisible.

Finalement, toutes ces analyses renvoient aux réflexions sur la notion de commun et aux possibilités d'accueillir ou d'exclure de nouveaux groupes d'usagers. Elles démontrent les spécificités d'un milieu lacustre pour la gestion foncière et sa gouvernance en général, mais ne dessinent pas d'avenir inéluctable en faveur ou non d'une institution locale idéale, ou au contraire totalement dépassée par les tensions et les conflits. C'est dans l'intermède de la définition des arènes sociales, où les acteurs agissent en fonction de stratégies propres et d'héritages historiques, que s'inventent les nouvelles formes de gouvernance. 


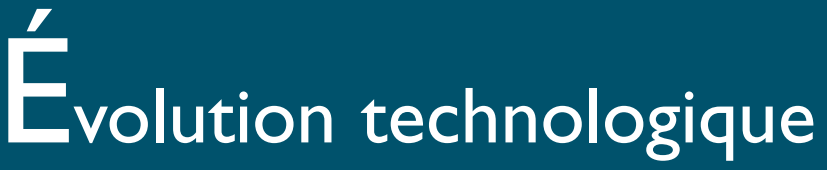

et gestion d'un espace halieutique dans la cuvette nord du lac Tchad

Hadiza KIARI Fougou, Jacques LEMOALLE

\section{Introduction}

La production annuelle de poisson du lac Tchad se situe entre 50000 et $150000 \mathrm{t}$ par an (KiARi FougOu et OUALBADET, 2015) avec une productivité de 25 à $75 \mathrm{~kg} /$ ha par an selon les années et les conditions de milieu. Depuis la sécheresse du début des années 1970, le fonctionnement hydrologique du lac est marqué par une variabilité de l'inondation de sa cuvette nord induisant une adaptation rapide des techniques de pêche. La pêche reste cependant très productive dans la partie nigérienne du lac, ce qui détermine l'occupation de cette zone par de nombreux pêcheurs de la région (tchadiens, maliens, nigérians, camerounais) qui représentaient plus de $55 \%$ de la population (KIARI FougOU, 2014). La pêche est une activité qui contribue à la fois à l'équilibre des recettes locales, à la résorption du chômage et à la sécurité alimentaire des populations riveraines du lac ou de la région. Durant les dernières décennies, la production annuelle de poisson pour la partie nigérienne a varié de $45000 \mathrm{t}$ en 2005 à 80000 t en 2014 (Kiari Fougou et Lemoalle, 2016).

L'introduction par les pêcheurs migrants de la technique de doumba (barrage de nasses) a permis d'augmenter rapidement les rendements de la pêche. Elle 
a permis de plus une appropriation foncière d'une portion de la mare et de l'eau et a offert l'assurance de disposer d'une portion de la ressource. Les droits des usagers de ces plans d'eau (pêcheurs et pisciculteurs) se limitent à l'accès, le prélèvement, la gestion et l'exclusion.

L'objectif de cet article est dans un premier temps de décrire l'évolution des techniques de pêche en fonction de l'évolution du lac Tchad, en particulier dans sa cuvette nord, et dans un second temps de comprendre comment les différents acteurs ont géré les ressources halieutiques et les conflits qui en découlent.

La partie nigérienne du lac Tchad qui constitue l'aire d'étude est une partie de la cuvette nord du lac essentiellement couverte d'eau libre et qui n'est plus alimentée en permanence par les apports d'eau du Chari. Plusieurs sites de pêche (fig. 1) caractérisés selon leur émergence ou submersion depuis la période de l'évolution récente du lac Tchad ont été visités dans les deux départements qui se partagent la partie nigérienne du lac et sa périphérie, à savoir les communes de Bosso et de N'Guigmi.

Les données collectées associent les sources, écrites, orales et observations de terrain. Parmi les données figurent nos travaux de recherche réalisés entre 2010 et 2013 qui correspondent à une situation d'avant l'interdiction de la pêche liée à la crise de Boko Haram dans le pourtour immédiat du lac Tchad (KIARI FougOU, 2014). D’autres données reposent essentiellement sur les enquêtes menées au

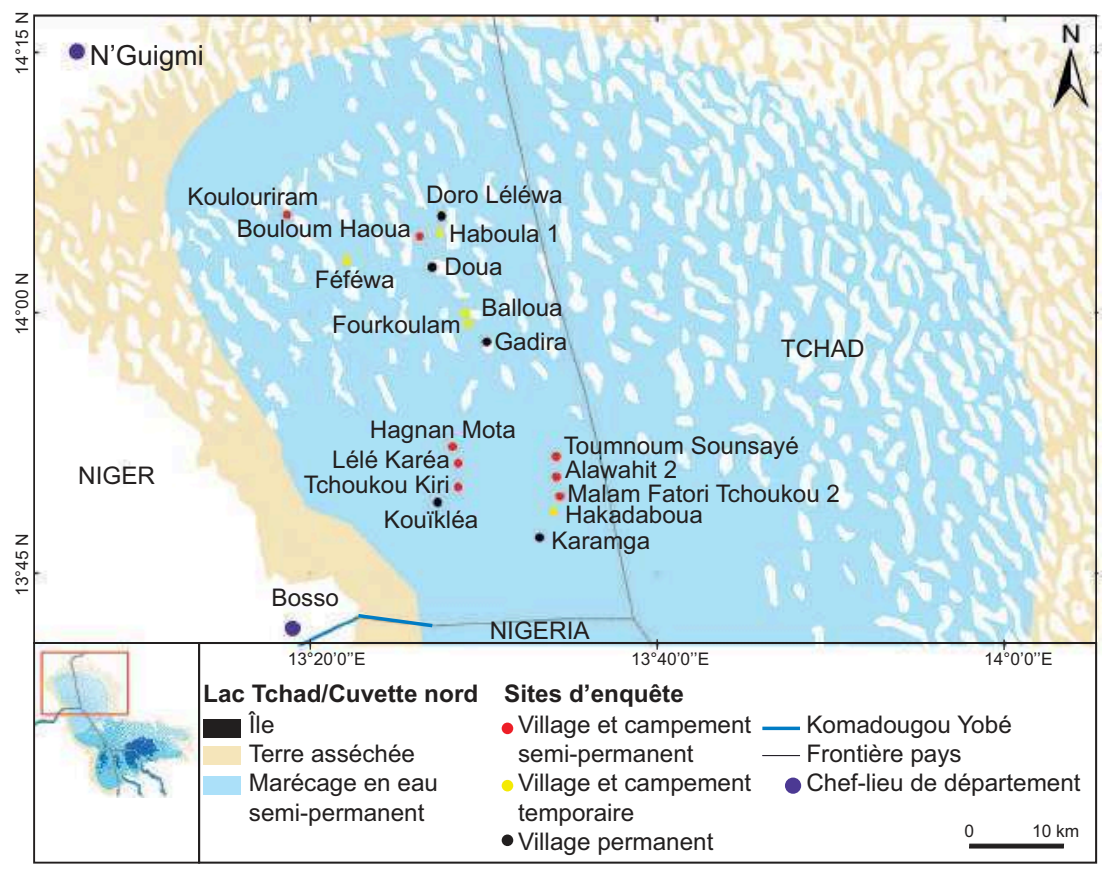

Figure I

Principaux sites de pêche dans la partie du lac Tchad au Niger. 
sein de la cuvette nord du lac entre 2010 et 2014. À cet effet, des personnes ressources et des acteurs clés identifiés ont été rencontrés pour des séances d'échanges. Il s'agit des pêcheurs et d'autres acteurs intervenant dans la gestion des ressources naturelles, en particulier les élites coutumières, ainsi que les autorités administratives et locales concernées.

\section{Fluctuations du lac Tchad et diversité de poissons}

La cuvette nord du lac est un espace de contraintes hydrologiques et d'imprévisibilité des ressources. Durant les années 1950 et 1960, en phase de Moyen Tchad, le lac présentait un grand plan d'eau unique d'environ $20000 \mathrm{~km}^{2}$ avec de grandes étendues d'eau libre peu accessibles aux pirogues des pêcheurs. Avec la diminution des pluies dans le Sahel et la baisse des débits des affluents du lac au début des années 1970, le lac Tchad a été scindé en plusieurs parties par l'émersion des hauts fonds. La cuvette nord, qui comprend la partie nigérienne, est irrégulièrement alimentée en eau et présente le plus souvent un paysage de marécage atteignant 6000 à $8000 \mathrm{~km}^{2}$, mais qui est parfois complètement asséchée (LEMOALLE, 2015). Seule l'importance de la crue du Chari, dont le maximum se produit fin octobre à N'Djamena, permet de prédire si la cuvette nord sera inondée à partir de février de l'année suivante. Le système est donc hautement imprévisible. Ces conditions hydrologiques jouent sur la composition du peuplement ichtyologique et génèrent un système écologique original. La survie de l'ichtyofaune qu'abrite ce milieu dépend étroitement de la disponibilité de l'eau dans le lac et des zones refuges de la Komadougou Yobé, rivière saisonnière qui alimente la cuvette nord. Lorsque la crue de cette rivière est importante, on observe face à son embouchure dans la partie nigérienne du lac une diversité de poissons qui attire les pêcheurs. En cas de faible crue, seules sont présentes les espèces de poissons sédentaires (Tilapias, Clarias, Heterotis) adaptées aux conditions difficiles (eaux de marécages faiblement oxygénées).

\section{Techniques de pêche et conditions de milieu}

Les conditions locales, et notamment la profondeur de l'eau ou l'encombrement par la végétation, influent sur la composition de l'ichtyofaune et exigent des 
techniques différentes. Chaque groupe de pêcheurs utilise des matériels de capture selon les habitudes de son groupe ethnique et le poisson recherché.

À l'échelle pluridécennale, on peut dire qu'il n'y a pas un brusque passage d'un ensemble de techniques à un autre, mais un recouvrement progressif qui s'étend à l'ensemble du lac.

Depuis le début des années 1950, en phase de Moyen ou Grand lac Tchad, on est passé de filets en fibres végétales à grandes mailles et des harpons à des filets en coton puis en nylon souvent à petites mailles et des lignes à hameçons industriels (fig. 2). En phase de Petit Tchad (avec des eaux moins profondes à partir de 1973) s'est répandue la dominance des nasses maliennes. Les engins de pêche ont suivi l'évolution technologique (nylon, etc.) mais aussi le niveau du lac et la composition des peuplements de poissons.

Le calendrier des activités de pêche au cours de l'année peut être défini selon l'amplitude de la crue lacustre qui elle-même dépend de la crue du Chari avec une distinction de trois années types : forte, moyenne et faible crue (fig. 3).

\section{Au cours d'une année humide}

Lors d'une année humide comme en 2011, on assiste à une dominance des trois techniques, le filet, la nasse doumba et les lignes d'hameçons (fig. 4). Ces types de matériels employés diffèrent selon les communautés des pêcheurs. La gamme la plus complète se rencontre chez les pêcheurs haoussa et boudouma. Par contre, les éperviers sont spécifiques aux professionnels de pêche djoukoune et quelques rares Haoussa.

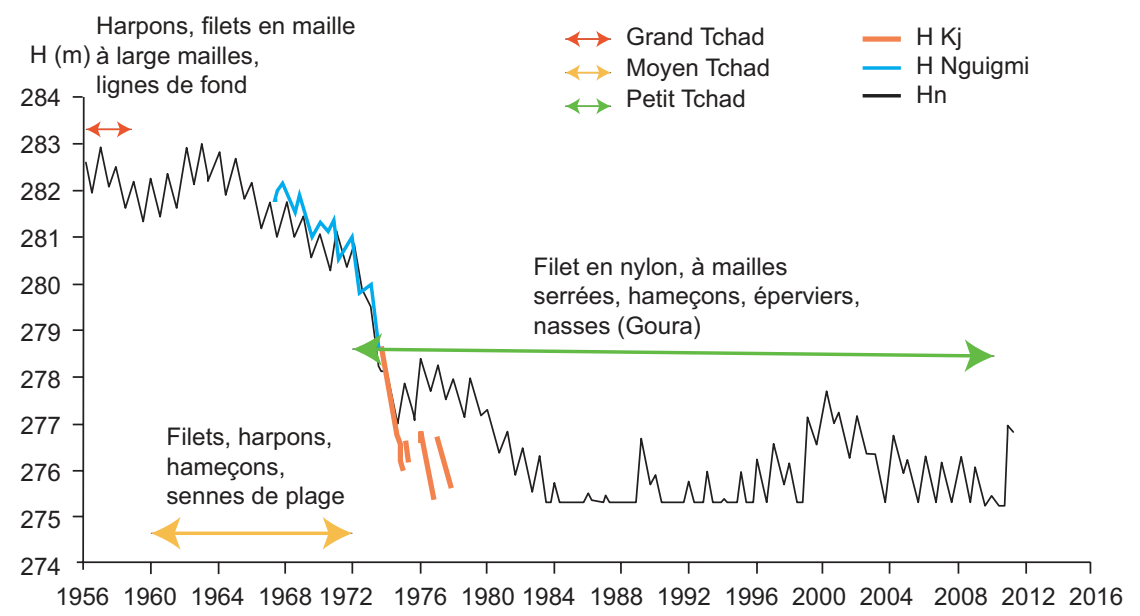

Figure 2

Les différentes techniques de pêche utilisées en fonction du temps et de l'évolution du niveau de l'eau dans la cuvette nord, observée à Kindjeria ( $H$ Kj) et N'Guigmi (H Nguigmi), et modélisées pour le reste de la période $(\mathrm{Hn})$. 


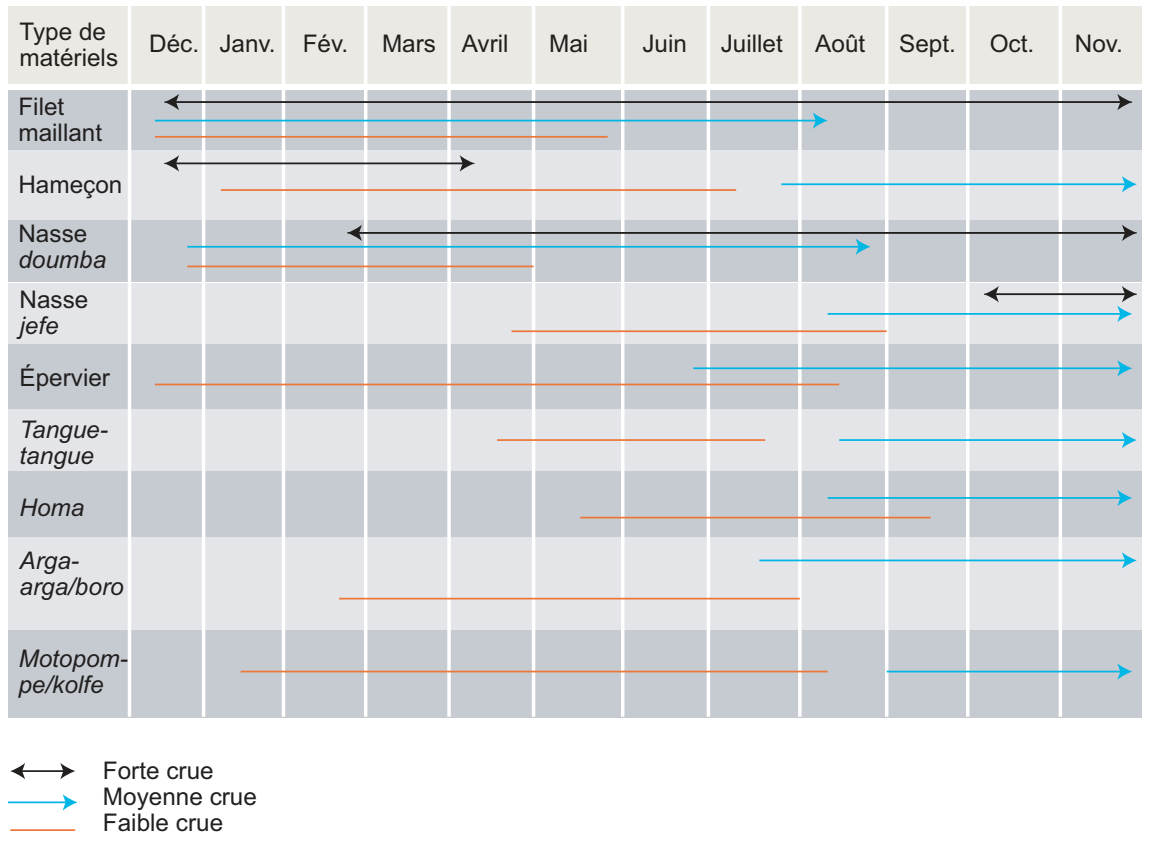

Figure 3

Les techniques de pêche suivant l'importance de la crue lacustre et de la saison.

En général, les pêcheurs boudouma et haoussa pratiquent en grand nombre la pêche aux nasses. Cette technique de pêche a gagné progressivement la partie nigérienne du lac Tchad depuis 1989 et a réactivé l'attractivité de la pêche compte tenu de la forte demande du poisson au Nigeria. Les hameçons sont utilisés surtout par les Haoussa et un peu par les autres groupes ethniques (Boudouma, Zarma, Kanouri). La campagne humide de pêche nécessite l'emploi de petites pirogues touke-touke et de goora ${ }^{3}$.

\section{Au cours d'une année de faible crue}

Il existe une diversité de matériels de pêche enregistrée pendant la période de mai à juin 2012 qui correspond à une année de faible crue comparée à la même période de 2011. Les différents groupes de pêcheurs ont associé plusieurs types

2. Petites pirogues en contre-plaqué dont les aires de construction sont concentrées à proximité des marchés au poisson dans les gros villages de Doro Léléwa, Gadira, Karamga, Blatoungour. D’une longueur moyenne de $4 \mathrm{~m}$, les touke-touke autorisent aussi le transport de marchandises légères, des petits ruminants sur de faibles distances. Elles permettent de transporter trois à quatre personnes au maximum mais peuvent être manœuvrées par une seule personne assise à l'arrière. Les petites pirogues sur les rives du lac se comptent aujourd'hui en centaines. Elles sont propulsées et dirigées par des pagaies (horami) en eau profonde par temps calme ou vent contraire et par des perches (djouom) dans des endroits peu profonds.

3. Grande calebasse de 0,8 à I $\mathrm{m}$ de diamètre, sur laquelle le pêcheur se couche à plat ventre pour en même temps pêcher et se déplacer. Elle sert d'embarcation pour installer les lignes d'hameçons dans les zones peu étendues ou encombrées de végétation qui limite le clapot. Le trou percé sur sa face supérieure permet d'y ranger le poisson. 
de matériels et techniques pour capturer le poisson compte tenu de la baisse rapide des eaux. Cette association varie d'une année à l'autre. Les principaux engins manœuvrés en 2011 ont été remplacés en 2012 par des techniques diverses comme la tangue-tangue, la boro, la motopompe, la kolfe et la homa (fig. 5). Cette période est marquée par la pêche à pied demandant plus d'effort physique.

Les petites sennes tangue-tangue sont utilisées par les jeunes pêcheurs âgés de 20 à 30 ans dont la plupart sont issus des communautés boudouma et haoussa. Cet engin de pêche est confectionné à l'aide d'une moustiquaire ou d'un filet à mailles serrées. Sa mise en œuvre se fait avec un équipage de deux personnes selon la longueur de la senne, puis le filet est tiré sur la berge. Ce matériel est

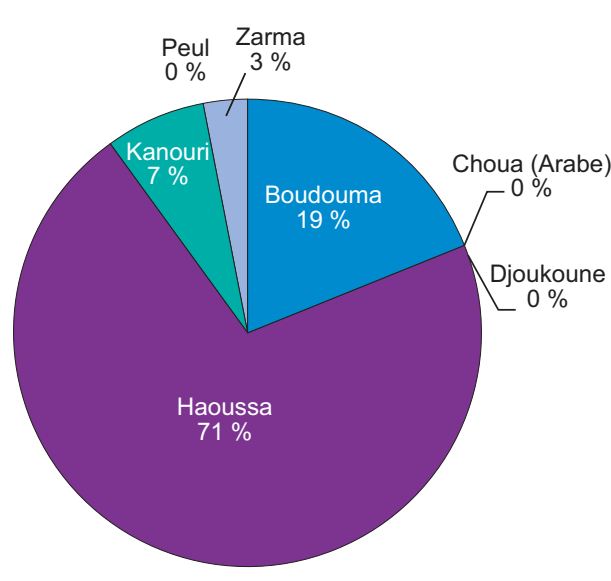

Hameçon

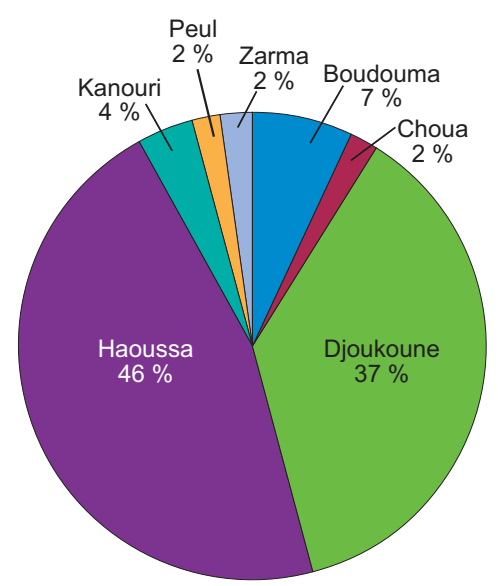

Filet

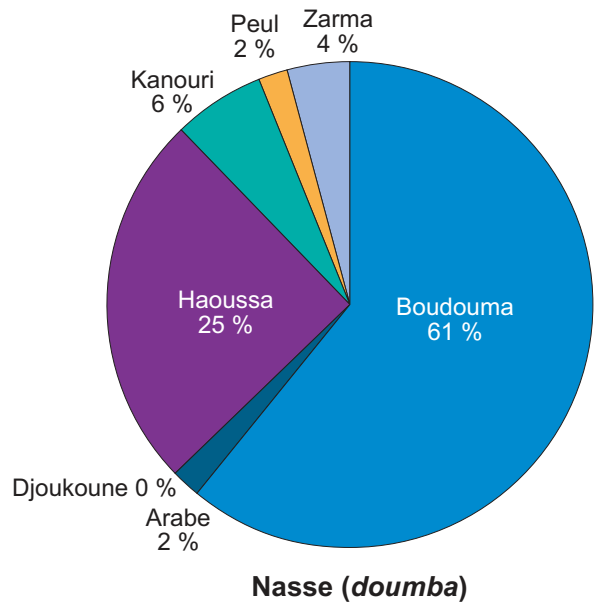

Figure 4

Principaux matériels utilisés lors d'une année humide et leur importance relative suivant les ethnies. 


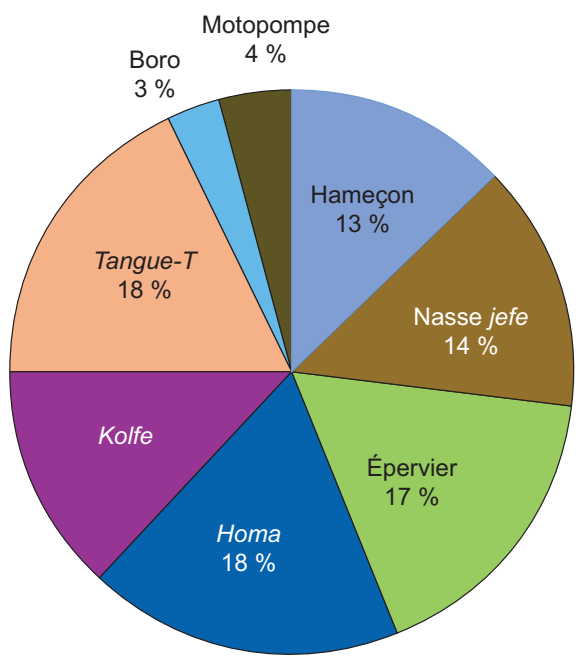

Figure 5

Diversité de techniques employées lors d'une année de faible crue.

appliqué en période de basses eaux quand les mares sont formées et nécessite des endroits qui ne sont pas encombrés de végétation.

La boro est un engin qui est conçu à l'aide de filets de mailles étroites formant une cage rectangulaire fixée par des piquets verticaux. La boro comporte sur l'un des côtés une grande porte d'entrée pour les poissons. La motopompe, à l'origine utilisée pour irriguer les cultures dans le lac, est devenue de plus en plus une technique de pêche. Cette technique permet de piéger les poissons dans les mares (des portions du lac). Elle consiste d'abord à barrer la mare avec des sacs remplis de sable humide ou avec des branches de Prosopis. Ensuite on place l'engin motorisé en bordure de l'eau afin de la pomper. Une fois que la mare est complètement vidée et l'eau rejetée plus loin, on ramasse les poissons. Cette technique moderne se fait lors de l'étiage ou durant les années de faible crue. Elle est pratiquée par de nombreux pêcheurs originaires du Nigeria pendant l'étiage, quand les eaux du lac forment des mares. L'usage de motopompe tend à se généraliser et se pratique de plus en plus par les migrants mais aussi par les pêcheurs locaux.

La technique de $k o l f e^{4}$ consiste à vider les eaux d'une portion du lac à l'étiage pour récolter les poissons. Cette technique proche de celle de la motopompe nécessite un effort physique du pêcheur. Ainsi le pêcheur divise le plan d'eau en deux parties par des branches de Prosopis et d'herbiers se trouvant aux abords de l'eau. Il utilise un morceau de bidon ou autre récipient et fait passer l'eau d'une partie à une autre afin de diminuer son niveau et ramasser le poisson. 
Une fois que les poissons sont ramassés, le pêcheur désinstalle cette barrière afin que le poisson puisse circuler librement.

La homa est un type de filet à armature triangulaire. Elle est maniée par une personne et utilisée à pied dans les endroits de faible profondeur.

\section{Émergence de nouvelles formes de pêche}

L'accès aux ressources dans la partie nigérienne du lac est le plus souvent réglementé par les autorités gouvernementales de l'État ainsi que par les autorités coutumières. Les règles étant le plus souvent associées au mode de pêche et à l'engin utilisé, nous analysons ici le mode d'accès pour les principaux engins.

\section{La gestion du système de doumba}

La doumba est une ligne de nasses permettant de barrer les eaux entre deux rivages, disposée généralement sur une ligne de 250 à $500 \mathrm{~m}$ de longueur à partir de la berge. Sa taille varie de 200 à 1000 nasses selon l'espace séparant deux villages et une kofa représente 7 à 10 nasses (fig. 6).

Elle est gérée par un pêcheur ou toute autre personne capable d'assurer la pérennité de son service. Malheureusement, la pauvreté croissante des pêcheurs limite leurs capacités d'investissement pour une doumba. Parmi les gestionnaires (ouban doumba), on retrouve une proportion importante des pêcheurs boudouma, des Haoussa migrants du Nigeria et des Maliens. Un seul ouban doumba peut également coordonner toute la ligne de doumba. Il est aussi de sa responsabilité d'attribuer une kofa au chef de village qui choisit lui-même l'endroit qui lui convient. Le chef de village a aussi le droit de désigner deux pêcheurs pour superviser une doumba. L'ouban doumba s'occupe également de la collecte de la somme à payer par chaque pêcheur concerné par la doumba et attribue les $k o f a$ aux différents pêcheurs. Chaque pêcheur utilise un morceau de tissu qu'il remet à l'ouban doumba qui s'en sert pour délimiter les propriétés. Un morceau

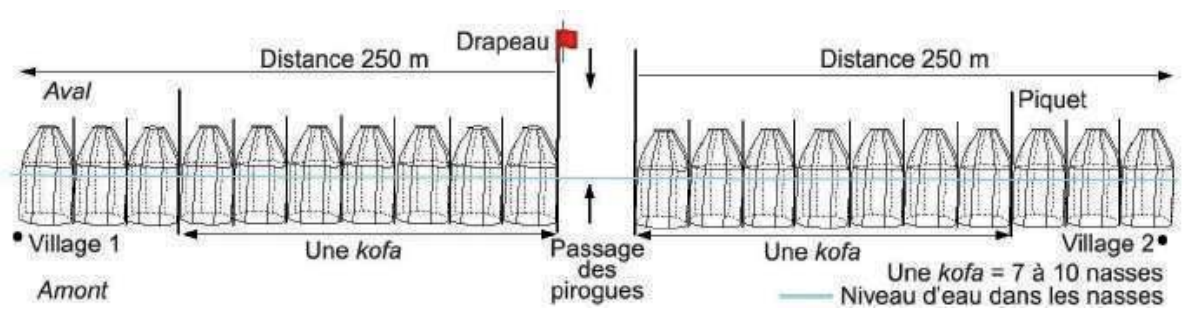

Figure 6

Description schématique du système de doumba. 
de tissu de même couleur accroché sur plusieurs kofa indique que celles-ci appartiennent à un même pêcheur.

Un délai de deux à sept jours est accordé aux pêcheurs pour installer leurs nasses sur la ligne de doumba. Au-delà de cette période, l'ouban doumba peut vendre cet espace à un autre pêcheur. En moyenne une kofa correspond à 10 nasses. Lorsque les nasses sont de grande taille, la kofa peut contenir 7 à 8 nasses. Si elles sont de petite taille, la kofa peut prendre 11 à 12 nasses. La limite entre deux kofa est matérialisée par un piquet. La longueur d'une kofa est en moyenne de $10 \mathrm{~m}$ et son prix fixé par l'ouban doumba varie de 2000 et 2500 nairas (10 à 16 euros). Un pêcheur peut avoir plus de $10 \mathrm{kofa}$, dans une ligne de doumba. Avant même la mise en place de la doumba, un barrage en filet est mis en place et les centaines de nasses sont ensuite alignées le long de ce filet. Il faut 5 bobines de filet à 10000 nairas pour fixer une ligne de doumba à 1000 nasses. À l'extrémité de la ligne de doumba, il y a parfois un espace libre. Dans ce cas, c'est le chef de doumba qui est censé compléter cet espace avec une ligne de filet.

À une distance de $100 \mathrm{~m}$ devant la doumba, un drapeau ou piquet est installé afin de signaler l'interdiction d'installer tout autre matériel de pêche. En fonction des endroits à occuper, l'ouban doumba verse au représentant du chef de canton un montant annuel qui varie entre 20000 et 50000 nairas (60 000 à 150000 FCFA, soit 90 à 230 euros) pour une petite doumba et de 100000 à 150000 nairas (300 000 à 450000 FCFA, soit 460 à 690 euros) pour une grande doumba.

En général, les nasses sont utilisées en eau peu profonde, mais de plus en plus, les pêcheurs développent des nouvelles stratégies pour les placer dans les parties profondes du lac. Ces stratégies consistent à augmenter leur taille ou à diminuer la profondeur du lac à en isolant des mares avec des sacs de sable. Ces derniers sont facturés à 2500 nairas l'unité. Pour une ligne de grande doumba, il faudra 500 sacs.

\section{L'accès aux autres techniques de pêche}

L'accès aux ressources par l'emploi d'autres techniques (filet maillant, ligne d'hameçons, épervier) se fait après le paiement d'une prestation symbolique au chef du village. En cas de bonne production, on peut offrir en récompense une petite somme d'argent à titre symbolique pour l'achat de sucre, du cola ou du thé. Tout comme pour la motopompe utilisée pour vider les mares résiduelles, l'autorisation pour l'utilisation des éperviers est fixée à une somme de 7000 nairas par pêcheur dans la zone de Bosso. Ce montant, imposé aux pêcheurs par les représentants des chefs de canton ou les chefs de village, est partagé entre le chef de canton et son représentant, le chef du village et les agents du service de pêche. Les pêcheurs étrangers migrants qui utilisent l'épervier jugent que les autorités coutumières veulent profiter d'eux. Ils se plaignent régulièrement du montant de cette taxe qu'ils considèrent comme illégale. De même pour la taxation des lignes qui sont autorisées si les distances entre les hameçons sont 
respectées $(30 \mathrm{~cm})$. Le montant perçu par les autorités coutumières pour l'utilisation d'une motopompe dépend de la taille de la portion du lac à assécher et se situe entre 2000 et plus de 20000 nairas. La mairie pour sa part avait taxé à 12000 nairas chaque pêcheur utilisateur de la motopompe.

\section{Des pratiques rituelles}

Bien que n'étant pas au sens strict des pratiques de pêche, les croyances et activités rituelles liées à la pêche sont à prendre en compte dans le cadre d'une description de cette activité.

De nombreuses sociétés du bassin tchadien considèrent que l'eau, comme d'autres éléments naturels, est imprégnée d'un esprit, d'une force vitale (SAMBO, 2010). Les Boudouma du clan Maïdjigodjia sont connus pour leurs forts pouvoirs mystiques et divinatoires. Il en est de même des Boudouma Baloua de la commune de Bosso dont l'influence sur la pêche est si importante que par leur incantation, ils peuvent favoriser une bonne ou une mauvaise campagne de pêche, selon les témoignages de plusieurs personnes interviewées.

En particulier, les Baloua sont considérés comme des gens de l'eau car, selon la légende, leur arrière-grand-père est né dans l'eau. Même le chef du village suit leur avis en cas de conflit. Ils ont le pouvoir d'empêcher le poisson de circuler et de réduire la chance d'un pêcheur d'en capturer après une dispute. Quand les Baloua manifestent un mécontentement suite à une dispute, ils obtiennent des excuses en raison de leur influence possible sur la campagne de pêche. Dans la portion nigérienne du lac, quand le poisson se fait rare, les Baloua font un sacrifice en jetant dans le lac une boule faite de farine de mil, de lait frais ou de beurre de vache. Quelque temps après, les poissons réapparaissent en abondance et se dirigent vers les filets. D'autres animaux aquatiques tels que les escargots ou les serpents font également leur réapparition à la surface du lac. C'est un vieillard qui est désigné comme maître de l'eau pour faire ce sacrifice. Une fois le sacrifice effectué, le maître de l'eau entre en premier dans le lac et effectue les premières prises de poissons. Lorsque le maître de l'eau meurt, c'est son fils qui lui succède. Au début de chaque saison de pêche, les danses de sacrifice au bord de l'eau durent pendant trois jours.

De nos jours, ces sacrifices se font plus rares chez les Boudouma à cause de l'influence de l'islam, de la cohabitation avec d'autres groupes ethniques et de l'implication de l'administration publique dans la gestion des ressources par l'attribution des permis de pêche. Cependant, chaque village fait des sacrifices au début de la campagne de pêche (début de la crue lacustre). Le chef de village (boulama), représentant le chef de l'eau, collecte une somme d'argent auprès des familles pour la donner aux marabouts du village pour implorer Dieu pour une meilleure production. En dehors du lac, des rites du même ordre se déroulent régulièrement et peuvent prendre un caractère d'événement public avec une nombreuse assistance. C'est le cas des deux grandes mares de Gaïdam Koura et Gaïdam Gana situées sur le cours de la Komadougou Yobé, qui donnent lieu 
à des grandes fêtes pour des pêches d'épuisement des mares au début de la saison de pêche avant la crue.

\section{Le système de réglementation}

Le système moderne de gestion de pêche vise surtout à conserver les ressources et à améliorer les rendements de pêche ainsi que les conditions de vie des pêcheurs. L'exercice de la pêche dans les eaux du domaine public de l'État et des collectivités territoriales est assujetti à la délivrance d'un permis de pêche sauf pour les bénéficiaires d'un droit d'usage. Le permis est accessible aux nationaux et aux étrangers. En ce qui concerne les engins de pêche autorisés, seules des contraintes de maille ou d'espacement sont stipulées ${ }^{5}$ (loi n ${ }^{\circ}$ 98-042 du 7 décembre 1998 portant régime de la pêche au Niger). La gestion des ressources se fait sous le contrôle des autorités coutumières représentées par les chefs de canton (Mai). Au Niger comme dans les autres pays riverains du lac Tchad, la décentralisation en vigueur laisse dans la réalité aux autorités locales une marge de manœuvre dans l'attribution des droits d'accès à la pêche.

\section{Une inégalité des droits d'accès à la pêche}

Il existe un grand nombre de circonstances sociales susceptibles de modifier l'accès d'un pêcheur, ou du groupe auquel il appartient, au lieu de production (PONCET et al., 1994). Il faut noter que l'accès à la pêche est aujourd'hui très inégalement réparti compte tenu des multiples techniques utilisées. L'appartenance des pêcheurs au groupe ethnique autochtone ou à une origine étrangère joue un rôle important dans l'accès à la pêche. Malam T. jeune pêcheur boudouma venant du village de Kindjeria (Tchad) dans la cuvette nord au Tchad (entretien du 22-05-2012) : « Nous sommes arrivés dans le village de Madouaram (à l'est de Doro Léléwa, au Niger) il y a deux semaines. Nous avions quitté Kindjeria au Tchad à cause de l'assèchement des eaux et la saison de culture est terminée. Ici au Niger nous avons eu un accès facile à la pêche, à l'amiable, car nous avons des liens de parenté avec le chef de canton de Kindjeria, donc nous n'avons rien payé pour installer nos matériels ». C'est en ce sens que PONCET et al. (1994) en étudiant le delta central du Niger au Mali disent que le partage de l'accès à l'espace productif n'est pas uniquement fondé sur ce que l'on possède mais aussi sur qui l'on est.

5. Au Niger, le code de la pêche interdit dans les eaux du domaine public de l'État et des collectivités locales : le filet épervier, la nasse, la senne, le filet maillant ou tout autre engin dont le côté de la maille est inférieur à 35 mm (3 doigts) ; la lance, la machette ou toute autre arme blanche ; la palangre dont les hameçons sont distants les uns des autres de moins de $30 \mathrm{~cm}$; la commercialisation du poisson immature pêché en territoire nigérien ou non, et la pratique, pendant la période de frai du poisson, de tous barrages non autorisés par le service de la pêche susceptibles d'empêcher le libre passage des poissons, crustacés, mollusques ou algues. 
Outre ce facteur, on note aussi l'ancienneté de l'installation dans un village, qui est un atout important pour l'attribution de l'espace de pêche. Après le retrait des eaux pendant un an sur le territoire d'un village, si le ouban doumba quitte ce village, il perd son espace l'année suivante même s'il y a inondation, alors qu'il aurait pu réoccuper sa doumba s'il était resté dans le village pendant cette période d'assèchement des eaux.

L'intégration au sein de la chefferie coutumière est importante dans les villages surtout parce que l'accès à la ressource est géré par les boulama qui sont en grande majorité des Boudouma alors que les wakil (les représentants) des chefs de canton de N'Guigmi ou de Bosso sont allochtones au lac. Ailleurs dans le lac, dans le delta du Chari et le long des côtes occidentales du lac Tchad, les études menées (BÉNÉ et al., 2003) montrent que l'acquisition des droits d'accès à la pêche entraîne systématiquement une certaine forme de paiement, soit en somme d'argent soit en équivalent en poisson (ou les deux). Les mêmes auteurs ont aussi montré l'existence des grands systèmes d'impositions illégales faites par des soldats des forces conjointes de patrouille ou d'autres agents de l'État. C'est en ce sens que QuENSIÈRE et al. (1994) estiment que les règles de gestion anciennes, basées sur le partage de la ressource et sa protection à long terme se sont considérablement affaiblies pour laisser place à une stratégie de profit immédiat. Certains pêcheurs migrants, en particulier ceux du Nigeria, peuvent corrompre le boulama pour obtenir un espace de pêche considéré comme plus poissonneux.

La répartition des doumba est très inégale. Ainsi, les familles de la chefferie boudouma détiennent les grandes doumba situées dans des grands espaces (dubel en langue kanouri) régulièrement en eau, alors que les pêcheurs migrants étrangers ne disposent que des petites doumba installées sur de petits dubel. La durée d'attribution varie d'un à deux ans pour un ouban doumba non issu de la famille du chef ou étranger migrant alors que si un ouban doumba est de la famille du boulama, la durée d'attribution de doumba est permanente. Cette situation engendre des conflits.

Une analyse sur l'accessibilité aux lieux de pêche relève que dans certaines parties du bassin du lac Tchad, seuls les plus riches ménages des pêcheurs ont accès à l'ensemble des plans d'eau disponibles, tandis que les ménages plus pauvres sont marginalisés, voire exclus de certains cours d'eau (NEILAND et BÉNÉ, 2003). Lors d'une année de faible crue environ un millier de pêcheurs locaux installés dans le village de Kanda Har, à l'ouest de Karamga, avaient porté plainte auprès des chefs coutumiers contre l'utilisation par les pêcheurs étrangers d'éperviers accusés d'avoir détérioré les lignes à hameçons et capturé des petits poissons. Les pêcheurs djoukoune d'une vingtaine de familles ayant utilisé les éperviers de façon illégale (sans prendre le permis) ont alors collecté entre eux une somme de 100000 nairas. Ce montant a été versé au service de l'environnement de Bosso d'une part en compensation pour avoir utilisé des éperviers et, d'autre part, pour régler les litiges avec les pêcheurs locaux. Ainsi, l'administration utilise la présence de pêcheurs étrangers corvéables pour justifier 
le niveau de prélèvement de taxe exigé auprès des pêcheurs riverains. De même, les pouvoirs locaux coutumiers feront appel à eux pour payer à l'administration des taxes dont le montant est jugé trop élevé (MiKOLASEK et al., 2000).

Le libre accès à la ressource n'est pas la règle et les bénéfices de la pêche restent entre les mains d'une minorité de privilégiés. La pauvreté associée à la pêche est donc plus le résultat d'un accès limité aux ressources et aux bénéfices des diverses activités liées à la pêche qu'aux stocks disponibles. Comme le notent également BÉNÉ et al (2003), notre étude montre une inégalité en matière de droit de pêche, les pêcheurs les plus riches ayant un accès facile au lieu de pêche tandis que les ménages plus pauvres sont marginalisés, voire exclus. Les autorités coutumières de la partie nigérienne du lac prélèvent une grande partie de ces frais auxquels s'ajoutent les prélèvements illégaux faits par des soldats des forces conjointes de patrouille ou encore d'autres agents de l'État au Tchad (BÉNÉ et al., 2003). On peut donc se demander dans quelle mesure la gestion par les autorités coutumières n'est pas un frein au développement social et économique du lac Tchad. La prédominance des autorités locales dans la gestion des pêcheries du lac Tchad a été critiquée par NEILAND et BÉNÉ (2003) repris par l'analyse Giwa (ForTNAm et al., 2004) où il est estimé que près de $40 \%$ des pêcheurs restent pauvres malgré la bonne productivité du lac Tchad (BÉNÉ et al., 2003). Ce système de gestion traditionnelle fonctionne bien pour réguler l'activité de pêche, mais crée des distorsions socioéconomiques.

On peut noter qu'il est difficile de définir un équilibre entre le respect des règlements actuels et une tolérance vis-à-vis des pêcheurs qui essaient simplement de gagner leur vie, d'où la nécessité de mettre en place des mécanismes de cogestion. Cette approche semble la plus adaptée aux défis actuels des pêcheries continentales africaines. Par conséquent, elle nécessite non seulement la présence d'acteurs sérieux au niveau local, mais aussi des arrangements préalables sur le plan juridique et pratique, auxquels sont associées les communautés, à l'appui de la gestion décentralisée et participative (BÉNÉ et al., 2008). Le département des pêches devrait, dans un environnement de cogestion, consacrer ses efforts à un suivi des pêches et à améliorer ses relations avec la communauté des pêcheurs afin de pouvoir donner des conseils fondés sur des résultats scientifiques. Le règlement des pêches devrait être revu pour tenir compte de la diversité des milieux.

\section{Pour une meilleure gestion des ressources halieutiques}

L'aménagement et la gestion des pêches demeurent des opérations complexes nécessitant la forte présence des acteurs de l'activité halieutique quant à leur soutien aux principes d'une cogestion. L'administration de pêche devrait également assurer une présence plus effective sur le terrain pour un meilleur suivi et contrôle des pêcheurs. Dans l'état actuel du lac, les agents affectés dans certaines zones, manquant de moyens et étant souvent considérés comme étrangers au lieu, ne sont à l'évidence pas en position de force pour faire respecter une réglementation mal acceptée par leurs voisins de tous les jours. Les propos 
recueillis auprès du responsable du service de pêche de N'Guigmi (entretiens du 3 juin 2011), montrent bien cet état de fait : « nous ne pouvons pas contredire les autorités coutumières sur certaines pratiques, ni parfois empêcher les pêcheurs d'utiliser certains matériels de pêche, comme la doumba. Il y a des années que les pêcheurs utilisent ces matériels, on essaie de fermer parfois les yeux car ils gagnent leur vie avec ça ».

$\mathrm{Au}$ lac Tchad, certaines caractéristiques méritent d'être prises en compte dans la gestion de pêche, principalement le peu de moyens pratiques pour faire respecter les règles, et donc le non-respect de la réglementation (matériels, mailles), la double hiérarchie (d'État/coutumière) avec risques de passe-droit pour l'accès aux zones de pêche et à la ressource, et l'imprévisibilité d'une année sur l'autre de l'hydrologie du lac Tchad et des ressources halieutiques.

\section{Conclusion}

Compte tenu de la diversité des techniques de pêche associée à l'imprévisibilité des variations du lac et de la ressource en poissons dans la cuvette nord du lac, les réglementations ne paraissent pas adaptées. Au contraire, le mode de gestion des services de l'État n'intègre pas suffisamment les variations des eaux, mais définit un ensemble de règles précises concernant l'accès à la ressource (par exemple via des règlements relatifs à la taille des mailles de filets ou à l'interdiction de certaines techniques). La gestion traditionnelle tient compte des situations écologiques locales, mais est souvent ambiguë en ce qui concerne l'accès à la ressource, avec un impact sur les revenus de certains pêcheurs. Il y a donc lieu de rechercher des règles de pêche adaptées aux différentes zones écologiques, à condition que celles-ci soient effectivement applicables et appliquées de façon claire.

\section{Bibliographie}

BénÉ C., NeILand A., Lewins R., 2008 Food security and poverty alleviation through improved valuation and governance of river fisheries in Africa. Policy analysis: Lake Chad basin and River Zambezi Basin synthesis report. Worldfish center, Cairo, 218 p.
Béné C., Neiland A., Jolley T., Ladu B., Ovie S., Sule O., Baba O., Belal E., Mindjimba K., Tiotsop F., DARa L., ZAKARA A., QUensière J., 2003

Natural resources institutions and property rights in inland African fisheries. The case of the Lake 
Chad Basin region. International Journal of Social Economics, 303 : 275-301.

Fortnam M. P., Oguntola J. A. (eds), 2004

Lake Chad Basin, GIWA Regional assessment 43. University of Kalmar, Kalmar, Sweden, Unep, $130 \mathrm{p}$.

\section{Kiari Fougou H., 2014}

Impacts des variations du niveau du lac Tchad sur les activités socioéconomiques des pêcheurs de la partie nigérienne. Thèse de doctorat de géographie, université Abdou-Moumouni, Niamey, Niger, 314 p.

\section{Kiari Fougou H., Lemoalle J., 2016}

« Le commerce de poisson dans la partie nigérienne du lac Tchad : analyse de l'évolution des circuits ». In : Actes du $1^{\text {er }}$ colloque international sur la culture de la paix et le développement dans le bassin du lac Tchad "Cléwa Salai », université de Diffa, Diffa du 2 au 4 nov. 2015, Niamey, Kashingo, p. $93-112$.

Kiari Fougou H., Oualdabet M. A., 2015

«Une pêche dynamique aux formes diversifiées ». In : Magrin G., Lemoalle J., Pourtier R. (dir.) : Atlas du lac Tchad. Paris, IRD Éditions/Passages, numéro spécial 183 : 92-94.

\section{LEMOALLE J., 2015}

«Les différents états du lac Tchad, un perpétuel changement ». In : Magrin G., Lemoalle J., Pourtier R. (dir.) : Atlas du lac Tchad. Paris, IRD Éditions/Passages, numéro spécial 183 : 23-27.

\section{Mikolasek O., Massou M., Allagbada E., 2000}

«Appropriation et gestion des espaces piscicoles nigériens par les populations villageoises riveraines ». In : Gascuel D., Chavance P., Bez N., Biseau A., éd. : Les espaces de l'halieutique. Paris, IRD, p. 517-526.

Neiland A., BÉNÉ C., 2003

Sustainable development of African continental fisheries: a regional study of policy options and policy formation mechanisms for the Lake Chad Basin. University of Portsmouth and European Commission, EU-Inco Project. Final Report, 286 p.

Poncet I., Kinzt D., Quensière J., 1994 « Systèmes transformables, ruralité durable au Mali. La ruralité dans les pays du Sud à la fin du $\mathrm{xx}^{\mathrm{e}}$ siècle ». In : Quensière J., éd. : La pêche dans le delta central du Niger. Approche pluridisciplinaire d'un système de production halieutique. Paris, Orstom/Karthala, vol. 1, $494 \mathrm{p}$.

Quensière J., Poncet Y., Fay C., Morand P., Kassibo B., Rey H., Baumann E., Bénech V., Bousquet F., Dansoko D., Herry C., Lä̈ R., Niaré T., Raffray J., Troubat J.-J., WEIGEL J.-Y., 1994

«Gestion des ressources deltaïques ». In : Quensière J., éd. : La pêche dans le delta central du Niger. Approche pluridisciplinaire d'un système de production halieutique. Paris, Orstom/Karthala, vol. 1 : 433-438.

\section{Sамво A., 2010}

Les cours d'eau transfrontaliers dans le bassin du lac Tchad : accès, gestion et conflits (XIX $-X X^{e}$ siècles). Thèse de doctorat, université de Ngaoundéré, 344 p. 



\section{e l'insécurité de Boko Haram} au conflit intercommunautaire dans le lac Tchad

\section{Le conflit Peul/Boudouma au Niger}

Mahamadou ABDOURAHAMANI, Maman WAZIRI MATO

\section{Introduction}

Le lac Tchad est un espace géographique potentiellement conflictuel, en raison de la diversité de ses ressources et de leurs fluctuations saisonnières, mais aussi par la pluralité des acteurs et la complexité des droits liés à leur exploitation. Cette situation est la conséquence du fonctionnement singulier de cet écosystème sahélosaharien.

Principalement alimenté par le sud avec le Chari qui lui apporte $90 \%$ de ses eaux, le lac Tchad est incisé par une barrière naturelle qui sépare la cuvette sud régulièrement alimentée en eau, de la cuvette nord plus profonde mais dont l'alimentation en eau dépend du seuil de franchissement de la barrière (LemoAlle, 1989). De ce fait, le remplissage de la cuvette nord est à la fois très aléatoire et plus variable que celui de la cuvette sud. La fluctuation des surfaces de marnage observée depuis la décennie 1970-1980, marquée par la baisse du niveau du lac et son passage durable au stade Petit lac (LuXEREAU et al., 2012), a des effets contrastés sur les activités économiques dans la région (MAGRIN, 2009). Le retour des eaux dans la cuvette nord depuis les années 2000 favorise les mises en valeur agricoles, pastorales et halieutiques au sein de la rive nigérienne. L'alternance et l'enchevêtrement de ces activités rurales engendrent une gestion complexe de droits d'accès aux ressources naturelles. Ainsi, la rive 
nigérienne est devenue au début $d u \mathrm{XXI}^{\mathrm{e}}$ siècle un espace convoité tant au niveau local par une diversité d'acteurs aux intérêts divergents, que national avec l'intérêt de l'État pour la production pétrolière. C'est dans ce contexte de vives convoitises sur les ressources naturelles que la crise liée à la secte islamiste Boko Haram intervient et amplifie les tensions déjà existantes (MAGRIN et PÉrouse De Montclos, 2018).

Le conflit Peul-Boudouma sur lequel porte la présente analyse est l'un des marqueurs de ces convoitises locales. Les Boudouma agropasteurs, pêcheurs et insulaires, vivent sur les îles du lac Tchad, auxquels s'ajoutent les Sougourti, éleveurs traditionnels de la vache kouri comme les Boudouma. Ces deux groupes font du lac leur véritable identité de par leur type d'élevage. Les Peuls Foulbé fonctionnent sur l'hinterland (zone dunaire des cuvettes pastorales) et élèvent des zébus rouges. Ils s'intéressent de plus en plus au lac Tchad qui leur offre à la fois du pâturage, mais aussi des possibilités de cultiver en saison sèche face à un élevage de moins en moins rentable. Les chameliers arabes mohamid sont les derniers éleveurs arrivant dans cet espace. À ces groupes d'éleveurs s'ajoutent les migrants agriculteurs majoritairement haoussa de plus en plus attirés par les cultures de décrue du lac.

Face à cette diversité d'acteurs aux logiques différentes dans un milieu aux ressources très fluctuantes, se posent les questions suivantes en termes de conflictualité qu'un tel melting pot prédispose : Quel rôle le lac Tchad a-t-il joué lors d'anciens conflits intercommunautaires ? Comment l'insécurité née de la crise de Boko Haram a-t-elle engendré le conflit actuel Peul-Boudouma? Quels sont les enjeux et les mobiles des affrontements sanglants et meurtriers entre ces deux groupes d'éleveurs sur la rive nigérienne du lac Tchad ?

Cet article montre comment, dans cette situation d'extrême confusion créée par Boko Haram, les positionnements avant-gardistes des groupes d'éleveurs pour l'accès et le contrôle des ressources naturelles du lac Tchad au Niger ont accentué et ravivé les tensions entre éleveurs, avec un éclairage sur le conflit PeulBoudouma. L'article est construit en deux grandes parties. La première présente les principaux groupes qui peuplent la rive nigérienne du lac Tchad, avec un accent particulier sur les groupes Peuls et Boudouma et leurs rapports au territoire. La seconde partie analyse l'influence de la crise née de l'insécurité de Boko Haram sur le conflit intercommunautaire Peul-Boudouma.

\section{Approche méthodologique}

La majorité des travaux scientifiques présentés dans des contextes de violences physiques extrêmes sont fondés sur des données collectées auprès des acteurs institutionnels. Or, les discours de ces bureaucrates, pour paraphraser OLIVIER 


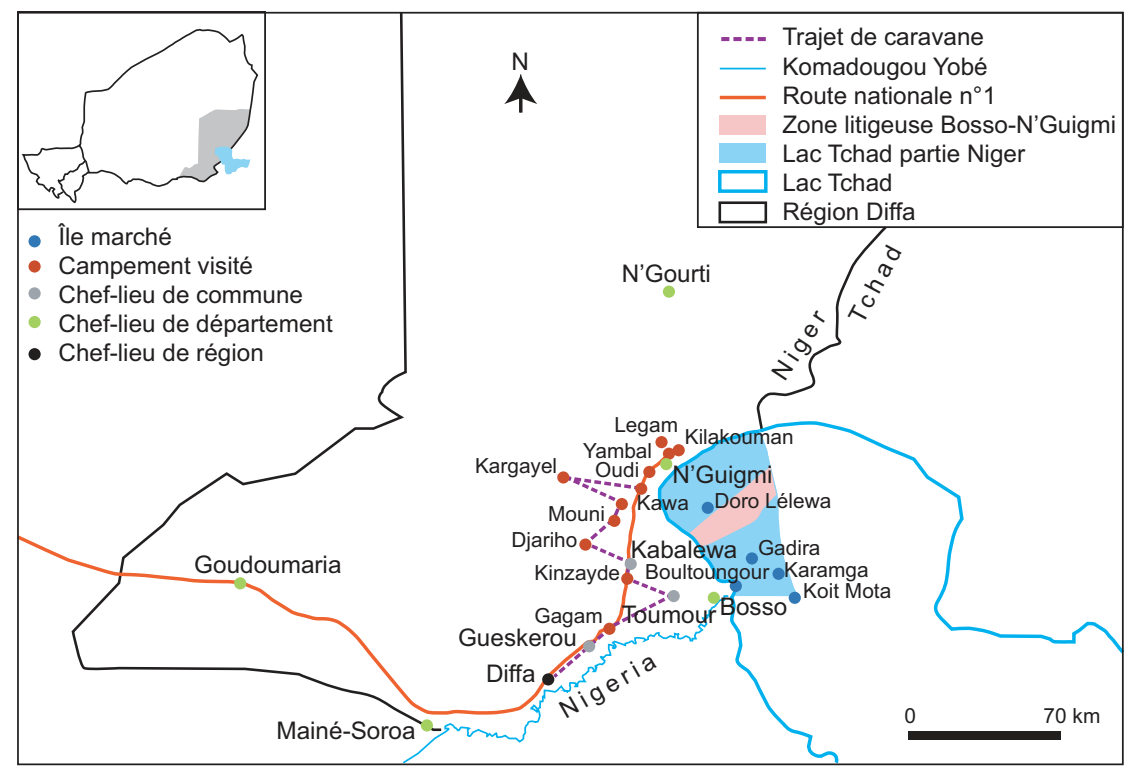

Figure I

Localisation de la zone d'étude.

Réalisation : Mahamadou Abdourahamani, 2017.

DE SARDAN (2012), sont parfois en déphasage avec ceux des acteurs véritablement concernés. La démarche méthodologique est essentiellement basée sur des entretiens individuels et collectifs, et apporte des témoignages in situ d'acteurs ruraux concernés, ce qui est original dans le contexte étudié de forte insécurité. En effet, profitant de l'opportunité d'une mission de terrain en août et septembre 2016 sur la médiation du conflit Peul-Boudouma, 20 campements situés dans la zone de Diffa ont pu être visités, dont 8 campements peuls et 12 boudouma (fig. 1). Participer aux assemblées générales organisées par cette mission, composées des élus locaux et des autorités coutumières ${ }^{1}$ ainsi que des responsables d'ONG œuvrant dans le domaine pastoral et de la paix ${ }^{2}$, a permis de comprendre de l'intérieur ce conflit complexe. En parallèle de ces grandes rencontres qu'un chercheur isolé ne pouvait pas mobiliser dans cette zone difficile d'accès, des entretiens individuels et des focus groupes de trois à une dizaine de personnes ont été organisés. Des compléments d'entretiens sur les mobiles de ce conflit avec des personnes ressources ont été aussi menés à Diffa.

I. La mission était composée des chefs de canton de Kawa, Toumour, N'Guigmi et N'Gourti et des maires de Kabalewa, Toumour et N'Guigmi.

2. Il s'agit de la Haute autorité à la consolidation de la paix (HACP), l'ONG Kouri et l'Association pour la redynamisation de l'élevage au Niger (Aren). 


\section{Peuplement de la rive nigérienne du lac Tchad et rapports des groupes au territoire}

La rive nigérienne du lac Tchad a connu un peuplement discontinu. Elle est composée de la partie intérieure du lac (partie insulaire), des rives très rarement inondées en cette phase de Petit lac, de l'arrière-pays ou la zone sableuse bordant le lac, et de la rive de la Komadougou Yobé. Cette partie de la rivière intégrée au système lac concerne uniquement la zone où la mobilité des populations est dépendante de la dynamique de la partie nigérienne du lac Tchad, de l'embouchure de la Komadougou Yobé à la commune de Gueskérou à l'est de Diffa. Les principaux groupes partageant la rive nigérienne du lac Tchad sont les Boudouma, les Kanouri, les Toubou, les Peuls et les Arabes. Parmi cette diversité de groupes ethniques, seule l'histoire des Boudouma et des Peuls est détaillée ici.

\section{Les Boudouma : les autochtones du lac}

De nombreux rapports d'archives coloniales du Niger présentent le lac Tchad comme un espace sociopolitique Yédina, communément appelé « Boudouma » ${ }^{3}$. Ils étaient alors les maîtres incontestés de la région du lac et considérés comme rebelles par l'administration coloniale (FOURREAU, 1902). Ils ont été longtemps écartés de tout contact avec la civilisation extérieure, pillant régulièrement les rives bornouanes (URVOY, 1949) et assurant l'hégémonie de la communauté boudouma sur les îles et les espaces inondables où ils élèvent des bœufs et des moutons. La période initiale de consolidation de cette influence demeure imprécise ${ }^{4}$, mais l'autorité boudouma sur le lac perdure jusqu'au cours du $\mathrm{XX}^{\mathrm{e}}$ siècle. Ils se singularisent des autres peuples de la région en ce sens que, retranchés dans leurs îles, ils n'ont jamais connu la tutelle de l'empire du Bornou, encore moins les razzias des Touareg, Toubou ou Arabes (ZAKARI, 1985).

Pendant les grandes sécheresses de 1974 et 1984, les insulaires boudouma suivent le retrait des eaux du lac Tchad et restent en perpétuel mouvement jusqu'au retour des eaux avec la grande crue de 1998. Les îles de Tchoukoudjani, Boultoungour et Koukléa représentent leurs plus anciennes zones de peuplement (Kiari FougOU, 2014). Jadis isolés et souverains sur ce territoire, les Boudouma sont confrontés depuis leur retour au début des années 2000 à un brassage forcé avec des arrivants de multiples origines (ANDERSON et MONIMART, 2009). Ils

3. D'après la tradition, les Boudouma viendraient d'un ancêtre du nom de Boulou appartenant à la tribu des Kanembou Yedina. Il aurait été installé dans l'île de Taguel sur les rives ouest du lac Tchad où il rencontra une tribu Sao dont il épousa une femme du nom de Sado Saoram. Pillards incorrigibles, les Boudouma sont de véritables écumeurs régnant en chef dans le lac Tchad qu'ils maîtrisent parfaitement (CHAPELLE, I957 ; ZAKARI, I985).

4. Nachtigal relève par exemple en décembre 1870, un gros village d'Arabes Shuwa surpris par les Boudouma : ces derniers massacrèrent une partie de la population mâle et emmenèrent femmes et enfants en captivité. Ces exactions demeurent impunies car, une fois dans leur domaine lacustre, les Boudouma défient toute poursuite (NACHTIGAL, I88I). 
restent fortement dépendants de la zone lacustre en raison de leurs vaches kouri, habituées aux parcours lacustres et supportant mal de longs déplacements en dehors du lac.

\section{Les Peuls : une arrivée récente appuyée par l'administration coloniale}

Les premières communautés peules s'installent au Niger oriental après la sécheresse de 1914. Elles arrivent dans une région nouvellement conquise par le pouvoir colonial, soumise auparavant à la domination du puissant empire Kanem-Bornou (VIII $-\mathrm{XIX}^{\mathrm{e}}$ siècles) d'une part et aux razzias des nomades toubou et arabes d'autre part. Selon Horowitz (1977) repris par THÉBAUd (2002), les premiers Foulbé à pénétrer dans le Niger oriental se fixent vers 1915 au nord de Maïné-Soroa sous la conduite de Kanta bii Baako, dont la famille détient encore la chefferie de Fulatari. Par la suite, d'autres Foulbé continuent à arriver en provenance de l'Ouest et sont surtout attirés par les cuvettes pastorales du Manga, où l'eau est facilement accessible (THÉBAUD, 1988).

L'arrivée et « l'insertion forcée » par l'administration coloniale française des Peuls Foulbé dans cette zone jadis parcourue par les Toubou et les Arabes Shuwa et Ouled Sliman désorganisent l'emprise territoriale de ces derniers (THÉBAUD, 2002). De plus, des structures administratives sont créées par cette même administration coloniale pour prendre en compte leur organisation politique spécifique basée sur la mobilité, qui contraste avec celle des sédentaires manga et mobeur organisés en villages et cantons. Ainsi sont mis en place des groupements peuls, comme celui de Toumour dans le cercle de N'Guigmi (MARTHÉ, 2015) qui demeure l'un de plus grands groupements peuls du Kadzell et de toute la région de Diffa. Ces dispositifs ont permis aux Peuls de renforcer leur présence au sein de cet espace pastoral Est nigérien. Cependant, l'ensablement et la dégradation continue des cuvettes pastorales sur lesquelles ils sont installés poussent un nombre croissant de groupes peuls à se réfugier sur la rive nigérienne du lac Tchad pour exploiter les ressources pastorales lacustres.

\section{Le rôle du lac Tchad dans les anciens conflits intercommunautaires du Niger oriental}

Le lac Tchad a joué un rôle majeur dans l'émergence et le développement des conflits qui ont secoué les groupes ethniques de l'est du Niger, dont le plus médiatisé est celui qui a opposé Peuls et Toubou entre 1985 et 2000. La descente et la réoccupation, pendant les années 1970-1980, des anciens territoires Toubou et Arabes au sud de la Dillia désertés par les Peuls Foulbé qui s'étaient réfugiés au sud du Nigeria en raison des années de sécheresse, est la cause de ce conflit (THÉBAUD, 2002). À leur retour, les Peuls se sont confrontés à la présence des groupes toubou et arabes sur leur ancien territoire. Au cours du conflit communautaire sanglant qui a duré plus de dix ans, le lac Tchad a servi aux 
milices peules de lieu d'entraînement, avec la caution de l'État qui a délibérément choisi de « fermer les yeux », voire d'armer les Peuls qui à l'époque combattaient à ses côtés les rebellions toubou du Front démocratique pour le renouveau (FDR). Ce conflit n'a pris fin qu'avec la médiation de l'Ardo Manzo, dignitaire peul très respecté des Toubou et occupant la cuvette Weltouma située à l'entrée de la zone toubou, appuyé par d'autres sages de la région (députés locaux et chefs de canton). Les accords négociés entre les groupes consistent à revenir à la situation d'avant la sécheresse de 1984, avec les Peuls au sud de la Dillia et les Toubou au nord. Dans ce conflit, la cuvette nord du lac Tchad a fourni une base arrière pour le groupe peul, en tant que zone peu accessible et non en raison de ses ressources naturelles.

Un autre conflit, moins ouvert, a opposé avant la crise liée à Boko Haram les insulaires Boudouma aux Mobeur agropasteurs de Bosso pour l'exploitation des ressources lacustres. Avec le retrait du lac Tchad suite à la sécheresse de 1984, les Boudouma ont été contraints de partir et de s'installer dans la cuvette sud. Beaucoup ne sont de retour sur les îles nigériennes qu'en 1999 avec la grande inondation de la cuvette nord et la reprise plus ou moins régulière des inondations depuis. Entre-temps, les plus grandes îles, et particulièrement les îles marchés (Boultoungour, Karamga, Koita Mota, Gadira), sont passées sous contrôle des Mobeur de Bosso qui se sont installés sur les terres vacantes. La cheffererie de Bosso installe ses représentants (boulama) qui assurent un contrôle foncier et distribuent les parcelles aux habitants de Bosso et aux migrants haoussa venus de plus en plus nombreux de l'ouest du Niger pour cultiver dans les zones de décrue du lac. En 2006, la chefferie de Bosso a voulu attribuer les terres de Tchilloua à ses populations mobeur de Baroua ; les Boudouma ont réagi par la force et des échauffourées ont éclaté et conduit à l'échec du contrôle de Tchilloua par Bosso, sans toutefois amener les Mobeur à renoncer à la colonisation des îles boudouma. Au cours des années 2007 et 2008, d'autres conflits violents explosent au sein de l'espace insulaire boudouma, provoqués par de forts différends fonciers du même type (Anderson et Monimart, 2009). Depuis ce temps, un conflit ouvert perdure sur fond d'autonomie boudouma vis-à-vis de la tutelle mobeur, et même des Kanouri de N'Guigmi. Les Boudouma, qui n'avaient jamais revendiqué de pouvoir au sein de l'organisation administrative nigérienne, posent comme objectif la création d'un canton boudouma, qui regrouperait toutes les îles de la partie nigérienne du lac Tchad. De fortes dissensions existent cependant au sein des Boudouma qui ralentissent le processus et empêchent d'arriver à un consensus. Des conflits territoriaux existent aussi entre les chefferies de Bosso et N'Guigmi : ils concernent, entre 1998 et 2012, 17 îles situées sur la frontière nord-est entre Bosso et N'Guigmi créant ainsi une véritable zone litigieuse entre les deux chefferies boudouma (ABDOURAHAMANi, 2013).

Au début des années 2010, quand éclate la crise Boko Haram, ces conflits fonciers liés au contrôle des ressources lacustres reconstituées depuis le retour des eaux ne sont pas résolus et sont fortement influencés par le différentiel démographique entre migrants haoussa et mobeur face aux Boudouma qui tend 
à s'amenuiser. S'ajoutent à cette situation complexe les Peuls de plus en plus nombreux qui exploitent les pâturages de décrue.

\section{La crise Boko Haram}

La crise née de l'insécurité liée à Boko Haram crée non seulement un bouleversement régional dans les pratiques pastorales habituelles des groupes, elle attise aussi les conflits intercommunautaires. Elle provoque des reconfigurations des rapports aux territoires au sein des groupes pastoraux d'une part, et entre groupes pastoraux et agropastoraux d'autre part.

\section{Boko Haram : genèse d'une insécurité régionale au sein du bassin tchadien}

La porosité des frontières dans la partie septentrionale du Nigeria a facilité l'expansion de la secte islamiste Boko Haram. Les frontières internationales ont permis aux insurgés de se réfugier dans les pays voisins et d'y établir des sanctuaires dans des zones où l'État était très peu présent (PÉROUSE DE Montclos, 2015a). Ceci a favorisé d'une part, l'expansion de la secte aux pays frontaliers des parties nord du Nigeria (Tchad, Niger et Cameroun) à partir de 2014 (PÉRouse De Montclos, 2015b) et, d'autre part, la transformation du lac Tchad en base arrière des islamistes de Boko Haram, dans un milieu très favorable aux insurgés du fait de son inaccessibilité (SEIGNOBOS, 2015). Depuis lors, l'influence et l'extension de Boko Haram se sont accentuées dans la région de Diffa, en particulier le long de la Komadougou Yobé servant de frontière entre le Niger et le Nigeria, et dans les îles de la partie nigérienne du lac Tchad. Pendant toute cette période, les exactions terroristes touchent les populations de plein fouet, les cas de violence physique, de kidnapping, de vol de bétail et de récolte se multiplient.

La fermeture de la frontière nigéro-nigérianne opérée en 2015 par le gouvernement du Nigeria, conjuguée aux débordements de la violence ${ }^{5}$ et au début des attaques de Boko Haram au Niger, a conduit les autorités nigériennes à instaurer l'état d'urgence $^{6}$. Fin avril 2015, le gouverneur de Diffa a imposé une évacuation forcée des populations des îles du lac Tchad au Niger. Cette mesure répressive pour limiter le contrôle de la zone lacustre par Boko Haram fait suite à la sanglante attaque (74 morts dont 46 militaires nigériens) perpétrée le 25 avril par le groupe terroriste sur la base militaire nigérienne de l'île de Karamga.

5. La psychose sécuritaire a commencé à gagner les populations de Diffa après les attaques de Damasak et surtout la prise de Malam Fatori en 2015, l'un des bourgs nigérians situé à seulement $3 \mathrm{~km}$ de Bosso.

6. L'état d'urgence proclamé le 10 février 2015, outre le plein pouvoir qu'il octroie à l'armée, impose l'interdiction de circuler de $21 \mathrm{~h}$ à $5 \mathrm{~h}$ du matin pour les populations et de $20 \mathrm{~h}$ à $5 \mathrm{~h}$ pour les véhicules. 
Dans ce contexte d'insécurité physique et matérielle, et de panique généralisée, les éleveurs et leurs animaux ont brutalement fait un mouvement d'ensemble vers le nord et nord-ouest du lac pour rejoindre les zones dites « de sécurité " par l'État (fig. 2). Or cette zone, de part et d'autre de la vallée de la Dillia, est peu pourvue en ressources pastorales au vu de la forte concentration d'animaux que ce mouvement spontané a engendrée. Face à l'arrivée massive de nouveaux éleveurs, les Peuls Foulbé renforcent leur appropriation des points d'eau et contrôlent l'accès aux ressources pastorales des autres pasteurs transhumants (Wodaabe, Bororo, Bokolodji, Boudouma, Sougourti). La concentration du bétail augmente le surpâturage et exacerbe les tensions liées à la cohabitation entre les groupes de pasteurs et d'agropasteurs, dans une zone où, même en année normale, l'exploitation des ressources pastorales est marquée par de fortes exclusions.

\section{Boko Haram, facteur d'exacerbation des tensions foncières}

Avant l'avènement de Boko Haram, la cohabitation était plutôt bonne entre Peuls et Boudouma. Ces deux groupes n'ont en effet jamais connu de guerres ouvertes, en dépit des multiples conflits ethniques qui ont secoué la partie nigérienne du lac Tchad. Les Boudouma ont même épaulé les Peuls dans les années 1990 en leur facilitant l'accès au lac et en les aidant à entraîner leurs milices dans ce milieu amphibie. Depuis l'installation de Boko Haram dans ce labyrinthe marécageux, cette entente entre Peuls et Boudouma est mise à rude épreuve avec plusieurs affrontements relevés (fig. 2).

L'antagonisme entre les deux communautés s'est creusé après l'évacuation forcée des populations insulaires par l'armée nigérienne (DIORI, 2015). Peu de temps après ce déguerpissement, les Peuls Foulbé et leurs milices d'autodéfense ${ }^{7}$ reviennent au lac et s'installent sur les îles en dépit de l'interdiction $\left(\mathrm{PCCN}^{8}\right.$, DifFA, 2016). Cet acte est très mal perçu par les Boudouma qui le considèrent comme un parti pris de la part des autorités de Diffa en faveur des groupes peuls (HASSANE et al., 2016).

La mobilisation de l'ethnie comme arme de défense de la cause commune est utilisée tant par les Peuls que par les Boudouma. Tous les discours tenus par l'une ou l'autre communauté sont centrés sur un relent identitaire fortement ethnicisé. Pour les Boudouma, il s'agit de défendre le milieu unique (le lac Tchad) qui incarne « l'identité boudouma » et asseoir leur suprématie sur les ressources lacustres grâce notamment à l'imposition de droits d'accès aux autres groupes, particulièrement aux Peuls. Les propos d'un leader boudouma interrogé en septembre 2016 sont illustratifs : «Les Peuls sont nos amis, nous leur donnons des champs, des zones pour pêcher et pâturons ensemble au lac. Sur les 18 boulama du lac, l'un d'eux est même un Peul de Weltouma ». Pour les Peuls, il s'agit de se procurer une portion de terres agricoles et pastorales et de se

7. Certaines sont des héritages des anciennes milices constituées contre les Toubou dans les années 1990.

8. Programme de cohésion communautaire au Niger. 


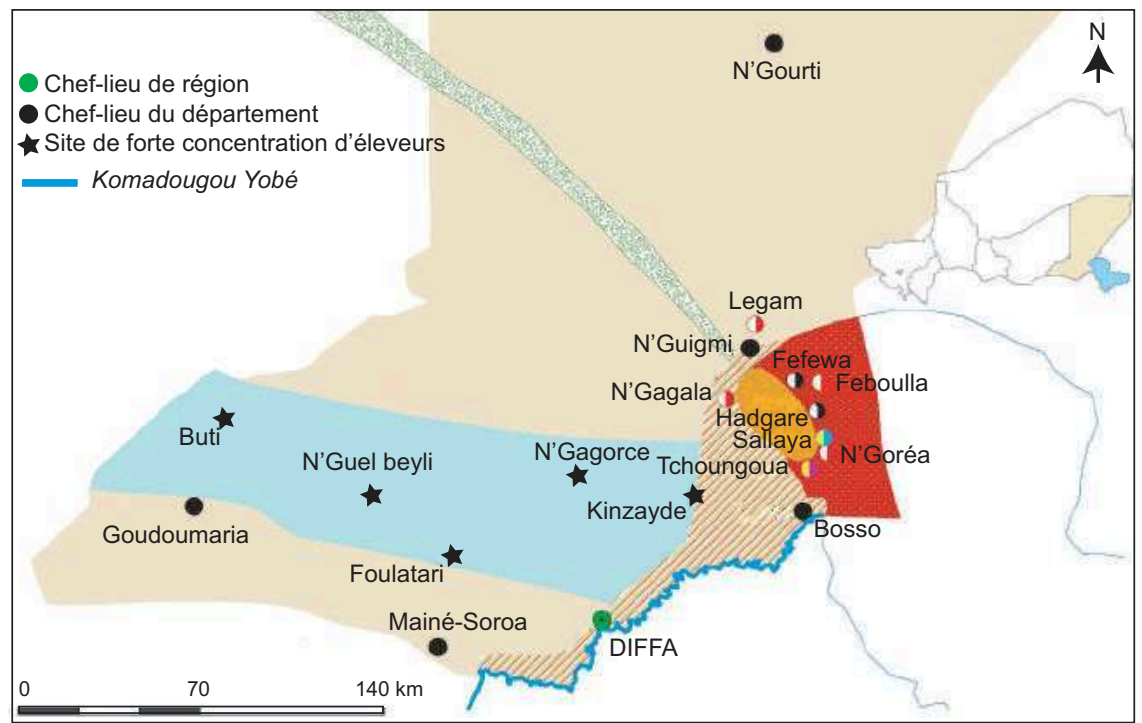

Lieu d'affrontements avec des groupes d'éleveurs ayant fait au minimum 5 morts

- Peul et Boudouma

- Peul et Boko Haram

- Arabe Mohamid et Boudouma

- Arabe Mohamid et Boko haram

Politique de sécurité d'État

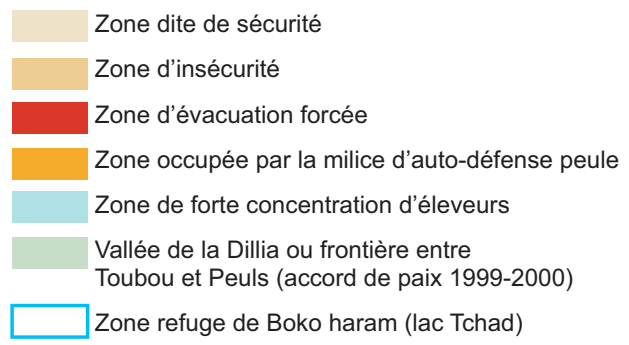

Figure 2

Les insécurités liées à la crise Boko Haram dans la rive nigérienne du lac Tchad.

Réalisation : Mahamadou Abdourahamani, 2016.

Source : enquêtes de terrain, saisons sèche et hivernale, 2016.

libérer des taxes et de la procédure de demande d'accès aux pâturages régulièrement refusé par les Boudouma. Les Peuls cherchent surtout à sécuriser des espaces de production dans la zone lacustre, qui représente la seule alternative face à l'ensablement et la dégradation des cuvettes pastorales : "Nous nous installons dans le lac. D'ici là, nous empêcherons tous les Boudouma de s'y installer, quand la paix reviendra nous serons maîtres de ces terres » (éleveur peul, août 2016).

Les groupes peuls ne sont pas les seuls à vouloir se positionner dans le lac Tchad vidé de ses habitants pour y faire reconnaître un terroir d'attache. Les Arabes Mohamid, grands transhumants chameliers, ont une stratégie moins marquée que celle des Peuls mais ils investissent aussi le lac interdit. Ils usent de la force, à l'arme à feu, contre les Boudouma en les assimilant aux éléments de Boko 
Haram. Ils recourent aussi à des alliances « contre-nature $»^{9}$, en s'alliant avec les Peuls contre les Boudouma.

Le projet d'aménagement et de mise en valeur du bassin de la Komadougou Yobé et du lac Tchad d'environ 120000 ha par la société saoudienne Al Horaish vient envenimer la question foncière de la rive nigérienne du lac Tchad (TCHANGARI et Diori, 2016 ; RANGÉ et al., 2018). Les terres convoitées par cette firme saoudienne via un bail de type emphytéotique correspondent à près de la moitié des superficies exploitables en irrigué et en décrue de la région. Ce sont en partie ces mêmes terres qui étaient occupées et exploitées avant que le lac ne soit évacué, que se disputaient les agriculteurs kanouri et haoussa et les Boudouma, et que revendiquent maintenant plusieurs groupes d'éleveurs transhumants.

\section{La production d'une territorialité par la violence}

Face au blocage des mobilités transnationales et à la quête de nouveaux espaces de production, de nombreux groupes d'éleveurs développent des stratégies pour justifier et légitimer leurs actions au sein de la partie nigérienne du lac Tchad. Les Boudouma incriminent les Peuls d'avoir des armes à feu au vu et au su des autorités administratives. Les Peuls, sans la nier, justifient cette situation par la nécessité de protéger leur famille et leurs animaux face aux Boudouma qu'ils accusent être de connivence avec les éléments de Boko Haram. Aussi, face aux enlèvements multiples de leurs troupeaux et des membres de leur famille (dont les jeunes femmes), les Peuls ont constitué des milices d'autodéfense pour se protéger et sécuriser leurs biens (AREN DifFA, 2016). L'absence quasi totale de l'État dans cette zone a fortement amplifié la circulation d'armes à feu à la fois dans les mains des éléments de Boko Haram et de leurs complices le long de la frontière avec le Nigeria, mais aussi dans les mains des milices d'autodéfense peules et dans celles des Boudouma qui se battent contre Boko Haram. Tous ces groupes se battent aussi entre eux à cause du vol d'animaux (VSF$\left.{ }^{10}, 2017\right)$.

Le conflit met face à face les communautés peules et arabes mohamid qui cherchent à créer de nouveaux terroirs d'attache d'un côté ; Boudouma et Kanouri Sougourti qui sont les anciens occupants du lac de l'autre. Ces groupes ont des liens contrastés avec Boko Haram. D'après l'association Aren, à forte connotation peule, la frontière entre Boudouma et Boko Haram n'est pas perceptible : « il est fortement établi que la quasi-totalité des membres de la communauté boudouma est membre de Boko Haram avec lesquels il ne peut y avoir de négociations » (AREN DifFA, 2016). « Si la présence boudouma au sein de Boko Haram est admise par les leaders boudouma, ceux de N'Guigmi en particulier, ils rejettent néanmoins l'idée d'une adhésion massive. De jeunes Boudouma

9. Dans la zone d'hinterland du lac, de graves antagonismes opposent les Peuls aux Mohamid pour l'accès aux points d'eau (forages, puits cimentés et traditionnels) où ces derniers ont souvent recours à la force pour $y$ abreuver leurs chameaux.

10 . Vétérinaires sans frontières. 
s'improviseraient comme guides, mariniers, receleurs ou même combattants pour Boko Haram » $\left(\mathrm{ICG}^{11}, 2017\right)$. Cependant, les groupes boudouma ne sont pas les seuls à être indexés à tort ou à raison de proximité et de sympathie avec les éléments de Boko Haram : les Kanouri Sougourti et les Peuls camerounais bokolodji sont aussi suspectés de forte complicité avec le groupe islamiste.

\section{Le vol de bétail : un autre facteur d'enlisement du conflit Peul-Boudouma}

Le vol des animaux constitue une préoccupation majeure des pasteurs. Historiquement dans cette région, les troupeaux les plus visés sont ceux des transhumants (DUPIRE, 1962). Chez certains groupes pasteurs comme les Toubou, le vol de bétail est une tradition et constitue un acte de bravoure du clan et de la tribu du voleur (BAROIN, 2009 ; BAROIN et Boutrais, 2009). En revanche pour les communautés arabes, le vol de bétail est un acte très blâmable. Le phénomène de vol de bétail a pris une dimension inquiétante avec la crise de Boko Haram, particulièrement entre les éleveurs peuls et boudouma. Il est en effet exacerbé avec le déplacement massif des populations lors de l'évacuation du lac qui n'a laissé que quelques heures aux populations pour quitter leur village ou campement, et elles ont souvent laissé leur bétail sur place. L'absence de tout contrôle a ensuite accentué les rivalités autour du bétail à travers des rapts mutuels d'animaux entre les groupes d'éleveurs. Les communautés boudouma et sougourti de N'Guigmi accusent les chefs peuls d'instrumentaliser la situation. Ces derniers constituent des milices d'autodéfense sous le prétexte d'appuyer les forces de l'ordre dans la lutte contre Boko Haram et de défendre leur famille. Cette position leur permet d'accaparer les richesses du lac en organisant un juteux trafic de bétail (ICG, 2017). En 2017, les animaux volés sont conduits sur les marchés lointains du Nigeria et du Niger (Mungono, Gudumbari, Zormodo) afin d'échapper à l'identification et la reconnaissance du bétail à travers le marquage, qui est le mécanisme de contrôle traditionnel et efficace des propriétés d'animaux au niveau local (DUPIRE, 1954 ; WAZIRI Mato et Souley, 2012). D'autres animaux sont abattus dans des abattoirs clandestins dans le lac et les produits sont transformés en viande boucanée avant d'être écoulés au Nigeria (VSF, 2017). Ainsi, cette guerre centrée sur la rente du bétail est l'un des nœuds gordiens de ce conflit intercommunautaire entre Peuls et Boudouma porté sous le chapeau de Boko Haram. 


\section{Conclusion}

La rive nigérienne, jadis espace répulsif pour de nombreux groupes du fait des razzias des insulaires boudouma et de la crainte des maladies animales pour les éleveurs, devient subitement avec les sécheresses un espace à grand enjeu de production pour tous les groupes. Les ressources lacustres apparaissent indispensables pour certaines catégories d'éleveurs, dans un environnement généralisé de raréfaction des ressources, de densification du parcellaire agricole et d'augmentation des cheptels.

Le contexte du retour des eaux dans la cuvette nord et de l'accroissement des possibilités de mise en valeur agricoles, pastorales et halieutiques a accentué les convoitises foncières d'une grande diversité d'acteurs aux intérêts divergents. Le lac et ses dédales marécageux ont joué le rôle de protecteur des groupes insoumis, permettant notamment aux éléments de Boko Haram de s'y réfugier. Le contexte particulier de l'état d'urgence et de l'évacuation forcée de la partie nigérienne du lac Tchad ouvre des perspectives inespérées aux acteurs opportunistes (Peuls Foulbé, Arabes Mohamid) qui cherchent à se positionner avant la normalisation de la situation sécuritaire. C'est dans ce contexte confus que pourraient aboutir les convoitises d'acteurs internationaux (société saoudienne Al Horaish) avec le concours de l'État central qui tentent une acquisition foncière de grande envergure. Tous ces éléments conjugués au sein cette zone lacustre nigérienne contribuent à exacerber, voire à maintenir dans la durée, aussi bien les tensions foncières locales que l'insécurité, plus ou moins liée à Boko Haram.

\section{Bibliographie}

Abdourahmani M., 2013

Foncier et agrobiodiversité sur les rives nigériennes du lac Tchad. Master 2, université Paris 1 Panthéon-Sorbonne, 83 p.

Anderson S., Monimart M., 2009

Recherche sur les stratégies d'adaptation des groupes pasteurs de la région de Diffa, Niger oriental. Rapport IIED, 102 p.

\section{Aren Diffa, 2016}

Situation pastorale de la région de Diffa. Rapport, 5 p.

\section{BAROIN C., 2009}

La circulation et les droits sur le bétail, clés de la vie sociale chez les Toubou (Tchad, Niger). Journal des Africanistes, 78 1/2 : 120-142.
Baroin C., Boutrais J., 2009

Bétail et société en Afrique. Journal des Africanistes, 78 1/2 : 9-52.

\section{Chapelle J., 1957}

Les Nomades noirs du Sahara. L'Harmattan, $449 \mathrm{p}$.

\section{DIORI I., 2015}

État d'urgence dans la région de Diffa : mission d'observation de la situation humanitaire et des droits de l'homme. Rapport, Alternative Espaces Citoyens, 16 p.

DUPIRE M., 1962

Des nomades et leur bétail. L'Homme, 2 : 22-39.

DUPIRE M., 1954

Contribution à l'étude des marques de propriété 
du bétail chez les pasteurs peuls. Journal des Africanistes, 24-2 : 23-143.

\section{Fourreau F., 1902}

D'Alger au Congo par le lac Tchad, mission saharienne Fourreau-Lamy. Paris, Masson, $618 \mathrm{p}$.

\section{Hassane M. L., Hassane M., Moustapha L., 2016 \\ Conflit intercommunautaire dans le canton de N'Guigmi. Rapport de mission, $4 \mathrm{p}$. \\ InTERnational Crisis Group, 2017 \\ Le Niger face à Boko Haram : au-delà de la contre-insurrection. Rapport, Bruxelles, $39 \mathrm{p}$.}

\section{KiARi Fougou H., 2014}

Impacts des variations du niveau du lac Tchad sur les activités socioéconomiques des pêcheurs de la partie nigérienne. Thèse de doctorat de géographie, université Abdou-Moumouni, Niamey, Niger, 314 p.

\section{Horowitz M., 1977}

« Les stratégies adaptatives avant et après la sécheresse ». In : Gallais J., éd. : Stratégies pastorales et agricoles des Sahéliens durant la sécheresse de 1969-74. Centre d'études de géographie tropicale, CNRS. Bordeaux, p. 219-233.

\section{LEMOALLE J., 1989}

Le fonctionnement hydrologique du lac Tchad au cours d'une période de sécheresse (1973-1989). Rapport Orstom 89-03, 29 p.

\section{Luxereau A., Genthon P., Karimou J.-M. A.,} WAZIRI MATO M., 2012

Fluctuations in the size of Lake Chad: consequences on the livelihoods of the riverain peoples in eastern Niger. Regional Environmental Change, 12 : 507-521.

\section{MAGRIN G., 2009}

« De longs fleuves tranquilles ? Les mutations des plaines refuges du bassin tchadien ». In : Raison J.-P., Magrin G., éd. : Des fleuves entre conflits et compromis. Essais d'hydropolitique africaine. Paris, Karthala, p. 125-172.

\section{Magrin G., Pérouse de Montclos M.-A.,} dir., 2018

Crise et développement. La région du lac Tchad à l'épreuve de Boko Haram. Paris, AFD, 291 p.
MARThÉ D. M., 2015

Dans les marches nord du Borno (Bornou) : les mutations politiques dans l'Est nigérien (1893-1960). Paris, L'Harmattan, 287 p.

\section{NaChtigal G., 1881}

Sahara et Soudan. Gallica, 577 p.

Olivier de SARDan J.-P., 2012

État, bureaucratie et gouvernance en Afrique de 1'Ouest francophone. Politique Africaine, 96 : 139-162.

\section{PCCN Diffa, 2016}

Forum de conciliation intercommunautaire de Kabléwa. Rapport, antenne Diffa, 5 p.

\section{Pérouse de Montclos M.-A., 2015a}

Boko Haram et la souveraineté du Nigeria : une histoire de frontières. Hérodote, 58-75.

\section{Pérouse de Montclos M.-A., 2015b}

Boko Haram et la mise en récit du terrorisme au « Sahelistan ». Afrique contemporaine, 255 : 21-41.

\section{Rangé C., Chauvin E., Raimond C.,} Zakinet D., Kiari Fougou H., Abourahamani M., Aoudou Doua S., MuHAMAD K., 2018

« La recomposition du système régional ». In : Crise et développement. La région du lac Tchad à l'épreuve de Boko Haram. Étude de l'Agence française de développement. Paris, AFD, 291 p.

\section{Seignobos C., 2015}

Boko Haram et le lac Tchad. Extension ou sanctuarisation? Afrique contemporaine, 255, p. $93-120$.

\section{TChangari M., Diori I., 2016}

Convoitises foncières dans le bassin du lac Tchad au Niger, Niamey, Alternative Espaces Citoyens, rapport de l'Observatoire du droit à l'alimentation au Niger, décembre, $45 \mathrm{p}$.

\section{THÉBAUd B., 2002}

Foncier pastoral et gestion de l'espace au Sahel : Peuls du Niger oriental et du Yagha burkinabé. Paris, Karthala, 343 p.

\section{THÉBAUd B., 1988}

Élevage et développement au Niger : quel avenir pour les éleveurs du Sahel ? Genève, BIT, 147 p.

\section{URVOY Y., 1949}

Histoire de l'empire du Bornou. IFAN, Mémoires de l'Institut français d'Afrique noire, $172 \mathrm{p}$. 
Vétérinaires Sans Frontières, 2017

Évaluation agropastorale et analyse des causes profondes des conflits dans la région de Diffa (Maine-Soroa, Diffa, Bosso, N'Guigmi et $N^{\prime}$ 'Gourti). Rapport, Conseil danois pour les réfugiés, $69 \mathrm{p}$.

WaZiri Mato M., Souley K., 2012 Importance sociale et impact territorial des marques des dromadaires chez les Toubou
Teda du Niger oriental. Revue Territoires,

Sociétés et Environnement, université de Zinder, Niger, $1: 139-151$.

\section{ZAKARI M., 1985}

Contribution à l'histoire des populations du Sud-Est nigérien. Le cas du Mangari $\left(X V I-X I X^{e} s\right.$ s.). Niamey, IRSH, Études nigériennes, $53,246 \mathrm{p}$. 


\section{Pratiques et enjeux de la sécurisation foncière autour du lac Fitri}

\section{Introduction}

Au cœur du Sahel tchadien à $12^{\circ} 50^{\prime} \mathrm{N}$ et $17^{\circ} 30^{\prime} \mathrm{E}$, le lac Fitri est considéré comme « le lac Tchad en miniature » (GILlet, 1962 ; CATERINA et MARzIO, 2005). Très variable, cet hydrosystème endoréique est principalement alimenté par le bahr ${ }^{1}$ Batha, un tributaire temporaire qui prend sa source à l'est dans le massif du Ouaddaï à plus de $600 \mathrm{~km}$. De faible profondeur $(1,5$ à $3 \mathrm{~m})$, sa superficie et son niveau sont très sensibles aux variations climatiques saisonnières et à la pluviométrie irrégulière. L'étendue du lac Fitri oscille entre $220 \mathrm{~km}^{2}$ et $420 \mathrm{~km}^{2}$ pendant l'étiage et entre $1300 \mathrm{~km}^{2}$ et plus de $1700 \mathrm{~km}^{2}$ pendant la crue, avec une moyenne de $800 \mathrm{~km}^{2}$ (LemOalle, 1989 ; Courel et al., 1997 ; YALIKUN et al., ce volume). Les apports annuels, qui assurent sa pérennité, sont estimés à plus d'un milliard de $\mathrm{m}^{3}$. Au cours des années particulièrement sèches (1901, 1913, 1973 et 1984), le lac se réduit à de nombreuses mares ou disparaît complètement (BIEP², 1989 ; DAGOU et al., 2005 ; P-SIDRAT $\left.{ }^{3}, 2013\right)$.

Zone refuge par excellence, le lac Fitri assure, par ses multiples ressources (eau, poisson, pâturages, terres agricoles, bois), un rôle vital pour les populations riveraines. Cette zone humide fait partie intégrante d'un ancien royaume qui a

\footnotetext{
I. Bahr : terme arabe désignant un fleuve ou une rivière importante.

2. Bureau interministériel d'études et de programmation.

3. Le Programme du système d'information pour le développement rural et l'aménagement du territoire.
} 
gardé son fonctionnement très structuré autour d'un pouvoir central. Les ressources naturelles, dont le foncier, sont gérées par ce pouvoir. Au Tchad, si la terre appartient à l'État d'après la législation foncière en vigueur (lois 23, 24, 25 du 22 juillet 1967), elle est au lac Fitri une propriété lignagère dont la gestion, acquise par droit de conquête au $\mathrm{XVI}^{\mathrm{e}}$ siècle, est placée sous le contrôle du sultan et ses relais au Fitri (ZELTNER, 1980 ; ZAKINET, 2015). Cependant, la pression démographique ainsi que l'installation récente de nouveaux acteurs (préfet, sous-préfet, gendarmerie) dans le cadre des réformes administratives viennent bouleverser le système foncier coutumier en place. Ces nouveaux acteurs sont vus par les autorités coutumières et la population comme une menace pour l'ordre traditionnel. Dans un contexte où les pratiques agraires s'adaptent à la variabilité saisonnière du milieu, les modes d'accès et de réglementation coutumière du foncier ne sont-ils pas devenus inadaptés face à la pression foncière et à la multiplication des instances de gestion?

Ce travail repose sur des enquêtes de terrain (70 entretiens individuels et collectifs) réalisées en deux phases (février-mars 2015 et juin 2016) dans quinze villages riverains et insulaires du lac Fitri. Les entretiens ont concerné les autorités locales (traditionnelles et administratives), les agents des services déconcentrés de l'État et la population. Le présent article expose les principaux résultats à travers l'analyse des pratiques foncières en lien avec l'organisation et l'occupation traditionnelle de l'espace d'une part et les enjeux actuels de la gestion foncière d'autre part.

\section{Le système foncier du lac Fitri}

Le foncier est considéré comme un ensemble de rapports entre les hommes impliqués dans l'utilisation de l'espace et des ressources (LE BRIS et al., 1982). Ainsi, le potentiel foncier du Fitri est déterminé par les conditions du milieu alors que les pratiques d'accès et d'exploitation de la terre sont régies par des règles traditionnelles.

\section{Un potentiel foncier très variable}

Les variations du niveau des eaux du lac Fitri consécutives aux apports annuels impliquent des modifications importantes des surfaces inondables affectant le potentiel foncier disponible. De ce fait, les superficies inondées changent largement d'une année à l'autre (YALIKUN et al., ce volume). En fonction de l'hydrologie du lac, l'étendue de la crue détermine les surfaces inondées et exploitables à la décrue ${ }^{4}$. En effet, un faible débordement des eaux du lac 
s'accompagne d'une faible extension des surfaces inondées, entraînant une forte concentration des activités agricoles et pastorales dans les secteurs jugés favorables. Cette situation se caractérise par la pression sur les ressources et parfois par des conflits entre usagers. En année de crue exceptionnelle, d'importantes superficies sont inondées puis découvertes et mises en valeur sans difficulté majeure d'accès au foncier, quel que soit le secteur considéré.

\section{Un régime foncier traditionnellement imbriqué dans l'organisation sociopolitique bilala}

Au Fitri, comme ailleurs en Afrique rurale, ce sont les règles coutumières qui assurent une gestion foncière efficace avec un accès à la terre basé sur des droits à la fois collectifs et individuels.

\section{Statut et gestion des terres par le sultanat du Fitri}

Comme dans les autres sultanats du Tchad, la légitimité du sultan sur les ressources du lac Fitri est reconnue par les usagers. En effet, le sultan est l'autorité suprême qui assure la gestion de la terre à travers ses relais (fig. 1). Les représentants du sultan dans les provinces (khalifa) et les administrateurs des territoires lignagers $\left(\right.$ kaïdala $\left.^{5}\right)$ veillent à l'application des règles d'accès et de

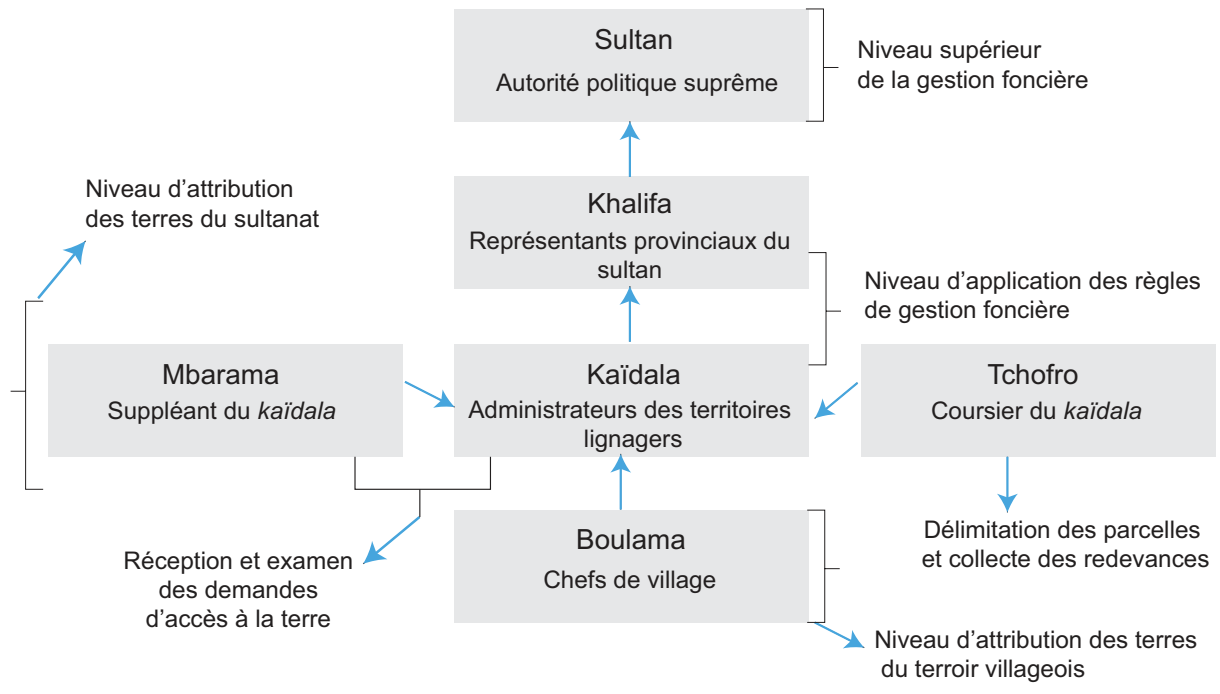

Figure I

Organisation politique et acteurs de gestion foncière traditionnelle au Fitri. Source : enquête de terrain (20I5).

5. Tout comme le khalifa, le kaïdala incarne l'autorité politique et administrative du sultan sur le territoire lignager. Nommé ou proposé par un clan ou une communauté lignagère, il peut contrôler jusqu'à plus d'une dizaine de villages. Le kaïdala assure à la fois le rôle d'administrateur territorial, de gestionnaire du foncier et même de gardien du patrimoine lignager dans certains cas. 
gestion des conflits fonciers. Ils sont chargés, à différents niveaux, de l'attribution des terres de la réserve foncière du sultanat.

Les terres vacantes de la zone d'inondation destinées principalement aux cultures de berbéré (sorgho de décrue) sont distribuées par le kaïdala ou son suppléant (mbarama) assisté d'un tchofro (coursier). Les premiers reçoivent et examinent les demandes d'accès à la terre alors que le second est chargé de la délimitation des parcelles et de la collecte des redevances. Contre une valeur symbolique ${ }^{6}$, anciennement appelée mâala (bilala/pain de sucre), soit en coro ${ }^{7}$ de mil soit en argent (5000 à $15000 \mathrm{FCFA})$, tout attributaire reçoit une parcelle à cultiver. Le bénéficiaire peut exploiter et transmettre les droits d'usage à ses ayants droit. Par ailleurs, ce sont les chefs de village (boulama) qui attribuent les terres exondées cultivées en saison pluvieuse et les terrains d'habitation au sein des terroirs villageois. Ils rendent compte de leur gestion au kaïdala et au khalifa. Si le sultan est le gardien du patrimoine foncier du Fitri au point d'en paraître le propriétaire, la terre est avant tout la propriété des communautés lignagères bilala.

\section{La terre, une propriété lignagère}

Les premières communautés bilala se sont d'abord concentrées dans les zones fertiles à proximité du lac au sein d'une douzaine de « villages-mères ${ }^{8}$ », avec le principe de double résidence alternant saison des pluies à l'extérieur du lac et saison sèche sur les rives (HAGENBUCHER, 1968 ; fig. 2). À l'origine, les « villages satellites » créés dans l'arrière-pays sont une stratégie d'adaptation aux variations saisonnières du lac (KOYOUMTAN, 2002). Les villages satellites se sont progressivement transformés en sites d'habitation permanente. Cette dynamique a permis au fil du temps de délester la concentration de la population sur le pourtour immédiat du lac. Certains villages-mères ou anciens villages des rivages du lac sont devenus des « villages fantômes » appelés localement koudou en bilala ou doungous en arabe. Ils sont occupés par des habitats temporaires seulement pendant la contre-saison pour la culture du berbéré (sorgho repiqué en arabe) lors des années de très forte crue.

Au cœur de cette organisation spatiale, ce sont les familles issues d'un même ancêtre qui sont propriétaires des terres selon le droit du premier occupant (droit de la hache ou du feu). En réalité, plusieurs droits de propriété foncière se superposent. Le foncier est avant tout un bien communautaire inaliénable, dont la gestion est assurée par le représentant du lignage. L'ancêtre fondateur joue le rôle de garant du patrimoine foncier commun ; et ses descendants ne peuvent se réclamer le droit de propriété éminente et exclusive. C'est le chef de terre

6. Cette contrepartie symbolique est généralement partagée entre le kaïdala et le tchofro.

7. Le coro est un récipient émaillé souvent utilisé comme unité principale de mesure des produits alimentaires notamment les céréales. Il correspond en moyenne à environ $2,5 \mathrm{~kg}$.

8. Le village-mère désigne le premier site sur lequel le père fondateur (aïeul du clan ou du lignage) s'est installé. Aujourd'hui, la plupart de ces villages originels sont faiblement peuplés ou complètement dépeuplés. 


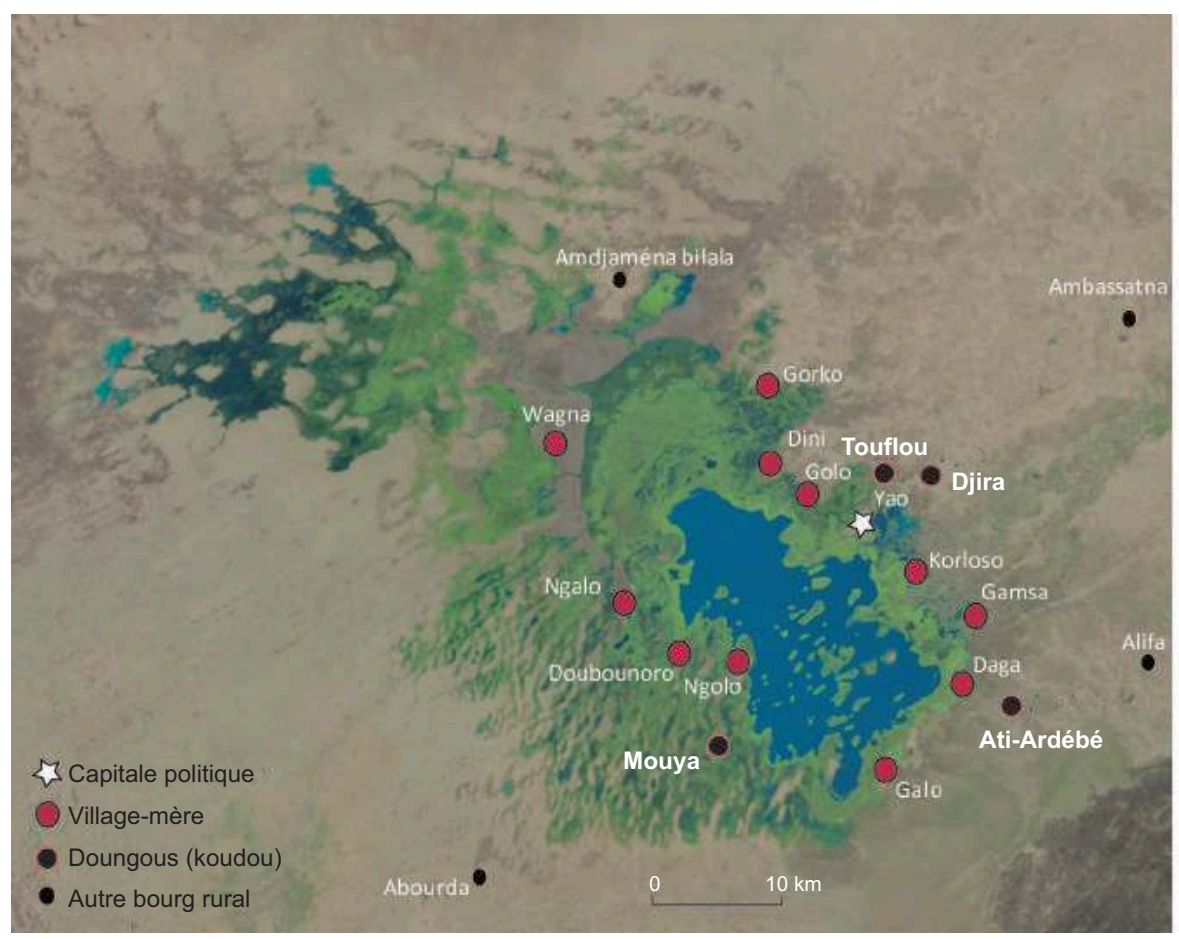

Figure 2

Implantation traditionnelle des villages-mères autour du lac Fitri.

Sources : données de terrain 2015 et image satellite, Nasa, 2014.

(dé-ardé ${ }^{9}$ issu du lignage fondateur d'un village-mère qui veille au respect des règles d'attribution et de transmission des terres au sein des segments lignagers. Les pouvoirs du chef de terre sont légués par l'ancêtre fondateur. Il peut disposer des terres non attribuées mais ne peut les vendre. C'est lui qui initie les rites avant les semis et la récolte. Avec la forte islamisation, ce sont les autorités religieuses (imam) qui prononcent les prières (lecture du coran) qui accompagnent désormais ces différents rituels.

Au niveau de la famille, le droit de propriété est aussi collectif. Il repose sur les relations sociales au sein du segment lignager (Colin, 2004). Chaque chef de famille reçoit un domaine de la réserve foncière du lignage qu'il distribue aux membres actifs et les droits de propriété familiale sont transmis par cession ou voie d'héritage de père en fils. Le chef de famille dispose du droit de regard et de gestion sur son domaine foncier mais il n'est pas propriétaire des terres. L'individu, quant à lui, ne bénéficie que du droit d'usage. Il a droit, en tant

9. Traditionnellement, le dé-ardé est considéré comme le propriétaire de la terre. Doté d'une personnalité spirituelle, c'est lui qui est chargé des rites fonciers. Cependant, cette responsabilité à la fois coutumière et religieuse est en voie de disparition car aujourd'hui le kaïdala assure la gestion des terres du sultanat et l'imam s'occupe des sacrifices à travers le récit des versets coraniques. 
qu'élément du lignage, à une parcelle de culture dont il dispose à sa guise en vue d'assurer sa survie. Il peut être héritier d'un parent défunt, successeur du droit familial à l'âge adulte par le truchement du mariage ou cultiver un champ directement octroyé par le kaïdala s'il en formule la demande, sous réserve de la disponibilité foncière. Il devient ainsi «propriétaire » de sa portion de terre (dé-djar) et jouit de l'usufruit, mais ne peut vendre la terre qui lui a été attribuée. Les femmes n'ont pas de droit sur les terres. Elles peuvent exploiter temporairement les parcelles de leurs parents ou de leur mari, ou à travers les droits légués à ses enfants à la mort de leur père. Un étranger à la communauté n'a pas de droit de propriété sur les terres et ne peut acheter la terre. Si on ne peut lui refuser une terre à cultiver, une fois la récolte terminée, il perd le droit d'usage et sa parcelle peut faire l'objet d'une nouvelle attribution ${ }^{10}$. Cependant, si la terre est une propriété lignagère qui ne peut être vendue, elle peut faire l'objet de location, de mise en gage ou de prêt au sein d'un lignage.

\section{Des pratiques foncières adaptées à la variabilité des ressources naturelles}

Selon le type de cultures pratiquées et en fonction des secteurs identifiés autour du lac Fitri, l'accès à la terre est défini par des règles coutumières plus ou moins strictement respectées. Les pratiques foncières se rapportent aussi aux redevances et rituels obligatoires qui garantissent les droits d'usufruit.

\section{Les règles d'accès aux terres de cultures}

La répartition spatiale des activités agricoles et de la double culture dans l'année amène à distinguer deux modes d'accès au foncier selon la fréquence de l'inondation. Les règles applicables aux terres de décrue en bordure du lac diffèrent de celles des terres sableuses et exondées de l'arrière-pays.

\section{L'accès aux terres de décrue}

Les surfaces cultivées à la décrue du lac étaient estimées en 1989 entre 5000 et 15000 ha en fonction de l'hydraulicité de l'année et de l'ampleur des crues (BIEP, 1989). Aujourd'hui, dans des conditions hydrologiques plutôt propices, ce potentiel est supérieur à 80000 ha (Kemsol NAGORNGAR et al., ce volume).

10. Lors des entretiens à Gorko avec le conseil du village, un vieux affirme en ces termes : « On ne refuse la terre à personne, on peut même la donner à un étranger sauf aux transhumants kréda et arabes car ils sont susceptibles de réclamer par tous les moyens la propriété définitive même dans le cas d'une location. » Ces propos corroborent ceux recueillis à Fourkouma par l'équipe de Marty (MARTY et al., 20I2:66). 
Ce sont des terres très fertiles qui permettent de bonnes récoltes $(900 \mathrm{~kg} / \mathrm{ha}) \mathrm{de}$ sorgho de décrue (berbéré) et de cultures maraîchères.

L'accès à ces terres est très organisé : elles sont minutieusement reparties au sein des segments lignagers suivant des règles coutumières strictes. Les terres de décrue ne doivent ni faire l'objet de marchandisation ni de donation définitive. Ce principe de gestion est tel que l'étranger ne peut bénéficier que d'un prêt temporaire à titre gratuit accordé par le kaïdala ou le boulama s'il remplit certains critères (durée de vie au village, mariage). Cependant, le prêt conditionnel ${ }^{11}$ entre les membres d'un même lignage est fréquent et n'implique pas nécessairement l'intervention du chef de terre. L'achat des terres de décrue est traditionnellement prohibé car cela pourrait se traduire par une appropriation définitive : " la terre au Fitri constitue une ressource très particulière, surtout la terre de culture de berbéré. Elle peut très rarement faire l'objet d'une donation définitive, mais juste une location pour une période ne dépassant pas dix ans. On change de terrain pour éviter que l'exploitant ne revendique la propriété » (MARTY et al., 2012 : 65). La location, un contrat de longue durée (trois à dix ans) sous forme de mise en gage ou d'hypothèque, est possible entre les Bilala. La parcelle est exploitée contre une caution de valeur variable selon sa taille. La caution est remboursable dès que le propriétaire réclame sa parcelle ou à la fin du contrat. Le prix de location d'un champ ${ }^{12}$ varie entre 7500 et 15000 FCFA en fonction des secteurs, de la taille et de l'emplacement de la parcelle.

\section{L'accès aux terres pluviales}

L'arrière-pays est le domaine par excellence des cultures pluviales sur terres sableuses. Ce sont de vastes superficies destinées notamment à la culture du petit mil, de l'arachide et du niébé. Elles sont exploitées pendant la saison pluvieuse par les membres des segments lignagers qui exploitent aussi les terres inondables en contre-saison. Du fait des contraintes liées aux caprices pluviométriques et en raison des récoltes hypothétiques, ces terres ne font pas l'objet d'une réglementation rigoureuse et de compétition particulière. Tout le monde, même l'étranger, y accède librement et de façon gratuite. Cependant, une demande est formulée à l'endroit du boulama. L'exploitant peut aussi disposer des terres de sa famille sans aucune condition préalable. Si une parcelle de cultures pluviales n'est pas valorisée pendant plus de trois années successives, le boulama peut la réattribuer à celui qui en exprime le besoin. Les terres sableuses ne peuvent être ni vendues ni louées.

II. Ces dernières années, malgré un retour à un niveau plus élevé du lac par rapport aux années de sécheresses, les terres de décrue disponibles sont rares du fait de l'expansion des Acacia nilotica et de la pression foncière due à la croissance démographique. Face à cette situation, ceux qui ont hérité ou occupent de grandes surfaces de décrue et ne peuvent exploiter la totalité donnent une partie sous forme de prêt contre une part de récolte ou toute autre forme de contrepartie (souvent de l'argent) suivant les termes de négociation convenus avec le bénéficiaire.

12. Les superficies d'un champ varient localement dans l'ordre de 900 à plus de $2000 \mathrm{~m}^{2}$. En moyenne, une parcelle couvre sensiblement plus d'I ha mais, pour des raisons de standardisation, on retient I ha. 


\section{Des pratiques foncières qui varient selon les crues}

Autour du lac Fitri, on distingue deux grandes zones de pratiques foncières suivant l'ampleur et la régularité des crues.

\section{L'accès aux terres régulièrement inondées}

Les secteurs des groupes lignagers de Galnoro et Djira au nord-est, Seïta et Zisoro à l'est et Sor-manmaf au sud-est forment la zone de forte extension des eaux du lac Fitri. Les terres régulièrement inondées de ces secteurs sont mises en valeur chaque année selon les règles de propriété collective. L'accès est fonction de l'importance de la crue. En année exceptionnelle comme en 2014 et 2015, ce sont de grandes surfaces qui sont inondées et exploitées par les familles détentrices des droits fonciers historiques. En revanche, au cours d'une année de faible crue (2009), les superficies cultivables sont réduites et le sultan veille, à travers ses relais (kaïdala et dé-ardé), à ce que chaque famille dispose d'une portion de terre.

\section{L'accès aux terres irrégulièrement inondées}

Les secteurs des groupes de Manga au nord-ouest, Tchémané à l'ouest et Djorto au sud-ouest sont soumis généralement à de faibles et irrégulières inondations. Ici, l'accès à la terre est strictement contrôlé et renouvelé. Le kaïdala procède chaque année à la redistribution des parcelles de culture de berbéré. Cette redistribution, organisée en fonction de l'étendue des superficies inondables, permet de partager collectivement les risques liés à la variabilité des crues et d'éviter la propriété définitive ainsi que les conflits récurrents entre les usagers. À Wagna tout comme dans la zone d'Abourda, la demande en terres est renouvelée au début de chaque campagne de décrue.

\section{Les redevances et les rituels fonciers}

L'exploitation des terres implique le respect des règles relatives aux redevances et aux rituels. Depuis la conversion des Bilala à l'islam, la zakhat (aumône religieuse) a remplacé l'impôt foncier traditionnel (wasak nanga ou tondolo en langue bilala). Cette redevance est obligatoire et correspond au dixième de la récolte céréalière versée au sultan. En principe, la zakhat est destinée aux œuvres de charité (assistance aux personnes nécessiteuses), à l'accueil des visiteurs étrangers au sultanat et à l'organisation des réceptions pour honorer un nouveau notable. En cas de non-paiement, le contrevenant peut s'exposer à des sanctions graves allant jusqu'au retrait de ses droits d'usage car la conscience sociale considère que ce refus attire des malheurs tels que les mauvaises récoltes. Jadis, même les produits de la cueillette comme le tingili (kreb ou fonio sauvage) et le gadé (graines du nénuphar) étaient aussi taxés.

Les cérémonies rituelles en lien avec la terre sont de même obligatoires et s'appliquent à tout exploitant, même aux étrangers. Chacun apporte une contribution en nature (mil) ou en argent pour les sacrifices offerts avant les 
semis, le repiquage et la récolte du berbéré. L'argent collecté permet d'acheter le mouton à offrir en guise de reconnaissance à Allah ou pour implorer la clémence des divinités ancestrales. Traditionnellement, un sacrificateur (ngarmané) issu du culte de la Margaï (esprit de l'eau) était chargé d'officier les rites agraires en lien avec le lac et de présider les différentes cérémonies rituelles (KARDIGUÉ, 2017). De nos jours, les prières musulmanes sont prononcées par un chef religieux (imam ou marabout) et c'est le sultan qui ordonne la récolte. Le respect et la participation aux sacrifices sont nécessaires même si dans les secteurs excentrés comme Garia, à l'extrême sud du lac, ces rituels sont quasi absents du fait de la faible production du berbéré. Une amende est imposée à tout contrevenant pour réparer le préjudice et ne pas impacter l'ensemble de la récolte.

\section{Les enjeux actuels de gestion foncière}

Pour Le Meur (2002), le terme d'enjeu foncier renvoie à la relation foncière, c'est-à-dire au rapport entretenu entre les individus vis-à-vis de la terre et des autres ressources. Cette relation est sous-tendue par des enjeux complexes. Ces enjeux sont portés par des acteurs d'une part en compétition pour l'accès aux ressources et d'autre part, par des instances de contrôle et de gestion de ces ressources. Au Fitri, les enjeux fonciers sont liés à la pression démographique, source de conflits récurrents et aux défis de gestion.

\section{Les enjeux démographiques}

Le canton du Fitri, devenu département administratif en 2004, couvre environ $10000 \mathrm{~km}^{2}$ avec une population résidente estimée vers la fin des années 1980 à 42000 sédentaires et 17500 transhumants, qui s'y installent durant la saison sèche de six à huit mois (BIEP, 1989). La population totale du Fitri était passée de 77000 habitants en 1993 à 110403 habitants en $2009\left(\mathrm{RGPH}^{13} 1\right.$ et 2). Aujourd'hui, le lac Fitri est considéré comme une zone sahélienne de forte concentration humaine avec près de $20 \mathrm{hab} / \mathrm{km}^{2}$ (P-SIDRAT, 2013). Cette situation démographique entraîne une forte compétition pour l'accès aux ressources naturelles et occasionne des conflits d'usage et d'usagers. Ainsi, l'une des difficultés majeures de gestion foncière au Fitri est consécutive à l'augmentation de la population. Les ressources foncières sont devenues insuffisantes et les modes traditionnels de régulation des terres agricoles ne sont plus efficaces. Certaines exploitations ayant beaucoup d'enfants sont obligées de louer des terres en complément du domaine foncier familial. En outre, les parcelles pour 
la culture du berbéré sont de plus en plus difficiles à trouver, surtout pour les familles, comme celles des îles de Moudo 1 et 2, qui ne dépendent pas des lignages gérant les zones de décrue.

Aussi, dans un contexte climatique sahélien caractérisé par le risque de sécheresse, la mauvaise répartition pluviométrique et l'irrégularité et la variabilité des apports au lac, chaque exploitant (agriculteur, éleveur, pêcheur) veut avoir accès aux ressources : l'eau et le bourgou ${ }^{14}$ pour les éleveurs, la terre pour les agriculteurs, le poisson pour les pêcheurs. La course à la ressource peut dégénérer en conflits violents prenant parfois une allure intercommunautaire entre sédentaires et transhumants. Cela se traduit non seulement par le paiement de la dia (prix du sang en langue arabe) mais aussi par l'effritement des rapports sociaux entre les anciens groupes alliés qui partagent cet espace commun (MARTY et al., 2012 ; ZAKINET, 2015). Face à la pression démographique, les enjeux liés à la gestion des conflits fonciers sont devenus importants et le système traditionnel de gestion des ressources semble dépassé.

\section{Les enjeux liés à la pluralité des gestionnaires des conflits fonciers}

En Afrique, les problèmes de la gestion foncière relèvent souvent du décalage entre les règles traditionnelles et la réglementation moderne (LAVIGNE DELVILLE, 2002). Au Fitri, la coexistence de plusieurs instances d'arbitrage des différends fonciers est la principale source d'insécurité et de menace qui pèse sur l'ancien système de gestion.

\section{La gestion traditionnelle}

Les mécanismes de gestion des conflits fonciers reposent sur des règles traditionnellement bien connues de tout le monde, notamment des sédentaires bilala et des transhumants arabes, qui exploitent les ressources du lac depuis des temps immémoriaux. Le premier niveau de réglementation est la conciliation à l'amiable. Les deux parties en conflit trouvent un terrain d'entente avant même que les autorités ne soient informées. En cas de non aboutissement, l'affaire est transmise aux autorités compétentes du village (boulama, kaïdala). À ce niveau, l'évaluation des dégâts, notamment à travers le constat de l'état du champ détruit par le bétail, permet de fixer les amendes conformément aux modalités locales de remboursement (tabl. 1). Si cette tentative d'arrangement échoue, le pouvoir juridictionnel du sultan est le dernier recours. En effet, il existe une cour de justice traditionnelle au palais du sultan. Le juge en charge s'appuie à la fois sur des règles coutumières et sur le droit musulman pour trancher les différends. Une fois le conflit réglé, le sultan prélève les droits correspondant au dixième du montant de l'amende payée à la victime et parfois, il perçoit une deuxième 
amende supplémentaire versée par le fautif. Si toutes ces tentatives n'ont pas permis de régler le problème de façon définitive, la sous-préfecture est saisie.

Tableau 1

Modalités coutumières de paiement des dégâts dans un champ.

\begin{tabular}{|l|l|}
\hline État de la culture & Montant de l'amende \\
\hline Pépinières de berbéré & I 250 à I 500 FCFA \\
\hline Berbéré nouvellement repiqué & Valeur des pépinières \\
\hline Berbéré en épiage (montaison) & Valeur du nombre de sacs escompté \\
\hline Pied de mil pénicillaire en épiaison & Valeur d'un coro (500 FCFA) \\
\hline Pied de gombo & 250 FCFA \\
\hline Pied de haricot & 125 à 250 FCFA \\
\hline Pied de tomate & 250 à 500 FCFA \\
\hline Pied de concombre & 500 FCFA \\
\hline
\end{tabular}

Source : données de terrain, 2015.

Cette procédure traditionnelle est considérée par certains usagers comme obsolète et inadaptée au nouveau contexte. Les éleveurs estiment que la réglementation coutumière en vigueur n'est ni juste ni équitable car elle est systématiquement favorable aux agriculteurs bilala. Ils considèrent que la gestion des ressources du Fitri doit tenir compte des changements dus à la perturbation du calendrier de transhumance au Sahel et du fait que les ressources naturelles sont devenues insuffisantes. En effet, l'arrivée des éleveurs au lac Fitri s'annonce plus tôt que d'habitude alors que leur départ retardé coïncide avec les semis de la saison pluvieuse. Cette situation pose problème par rapport à l'occupation de l'espace. Les champs se trouvent dans les zones de pâturage et les animaux divaguent dans les champs non récoltés ou à peine ensemencés. De même, du côté des sédentaires, les agriculteurs s'accusent entre eux par rapport à l'élargissement des limites des champs et certains ne s'entendent plus sur le partage des terres lignagères. Dans ces conditions, les éleveurs et les agriculteurs qui se croient lésés préfèrent, depuis ces dernières années, avoir directement recours aux instances de régulation étatique.

\section{Le système de régulation étatique}

L'implication de nouveaux acteurs institutionnels (préfecture, sous-préfecture, brigade de gendarmerie) depuis le début des années 2000 dans la gestion des conflits fonciers fragilise le pouvoir traditionnel et attise les rivalités entre les autorités coutumières et administratives. Même si ces instances étatiques ne contrôlent pas encore l'accès aux ressources foncières du Fitri (eau, pâturages et terre), elles s'impliquent davantage dans la gestion des conflits d'usage. En tant que représentantes de l'État, elles s'imposent comme gestionnaires du domaine foncier national. L'apparition d'une diversité d'acteurs de la gestion 
foncière s'accompagne souvent de pratiques variées et floues qui combinent à la fois le droit moderne, la coutume, les conventions locales ou la force (LE Meur, 2002). On assiste dès lors à des procédures parfois contradictoires et fondées sur des négociations dont les mécanismes alimentent le clientélisme et accentuent les tensions sociales. Cette situation crée un climat de méfiance et un espace de contestation alimenté par l'indétermination des règles légitimes, le jeu des pouvoirs locaux et leur manipulation, que les acteurs investissent de façon opportuniste.

\section{Conclusion}

Au Fitri, tout comme ailleurs au Tchad et au Sahel, la problématique de la gestion foncière se pose aujourd'hui avec acuité. Les questions liées à la dualité des régimes fonciers, aux conflits d'usage et aux enjeux de gestion demeurent partout presque identiques. Autour du lac Fitri, les pratiques foncières varient en fonction de la valeur agricole des terres et des secteurs déterminés par la régularité de l'inondation. Si les modes traditionnels d'accès à la terre sont encore restés en marge de la marchandisation, les mutations en cours (pression démographique, désenclavement, présence croissante de l'État et scission du sultanat en plusieurs cantons dans le cadre de la décentralisation) s'accompagnent de changements susceptibles de modifier les mécanismes de régulation d'autrefois. On assiste à une diversification des instances de gestion des conflits d'usage, qui pose le problème des interférences et de la transparence dans la gestion foncière. Cette pluralité des acteurs de la gestion foncière alimente le jeu du clientélisme et de la corruption, et accentue les tensions entre les différentes communautés qui exploitent les ressources du lac. Si le sultan et ses relais souhaitent maintenir les anciennes règles de gestion autour de l'autorité traditionnelle, l'administration moderne qui est censée faire appliquer une réglementation plus équitable en affaiblit la légitimité sans avoir les moyens de jouer pleinement son rôle. La gestion « moderne » par les représentants de l'État dans un contexte comme celui du Tchad s'accompagne de dysfonctionnements multiples et constitue un facteur de risque important dans des situations sensibles comme celle du Fitri. Face au flou et aux incertitudes de la gestion foncière actuelle, le défi est d'arriver à définir un cadre consensuel permettant de fixer de nouvelles modalités d'accès, d'exploitation et de réglementation des conflits autour des ressources foncières sous pression, dont la gestion organisée est indispensable à l'essor de l'économie rurale. Face à cet enjeu de la gouvernance hybride entre l'administration et les autorités coutumières à mettre en place, il est d'un grand avantage de pouvoir composer avec un sultan moderne qui comprend les mutations en cours et qui représente une instance de régulation respectée, capable de gérer au mieux les conflits d'usages. Au-delà, il est 
important d'articuler les diverses formes de gestion tout en respectant les droits des exploitants pour une meilleure cohabitation et une bonne gouvernance foncière locale.

\section{Bibliographie}

BIEP, 1989

Étude du développement intégré du lac Fitri. Min. Agric., BID, Cedrat-SA, N'Djamena, 61 p.

\section{Caterina B., Marzio M.,} Adamou Harouna T., 2005

Le futur est un ancien lac: savoirs traditionnels, biodiversité et ressources génétiques pour l'alimentation dans les écosystèmes du bassin du lac Tchad. FAO, 320 p.

\section{Colin J.-P., 2004}

Droits fonciers et dimension infra-famille de la gestion foncière. Note méthodologique pour une ethnographie économique de l'accès à la terre en Afrique. IRD REFO, UR 095, document de travail, $8,31 \mathrm{p}$.

Courel M.-F., Morin S., Raimond C., 1997 «Intégration modèle ou modèle d'intégration. La gestion de l'environnement au lac Fitri (Tchad) ». In : Singaravelou P., éd. : Gestion de l'environnement dans les pays tropicaux, Bordeaux, Dymset-Cret, p. 311-326.

Dagou P., Mustapha M. A.,

Goltob Mbaye N., Passiring K.,

NGAR-ODJILO M., 2005

« La pêche dans les lacs Fitri et Léré au Tchad.

Techniques de capture, conservation des produits et enjeux de protection ». In : Raimond C., Garine E., Langlois O., éd. : Ressources vivrières et choix alimentaires dans le bassin du lac Tchad. Paris, IRD Éditions/Prodig, p. 87-112.

\section{Gillet H., 1962}

«Végétation, agriculture et sol du Centre Tchad. Feuilles de Mongo, Melfi, Bokoro, Guéra ». Journal d'agriculture tropicale et de botanique appliquée, 9 (11-12) : 451-501.

\section{Hagenbucher F., 1968}

Notes sur les Bilala du Fitri. Cahiers Orstom Sciences Humaines, ${ }^{\circ}$ 5(4) : 39-76.

\section{KARDIGUÉ J., 2017}

L'homme et l'eau autour du lac Fitri. Master 2 d'anthropologie, université de N'Djamena, $121 \mathrm{p}$.

\section{Kemsol Nagorngar A., Raimond C., Madjigoto R., JofaCk S. V., DJimassal D., Libar J., Kouame K. F. \\ «Fluctuation des récoltes de sorgho repiqué et potentialités de culture. Une analyse par télédétection dans la région du lac Fitri ». Ce volume.}

\section{Koyoumtan A., 2002}

Activités rurales et gestion foncière autour du lac Fitri. Mémoire de maîtrise de géographie, université de N'Djamena, 109 p.

\section{LaVigne Delville P., 2002}

Les pratiques populaires de recours à l'écrit dans les transactions foncières en Afrique rurale. Éclairages sur les dynamiques d'innovation institutionnelle. IRD REFO, UR 095, document de travail, 7, $21 \mathrm{p}$.

\section{Le Bris E., Le Roy E., Leimdorfer F., GRÉGOIRE E., 1982}

Enjeux fonciers en Afrique Noire. Orstom, Karthala, coll. Hommes et Sociétés, 430 p.

\section{Le Meur P.-Y., 2002}

Approche qualitative de la question foncière. Note méthodologique. IRD Refo, UR 095, document de travail, 4, $23 \mathrm{p}$.

\section{Lemoalle J., 1989}

Le fonctionnement hydrologique du lac Tchad au cours d'une période de sécheresse (1973-1989). Rapport Orstom 89-03, 29 p.

\section{Marty A., Zakinet D., Khamis D. D.,} BERNARD C., 2012

Almy al Afia 2. Analyse de l'évolution des ressources dans le département du Fitri. Document principal. République du Tchad, 
programme d'hydraulique pastorale au Tchad central, Phase II. Antea-Iram, 128 p.

\section{P-SidRAT, 2013}

Schéma régional d'aménagement du territoire (SRAT) du Batha. Rapport final, consortium Agrer-I-mage, Matuh, 49 p.

YALIKUN T., RAIMOND C.,

Kemsol Nagorngar A., Zakinet D.,

Schuster M., Sylvestre F, 2017

«Variabilité des crues et paysages du Fitri depuis les grandes sécheresses des années 1970-1980». Ce volume.

\section{ZAKINET D., 2015}

Des transhumants entre alliances et conflits.

Les Arabes du Batha (Tchad) : 1635-2012. Thèse de doctorat en histoire, Aix-Marseille Université, $466 \mathrm{p}$.

\section{Zeltner J.-C., 1980}

Page d'histoire du Kanem, pays tchadien. Paris, L'Harmattan, 278 p. 


\title{
É conomie des échanges au lac Fitri
}

\author{
Un déficit récurrent \\ en produits alimentaires
}

\section{Introduction}

Du fait de ses ressources naturelles importantes, le lac Fitri offre des conditions favorables à l'exercice de plusieurs activités économiques. La fluctuation des crues et les pluies permettent un dualisme agricole (Koyoumtan, 2002), avec la culture de décrue aux abords immédiats du lac (berbéré, maraîchage) et la culture pluviale sur les terres exondées (sorgho, mil pénicillaire, niébé). De plus, la présence du plan d'eau pérenne rend possible les activités de pêche, d'élevage, de cueillette et d'artisanat.

Si l'on considère son potentiel halio-agropastoral, le lac Fitri devrait être un pôle exportateur de produits alimentaires comme le lac Tchad (MAGRIN et MBAYE, 2014). Paradoxalement, cette région importe régulièrement des céréales pour nourrir sa population. Pourquoi cette région se trouve-t-elle dans une situation d'échanges déficitaires réguliers et ce, malgré des ressources naturelles abondantes ? La création de nouveaux marchés de produits alimentaires dans le cadre du processus de désenclavement en cours va-t-il contribuer, comme d'autres contextes (MAGRIN, 2001), à un excès de commercialisation susceptible de renforcer l'insécurité alimentaire ? Nous éprouverons l'hypothèse suivante : la vulnérabilité vis-à-vis de l'inondation, la croissance rapide de la population, et les facteurs secondaires tels que les méfaits des oiseaux granivores et des 
animaux en divagation, expliquent les déficits vivriers et le fait que le Fitri n'est pas toujours exportateur de céréales et de vivres.

La présente analyse s'inscrit dans le courant de la géographie rurale, qui depuis les années 1980, « retrouve » les villes et s'intéresse à l'émergence d'une agriculture vivrière marchande destinée à satisfaire la demande urbaine (ChaléARD, 1996). Nous utilisons ici l'expression « vivrier marchand » pour désigner l'ensemble des produits agricoles destinés à la fois à l'autoconsommation et à la vente.

Les résultats sont basés sur l'exploitation des rapports de plusieurs projets de développement qui sont intervenus dans la région depuis les années 1960, sur les observations directes des pratiques commerciales autour du lac Fitri et sur des entretiens individuels réalisés auprès des différents acteurs. Au total, 11 focus groupes ont été réalisés avec les chefs de village, leurs notables et les responsables de marchés de village. Nous avons aussi réalisé 291 entretiens individuels sur les différents marchés autour du lac avec les vendeurs de produits agricoles, artisanaux, de cueillette ; avec les pêcheurs, les négociants, les rabatteurs ou collecteurs, les stockeurs de poisson fumé ; avec les éleveurs, les secrétaires de marchés à bétail, les collecteurs de taxes, les collecteurs de bétail ; enfin avec les conducteurs de véhicules, les dignitaires du sultanat et les chefs de soussecteur de pêche.

Nous présentons en premier lieu la diversité des produits du Fitri et des lieux de commercialisation, puis nous évaluons les excédents céréaliers potentiels en fonction des niveaux de crue. Enfin, les contraintes liées au développement des échanges sont analysées et mettent en évidence une forte augmentation des productions depuis la fin des années 1990, qui ne suit toutefois pas le rythme de l'accroissement démographique local.

\section{Une diversité de ressources naturelles pour une diversité de productions}

Comme les autres zones humides sahéliennes, le lac Fitri concentre une grande diversité de ressources qui attire de nombreuses populations.

\section{L'eau, la terre, le fourrage et le poisson}

L'écosystème lacustre du Fitri fournit les ressources naturelles suivantes :

- un plan d'eau pérenne et poissonneux qui constitue la principale source d'abreuvement pour les animaux sauvages et domestiques ; 
- des ressources fourragères abondantes et diversifiées dominées par les pâturages aquatiques sur près de 25000 ha composés surtout de bourgou (Echinochloa stagnina), qualifié d'excellent par les éleveurs et les agropastoralistes (RÉPUBLIQUE DU TCHAD, 2011);

- des sols cultivables repartis sur les terres inondées (domaine de la culture de décrue) et les terres exondées (domaine de la culture pluviale) ;

- des arbres diversifiés utilisés pour leur bois, leurs feuilles et leurs fruits et destinés à des usages alimentaires et domestiques (feu, construction), ainsi qu'à la confection des objets artisanaux : principalement Acacia seyal, Acacia senegal, Ziziphus mauritania, Tamarindus indica, Balanites aegyptiaca, Hyphaene thebaica, Borassus aethiopium;

- et des graminées, dont Nymphea aquatica, Panicum laetum, Hibiscus asper qui font régulièrement l'objet de cueillette pour l'alimentation humaine et sont les plus commercialisées.

Ces ressources naturelles favorisent l'imbrication de plusieurs types d'activités économiques autour du lac Fitri.

\section{Une pluriactivité favorisée par le milieu}

La pluriactivité d'un espace est la compatibilité d'un milieu multifonctionnel à fournir une panoplie d'activités économiques que sont, pour le cas du Fitri : l'agriculture, l'élevage, la pêche, la cueillette, l'artisanat (MARTY et al., 2012).

L'agriculture repose sur deux types de cultures pratiquées en alternance dans l'année. La culture pluviale sur les terres exondées concerne le sorgho rouge (Sorghum caudatum) et le mil pénicillaire (Pennisetum typhoides). La culture de décrue porte principalement sur le sorgho repiqué (Sorghum bicolor) appelé berbéré en arabe local. Il est notamment cultivé dans la zone de marnage du lac. Le maraîchage est pratiqué sur les îles, autour du lac et dans le delta du Batha. Il concerne le gombo (Abelmoschus esculentus), les cucurbitacées (pastèques, melons, concombres, calebasses), la tomate (Lycopersicum esculentum), le niébé (Vigna unguiculata), la patate douce (Ipomea batatas), la laitue (Lactuca sativa), la carotte (Daucus carota), l'oseille de Guinée (Hibiscus sabdariffa), le piment (Capsicum spp.), l'aubergine amère (Solanum aethiopicum). Au-delà de leur usage pour l'alimentation familiale, ces produits agricoles font tous l'objet de vente sur les marchés.

L'élevage est de type extensif et caractérisé par une mobilité constante des animaux et des bergers. Les éleveurs du Fitri sont principalement des transhumants qui fréquentent la zone du Fitri en saison sèche puis reconduisent les animaux en saison des pluies vers le nord de la région du Batha, notamment dans la zone de Djedda et Abou Hedjilidji (AuBAgue et al., 2007). On peut estimer globalement à 2,2 millions de têtes l'effectif du cheptel dans le Fitri, dont 2 millions de têtes de bovins, caprins et ovins ; 4000 têtes de camelins et 82000 têtes d'asins et d'équins (République DU TCHAD, 2011). 
La pêche artisanale est pratiquée au Fitri à partir de novembre et prend fin en juin. Cette saisonnalité s'explique par l'influence de l'agriculture, considérée comme l'activité principale des Bilala, population riveraine majoritaire du lac. On distingue deux catégories de pêcheurs dans le lac Fitri : les pêcheurs professionnels qui sont des étrangers (nigérians, camerounais, nigériens, maliens) et les pêcheurs saisonniers qui sont les agriculteurs bilala sédentaires et les éleveurs arabes transhumants.

La cueillette est une activité pratiquée par tous les groupes qui fréquentent le Fitri pour se prémunir des situations de famine (KoYOUMTAN, 2002) ou de déficit alimentaire. Ainsi les fruits de Ziziphus mauritania, Tamarindus indica et Balanites aegyptiaca, les gousses et racines de Nymphea aquatica, les graines de Panicum laetum, sont cueillis et utilisés pour l'alimentation familiale et une partie est aussi vendue sur les marchés. Les exsudats d'Acacia seyal et Acacia senegal sont collectés pour être spécifiquement commercialisés, de même que les noix de doum (Hyphaene thebaica).

L'artisanat est aussi une activité traditionnelle, presque exclusivement féminine. Les femmes sont spécialisées dans le tissage des nattes, cordes, paniers, la poterie et la tannerie. Quelques hommes sont des forgerons. Ils fabriquent principalement l'outillage agricole (houe, hache, coupe-coupe) et des couteaux. Les artisans sont communément appelés haddad en arabe. L'artisanat procure à ceux qui le pratique un revenu supplémentaire non négligeable permettant de subvenir à leurs besoins élémentaires, notamment l'achat de la nourriture et des habits.

Les produits de ces divers secteurs d'activités, à savoir les céréales, le bétail sur pied et le poisson, favorisent des échanges marchands. La volonté de vendre ces produits, conjuguée au besoin de gagner de l'argent pour pouvoir satisfaire les besoins essentiels (dont les habits, le sucre, le thé), est à l'origine de la création de plusieurs types de marchés autour du lac Fitri.

\section{La typologie des marchés autour du lac Fitri}

Il existe 24 marchés autour du lac Fitri. Nous avons pu en visiter 17. La majorité de ces aires d'échanges est animée une fois dans la semaine. Pour établir la typologie des marchés, nous nous sommes servis des critères suivants : la saisonnalité, le rayon de fréquentation, les infrastructures et le niveau du flux de marchandises. Nous distinguons trois principaux types de marchés autour du lac Fitri, conformément aux autres zones rurales tchadiennes (MAGRIN, 2001). 


\section{Les marchés ruraux hebdomadaires}

Ils se localisent dans les villages de 400 à 1700 habitants. Ce sont de petits marchés qui se tiennent une fois dans la semaine. Leurs infrastructures d'accueil sont dominées par des hangars construits à base de tiges de mil. Ils sont fréquentés par les habitants des villages et ceux des environs venus pour vendre une partie des récoltes en vue de satisfaire leurs besoins élémentaires, sur un rayon de fréquentation allant de 20 à $40 \mathrm{~km}$.

Du point de vue de la saisonnalité, on distingue deux sous-groupes. Les marchés ruraux hebdomadaires saisonniers sont complètement inondés cinq à six mois dans l'année par les crues du lac, comme par exemple les marchés d'Ati Ardep (1 750 habitants en 2015) et de Doubounoro (450 habitants), respectivement à moins de $3 \mathrm{~km}$ et $1 \mathrm{~km}$ du lac. Les marchés ruraux hebdomadaires mobiles se tiennent toute l'année mais oscillent entre deux espaces commerciaux différents. Une aire d'échange est située dans un village riverain du lac et l'autre dans un village, un peu éloigné du lac, dans la plaine exondée. Lorsque le débordement du lac inonde la route menant vers le village riverain, le marché est automatiquement déplacé dans le village plus éloigné de la crue. Les marchés se déplacent donc entre deux villages. C'est le cas du marché mobile de Kassaba (400 habitants) qui est éloigné d'environ $5 \mathrm{~km}$ du lac et du marché mobile de Dogo (900 habitants) près du lac dans sa partie sud (fig. 1).

Ces marchés ruraux sont des aires de distribution ou d'approvisionnement en vivres par le biais d'achats entre les éleveurs arabes demandeurs de produits agricoles et les agriculteurs bilala demandeurs de produits et sous-produits de l'élevage. Des véhicules (surtout des pick-up), des motos et des charrettes hippomobiles assurent la livraison des marchandises. Ainsi, on a observé le jour du marché en mars 2015 : 1 à 3 pick-up sur certains marchés comme Taba (1 pick-up), 5 à 10 pick-up sur d'autres à savoir Gorko (9 pick-up), Seita Korlosso (6 pick-up) et Galo (10 pick-up).

\section{Les marchés semi-urbains de rassemblement}

Les infrastructures de ces marchés sont mixtes, c'est-à-dire semi-modernes et traditionnelles. Elles sont constituées d'une part de boutiques et magasins de céréales construits en banco qui ouvrent leurs portes quotidiennement; et d'autre part de hangars destinés à accueillir les vendeurs d'ail, oignon, tomates fraîches, céréales, laitues, patates douces, mais aussi des vêtements et divers produits manufacturés. En réalité, ces marchés peuvent être aussi appelés « marchés semi-urbains régionaux » puisqu'en saison sèche, des véhicules (pick-up et parfois des gros porteurs) en provenance d'autres régions voisines y arrivent. C'est par exemple le cas du marché semi-urbain d'Ambassatna (4 000 habitants en 2015) et celui d'Abourda (3000 habitants) qui sont alimentés en produits manufacturés et en produits alimentaires (gombo séché, piment séché, tomate séchée, huile, etc.) par les véhicules en provenance de Bitkine, dans la région du Guéra. En dépit des difficultés d'accès en saison des pluies, l'animation de 


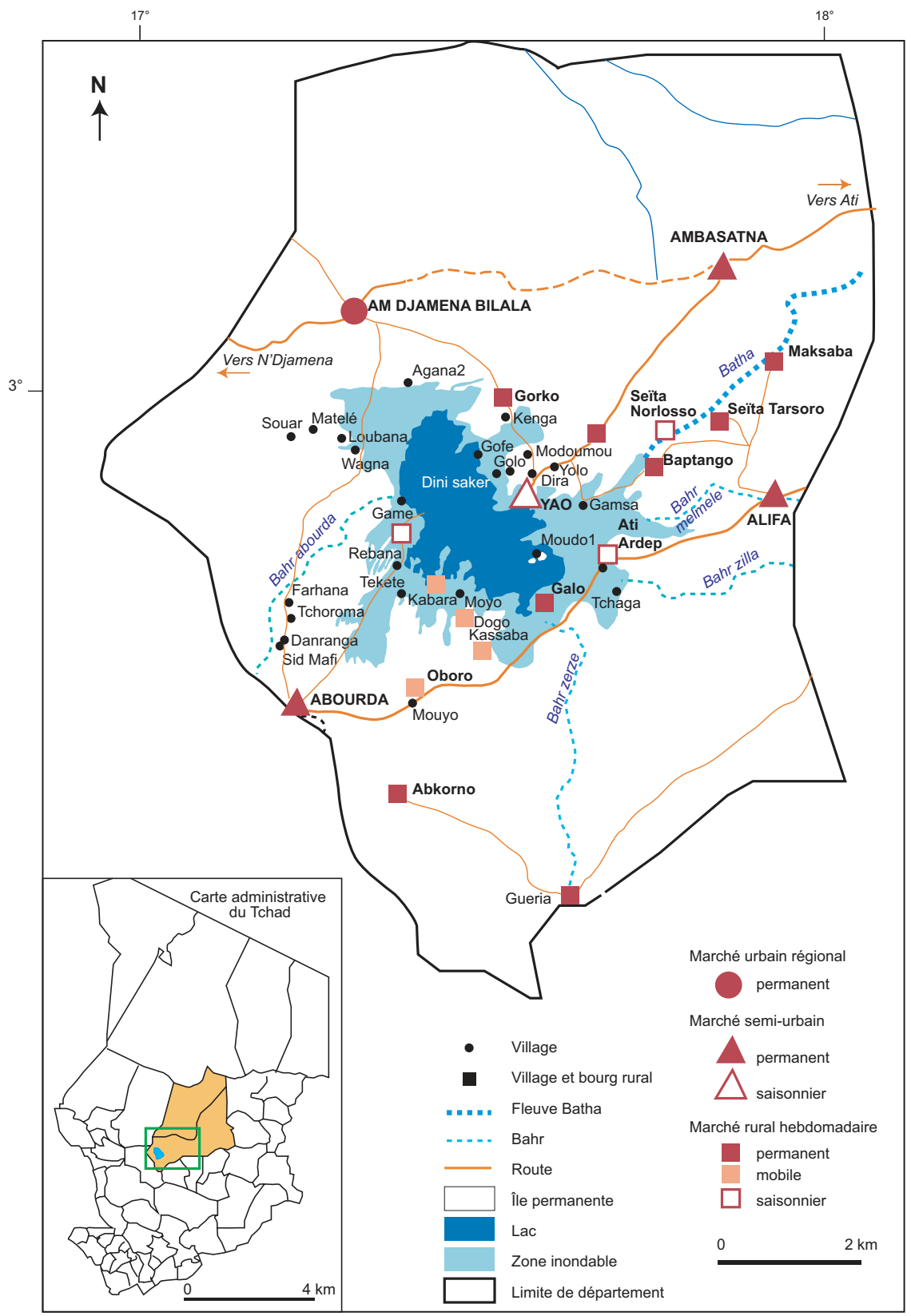

Figure I

Localisation et typologie des marchés autour du lac Fitri.

Source : P-Sidrat.

Réalisation : Blaise Bémadji, 21/02/2017.

ces marchés demeure quasi permanente du fait de l'importance de la population des bourgs qui les hébergent et de leur position par rapport au lac. Toutefois, certains souffrent de l'inondation des routes par les eaux des pluies et les crues du lac. C'est le cas par exemple du marché de Yao (5 000 habitants) qui est inaccessible d'août à septembre. 


\section{Le marché urbain régional}

C'est le marché d'Am Djamena Bilala (9 000 habitants en 2015). Les infrastructures d'accueil dominantes sont les boutiques construites en matériaux non durables (briques de terre crue, toits en tôle ou en banco). Ce marché connaît une animation quotidienne remarquable. Les boutiques font fonction de lieux de conservation (magasins céréaliers) et de vente de produits de consommation courante tels que boissons sucrées, huile, sucre, thé, savons, piles. Les hangars sont ici moins importants que dans les marchés semi-urbains. Situé à $45 \mathrm{~km}$ au nord-ouest du lac, ce marché est le grand débouché des marchés semi-urbains et des marchés ruraux hebdomadaires. Il est l'espace commercial où s'opère la revente des céréales, des produits maraîchers, du poisson séché ou fumé, des peaux et cuirs collectés sur les marchés ruraux et semi-urbains. Ce marché a une envergure régionale puisqu'en dehors du Batha, les commerçants d'autres régions comme le Salamat y envoient régulièrement leurs marchandises. C'est un marché permanent malgré l'enclavement en années de bonne pluviométrie. Le bitumage de la route, en cours depuis 2014, en facilitera l'accès. Il est difficile d'avoir le nombre exact de véhicules aperçus sur ce marché parce que certains pick-up appartiennent à des commerçants locaux. Des véhicules sortent avec des sacs de berbéré, chaque samedi pendant les récoltes. En revanche, pendant la période de soudure, environ 5 véhicules débarquent par semaine à Am Djamena Bilala des céréales importées d'Am Timan.

\section{Les marchés à bétail}

Il faut noter que les marchés à bétail sont hétérogènes. Les petits ruminants sont spécifiquement proposés sur les marchés ruraux hebdomadaires tandis que sur les marchés semi-urbains de rassemblement, on associe quelques têtes de bœufs, de chevaux et ânes avec les petits ruminants et avec les produits agricoles et manufacturés. Il existe un seul marché spécialisé pour le bétail dans le département de Fitri, c'est celui d'Am Djamena Bilala.

\section{Des excédents aléatoires}

Pour évaluer le système des échanges commerciaux du Fitri, nous nous focalisons sur les céréales en proposant en premier lieu une évaluation du bilan céréalier annuel, puis en observant sa variabilité interannuelle.

\section{Le bilan céréalier}

Pour estimer le bilan céréalier, nous avons utilisé la méthode suggérée par la division des statistiques agricoles du ministère de l'Agriculture. Elle consiste à 
soustraire les besoins céréaliers de la population locale de la production totale. Le besoin en céréales par personne est estimé à $159 \mathrm{~kg}$ par l'Inseed ${ }^{1}$. Pour évaluer le besoin en consommation en tonnes de la population, il faut multiplier l'effectif de la population de l'année considérée par 0,159 t. Ainsi, si nous prenons la production céréalière de 2011 qui est de $18658 \mathrm{t}$ (données statistiques de 1'ONDR Yao, 2015) et la population de 2011 du Fitri, estimée à environ 118462 habitants (projection à partir des données du RGPH, 2009), le besoin en consommation de céréales est de $18835 \mathrm{t}$ : la production de l'année est inférieure au besoin en consommation de céréales. La différence entre besoin en consommation de céréales et production est de -177 t, qu'il faudra importer d'autres régions pour que le besoin en consommation céréalière soit assuré pour l'année considérée. Cette année-là, les céréales ne peuvent théoriquement pas être exportées du Fitri puisque la production ne couvre pas le besoin alimentaire de la région. De ce fait, l'assertion selon laquelle le Fitri est un « grenier à mil » est à employer avec prudence (MARTY et al., 2012).

À l'inverse, si nous considérons la production céréalière de 2014 qui est de 119687 t et la population de 2014 du Fitri estimée à environ 131755 habitants, le besoin en consommation de céréales est de 20950 t. La production de l'année est ici très nettement supérieure au besoin local en céréales : il se dégage un surplus, entre le besoin en consommation de céréales et la production, de $98737 \mathrm{t}$ qui pourrait être exporté.

Au cours des cinq dernières années (2010-2014), on a constaté deux bonnes années de production céréalière avec des excédents exportables contre trois mauvaises années de production céréalière.

Les bonnes années correspondent aux années de bonne production céréalière comme en 2014 (119000 t de céréales), 2012 (112000 t) et les mauvaises années correspondent à une médiocre production comme en 2010 (76 $000 \mathrm{t})$, $2011(18000 \mathrm{t})$ et $2013(95000 \mathrm{t})$.

\section{Les stratégies d'adaptation des commerçants}

Les commerçants-grossistes adoptent des stratégies en fonction de la situation. Lors des bonnes années, les commerçants achètent d'importantes quantités de sacs de céréales, les stockent dans leurs magasins construits à cet effet dans les centres semi-urbains comme Ambassatna, Alifa et urbain comme Am Djamena Bilala. D'après les commerçants-grossistes interrogés, le stock se fait généralement pendant la période des récoltes, c'est-à-dire au moment où les céréales sont abondantes et à bon prix. On achète le sac de $100 \mathrm{~kg}$ de 18000 à $20000 \mathrm{CFA}$ soit 450 à $575 \mathrm{CFA}_{\text {le }}$ coro $^{2}$, de volume variable selon les types de céréales. On le revend en période de soudure sur le même marché, à partir de juillet, de 26000 à 30000 CFA le sac soit 650 à 750 CFA le coro.

I. Institut national de la statistique, des études économiques et démographiques, organisme de production des données statistiques du ministère du Plan au Tchad.

2. Unité de mesure utilisée sur le marché équivalant à $2,5 \mathrm{~kg}$. 
Les mauvaises années, les céréales sont importées des régions voisines et/ou lointaines pour combler le déficit local. Les commerçants-grossistes disent avoir souvent importé principalement le berbéré du Salamat. Pendant certaines très mauvaises années comme en 2011, les céréales sont importées de Ba-Illi dans le Chari-Baguirmi (mil pénicillaire et sorgho pluvial uniquement), voire le berbéré de Kousseri au Cameroun. Ces commerçants-grossistes s'organisent en petits groupes de 4 à 6 personnes pour louer un camion gros porteur afin d'acheminer ces produits au Fitri. Les céréales sont directement vendues sur les marchés mais une partie est stockée dans les magasins pour n'être revendue qu'en période de soudure, au moment où les gros porteurs ne peuvent pas accéder au Fitri.

\section{Les contraintes au développement de la production et aux échanges des produits alimentaires}

\section{L'enclavement, un défi permanent}

La région du Batha demeure l'une des régions les plus enclavées du Tchad et ce, malgré le récent investissement dans les infrastructures routières par l'État. Le réseau routier régional est constitué essentiellement de pistes (RÉPUBLIQUE DU TCHAD, 2011). La plupart des chefs-lieux de départements et sous-préfectures sont inaccessibles pendant une grande partie de l'année et coupés d'Ati, le cheflieu de la région. Ainsi, le département du Fitri, zone de grande production agropastorale, est quasiment enclavé de quatre à cinq mois dans l'année (Aubague et al., 2007). Yao, le chef-lieu du département n'est desservi que par des routes à praticabilité intermittente. D'août à septembre, la circulation est impossible sur les axes Yao-Am Djamena Bilala ou Ambassatna-Am Djamena Bilala qui traversent les plaines argileuses. D'autres pistes reliant Yao sont coupées par le fleuve Batha (fig. 1).

L'impraticabilité des routes a une incidence sur l'exportation de certains produits du lac Fitri. Ainsi, jusqu'en 2009, il était impossible d'approvisionner Abéché en poisson frais du lac Fitri, le plus grand marché de consommation de l'est du Tchad (97 900 habitants). En 2016, cet approvisionnement est rendu possible grâce au bitumage de l'axe Oum Hadjer-Abéché (entre 2005-2009) et du pont de Koundjourou (en 2013). Le bitumage de l'axe Ngoura-Am Djamena Bilala dont les travaux ont commencé depuis 2014 et se poursuivent, permet aussi d'approvisionner la capitale du Tchad, N'Djamena. Environ 6,6 t de poisson frais du Fitri sont acheminées par jour vers N'Djamena et $11 \mathrm{t}$ de poisson frais par semaine vers Abéché (enquête, février 2015). 


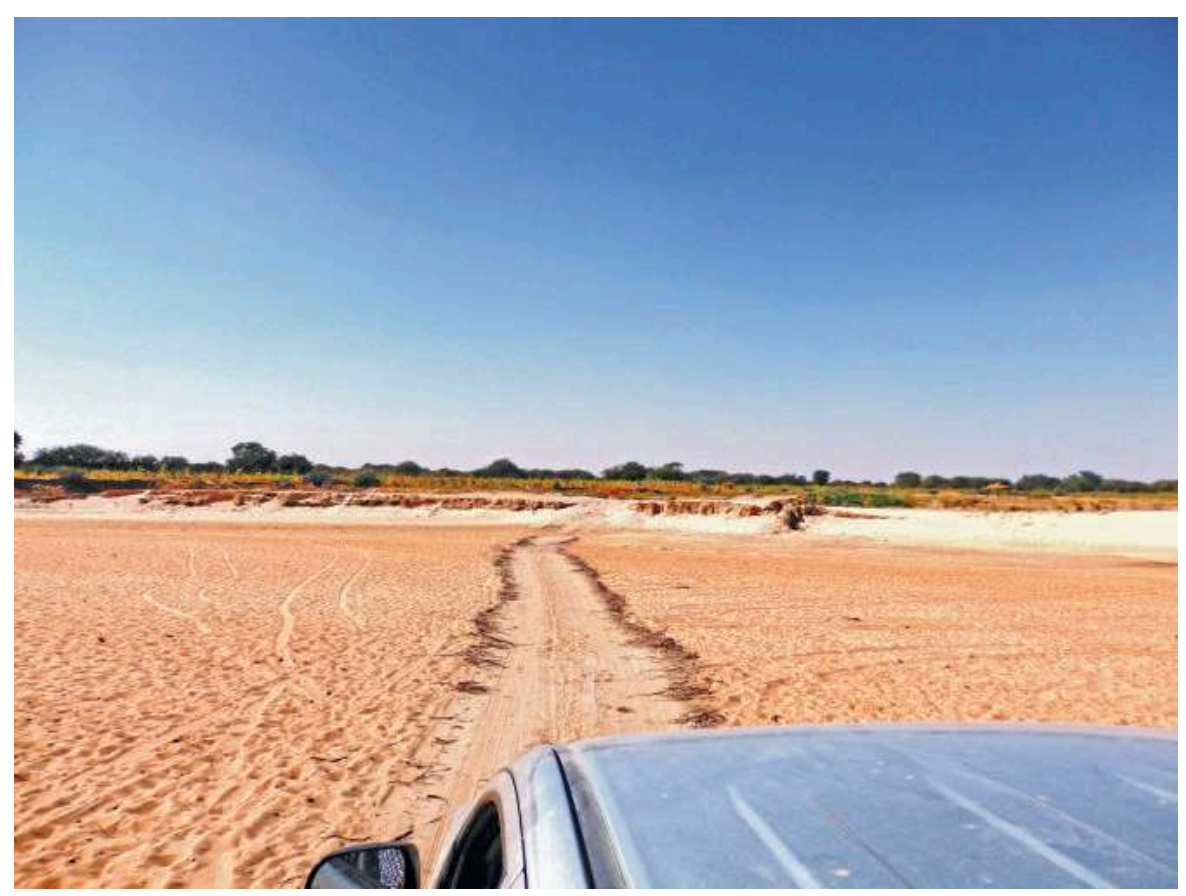

\section{Photo I}

Une piste traversant le lit du fleuve Batha.

Pour accéder aux marchés situés sur la rive orientale (Alifa ou Ati Ardep), il faut attendre le tarissement total des eaux du fleuve qui coule de juillet à octobre.

(c) Blaise Bémadji, février 2015.

Le mauvais état des routes a des répercussions sur la commercialisation des produits halieutiques. Ainsi, l'inaccessibilité des véhicules à certains débarcadères oblige les pêcheurs et les commerçants à sécher les poissons pour attendre que les pistes soient à nouveau praticables à partir de janvier.

La méconnaissance des techniques de conservation post-récolte constitue également une contrainte à la commercialisation de produits maraîchers, notamment la tomate fraîche (MoussA et YACOUB, 2012).

L'impraticabilité des routes une partie de l'année est aussi un obstacle à la permanence des marchés de bétail. En effet, pendant la saison des pluies, on observe un temps mort dans les activités de vente de bétail sur pied. Par ailleurs, plusieurs marchés fonctionnent au rythme des crues (fig. 1). Pendant les crues, certains marchés ruraux hebdomadaires sont inondés tandis que d'autres sont coupés durant la période d'écoulement du Batha et des autres rivières. Ainsi, certains marchés ruraux hebdomadaires sont animés quatre mois sur douze, d'autres sept à huit mois sur douze selon la position de ces derniers par rapport au lac. Pour accéder en véhicule ou en charrette hippomobile aux marchés situés de part et d'autre des rives du fleuve Batha, il faut attendre début novembre quand le fleuve est à sec (photo 1). 


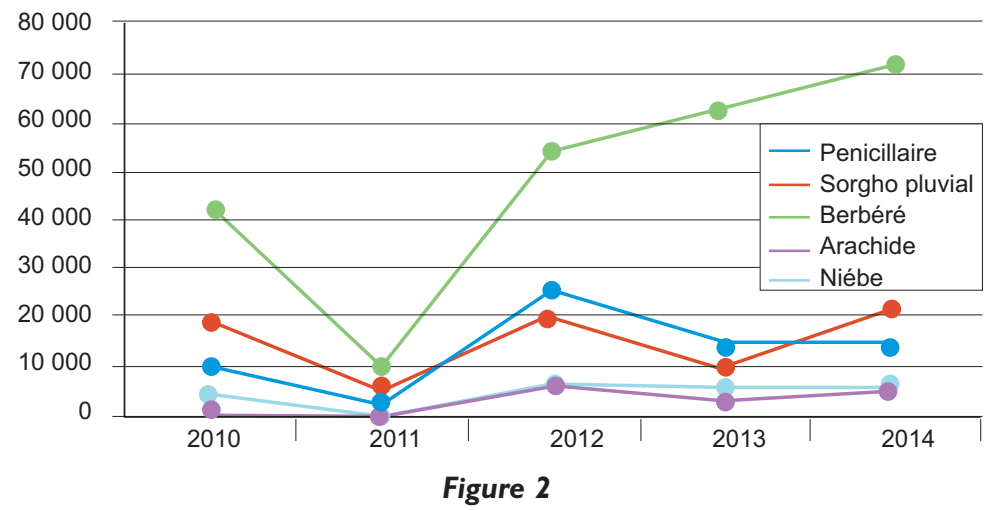

Production agricole totale en tonnes de 2010 à 2014 au Fitri. Source : sous-secteur ONDR de Yao, décembre 2015.

\section{L'influence des crues et de la pluviométrie sur la production et les échanges de céréales}

Les fluctuations du lac et la variation de la pluviométrie influent sur la production et les échanges de céréales. Les années de forte production se caractérisent par des précipitations abondantes et de fortes crues tandis que celles de faible pluviométrie et faibles crues entraînent une production médiocre. En effet, on observe suivant les années une alternance des crues de plus ou moins importante amplitude et une variabilité des précipitations. Ainsi, quand les pluies et les crues sont importantes, elles inondent de vastes superficies cultivables en décrue autour du lac, estimées de 29760 ha à 53900 ha (KOYOumTAN, 2002), et jusqu'à 100000 ha en 2015 (Kemsol Nagorngar et al., ce volume). Ces années représentent une production céréalière considérable dont l'excédent est exporté. En revanche, les années de faible pluviométrie et crue ne dégagent pas suffisamment de surfaces cultivables (8 520 ha à 17460 ha). La production céréalière est alors moins importante, plongeant la région dans un déficit de berbéré d'une part et d'autre part dans une insécurité alimentaire relative.

La production céréalière est donc très variable d'une année à l'autre, particulièrement le berbéré étroitement dépendant de l'ampleur de la crue (fig. 2). Ainsi, la production de berbéré est multipliée par sept entre 2011 (9845 t) et 2014 (71 $700 \mathrm{t}$ ). Les cultures pluviales sont moins importantes et dépendantes de la pluviométrie : la production de mil pénicillaire était de $2256 \mathrm{t}$ en 2011, $15520 \mathrm{t}$ en 2014, le sorgho de $5440 \mathrm{t}$ en 2011 et $21820 \mathrm{t}$ en 2014.

L'importante production de berbéré va de pair avec la production de mil pénicillaire et de sorgho pluvial parce que c'est la bonne pluviosité qui conditionne les fortes crues par le déclenchement très fort des écoulements du fleuve Batha et autres barhs qui alimentent le lac.

Les faibles crues et précipitations sont les principales responsables de la sousproduction de céréales induisant un déficit dans le Fitri. Selon l'enquête, les 
commerçants-grossistes importateurs interrogés disent n'avoir pas importé de céréales en 2014 parce que la production de cette année est considérée comme excédentaire, avec de fortes précipitations et crues, donc d'importantes superficies cultivables.

Le déficit céréalier très important enregistré en 2011 a créé une situation de disette dans la région du Batha (MARTY et al., 2012), comme il en a existé d'autres auparavant en 1973 ou 1984 (Courel et al., 1997) et a entraîné l'importation de céréales pour nourrir la population.

\section{Les facteurs secondaires limitant la production et les échanges des produits alimentaires}

La croissance rapide de la population exerce un poids non négligeable sur l'exportation des produits alimentaires. En effet, l'augmentation de la population implique l'augmentation des besoins en consommation. Bien qu'on ne dispose pas actuellement de données précises sur la quantité de céréales exportées, on sait que le Fitri était une zone de production excédentaire (BIEP, 1989) qui exportait dans les années 1990 des céréales : environ 2000 t vers le BorkouEnnedi-Tibesti, 5000 t vers N'Djamena (SNV, 1991). Aujourd'hui, on constate que les céréales sont beaucoup plus consommées localement qu'exportées. Cet accroissement de la demande locale s'explique par le nombre élevé des habitants enregistré ces dernières années, probablement sous-estimé en raison de la grande mobilité des populations : 77000 habitants en 1993 et 110403 habitants en 2009. Ainsi, la demande locale en céréales devient considérable et limite les excédents commercialisables.

Le comportement des bouviers conduisant les animaux dans les champs de sorgho influe également sur la production céréalière dans le Fitri. En effet, le problème de la destruction des champs par les animaux en divagation découle du non-respect des droits traditionnels d'accès aux ressources pastorales. Ces droits étaient fortement observés jadis par les éleveurs transhumants (ZAKINET, 2015). Aujourd'hui ceux-ci reviennent très tôt dans le Fitri, avant la récolte des cultures pluviales, entraînant des conflits entre les Bilala agriculteurs sédentaires et les Arabes éleveurs transhumants. Ces heurts se soldent par des pertes en vies humaines et des dégâts matériels. Nombre de paysans, notamment de la zone de Seita, disent avoir réduit le nombre d'hectares de terres qu'ils cultivaient afin de mieux assurer la surveillance des champs et d'éviter les dégâts causés par les animaux. Cette situation a des répercussions sur la production céréalière.

Par ailleurs, les oiseaux granivores (mange-mil et moineaux dorés) dévastent les champs de sorgho et causent d'énormes pertes agricoles. D'après nos entretiens avec les paysans, ces oiseaux peuvent détruire un champ en une journée. Ainsi, en 2014 à Gueria, les oiseaux ont presque tout mangé. Un hectare de champ n'a pu donner cette année-là qu'un sac de $100 \mathrm{~kg}$. Ce phénomène crée une situation insupportable chez les victimes, les contraignant à se livrer à la cueillette. À l'approche des récoltes, pour surveiller les champs de berbéré, 
il n'est pas rare de voir les femmes et les enfants passer toute la journée dans les champs pour chasser les oiseaux. Cette situation compromet non seulement la production mais aussi l'éducation des enfants, qui désertent les salles de classe pour surveiller les champs.

En 2014, selon l'ONDR et le Programme national de sécurité alimentaire de Yao, 2150 ha de champs de mil pénicillaire, sorgho et berbéré ont été dévastés par les oiseaux granivores et les insectes. Au cours de cette même année, seulement 165 ha de champs de niébé ont été détruits. Ceci a déclenché la formidable reconversion des paysans dans la culture de niébé. C'est la ville de Yao qui a subi les plus grosses pertes, où 412 ha de champs ont été détruits, 213 ha à Sartoua, 214 ha à Abragna, 178 ha à Baptango. Ces villages se situent tous au nord du lac, et les rendements sont compris entre $500 \mathrm{~kg}$ et $700 \mathrm{~kg} / \mathrm{ha}$, soient 5 à 7 sacs de $100 \mathrm{~kg}$ (enquête). Si l'on considère un rendement moyen de $600 \mathrm{~kg} / \mathrm{ha}$, soit 6 sacs de $100 \mathrm{~kg}$, multiplié par le nombre total de champs dévastés (2 147), ces villages ont perdu 12882 t. Avec un prix par sac pendant cette période de 20000 FCFA, on estime la perte à 257640000 FCFA pour les agriculteurs.

\section{Conclusion}

L'écosystème du lac Fitri possède d'importantes ressources naturelles favorisant une pluriactivité économique. La nécessité de vendre une partie de la production issue de ces diverses activités afin de subvenir aux besoins élémentaires des populations riveraines induit la création de plusieurs types de marchés autour du lac. Cependant, l'enclavement du Fitri demeure une contrainte majeure au développement des échanges des produits alimentaires (MARTY et al., 2012 ; RÉPUBliQue DU TCHAD, 2011).

Les enquêtes montrent qu'il existe d'autres contraintes non négligeables affectant la production céréalière et partant, le déficit d'échanges. Il s'agit de la vulnérabilité aux crues, de la croissance rapide de la population, de la dévastation des champs par les animaux en divagation et des oiseaux granivores.

Au cours des cinq dernières années (2010-2014), on a constaté deux bonnes années de production céréalière avec des excédents exportables contre trois mauvaises années de production céréalière.

L'aménagement des routes et des pistes rurales autour du lac favorisera la fluidité dans l'écoulement des produits (céréales, poissons et bétail sur pied) vers les marchés urbains de consommation plus rémunérateurs. Il sera aussi un facteur incitatif qui poussera les paysans à augmenter la production. 


\section{Bibliographie}

Aubague S., Duimadoum D., Ali A. M., 2007 Le Fitri : Diagnostic pastoral. République du Tchad, ministère de la Pêche, de l'Hydraulique pastorale et villageoise, programme d'hydraulique pastorale au Tchad «Almy Al Afia », Antea-Iram, 91 p.

\section{BIEP, 1989}

Étude de développement intégré du lac Fitri. N'Djamena, ministère de l'Agriculture, Banque islamique de développement, Cedrat-SA, 61 p.

\section{Chaléard J.-L., 1996}

Temps des villes, temps des vivres.

L'essor du vivrier marchand en Côte d'Tvoire. Paris, Karthala, 661 p.

Courel M.-F., Morin S., Raimond C., 1997 «Intégration modèle ou modèle d'intégration. La gestion de l'environnement au lac Fitri (Tchad) ». In : Singaravelou P., éd. : Gestion de l'environnement dans les pays tropicaux, Bordeaux, Dymset-Cret, p. 311-326.

Kemsol Nagorngar A., Raimond C., Madjigoto R., Jofack S. V., DJimassal D., Libar J., Kouame K. F.

«Fluctuation des récoltes de sorgho repiqué et potentialités de culture. Une analyse par télédétection dans la région du lac Fitri ». Ce volume.

\section{Koyoumtan A., 2002}

Activités rurales et gestion foncière autour du lac Fitri. Mémoire de maîtrise de géographie, université de N'Djamena, 109 p.

\section{MAGRIN G., 2001}

Le sud du Tchad en mutation. Des champs de coton aux sirènes de l'or noir. Paris, Cirad-Prasac-Sepia, 428 p.

\section{Magrin G., Mbaye N. G., 2014}

«Le lac Tchad et les échanges : un pôle agricole exportateur ». In : Lemoalle J., Magrin G., dir. : Le développement du lac Tchad : situation actuelle et futurs possibles. CBLT, N'Djamena, Marseille, Expertise collégiale IRD, AFD-FFEM, rapport de synthèse, $67 \mathrm{p} .+$ traduction anglaise et contributions intégrales des experts (CD, 20 chapitres, 620 p.) : 539-580.

\section{Marty A., Zakinet D., Khamis D. D.,} BERNARD C., 2012

Almy al Afia 2. Analyse de l'évolution des ressources dans le département du Fitri. Document principal. République du Tchad, programme d'hydraulique pastorale au Tchad central, Phase II. Antea-Iram, 128 p.

\section{Moussa A. K., YACOUB A., 2012}

Suivi et évaluation des cultures maraîchères dans la région du Batha : cas de la tomate dans la ville d'Ati. Rapport de fin de stage, ISTD, $40 \mathrm{p}$.

\section{RÉPUblique DU TCHAD, 2011}

Programme de système d'information pour la région du Batha. Rapport préliminaire, ministère de l'Aménagement du territoire, de l'Urbanisme et de l'Habitat, 124 p.

\section{SNV, 1991}

Programme de développement régional/préfecture du Batha. Rapport de synthèse, République du Tchad, ministère du Plan et de la Coopération internationale, $114 \mathrm{p}$.

\section{ZAKINET D., 2015}

Des transhumants entre alliances et conflits. Les Arabes du Batha (Tchad) : 1635-2012. Thèse de doctorat en histoire, Aix-Marseille Université, $466 \mathrm{p}$. 


\section{es territoires d'eau}

\section{ou comment gérer les ressources}

\section{halieutiques dans les espaces \\ lacustres?}

\section{Les lacs Fitri et Iro}

Marguerite SAUNIER, Christine RAIMOND,

Brahim ABBA AMBAR

\section{Introduction}

Un « territoire d'eau » est un espace aquatique délimité de manière symbolique et/ou concrète. Il est qualifié de territoire lorsqu'il est approprié par les populations qui utilisent ses ressources halieutiques. Comme tout territoire au sens politique, ses limites sont parfois contestées et revendiquées (FAY, 1989 : 173, cité par De LA CROIX, 2015). Alors que la réglementation internationale des pêcheries maritimes est devenue un impératif majeur face à la menace que celles-ci font peser sur les écosystèmes marins, les conséquences écologiques et humaines des pratiques de pêche continentales demeurent difficiles à évaluer car souvent peu contrôlées (FAO, 2012 : 68). C'est le cas notamment au Tchad, où nous avons étudié en 2015 et $2016^{1}$ la gestion de la ressource halieutique au lac Fitri et au lac Iro.

Dans les années 1970, les pêcheries artisanales de milieu fluviolacustre en région sahélienne connaissent une pression anthropique sans précédent suite à plusieurs années de sécheresse exceptionnelles (QuENSIÈRE, 1994). En parallèle, au Tchad, la pêche s'inscrit dans le cadre de changements socioéconomiques majeurs, avec 
l'amélioration technique de la filière, l'augmentation de la demande alimentaire de la population urbaine et plus généralement, la croissance démographique. À l'échelle des zones de pêche, ces mutations posent des questions de paix sociale, de partage équitable des ressources et de durabilité.

En comparant les lacs Fitri et Iro, deux zones humides enclavées qui s'intègrent à présent aux marchés urbains et aux nouvelles infrastructures, nous cherchons à comprendre les processus institutionnels en œuvre pour gérer la compétition croissante sur les ressources halieutiques ainsi que les formes de collaboration et de résistances communautaires. Nous voulons mettre en évidence l'existence d'une gestion institutionnelle et territorialisée de la ressource halieutique sur ces deux lacs. Ces espaces lacustres ne sont pas de vastes ensembles homogènes, ils révèlent au contraire de multiples manières de gérer des territoires remettant parfois en cause l'équité de l'accès aux ressources naturelles. Notre démarche s'inspire des approches du développement participatif, devenues credo de la résilience des projets de développement depuis les travaux conduits par Elinor Ostrom sur les systèmes socioécologiques (BRONDIZIO et al., 2009).

Nous posons l'hypothèse que la pression anthropique et la diversification des activités qui touchent ces deux lacs réactivent des principes de gestion plus anciens. La présence d'un pouvoir traditionnel fort au lac Fitri peut pallier les manquements de l'État dans un pays classé parmi les plus pauvres, toutefois avec le risque de servir son propre intérêt. En revanche, un pouvoir traditionnel moins unifié et territorialisé sur le lac Iro semble exacerber des tensions économiques et environnementales, tout en préservant des espaces de liberté éventuellement plus propices à des arrangements institutionnels novateurs.

Nous nous appuierons sur les notes recueillies au cours d'une centaine d'entretiens semi-directifs menés entre 2015 et 2016 avec des pêcheurs, des chefs coutumiers, des agents de l'État, des membres de groupements de pêche et d'associations. Nos bases locales d'études furent : au Fitri, l'île de Moudo,

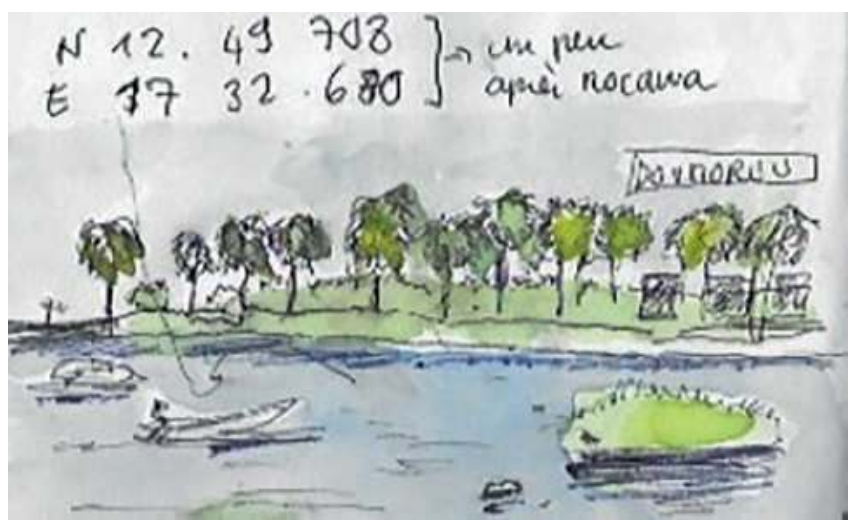

Figure I

Croquis des îles Doumourou.

Réalisation : Marguerite Saunier, 2015. 
habitée par des pêcheurs, et le sultanat ; au lac Iro, les locaux du Prodepêche en compagnie de l'équipe du programme Gelt. Les entretiens étaient le plus souvent menés en groupe avec des interprètes pêcheurs ou enseignants, autochtones ou allochtones, qui nous ont guidés sur les lacs et dans nos analyses. Notre sujet s'appuie sur l'observation de la répartition des pêcheurs autour du lac, de leur appropriation du territoire au contact d'autres communautés, ainsi que de leur rapport à la réglementation de la pêche.

Nous présenterons d'abord les modèles de gestion anciens des lacs Fitri et Iro, puis nous verrons comment les récentes évolutions démographiques et socioéconomiques ont entraîné une mutation de la gestion de la pêche. Enfin, nous nous intéresserons à la territorialisation des espaces lacustres et au rôle des institutions dans la régulation des activités halieutiques.

\section{Poissons et coutumes : les lacs Fitri et Iro, deux modèles de gestion anciens des ressources halieutiques}

\section{La modernisation de la pêche en territoire bilala}

Le lac Fitri, situé en région sahélienne, est un lac endoréique peu profond dont la superficie peut être multipliée par trois entre la saison sèche et la saison pluvieuse (COUREL et al., 2005). La situation de ce lac en zone semi-aride et la répétition d'épisodes de sécheresses totales en 1984 et 1996, limitent la quantité et la variété des espèces halieutiques. En 2002, on estimait la pêche entre 50 et $75 \mathrm{~kg}$ par jour (CIMA-SogEC, 2002), une production largement en deçà de son potentiel. En 2016, cette production a probablement augmenté et nous en verrons les facteurs et les conséquences.

La pêche au lac Fitri est une activité ancienne pour les populations locales, au même titre que la chasse et la cueillette en complément de l'agriculture. Les Bilala sont considérés comme les autochtones du lac Fitri. Ils ont construit leur identité autour du territoire et de l'organisation politique et religieuse d'un sultanat dont le siège se situe à Yao. Avant la colonisation, et avant l'État tchadien, le sultanat possédait les pleins pouvoirs sur la région du lac Fitri.

Le pouvoir du sultanat repose sur une structure centralisée : le sultan, représentant suprême, gouverne avec l'aide de sa cour et un système hiérarchisé. Il est représenté par des khalifa dont l'autorité couvre un territoire spécifique. Ces territoires comptent ensuite des kaïdala responsables de plusieurs villages et de leurs territoires. Les chefs de village appelés boulama sont élus par les villageois et dépendent des kaïdala. 
Ces différentes instances de pouvoir ont un rôle en matière de pêche. En principe, les pêcheurs allochtones sont introduits sur les différents territoires de pêche par le sultan. Aujourd'hui, ils se présentent plus communément auprès des boulama ou kaïdala responsables des îles et campements (MARTY et al., 2012). D'autres règles anciennes sont issues de cultes de la Margaï, déesse de l'eau. Ainsi les prêtres de l'eau, N'Garboulou, veillaient au respect des cérémonies de la pêche en son honneur (HAGENBUCHER, 1968). Lors du sacrifice, la pêche était interdite pendant plusieurs jours. Depuis les années 1970, ces traditions sont plus ou moins secrètes ou oubliées ; elles ont été en partie concurrencées par l'autorité religieuse du sultanat musulman.

Par ailleurs, jusque dans les années 1990, les palangres ${ }^{2}$ étaient les seuls outils autorisés sur le lac par le sultan, les pirogues à fond plat étaient interdites et la pêche se pratiquait uniquement durant la saison sèche pour permettre au stock de se régénérer en saison des pluies (COUREL et al., 1997). Depuis, les outils de pêche se sont diversifiés sur le lac avec l'utilisation des nasses et des filets et l'augmentation du nombre de pêcheurs.

\section{Les ressources halieutiques au cœur du pays d'Iro}

Le lac Iro est situé en région soudano-guinéenne. De forme circulaire, sa superficie moyenne est de $105 \mathrm{~km}^{2}$, tandis que ses environs abritent des forêts denses arbustives et des plaines d'inondation alimentées par le Chari et le Bahr Salamat. Grâce à la pluviométrie plus importante et une superficie moins variable, on recense plus d'une vingtaine d'espèces de poissons (PAIGBC, 2012). La pêche est plus conséquente qu'au lac Fitri, avec une production moyenne de $175 \mathrm{~kg}$ / jour par an (tabl. 1).

\section{Tableau I}

Comparatif des principales caractéristiques entre les lacs Fitri et Iro.

\begin{tabular}{|lll|}
\hline Caractéristiques & Lac Fitri & Lac Iro \\
\hline $\begin{array}{l}\text { Région } \\
\text { Population du département }\end{array}$ & $\begin{array}{l}\text { Batha } \\
\text { II0 } 403 \text { habitants (Fitri, } \\
\text { chef-lieu Yao) }\end{array}$ & $\begin{array}{l}\text { Moyen-Chari } \\
\text { I74 I95 habitants (lac Iro, } \\
\text { chef-lieu Kyabé) }\end{array}$ \\
\hline $\begin{array}{l}\text { Superficie en eau moyenne } \\
\text { Lieux de stockage } \\
\text { et d'exportation locaux }\end{array}$ & $\begin{array}{l}\text { Yoo, Galo,Am Djamena Bilala } \\
\text { (saison pluvieuse) }\end{array}$ & $\begin{array}{l}\text { Malam, Masidjanga, Moundourou } \\
\text { (saison pluvieuse), Roro }\end{array}$ \\
\hline Marchés locaux & $\begin{array}{l}\text { Yao, Galo,Ati Ardep, Dogo, } \\
\text { Doubonoro }\end{array}$ & Boum Kebir, Roro \\
\hline
\end{tabular}

Sources: PAIGBC, 2012 ; RGPG2, 2009.

La communauté goula est une communauté culturelle et linguistique présente dans le sud du Tchad et en République centrafricaine. Les Goula Iro sont parmi

2. Palangre : ligne fixée à la surface ou en profondeur, posée durant plusieurs heures ou journées sur laquelle des hameçons sont attachés. 
les plus anciens habitants du lac Iro et, comme pour les Bilala au Fitri, pratiquent la pêche pour leur propre consommation depuis plusieurs siècles. Au début du $\mathrm{XX}^{\mathrm{e}}$ siècle, pendant la colonisation française, les autorités ont désigné parmi les dignitaires du village de Boum Kebir un responsable traditionnel du canton Iro, ce qui a conféré à ce village ainsi qu'à la communauté goula une responsabilité dans la gestion des ressources sur l'ensemble du lac (PAIRAULT, 1994).

Dans les années 1960, les Goula pêchaient à l'aide de pièges et de sagaies. Tout comme au lac Fitri, les épisodes de sécheresse des années 1970 et 1980 marquent l'arrivée de nouveaux pêcheurs et de nouvelles techniques (PAIRAULT, 1994). Contrairement au lac Fitri, la pêche n'est pas centralisée autour d'une figure traditionnelle ancienne ${ }^{3}$ comme le N'Garboulou ou le sultan du lac Fitri. D'après Claude Pairault et nos entretiens, le chef traditionnel du lac Iro s'est effacé au profit du chef de canton.

Par ailleurs, il n'y a pas d'organisation supra-villageoise, les villages sont indépendants les uns des autres et parlent parfois des langues différentes. On remarque cependant que des chefs de mare sont en charge du contrôle des bras et des mares résiduelles durant la saison sèche du Bahr Salamat. Ces autorités coutumières, dont le pouvoir se transmet de génération en génération, émettent des règles spécifiques telles que la périodisation de la pêche et la délimitation des zones de mise en défens.

\section{La filière pêche en développement et le dérèglement du système traditionnel}

Alors que la région du Batha demeure parmi les moins peuplées au Tchad, entre 1989 et 2009 la population du département du lac Fitri est passée de 59000 à 110403 habitants (RGPH2, 2009). Dans le sud du Tchad, le lac Iro bénéficie de sa proximité avec des régions plus dynamiques en termes de desserte, d'urbanisation et de croissance de la population. Les deux lacs connaissent une pression grandissante sur leurs ressources naturelles qui pèse sur les systèmes de gestion locaux et coutumiers. Ces évolutions sont démographiques et sociales, liées à l'arrivée de plus en plus importante de migrants ; elles sont aussi économiques et géographiques, par le désenclavement de ces espaces et l'amélioration de la commercialisation du poisson ; et enfin politiques, avec l'imposition de dispositifs de protection environnementale dans les politiques nationales sous l'influence des bailleurs de fonds internationaux et le renforcement du pouvoir étatique à l'échelle locale. 


\section{Deux lacs aux trajectoires migratoires différentes}

Dans les années 1970, la sécheresse provoque la migration d'éleveurs transhumants et de pêcheurs vers les lacs et les zones humides. Au Tchad, cette période est marquée par l'arrivée importante de pêcheurs kanouri et haoussa venus du Nigeria, comme ce fut le cas au lac Tchad et aux lacs Léré, Fitri et Iro (MBAYE, 2009). Ces pêcheurs ont apporté leurs pratiques de pêche et se sont parfois sédentarisés, en particulier au lac Iro où ils ont été rejoints par leur famille. D'autres populations de pêcheurs sont arrivées ensuite, comme les Kotoko et les N'Gambaye. Cette immigration - d'après nos entretiens - semble inscrite dans la durée au lac Iro. Au lac Fitri, d'après le recensement opéré par le préfet de Yao en janvier 2015, on comptait 5000 pêcheurs professionnels non bilala. D'après nos interlocuteurs, ces pêcheurs sont arrivés de manière soudaine et importante suite aux violences perpétrées par Boko Haram au lac Tchad. Les articles parus sur le lac Fitri en 2005 valident cette thèse car ils ne mentionnent pas encore la présence des nasses - apanage des pêcheurs venus du lac Tchad alors qu'on les trouve sur une grande partie du lac aujourd'hui (COUREL et al., 2005 ; DAgOU et al., 2005). La proportion de plus en plus importante d'allochtones par rapport aux autochtones entraîne non seulement une mutation des techniques de pêche, mais aussi des modifications socioculturelles importantes parmi les pêcheurs.

\section{Émergence du commerce du poisson}

Le lac Fitri n'a pas toujours été réputé pour son commerce de poisson frais. À N'Djamena, il est largement concurrencé par la pêche en provenance du lac Tchad (Couty et Durand, 1968 ; Koussou et al., 2012). Situé à $430 \mathrm{~km}$, le lac Fitri a la réputation d'entretenir une pêche artisanale de poisson séché destinée à la consommation locale et l'exportation vers le Nigeria (CIMA-SOGEC, 2002).

La pêche connaît des changements importants liés à la route, désormais en partie goudronnée entre N'Djamena et le lac Fitri. Le débarcadère de Yao n'est plus qu'à $50 \mathrm{~km}$ de l'axe routier national N'Djamena-Abéché depuis 2016, ce qui permet d'exporter le poisson frais du lac Fitri jusqu'à la capitale en quatre heures. Les tilapias du lac Fitri sont transportés via des camionnettes remplies de glace et vendus au marché de Dembé à N'Djamena.

Le lac Iro est réputé pour sa pêche d'exportation, sa production de poisson fumé étant transportée par les pêcheurs et commerçants nigérians depuis les années 1970, et par des pêcheurs de toutes les communautés depuis les années 1990 (PAIRAUlt, 1994). Pourtant, le lac demeure difficile d'accès : la réserve naturelle de Zakouma limite le commerce de la pêche vers le nord et les inondations coupent l'ensemble du lac des voies terrestres pendant presque cinq mois dans l'année. Sur place, la pêche semble mieux organisée à des fins d'exportation commerciale qu'au lac Fitri. En effet, les campements de pêche autour du lac sont relativement accessibles en saison sèche. À partir du mois de décembre, les commerçants de poisson circulent le long du lac et récupèrent 
les productions de chaque campement pour les acheminer jusqu'au marché de Roro puis vers Kyabé en direction de Sahr, du sud du Tchad, de la capitale et éventuellement des pays voisins. En saison des pluies, la pêche se pratique dans les bras du Salamat, tandis que la production du lac Iro est acheminée par voie fluviale jusqu'au village de Moundourou (fig. 3). Comme au lac Fitri, la route goudronnée - accessible depuis Kyabé - se rapproche petit à petit du lac. La commercialisation de la pêche façonne l'espace et le mode vie des habitants du lac Iro. Conformément aux observations de Claude Pairault en 1994, les pêcheurs dépendent des commerçants avec lesquels ils échangent leur capture contre du matériel. Ce système joue au détriment de certains pêcheurs qui se retrouvent endettés et dans une situation précaire.

\section{La loi I4, contestation et revendication du pouvoir coutumier}

Le commerce de la pêche est contrôlé au Tchad depuis les années 1920 et l'époque de la colonisation française. Les premières formes de régulation des ressources halieutiques datent seulement des années 1990 (CHAUVEAU et al., 2000). En 2008, la loi 14/PR/2008 est mise en place et reprend le code de pêche publié par la FAO en 1995 sur la pêche maritime et continentale, soulevant des critiques quant à son caractère coercitif et intempestif (MuGELÉ, 2013). Elle bannit les engins de pêche les plus dangereux et limite la taille des hameçons et des mailles de filets. Elle installe aussi la taxation du commerce en aval de la pêche, rend le permis de pêche obligatoire et permet d'instaurer des politiques communes de surveillance entre les pays frontaliers du lac Tchad appuyées par la Commission du bassin du lac Tchad.

Le processus de déconcentration de la gestion de l'environnement au Tchad change la réglementation à l'échelle locale, comme c'est le cas aux lacs Fitri et Iro avec l'implantation de bureaux des Eaux et Forêts dont le pouvoir se juxtapose avec celui des autorités coutumières reconnues par la Constitution tchadienne. $\mathrm{Au}$ lac Fitri, le sultanat bilala limitait l'accès aux ressources halieutiques en interdisant les filets, mais l'arrivée de plus en plus importante de pêcheurs émigrés a amené de nouvelles pratiques de pêche. Le filet s'est diffusé au sein de la plupart des communautés. Parallèlement, les autorités étatiques, le préfet et les agents des Eaux et Forêts prennent le pas sur les autorités coutumières plus anciennes. En 2016, plusieurs campagnes « coup de poing » sur le lac Fitri sont destinées à affirmer l'autorité du pouvoir central sur les populations et autorités locales. Sur le campement de Goffé, dépendant de l'autorité traditionnelle du N'Garboulou, des nasses ${ }^{4}$ ont été brûlées en décembre 2015, des filets confisqués et détruits parce qu'ils n'étaient pas conformes aux normes de la loi 14.

Au lac Iro, le chef de canton a interdit l'utilisation des sennes de plage. Quelques « chefs de mares » résistent et évoquent une réglementation spécifique de leurs mares. Ils expliquent aussi leurs difficultés face à l'afflux de pêcheurs et 
l'affaiblissement des coutumes. Le chef de canton, issu de la communauté goula, joue de son statut mi-traditionnel et mi-étatique. En 2015, il a autorisé l'utilisation de la senne de plage sur l'ensemble du lac pour permettre à la communauté goula de Boum Kebir de rembourser un cas de diya ${ }^{5}$.

\section{Quel rôle pour les institutions locales dans la régulation de la pêche?}

Une régulation de l'accès aux ressources s'impose dans les zones lacustres pour prendre en compte les besoins essentiels des utilisateurs, le développement économique de ces espaces, la préservation du milieu et la prévention des conflits entre autochtones et allochtones. Ces enjeux appellent les acteurs institutionnels à considérer la gestion de ces espaces afin de garantir l'accès équitable aux ressources.

\section{D'un système mobile, structuré par les campements de pêche, au zonage de pêche}

Les pêcheurs circulent aussi bien sur et autour du lac, selon des mouvements saisonniers. Cette mobilité suit le déplacement des poissons. Les pêcheurs d'une part et les institutions politiques anciennes, de l'autre, ont développé un mode de production adapté à la saisonnalité de la pêche et à la topographie des lacs. Les pêcheurs de ces zones lacustres ayant l'habitude de travailler dans des eaux peu profondes, la pêche a tendance à être moins abondante en saison pluvieuse et jusqu'au mois d'octobre, lorsque le niveau d'eau est élevé. Beaucoup de pêcheurs pratiquent aussi l'agriculture et certains rentrent dans leur village à l'extérieur du lac le temps de la saison de culture. Les territoires d'eau anciens (fig. 2) sont des repères pour localiser l'emplacement des pêcheurs, guider leurs déplacements et faciliter la gestion des ressources sous la surveillance des autorités coutumières.

En 2003, le projet Prodalka ${ }^{6}$ au lac Léré, visait à rassembler les utilisateurs des ressources sur la base d'une gestion traditionnelle ancienne. Des zones de mise en défens pour la pêche ont été délimitées et appuyées par l'autorité traditionnelle du gong de Léré et se sont révélées efficaces en matière de renouvellement du

\footnotetext{
5. En langue arabe, la diya, ou prix du sang, est une compensation financière donnée en cas d'homicide dans le droit traditionnel musulman.

6. Le Programme de développement rural décentralisé du Mayo Dallah du lac et Kabia, issu de la coopération tchado-allemande GIZ.
} 


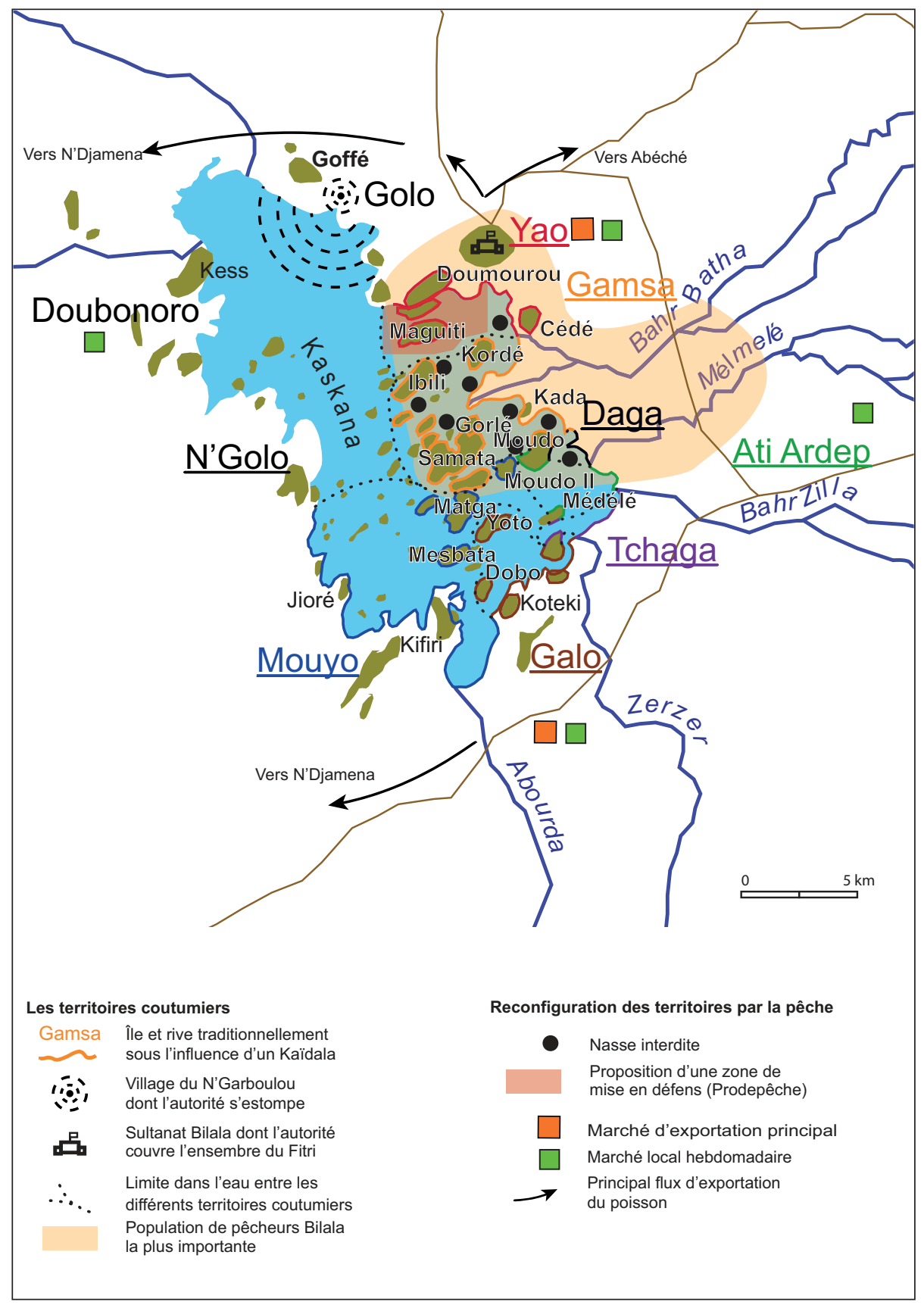

Figure 2

Les territoires d'eau au lac Fitri, une construction politique (SAUNIER, 20I6).

Source : ESRI Topography Map. 
stock halieutique. Le projet Prodepêche ${ }^{7}$ entre 2009 et 2014 s'est inspiré de ce succès rapide pour l'appliquer au lac Iro, puis au lac Fitri.

Contrairement au lac Fitri, il n'existe pas au lac Iro de limites entre les villages ni de chefs traditionnels sur le lac. Le Prodepêche, en 2009, a donc tenté d'établir un zonage partageant le lac en trois parties distinctes : une zone banale, une zone tampon et une zone de mise en défens de la pêche pour protéger le stock halieutique et permettre sa reproduction (fig. 3). Ce partage reposait sur les pratiques locales de pêche, avec la mise en défens d'une zone en principe moins fréquentée. Il s'est avéré que le positionnement de la zone de mise en défens entravait surtout la pêche d'un grand nombre de pêcheurs allochtones. Les conflits entre usagers et la pression commerciale ont finalement eu raison de ce zonage. Le comité de surveillance des pêcheurs mis en place par les Ilod (Instances locales d'orientation et de décision), sur le même modèle qu'au lac Léré, n'a eu ni les moyens ni le soutien des autres pêcheurs pour rendre effective la limitation de l'accès à la zone. Au lac Fitri, une zone de mise en défens a aussi été proposée dans le nord du lac mais sa matérialisation n'a pas abouti. Selon nos entretiens, il se pourrait que le N'Garboulou ait été à l'origine de l'opposition à ce zonage, en plus des résistances de la part des usagers et des difficultés à trouver les membres d'un comité de surveillance.

\section{Une nouvelle construction politique des territoires dans le lac Fitri}

Les pêcheurs bilala du lac Fitri se réfèrent à un passé commun marqué par des sécheresses pour entretenir un discours autour de la préservation du milieu, présent aussi dans la rhétorique du sultanat. Ces éléments ont permis la construction d'un discours politique, notamment par les autochtones, afin de se prémunir face à l'augmentation des pêcheurs allochtones sur le lac. Ces arguments servent localement à revendiquer des règles d'usage différenciées selon les secteurs du lac (fig. 2).

Les limites dans l'eau ont surtout été appropriées par un groupe de population représentée par le kaïdala de Gamsa afin de garantir l'usage exclusif des palangres dans sa zone, ce qui en bannit les pêcheurs allochtones qui ne les utilisent pas. Cette limitation de l'accès aux ressources a suscité un mouvement de contestation de la part d'autres Bilala, notamment à Moudo, un village insulaire qui possède une limite traditionnelle dans l'eau avec celle de Gamsa. En effet, les filets ne sont plus aujourd'hui l'apanage des allochtones mais sont aussi communément utilisés par les Bilala. Pour cette raison, des filets de plusieurs pêcheurs de Moudo ont été détruits par des pêcheurs de Gamsa. D'autres kaïdala, comme celui de Daga, se sont inspirés des méthodes du village de Gamsa pour interdire l'usage des nasses dans leur zone, technique jugée parmi les plus prédatrices pour la pêche au lac Fitri selon les Bilala. 
Ces initiatives coercitives sont plus ou moins soutenues par les associations existantes au lac Fitri $^{8}$ en raison de leur objectif pour la préservation des ressources halieutiques.

Le sultan est partagé entre l'encouragement à ces initiatives locales et leur interdiction. En 2014, plusieurs kaïdala, comme celui de Galo, ont rendu l'accès aux ressources payant pour les pêcheurs allochtones. Cette pratique a été rapidement condamnée par le sultan parce qu'elle remettait en cause son autorité et les interdits fondamentaux quant à la possession foncière d'un territoire dans l'eau.

\section{La construction d'un territoire économique au lac Iro}

$\mathrm{Au}$ lac Iro, les Goula n'ont pas la même influence politique pour interdire aux autres communautés de pêcher dans leur espace. D'abord parce que la communauté est réduite, mais surtout parce que la pression économique et commerçante s'impose plus fortement qu'au lac Fitri. En effet, avec une pression anthropique et animale très importante, la compétition entre les utilisateurs des ressources semble catalysée par la promiscuité des acteurs. Contrairement au lac Fitri, dont le système repose sur la multiactivité et la multifonctionnalité grâce à l'alternance d'activités telles que l'agriculture, le maraîchage, la transhumance et la pêche, au lac Iro il est difficile de maintenir cet équilibre des activités. L'agriculture sur les rives, surtout le maraîchage en décrue, est très délicate en raison de l'importance du cheptel de saison sèche. Les hippopotames présents dans la zone, et protégés, occasionnent des dégâts importants sur les rives. Cette pression humaine et animale au lac Iro amène les populations à se spécialiser dans les deux activités possibles, à savoir la pêche ou l'élevage, et à laisser de côté l'agriculture.

La pêche obéit à la loi de l'offre et de la demande dictée par les commerçants, plutôt qu'aux injonctions des institutions politiques existantes mal comprises par les usagers. Ainsi, l'espace semble se structurer d'abord par rapport aux acteurs de la filière, marchés et routes d'exportation du poisson (fig. 3).

Même si l'accès au lac Iro demeure plus difficile qu'au lac Fitri aujourd'hui (200 km de pistes en partie inondée durant la saison pluvieuse) et que la vente de poisson frais n'est pas développée, il semble que la commercialisation du poisson se soit tournée vers l'extérieur depuis plus longtemps et plus intensément qu'au lac Fitri. Sans autre alternative productive, les populations allochtones comme autochtones puisent dans la ressource piscicole pour obtenir un moyen d'échange et assurer leurs besoins, sans se soucier de la durabilité du système. Cette homogénéité plus grande des intérêts entre les autochtones et les allochtones n'exclut pas la présence de conflits. Au contraire, la compétition

8. Par exemple : La Faîtière (« l'organisation tchadienne de la promotion de la pêche, de l'aquaculture et de l'environnement » qui chapeaute l'ensemble des groupements de pêche tchadien dont le siège se trouve à N'Djamena), les associations paysannes du lac Fitri (associations apparues à la suite de la tentative de mise en place d'llod au lac Fitri par le Prodepêche), l'ATPDI ('’Association tchadienne pour la promotion du développement intégré). 
pour les ressources entretient des discours basés sur l'appartenance ethnique et les techniques de pêche malgré l'ancienneté des pêcheurs allochtones dans la zone.

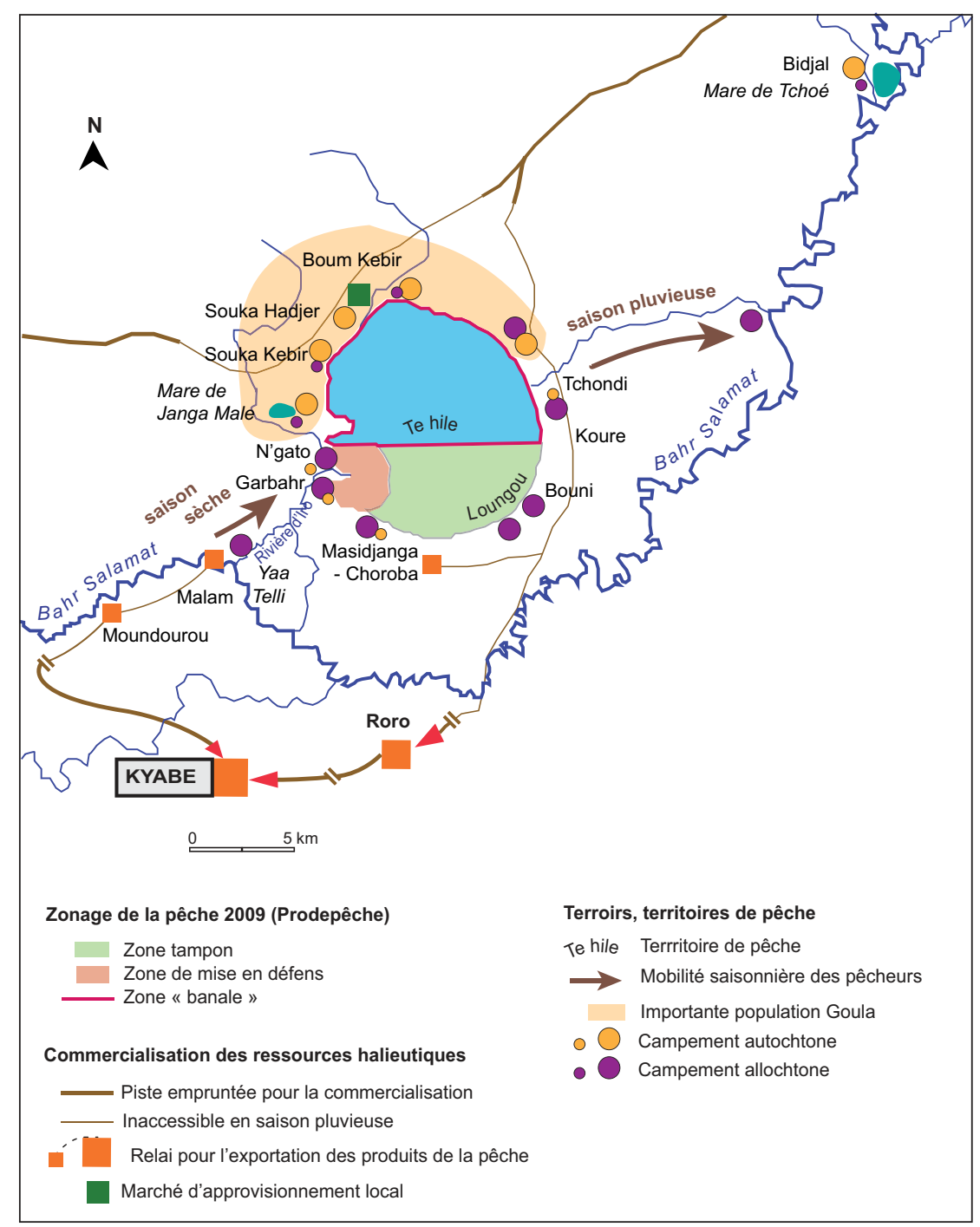

Figure 3

Le lac Iro : un espace organisé par le commerce de la pêche (SAUNIER, 20l6). Source : ESRI World Topographic Map. 


\section{Conclusion}

Nous avons étudié la place des modèles anciens de gestion des ressources et dans quelle mesure ils pourraient soutenir la préservation et le développement des espaces lacustres de la région sahélosoudanienne.

$\mathrm{Au}$ lac Fitri, les territoires dans l'eau sont des références communes pour l'ensemble des pêcheurs pour se repérer dans l'espace. Pour certains, elles ont seulement une valeur historique et il est tout à fait anodin de les transgresser. Ces limites ont toutefois le mérite d'être connues de tous, ce qui pourrait leur donner une certaine légitimité dans le cadre d'une gestion partagée des ressources halieutiques sur le lac. Pourtant, les kaïdala responsables de ces espaces dans l'eau, appuyés sur le prestige du sultanat, soutiennent d'abord les intérêts de leur communauté fragilisée par l'arrivée de nouveaux pêcheurs.

$\mathrm{Au}$ lac Iro, il n'existe pas de système de gestion territorialisée appuyée par une autorité coutumière légitime. Une gestion ancienne des ressources existe, mais pour pouvoir la comprendre et la comparer au lac Fitri, il faudrait considérer les chefs de mare et donc étudier le lac Iro dans l'ensemble fluviolacustre régional. Dans ce lac, l'absence d'institution centralisée n'introduit pas une plus grande équité dans l'accès aux ressources entre les autochtones et les allochtones. D'autres acteurs se sont approprié la gestion de la ressource halieutique : ce sont les commerçants, qui, par le prêt de matériel, les crédits et l'organisation des filières structurent l'espace et orientent l'économie de la région. Leurs intérêts s'imposent sans régulation des conflits ni gestion de la ressource.

Dans le cas de ces deux lacs tchadiens, qu'il s'agisse d'une institution coutumière forte et reconnue, ou fragile car issue d'une construction administrative coloniale, elle se révèle impuissante à relever seule le défi de la gestion environnementale et des conflits d'usages face à une pression anthropique croissante et à la multiplication des acteurs du territoire. Une territorialisation est effectivement en cours et elle ne renvoie pas de la même manière à la notion des communs (Ostrom, 1990). C'est dans le territoire où les règles de gestion sont les plus explicites que s'expriment le plus clairement les exclusions et les replis identitaires, et que s'observe une appropriation différenciée de l'espace par différents types d'acteurs au détriment d'une gestion environnementale concertée. A contrario, dans le lac Iro où les règles d'accès sont moins claires, le territoire lacustre reste un bien commun où aucune partition spatiale n'est tolérée ni appropriée. Cela n'évite pas les conflits intercommunautaires et questionne la durabilité du système et les rapports de force entre pêcheurs et commerçants. Cette situation originale permet toutefois d'envisager plus facilement une gestion intégrée de l'ensemble de l'espace lacustre et la négociation de conventions locales modernes qui ne reposent pas obligatoirement sur une revitalisation de structures traditionnelles affaiblies et contestées. 


\section{Bibliographie}

Brondizio E., Ostrom E., Young O., 2009 Connectivity and the Governance of Multilevel Social-Ecological Systems: The Role of Social Capital. The Annual Review of Environment and Resources, 34: 253-278.

\section{Chauveau J.-P., Jul-Larsen E., Chaboud C., 2000}

Les pêches piroguières en Afrique de l'Ouest : dynamiques institutionnelles : pouvoirs, mobilités, marchés. Paris, Karthala, 397 p.

\section{Cima-Sogec, 2002}

Étude du sous-secteur de la pêche et de l'aquaculture au Tchad (ESSPA). Bilan diagnostic. Partie 3. Ressources halieutiques et production. $151 \mathrm{p}$.

Courel M.-F., Bachimon P., Riser J., Dagou P., Mustapha M. A., Raimond C., 2005 Géosystèmes lacustres, étude des régions des lacs Léré et Fitri, Tchad. Ministère des Affaires étrangères, France, rapport scientifique, projet Campus 5 : 97319 107, fac $n^{\circ} 99002$ 600. Corus (MAE), 318 p.

\section{Courel M.-F., Morin S., Raimond C., 1997} « Intégration modèle ou modèle d'intégration. La gestion de l'environnement au lac Fitri (Tchad) ». In : Singaravelou P., éd. : Gestion de l'environnement dans les pays tropicaux, Bordeaux, Dymset-Cret, p. 311-326.

\section{Couty P., Durand P., 1968}

Le commerce du poisson au Tchad.

Paris, Orstom, 252 p.

Dagou P., Mustapha M. A., Goltob Mbaye N., Passiring K., NGAR-ODJILO M., 2005

« La pêche dans les lacs Fitri et Léré au Tchad. Techniques de capture, conservation des produits et enjeux de protection ». In : Raimond C., Garine E., Langlois O., éd. : Ressources vivrières et choix alimentaires dans le bassin du lac Tchad. Paris, IRD Éditions/Prodig, p. 87-112.

\section{De la Croix K., 2015}

Les territoires-temps des communautés de pêcheurs bozo et somono sur le Niger supérieur (Mali, Guinée). Thèse doctorat, université Paris-Ouest, 463 p.

\section{FAO, 1995}

Code de conduite pour une pêche responsable. Rome, 46 p.

\section{FAO, 2008}

Profils des pêches et de l'aquaculture par pays : La République du Tchad. [En ligne]

Http:/www.fao.org/fishery/facp/TCD/fr

\section{FAO, 2012}

La situation des pêches et de l'aquaculture. Rome, $241 \mathrm{p}$.

\section{FAY C., 1989}

«La production de pêche dans le delta central du Niger (Mali) : systèmes de perception et d'appropriation des territoires ». In : Durand J.-R., Lemoalle J. et Weber J., éd. : La Recherche face à la pêche artisanale, symp. int. Orstom-Ifremer, Montpellier, France, 3-7 juillet 1989, Paris : Orstom, 1991, p. 881-888.

\section{Hagenbucher F., 1968}

Notes sur les bilala du Fitri. Cahiers Orstom, Sciences Humaines, $\mathrm{n}^{\circ}$ 5(4) : 39-76.

Koussou M. O., Mbaye N. G., KeILAR A. T., 2012

La filière d'approvisionnement de N'Djamena en poisson frais du lac Tchad. Revue Scientifique du Tchad, $13 \mathrm{p}$.

\section{Marty A., Zakinet D., Khamis D. D., BERNARD C., 2012 \\ Almy al Afia 2. Analyse de l'évolution des ressources dans le département du Fitri. Document principal. République du Tchad, programme d'hydraulique pastorale au Tchad central, Phase II. Antea-Iram, 128 p.}

\section{Mbaye N. G., 2009}

"Les immigrés haoussa de Fouli, au bord du lac de Léré (Tchad) ». In : Tourneux H., Woïn N., éd. : Migrations et mobilité dans le bassin du lac Tchad. Marseille, IRD Éditions, p. 395-416.

\section{Mugelé R., 2013}

Enjeux et conséquences de la réglementation sur le bois-énergie au Tchad. EchoGéo [En ligne], 26 | 2013. URL : http://journals. openedition.org/echogeo/13620; DOI : 10.4000/echogeo.13620. 


\section{Ostrom E., 1990}

Governing the commons: the evolution of institutions for collective action. Cambridge University Press, 280 p.

\section{RÉPUBLIQUE DU TCHAD, 2008}

"Du régime des ressources halieutiques ». Loi $n^{\circ}$ 14/PR/2008 portant régime des forêts, de la faune et des ressources halieutiques. République du Tchad : 30-40.

\section{Pairault C., 1994}

Retour au pays d'Iro, chronique d'un village au Tchad. Paris, Karthala, 292 p.

\section{Paigbc, 2012}

Plan d'aménagement intégré à base communautaire, site de Boum Kebir. Ministère de 1'Environnement et des Ressources halieutiques, direction des pêches et de l'aquaculture, $110 \mathrm{p}$.

\section{Quensière J., 1994}

La pêche dans le delta central du Niger.
Approche pluridisciplinaire d'un système de production halieutique. Paris, Orstom/Karthala, vol. 1, 495 p.

\section{RGPH2, 2009}

Deuxième recensement général de la population et de l'habitat, résultats définitifs par sous-préfecture, mars 2012. Inseed, ministère du Plan, de 1'Économie et de la Coopération internationale, annexes : 44-164.

\section{SAUNIER M., 2015}

L'accès aux ressources halieutiques en lien avec l'évolution des techniques de pêche au lac Fitri, Tchad. Mémoire de master 1, université Paris 1 Panthéon-Sorbonne, 146 p.

\section{SAUNIER M., 2016}

Les territoires d'eau : enjeux et jeux de pouvoir pour la gestion des ressources halieutiques dans les lacs Fitri et Iro au Tchad. Mémoire de master 2 de géographie, université Paris 1 Panthéon-Sorbonne, 128 p. 

Partie 4

\section{Des territoires en mutation : le défi du développement durable}

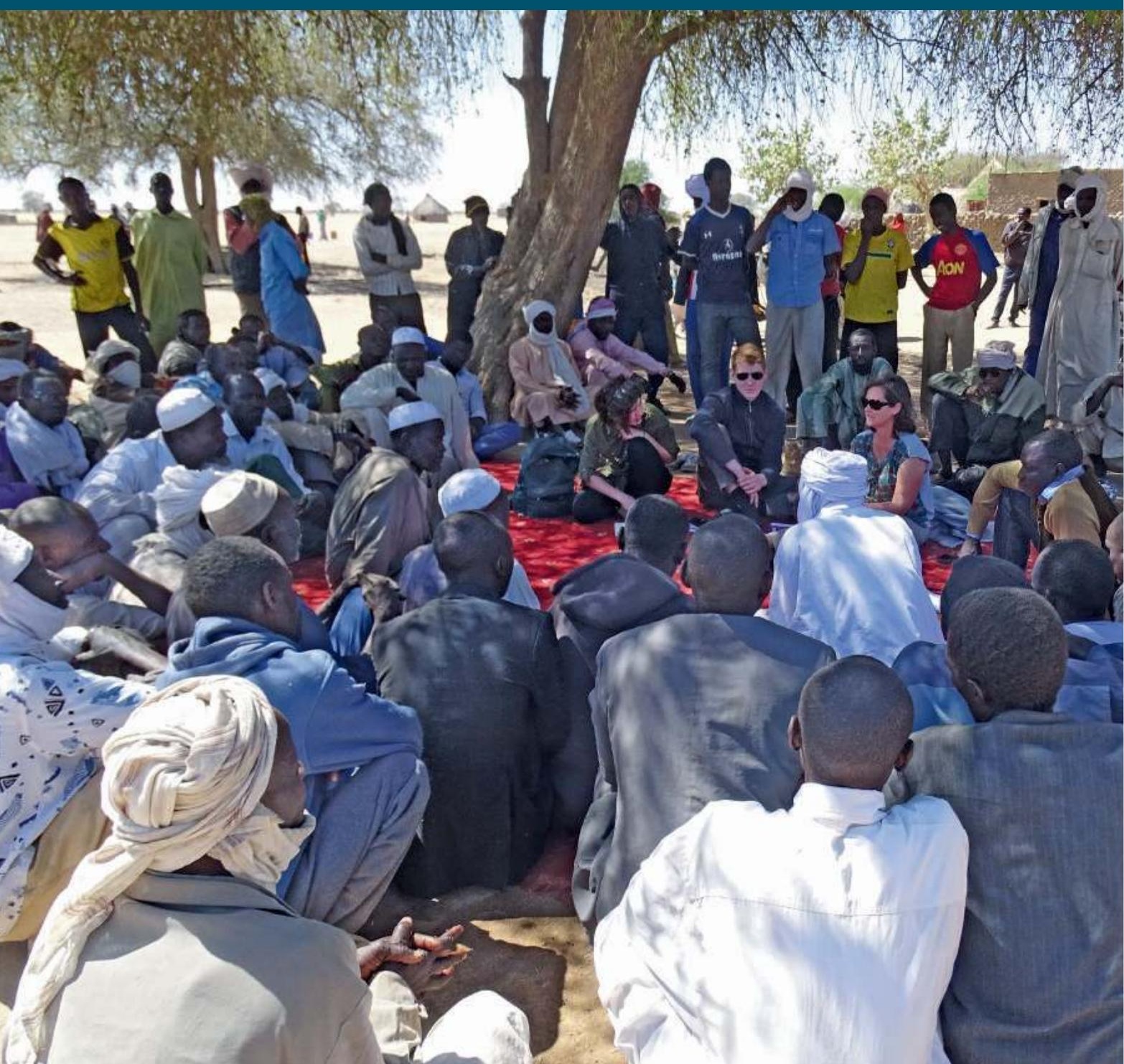


De l'or a été découvert au Fitri en 2016. Les habitants du lac s'interrogent sur l'avenir de cette activité et le partage des ressources avec les migrants et les investisseurs extérieurs (Ati Ardep, janvier 2016). 


\section{Introduction}

Christine RAIMOND, Florence SYLVESTRE,

Dangbet ZAKINET

Les zones humides sahariennes et sahéliennes sont des systèmes complexes, dont l'avenir est difficile à anticiper. Leur équilibre écologique est fragile du fait de leur localisation en zone aride et semi-aride et de la variabilité d'une année à l'autre des apports en eau. Malgré les contributions scientifiques récentes, les échanges avec le sous-sol (aquifères inclus) et l'atmosphère sont encore peu connus, et moins encore leur évolution dans le cadre du changement climatique actuel. Si la compréhension des paléoenvironnements peut aider les scientifiques à envisager les modifications environnementales à venir, les incertitudes liées aux prédictions climatiques sont encore trop grandes pour définir une trajectoire unique d'évolution. La pression anthropique croissante, l'évolution des techniques et les politiques d'encadrement constituent de nouvelles variables à intégrer sur des pas de temps beaucoup plus courts et qui peuvent influer de manière beaucoup plus radicale sur l'écosystème et la disponibilité en ressources.

Dans les pays en développement, les dispositifs de protection environnementale ne sont pas viables s'ils ne tiennent pas compte des besoins vitaux des sociétés tout en prévenant la reproduction des écosystèmes pour les générations futures. Conserver ou développer ne constitue donc pas une alternative pour les zones lacustres, comme en attestent les dispositifs de protection environnementale octroyés depuis les années 2000 au Tchad : Patrimoine mondial de l'Unesco, Réserve de Biosphère, zone Ramsar ne sont pas des aires protégées qui excluent les sociétés lacustres, mais qui prévoient au contraire leur participation à la gestion environnementale et intègrent les activités anthropiques. Près de 20 ans après leur mise en place, le constat de la surexploitation des ressources lacustres et la crise de gouvernance reposent la question d'un développement adapté au fonctionnement très particulier des zones humides africaines dans le contexte du changement global. Il rappelle aussi l'impérieuse nécessité de l'intégration des populations à l'établissement des diagnostics de changements, à la gestion 
territoriale et environnementale ainsi qu'aux discussions avec les experts et les gestionnaires pour définir un avenir souhaitable.

Les pistes d'avenir explorées dans cette dernière partie éprouvent le concept du développement durable. Elles rappellent l'importance de la prise en compte des jeux d'échelles, du local à l'international, de l'individuel au collectif, pour appréhender les trajectoires possibles. Dans les zones lacustres, comme dans le reste des zones humides sahéliennes, les questions de l'aménagement et du désenclavement sont récurrentes pour stimuler la production, dégager des revenus pour les sociétés rurales et participer à la sécurité alimentaire nationale et régionale. Les rétroactions des aménagements sur le fonctionnement des socioécosystèmes sont envisagées et questionnent leur résilience sur le temps long. Leur efficience écologique et économique est aussi éprouvée au regard des systèmes endogènes et de la productivité des espaces, mais aussi des enjeux liés à la sécurité et à l'emploi. Pour le lac Tchad et sa sortie de crise, trois trajectoires sont envisagées entre chaos et régime humanitaire, grands aménagements et agrobusiness, et développement inclusif reposant sur l'agriculture familiale.

Les modalités d'accès aux ressources naturelles et de gouvernance sont au cœur des débats, car ce sont ces règles et la régulation des conflits qui sont la base d'une gestion partagée des ressources et de la paix sociale. Dans ce domaine, les études récentes mettent l'accent sur des innovations endogènes basées sur une auto-organisation des acteurs, qui connaissent intimement les flux du socioécosystème, particulièrement bien les rythmes et l'étendue des crues et des décrues, et les usagers qui les exploitent. Ces derniers s'entendent pour occuper l'espace et en aménager collectivement certaines portions au bénéfice de tous. Ces organisations particulières, observées dans le lac Tchad, dans la zone des Yaéré au Nord Cameroun, et aussi dans une certaine mesure dans le lac Iro, dépassent la vision " ostromienne » des communs basée sur l'existence d'un groupe d'ayants droit en capacité d'exclure les autres usagers dans le cadre d'une régulation intentionnelle. Le mode de gouvernance observé repose au contraire sur une coordination fluide et inclusive des acteurs, qui ont permis une intensification des systèmes agricoles et pastoraux. Ce résultat remarquable repose sur la très grande finesse de la connaissance empirique du socioécosystème par les acteurs, résultat d'un apprentissage continu et loin des simplifications technicistes et modernistes des institutions étatiques qui peinent à intervenir dans ces milieux.

Le dernier texte revient sur la crise de gouvernance au lac Fitri, marquée par un système de régulation dépassé, une crise de légitimité du sultanat bilala et le démantèlement du canton historique du Fitri par l'administration tchadienne depuis 2016 pour consolider son contrôle sur le territoire national. Le double processus à l'œuvre, par le bas de construction territoriale et nouveaux modes de gouvernance à l'intérieur de la zone humide et, par le haut, la fragmentation $\mathrm{du}$ territoire du sultanat, remet aussi en cause l'idée tentante de s'appuyer 
totalement sur une institution coutumière jadis puissante pour assurer l'équité dans l'accès aux ressources et la régulation des conflits.

Il n'existe pas un seul modèle de gouvernance idéal pour les zones humides, mais une grande diversité d'alternatives qu'il convient de mieux documenter pour appuyer les expérimentations indispensables dans les territoires en crise. Du modèle ostromien typique, représenté par le lac Fitri et le sultanat bilala, aux modèles d'auto-organisation décrits ci-dessus, le panel est large pour penser des innovations institutionnelles originales support de progrès et de développement social et économique durable. Surtout, l'observation de ces institutions renouvelle la question des communs, qui peine encore à être intégrée des législations nationales inadaptées.

Comprendre ces mutations est un enjeu fondamental pour la conservation des zones humides sahéliennes et la viabilité des systèmes mis en place. C'est aussi une contribution majeure aux éclairages locaux indispensables à toute prise de décision en matière de gestion territoriale d'une part, et à la conception de politiques publiques et lois nationales adaptées aux réalités locales d'autre part, à l'heure où le changement climatique invite à penser de nouveaux rapports entre milieux et sociétés. 



\section{Chapitre 16 \\ $L_{\text {avenir du lac Tchad : }}$ les échelles de l'incertitude}

Géraud MAGRIN,

Jacques LEMOALLE

\section{Introduction}

La question du lac Tchad, telle qu'elle est posée au niveau de la scène internationale à travers de nombreuses conférences et sommets ${ }^{1}$, a beaucoup évolué au cours des dernières années. Après les sècheresses des décennies 19701980, le lac Tchad a longtemps été abordé sous l'angle de sa supposée dynamique régressive, avec comme solution un projet de transfert interbassin proposé sous de multiples avatars à partir de la fin des années 1980, occultant toute autre réflexion (MAGRIN, 2016). Or le diagnostic des chercheurs pour la période 19952014 est celui d'une situation relativement favorable du point de vue de l'hydrologie et des services écosystémiques associés (LEMOALLE et MAGRIN, 2014). Après une phase de sécheresse correspondant à un «Petit lac Tchad irrégulier », le lac fonctionne depuis le milieu des années 1990 sous un régime de « Petit lac Tchad régulier ». La cuvette nord est inondée chaque année, dans des proportions variables. Cette situation permet notamment la pêche et une bonne valorisation des activités de décrue (agriculture, élevage). Le lac Tchad apparaît comme une région agricole productive (céréales, produits maraîchers, 
produits de la pêche et de l'élevage), un pôle émetteur de ressources alimentaires vers son arrière-pays rural et les villes régionales, notamment N'Djamena et Maiduguri. En 2014, tout s'effondre. La crise Boko Haram, qui s'est diffusée à partir de Maiduguri vers le Bornou et les zones frontalières du Niger et du Cameroun, atteint le lac Tchad. Son ampleur occulte toutes les autres réflexions. L'urgence prend le pas sur le temps long, le politique sur l'hydrologie.

Nous souhaitons proposer ici une réflexion prospective sur l'avenir du lac Tchad, avec comme point de départ les acquis de l'expertise collégiale réalisée par l'Institut de recherche pour le développement (IRD) pour la Commission du bassin du lac Tchad (CBLT) en 2012-2013 (LEMOALlE et MAGRIN, 2014). Intitulée Le développement du lac Tchad. Situation actuelle et futurs possibles, cette expertise présentait un état des lieux des connaissances scientifiques disponibles sur l'environnement et les sociétés du lac Tchad. Son dernier chapitre, prospectif, proposait des visions de l'avenir du lac Tchad suivant trois principaux facteurs d'évolution : l'hydrologie, la démographie, la trajectoire du développement (MAGRIN et al., 2014). L'incertitude semblait la plus forte sur l'hydrologie en raison des divergences entre les modèles de prédiction climatique du Groupe d'experts intergouvernemental sur l'évolution du climat (Giec). Notre objectif est ici d'actualiser cette réflexion dans un contexte renouvelé par la précision de certaines données du Giec, mais surtout par le changement du contexte régional du développement. Celui-ci est marqué d'une part par la crise Boko Haram, qui se diffuse dans le Borno à partir de 2009 et affecte directement le lac Tchad en 2014. Il se traduit d'autre part par la baisse des prix du pétrole, qui entraîne des difficultés économiques importantes mais variables dans les pays riverains du lac à partir de fin 2014.

Pour penser l'avenir du lac, il importe en outre de prendre en compte trois échelles d'analyse : celle du lac stricto sensu (dynamique des paysages suivant la crue, évolution de l'occupation de l'espace par les sociétés, et notamment des règles d'accès aux ressources et des relations entre activités et entre groupes...), celle du bassin dans son entier (qui détermine les apports en eau, la régulation environnementale, les trajectoires régionales du développement, donc l'urbanisation, les mobilités, flux commerciaux...), enfin l'échelle globale du climat et des interventions extérieures.

Nous éprouverons deux hypothèses :

- la cuvette nord du lac pourrait bien connaître un assèchement répété et durable, même si la disparition du lac Tchad entier n'est pas plausible, la pérennité de l'alimentation de la cuvette sud paraissant assurée au moins à moyen terme ;

- la gestion des équilibres entre les ressources en eau et les populations à l'échelle $\mathrm{du}$ bassin est centrale pour penser l'avenir du lac. Il dépendra moins du changement climatique que des choix politiques en matière de modèle de développement agricole et de leurs implications en termes d'utilisation de l'eau.

Nous proposons d'abord d'étudier les deux variables clés que sont le climat et la démographie. Puis nous donnerons un aperçu de la gamme des choix politiques 
disponibles qui peuvent se combiner, dans chacun des pays riverains du lac. Enfin nous proposerons trois photographies de futurs envisageables, parmi le très grand nombre d'agencements possibles.

\section{L'étude des deux variables clés}

Il s'agit ici d'évoquer deux variables de fond, dont le comportement est largement hors de portée des décisions politiques à l'échelle des pays du bassin : le climat et la démographie.

\section{Le climat}

L'évolution du climat, et son impact sur les ressources en eau et les ressources naturelles, est à prendre en compte en premier lieu. Les deux variables climatiques principales considérées ici sont celles retenues par le Giec (GIEC, 2014) à savoir la température et la pluviométrie.

\section{L'environnement du lac Tchad : situations au $X X^{\mathrm{e}}$ siècle et observations récentes}

Au cours des décennies 1970 et 1980 marquées par des années de sécheresse sévère, le débit du Chari a diminué et le niveau d'eau dans le lac Tchad s'est abaissé. Ce qui s'est traduit par l'exondation d'un haut fond, la Grande Barrière, qui a scindé le lac en deux parties, la cuvette nord et la cuvette sud. Cet état particulier a été qualifié de «Petit Tchad» par Tilho qui avait observé un épisode de ce type au début du $\mathrm{XX}^{\mathrm{e}}$ siècle (TiLho, 1928). La cuvette nord ne reçoit de l'eau du Chari via la cuvette sud que lorsque celle-ci se remplit jusqu'à une altitude de l'ordre de 280-281 m, correspondant à au moins $15 \mathrm{~km}^{3}$ d'apport annuel du Chari. La cuvette nord du lac peut alors conserver de l'eau toute l'année, comme de 1998 à 2017, ou s'assécher pendant une courte période. Lors des années de forte sécheresse (1985, 1987, 1988 et 1991), l'apport en eau au lac n'est pas suffisant et la cuvette nord n'est pas alimentée en eau : elle s'assèche complètement. C'est un état que l'on propose ici de qualifier de « Petit Tchad sec », ou « Petit lac Tchad irrégulier ».

Depuis la fin des années 1990, le lac fonctionne sous un régime que l'on peut désigner comme « Petit Tchad non sec », ou « Petit lac Tchad régulier », avec chaque année une inondation plus ou moins complète de la cuvette nord, ce qui permet une bonne exploitation des ressources naturelles du lac (culture, élevage, pêche) du fait de la grande extension des zones de décrue saisonnières et des pâturages marécageux propres à cet état du lac (fig. 1). 


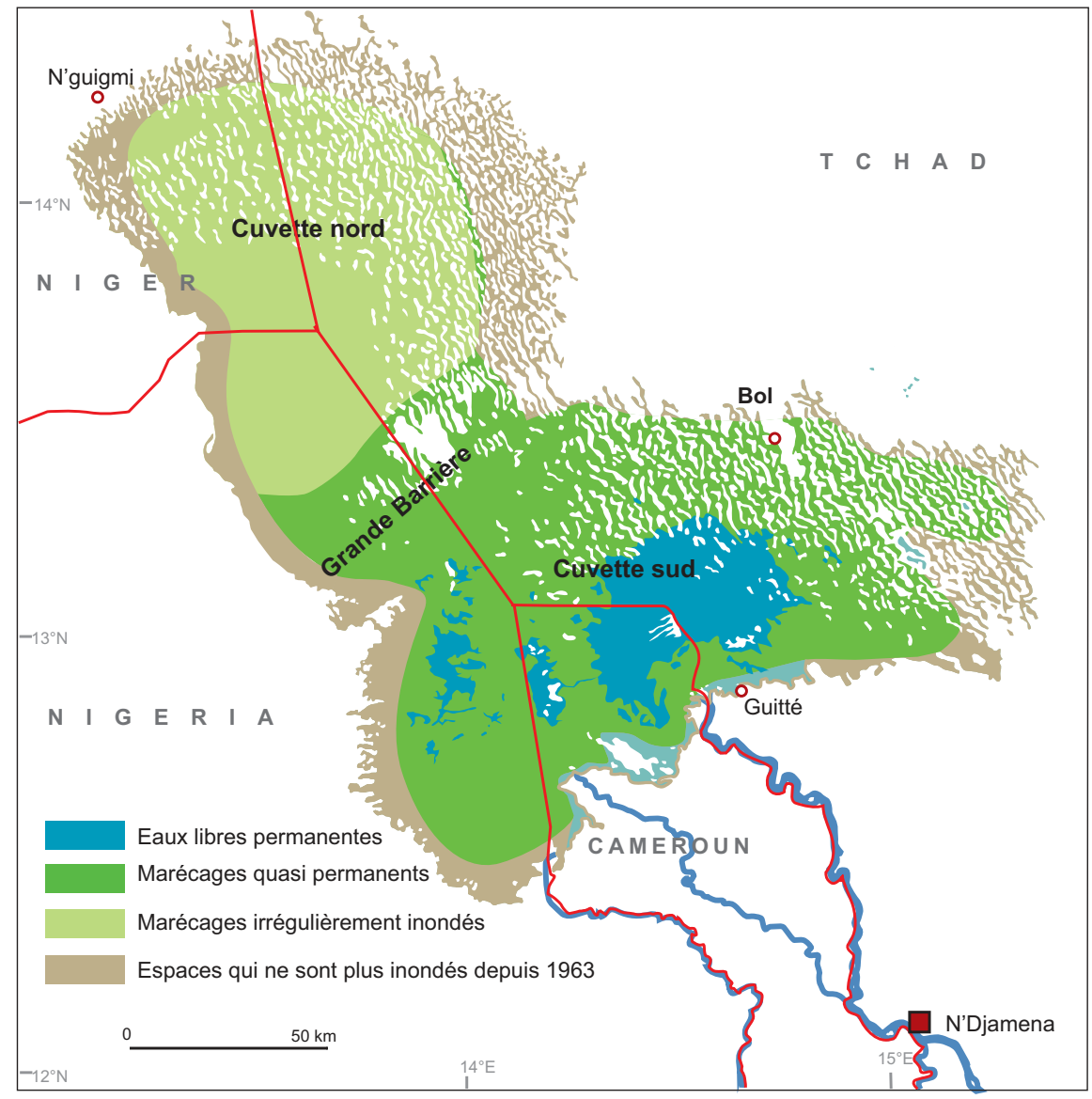

Figure I

Carte schématique des paysages du lac Tchad dans les années 2010 (d'après LemOalle et MAGRIN, 20l4).

L'évolution du climat dans le bassin du lac Tchad depuis 1950 peut se résumer schématiquement en trois phases selon la résultante sur l'état du lac Tchad :

- une période humide de 1950 à 1970, avec un « Moyen Tchad » constitué d'un seul plan d'eau de niveau moyen supérieur à $281 \mathrm{~m}$;

- une période sèche de 1970 à 1990, avec une forte migration des populations rurales de la région vers le lac et parfois de la cuvette nord vers la cuvette sud;

- une période moyenne de 1995 à nos jours. La pluie annuelle sur le bassin est très proche de la moyenne du siècle dernier.

Les données du Climate Research Unit (CRU ; HARRIS et al., 2014) indiquent une augmentation de la température dans la région de $\mathrm{N}^{\prime}$ Djamena de $1,4{ }^{\circ} \mathrm{C}$ au cours des cinquantes dernières années. Mais il faut noter que cette augmentation est moins nette dans d'autres parties du bassin (fig. 2). Un rapport de l'Usaid 


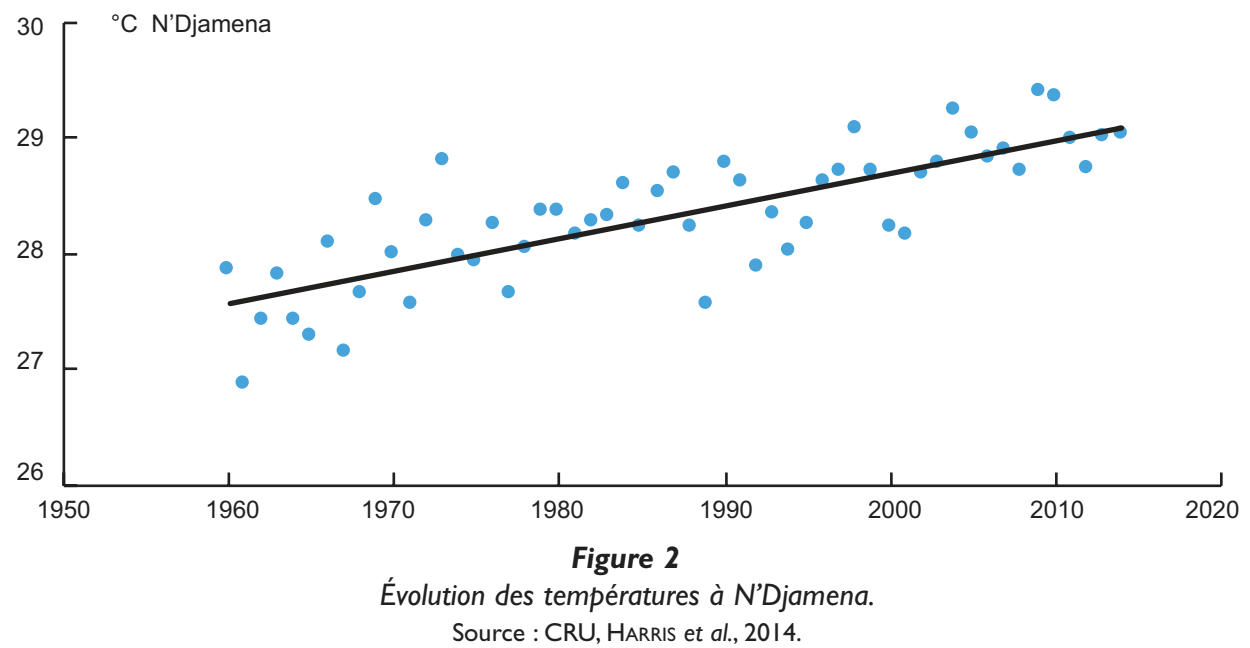

chiffre quant à lui l'augmentation de température entre 0,8 et $1{ }^{\circ} \mathrm{C}$ dans la partie sud-ouest du Tchad pour la même période (FunK et al., 2012). Selon le Giec, l'augmentation de température devrait se poursuivre.

L'évolution de la pluviométrie sur le bassin est moins régulière, avec un minimum au cours de la période 1970-1990 et un rétablissement à des valeurs intermédiaires de 1990 à 2015. Le coefficient de ruissellement ${ }^{2}$ du Chari est cependant sensiblement plus faible depuis 1979 par rapport à la période antérieure : à pluie égale sur le bassin, l'apport en eau au lac est environ $30 \%$ plus faible 3 .

\section{Les impacts futurs prévisibles du changement climatique}

Les prévisions récentes du Giec (GIEC, 2014) concernant la région du lac Tchad et son bassin pour l'horizon 2050 sont une augmentation de la température et, avec une assez large imprécision, une stabilisation de la pluie autour de la valeur observée au cours de la période 2000-2015.

Les modèles agronomiques indiquent que l'augmentation de la température abaisse les rendements du sorgho et du mil dans les savanes sahéliennes et soudaniennes de l'Afrique de l'Ouest. À pluie égale, la baisse est de 10 à $20 \%$ pour un réchauffement de $2{ }^{\circ} \mathrm{C}$, et de 20 à $30 \%$ pour $4{ }^{\circ} \mathrm{C}$ sans qu'un apport supplémentaire d'eau puisse contribuer à compenser complètement cette perte (Sultan et al., 2013). Ce résultat peut s'appliquer à l'ensemble du bassin du lac Tchad. Compte tenu de la croissance démographique prévue pour cette région de l'Afrique (cf. infra), il est important d'appuyer dès à présent l'adaptation au changement de température, avec l'emploi de variétés adaptées ${ }^{4}$, des techniques

2. Débit du fleuve divisé par la quantité de pluie sur le bassin.

3. D'après les données de la Direction des ressources en eau et de la météorologie (Drem) du Tchad et du CRU.

4. Sans négliger la valorisation des savoirs et des semences locales. 
culturales économes d'eau, l'utilisation raisonnable d'intrants et une amélioration des services d'appui à l'agriculture.

Si l'on suppose que la pluie sur le bassin ne changera pas de façon significative, l'évaporation directe sur le lac du fait de la température sera augmentée d'une façon encore difficile à estimer mais qui pourrait être de l'ordre de $6 \%$ en 2050 et de $11 \%$ en 2100 (GIZ, 2015). L'impact d'une augmentation de $10 \%$ de l'évaporation sur le niveau de la cuvette nord du lac a été simulé avec le modèle hydrologique du lac (BADER et al., 2011 ; LeMOALle et al., 2012) (fig. 3). À cela s'ajoute la diminution du coefficient de ruissellement du Chari (la part de la pluie reçue par le bassin qui est transportée par le fleuve), qui est observée depuis la fin des années 1970 et dont on ne sait pas si elle est réversible. Ces deux facteurs conjoints, évaporation et coefficient de ruissellement, contribuent à augmenter la probabilité de bas niveaux de la cuvette nord du lac, et donc des épisodes de "Petit Tchad sec », avec ses conséquences désastreuses sur les populations qui habitent cette partie du lac et exploitent ses ressources. Rappelons que la cuvette nord abrite en 2014 environ $25 \%$ des 2 millions d'habitants du lac Tchad, soit 500000 habitants environ (MAGRIN, 2015).

Le Giec souligne toutefois que d'autres facteurs contribuent à la détérioration de l'environnement global, et en particulier l'augmentation de la population en Afrique centrale.

Pour s'adapter à ces impacts, ainsi qu'à l'augmentation de la population, il convient de réfléchir avec la participation des populations concernées à l'amélioration de la gestion de l'eau de surface ou souterraine à toutes les échelles, depuis le terroir villageois jusqu'au bassin avec ses immenses plaines d'inondation et éventuellement l'interbassin.

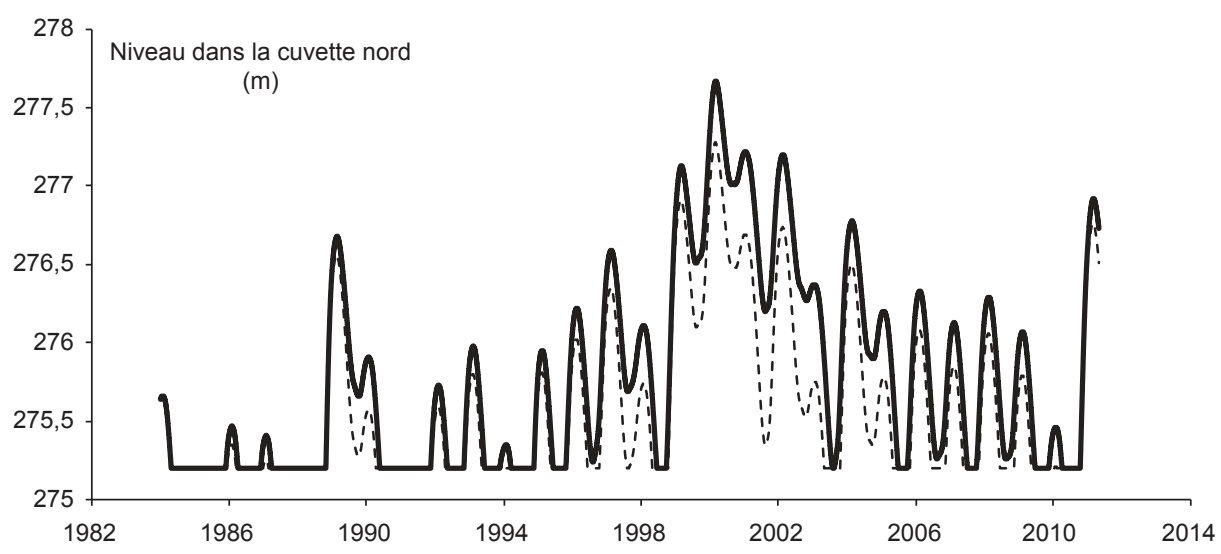

Figure 3

Simulation de l'évolution du niveau de l'eau dans la cuvette nord du lac Tchad (courbe noire) avec une évaporation augmentée de 10 \% (tireté) qui accroît la probabilité d'assèchement temporaire de la cuvette. 


\section{La démographie et le peuplement}

À l'échelle du lac, on peut s'interroger sur la profondeur des changements dans la répartition du peuplement induits par la crise Boko Haram. Au sein d'un peuplement régional largement perturbé par l'insurrection et sa répression - on enregistre 2 millions de réfugiés et déplacés dans le Borno et dans les régions frontalières des trois pays riverains -, le lac Tchad et ses rives ont connu aussi une certaine modification de leur peuplement par rapport au tableau que l'on pouvait proposer en 2014 (MAGRIN, 2015). Les zones les plus densément occupées demeurent les rives méridionales nigérianes, camerounaises et tchadiennes, les mieux connectées aux systèmes urbains méridionaux. Les violences de Boko Haram puis les mesures contre-insurrectionnelles prises par les pays riverains du lac, en particulier le Tchad et le Niger, ont amené à vider les îles de l'essentiel de leurs populations ${ }^{5}$, regroupées au Tchad dans les camps de réfugiés des environs de Baga Sola et Bol, au Niger dans de nombreux villages et implantations le long de la Komadougou Yobé de part et d'autre de Diffa, ainsi qu'autour de Kabalewa ; au Cameroun dans les villes situées en arrière du littoral lacustre (Mada, Blangoa) (fig. 4). Au Nigeria, les populations des rives les plus exposées se sont largement regroupées autour des chefs-lieux de Local Government (Magrin et Pérouse De Montclos, 2017). Dans certains cas, les terroirs évacués ont été occupés par de nouveaux acteurs, par exemple, dans certaines îles de la cuvette nord, des éleveurs arabes remplaçant les autochtones boudouma après avoir été autorisés par le gouvernement nigérien à occuper les lieux grâce à leur organisation en milices anti-Boko Haram.

L'arrêt forcé de l'économie du lac causé par l'évacuation des zones les plus productives et par l'interdiction de certains échanges commerciaux soupçonnés de nourrir la rébellion (commerce de bétail, de poisson, de poivron) est à l'origine d'un marasme économique profond dans une région récemment relativement prospère. Si Boko Haram et sa répression perdurent, on peut imaginer que le lac Tchad perdra de son attractivité et que la pression sur les ressources (terres agricoles, pâturages, poisson) diminuera.

Ce n'est cependant pas l'hypothèse la plus probable. L'amélioration sécuritaire qui se dessine en 2017 - la mobilisation des armées régionales ayant réduit les capacités de nuisance de Boko Haram - devrait au contraire se révéler favorable à un retour massif vers les zones abandonnées. La régulation de la course aux ressources - entre anciens ayants droit et nouveaux occupants - constituera alors un enjeu essentiel de l'après-crise.

À l'échelle du bassin et à plus long terme, les incertitudes sont moins importantes du fait de la grande inertie des structures démographiques. Malgré les appels des bailleurs internationaux à la mise en place de politiques de population, la population va augmenter dans de fortes proportions. En retenant des taux de

5. Les îles et les marécages qui les environnent ont servi de sanctuaire aux combattants de Boko Haram. De plus, l'enrôlement de certains insulaires boudouma dans l'insurrection a nourri la suspicion des États tchadien et nigérien envers ce groupe tout entier (SEIGNOBOS, 2015 et 2016). 


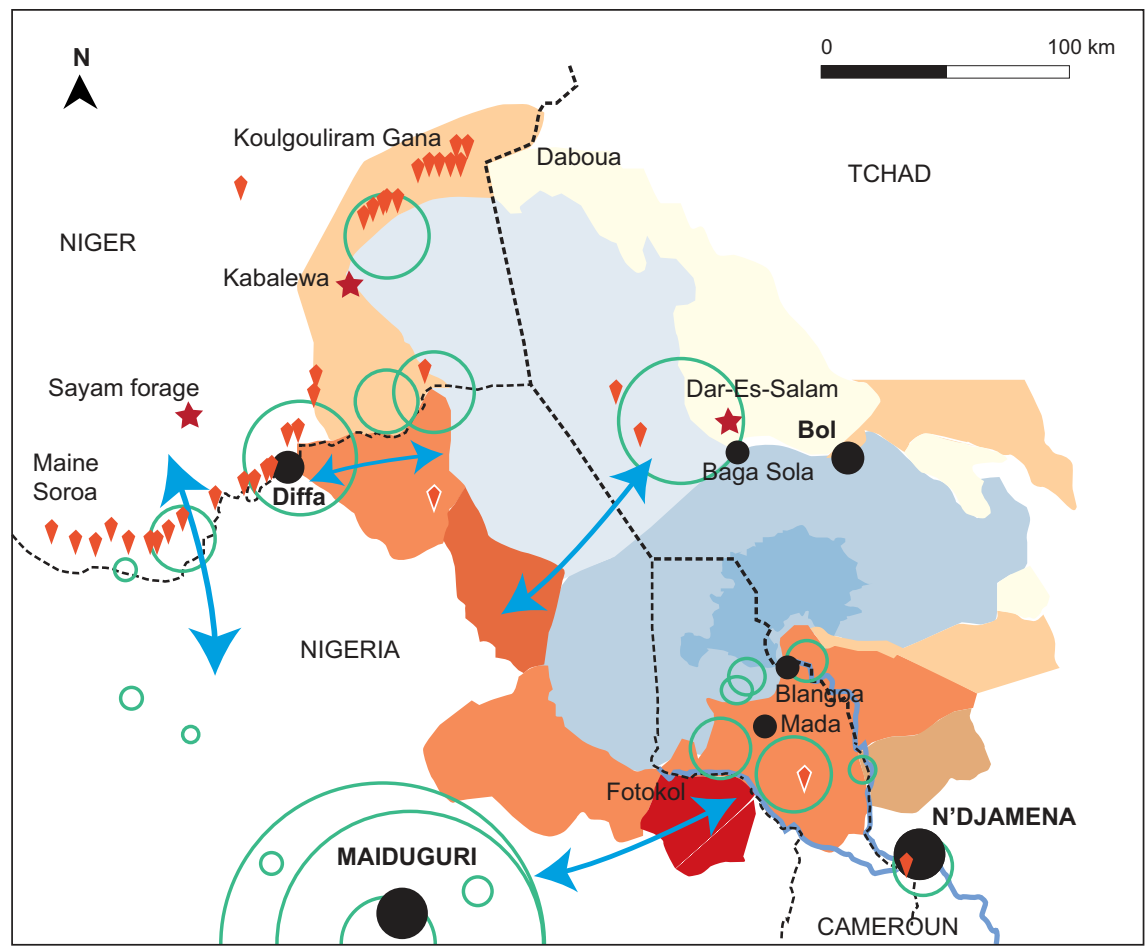

Déplacés internes

Réfugiés
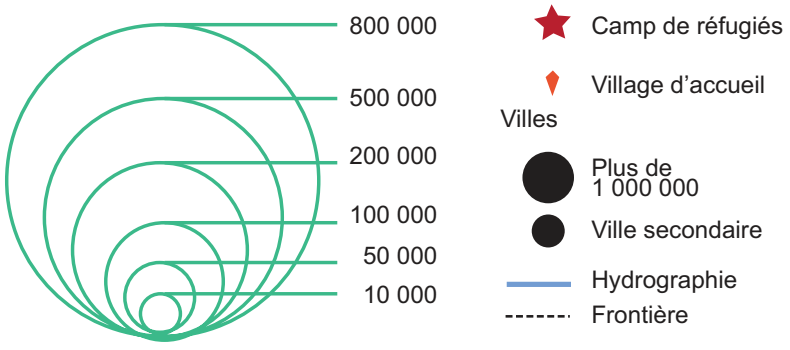

Densité en 2013 hab/km²
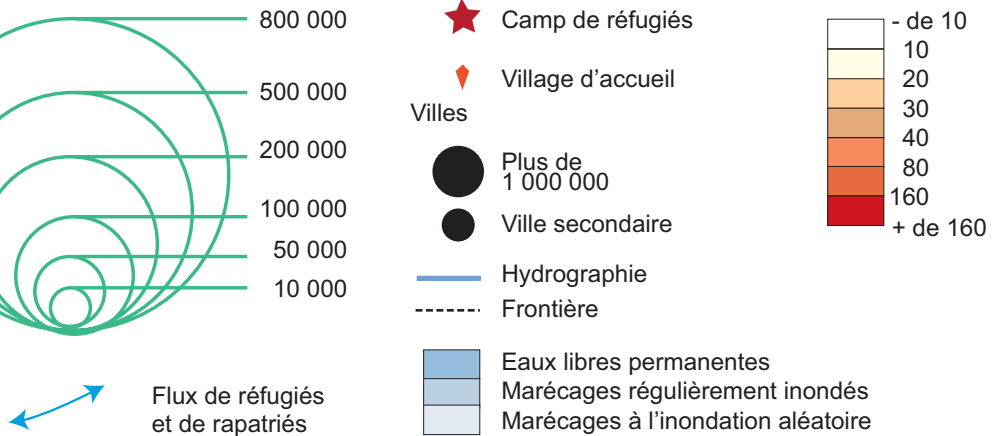

\section{Eaux libres permanentes}

Marécages régulièrement inondés

Marécages à l'inondation aléatoire

\section{Figure 4}

Densité de peuplement (20/3) et déplacements liés à la crise Boko Haram et à sa répression (d'après MAGRIN, 20I5).

Réalisation : P. G. UMR Prodig, 2018.

Sources : IOM ; UNHCR ; ACLEDUNHCR, 2016 ; Atlas du lac Tchad, 2015 ;

Magrin G., Pérouse de Montclos M.-A., 2018.

croissance annuelle moyens inférieurs à ceux actuels $(2,7 \%$, contre plus de $3 \%$ de solde naturel régional moyen), la population du lac et de son arrière-pays étendu (dans un rayon de $300 \mathrm{~km}$ environ) passera de 13 millions d'habitants 
en 2013 à 35 millions en 2050 ; celle du bassin conventionnel géré par la CBLT passera de 50 millions en 2013 à près de 130 millions en 2050 (LEMOALLE et MAGRIN, 2014).

Du fait notamment de l'échec des grands projets d'irrigation menés dans les années 1970-1980, les prélèvements en eau actuels sur le bassin (tous usages confondus) ont peu d'effet sur le niveau du lac : ils représentent moins de $3 \mathrm{~km}^{3}$ sur 18-20 km³ d'apports moyens au cours de la période 1991-2013 (LEMOALLE et MAGRIN, 2014). Un doublement de la consommation par habitant à partir des eaux de surface du bassin Chari-Logone, rendu possible par exemple par les avancées de l'urbanisation, du développement, de l'agriculture irriguée en réponse à l'élévation des températures, amènerait ces prélèvements à $15 \mathrm{~km}^{3}$ par an, ce qui occasionnerait un assèchement de la cuvette nord du lac, dans le scénario probable d'une relative stabilité de l'hydraulicité naturelle.

L'évolution du peuplement - plus ou moins polarisé par les villes, plus ou moins équilibré - dépendra des politiques de développement mises en œuvre (cf. infra).

\section{Les choix possibles et les scenarii}

Il s'agit ici de présenter les types de choix disponibles pour les décideurs nationaux, régionaux et internationaux, puis de décrire trois exemples de futurs envisageables, aussi contrastés que possible, résultant de choix différents.

\section{L'éventail des choix}

\section{Les choix possibles et leur combinaison}

L'avenir du lac Tchad dans son bassin dépendra de la conjugaison des variables structurelles présentées avec les choix politiques réalisés dans les pays riverains. Ces choix concernent de multiples domaines et s'établiront, dans chacun des quatre pays riverains, à des niveaux différents entre différents pôles (LEMOALLE et MAGRIN, 2014) :

- l'environnement (priorité à la conservation ou au développement) ;

- l'échelle des politiques (priorité au cadre national ou à l'intégration régionale, dans une aire historiquement et fortement structurée par les mobilités et les échanges régionaux transfrontaliers);

- la définition d'une vocation du lac unique (spécialisation sectorielle, par exemple en céréaliculture irriguée) ou reconnaissant la multifonctionnalité (agriculture, pêche, élevage, biodiversité) ;

- le choix de modèles agricoles reposant sur l'agribusiness ou l'agriculture familiale ; 
- la priorité accordée à une gestion sécuritaire (militarisation du lac Tchad et gestion humanitaire des victimes de violence);

- le choix de mettre rapidement l'accent sur le développement rural comme mécanisme de stabilisation sociopolitique.

Enfin, il conviendra de choisir entre une logique de transformation et d'aménagement lourd du milieu pour répondre à la baisse des ressources (par exemple par un transfert des eaux et de grands aménagements d'irrigation) et une option qui consisterait à mieux gérer les ressources existantes en diversifiant les approvisionnements (par le recours aux eaux souterraines) et par des usages plus économes de l'eau (goutte à goutte ou autres systèmes).

\section{Toujours et encore la tentation du transfert interbassin}

Face aux défis du lac Tchad et de son bassin - qui déclinent dramatiquement le nexus sahélien population/alimentation/ressources/pauvreté/insécurité -, le recours à un transfert des eaux depuis le bassin de l'Oubangui est toujours sur la table. Au-delà de son objectif explicite permanent - assurer la sauvegarde du lac Tchad -, le projet consiste à maîtriser le niveau de l'eau dans le lac Tchad et à permettre le développement de l'irrigation dans le lac et le bassin, au moyen de barrages, de prises d'eau, de l'aménagement de canaux et de la chenalisation d'une partie du tracé de cours d'eau existants. Ce projet a de fait connu de multiples avatars et trois étapes principales : de premières ébauches, appelées Transaqua, proposées en 1982 (transfert annuel moyen de $100 \mathrm{~km}^{3}$ à partir du Congo) puis 1989 (transfert annuel moyen de $40 \mathrm{~km}^{3}$ à partir de l'Oubangui) par la firme italienne Bonifica. En 2011, la CBLT a fait réaliser par Cima International une étude de faisabilité pour un transfert annuel moyen de $6,4 \mathrm{~km}^{3}$. Or, malgré le coût considérable estimé (14 milliards de dollars, soit trois fois le projet pétrolier Tchad-Cameroun !), un tel volume de transfert ne permettrait pas de sécuriser la cuvette nord en cas de baisse des apports naturels ou de hausse des prélèvements. Le Sommet des chefs d'État a alors décidé en 2012 d'entreprendre dans un premier temps l'amélioration de l'hydraulicité du système Chari-Logone et l'aménagement du lac Tchad (désensablement et désherbage) (CBLT-GIZ, 2016). En décembre 2016, un accord entre la CBLT et la société Power China a été conclu pour l'étude de faisabilité d'un transfert de $50 \mathrm{~km}^{3}$ par an (CBLT, 2017). De très nombreuses questions sont posées par l'éventualité d'un transfert (viabilité financière, impacts environnementaux notamment sur les plaines d'inondation appelées à être chenalisées, gestion de la décrue essentielle au fonctionnement actuel de l'économie du lac Tchad). La moindre n'est pas celle de la position des pays du bassin émetteur, et notamment de la République démocratique du Congo, où la question de l'exportation des ressources naturelles au bénéfice d'acteurs étrangers est d'une sensibilité particulière.

De notre point de vue, le principal problème posé par le projet de transfert interbassin ne réside pas dans sa réalisation effective - les obstacles sont tels qu'elle paraît hautement improbable, sauf conjonction de facteurs difficiles à 
imaginer aujourd'hui ${ }^{6}$. Il réside dans le fait que la fascination qu'il exerce sur les gouvernants, les institutions comme la CBLT, les médias et donc l'opinion publique occulte toute réflexion stratégique alternative sur la manière de répondre, par des utilisations de l'eau et des politiques de développement régional (nationales et transfrontalières) nouvelles, inventives et innovantes, aux défis à venir.

\section{Les trois tableaux de l'avenir du lac (et de son bassin)}

En considérant une hausse des températures, une relative constance des précipitations et la poursuite de la croissance démographique, trois images de futurs possibles peuvent être présentées, suivant la déclinaison chromatique usuelle en matière prospective (noir, gris, blanc).

\section{Le chaos et l'humanitaire}

Un scénario tendanciel verrait la poursuite de la dégradation des conditions de production agricole. À l'échelle du bassin, les effets du réchauffement climatique sur les rendements agricoles ne seraient pas compensés par des politiques publiques plus efficaces que celles mises en œuvre jusqu'à présent. Un tel échec de la régulation régionale (non respect de la Charte de l'eau de la CBLT) et nationale conduirait à d'importants gaspillages d'eau. Au niveau du lac, les épisodes de Petit Tchad secs seraient plus fréquents avec la hausse de prélèvements sauvages, non régulés et non coordonnés sur le bassin amont, conduisant à un abandon de la cuvette nord.

Il en découlerait un accroissement de la pression sur les ressources de la cuvette sud (terres, poissons, pâturages), et donc des tensions sociopolitiques et des violences latentes. Le sous-emploi d'une jeunesse en nombre croissant trouverait un exutoire dans la violence (terrorisme, banditisme), qui s'inscrirait à la fois dans la longue histoire de banditisme rural du bassin du lac Tchad (SAÏBOU, 2010) et dans les survivances du mouvement Boko Haram. Il en résulterait une gestion nationale et internationale à la fois militaire et humanitaire de la région du lac Tchad, qui en entretiendrait la crise.

\section{Le développement par de grandes infrastructures et l'agrobusiness}

Un deuxième tableau verrait la mise en œuvre d'une politique de développement volontariste fondée sur les grandes infrastructures de contrôle de l'eau et sur l'agrobusiness. Elle prendrait la suite de premières tentatives réalisées dans les décennies 1970-1980, où de grands investissements hydroagricoles publics avaient été réalisés sur les rives du lac Tchad (Nigeria, Tchad) et ailleurs dans le bassin, avec des résultats très inégaux - les plus grands projets, le South Chad

6. Utilisons la liberté de la réflexion prospective pour dire l'improbable : un projet de transfert qui semblerait soudain essentiel à un acteur majeur comme la Chine en échange d'accès à des ressources naturelles dont on découvrirait la soudaine abondance (à travers de nouveaux gisements majeurs de pétrole, d'uranium ou autres qui seraient mis à jour), en plus de contrats pour ses entreprises (travaux publics, énergie, agriculture)... ce pays userait alors de son influence diplomatique et de sa puissance technique et financière pour sa réalisation. 
Irrigation Project et le Baga Polder Project au Borno ayant tourné au fiasco (Bertoncin et Pase, 2012).

Depuis le début des années 2000, le lac Tchad n'a pas attiré d'investissements étrangers, contrairement à d'autres régions humides sahéliennes comparables comme la zone Office du Niger ou le delta du fleuve Sénégal. Des tentations de développement de l'irrigation appuyées sur le secteur privé ou des partenariats public-privé existent cependant. Au Tchad et au Nigeria, depuis 2014, la situation de crise économique nationale liée au bas niveau des cours du pétrole est défavorable au volontarisme public, et l'agenda sécuritaire a pris le dessus. Cependant, les plans nigérians de redressement du pays font une part belle à l'appel aux capitaux privés (NESTS, 2014). La plus grande entreprise privée africaine, le groupe Dangote, a notamment annoncé son intention d'investir dans l'agriculture irriguée dans le nord-est. Au Cameroun, des transactions foncières à grande échelle ont été enregistrées avant la crise Boko Haram dans le département du Logone et Chari concernant des secteurs de terres de décrue du lac Tchad (entre le Chari et l'El Beïd) en faveur d'hommes d'affaires régionaux proches du pouvoir (RANGÉ, 2016). Au Niger, la société Fleuve Niger, à capitaux saoudiens, envisage de mettre en culture 74000 ha dans la région de Diffa, y compris dans le lit du lac Tchad (TCHANGARI et DiORI, 2016).

Comme partout en Afrique, les retombées mises en avant par les promoteurs de tels projets et les États qui y sont très favorables sont une hausse rapide de la production agricole et des recettes fiscales publiques. Les risques associés à ce modèle sont également classiques : aggraver l'insécurité alimentaire si les productions ne sont pas tournées vers les marchés intérieurs, mais surtout - puisqu'apparemment, vu le contexte d'enclavement, il s'agit ici de produire pour les marchés intérieurs -, s'accompagner de tensions sociopolitiques si ces modèles se révèlent générateurs d'exclusion sociale (des jeunes, des femmes et des agriculteurs pauvres) et foncière (des agriculteurs sans terre, des éleveurs et des pêcheurs). En outre, le développement d'une grande agriculture sur le lac ou en amont sans régulation ni concertation, donc sans prise en compte de la Charte de l'eau, pourrait accélérer l'assèchement du lac. La modernisation apparente de l'agriculture risquerait donc de s'accompagner d'une aggravation des tensions sociopolitiques, sauf si des innovations permettant le maintien de la multifonctionnalité de l'espace et des articulations inclusives entre agrobusiness et formes d'agriculture familiale existante étaient mises en place.

\section{Un développement inclusif?}

Enfin, le tableau d'un développement plus inclusif serait le résultat de politiques ambitieuses et coordonnées entre les quatre États riverains du lac d'appui à la valorisation du potentiel de l'agriculture familiale, qui a prouvé son efficacité en termes de productivité et d'emploi (Lemoalle et Magrin, 2014 ; RanGé, 2016). Cela supposerait une relance efficace des services d'appui à l'agriculture (vulgarisation, conseil, crédit, assurance, intrants), la réalisation d'aménagements légers à l'échelle du terroir permettant de mieux gérer l'irrégularité climatique. 
Cela impliquerait aussi des politiques de développement territorial permettant la sécurisation foncière, des investissements dans les petites villes et les infrastructures à l'articulation de l'économie agricole et de la demande urbaine (conservation et transformation des produits agricoles, transports, services urbains de base), une meilleure valorisation de l'eau sous toutes ses formes sur les rives du lac et sur l'amont du bassin (mobilisation des eaux souterraines, aménagement des petites vallées, mise en place de systèmes d'irrigation économes en eau), des progrès de l'intégration régionale, pour gérer à cette échelle les ressources en eau, les échanges, les mobilités et la sécurité.

\section{Conclusion}

L'avenir du lac Tchad ne peut être pensé qu'à l'échelle de son bassin. En fonction des choix qui seront réalisés, la probabilité de l'assèchement de la cuvette nord sera plus ou moins forte, celle d'un assèchement complet du lac étant limitée. Le laisser-faire concernant l'utilisation non régulée des ressources sera une cause beaucoup plus directe d'assèchement de la cuvette nord que le changement climatique. Face à ce risque, le transfert interbassin depuis l'Oubangui n'est qu'une option volontariste parmi d'autres. Ces autres options de gestion et de mobilisation efficaces des ressources à l'échelle du bassin méritent tout autant d'être étudiées. Ainsi, à moyen terme, une question majeure est de savoir si des régulations concernant l'usage des ressources naturelles - ressources foncières à l'échelle locale et ressources en eau à celle du bassin - permettront de mieux valoriser le potentiel productif et en termes de développement du lac Tchad, ou si au contraire l'absence de régulation s'accompagnera d'une crise socioéconomique profonde transformant ce qui fut il y a peu une oasis à fort pouvoir polarisant en zone de crise et d'émigration.

Dès lors, les chercheurs doivent continuer à alimenter le débat public à partir d'informations, de connaissances et d'analyses sur les choix possibles - par des études qui pourraient porter par exemple sur l'optimisation des usages de l'eau, sur les possibilités de mobilisation des eaux souterraines, sur la redéfinition d'une agriculture familiale adaptée au changement climatique (et aux aléas des marchés), sur la conception de recherches d'accompagnement en appui au développement territorial (gestion de l'espace et du foncier, aménagement du territoire, développement local), par exemple à travers des observatoires pilotes impliquant les utilisateurs de la recherche dès la conception des projets, en réponse aux enjeux locaux du développement. 


\section{Bibliographie}

Bader J.-C., Lemoalle J., Leblanc M., 2011 Modèle hydrologique du lac Tchad, Hydrological Sciences Journal, 56 (3) : 411-425.

\section{Bertoncin M., Pase A., 2012}

Autour du lac Tchad. Enjeux et conflits pour le contrôle de l'eau. Paris, L'Harmattan, $354 \mathrm{p}$.

\section{CBLT-GIZ, 2016}

Rapport sur l'état de l'écosystème du bassin du lac Tchad, GIZ Bonn, Germany, 233 p.

\section{CBLT, 2017}

Après la signature du Mémorandum d'entente sur le transfert des eaux, PowerChina se rend à N'Djamena. http://www.cblt.org/fr/actualites/ apres-la-signature-du-memorandum-dentente-surle-transfert-des-eaux-powerchina-se-rend (20 avril 2017).

Funk C. C., Rowland J., Adoum A. A., Eilerts G., White L., 2012

A climate trend analysis of Chad. Famine Early Warning Systems Network - Informing Climate Change Adaptation Series Fact Sheet 2012-2070, 4 p.

\section{GIEC, 2014}

Changements climatiques 2014. Rapport de synthèse. Contribution des groupes de travail I, II et III au cinquième rapport d'évaluation du Groupe d'experts intergouvernemental sur l'évolution du climat [sous la direction de l'équipe de rédaction principale, R. K. Pachauri et L. A. Meyer]. Giec, Genève, Suisse, $161 \mathrm{p}$.

\section{GIZ, 2015}

Africa Supraregional Adaptation to Climate Change in the Lake Chad Basin Climate change study. GIZ Bonn, Germany, 69 p.

\section{Harris I., Jones P. D., Lister D. H.,} Osborn T. J., 2014

Updated high-resolution grids of monthly climatic observations of the CRU TS3.10 Dataset. Int. J. Climatol., 34: 623-642.

\section{Lemoalle J., Bader J.-C., LeblanC M.,} SEDICK A., 2012

Recent changes in Lake Chad: observations, simulations and management options
(1973-2011), Global and Planetary Change, 80-81: 247-254.

Lemoalle J., Magrin G., dir., 2014

Le développement du lac Tchad : situation actuelle et futurs possibles. CBLT, N'Djamena, Marseille, Expertise collégiale IRD, AFD-FFEM, rapport de synthèse, $67 \mathrm{p}$. + traduction anglaise et contributions intégrales des experts (CD, 20 chapitres, 620 p.).

Magrin G., Lemoalle J., Raimond C., 2014

«Les futurs du lac Tchad : réflexions prospectives, en guise de conclusion ». In Lemoalle J., Magrin G. (dir.) : Le développement du lac Tchad. Situation actuelle et futurs possibles, Marseille, IRD Éditions, coll. Expertise collégiale, p. 600-638 (clé USB).

\section{MAGRIN G., 2015}

« Démographie du lac Tchad. Incertitude, croissance différenciée et perspectives ». In Magrin G., Lemoalle J., Pourtier R. (dir.) : Atlas du lac Tchad, Paris, IRD Éditions/Passages, p. 74-77.

\section{MAGRIN G., 2016}

The disappearance of Lake Chad: history of a myth. Journal of Political Ecology 23, p. 204-222.

\section{Magrin G., Pérouse de Montclos M.-A., dir., 2018 \\ Crise et développement. La région du lac Tchad à l'épreuve de Boko Haram. Paris, AFD, 291 p.}

\section{NeSTS, 2014}

North East States Transformation Strategy. Abuja, Shamsuddeen Usman \& Associates.

RANGÉ C., 2016

Multi-usage des ressources et mobilités :

l'intensification dans une zone humide sahélienne. Le lac Tchad vu par sa fenêtre camerounaise. Thèse de doctorat, AgroParisTech, $675 \mathrm{p}$.

\section{SAÏвOU I., 2010}

Les coupeurs de route. Histoire du banditisme rural et transfrontalier dans le bassin du lac Tchad. Paris, Karthala, 276 p.

\section{Seignobos C., 2016}

Et si Boko Haram faisait du lac Tchad son sanctuaire? Tout comprendre de la stratégie 
des terroristes de Boko Haram, Le Monde, 29 avril 2016 https://www.lemonde.fr/afrique/ article/2016/04/29/et-si-boko-haram-faisait-dulac-tchad-son-sanctuaire-tout-comprendre-de-lastrategie-des-terroristes_4911132_3212.html.

\section{Seignobos C., 2015}

Boko Haram et le lac Tchad. Extension ou sanctuarisation? Afrique contemporaine, 255, p. 93-120.

\section{Sultan B., Roudier P., Quirion P.,} Alhassane A., Muller B., Dingkuhn M., Ciais P., Guimberteau M., Traore S.,

\section{BARON C., 2013}

Assessing climate change impacts on sorghum and millet yields in the Sudanian and Sahelian savannas of West Africa, Environmental

Research Letters, 8 (1), 014040.

TChangaRi M., DioRI I., 2016

Convoitises foncières dans le bassin du lac Tchad au Niger. Niamey, Alternative Espaces Citoyens, rapport de l'Observatoire du droit à l'alimentation au Niger, décembre, 45 p.

\section{TiLho J., 1928}

Variations et disparition possible du Tchad. Annales de géographie, vol. 207, p. 238-260. 



\section{es communs non excluant?}

\section{La gouvernance des ressources naturelles dans la partie camerounaise du lac Tchad}

\section{Introduction}

La croissance démographique soutenue et les changements environnementaux rapides questionnent la durabilité des systèmes lacustres sahéliens reposant sur les mobilités et le multi-usage de l'espace et leur capacité à répondre aux défis de l'emploi et de l'alimentation des villes. En Afrique de l'Ouest, depuis les sécheresses des années 1970 et 1980, le développement du delta intérieur du Niger et de la vallée du fleuve Sénégal repose principalement sur les aménagements de périmètres irrigués. Au lac Tchad au contraire, le multi-usage de l'espace a supporté un véritable processus de densification démographique et d'intensification halio-agropastorale (RANGÉ, 2016a ; RANGÉ et COCHET, 2018) qui peut être qualifié de développement territorial (LEMOALLE et MAGRIN, 2014).

En se focalisant sur la partie camerounaise du lac Tchad, ce chapitre analyse l'originalité du mode de gouvernance des ressources naturelles sur lequel a reposé cet exceptionnel développement économique. Contrairement au modèle de l'école des communs qui conditionne l'efficacité et la durabilité de la gouvernance locale des ressources naturelles à l'existence d'un groupe d'ayants droit en capacité d'exclure les autres usagers de la ressource et à une régulation intentionnelle élaborée (OSTROM, 1990), ce mode de gouvernance a reposé sur la fluidité des formes de coordination entre usagers et sur l'absence d'exclusion. 
Analyser l'originalité de ce mode de gouvernance revêt un intérêt particulier à un moment où l'insurrection de Boko Haram a en grande partie vidé le lac Tchad de sa population et fortement perturbé son mode d'exploitation (MAGRIN et PÉrouse De Montclos, 2018). On sait en effet que les périodes de conflit sont favorables aux recompositions foncières et qu'en contexte postconflit les enjeux de reconstruction de l'État sont propices aux politiques foncières et agricoles volontaristes $\left(\mathrm{CTFD}^{1}, 2015\right)$. L'analyse des dynamiques qui prévalaient avant le conflit constitue donc un enjeu majeur pour penser les formes du « retour du développement » dans la région.

Après avoir caractérisé le cadre géographique de l'étude, nous analysons l'émergence de principes et droits fonciers égalitaires durant la période de colonisation halio-agropastorale, entre les années 1950 et 1980, et ses conséquences sur les dynamiques d'exploitation. Nous décrivons ensuite la fluidité de la régulation pastorale, puis nous caractérisons les quelques formes d'action collective sur lesquelles ont reposé les processus d'intensification du multi-usage de l'espace entre les années 1990 et 2000. La conclusion discute la durabilité de ce mode original de gouvernance des ressources naturelles en questionnant la montée récente des tensions foncières et les recompositions foncières liées à l'insurrection.

Le travail de terrain a été réalisé juste avant l'insurrection de Boko Haram, entre 2011 et 2013, sur seize mois en cumulé dans le cadre d'une thèse de doctorat (RANGÉ, 2016a). Les enquêtes ont principalement été menées dans quatre villages situés dans le prolongement les uns des autres (de l'extérieur vers l'intérieur du lac), mais des observations et entretiens plus extensifs ont été réalisés dans l'ensemble des villages camerounais du lac Tchad. Nous avons procédé à des observations de paysage et de pratiques, à la consultation des archives préfectorales (procès-verbaux de conflits fonciers, correspondances, rapports des services de l'agriculture) et à 589 entretiens avec les usagers et les autorités locales. D'une durée très variable allant de la demi-heure à la journée, les entretiens consistaient en conversations conduites avec un interprète en langue véhiculaire (arabe shuwa et haoussa) à partir d'un canevas d'entretien, le plus souvent sur les lieux de travail (parcelles, pâturages, sites de pêche). Le choix des interlocuteurs résulte d'un échantillonnage raisonné construit au fil de l'enquête et visant à analyser la diversité des situations et des discours sur un même sujet. 


\section{Le lac Tchad et sa fenêtre camerounaise, des marges dynamiques}

Espace quasi vierge dans les années 1940, l'essor du $\mathrm{Lac}^{2}$ ne remonte qu'au milieu du $\mathrm{XX}^{\mathrm{e}}$ siècle, quand le développement des infrastructures de transport et des marchés urbains du sud Nigeria impulsa un véritable « boom de la pêche », attirant des migrants aux origines très diverses. Dans les années 1970-1980, à l'époque des sécheresses sahéliennes, la baisse du niveau du lac a découvert de vastes terres de décrue qui furent le lieu d'une colonisation agropastorale d'ampleur. Aujourd'hui, les rives du Lac sont devenues des régions densément peuplées $^{3}$ et cosmopolites. Jusqu'à ce que la crise de Boko Haram vienne déstabiliser l'économie régionale, les rives sud exportaient d'importants surplus vivriers vers les marchés urbains du sud Nigeria, de Maiduguri (Nigeria) et de N'Djamena (Tchad) (LemOALle et Magrin, 2014).

Le Lac se caractérise par de fortes disparités dans les formes du multi-usage (RAIMOND et RANGÉ, 2015). Une zone de hauts-fonds individualise une cuvette nord et une cuvette sud : les années où la crue est trop faible, la cuvette nord peut s'assécher en totalité alors que la cuvette sud est inondée quel que soit le niveau des eaux (fig. 1, chap. 16, p. 292). La fenêtre camerounaise, large seulement de $50 \mathrm{~km}$, bénéficie de conditions hydroécologiques favorables : elle offre l'assurance d'une poche d'eau permanente et des espaces de décrue, seules changent leur étendue et leur localisation. Elle joue de ce fait un rôle central dans les mobilités transfrontalières, en particulier halieutiques et pastorales, impulsées par les variations du niveau des eaux (RANGÉ, 2015). Relativement bien relié aux marchés des métropoles régionales, le parcellaire agricole y est dense, sans que cela ne s'oppose à la venue saisonnière d'un grand nombre de pasteurs. À la population résidente, déjà importante ${ }^{4}$, s'ajoutent chaque année de nombreux migrants saisonniers (pour l'agriculture, la pêche et l'élevage) venant en majorité de l'arrière-pays et dont le nombre et la durée du séjour sont très variables d'une année sur l'autre.

2. Nous écrivons « Lac » pour faire référence à l'ensemble constitué par les eaux, les îles, les marécages et la bande de terre aujourd'hui asséchée mais qui était inondée dans les décennies 1950 et 1960.

3. La population résidant au Lac ou dans son arrière-pays proche en 2013 a été estimée à 2000000 habitants pour une surface moyenne du Lac de $8000 \mathrm{~km}^{2}$ sur les deux dernières décennies. La densité de population était évaluée à plus de $60 \mathrm{hab} / \mathrm{km}^{2}$ sur les rives méridionales (LEMOALLE et MAGRIN, 20I4).

4. Nous l'estimons en 2013 à III 400 habitants, pour une densité moyenne de IOI hab/km² (LEMOALLE et MAGRIN, 20I4). 


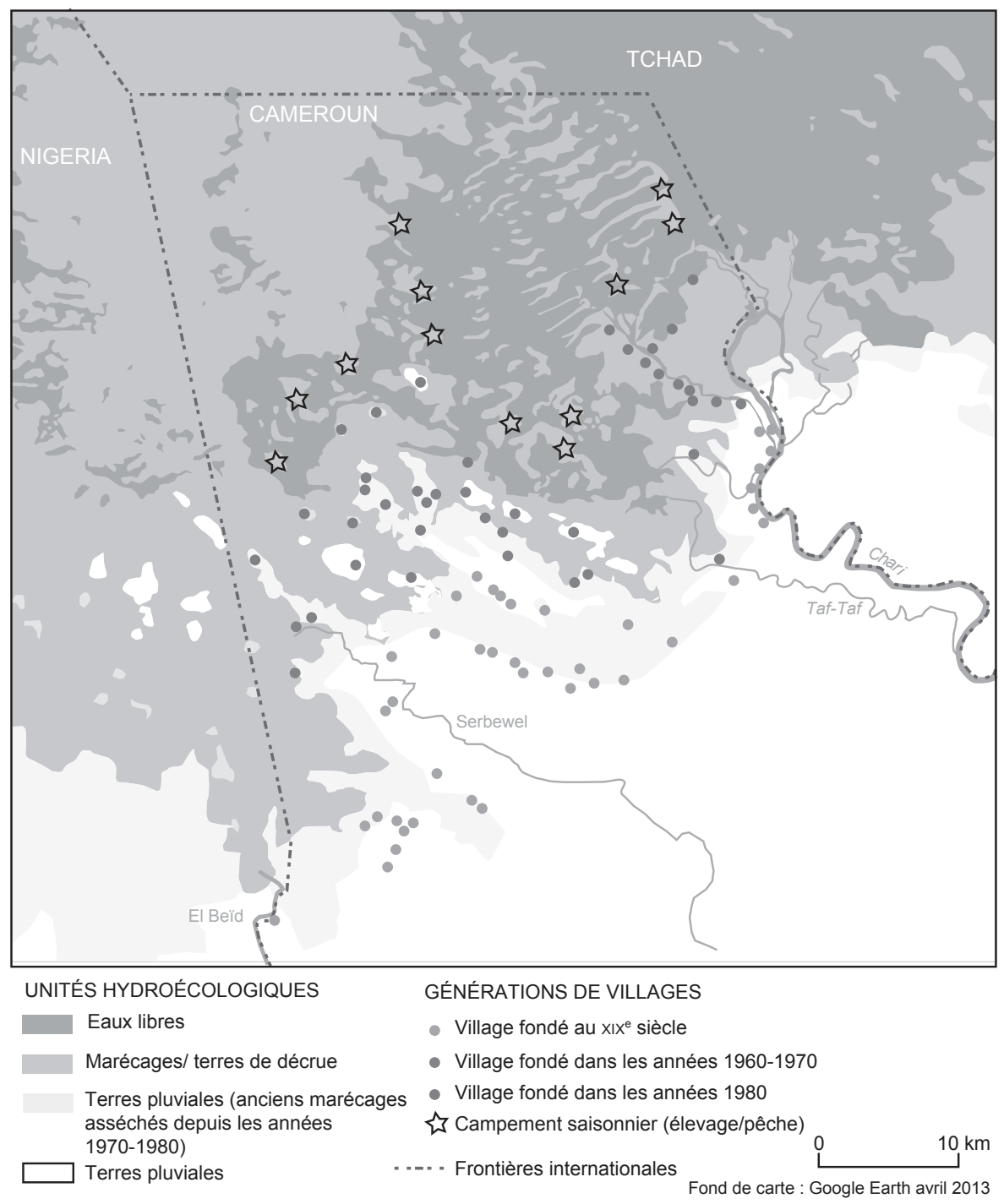

Figure I

Organisation des villages dans la topographie lacustre, Cameroun

(d'après RANGÉ et LAVIGNE Delville, 2019). 


\section{Égalitarisme des principes fonciers et densification du peuplement}

\section{Droits et principes fonciers associés à la colonisation halio-agropastorale}

En Afrique subsaharienne, les droits sur les ressources sont fondés sur les appartenances (lignagères, ethniques, statutaires). Peuplement et mobilités sont régulés par la détention par un ensemble de groupes lignagers, reconnus comme autochtones, d'une maîtrise foncière exclusive. Ici réside l'originalité première des rives camerounaises du lac Tchad : au niveau de cet ancien no man's land, aucun groupe ne jouissait de maîtrise foncière exclusive, que ce soit sur les eaux et le poisson, ou sur les terres et les pâturages découverts à partir des années 1970 . Les migrants ont ainsi installé leurs campements de pêche, puis mis en culture les premières parcelles en dehors du contrôle des autorités. Le sultan kotoko qui contrôlait l'arrière-pays a rapidement revendiqué le contrôle des eaux, aménageant des débarcadères et installant des représentants dans les campements dès les années 1960, en bonne entente avec l'administration qui déploya timidement ses agents pour prélever les taxes aux frontières. Tout en restant sous l'autorité du sultan, les chefs arabes shuwa des villages du Lac fondés au $\mathrm{XIX}^{\mathrm{e}}$ siècle ont étendu leur territoire plus en aval, au fur et à mesure du retrait des eaux, afin de contrôler les mises en culture sur les terres de décrue nouvellement découvertes (fig. 1).

Passée la courte période d'accès libre du début de la colonisation, pêcheurs, agriculteurs et éleveurs se présentaient au sultan ou à ses représentants et, pour les éleveurs, à l'administration avant de s'installer. À condition de reconnaître leur autorité, tout individu, quelle que soit son appartenance ethnique ou nationale, avait accès à la terre et aux ressources naturelles dans les mêmes conditions. Les principes du travail créateur de droit et du droit à la subsistance pour tout individu et sa famille se conjuguaient pour structurer les bases d'une société dont tous les membres partageaient une même expérience de migration et se reconnaissaient mutuellement une légitimité égale à exploiter les ressources, selon le principe du « tous étrangers, donc tous égaux $»^{5}$. Ce que les usagers du Lac expliquent par des formules telles que «Avant, au Lac, c'était l'eau. La terre n'appartient à personne ». Les relations entre groupes d'usagers et d'appartenance se sont dès lors fondées sur un rapport de force équilibré, des interdépendances économiques croissantes et un dense réseau d'interconnaissance. Le retour d'années en années des mêmes éleveurs et migrants, l'installation des campements à proximité des villages, l'accueil des migrants venus pêcher ou cultiver chez leurs tuteurs résidents, la sédentarisation de parents dans les villages du Lac pour les pasteurs, la fréquentation des mêmes marchés et autres lieux 
publics, tout cela a contribué à créer des relations d'obligation et d'interdépendance mutuelle.

« C'est bien que les éleveurs viennent au Lac. Ils viennent gagner leur vie comme nous. Ils nous apportent du lait et achètent les céréales. Leurs femmes font du petit commerce et travaillent dans nos champs. Je ne fais pas de différence entre les Peuls et les Arabes, ce sont tous des bergers et tous des musulmans comme moi. » (résident agropêcheur arabe shuwa, env. 35 ans, 2013)

\section{Adaptation à la variabilité environnementale et densification du peuplement}

L'absence de critère socioethnique dans l'accès aux ressources halieutiques, agricoles et pastorales a été au fondement du peuplement et de la capacité des populations à s'adapter aux changements majeurs de l'environnement et de l'économie des années $1980^{6}$, en leur permettant de diversifier leurs activités ou de se reconvertir (RANGÉ, 2016b). Cela favorisa également les échanges entre groupes d'origine géographique et d'appartenance ethnoculturelle très diverses, conférant à l'agrosystème lacustre un caractère cosmopolite et innovant (RANGÉ et ABDourahamani, 2014).

Dans la pêche, seule la transformation du poisson faisait l'objet de prélèvements limités. Dans l'agriculture, des droits d'usage et de transfert étaient attribués sur les parcelles contre un tribut symbolique et les parcelles abandonnées plusieurs années pouvaient être réallouées par le chef de terre. Cette dernière règle a conféré une grande fluidité à l'accès à la terre et en a limité la monétarisation. Elle a ainsi facilité l'accès à la terre aux nouveaux migrants et aux jeunes. Contrairement aux droits et pratiques en cours dans l'arrière-pays, l'accès aux différents pâturages pouvait être considéré comme libre, le sultan et l'administration contrôlant seulement les mobilités de l'arrière-pays vers le Lac et de part et d'autre des fleuves-frontières. Cet accès libre de fait aux pâturages lacustres camerounais permit, notamment dans les années 1980, à une grande diversité d'éleveurs d'ajuster leurs parcours aux changements environnementaux. Cette situation contraste avec les conflits qui animèrent les autres grandes zones humides sahéliennes dans la même décennie et qui trouvaient leur source dans la complexité des maîtrises foncières anciennes sur les ressources pastorales (FAY, 1999). 


\section{Régulation « de fait» et intensification pastorale}

L'importance de la régulation de fait - « lorsque les régularités observées sont issues d'une somme de comportements et d'interactions sans résulter d'un processus explicite et intentionnel » (LAVigne Delville et Hochet, 2005) contraste avec le caractère sophistiqué de la régulation intentionnelle - « clairement formulée par les acteurs et mettant en œuvre le couple règles/ autorités » (ibid.) - qui a présidé à la coordination des usages dans le delta intérieur du Niger et la vallée du Sénégal par exemple, et qui puise ses racines dans l'histoire longue du peuplement et des pouvoirs (BARRIÈRE et BARRIÈRE, 2002). On peut distinguer la régulation de fait par interaction «lorsque les procédures d'accès se stabilisent au fil d'une histoire sociale et politique des interactions autour de la ressource " de la régulation de fait par évitement « lorsque les acteurs évitent effectivement l'interaction en connaissance des potentialités conflictuelles qu'elle contient » (LAVIGNE Delville et HocheT, 2005). Au Lac, on observe ces deux formes de régulation.

\section{Évitement et intensification pastorale}

Jusqu'au début des années 1980, les éleveurs profitaient d'un bras du Lac toujours en eau pour envoyer leurs animaux paître sans surveillance à l'écart des champs. À partir des années 1980, l'extension des surfaces cultivées, la hausse du nombre de troupeaux et l'importance nouvelle prise par la pêche dans les marécages ont induit le chevauchement des espaces agricoles, pastoraux et halieutiques. En s'avançant plus à l'intérieur du Lac, les éleveurs trouvaient encore de vastes pâturages de décrue à l'écart des parcelles. À partir du milieu des années 1990, avec la forte densification du parcellaire, les espaces libres de champs se sont progressivement réduits aux seuls marécages. Poursuivant une stratégie d'évitement des parcelles, un nombre toujours croissant d'éleveurs a fait le choix d'envoyer son troupeau dans les marécages, toujours plus tôt et plus longtemps dans la saison, dans des hauteurs d'eau toujours plus importantes (photo 1).

Seuls ceux exploitant les plus gros troupeaux ont néanmoins intérêt à le faire et seuls ceux disposant de suffisamment de main-d'œuvre jeune et expérimentée peuvent effectivement le faire. Les autres sont au contraire amenés à adapter la conduite des animaux pour valoriser le pâturage interstitiel entre les parcelles et les résidus de culture. L'exploitation du pâturage marécageux exige en effet un important travail, une bonne connaissance des animaux, et une capacité à supporter des conditions de travail éprouvantes, trois conditions que seuls les jeunes âgés d'une quinzaine à une trentaine d'années réunissent.

L'exploitation toujours plus poussée du pâturage marécageux a fait émerger de nouvelles complémentarités entre différents systèmes d'élevage qui permettent 
les fortes charges pastorales à l'hectare observées sur les pâturages lacustres camerounais. L'évolution des conditions d'exploitation du pâturage (hauteur d'eau, degré d'insalubrité, densité du parcellaire) et sa régénération tout au long de l'année permettent en effet à différents éleveurs d'exploiter successivement le même espace. Au-delà, cette pratique a fait naître une profonde synergie entre agriculture et élevage autour du contrôle de l'enherbement, assurant ainsi l'augmentation parallèle des surfaces cultivées et des troupeaux. Au moment de l'exondation de la parcelle, le couvert a en effet été éliminé sous l'effet du passage des troupeaux, permettant aux agriculteurs d'étendre considérablement les parcelles cultivées.

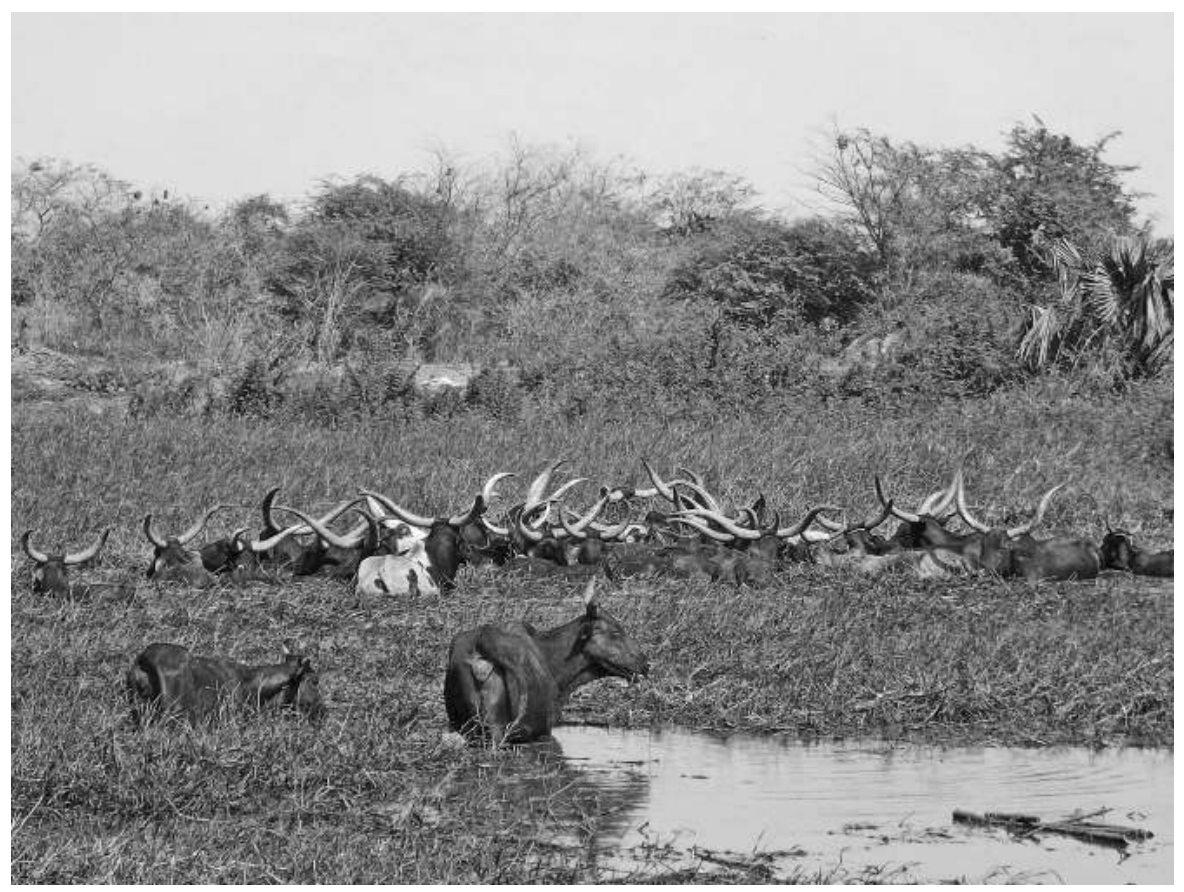

Photo I

Troupeau de zébus mbororo pâturant dans les marécages en saison sèche, Ndili, arrondissement de Blangoua, Cameroun, février 2012.

(C) Charline Rangé

\section{La dimension temporelle des territoires pastoraux}

Avec la forte augmentation du nombre de troupeaux à partir du milieu des années 1990, les éleveurs ont développé des stratégies d'évitement consistant à changer de campement et de zone de pâturage en fonction des mobilités des autres éleveurs. L'efficacité de ces stratégies tient au fait que tous les éleveurs n'entrent pas en concurrence pour le même espace au même moment. Certains éleveurs exploitent les riches pâturages des marécages, à l'écart des champs mais infestés d'insectes ; d'autres attendent la décrue pour valoriser les interstices 
non cultivés entre les parcelles, moins insalubres. Ces stratégies d'évitement permettent la succession d'un grand nombre d'éleveurs sur un même espace au cours de l'année. Loin de l'impression de parcours anarchiques à laquelle l'observateur trop pressé serait tenté de conclure, la fonctionnalité des stratégies d'évitement dans un contexte de forte densité aussi bien agricole que pastorale signifie que les éleveurs connaissent très bien leurs pratiques d'élevage respectives et leurs impacts sur le pâturage. Ils savent quels groupes ont pour habitude de les précéder et de leur succéder sur un même campement ou dans un même pâturage, et ajustent leurs mobilités en fonction.

« En ce moment on ne pâture pas dans l'eau. Dès que les Peuls vont arriver, on devra y aller. On ira à Djibouniba, on fera un mois, puis on partira à Darak. Dès qu'on apprend l'arrivée des Peuls à Blangoua, on quitte pour Darak Klaescou. » (éleveur arabe shuwa, 2011)

La situation est différente lorsque les groupes d'éleveurs partagent les mêmes pratiques. C'est le cas notamment de la majorité des éleveurs peuls tchadiens qui ont gagné les pâturages camerounais à partir du milieu des années 1990 . Des arrangements territoriaux implicites existent, qui voient ces groupes de taille importante fréquenter des campements distincts, et y revenir d'année en année. Chaque groupe dispose ainsi d'un chapelet de campements de l'extérieur vers l'intérieur des rives qu'il occupe successivement au fur et à mesure de la baisse du niveau des eaux. Ces arrangements restent de l'ordre de l'implicite et leur légitimité est restreinte au seul ensemble d'éleveurs partageant les mêmes pratiques pastorales.

\section{Souplesse et efficacité des formes d'action collective}

\section{Le partage de l'usage de l'espace entre agriculture et élevage}

La régulation intentionnelle sur laquelle repose le partage de l'usage de l'espace entre agriculture et élevage s'est construite progressivement dans la résolution des conflits, à mesure que la densification du parcellaire et des troupeaux générait de nouvelles formes d'interactions entre usagers. Jusque dans les années 1990, le système d'autorité - sultan, sous-préfet, chefs de terre et représentants des éleveurs - jouissait d'une légitimité et d'une capacité de sanction suffisante pour jouer un rôle de médiateur et d'arbitre dans la négociation, et aussi pour permettre la définition de nouvelles règles. Quand les agriculteurs et les éleveurs ont commencé à exploiter les mêmes espaces, les inévitables dégâts sur les parcelles ont posé de manière inédite la question du partage de l'usage de l'espace. À la suite de l'arbitrage du sultan et du sous-préfet, les éleveurs se 
sont vus reconnaître des droits sur l'ensemble des espaces non travaillés, en particulier sur les parcelles jusqu'à leur semis et après la récolte. La hiérarchie des droits d'usage entre agriculteurs et éleveurs sur un même espace change d'une année sur l'autre en fonction de la densité du parcellaire : les agriculteurs ne sont indemnisés par les éleveurs que dans les espaces densément cultivés ; lorsque la parcelle est isolée, ils ne le sont pas. En parallèle, des couloirs à bétail ont été progressivement délimités, permettant aux éleveurs de traverser les blocs de parcelle pour mener les animaux aux pâturages.

Les chefs de terre et les agriculteurs sont ainsi incités à distribuer et à cultiver des parcelles intégrées à des blocs de parcelles, et donc à laisser de vastes espaces non cultivés aux éleveurs. Inversement l'éleveur qui se risque à faire paître son troupeau près des parcelles (photo 2) est incité à mettre en œuvre un gardiennage efficace. Le regroupement des parcelles permet une entraide entre les agriculteurs pour éloigner les animaux s'approchant trop près des parcelles et, le cas échéant, identifier le berger fautif. Les rares mais inévitables dégâts sur les parcelles voient le voisin de champs - et parfois un parent - jouer le rôle de médiateur entre l'agriculteur lésé et le berger pour trouver un compromis se traduisant par une sanction, limitée pour l'éleveur de bonne volonté.

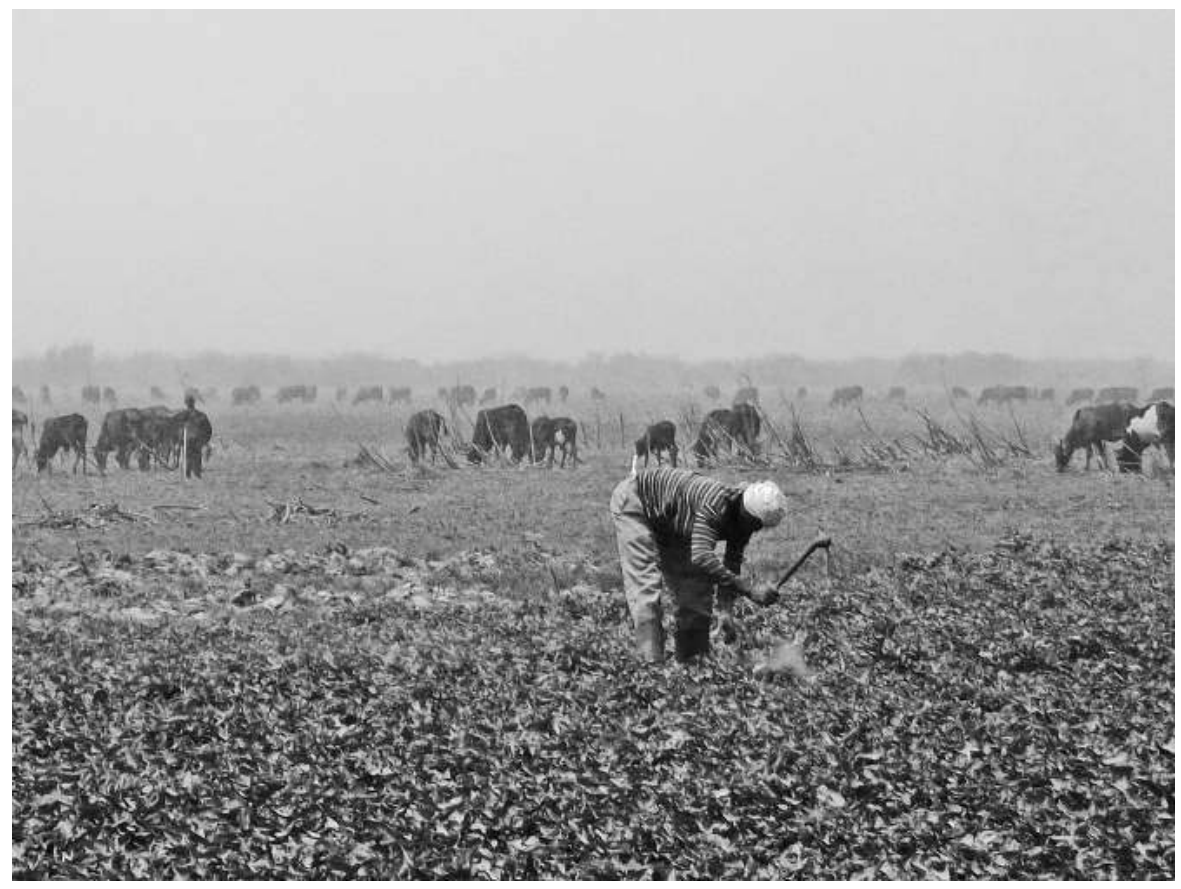

Photo 2

Troupeau de zébus arabes pâturant entre les parcelles, Ndili, arrondissement de Blangoua, Cameroun, mars 20I2.

(C) Charline Rangé 
Dans ces conditions, l'ouverture de nouvelles parcelles ne peut résulter que d'une initiative collective des agriculteurs, limitant d'autant les phénomènes de course à la terre et les contraintes posées par l'agriculture à l'élevage.

« Je pourrais avoir d'autres parcelles maintenant mais aller cultiver seul, c'est pas possible. Les boeufs vont causer des problèmes. En groupe, on peut aller. Si on voit que l'inondation est mauvaise, on peut se déplacer en groupe. Si c'est un déplacement collectif, même si les boeufs viennent, ils vont contourner. » (résident kotoko, env. 70 ans, 2012)

Cette fluidité des modes de coordination entre agriculteurs et éleveurs a été au fondement de la réorganisation spatiotemporelle des activités agricoles et pastorales sous l'effet des changements du milieu, de la densification du parcellaire et de l'augmentation du nombre de troupeaux.

\section{Organisation paysanne et construction d'ouvrages hydroagricoles}

Avec la densification démographique, les agriculteurs ont cherché à gagner des espaces cultivables en retardant l'arrivée de la crue par l'aménagement de digues. Barrant tout un bras du lac, elles permettent de protéger plusieurs centaines à milliers d'hectares répartis sur plusieurs villages?

Constituées de sacs en nylon remplis de sable, ces digues sont ouvertes chaque année une fois les récoltes assurées pour laisser entrer l'eau et les poissons. Chaque année, leur aménagement et leur entretien exigent un travail important répété tous les deux à trois jours, de la montée de l'eau jusqu'à la récolte (soit durant un à trois mois), ainsi qu'un certain niveau de capital (sacs, pelles, brouettes). Le choix de l'emplacement de la digue résulte d'un compromis entre la superficie qui peut être gagnée et le travail qui peut collectivement être investi. Il mobilise une connaissance fine du système de propagation de la crue ainsi qu'une certaine capacité d'anticipation de son niveau et de son calendrier ${ }^{8}$.

Un tel ouvrage suppose des modes d'organisation supra-villageois. Pour faire face aux défauts d'engagement et assurer la coordination avec les autres usages de l'espace, un ensemble de rôles a été progressivement défini par les usagers eux-mêmes : un responsable technique surveille la montée de l'eau et décide, en accord avec un représentant des pêcheurs, de la date de début des travaux et de celle de l'ouverture de la digue ; une personne est responsable de l'information et de la mobilisation des agriculteurs concernés ; une dernière dispose du droit de délivrer des amendes en cas de non participation. L'efficacité de cette organisation tient à la rapidité de circulation de l'information dans les villages et à la surveillance mutuelle entre producteurs, chacun pouvant s'assurer de la présence effective de son voisin de champs. La réprobation qui pèse dans les

7. Une étude réalisée en $200 \mathrm{I}$ par le Programme national de vulgarisation et de recherche agricoles recensait 79 digues, protégeant chacune entre 40 et 7000 ha, sur l'ensemble du territoire lacustre camerounais.

8. L'évolution du niveau du Chari dans les villages situés en aval de N'Djamena (Tchad) est ainsi suivie avec intérêt, et les marchés constituent des lieux propices aux échanges d'informations. 
villages sur les « passagers clandestins » constitue la première des sanctions, renforcée par la délivrance d'une amende dont le montant est indexé sur les moyens humains et financiers du fautif.

Tous les usagers dont le champ est protégé par la digue ont l'obligation de participer au travail et aux frais d'aménagement. Ceux qui estiment le travail trop lourd doivent chercher une parcelle en dehors de l'espace protégé par la digue. Quand un nombre trop important d'usagers fait ce choix, l'aménagement et l'entretien collectif de l'ouvrage cessent. C'est en particulier le cas les années où les pluies ont été importantes dans l'arrière-pays (soit environ une année sur trois sur les quinze dernières années) et que les migrants saisonniers sont peu nombreux.

«Cette année, les cultivateurs sont peu nombreux, sinon à pareil moment on fait la digue de Dor Imar. Avant il n'y avait pas de pluies donc les gens de l'extérieur étaient obligés de venir ici. L'an passé, il y a eu des pluies à l'extérieur et des problèmes d'inondation ici, donc les gens ne sont pas venus cette année. C'est bien pour nous quand les gens de l'extérieur viennent car cela nous permet de faire la digue. » (résident arabe shuwa, env. 60 ans, 2011)

Dans ces conditions, l'espace cultivable n'est gagné sur les pâturages qu'à la condition que les agriculteurs soient suffisamment nombreux, la situation pouvant s'inverser d'une année sur l'autre.

\section{Conclusion. La conflictualisation de la gouvernance des ressources naturelles : quel avenir pour le multi-usage au Lac?}

En réaction aux limites de la gestion étatique des ressources naturelles, le principe d'une gestion participative et décentralisée a été imposé sous l'influence des bailleurs de fonds en Afrique subsaharienne. Largement influencés par le modèle ostromien, des projets ont été mis en place selon une vision très standardisée de la gouvernance des ressources qui cadre mal avec les dynamiques foncières locales (Turner, 1999 ; Lavigne Delville et Hochet, 2005 ; Moritz, 2017). Il s'agit très souvent d'élire un comité de gestion censé représenter un ou plusieurs groupes d'ayants droit et chargé d'élaborer et/ou de faire respecter un ensemble de règles définissant les conditions d'accès aux ressources des différents usagers dans le temps et dans l'espace. La réalité des régulations foncières est toute autre dans la partie camerounaise du lac Tchad. D'une part, à rebours de règles définissant les droits de manière fixe dans l'espace et dans 
le temps, les coordinations pratiques entre usagers se révèlent d'une remarquable efficacité et équité, offrant la fluidité nécessaire à l'adaptation aux changements environnementaux et à la croissance démographique. D'autre part, c'est bien l'incapacité des différents groupes à exclure les autres qui a permis de pousser aussi loin le partage de l'usage de la ressource et de l'espace.

Toutefois, entre le milieu des années 2000 et jusqu'à l'insurrection de Boko Haram en 2014, les conflits à propos des ressources se sont multipliés dans cette partie du Lac. Les changements techniques (usage des herbicides, développement massif de techniques de pêche capitalistiques) d'une part, la pression pastorale et la densification du parcellaire d'autre part, avaient créé de la compétition entre pêcheurs, éleveurs et, par endroits, entre agriculteurs et éleveurs. Cependant, les tensions foncières ne découlaient pas des seuls changements dans les conditions d'exploitation du milieu. Elles résultaient aussi des jeux de pouvoir qui caractérisaient la phase de recomposition sociopolitique impulsée par les politiques de démocratisation et de décentralisation (RANGÉ, 2016b). Ces changements se traduisaient par une instrumentalisation politique du foncier par les notables locaux et régionaux et par une clientélisation croissante de l'accès aux ressources. Les éleveurs de l'arrière-pays se mobilisaient à travers les réseaux clientélistes pour que les autorités préfectorales et communales prennent des arrêtés retardant l'entrée des éleveurs non nationaux au Cameroun ou sur le territoire des communes. Inversement, les éleveurs non nationaux faisaient pression sur les communes et sur l'administration pour que soient délimitées des aires de campement à usage pastoral exclusif, au motif qu'ils payaient une taxe à ces autorités pour accéder aux pâturages. Des conflits émergeaient aussi autour du contrôle et de l'accès aux ressources agricoles et aux sites de pêche qui voyaient des réseaux de clientèle en compétition pour le contrôle des territoires lacustres remettre en cause la légitimité des autorités à autoriser l'accès aux ressources.

Avec l'insurrection de Boko Haram, les populations ont été nombreuses à quitter le Lac et la gouvernance locale s'est militarisée et « miliciarisée », ouvrant la voie à d'importantes recompositions dans les arènes de pouvoir (MAGRIN et PÉrouse de Montclos, 2018). Suite à l'installation des forces de Boko Haram dans la partie camerounaise du Lac, les migrants saisonniers - agriculteurs, pêcheurs et éleveurs - ont dans leur grande majorité cessé de venir et de nombreux résidents ont fui. Pour ceux qui sont restés ou ont continué à fréquenter cette partie du Lac, les arrangements avec Boko Haram ont très vraisemblablement consolidé le pouvoir des big men ${ }^{9}$. La crise Boko Haram risque ainsi d'autant plus de renforcer la tendance à la clientélisation de l'accès aux ressources que le foncier devient un enjeu politique majeur en période postconflit (CTFD, 2015). C'est donc en pleine conscience de ces enjeux de pouvoir qu'il faudra penser le « retour du développement » dans la région. 


\section{Bibliographie}

\section{BARriÈre O., BARrière C., 2002}

Un droit à inventer. Foncier et environnement dans le delta intérieur du Niger. Paris, IRD, $476 \mathrm{p}$.

\section{Chauveau J.-P., Jacob J.-P., Le Meur J.-Y., 2004}

L'organisation de la mobilité dans les sociétés rurales du sud. Autrepart, 30 : 3-23.

\section{CTFD, 2015}

Enjeux fonciers en milieu rural, conflits civils et développement. Note de synthèse $\mathrm{n}^{\circ} 20,11 \mathrm{p}$.

\section{FAY C., 1999}

« Pastoralisme, démocratie et décentralisation au Maasina (Mali) ». In : Bourgeot A., éd. : Horizons nomades en Afrique sahélienne: sociétés, développement et démocratie. Paris, Karthala : 115-136.

\section{Lavigne Delville Ph., Hochet P., 2005}

Construire une gestion négociée et durable des ressources naturelles renouvelables en Afrique de l'Ouest. Rapport final de la recherche. Paris, Gret/Claims/AFD, 183 p.

Lemoalle J., Magrin G., dir., 2014

Le développement du lac Tchad : situation actuelle et futurs possibles. CBLT, N'Djamena, Marseille, Expertise collégiale IRD, AFD-FFEM, rapport de synthèse, $67 \mathrm{p}$. + traduction anglaise et contributions intégrales des experts (CD, 20 chapitres, 620 p.).

\section{Magrin G., Lemoalle J.}

« L'avenir du lac Tchad : les échelles de l'incertitude ». Ce volume.

\section{Magrin G., Pérouse de Montclos M.-A., dir., 2018}

Crise et développement. La région du lac Tchad à l'épreuve de Boko Haram. Paris, AFD, 291 p.

\section{Magrin G., Lemoalle J., Pourtier R.,} éd., 2015

Atlas du lac Tchad. Paris, IRD Éditions/Passages, $225 \mathrm{p}$.

\section{Moritz M., 2017}

Misreading a pastoral property regime in the Logone floodplain, Cameroon. Ecology and Society, 22 (1) : 1-13.

\section{Ostrom E., 1990}

Governing the Commons: The Evolution of Institutions for Collective Action. Cambridge, Cambridge University Press, 280 p.

\section{RaIMond C., RANGÉ C., 2015}

« Les systèmes d'activité des populations riveraines ». In : Magrin G., Pourtier R., Lemoalle J., éd. : Atlas du lac Tchad. Paris, IRD Éditions/Passages : 120-123.

\section{RANGÉ C., 2016a}

Multi-usage des ressources et mobilités : l'intensification dans une zone humide sahélienne. Le lac Tchad vu par sa fenêtre camerounaise. Thèse de doctorat, AgroParisTech, $675 \mathrm{p}$.

\section{RANGÉ C., 2016b}

Gouvernance foncière et intensification du multi-usage de l'espace. Le cas de la fenêtre camerounaise du lac Tchad. Économie rurale, 353-354 : 45-63.

\section{RANGÉ C., 2015}

«Variations lacustres et mobilités humaines. De la résilience à la performance ". In : Magrin G., Pourtier R., Lemoalle J., éd. : Atlas du lac Tchad. Paris, IRD Éditions/ Passages : 78-80.

\section{Rangé C., Abdourahamani M., 2014}

Le lac Tchad, un agrosystème cosmopolite centré sur l'innovation. Les Cahiers d'Outre-Mer, 265 : 43-66.

\section{Rangé C., Cochet H., 2018}

Multi-usage familial et agriculture de firme sur les rives du lac Tchad : une comparaison des performances économiques. Natures Sciences Sociétés, $26:$ 33-48.

Rangé C., Lavigne Delville Ph., 2019.

« Gestion en commun » ou accès ouvert autorisé ? La gouvernance du pluri-usage sur les rives du lac Tchad (Cameroun), $n^{\circ}$ 202-203 Études rurales, $\mathrm{n}^{\circ}$ spécial Zones humides, conquêtes et colonisation.

\section{TURner M. D., 1999}

Conflict, environmental change, and social institutions in dryland Africa: Limitations of the community resource management approach. Society \& Natural Resources, 12 (7) : 643-657. 


\section{Entre auto-organisation et planification dans la plaine d'inondation du Logone (Cameroun)}

Sarah LABORDE, Aboukar MAHAMAT, Mark MORITZ

\section{Introduction}

« La plaine est comme un corps humain ; le fleuve et les mayos ${ }^{1}$ son système vasculaire. Si le flux est perturbé, le corps tombe malade »-c'est ainsi qu'Aboukar Mahamat décrit la plaine inondable du Logone dans l'Extrême-Nord $\mathrm{du}$ Cameroun, dans laquelle il a grandi. Cette métaphore évoque deux caractéristiques qui distinguent les plaines inondables d'autres systèmes socioécologiques. D'une part, les cycles d'inondation et d'étiage sont clé pour la productivité écologique des plaines inondables naturelles. D'autre part, ces systèmes sont très connectés d'un point de vue hydraulique en raison de leur topographie plane, ce qui entraîne une forte connectivité écologique et sociale : une faible variation de l'écoulement des eaux peut avoir des répercussions très étendues (Krause, 2016 ; Di BALdASSARRE et al., 2013).

Cet article s'intéresse à deux stratégies d'adaptation à la variabilité hydroclimatique dans la plaine du Logone : la planification environnementale et l'autoorganisation socioécologique. La planification se réfère dans cet article aux transformations à grande échelle du paysage écologique et social, basées sur une considération de la gestion de l'eau et du territoire comme un défi largement 
technique. Dans la plaine du Logone, ce type de planification repose largement sur le contrôle hydraulique, planifié depuis Yaoundé.

Par auto-organisation, nous entendons un type d'organisation émergente à l'échelle du système, qui au contraire de la planification gouvernementale ne repose pas sur des règles explicites ou sur le contrôle d'un pouvoir central. Le concept a été développé dans les années 1970 par des chercheurs en physique et cybernétique, et a plus récemment été appliqué aux dynamiques sociales, à la fois chez les animaux (i.e. BonABEAU et al., 1997) et les humains (i.e. LANSING et al., 2017).

Dans le contexte de l'usage des ressources dans les plaines inondables, G. Magrin, C. Rangé et leurs collègues ont récemment montré qu'un mode d'organisation flexible et tacite caractérisé par la mobilité et la multifonctionnalité peut s'avérer plus productif que la planification centralisée de la production (Lemoalle et Magrin, 2014 ; Rangé, 2016). Dans les plaines inondables du lac Tchad, Charline Rangé a décrit un mode de production émergeant de l'ajustement constant des individus aux mouvements des eaux, des animaux et des autres individus, clairement distinct de l'anarchie mais souvent sans règle explicite (la « régulation de fait »; RANGÉ, ce volume). C'est ce type de dynamique que nous appelons auto-organisation dans cet article.

Nous nous intéressons ici à la planification et à l'auto-organisation comme deux modes d'organisation socioécologique en réponse aux inondations dans la plaine du Logone. La plaine s'étend de chaque côté du fleuve Logone, qui constitue la frontière entre le Cameroun et le Tchad. Cet article concerne un projet de recherche effectué côté camerounais, où la plaine s'étend sur environ $10000 \mathrm{~km}^{2}$ et supporte des communautés sédentaires et nomades au travers de pratiques de pêche, d'élevage et d'agriculture (SEIGNOBOS et IYÉBI-MANDJEK, 2000), avec un accès aux ressources relativement libre (MORITZ et al., 2016).

\section{Méthodologie}

Notre analyse se base sur de multiples sources de données. Un travail de recherche ethnographique est à la base de la description des activités humaines et des mobilités dans la plaine, en particulier le travail de Mark Moritz avec les éleveurs, celui de Sarah Laborde avec les pêcheurs et l'expérience d'Aboukar Mahamat des dynamiques de sa plaine natale. Nous avons également collecté et analysé : 1) des données bibliographiques sur les projets d'infrastructure hydraulique en cours ; 2) des données de débit du Logone et des images satellite de la plaine inondée en 2014 et 2015 ; 3) des données issues de groupes de discussion menés par Mahamat dans six villages du sud de la plaine en 2016. Ces groupes de discussion ont réuni en moyenne pour une journée 26 participants 
par village, des personnes reconnues dans leurs communautés pour leur connaissance de la plaine. Les participants y ont décrit leurs observations concernant les changements récents d'écoulements des eaux, leurs conséquences et leurs causes possibles. Ils ont également détaillé comment eux-mêmes et d'autres membres de leurs communautés se sont adaptés à ces changements.

\section{Résultats}

Nous présentons nos analyses en trois sections. D’abord, nous décrivons les interactions entre le cycle d'inondation et d'étiage de la plaine et les principales activités de subsistance et de production - interactions que KRAUSE (2016) a appelées « hydrosociales » pour mettre en évidence les codépendances entre flux sociaux et aquatiques. Nous décrivons ensuite brièvement les travaux de génie civil en cours sur les berges du Logone et le discours qui les sous-tend, avant de présenter les résultats des groupes de discussion sur les modes d'adaptation endogène aux changements hydrauliques en cours.

\section{Cycles hydrosociaux dans la plaine du Logone}

La figure 1 présente le cycle couplé de l'hydrologie et des principales activités humaines dans la plaine d'inondation du Logone.

En saison sèche (janvier), dans la plaine (également appelée yaéré), les eaux de crue se sont tout juste retirées. L'herbe est abondante, il y a de l'eau dans les mares et la plaine constitue l'une des plus importantes zones de pâturage du bassin du lac Tchad, attirant de nombreux éleveurs transhumants arabes et foulbé. Les troupeaux arrivent dès que les inondations commencent à se retirer et restent jusqu'aux premières pluies si les conditions le permettent. MorITz et al. (2014) ont montré que leur répartition dynamique dans la plaine est auto-organisée : elle résulte des décisions individuelles des éleveurs sans contrôle central ni règle explicite, dans un contexte d'accès libre aux pâturages dont est fait un usage optimal. Les pêcheurs et agriculteurs mousgoum et kotoko, sédentaires, dépendent des crues annuelles pour leur subsistance (DelClaUX et al., 2010). Les possibilités de pêche sont limitées pendant la saison sèche, ce qui pousse de nombreux jeunes pêcheurs à quitter la zone pour aller pêcher pendant quelques semaines au lac Tchad, vers les lacs artificiels de Lagdo ou Dom-Pangar, ou jusqu'à l'océan.

Avec l'arrivée de la saison des pluies, les premières précipitations se produisent généralement en mai, et sont les plus abondantes en juillet et août. Les pluies sont un signal de départ pour les éleveurs qui retournent vers des pâturages plus élevés. Certains pêcheurs et agriculteurs qui avaient émigré pendant la saison sèche reviennent, car c'est le moment de planter le riz de saison pluvieuse. 
Entre fin août et fin octobre, le fleuve Logone déborde et inonde jusqu'à 800000 ha de la plaine. Les poissons frayent dans la plaine immergée. Les déplacements entre villages se font en pirogue. Si les inondations sont particulièrement importantes comme en 2012, elles peuvent causer des pertes d'infrastructure, de cultures et de bétail.

Les eaux de crue se retirent en novembre et décembre. C'est le moment de la récolte du riz, et le temps de la Grande Pêche : la capture des poissons s'effectue lors de leur retour vers le lit des rivières. La pêche est abondante et certains pêcheurs viennent de loin pour y prendre part. De nombreux pêcheurs et leurs familles vivent temporairement sur leurs lieux de pêche. À partir de janvier, la plaine s'assèche, le revenu et les récoltes générés pendant les périodes productives sont consommés, et le cycle recommence.

Les mouvements et activités saisonniers décrits ci-dessus reflètent une forme d'auto-organisation : un ajustement à l'échelle de la plaine de la pression sur
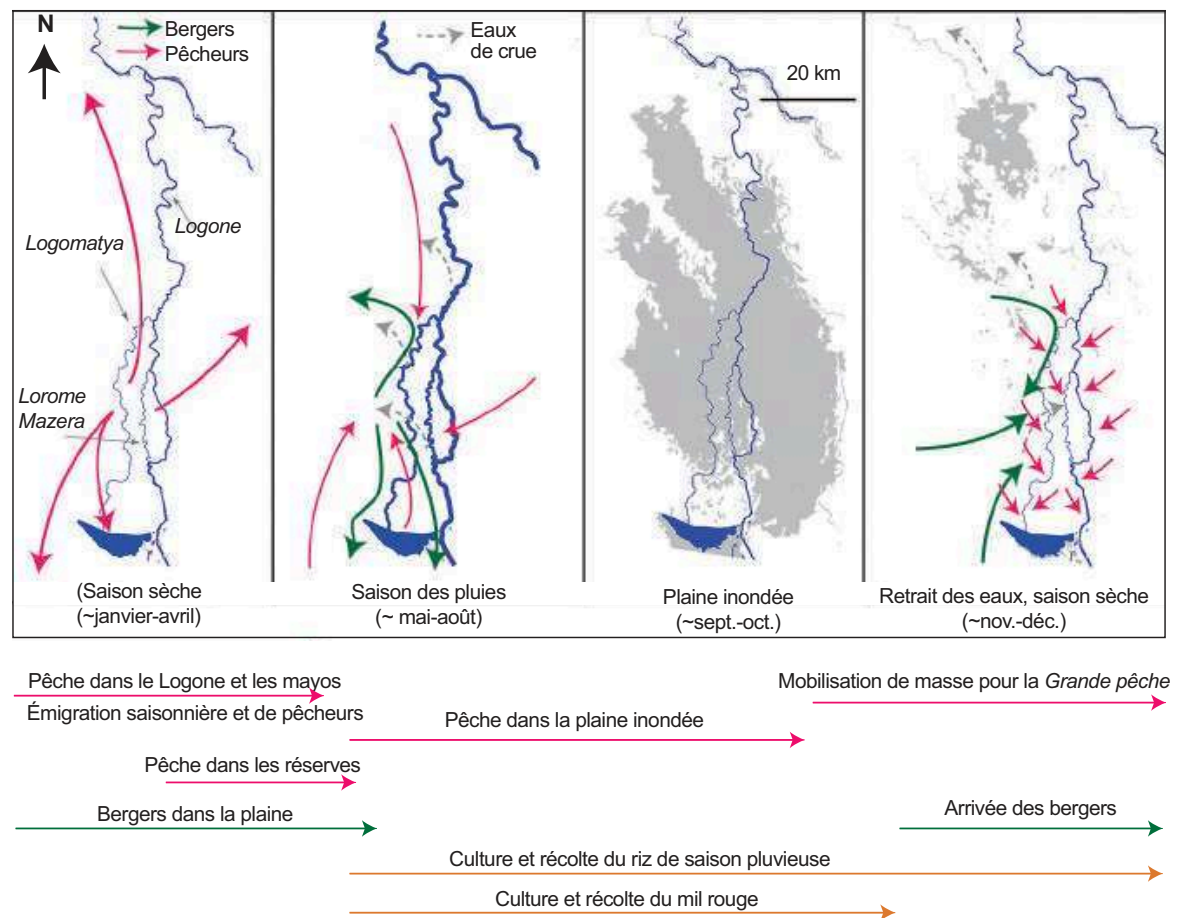

Figure I

Cycle couplé de l'hydrologie et des principales activités humaines dans la plaine d'inondation du Logone.

Zones grises : zones inondées (Landsat).

Flèches roses : mouvements de pêcheurs.

Flèches vertes : mouvements des éleveurs et leurs troupeaux.

(Adaptée de LABORDE et al., 20I8) 
les ressources à leur abondance. Ces mouvements n'émergent pas de règles explicites mais des décisions individuelles des pêcheurs, éleveurs et agriculteurs, basées sur une connaissance tacite du milieu socioécologique, des habitudes, et des règles implicites d'accès aux ressources et d'interactions entre usagers. Comme l'a observé RANGÉ (2016) au lac Tchad, dans ce contexte, un certain degré d'incertitude hydroécologique fait partie du fonctionnement « normal » de la plaine pour ses habitants.

\section{Planification en cours, y compris en réponse aux inondations de 2012}

Une analyse de documents de planification issus du gouvernement camerounais suggère une vision impliquant un plus grand contrôle des flux hydrauliques et économiques de la plaine du Logone au moyen de digues, du développement de la riziculture irriguée et de l'ouverture au développement industriel (République du Cameroun, 2014a et b ; Biya, 2012). Le gouvernement a entamé en 2016 un nouveau projet de riziculture au milieu de la plaine inondable, et signé en 2009 un contrat de partage de production permettant l'exploration pétrolière sur une zone couvrant environ $85 \%$ de la plaine inondable du Logone.

Immédiatement après une forte inondation en 2012, le président Biya s'est engagé à lutter contre les crues du Logone à travers la construction d'une digue de $330 \mathrm{~km}$ séparant le fleuve et sa plaine d'inondation côté camerounais (BIYA, 2012) afin de « sécuriser les populations, leurs biens, l'environnement et les limites frontalières »(RÉPublique DU CAMERoun, 2014a: 3). Des études préliminaires sont menées, et un projet d'accompagnement pour la restauration des digues existantes est en cours (Projet d'urgence de lutte contre les inondations, Pulci). Simultanément, au cours des deux dernières années, le Tchad a commencé les travaux de construction d'une digue le long de la rive droite du Logone, dans le cadre du projet de gestion et de valorisation des ressources naturelles dans les zones soudaniennes (Fonds africain de développement, FAD).

Ces travaux d'endiguement ont causé des changements d'écoulement des eaux de crue entre 2014 et 2015 : une augmentation de la surface inondée côté camerounais est évidente sur les images satellite (fig. 2A, B, E), malgré le fait que 2015 ait été une année similaire à 2014 en termes de précipitations et de débit du Logone (fig. 2D). Les participants des groupes de discussion ont souligné cette augmentation des inondations en 2015. Dans certains villages, les fortes inondations ont conduit à une bonne saison de pêche mais aussi d'importantes difficultés : 755 habitations ont été endommagées par les inondations de 2015 dans les six villages enquêtés (fig. 2C). Les habitants ont également signalé des dommages aux points d'eau potable, aux magasins de stockage et aux infrastructures religieuses (plusieurs mosquées et une église), ainsi que la détérioration de la qualité des fourrages et de la santé du bétail, et la perte de toute récolte de riz de saison pluvieuse. 

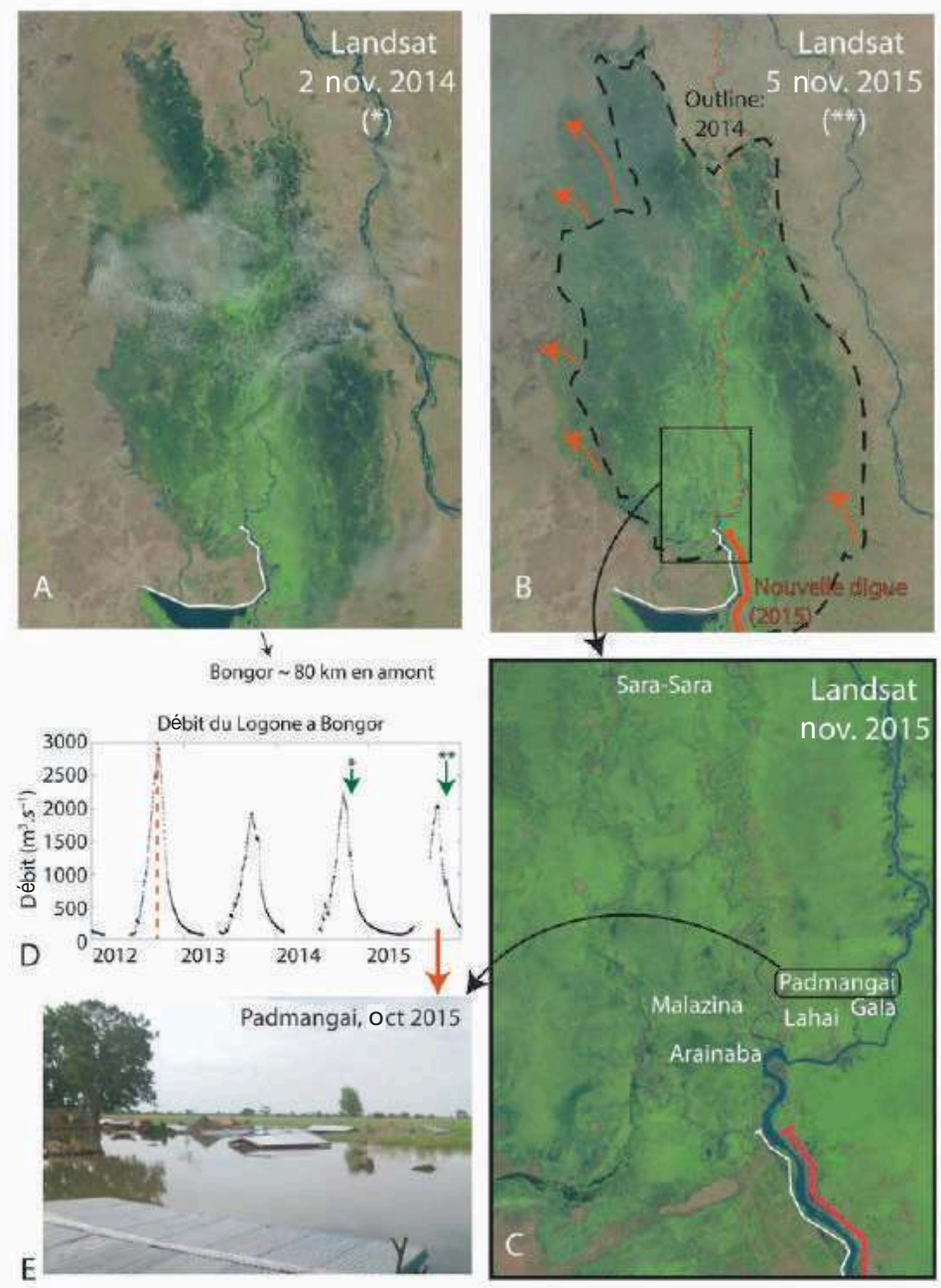

Figure 2

A : Image satellite (Landsat) de la plaine en novembre 20I4. Les lignes blanches sont les digues existantes, en cours de rénovation. B : Image satellite (Landsat) de la plaine en novembre 2015. La ligne rouge est la digue construite en 2015. Le contour pointillé est le contour de végétation aquatique (proxy pour l'inondation) en novembre 20I4, pour comparaison. Les flèches rouges montrent le déplacement de la zone inondée entre 2014 et 2015. C : Zoom du cadre noir en B et localisation des villages où les groupes de discussion ont été tenus. $\boldsymbol{D}$ : Débit du Logone à Bongor, environ $80 \mathrm{~km}$ en amont de la plaine $\left(\mathrm{m}^{3} \cdot \mathrm{s}^{-1}\right.$, données Drem Tchad). La ligne pointillée verticale rouge marque le discours du président Biya à Guirvidig. Les flèches vertes marquent les dates des images Landsat de novembre 2014 (*) et 2015 (**). La flèche rouge sous le graphique marque la date de la photo en $E$ (le lieu de la photo est montré en C).

(Adaptée de LABORDE et al., 2018) 


\section{Adaptation endogène en réponse aux inondations de 2015}

Dans tous les villages enquêtés, les ménages ont pris en 2015 une série de mesures pour prévenir ou atténuer les conséquences des inondations de l'année suivante, par exemple en construisant des diguettes autour de leurs maisons. Suite à la perte des récoltes en 2015, la culture du riz de saison pluvieuse a été suspendue pour la saison 2016. Certains ont également rapporté avoir détruit une partie des constructions sur leurs concessions afin de réutiliser le matériau pour des remblais. La décision de ne pas reconstruire les maisons détruites en 2015 afin de ne pas risquer une nouvelle destruction en 2016 a également été rapportée par 25 ménages. Mahamat a effectué des visites dans la plaine pendant et après les inondations de 2016 et 2017. En 2016, à la suite d'une inondation moins importante qu'en 2015, les dommages furent moindres. En 2017, les mesures de protection se sont avérées insuffisantes : bien que l'inondation soit jugée « moyenne » par Mahamat, elle a tout de même donné lieu à des dommages très importants et localisés comme en 2015 dans les villages à proximité de l'extrémité des digues sur le Logone.

\section{Discussion}

Notre recherche a mis en évidence les interactions entre deux modes d'organisation hydrosociale dans la plaine du Logone en réponse aux inondations : la planification à large échelle et l'auto-organisation. Le discours actuel du gouvernement sur le futur de la plaine est focalisé sur la notion de contrôle, contrastant avec la métaphore " organique » de Mahamat avec laquelle nous avons ouvert cet article, qui illustre l'auto-organisation fluide qui a prévalu jusqu'à maintenant à l'échelle de la plaine.

Contrairement à ses objectifs, la logique gouvernementale de protection contre les inondations a contribué à court terme à une augmentation de l'incertitude hydroécologique pour les populations de la plaine, d'autant que les projets de digue ont été mis en œuvre sans réel engagement des populations locales. Il est utile de rappeler ici le travail de James Scott (1998), qui explique l'échec fréquent des projets de transformation étatique du paysage écologique et social par trois caractéristiques :

- La simplification des interactions socioécologiques par les institutions étatiques : la catégorisation des inondations comme un phénomène qui doit être simplement « combattu » (le « 1 » dans Pulci est pour « lutte ») ignore les conséquences multiples, dynamiques et complexes des inondations dans la plaine. Ce discours simplificateur promeut une transition de la situation actuelle de « vie avec les inondations » vers une plaine espérée plus productive au travers d'une « lutte contre les inondations » (Di BALDASSARRE et al., 2013) qui 
faciliterait les activités agroindustrielles et les cadres administratifs tels que les inventaires fonciers ou bilans de production. La vision d'un fleuve Logone contrôlé et sédentaire est associée à celle de la sédentarisation et de l'encadrement par le pouvoir central des pratiques de production - une vision difficilement compatible avec la multiactivité et la mobilité décrites en figure 1.

- L'idéologie moderniste qui sous-tend les grands projets de génie civil comme la digue-route, basée sur une confiance totale en la maîtrise de la nature par des moyens technologiques : le fleuve Logone a un débit qui atteint une moyenne de $1800 \mathrm{~m}^{3}$. $\mathrm{s}^{-1}$ à la fin de la saison des pluies. Suggérer que priver le fleuve de ses milliers de kilomètres carrés de plaine d'inondation - sur les deux rives permettra un contrôle total des eaux semble plus qu'ambitieux, et ignorant des risques de rupture qui sont inhérents à ce type d'aménagement (DI BALDASSARRE et al., 2016).

- Le manque d'implication de la société civile dans ce type de projet à grande échelle orchestré par l'État : les projets tels que l'endiguement du Logone ont tendance à s'effectuer avec peu ou pas de consultation préalable des populations locales.

La solution pour Scott (1998) est une meilleure prise en compte des connaissances locales. Nous soutenons ici qu'il est nécessaire d'aller au-delà de la division entre connaissances locales et technoscientifiques, et qu'une des clés de la planification environnementale est de maintenir les rythmes (i), la connectivité (ii) et l'apprentissage en continu pour une auto-organisation durable (iii).

\section{Rythmes}

Les réseaux auto-organisés sont adaptables aux changements progressifs (LANSING, 2012), mais ils dépendent d'une régularité relative, à long terme, des rythmes socioécologiques. Par exemple dans la plaine du Logone, les éleveurs transhumants se basent sur leurs connaissances spatiales et temporelles des inondations pour décider de leurs déplacements, dans l'expectative qu'elles seront suffisamment régulières d'année en année. Les pêcheurs ajustent leurs mouvements en fonction du mouvement d'autres pêcheurs, et d'indices hydroécologiques liés de manière relativement fiable à certains phénomènes (tels que le mouvement des poissons). Les agriculteurs anticipent une montée des eaux favorable à la croissance des cultures. Lorsque les rythmes de la plaine sont perturbés, la capacité d'adaptation peut être compromise car l'incertitude devient trop importante pour qu'un nouveau régime hydraulique émerge comme nouvelle « norme » (Dumas et al., 2010). Les groupes de discussion ont confirmé que les communautés de la plaine sont très adaptables et réactives, changeant leurs pratiques d'une année à l'autre pour atténuer l'impact des inondations à venir. Cependant, ce type de réponse n'est pas toujours suffisant lorsque la variabilité hydroécologique est importante, comme cela a été le cas depuis 2015. 


\section{Connectivité}

La considération de la connectivité sociale et écologique est une clé de l'autoorganisation durable, en particulier dans les plaines inondables. Le projet de digue-route propose une modification drastique des flux hydrologiques, sans analyse de la modification tout aussi drastique des relations sociales et écologiques que celle-ci entraînerait (Krause et Strang, 2016) : le blocage dans un premier temps, puis la modification à grande échelle des cycles actuels de pêche, d'agriculture et d'élevage.

\section{Apprentissage continu}

Enfin, l'auto-organisation implique une rétroaction constante entre les processus sociaux et écologiques qui nécessite un apprentissage continu. Le paradigme moderniste tend à proposer des schémas normatifs (e.g. « la construction d'une digue suffisamment élevée arrêtera les inondations ») et à réprimer du même coup l'apprentissage expérientiel et continu (« à tout moment, en fonction des changements hydrologiques, devrions-nous changer nos pratiques ?»). Dans la plaine du Logone, l'intention des gouvernements de canaliser le fleuve risque de conduire à une prophétie autoréalisatrice de nécessité grandissante d'interventions technologiques; comme pour le Mississippi, où la tentative de contention du fleuve a entraîné une augmentation des niveaux d'eau et d'érosion après la saison des pluies et, en réaction, une augmentation graduelle de la hauteur des digues, avec pour conséquence une vulnérabilité accrue aux inondations catastrophiques (PICKERING, 2006, Di BALDASSARRE et al., 2010). Une telle escalade reflète un déni de la connectivité socioécologique, déjà apparent dans la préconisation pour la digue camerounaise d'être rehaussée à une hauteur « au moins $20 \mathrm{~cm}$ plus haute » que celle côté tchadien (RÉPUBLIQUE Du Cameroun, 2014a: 47, 61).

\section{Conclusion}

Nous avons présenté les dynamiques hydrosociales dans la plaine d'inondation du Logone dans le cadre des plans actuels de modification des berges du fleuve à des fins de développement économique, mettant en évidence les interactions entre planification étatique et auto-organisation en réponse aux inondations. Nous avons montré que les aménagements issus de la planification étatique ont augmenté entre 2015 et 2017 l'incertitude liée aux écoulements des eaux de crue, augmentant du même coup la pression sur la capacité d'adaptation des populations locales. 
Nous soutenons que le risque d'un projet comme la digue-route est celui d'un déni de la complexité socioécologique et des propriétés d'auto-organisation de la plaine inondable. Nous ne suggérons pas qu'aucune planification ou modification hydraulique soit appropriée. Cependant, de tels changements doivent prendre en compte les rythmes et la connectivité dans le système, et s'inscrire dans un processus d'apprentissage continu au travers du suivi des changements sur le terrain. Un soutien scientifique peut jouer un rôle important dans ce processus, par exemple au travers de l'étude et la modélisation des rétroactions hydrosociales (i.e. LANSING, 2012 ; LANSING et al., 2017), la mise en place collaborative et participative de programmes de monitoring et d'alerte hydroclimatiques (DI BALDASSARRE et al., 2016), y compris transfrontaliers dans un contexte où, pour reprendre l'expression de KRAUSE (2016), « la digue des uns fait l'inondation des autres ».

\section{Bibliographie}

\section{Adams W. M. A., 2014}

Wasting the Rain (Routledge Revivals): Rivers, People and Planning in Africa. Routledge, 256 p.

\section{BIYA P., 2012}

Allocution du chef de l'État à l'occasion de sa visite aux populations victimes des inondations dans les régions du Nord et de l'Extrême-Nord. https://www.prc.cm/fr/ multimedia/documents/524-allocution-du-chefde-1-etat-guirvidig-20-09-2012 (accessed 1 March 2017).

Bonabeau E., Theraulaz G., Deneubourg J. L., Aron S., Camazine S., 1997

Selforganization in social insects. Trends in Ecology \& Evolution, 12 (5) : 188-193.

Delclaux F., Seignobos C., Liénou G., Genthon P., 2010

« Water and people in the Yaéré floodplain (North Cameroon). » Floodplains: Physical Geography, Ecology and Societal Interactions. Nova publishers, Hauppauge (NY) p. 1-27.

Di Baldassarke G., Montanari A., Lins H., Koutsoyiannis D., Brandimarte L., BLÖSCHL G., 2010

Flood fatalities in Africa: from diagnosis to mitigation. Geophysical Research Letters, 37 (22).
Di Baldassarre G., Kooy M.,

Kemerink J. S., Brandimarte L., 2013

Towards understanding the dynamic behaviour of floodplains as human-water systems.

Hydrology and Earth System Sciences, 17 (8), p. 3235 .

\section{Di Baldassarre G., Brandimarte L., BEVEN K., 2016 \\ The seventh facet of uncertainty: wrong assumptions, unknowns and surprises in the dynamics of human-water systems. Hydrological Sciences Journal, 61 (9), p. $1748-1758$.}

Dumas D., Mietton M., Hamerlynck O., Pesneaud F., Kane A., Coly A., Duvall S., BABA M. L. O., 2010

Large dams and uncertainties: the case of the Senegal River (West Africa). Society and Natural Resources, 23 (11) : 1108-1122.

KraUSE F., 2016

« One Man's Flood Defense Is Another Man's Flood » : Relating Through Water Flows in Gloucestershire, England. Society \& Natural Resources, 29 (6), p. 681-695.

Krause F., Strang V., 2016

Thinking relationships through water. Society \& Natural Resources, 29 (6), p. 633-638. 
Laborde S., Mahamat A., Moritz M., 2018 The interplay of top-down planning and adaptive self-organization in an African

floodplain. Human Ecology, 46 (2) : 171-182.

LANSING J. S., 2012

Perfect order: Recognizing complexity in Bali. Princeton University Press, 248 p.

Lansing J. S., Thurner S., Chung N. N., Coudurier-Curveur A., Karakaș Ç.,

Fesenmyer K. A., Chew L. Y., 2017

Adaptive self-organization of Bali's ancient rice terraces. Proceedings of the National Academy of Sciences, p. 6504-6509.

\section{Lemoalle J., Magrin G., dir., 2014}

Le développement du lac Tchad : situation actuelle et futurs possibles. CBLT, N'Djamena, Marseille, Expertise collégiale IRD, AFD-FFEM, rapport de synthèse, $67 \mathrm{p}$. + traduction anglaise et contributions intégrales des experts (CD, 20 chapitres, 620 p.).

Moritz M., Laborde S., Phang S. C., Ahmadou M., Durand M., Fernandez A., Hamilton I. M., Kari S., Mark B., Scholte P., XiaO N., 2016

Studying the Logone floodplain, Cameroon, as a coupled human and natural system. African Journal of Aquatic Science, 41 (1) : 99-108.

\section{Moritz M., Hamilton I. M., Chen Y. J.,} SCHOLTE P., 2014

Mobile pastoralists in the Logone Floodplain distribute themselves in an Ideal Free Distribution. Current Anthropology, 55 (1) : 115-122.

\section{Pickering A., 2006}

New ontologies. Andrew Pickering. https://ore. exeter.ac.uk/repository/bitstream/ handle/10036/29882/newontols1006. pdf? sequence $=3$ (accessed 1 Feb 2017).

\section{RANGÉ C., 2016}

Multi-usage des ressources et mobilités : l'intensification dans une zone humide sahélienne. Le lac Tchad vu par sa fenêtre camerounaise. Thèse de doctorat, AgroParisTech, $675 \mathrm{p}$.

\section{RANGÉ C.}

« Des communs non excluant? La gouvernance des ressources naturelles dans la partie camerounaise du lac Tchad ». Ce volume.

\section{République du CAMERoun, 2014a}

Études techniques en vue de la construction d'une digue-route, la protection des berges et l'aménagement des infrastructures hydroagricoles le long du fleuve Logone entre Gobo et Kousseri, dans la région de l'Extrême-Nord. - Termes de Référence.

République du Cameroun, 2014b

Études d'impact environnemental et social $d u$ Projet d'urgence de lutte contre les inondations (Pulci) dans la région de l'Extrême-Nord du Cameroun.

ScotT J. C., 1998

Seeing like a state: How certain schemes to improve the human condition have failed. Yale University Press, 460 p.

Seignobos C., IyÉbi-MandJek O., éd., 2000 Atlas de la province Extrême-Nord Cameroun. Yaoundé (CMR), Paris, MINREST, IRD, 172 p. 



\section{e lac Fitri, un modèle} de gestion en crise?

Ronan MUGELÉ

\section{Introduction : un petit lac en mutation rapide}

Si le lac Fitri se singularise par des conditions écologiques particulières - un lac à fond plat à forte variabilité situé en zone semi-aride -, il comporte également une unité politique fondée sur le pouvoir historique de la chefferie coutumière bilala établie à Yao. Longtemps, il fut le centre de gravité d'un puissant royaume sahélien constitué à partir du $\mathrm{XVI}^{\mathrm{e}}$ siècle et qui a su valoriser la fonction de refuge de ce milieu marécageux tout en encadrant l'exploitation de ses abondantes ressources agrosylvopastorales et halieutiques (HAGENBUCHER, 1967). Chercheurs et « aménageurs » se sont notamment penchés sur l'existence au lac Fitri d'un système traditionnel efficace de gestion des ressources naturelles (BIEP, 1989 ; COUREL et al., 1997). Celui-ci est fondé sur le monopole du sultanat dans la définition de règles communes et de droits d'accès régissant les pratiques d'exploitation des ressources. Ce système contrôlant étroitement les rapports entre les différentes catégories d'usagers s'accompagne également de mécanismes de régulation des conflits.

De toute évidence, la viabilité de ce système reposait sur la faiblesse de l'occupation de l'espace, la relative homogénéité du peuplement, la disponibilité importante des ressources et, surtout, le consentement de tous à l'égard d'une autorité coutumière bilala demeurée, après l'indépendance nationale en 1960, largement 
autonome vis-à-vis du pouvoir central. Or le lac Fitri connaît ces dernières années d'importantes transformations démographiques, socioéconomiques et territoriales qui entraînent une profonde reconfiguration de cet espace longtemps enclavé. En effet, on assiste depuis le début du $\mathrm{XXI}^{\mathrm{e}}$ siècle à une densification et à une diversification du peuplement, à une intégration croissante de l'espace lacustre au reste du territoire national et à l'introduction de nouvelles techniques d'exploitation du milieu qui semblent fragiliser le fonctionnement de ce système centralisé. Déjà mis en évidence par des enquêtes antérieures (MARTY et al., 2012), ce constat paraît d'autant plus valable que plusieurs événements conjoncturels récents viennent aggraver les pressions sur les ressources et les tensions autour de leur exploitation, comme la ruée vers l'or débutée au lac Fitri en janvier 2016 et les effets à retardement de l'insécurité qui prévaut dans la région du lac Tchad ${ }^{1}$.

À l'heure où les débats au sujet des communs (OsTROM, 1990) portent sur la recherche de modèles innovants de gestion décentralisée des ressources naturelles (LAVIGNE Delville, 2006) et sur leur articulation avec les enjeux environnementaux globaux qui renouvellent la pensée du développement (LEYRONAS et BAMBRIDGE, 2018), le lac Fitri apparaît comme un exemple pertinent pour analyser la crise d'un système traditionnel qui, sous l'effet combiné de mutations endogènes et d'influences extérieures croissantes, ne parvient plus à encadrer efficacement la gestion collective des ressources du milieu. Issu d'observations et entretiens menés au lac Fitri (février 2015 et février 2016) et enrichis par les enquêtes des autres participants au programme Gelt, cet article vise ainsi à montrer que le système intégré de gestion des ressources naturelles du lac Fitri est remis en cause sous l'effet de la transformation des pratiques d'exploitation des milieux (« par le bas ») et des rapports de pouvoir («par le haut») qui limitent à la fois l'efficacité et la légitimité de l'encadrement coutumier. Une discussion sur le devenir de la gouvernance dans l'espace lacustre est proposée en conclusion, à la lumière des enjeux contemporains du développement des zones humides situées en milieu semi-aride.

\section{Une crise d'efficacité : des dynamiques incontrôlables par un encadrement dépassé ?}

Depuis deux décennies environ, on assiste à une mutation rapide de l'espace lacustre et de ses abords : dynamiques démographiques, innovations techniques et apparition de nouvelles activités économiques se conjuguent pour expliquer les transformations des pratiques d'exploitation des ressources naturelles.

\footnotetext{
I. Les violences perpétrées par la secte Boko Haram et les stratégies contre-insurrectionnelles menées par les États riverains (Tchad, Cameroun, Niger et Nigeria) ont entraîné une réduction drastique des activités de pêche et des échanges transfrontaliers dont dépend le secteur de l'élevage (MAGRIN et PérouSE de MONTCLOS, 2018).
} 


\section{Une pression accrue sur les ressources naturelles}

On observe tout d'abord une forte augmentation de la population : le croît naturel est renforcé par d'importants apports de populations allochtones si bien que la population du département du Fitri est passée entre 1989 et 2009 de 59500 à 116157 habitants (RGPH2, 2009). Ce quasi-doublement entraîne une densification des terroirs et une réduction des disponibilités foncières du fait, d'une part, de l'extension des cultures de berbéré (sorgho repiqué de décrue) et, d'autre part, de l'essor des cultures maraîchères, très productives sur les rives et dans les îles habitées, comme à Moudo. Ces dynamiques peuvent générer localement diverses revendications foncières ainsi qu'une course aux meilleures terres, la valeur des parcelles étant fonction de l'inondation lors de la crue du lac à l'échelle intra et interannuelle. De même, les activités de pêche connaissent ces dernières années un développement très rapide, qui est renforcé par la crise au lac Tchad (insécurité liée à Boko Haram et interdictions plus ou moins durables de pratiquer la pêche). Dans ce contexte, l'installation au moins temporaire au lac Fitri de nombreux pêcheurs professionnels issus d'autres régions du Tchad et des États riverains (Cameroun et Nigeria) provoque une forte augmentation de l'effort de pêche afin de répondre à la demande croissante en poissons frais ou séchés émanant des grands centres urbains (N'Djamena et Abéché).

Par ailleurs un pôle incontournable du pastoralisme transhumant au Tchad central, le Fitri constitue de longue date un point de passage pour les éleveurs de bovins et de petits ruminants, du fait de la disponibilité pérenne des ressources pastorales (eau et pâturages abondants). Longtemps exploité par des groupes restreints et bien identifiés tels que les Arabes Djaatné avec lesquels les Bilala ont noué de solides alliances pour coexploiter pacifiquement les ressources lacustres (ZAKINET, 2015), le lac voit aujourd'hui converger des troupeaux sans cesse plus nombreux, appartenant à des groupes d'éleveurs plus variés et dont la durée de stationnement tend à s'allonger (photo 1). Il en résulte une intensification de la pression sur les pâturages herbacés, aériens et post-récoltes, et ce d'autant plus que le Fitri subit ces dernières années les répercussions des crises sécuritaires de son environnement régional ayant fortement réduit le commerce de bétail ${ }^{2}$.

Enfin, le développement rapide d'une activité d'orpaillage de type artisanal au lac Fitri a encore un peu plus perturbé la vie socioéconomique locale. Les autorités (gouverneur du Batha, préfet et sultan du Fitri) sont averties le 6 janvier 2016 de la découverte de gisements aurifères alluvionnaires subaffleurants au sud-est du lac, où s'écoule un ouadi depuis les contreforts du massif granitique de l'Ab Touyour (MAGRIN, 2017). L'annonce de cette découverte a suscité au cours des jours suivants un afflux brutal d'orpailleurs de tous horizons qui, malgré le déploiement rapide de forces de sécurité venues de N'Djamena pour sanctuariser le site initial d'extraction, ont implanté plusieurs dizaines de campements autour du village d'Ati Ardébé pour regrouper probablement plus

2. Depuis 2013, outre l'insécurité au lac Tchad (Boko Haram), la crise centrafricaine (combats, fermeture des frontières) perturbe elle aussi l'exportation de bétail sur pied tchadien dont dépend l'économie locale, tout particulièrement au Batha qui est un pôle central du pastoralisme à l'échelle nationale. 
de 30000 personnes $^{3}$. Cette authentique ruée vers l'or est lourde de conséquences pour les habitants de l'espace lacustre (insécurité alimentaire, hausse des prix sur les marchés, déplacements massifs de populations, multiplication des conflits, présence accrue des militaires) et pour les fragiles ressources du milieu (prélèvements massifs en poissons et en bois de feu, braconnage, utilisation du mercure). Elle constitue de toute évidence un facteur supplémentaire de déstabilisation de l'espace lacustre.

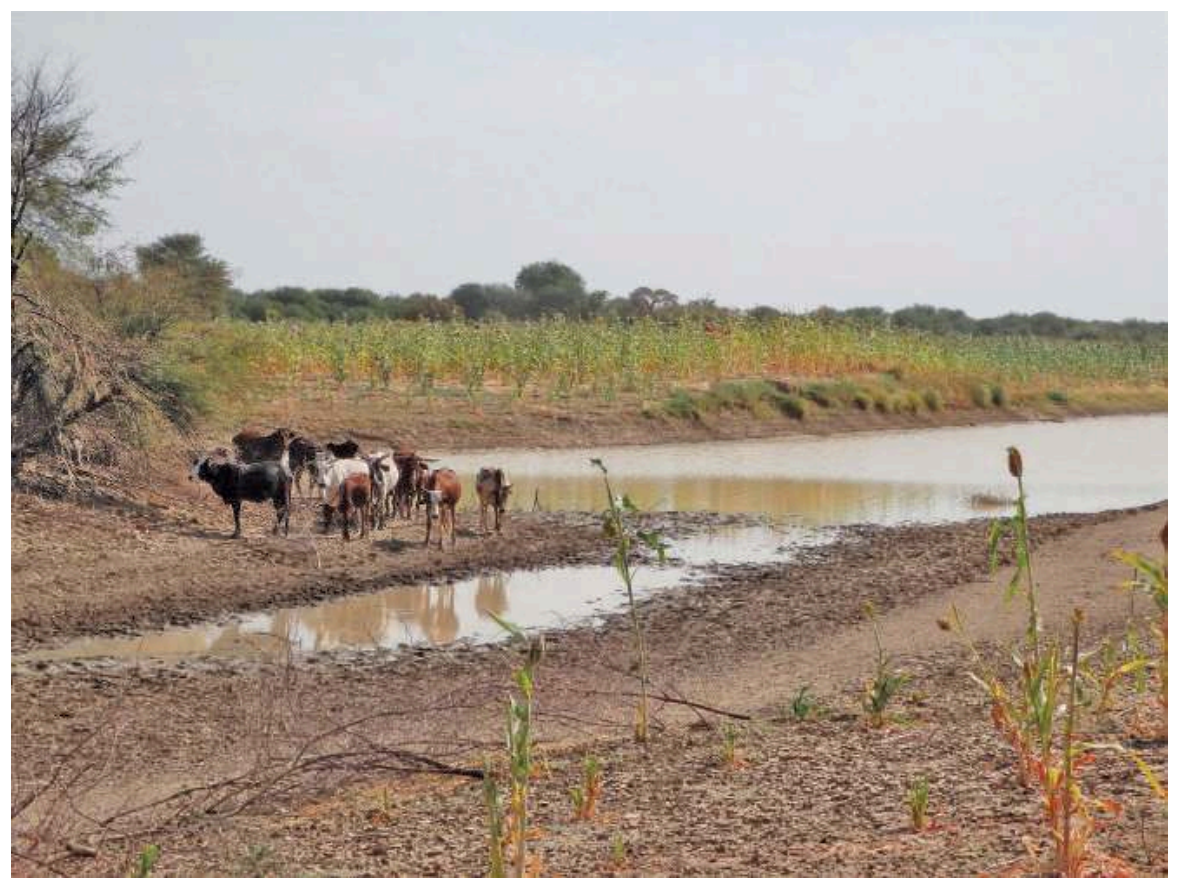

\section{Photo I}

Les rivages lacustres concentrent les enjeux de la coexploitation des ressources naturelles sur laquelle repose la multifonctionnalité des espaces : au gré de l'inondation se succèdent les pratiques de pêche, les cultures de berbéré et l'abreuvement du bétail.

Le développement rapide et conjoint des activités halio-agropastorales tend à fragiliser leurs complémentarités dans le temps comme dans l'espace.

(c) Ronan Mugelé, 2015.

\section{Des mécanismes de régulation dépassés}

Une manifestation évidente de cette crise d'efficacité réside dans la multiplication des conflits entre usagers pour les ressources. En effet, de nouvelles pratiques viennent fragiliser les complémentarités qui étaient au fondement de la multiactivité des systèmes de production et de la multifonctionnalité des espaces, 
ce qui amène les différents groupes (et notamment les autochtones bilala) à réclamer des droits exclusifs pour l'accès aux ressources.

La multiplication des conflits s'explique largement par une compétition accrue autour de l'accès aux ressources et par les difficultés à concilier les besoins de chaque catégorie d'acteurs. Ainsi l'arrivée toujours plus précoce des éleveurs transhumants occasionne souvent des destructions des parcelles mises en culture, mais également des filets de pêche lorsque les troupeaux pénètrent à l'intérieur du lac. C'est la rançon de la réduction drastique des parcours et de l'accès aux rives du lac occupées par les cultures maraîchères permanentes. De plus, il existe des conflits internes au sein de chaque catégorie d'acteurs : ainsi les groupes de pêcheurs sont en concurrence pour la localisation des campements et des filets, les cultivateurs rivalisent pour l'accès aux meilleures terres tandis que certains éleveurs arabes importent au lac Fitri des querelles anciennes, parfois meurtrières, ayant pour origine le vol du bétail dans les confins de leurs terroirs d'attache au nord-Batha (MARTY et al., 2012). Cette recrudescence des conflits au Fitri contribue à confirmer tout en la perpétuant la représentation d'une terre de violences où s'affrontent continuellement des communautés armées antagonistes.

Néanmoins, le constat de la multiplication des conflits doit être appréhendé dans sa dimension sociopolitique, et non seulement démographique ou environnementale : elle résulte aussi de la défaillance des encadrements coutumiers et de la remise en cause du monopole dont ils bénéficiaient jusque lors. En effet, le contrôle qu'opérait le sultanat sur l'accès aux ressources n'est plus que partiellement respecté. Ainsi, l'interdiction des engins de pêche jugés trop prédateurs (filets à petites mailles, nasses) est-elle contournée par une partie significative des pêcheurs professionnels (SAUNIER, 2015), tandis que les vieilles pratiques de signalement des éleveurs transhumants contre octroi d'espaces de stationnement tendent à disparaître. Non seulement les mesures émanant du sultanat sont transgressées par des groupes qui ne consentent pas à reconnaître son pouvoir, mais de surcroît la justice traditionnelle ne semble plus à même de pouvoir gérer l'intégralité des conflits, si bien qu'une partie croissante des litiges se règle désormais au tribunal civil à Ati ${ }^{4}$. Cette marginalisation relative de l'encadrement coutumier s'effectue enfin également «par le bas », lorsque de nouvelles pratiques échappant au contrôle du sultanat s'effectuent directement entre groupes d'usagers, comme par exemple la monétarisation croissante de l'attribution des terres entre cultivateurs, de l'accès aux zones de pêche ou aux résidus des récoltes pour les troupeaux.

Ainsi certains seuils quantitatifs ont été franchis concernant l'exploitation des ressources lacustres ${ }^{5}$, plus conflictuelle car moins régulée par un pouvoir coutumier dont la structure centralisée elle-même est remise en cause.

4. Entretien au gouvernorat d'Ati en février 2016.

5. Les modalités de l'intensification des modes d'exploitation et la dynamique des conflits commencent à être mieux documentées au Fitri grâce aux enquêtes des étudiants ayant participé au programme Gelt. 


\section{Une crise de légitimité : une fronde des notables confortée par l'intervention de l'État?}

La remise en cause de l'efficacité de ce modèle intégré se double d'une contestation de la légitimité même de la chefferie coutumière. Elle débouche au début de l'année 2016 sur le démembrement du canton historique du Fitri, décision emblématique de l'ambiguïté des rapports que noue l'État tchadien avec les pouvoirs locaux aux racines précoloniales.

\section{Le démembrement du canton historique du Fitri}

L'organisation territoriale du Fitri est un véritable casse-tête pour le chercheur de passage, l'hybridation entre pouvoir coutumier et pouvoir étatique entraînant une certaine confusion administrative. Créé en 1967, le canton de Fitri est l'un des plus grands du pays. Il dépend de la sous-préfecture d'Ati jusqu'en 2000, date à laquelle il est promu en sous-préfecture de Yao. Puis en 2004, il est érigé en département comportant deux sous-préfectures (Yao et Amjéména Bilala) mais un unique canton, pourtant l'unité administrative la plus petite. De son côté, le pouvoir coutumier bilala est organisé selon un modèle pyramidal : le sultan - qui s'identifie pour l'État au chef de canton - est à la tête d'une hiérarchie comportant divers représentants à l'échelle locale (sept khalifa à la tête de plusieurs kaïdala eux-mêmes commandant à plusieurs chefs de villages). Ils sont chargés de transmettre et de faire respecter les décisions émanant du sultanat, tout en veillant à la sécurité des biens et des personnes (BIEP, 1989).

Cette configuration héritée a été bouleversée au début de l'année 2016, quand le canton du Fitri a été démembré au terme de longues tractations entre les différents chefs coutumiers, sous la supervision des représentants de l'État au gouvernorat d'Ati : le canton unique se trouve alors subitement subdivisé en sept nouveaux cantons (RÉPUBLIQUE DU TCHAD, 2015). Comme ailleurs au Tchad, ces cantons nouvellement créés n'ont pas d'assise territoriale formellement délimitée mais regroupent autour du chef-lieu, un bourg rural, plusieurs villages (fig. 1). De même, ils ne bénéficient officiellement d'aucun transfert de ressources financières, techniques ou matérielles. Comment dès lors comprendre la décision prise concernant la réforme du statut du canton du Fitri ?

\section{Les raisons du découpage}

S'il procède certes de la remise en cause des mécanismes de gestion des ressources naturelles évoquée précédemment, le découpage administratif constitue surtout la traduction territoriale d'une crise interne à la chefferie ayant des ramifications jusque dans la capitale. 


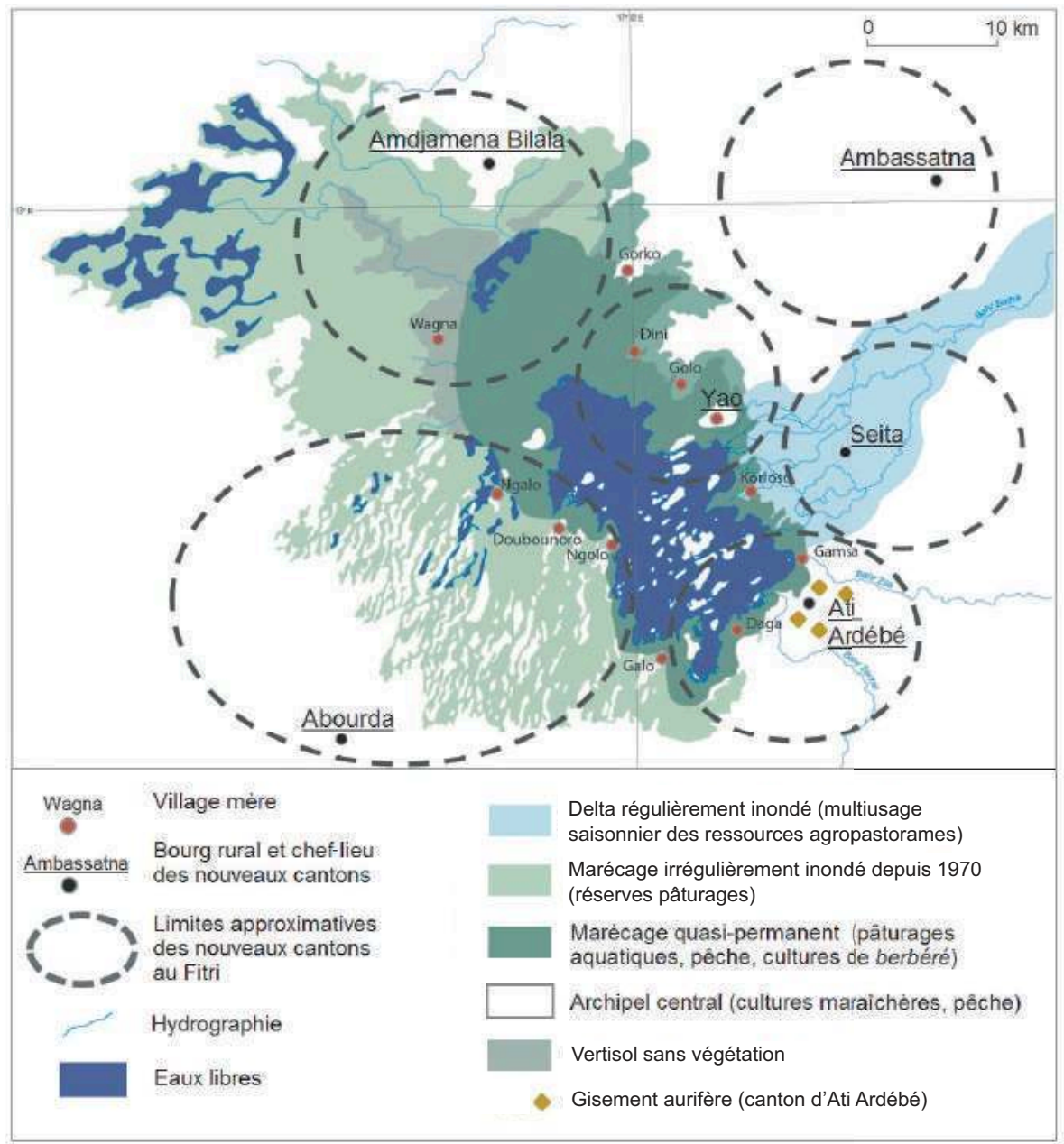

Figure I

À défaut de territoires formellement délimités, le nouveau maillage de l'espace lacustre dessine les zones d'influence correspondant aux six cantons créés au Fitri, auxquels s'ajoute un septième localisé au Batha et non représenté ici. Les enjeux du contrôle des pratiques d'exploitation des ressources naturelles sont plus ou moins grands selon les conditions du milieu. Source : RAIMOND et al., à paraître.

Le déclenchement d'une fronde des khalifa en 2014 s'inscrit dans la continuité historique des rivalités de pouvoir internes au sultanat bilala qui, malgré une unité politique apparente, regroupe plusieurs communautés anciennement divisées et disséminées ${ }^{6}$ (HAGENBUCHER, 1967). Cette contestation procède largement de la crise d'efficacité du modèle de gestion des ressources naturelles, 
qui ne parvient plus à faire respecter les règles en vigueur, notamment en matière de régulation des conflits. Jugées trop partisanes et trop lentes, les décisions des vieux juges coutumiers à Yao exacerbent les rancœurs contre la cour du sultan. C'est donc la structure centralisée même qui est remise en cause. Enfin, outre des rivalités entre des individus pourtant issus d'un même lignage et diverses considérations de prestige, c'est la perspective pour les khalifa et leur entourage de capter de nouvelles ressources qui exalte les revendications locales : la taxation des flux commerciaux de poissons frais ou séchés est une manne potentielle appelée à se développer, tandis que l'appropriation des gisements aurifères fait l'objet de toutes les convoitises.

Cette fronde des chefs coutumiers n'aurait toutefois pas débouché sur le démembrement du canton sans l'intervention du pouvoir central. En effet, les notables du Fitri ont trouvé à N'Djamena des relais puissants en s'appuyant sur le cadre juridico-légal de la décentralisation. Inscrite dans la constitution de 1996 mais encore balbutiante quoique fréquemment mobilisée par le pouvoir présidentiel, la décentralisation se limite pour l'heure en milieu rural à la création décrétée de nouveaux cantons ${ }^{7}$. L'absence de véritable transfert de compétences et de la modestie des ressources financières attribuées aux autorités locales ${ }^{8}$ laisse entrevoir les motivations réelles du découpage, entre outil de promotion et de sanction : il s'agit de consolider le contrôle territorial de l'État en limitant l'influence des pouvoirs locaux parfois rivaux, tout en multipliant les postes donnant accès aux ressources liées au statut de fonctionnaire et mobilisables en temps de campagnes électorales.

Cette volonté exprimée par l'État central de ne pas exempter le Fitri de ce processus de décentralisation a certes été entendue par les notables locaux, mais également par certaines élites bilala résidant à N'Djamena et entretenant un rapport de défiance avec le sultanat. En effet, longtemps sous-représentés dans la population n'djaménoise (où ils étaient réduits dans les représentations collectives au sociotype méprisant de «porteurs d'eau »), les Bilala comptent aujourd'hui un nombre significatif de cadres dans l'appareil d'État, l'armée ou les entreprises publiques. Beaucoup stigmatisent le conservatisme d'un sultanat jugé otage de ses notables les plus rétrogrades et regrettent la marginalisation dont ils se disent victimes quant aux prises de décisions affectant l'ensemble de la communauté bilala9 . L'activisme de cette fraction contestataire et bénéficiant d'un solide réseau dans l'appareil d'État a semble-t-il fortement accéléré la décision hautement politique de procéder au démembrement du canton historique.

7. Ce processus se traduit localement par une véritable inflation cantonale comme dans les départements du Batha comptant plus d'une trentaine de cantons, créés pour la plupart après 2005.

8. Elles se limitent aux salaires et indemnités versées aux nouveaux chefs de canton et à leurs proches auxiliaires administratifs.

9. Entretien à N'Djamena en février 2015 . 


\section{Nouveaux pouvoirs, nouveaux acteurs : quelle gouvernance pour le lac Fitri?}

L'installation ou la consolidation d'autres pouvoirs intervenant aussi en matière de gestion des ressources naturelles redessine finalement le rôle de l'encadrement coutumier mais nécessite de redéfinir un système de règles et d'usages pour l'ensemble des populations riveraines.

\section{Une nouvelle distribution des pouvoirs}

S'il a vu ses pouvoirs modifiés, le sultan n'a pas subitement perdu toute son influence, loin de là. Le sentiment de déclassement exprimé ne doit pas faire oublier le prestige et la protection qu'il apporte encore à la population bilala, fonctions plus essentielles encore à son autorité que le ressort territorial rogné de sa chefferie. Paradoxalement, son prestige apparaît même renforcé par le découpage du canton historique puisque celui-ci s'accompagne d'une contrepartie symbolique, la reconnaissance officielle du titre honorifique de sultan par l'administration étatique ${ }^{10}$. Le sultanat du Fitri semble ainsi suivre une trajectoire comparable à d'autres pouvoirs « néotraditionnels » sachant valoriser les ressources de l'interface avec l'État tchadien. Un État qui depuis peu s'emploie à accompagner les dynamiques de développement en cours au lac Fitri.

Longtemps marginalisé dans les politiques nationales de développement malgré sa position géographique centrale, le lac Fitri fait l'objet ces dernières années d'une attention nouvelle de la part de l'État. Priorité est donnée au désenclavement, afin d'accélérer l'intégration de cet espace au reste du territoire national. La finalisation d'axes routiers goudronnés facilite les circulations de personnes et de marchandises vers l'arrière-pays (Batha et Tchad central) et les principaux centres urbains ${ }^{11}$, même si l'inondation périodique de la cuvette lacustre lors de la saison pluvieuse continue d'entraver fortement les échanges plusieurs mois durant. De plus, les autorités cherchent à poursuivre au lac Fitri le déploiement des services déconcentrés (administration civile et forces de sécurité) pour affirmer la présence étatique tout en s'efforçant de palier le dénuement d'un territoire longtemps sous doté en équipements élémentaires de santé et d'éducation. Surtout, depuis 2011 et le lancement d'un quinquennat présidentiel consacré au développement agricole et rural, l'État a concrétisé son volontarisme par plusieurs interventions au Fitri. Le projet Prodepêche, lancé dès 2007, visait à améliorer l'organisation des activités de pêche (création de groupements, construction de débarcadères frigorifiques, attribution de permis de pêche individuels et collectifs et création de zones mises en défens). Le Projet énergie

10. Des nombreuses chefferies traditionnelles que compte le Tchad, l'État ne reconnaît comme sultanats que les héritières des principaux royaumes précoloniaux (Ouaddaï, Kanem, Baguirmi, Moundang).

II. Le Fitri est contourné au nord (via Ati) et au sud (via Mongo) par deux grands axes goudronnés reliant N'Djamena à Abéché. Si quelques tronçons demeurent inachevés depuis le début des travaux en 20II faute de financements, les temps de trajets ont fortement diminué. 
domestique de l'Aéde (2013-2015) quant à lui a créé à proximité de Gueria, au sud-est du lac, plusieurs zones homologuées de récolte et de production de bois-énergie pour améliorer l'approvisionnement de N'Djamena (rationalisation des pratiques de production de bois et du transport via un système de quittances partiellement reversées aux autorités locales). Enfin, le Programme national de sécurité alimentaire (PNSA) a achevé en 2012 l'aménagement de trois périmètres hydroagricoles (500 ha au total) au nord de Yao afin d'y expérimenter la riziculture. Ces trois projets ont notamment eu pour effet de consolider le rôle des agents forestiers qui, en faisant appliquer la législation environnementale en vigueur à l'échelle nationale, ont contribué eux aussi à faire évoluer les pratiques en matière de gestion des ressources naturelles (réglementation de la coupe de bois, interdiction de la chasse des espèces menacées). Toutefois, ces projets ont rencontré de multiples difficultés (faiblesse des moyens, défauts de conception et oppositions locales aux projets) et n'ont eu au mieux qu'un impact très localisé quand ils n'ont pas été purement abandonnés.

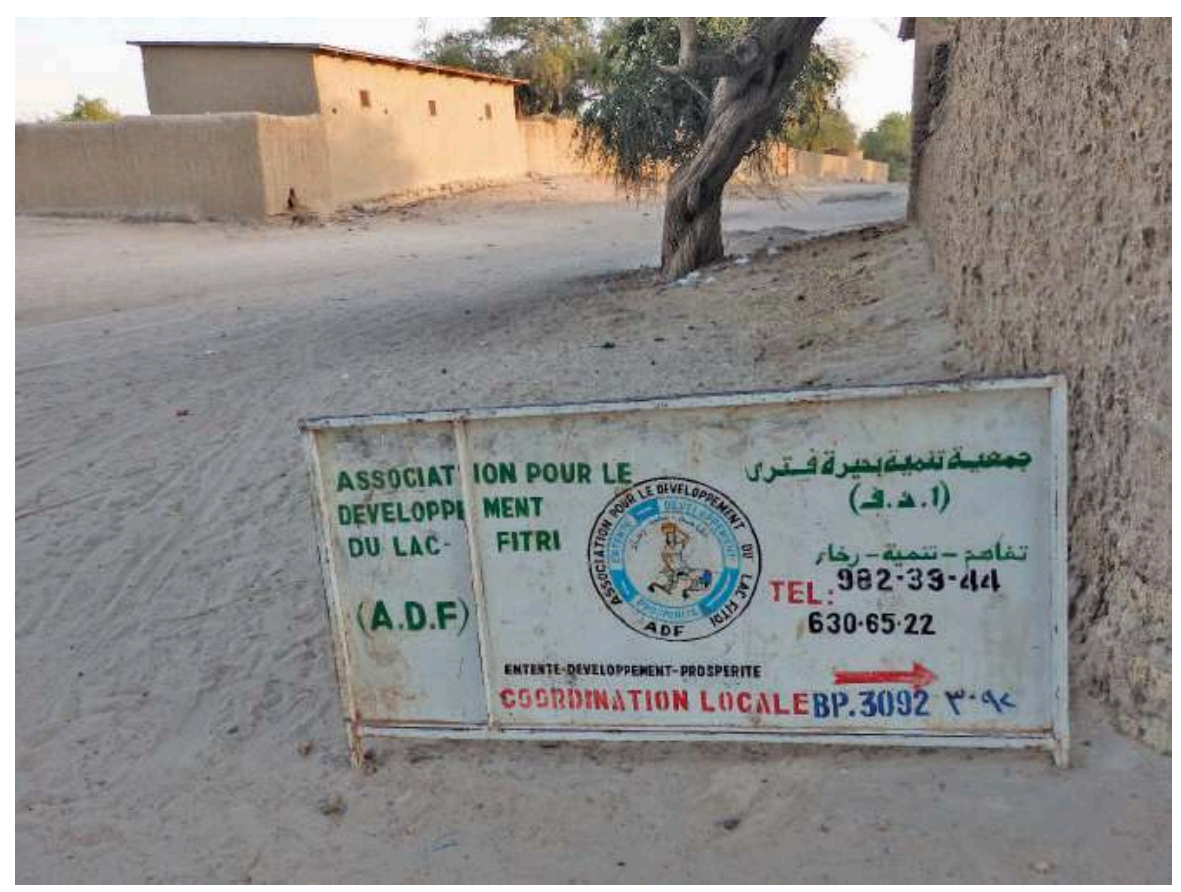

Photo 2

Le siège de l'Association pour le développement du lac Fitri (ADF) se situe entre le palais du sultan (dont une partie est visible à l'arrière-plan) et la sous-préfecture de Yao. Créée au milieu des années 2000 par des habitants locaux, elle peine à exister malgré la multiplication des projets de développement exogènes mis en œuvre au lac Fitri depuis le milieu des années 2000.

(c) Ronan Mugelé, 2016. 
Enfin émerge un troisième pouvoir, qui ne relève directement ni de l'État ni du sultanat : il s'agit des acteurs du développement. La présence des ONG au Fitri n'est pas nouvelle - le Secours catholique pour le développement (Secadév) y a joué un rôle important pour le développement agricole entre 1984 et 1996 mais elle tend à se renforcer sous l'effet de la multiplication des projets de coopération internationale. Ceux-ci concernent principalement l'hydraulique pastorale (Projet d'hydraulique pastorale au Tchad central financé par l'AFD), la sécurité alimentaire (Programme alimentaire mondial financé par la FAO), l'appui au développement local et à la gestion des ressources naturelles (PADLGRN financé par l'UE). La diversification des promoteurs du développement (acteurs extérieurs aux compétences et aux ressources très variables) s'accompagne également de la création d'associations locales même si leur rôle demeure pour l'heure encore marginal (photo 2). Autant d'acteurs qui, par leurs interventions et les ressources dont ils disposent, renouvellent les rapports de pouvoir à l'échelle locale.

Ces dynamiques, si elles ne sont pas spécifiques au lac Fitri, entraînent donc une redistribution du pouvoir entre les instances étatiques, coutumières et " développementistes ». Comment dès lors articuler ces pouvoirs aux compétences parfois concurrentes et aux priorités difficilement conciliables?

\section{Un lac en quête de gouvernance}

Pressions accrues sur les ressources et fragilisation des encadrements locaux : comme le lac Tchad (LemoAlle et MAGRIN, 2014), et selon des modalités différentes au lac Léré, le lac Fitri semble concentrer les enjeux politiques et environnementaux concernant la gestion des zones humides à l'heure où la perspective du changement climatique invite à penser de nouvelles formes de rapport entre milieux et sociétés.

On l'a vu, l'irruption de nouveaux acteurs instaure de facto une forme de gouvernance qui pour l'heure paraît peu à même d'accompagner et d'orienter les dynamiques de développement du lac Fitri et de son arrière-pays. Or, pour garantir à tous les usagers l'accès inclusif aux ressources, la sécurisation des systèmes d'activité et la prévention des conflits, il semble indispensable d'achever la construction d'une gouvernance institutionnalisée afin de réunir autour d'un projet commun des pouvoirs aujourd'hui concurrents. Il y a là pour la chefferie coutumière une opportunité à réinventer son pouvoir, non plus fondé sur un contrôle exclusif des ressources et des populations, mais davantage sur des fonctions de coordination et de concertation.

Dans cette perspective, le lac Fitri pourrait largement s'inspirer de la longue expérience de la coopération tchado-allemande menée au Mayo Kébbi avec le Projet de conservation et de gestion des ressources naturelles (PCGRN, 19942003) puis le Programme de développement rural décentralisé du Mayo-Dallah, du lac Léré, Mont-Illi et de la Kabbia (ProdalKA, 2003-2011). Ces deux initiatives ont significativement contribué à améliorer au lac Léré et dans sa 
proche périphérie la cogestion des ressources et la régulation des conflits locaux via l'action des Ilod (Instances locales d'orientation et de décision), organes reconnus tant par l'État que par l'ensemble des usagers, afin de gérer collectivement les ressources lacustres (DAGOU et al., 2005).

À l'échelle nationale enfin, le Fitri est amené à sortir du relatif anonymat qui l'a longtemps caractérisé. Son potentiel productif et sa contribution à la sécurité alimentaire régionale interpellent l'État quant aux politiques de développement et d'aménagement à mettre en œuvre. Les choix politiques qui seront faits pour encadrer l'orpaillage (réglementation et octroi de permis individuels, taxation de la production voire semi-industrialisation) sont l'opportunité de clarifier la stratégie étatique concernant la gestion et l'exploitation de l'espace lacustre. Mais l'État peut également privilégier l'alternative d'une réactivation des mesures de conservation de l'espace lacustre et de ses ressources : le lac Fitri, pourtant inscrit en 1989 en tant que réserve de biosphère et placé sous le régime de la Convention de Ramsar, n'a pour l'heure que très peu bénéficié de l'intérêt que prêtent les acteurs de la globalisation environnementale aux zones humides à forte valeur écologique, notamment en termes de biodiversité. Conserver, exploiter ou aménager : le lac Fitri a surtout besoin d'une vision politique du rôle qui lui revient dans la stratégie nationale de développement et qui tienne compte aussi des attentes exprimées par les acteurs locaux légitimes.

\section{Conclusion : I'urgence d'une vision politique}

Le lac Fitri est-il rentré dans le rang ? Progressivement intégré au territoire national, fragmenté à l'échelle locale et confronté à des dynamiques de développement difficilement contrôlables, le modèle de gestion intégrée des ressources naturelles semble avoir vécu. La différenciation de l'espace lacustre par les forces centrifuges s'exprime autant «par le haut» (rivalités de pouvoir) que « par le bas » (diffusion de nouvelles pratiques) dans un contexte où des seuils qualitatifs et quantitatifs sont en passe d'être irréversiblement franchis dans l'exploitation des ressources lacustres. Certes, les facteurs de déstabilisation du système sont aussi potentiellement les leviers potentiels de sa résilience : la décentralisation et l'orpaillage pourraient être deux vecteurs décisifs d'un meilleur partage des compétences et des richesses. Mais l'interventionnisme du pouvoir central au lac Fitri peut-il s'accommoder de l'émergence d'acteurs locaux légitimes et capables de promouvoir des formes de gouvernance adaptées au contexte socioécologique original de l'espace lacustre? 


\section{Bibliographie}

BIEP, 1989

Étude de développement rural intégré du lac Fitri. République du Tchad, ministère de l'Agriculture, Banque islamique de développement, Cedrat-SA, Biep, document de synthèse et annexes, 61 p., multigr.

Courel M.-F., Morin F., Raimond C., 1997 «Intégration modèle ou modèle d'intégration. La gestion de l'environnement au lac Fitri (Tchad) ». In : Singaravelou P., éd. : Gestion de l'environnement dans les pays tropicaux, Bordeaux, Dymset-Cret, p. 311-326.

\section{Dagou P., Mustapha M. A., Goltob Mbaye N., Passiring K., Ngar-Odjilo M., 2005}

« La pêche dans les lacs Fitri et Léré au Tchad. Techniques de capture, conservation des produits et enjeux de protection ». In : Raimond C., Garine E., Langlois O., éd. : Ressources vivrières et choix alimentaires dans le bassin du lac Tchad. Paris, IRD Éditions/Prodig, p. 87-112.

\section{Hagenbucher F., 1967}

Notes sur les Bilala du Fitri. Cahier Orstom, Sciences humaines $\mathrm{n}^{\circ} 5(4):$ 40-75.

\section{Lavigne Delville P., 2006}

«Conditions pour une gestion décentralisée des ressources naturelles. Entre «community failures», «market failures» et «state failures», construire de nouveaux «communs»».

In : Bretrand A., Karsenty A., Montagne R., éd. : L'État et la gestion locale durable des forêts en Afrique francophone et à Madagascar, Montpellier/Paris, Cirad/L'Harmattan, 470 p.

\section{Leyronas S., Bambridge T., 2018}

Communs et développement : une approche renouvelée face aux défis mondiaux. Revue internationale des études du développement. Vol. 233 (1) : 11-29.

\section{Lemoalle J., Magrin G., dir., 2014}

Le développement du lac Tchad : situation actuelle et futurs possibles. CBLT, N'Djamena, Marseille, Expertise collégiale IRD, AFD-FFEM, rapport de synthèse, $67 \mathrm{p}$. + traduction anglaise et contributions intégrales des experts (CD, 20 chapitres, 620 p.).

\section{MAGRIN G., 2017}

Orpaillage illégal au Fitri (Tchad central). Géoconfluences, image à la une, octobre 2017. http://geoconfluences.ens-lyon.fr/informationsscientifiques/a-la-une/image-a-la-une/ magrin-orpaillage-tchad.

\author{
Magrin G., Pérouse de Montclos M.-A., \\ dir., 2018 \\ Crise et développement. La région du lac Tchad \\ à l'épreuve de Boko Haram. Paris, AFD, 291 p.
}

Marty A., Zakinet D., Khamis D. D., BERNARD C., 2012

Almy al Afia 2. Analyse de l'évolution des ressources dans le département du Fitri. Document principal. République du Tchad, programme d'hydraulique pastorale au Tchad central, Phase II. Antea-Iram, 128 p.

\section{OSTRom E., 1990}

Governing the Commons: The Evolution of Institutions for Collective Action. New York, Cambridge University Press, 270 p.

\section{Raimond C., Mbagogo A., Madjigoto R., ZAKINET D., à paraître}

Repenser la gouvernance d'une zone humide autour d'un pouvoir local fort : l'exemple du lac Fitri (Tchad) face à la montée des insécurités. Colloque Méga-Tchad Les insécurités dans le bassin du lac Tchad, Nice, XVII ${ }^{\mathrm{e}}$ colloque Méga-Tchad, 14-16 juin 2017 (à paraître).

\section{RGPH2, 2009}

Deuxième recensement général de la population et de l'habitat, résultats définitifs par souspréfecture, mars 2012. Inseed, ministère du Plan, de l'Économie et de la Coopération internationale, annexes : 44-164.

\section{RÉPUBLIQUE DU TCHAD, 2015}

Décret $\mathrm{n}^{\circ}$ 2370/PR/PM/MAT/2015

du 8 décembre 2015 portant création de sept (07) cantons dans les sous-préfectures de Yao, Amdjamena Bilala et Ati rural, départements de Fitri et Batha Ouest, région du Batha.

\section{SAUNIER M., 2015}

L'accès aux ressources halieutiques en lien avec l'évolution des techniques de pêche au lac Fitri, Tchad. Mémoire de master 1, université Paris 1 Panthéon-Sorbonne, 146 p.

\section{ZAKINET D., 2015}

Des pasteurs transhumants entre alliances et conflits au Tchad. Les Arabes Salamat Sifera et les Arabes Djaatné au Batha. Afrique contemporaine 2015/3 ( $\mathrm{n}^{\circ} 255$ ) : 127-143. 



\section{Conclusion Pour un observatoire au lac Fitri. Une exigence d'intégration de points de vue et d'enjeux différents}

\section{Introduction}

Les lacs du Tchad sont révélateurs des effets du changement global sur les territoires, par l'effet amplificateur de leur réponse aux variations du climat et la concentration d'enjeux différents pour une gamme variée d'acteurs et d'opérateurs. La complexité du fonctionnement de ces socioécosystèmes, dévoilée dans les pages de cet ouvrage, plaide pour la mise en place d'observations longitudinales afin de saisir les mutations en cours et pour alimenter les réflexions sur la gestion territoriale et la conception de politiques publiques adaptées aux réalités locales.

Chaque lac est particulier, de par ses caractéristiques géologiques, son alimentation en eau, les sociétés qui l'occupent et les institutions qui les encadrent. Les différents chapitres révèlent la diversité des réponses locales et nationales aux changements en cours. S'ils plaident pour le renouvellement des questionnements sur les biens communs, la gestion participative et l'aménagement du territoire, ils démontrent aussi l'absence d'un modèle unique de gouvernance et de développement pour ces zones lacustres hautement vulnérables. L'enjeu est au contraire d'appréhender chaque socioécosystème dans toute sa complexité pour comprendre les interrelations entre les composantes du système et anticiper les conséquences des évolutions en cours. Celles-ci s'exprimant à toutes les échelles, de la parcelle et du terroir villageois à l'échelle globale, et selon des points de vue disciplinaires très différents, il convient de réfléchir à l'intégration 
des résultats de la recherche et leur utilisation par les acteurs du territoire. Pour comprendre les évolutions passées et réfléchir à l'avenir, l'acquisition de données régulières et actualisées sur le climat et l'hydrologie, sur les évolutions des sociétés, de leurs activités et de leurs institutions est primordiale. C'est sur ce besoin d'observatoires mettant à disposition les connaissances et associant les acteurs du développement que se focalise la conclusion de cet ouvrage.

Nous présentons ici une synthèse de la contribution des recherches, conduites par les chercheurs et étudiants du programme Gelt, à la compréhension du socioécosystème du Fitri et des enjeux de la gestion environnementale. Le lac Fitri est peu connu du grand public mais concentre les défis des zones humides tout en ayant été longtemps préservé par son enclavement : reconnu par l'IUCN pour sa biodiversité, avec un système basé sur la multifonctionnalité et la pluriactivité régi par des règles ancestrales négociées entre acteurs, il connaît aujourd'hui une forte croissance démographique, une diversification des acteurs du territoire et une montée des insécurités représentatives de l'ensemble sahélien (RAImOND et al., 2017). La multiplication des conflits et la dérégulation des droits d'accès aux ressources naturelles révèlent une crise profonde de la gouvernance. Nous montrons les changements du socioécosystème à l'aide de chorèmes, qui constituent une modélisation spatiale (BRUNET, 1986), et l'intérêt de comprendre conjointement les dynamiques écologiques et sociales pour penser l'avenir de ce territoire lacustre représentatif des enjeux contemporains des zones humides au Sahel.

\section{Le lac Fitri, un observatoire}

Le lac Fitri est un lac endoréique peu profond, dont le bassin versant est entièrement inclus dans la zone sahélienne. Il est souvent comparé au lac Tchad, dont il partage l'histoire en tant que vestige de l'ancien Mégalac Tchad (SCHUSTER et al., ce volume) ainsi que certaines caractéristiques. Sa superficie varie fortement en fonction de l'ampleur des crues, ses eaux recouvrent un système dunaire ancien qui forment les îles et l'archipel ; elles inondent un système de mares plus ou moins connectées où s'installe une végétation aquatique poissonneuse et très bien valorisée par le bétail, dont une partie est mise en culture à la décrue (fig. 1 et 2). Comme au lac Tchad, cet écosystème est attractif pour les populations riveraines, qui y développent des systèmes de production basés sur les mêmes principes de mobilité, de multiactivité (pêche, agriculture de décrue, élevage) et de multifonctionalité des espaces (RAIMOND et al., 2014).

La baisse des précipitations enregistrée sur le bassin versant dans les décennies 1970-1980 a immédiatement et fortement réduit la superficie en eaux libres, concentré l'exploitation agricole dans les zones de décrue et renforcé la fréquentation par les troupeaux transhumants (fig. 1). Contrairement au lac 
Tchad, on n'observe pas d'installation de nouveaux agriculteurs et les arrivées de pêcheurs sont peu nombreuses en raison de l'enclavement de la région qui limite les débouchés commerciaux.

Depuis les années 2000 et un retour à un régime de pluies plus abondantes, la cuvette principale du Fitri se remplit à nouveau et se connecte avec les mares à l'ouest. La zone de décrue plus étendue permet d'augmenter les superficies cultivées en décrue (berbéré et maraîchage) et les pâturages (fig. 2). Une ceinture de berbéré se met en place en auréole autour des pâturages à l'intérieur du lac et sur les îles, constituant un obstacle majeur à la mobilité des troupeaux entre novembre et février. La progression du maraîchage en bordure du lac et sur les îles prolongent cet obstacle jusqu'en fin de saison sèche. L'arrivée de pêcheurs plus nombreux depuis la crise liée à Boko Haram sur le lac Tchad, puis des orpailleurs en 2016, multiplie les types de conflits.

Le lac Fitri possède aussi ses caractéristiques propres, qui en font un modèle particulier. Le régime exclusivement sahélien des pluies qui alimentent son bassin versant différencie les rythmes de fluctuation des lacs Fitri et Tchad, géographiquement proches. De plus, avec sa taille modeste $(30 \mathrm{~km}$ de long sur $10 \mathrm{~km}$ de large), le lac Fitri est compris dans le territoire du sultanat Bilala qui gère, depuis le $\mathrm{XVI}^{\mathrm{e}}$ siècle, l'accès des populations aux ressources lacustres. Cette unité de gestion exercée par un pouvoir fort et centralisé a pendant longtemps assuré la régulation des activités exercées dans le lac et la résolution des conflits d'usage. Malgré les nombreux changements opérés au XIX ${ }^{e}$ siècle - colonisation française, Indépendance, sécheresses des années 1970-1980 - la résilience du système a reposé, d'une part, sur la complémentarité des activités dans l'espace et dans le temps (ILYADE, 2016*), et, d'autre part, sur la reconnaissance de l'autorité coutumière par l'ensemble des usagers. Cette situation fait de cette autorité coutumière un atout majeur dans la gouvernance à mettre en place pour faire face aux défis de la croissance démographique et de l'augmentation de la pression sur les ressources.

\section{Une région qui se développe}

Si les idées sur la désertification et la dégradation de l'environnement sont tenaces parmi les gestionnaires du territoire en Afrique, les populations locales saisissent les opportunités de valorisation des ressources dans les régions les plus favorables, notamment dans les zones humides. Au lac Fitri, la superficie des eaux libres et de la zone humide a doublé depuis 1972 et fournit d'autant plus de ressources pastorales et halieutiques pour les populations plus nombreuses

\footnotetext{
* Les références indiquées par* sont rassemblées dans la liste des travaux académiques réalisés dans le cadre du programme Gelt sur le Fitri, située à la fin de la conclusion.
} 

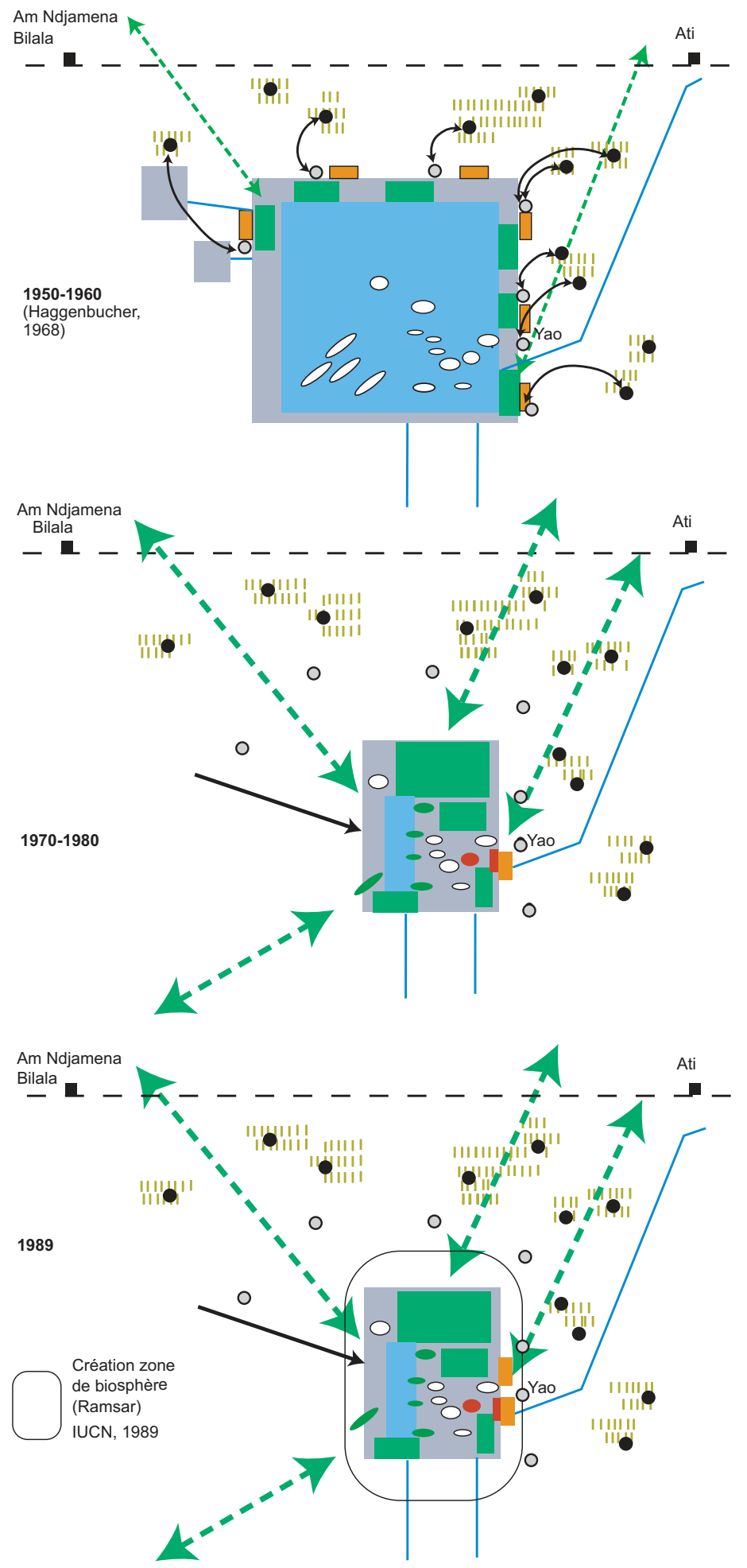

Figure I

Évolution du système lacustre du Fitri entre 1950 et 1990 (chorèmes). 

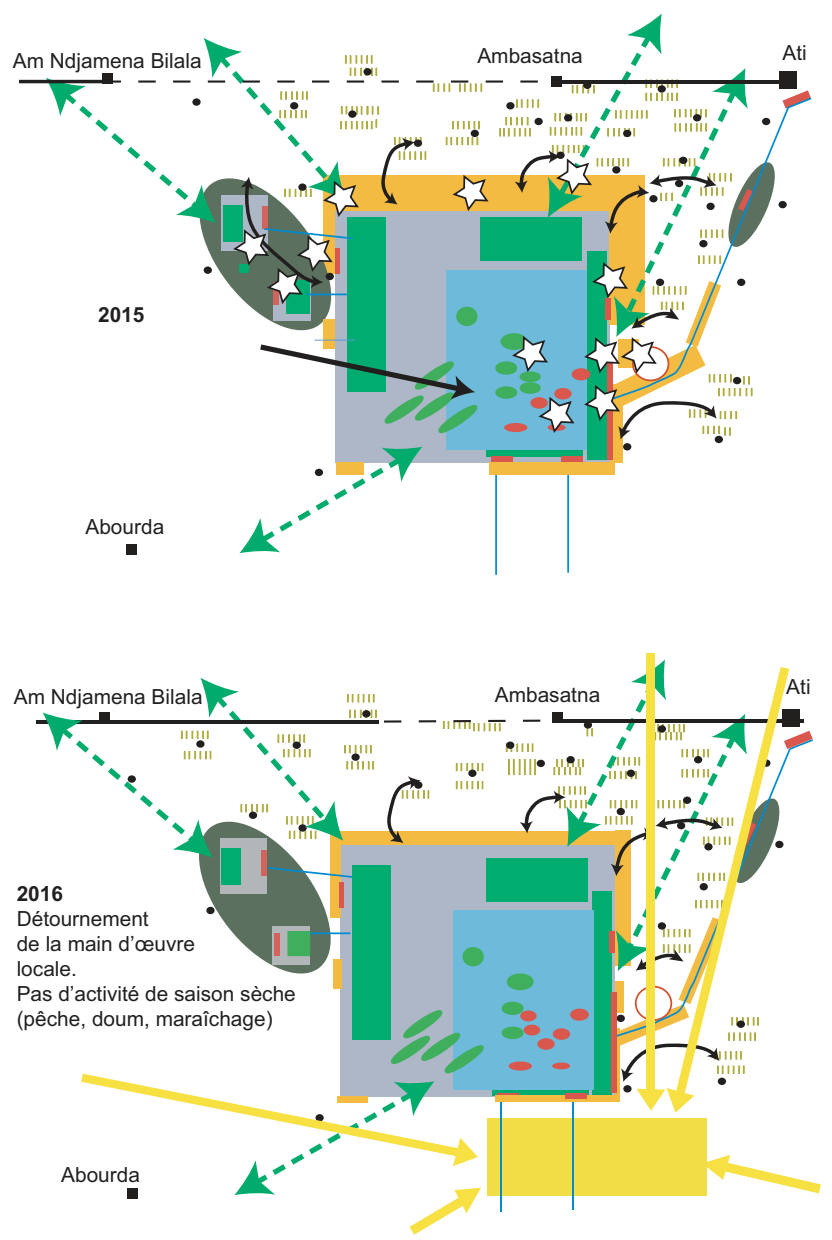

Zone d'orpaillage

Eaux libres

- Cours d'eau

Marécages

Route à praticabilité permanente (bitumée)

Agriculture de décrue (berbéré)

Maraîchage

Agriculture pluviale très aléatoire

- - Route à praticabilité saisonnière

- Ville/bourg rural

- Village mère

- Village satellite

$\int$ Migrations saisonnières

Pâturage de décrue

Doumeraie

Migration de pêcheurs (peu nombreux)

Migration d'orpailleurs (très nombreux)

Conflits sur les ressources

(eau, cultures de décrue, pâturage)

Yao Centre politique du sultanat Bilala

$<\rightarrow$ Transhumances

$\longrightarrow$ (cheptel nombreux/peu nombreux)

Figure 2

Le système lacustre en 2015 et 2016 (chorèmes). 
qui s'y installent (YALIKUN et al., ce volume). Ce lac n'est pas encore devenu une grande région densément peuplée et exportatrice comme le lac Tchad, mais pourrait le devenir. Son insertion dans l'économie nationale est croissante et s'accélère avec l'intervention de plusieurs projets de développement et la construction, depuis 2011, de la route bitumée au nord du lac.

Le Secadév (Secours catholique et développement, ONG caritative), qui est intervenu entre 1984 et 1996, a proposé plusieurs activités dans le cadre du développement rural. Les variétés « améliorées » de sorgho repiqué berbéré (en arabe tchadien), peu adaptées et moins productives que les variétés locales, sont encore présentes dans la région mais n'ont pas changé les rendements en céréales. En revanche, le maraîchage a été adopté par les agriculteurs (DJEKORNOM, 2016*). Déjà habitués à cultiver des légumes dans les jardins, l'accès à du matériel et des semences a permis aux Bilala de produire des aliments de complément, ou de soudure (la patate douce notamment) lors des années de mauvaise pluviométrie (TogasRa, 2016*). Un marché local s'est développé, centré sur les villes de Yao et Abourda, et contribue à la sécurité alimentaire locale.

Deux autres innovations majeures datant de cette période renforcent la mobilité et intensifient le système. La charrette, couramment vulgarisée dans les années 1990 avec très peu de succès dans les autres zones rurales africaines, a connu dans la région du Fitri un engouement d'autant plus rapide que les Bilala possédaient déjà les chevaux pour les tracter. Elle répondait à un besoin spécifique dans cette région où tout est mobile : les ressources, les activités et les personnes qui se déplacent saisonnièrement de villages temporaires en campements agricoles et de pêche. La charrette permet d'intensifier les déplacements, d'allonger les distances parcourues et d'augmenter les charges transportées, notamment entre les lieux de production, de consommation et de vente. Avec le téléphone portable et l'installation des antennes à Am Djamena Bilala, Ambassatna, Yao et Abourda, les informations circulent plus vite et facilitent les interactions entre différents types d'acteurs.

Deux filières commerciales émergent de manière endogène : l'exportation de poissons frais vers les marchés urbains (SAUNIER, 2015*) et celle de la noix de doum comme combustible à N'Djamena (GuEDON, 2016*). Le marché local s'est aussi développé avec la création de nombreux marchés hebdomadaires commercialisant les produits locaux, notamment les céréales en période de récolte (BÉMADJI et MBAYE, ce volume). Lors des très bonnes années (e.g. 20142015), le lac Fitri est exportateur en berbéré, mais les années très déficitaires imposent une aide alimentaire (e.g. 2009, 2016 ; Minguedjim, 2016* ; KeMSOL NAGORNGAR et al.*, ce volume).

Jusqu'en 2018, les projets de développement visant une filière particulière n'ont pas fonctionné. Les trois périmètres rizicoles du Programme national de sécurité alimentaire aménagés en 2012 sont un échec car, mal conçus et inadaptés, ils ne rentraient pas dans les objectifs de production des agriculteurs. Les aménagements ont imposé un remembrement de parcelles appropriées de longue date (MBagogo KoumbraïT, ce volume), qui ont été redistribuées et dont le 
fonctionnement hydrique a été fortement perturbé. La production de riz s'est révélée impossible, le sorgho repiqué y pousse à nouveau avec un rendement plus faible qu'avant. Les aménagements du projet Prodepêche, lancé en 2007, ne sont pas non plus utilisés car ils sont localisés sur une presqu'île inaccessible aux véhicules pendant une grande partie de la saison de pêche. Cela n'empêche toutefois pas l'essor de cette filière, qui s'appuie sur un réseau de commerçants bilala bien connectés à la ville. Aujourd'hui, ce système basé sur la multiactivité et la multifonctionnalité a un potentiel productif très élevé, tout en se basant sur le fonctionnement écologique lacustre et en maintenant une forte biodiversité, sur un modèle similaire à celui décrit pour le lac Tchad (RANGÉ et CocHET, 2018).

\section{Un espace en crise?}

La dernière évaluation de la réserve de biosphère en 2016 est alarmante vis-àvis de la pression anthropique observée dans la région du lac Fitri (BEGC, 2016 ; BÉCHIR et al., ce volume). La croissance démographique touche non seulement les populations sédentaires, mais aussi les éleveurs transhumants qui fréquentent la région plus nombreux, pour des temps de séjour plus longs (MARTY et al., 2012). La population a doublé entre les recensements de 1989 et 2009, même si la mobilité et la répartition de la population sur le territoire, résidant dans des campements temporaires pendant une grande partie de l'année, questionne la fiabilité de ces recensements ainsi que les aménagements à prévoir pour accompagner le développement de cette région (BAIKOUMA*, en cours). Avec la concentration des hommes et des troupeaux autour du lac, les conflits se multiplient (fig. 2) entre agriculteurs (MBAGOGO KOUMBRAïT*, ce volume), entre agriculteurs et éleveurs (OULONA*, 2016), entre éleveurs (MAMADI*, en cours), entre éleveurs et pêcheurs, et entre pêcheurs (SAUNIER* 2016).

Les recherches mettent en évidence les facteurs qui remettent en cause la résilience du système :

- la pression anthropique croissante sur le milieu et la dégradation des ressources naturelles, malgré un retour à des conditions hydrologiques et climatiques plus favorables ;

- l'extension des superficies de sorgho repiqué en auréole autour du lac qui entrave la mobilité des troupeaux et leur accès aux pâturages aquatiques pendant la saison sèche ;

- la diffusion très rapide des cultures maraîchères sur les pourtours des îles, en réponse à une demande locale croissante, condamne ces îles aux éleveurs qui ne peuvent plus y accéder ; 
- l'accroissement des cheptels et le changement du calendrier de transhumance qui augmentent la durée annuelle de fréquentation des troupeaux au lac Fitri ;

- la réduction des complémentarités entre sédentaires et transhumants (paiement des résidus post-récolte, disparition du transport de récolte à dos de dromadaire en raison de l'utilisation de la charrette ; garde réciproque d'animaux et de matériel de campement) ;

- la sédentarisation des éleveurs en bordure du lac accroît la pression pastorale par des troupeaux qui restent sur place presque toute l'année ;

- l'arrivée de nouveaux pêcheurs professionnels attise les stratégies d'exclusion et de construction territoriale à l'intérieur des eaux du lac, en contradiction avec le pouvoir du sultanat (SAUNIER et al., ce volume) ;

- l'arrivée massive d'orpailleurs s'inscrit dans une activité extractive minière et ne dépend d'aucun des quatre principes qui font la résilience du socioécosystème lacustre du Fitri, tout en augmentant considérablement les besoins en nourriture et en ressources naturelles locaux (eau, bois de construction et énergie) ;

- l'affaiblissement du pouvoir du sultanat bilala et la remise en cause des anciennes règles d'accès aux ressources naturelles par les nouveaux acteurs du territoire ;

- l'émergence de nouveaux pouvoirs, services déconcentrés de l'État et ONG, qui reconfigurent les rapports de pouvoir à l'échelle locale.

Les anciens systèmes de régulation fonctionnent de plus en plus mal, et les liens entre les hommes et la nature se distendent (KARDIGUÉ, 2017* ; TOTOUDJIMBAYE, $\left.2017^{*}\right)$. Les alliances séculaires entre agriculteurs et éleveurs sont remises en causes par de nouvelles pratiques (ZAKINET, 2015). Le système foncier, qui reposait sur la répartition de deux parcelles plus ou moins inondées par agriculteur, complété par un système de redistribution foncière en cas de crue très faible, est bloqué par la mise en valeur de la quasi-totalité de l'espace cultivable en décrue et la disparition de la souplesse qu'il imposait (MBAGOGO KOUMBRAÏT, ce volume ; YOMSINGAR*, en cours).

La multiplication des conflits met en évidence la défaillance des pouvoirs coutumiers, qui ne sont plus capables de concilier les intérêts divergents des usagers et souffrent d'une crise de légitimité (Mugelé, ce volume). Celle-ci ne date pas seulement de la dernière réforme administrative : la suppression de la taxe sur le pâturage ( hag al gesh en arabe tchadien) par l'administration coloniale en $1947^{1}$ marque le premier recul du pouvoir sultan sur la gestion du territoire et sur sa légitimité à réguler la transhumance.

\footnotetext{
I. Cette mesure a été prise pour l'ensemble du Tchad en raison de pratiques abusives de la part de goumiers au service des chefs traditionnels surtaxant les éleveurs en transhumance. Elle s'inscrit dans une réflexion plus large des administrateurs coloniaux face à la transhumance, qui pose encore question au début du $\times \mathrm{XI}^{\mathrm{e}}$ siècle.
} 


\section{Conclusion}

Comme dans de nombreuses zones humides africaines, la pression anthropique croît dans le lac Fitri et remet en cause les équilibres anciens du socioécosystème. Nous montrons que la région s'est développée grâce à une reprise, depuis les sécheresses des années 1970-1980, de la pluviométrie dans l'ensemble du bassin versant et en puisant dans les innovations proposées par les projets de développement, tout en conservant les principes qui fondent la résilience $\mathrm{du}$ système lacustre. C'est en grande partie une crise institutionnelle qui remet en cause la régulation coutumière et entraîne une réduction des complémentarités entre les activités, par une spécialisation des espaces et une exclusion de catégories d'acteurs de certaines parties du lac et de sa périphérie. Le principe d'équité entre les acteurs n'est plus respecté et remet en cause la capacité du sultanat à réguler les activités et résoudre les conflits. Ainsi, l'exemple du lac Fitri est emblématique des nouvelles modalités d'une gouvernance hybride à inventer, impliquant acteurs publics, privés et communautés (LEYRONAS et BAMBRIDGE, 2018). Il est aussi représentatif des enjeux liés aux eaux continentales vulnérables dépendant directement du changement climatique (CREED et al., 2017) et des systèmes complexes qui régissent les liens imbriqués entre nature et société dans ces milieux très variables (MORITZ et al., 2016), dans un contexte sahélien marqué par la montée des insécurités (MAGRIN et PÉROUSE DE MontClos, 2018 ; CHAUvin et al., à paraître).

Les chapitres de cet ouvrage mettent en évidence les particularités de ces zones humides sahéliennes où la variabilité environnementale est un fondement structurel du système, avec des interactions constantes entre flux sociaux et flux aquatiques qu'il faut considérer conjointement. La taille modeste du lac Fitri facilite l'appréhension de ces interactions entre la climatologie, l'hydrologie, l'évolution des ressources naturelles et des activités, ainsi que celle des impacts des innovations et changements sur l'ensemble du socioécosystème. C'est une sorte de modèle « de poche » qui constitue un indicateur fiable des évolutions en cours et des effets des politiques publiques. Un suivi de ses caractéristiques physiques (climat, hydrologie, hydrogéologie) fournirait des informations précieuses sur le climat de l'ensemble du Sahel oriental en contexte de changement climatique, bien moins connu qu'en Afrique de l'Ouest. Une meilleure compréhension de son histoire, lors de périodes plus humides notamment, ainsi qu'un suivi régulier permettrait de mieux envisager les trajectoires de développement de ce lac et son accompagnement par les politiques publiques. Un tel dispositif ne peut que s'appuyer sur une alliance de disciplines, en sciences de la vie et de la terre et sciences humaines et sociales, permettant d'appréhender leurs points de vue, priorités et enjeux différents. Mais c'est en intégrant aussi les sociétés lacustres et les gestionnaires des territoires que pourront se construire l'hybridation des savoirs et l'articulation des temporalités des crises et des changements, indispensables à la définition d'un modèle d'avenir pour ces zones humides au défi du changement global. 


\section{Bibliographie}

\section{Béchir A. B., Mian-Oudanang K.,} Mahamat M. A., Bourdjolbo T. « La végétation pastorale du Lac Fitri : état des lieux et dynamique ». Ce volume.

BEGC, 2016

Étude diagnostique de la diversité biologique de la réserve de biosphère du lac Fitri.

République du Tchad, ministère

de l'Environnement et des Pêches, FSE, 81 p.

Bémadji B., Mbaye N. G.

«Économie des échanges au lac Fitri : un déficit récurrent en produits alimentaires ». Ce volume.

\section{BRUNET R., 1986}

La carte modèle et les chorèmes. Mappemonde, $\mathrm{n}^{\circ} 4$, p. 2-6.

\section{Chauvin E., Langlois O., Seignobos C.,} BAROIN C., à paraître

Les insécurités dans le bassin du lac Tchad. $\mathrm{XVII}^{\mathrm{e}}$ colloque Méga-Tchad, Nice,

14-16 juin 2017.

Courel M.-F., Morin F., Raimond C., 1997 « Intégration modèle ou modèle d'intégration. La gestion de l'environnement au lac Fitri (Tchad) ». In : Singaravelou P., éd. : Gestion de l'environnement dans les pays tropicaux, Bordeaux, Dymset-Cret, p. 311-326.

I. F. Creed, C. R. Lane, J. N. Serran, L C. Alexander, N. B. Basu,

A. J. K. Calhoun, J. R. Christensen, M. J. Cohen, C. Craft, E. D'Amico, E. DeKeyser, L. Fowler, H. E. Golden, J. W. JaWitz, P. Kalla, L. K. KiRKMAN, M. Lang, S. G. Leibowitz, D. B. LeWIS, J. Marton, D. L. McLaughlin,

H. RaAnan-Kiperwas, M. C. Rains, K. C. Rains \& L. SMith, 2017

Enhancing protection for vulnerable waters. Nature geoscience, vol. 10 : 809-815. DOI: 10.1038/NGEO3041

Kemsol Nagorngar A., Raimond C., Madjigoto R., Jofack S. V., DJimassal D., Libar J., Kouame K. F.

«Fluctuation des récoltes de sorgho repiqué et potentialités de culture. Une analyse par télédétection dans la région du lac Fitri ». Ce volume.
Leyronas S., Bambridge T., 2018

Communs et développement : une approche renouvelée face aux défis mondiaux. Revue internationale des études du développement. Vol. 233 (1) : 11-29.

\section{Magrin G., Pérouse de Montclos M.-A., dir., 2018 \\ Crise et développement. La région du lac Tchad à l'épreuve de Boko Haram. Paris, AFD, 291 p.}

\section{Marty A., Zakinet D., Khamis D. D., BERNARD C., 2012 \\ Almy al Afia 2. Analyse de l'évolution des ressources dans le département du Fitri. Document principal. République du Tchad, programme d'hydraulique pastorale au Tchad central, Phase II. Antea-Iram, 128 p.}

\section{Mbagogo Koumbraït A.}

« Pratiques et enjeux de la sécurisation foncière autour du lac Fitri ». Ce volume.

Moritz M., Laborde S., Ahmadou M., Durand M., Fernandez A., Hamilton I. M., Kari S., Scholte P., Xiao N., Ziebe R., 2016 Studying the Logone Floodplain, Cameroon, as a coupled human and natural System. African Journal of Aquatic Science, 41-1 : 99-108.

\section{Mugelé R.}

« Le lac Fitri, un modèle de gestion en crise ?». Ce volume.

\section{Raimond C., Mbagogo A ., Madjigoto R ., ZAKINET D ., (à paraître)}

Repenser la gouvernance d'une zone humide autour d'un pouvoir local fort : l'exemple du lac Fitri (Tchad) face à la montée des insécurités. Colloque Méga-Tchad Les insécurités dans le bassin du lac Tchad, Nice, XVII ${ }^{\mathrm{e}}$ colloque Méga-Tchad, 14-16 juin 2017.

\section{Raimond C., Rangé C., Guérin H., 2014}

La multiactivité et la multifonctionnalité, principes d'un fonctionnement durable pour le lac ? In : Lemoalle J., Magrin G., dir. : Le développement du lac Tchad : situation actuelle et futurs possibles. CBLT, N'Djamena, Marseille, Expertise collégiale IRD, AFD-FFEM, p. 423-474 (clé USB). 
Rangé C., Cochet H., 2018

Multiusage familial et agriculture de firme sur les rives du lac Tchad : une comparaison des performances économiques. Natures Sciences Sociétés, 26 : 33-48.

Saunier M., Raimond C., Abba Ambar B. «Les territoires d'eau ou comment gérer les ressources halieutiques dans les espaces lacustres (lacs Fitri et Iro) ». Ce volume.

\section{Schuster M., Sylvestre F., Raimond C.,} Moussa A., Abakar Y., Yalikun T.

« Changements environnementaux et climatiques à la période humide africaine Holocène dans le bassin du lac Fitri (Tchad) ». Ce volume.

YALIKUN T., RAIMOND C., Kemsol Nagorngar A., Zakinet D., Schuster M., Sylvestre F.

«Variabilité des crues et des paysages du Fitri depuis les grandes sécheresses des années 1970-1980 ». Ce volume.

\section{ZAKINET D., 2015}

Des pasteurs transhumants entre alliances et conflits au Tchad : Les Arabes Salamat Sifera et les Arabes Djaatné au Batha. Afrique contemporaine, 2015/3 ( $\left.{ }^{\circ} 255\right)$ : 127-143. 



\section{Travaux académiques réalisés sur le lac Fitri dans le cadre du programme Gelt}

Abdelnassir K., 2016

Le pluralisme médical et les itinéraires thérapeutiques des populations autour de Yao. Master 1 d'anthropologie, université N'Djamena, rapport de stage.

\section{BaIKoUMA K.}

Population et aménagement du territoire dans le département $d u$ Fitri. Master 2 de géographie, université N'Djamena, en cours.

\section{BÉMADJI B., 2016}

Économie des échanges de produits alimentaires autour du lac Fitri. Master 2 de géographie, université N'Djamena, $168 \mathrm{p}$.

\section{DJEKORNOM O., 2016}

Dynamique des pratiques maraîchères autour du lac Fitri: Modo, Doumo, Mafé, Yao. Master 2 de géographie, université de N'Djamena, $180 \mathrm{p}$.

\section{GUEDON J., 2016}

Origines et évolution de la filière des noix de doum du Fitri : nécessité alimentaire et opportunité énergétique. Master 1 de géographie, université Paris 1 Panthéon-Sorbonne, 152 p.

ILYADE H., 2016

La multifonctionnalité et la pluriactivité dans le terroir de Fourkouma, département du Fitri (région du Batha). Master 2 de géographie, université N'Djamena, $108 \mathrm{p}$.

\section{KARGIGUÉ J., 2017}

L'homme et l'eau autour du lac Fitri. Master 2 d'anthropologie, université de N'Djamena, $121 \mathrm{p}$.

\section{Kemsol Nagorngar A., 2018}

Dynamique spatiale des cultures de décrue dans les zones lacustres soudano-sahéliennes de 1985 à 2015 : le cas des lacs Fitri et Iro au Tchad. Doctorat de géographie, université 
Félix-Houphouët-Boigny, Abdidjan, $178 \mathrm{p}$.

\section{LOUKMaN B.}

Caractérisation chimique des eaux de surface et souterraines : relations lac-aquifères dans le Département $d u$ Fitri. Doctorat en hydrogéologie, université de Ouagadougou, Burkina Faso, en préparation.

\section{Mahamat Al-Hassine A., 2016}

La transhumance dans le Batha Ouest : le cas des arabes Djaatne dans la zone $d u$ Fitri. Master 2 de géographie, université N'Djamena, $83 \mathrm{p}$.

\section{MinguedJIM M., 2016}

La variabilité des crues et la dynamique de la culture du sorgho repiqué dans les plaines d'inondation de la rive nord-est du lac Fitri. Master 2 de géographie, université N'Djamena, $152 \mathrm{p}$.

\section{Ngossassou M.}

Femme et pouvoir dans la société traditionnelle Bilala. Master 2 d'histoire, université N'Djamena, en cours.

\section{Mamadi M.}

Sédentaires Bilala et transhumants Arabes Djaatne, entre accès pacifique et conflictuel aux ressources naturelles: le cas du Khalifa de Am Djamena Bilala. Master 2 d'histoire, université N'Djamena, en cours.

\section{NdJiglembaye A., 2016}

Pouvoir local autour du lac Fitri. Reconstitution anthropologique et historique de leurs fondements et caractéristiques. Master 1 d'anthropologie, université N'Djamena, rapport de stage.

\section{Oulona E., 2016}

Les conflits d'usage des ressources naturelles dans le système lacustre du Fitri : résultante d'une multi-activité et d'une multifonctionnalité de l'espace? Master 2 de géographie, université N'Djamena, $110 \mathrm{p}$.

\section{Poulin C., 2019}

Bilans hydrologiques et étude de la recharge de trois systèmes lac-aquifère du bassin du lac Tchad par une approche géochimique multitraceurs (18O, 2H, 36CI, 14C). Thèse de doctorat, Aix-Marseille Université, $145 \mathrm{p}$.

\section{Bourdjolbe T., 2018}

Diversité floristique et dynamique de la végétation ligneuse dans le lac Fitri. Master 2 de géographie, université de N'Djamena, 79 p.

\section{Togasra E., 2016}

Dynamique alimentaire chez les Boulala du lac Fitri. Master 2 d'anthropologie, université N'Djamena, $101 \mathrm{p}$.

\section{Tondandi A., 2016}

La mobilité en milieu bilala : motivations, trajectoires et réseaux. Master 1 d'anthropologie, université de N'Djamena, rapport de stage.

Totoudjimbaye Betoloum M., 2018 Rapport homme/animal sauvage autour du lac Fitri. Master 2 d'anthropologie, université N'Djamena, 100 p. 


\section{RIRONGARTI R.}

La flore des diatomées des lacs du Tchad: inventaire, biodiversité, et calibration des conditions physicochimiques des milieux aquatiques. Thèse de doctorat, Aix-Marseille Université, en préparation.

\section{SAUNIER M., 2015}

L'accès aux ressources halieutiques en lien avec l'évolution des techniques de pêche au lac Fitri, Tchad. Master 1 de géographie, université Paris 1 Panthéon-Sorbonne, 146 p.

\section{SAUNIER M., 2016}

Les territoires d'eau : enjeux et jeux de pouvoir pour la gestion des ressources halieutiques

dans les lacs Fitri et Iro au Tchad.

Master 2 de géographie, université

Paris 1 Panthéon-Sorbonne, 128 p.

YASSINe A. O., 2017

Etude sédimentologique et géomorphologique du bassin du lac Fitri (Tchad). Master 2 Sciences de la Terre, université de Strasbourg.

\section{YOMSINGAR K.}

Le foncier à berbéré dans le département du Fitri. Master 2 de géographie, université N'Djamena, en cours. 



\section{Autres travaux académiques réalisés dans le cadre du programme GELT}

Amngar G., 2017

Caractérisation physico-chimique des eaux souterraines. Implications pour l'évaluation des potentialités des ressources en eau du bassin hydrogéologique de la région du Lac Iro. Master 2 HydroSIG, université de N'Djamena.

\section{BAOUYÉ K.}

Peuplement des macroinvertébrés aquatiques des lacs du Tchad. Thèse de doctorat, université de Sarh, en préparation

\section{BianPambe D., 2017}

Caractérisation physico-chimique des eaux d'Ounianga. Master 2 HydroSIG, université de N'Djamena.

Klatolna C. R., 2017

Caractérisation physico-chimique des aquifères et systèmes lacustres d'Ounianga, nord-est du Tchad. Master 2 HydroSIG, université de N'Djamena.

\section{Mahamat S., 2017}

Étude piézométrique de la nappe des grès de Nubie dans la zone
d'Ounianga. Master 2 HydroSIG, université de N'Djamena.

Mahamat A. M., 2017

Étude bathymétrique des lacs d'Ounianga. Cas des lacs Yoan et Téli. Master 2 HydroSIG, université de N'Djamena.

Mahamat Nour A., 2019

Fonctionnement hydrologique, chimique et isotopique du principal affluent du lac Tchad : le système Chari-Logone. Thèse de doctorat, Aix-Marseille Université, 176 p.

NARMADJI L., 2017

Géomorphologie des bassins lacustres d'Ounianga. Master 2 Sciences de l'Environnement Terrestre, Aix-Marseille Université.

Yacoub A. N., 2018

Reconstitution des paléoenvironnements à l'Holocène récent à partir des archives sédimentaires des lacs d'Ounianga (Tchad). Master 2 Sciences de l'Environnement Terrestre, Aix-Marseille Université. 



\section{Liste des auteurs}

Yassine Abakar, sédimentologue, étudiant, université de Strasbourg, UMR 7516, Strasbourg, France.

Brahim Abba Ambar, hydrobiologiste, enseignant-chercheur, université de Sarh, département de biologie, Sarh, Tchad.

\section{Mahamadou Abdourahamadi,} géographe, doctorant, université Abdou-Moumouni, département de géographie, Niamey, Niger, UMR Prodig, Paris, France.

Guinbé Amngar, hydrologue, étudiant, université de N'Djamena, département de géologie, N'Djamena, Tchad.

Ali Brahim Béchir, pastoraliste, enseignant-chercheur, institut national d'élevage de Moussoro (Insem), Abéché, Tchad.

Blaise Bémadji, géographe, étudiant, université de N'Djamena, département de géographie, N'Djamena, Tchad.

Camille Bouchez, hydrogéochimiste, post-doctorante, Aix-Marseille université, Cerege, Aix-en-Provence, France.

Tchago Bouimon, archéologue, enseignant-chercheur, université de N'Djamena, N'Djamena, Tchad.
Tchoudiba Bourdjolbo, géographe, étudiant, université de N'Djamena, département de géographie, N'Djamena, Tchad.

Marie-Pierre Coustures, archéologue, enseignante-chercheure, université Jean-Jaurès, UMR Traces, Toulouse, France.

Jean-François Crétaux, hydrologue, chercheur, Cnes-Legos, Toulouse, France.

Pierre Deschamps, géochimiste, chercheur IRD, Cerege, Aix-en-Provence, France.

Datoloum Djimassal, géographe, chercheur, CNRD, N'Djamena, Tchad.

Guemona Djimet, archéologue, doctorant, université Jean-Jaurès, UMR Traces, Toulouse, France.

Paula do Amaral, palynologue, post-doctorante IRD, Cerege, Aix-en-Provence, France.

Jean-Claude Doumnang, géologue, professeur, université de N'Djamena, département de géologie, N'Djamena, Tchad.

Barbara Eichhorn, anthracologue, chercheure, Goethe Universität Frankfurt, Institut für Archäologische Wissenschaften, Abteilung Vor-und Frühgeschichte, Archäologie und 
Archäobotanik Afrikas, Francfort, Allemagne.

Christine Gauthier, archéologue, retraitée.

Yves Gauthier, archéologue, retraité.

Bruno Hamelin, géochimiste, professeur, Aix-Marseille université, Cerege, Aix-en-Provence, France.

Abderamane Hamit, hydrogéochimiste, enseignant-chercheur, université de N'Djamena, Insem, N'Djamena, Tchad.

Valère Jofack Sokeng, hydrogéologue, enseignant-chercheur, Curat, université Félix-Houphouët-Boigny d'AbidjanCocody, Abidjan, Côte d'Ivoire.

Baouyé Kemkong, biologiste, enseignant-chercheur, université de Sahr, département de biologie, Sahr, Tchad.

Angeline Kemsol Nagorngar, géographe, chercheure, CNRD, N'Djamena, Tchad.

Hadiza Kiari Fougou, géographe, enseignante-chercheure, université de Diffa, Diffa, Niger.

Fernand Kouamé Koffi, géographe, professeur, Curat, université FélixHouphouët-Boigny d'Abidjan-Cocody, Abidjan, Côte d'Ivoire.

Sarah Laborde, anthropologue, postdoctorante, institut australien des Rivières, université de Griffith, Nathan, Australie.

Jacques Lemoalle, hydroécologue, directeur de recherche émérite, IRD, UMR G-EAU, Montpellier, France.

Joseph Libar, géographe, ingénieur, CNRD, N'Djamena, Tchad.

Bichara Loukman, hydrologue, enseignant-chercheur, université de N'Djamena, département de géologie, N'Djamena, Tchad.

Robert Madjigoto, géographe, enseignant-chercheur, université de N'Djamena, département de géographie, N'Djamena, Tchad.

Géraud Magrin, géographe, professeur, université Paris 1 Panthéon-Sorbonne, UMR Prodig, Paris, France.

Aboukar Mahamat, association camerounaise pour l'éducation environnementale, Maroua, Cameroun.

Alhassine Mahamat, géographe, étudiant, université de N'Djamena, département de géographie, N'Djamena, Tchad.

\section{Abdallah Mahamat Nour,} hydrogéochimiste, doctorant, AixMarseille université, Aix-en-Provence, France ; université de N'Djamena, département de géologie, N'Djamena, Tchad.

Jean-Charles Mazur, biologiste, technicien CNRS, Cerege, Aix-en-Provence, France.

\section{Audrey Mbagogo Koumbraït,} géographe, enseignante-chercheure, université d'Ati, Ati, Tchad.

Jean Mbairo, archéologue, enseignantchercheur, université de N'Djamena, N'Djamena, Tchad.

Ngaressem Goltob Mbaye, géographe, enseignant-chercheur, université de N'Djamena, département de géographie, N'Djamena, Tchad.

Guillemette Menot, géochimiste, professeure, université Lyon 1, ENS de Lyon, LGL-TPE, Lyon, France.

\section{Koussou Mian-Oudanang,}

zootechnicien, enseignant-chercheur, institut de recherche en élevage 
pour le développement, N'Djamena, Tchad.

Mark Moritz, anthropologue, professeur, département d'anthropologie, université d'État de l'Ohio, Columbus, États-Unis d'Amérique.

Vincent Mourre, archéologue, chercheur Inrap, UMR Traces, Inrap Méditerranée, Nîmes, France.

Abderamane Moussa, géologue, enseignant-chercheur, université de N'Djamena, département de géologie, N’Djamena, Tchad.

Ronan Mugelé, géographe, docteur, université Paris 1 Panthéon-Sorbonne, UMR Prodig, Paris, France.

Chloé Poulin, géochimiste, docteure, Aix-Marseille université, Cerege, Aix-en-Provence, France.

Christine Raimond, géographe, directrice de recherche, CNRS, UMR Prodig, Paris, France.

Charline Rangé, géographe, docteure, AgroParisTech, UMR Prodig, Paris, France.

Remadji Rirongarti, biologistediatomiste, doctorante, Aix-Marseille université, Cerege, Aix-en-Provence, France ; université de N'Djamena, département de biologie, N'Djamena, Tchad.

Caroline Robion-Brunner, archéologue, chercheure, CNRS, UMR Traces, Toulouse, France.

Marguerite Saunier, géographe, étudiante, université Paris 1 PanthéonSorbonne, Paris, France.

Mathieu Schuster, géologue, chercheur, CNRS, UMR 7516, Strasbourg, France.
Adoum Bari Sinine, hydrologue, étudiant, Aix-Marseille université, Cerege, Aix-en-Provence, France.

Florence Sylvestre, paléoclimatologue, directrice de recherche IRD, Cerege, Aix-en-Provence, France.

Margareta Tengberg, carpologue, chercheure, MNHN, UMR 7209, Paris, France.

\section{Christine Vallet-Coulomb,} hydrogéochimiste, enseignantechercheure, Aix-Marseille université, Cerege, Aix-en-Provence, France.

Thijs Van der Meeren, paléolimnologue, post-doctorant, université de Gand, unité de limnologie, département de biologie, Gand, Belgique.

Dirk Verschuren, paléolimnologue, professeur, université de Gand, unité de limnologie, département de biologie, Gand, Belgique.

Nicolas Waldmann, paléoclimatologue, professeur, université de Haifa, département de géosciences marines, Haifa, Israel.

Maman Waziri Mato, géographe, enseignant-chercheur, université Abdou-Moumouni, département de géographie, Niamey, Niger.

Tashi Yalikun, géographe, ingénieur de recherche, CNRS, UMR Prodig, Paris, France.

Dangbet Zakinet, historien, enseignant-chercheur, directeur de la recherche et de l'innovation (Mesri), université de N'Djamena, département d'histoire, N'Djamena, Tchad. 


Imprimé en France sur les presses d'Estimprim - 25I 10 Autechaux

Dépôt légal : juin 2019 